






\title{
LIVING ANATOMY AND PATHOLOGY
}

THE DIAGNOSIS OF DISEASES

IN

EARLY LIFE

BY THE

ROENTGEN METHOD

\author{
BY \\ THOMAS MORGAN ROTCH, M.D. \\ PROFESSOR OF PEDIATRICS, HARVARD UNIVERSITY
}

THREE HUNDRED AND THREE ILLUSTRATIONS

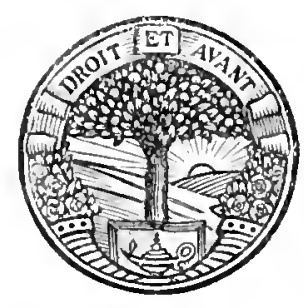

PHILADELPHIA E LONDON

J. B. LIPPINCOTT COMPANY 


\section{Coprkinit, 1910}

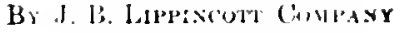

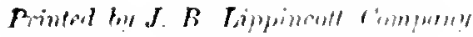

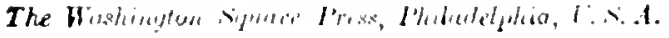


TO

WILLIAM OSLER, M.D., F.R.S.

REGIUS PROFESSOR OF MEDICINE, OXFORD UNIVERSITY

IN RECOGNITION OF THE AID WHICH

HE HAS GIVEN TO THE DEVELOPMENT

OF THE SUBJECT OF PEDIATRICS 



\section{PREFACE}

THE Roentgen ray has been largely used as an aid to diagnosis in both early and later life. Its mechanism has been fully explained in various books, but a systematic exposition of the practical results of the Roentgen method of examination has not yet appeared.

The purpose of this book is to deal as little as possible with the questions of apparatus and technic and to devote the entire space to the actual clinical teaching of the subject.

This teaching is accomplished by means of illustrative plates, by legends corresponding to them, and by a text explanatory of what can really be seen in health and in disease in early life.

The book is devoted to the diagnosis of disease and does not deal to any extent with treatment. It is intended to provide a means by which a fair knowledge of the Roentgen method can be acquired by the student when the personal instruction of a skilled Roentgenologist is not available.

I believe that in teaching Roentgenology it is of the utmost importance to present illustrations of evident conditions and not to mislead the student with vague descriptions. In the plates I have been careful not to allow any retouching whatever, and I have discarded those which would have to be altered in order to show what would be described in the legends. Unless this is done the plates are reduced to diagrams and lose their value for accuracy and for teaching the student to interpret them independently.

The plates are placed opposite to the legends, and the illustrations and details are given to the student as would be done if an 
expert Roentgenologist were standing beside him describing the plate by means of an illuminator. The different parts of the plate, whether normal or abnormal, are designated by leaders and by letters just as the Roentgenologist would designate them with his pointer.

The two methods are in this way identical, excepting that the questions which the student may ask have to be anticipated in the legends. It is, therefore, important to make the legends cover what a fairly intelligent student can reasonably be expected to ask. It is important to explain in the text whether the different parts of the illustrations are normal or abnormal. This is not only to anticipate possible questions, but in order that the student may learn to distinguish normal conditions, and by studying the reproductions methodically, recognize the normal in his search for the abnormal.

In this sense the recognition of the normal becomes as important as does that of the abnormal, and the student is taught not to overlook anything - not even an artifact.

By this method, if reference is made to previous normal illustrations, the abnormal can readily be compared with the normal conditions of the same part. The knowledge acquired from this book will thus approach very closely to that obtained from the living instructor, and the student's power of original observation will be strengthened and improved.

Believing, as I do, that in the study of diseases a knowledge of normal conditions should first be mastered, I have begun my illustrations by showing a set of normal Roentgenographs covering the different stages of development from a late period of intra-uterine life through childhood.

I have then given the results of my study of the bones of the wrist, so as to exemplify what especial practical use ean be made from an exact knowledge of the living anatomy of an especial part. 
I have next given examples of what can be seen in diseases of the new-born, such as the various congenital malformations and such abnormal conditions as are present at birth.

By this portrayal of normal living anatomy and the grosser forms of the abnormal eonditions the student is prepared to understand the finer pathologic lesions in the living subject.

I have next shown the characteristic living lesions of the diseases of nutrition. I have then divided the book into certain groups which represent living pathologie conditions. Thus, in one division I have described the head and spine. In the next division I have given in detail what can be seen in Roentgenographs of the thorax, describing in succession abnormal conditicns of the bronchial nodes, the bronchi, the lungs, the pleura, the pericardium, the heart, and aneurism.

In the next division, the abdomen, I have been able to illustrate the various conditions to only a limited degree, as this part of the infant and child presents great difficulties for the technic of the Roentgen method.

Next I have shown how foreign bodies can be detected in different parts of the trunk and extremities. Finally I have grouped under diseases of the extremities, for obvious tcchnical reasons, a number of conditions of varying etiology.

The material which illustrates the book has in most cases been taken from the Roentgen records of the Children's Hospital, and I wish to express my appreciation of the eourtesy of the Managers, who have permitted me to use what has amounted in the past ycar to over two thousand and three hundred cases.

The plates of the Roentgen illustrations were taken by Dr. Arial W. George, and in their unusual excellence speak for themselves. To Dr. George I can only express my appreciation of his skill and the ever new light which he has thrown upon a somewhat difficult subject by his clear and original interpretation. 
To Dr. Perey Brown I owe my thanks for much expert advice on stuljects eonneeted with the writing of the book and for the plates illustrating long and short exposures.

To Dr. Walter Curtis Miner I wish to express my appreciation of his work in connection with those illustrations which represent the development of the teeth and their anomalies. What is shown in these dental Roentgenographs will be of great service not only to the oral surgeon but to the orthodontist and to the general practitioner.

Dr. Willian Palmer Lucas has given much practical aid in many ways connected with the book, and to him are due my thanks.

I offer this work to the Medieal Profession in the hope that it may be of assistance in solving many of the more diffieult problems which arise in the diagnosis of disease in early life.

THOMAS MOMRAN ROTCH.

197 Commonfealth Avenue, Boston, Massachuskitis,

January, 1910. 


\section{TABLE OF CONTENTS}

\section{DIVISION I.}

LIVING NORMAL ANATOMY.

Bone; Periosteum; The Constituents of Bone; Diaphyses and Epiphyses; Time of the Appearance of the Epiphyses; Development of Bone; Humerus; Radius; UIna; Carpus; Metacarpus; Phalanges; Femur; Patella; Tibia; Fibula; Tarsus; NLetatarsus; Phalanges; Os Innominatum; Teeth; Temporary Teeth; Permanent Teeth; Chronologic Examples of Normal Living Anatomy.

\section{DIVISION II.}

ILLUSTRATIVE USE OF LIVING NORMAL ANATOMY

Key to Index Development; State Laws regarding Child Labor.

\section{DIVISION III.}

DISEASES OF THE NEW-BORN

Anomalies of the Head, Spine, and Ribs; Spina Bifida; Intra-thoracic and Intraabdominal Anomalies; Anomalies of the Extremities and Pelvis; Backward Mental Development; Iyxœdema; Cretinism; Chondrodystrophia Fætalis; Achondrophasia; Osteogenesis Imperfecta; Fetal Rhachitis; Obstetrical Paralysis.

\section{DIVISION IV.}

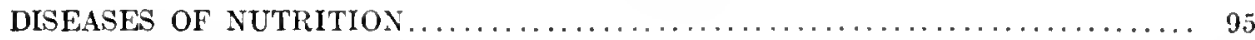

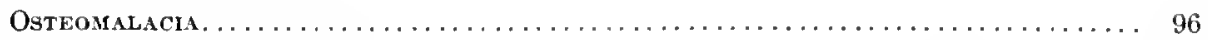

INFANTILE ATROPHY . . . . . . . . . .

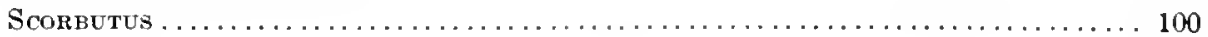

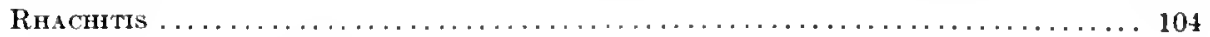

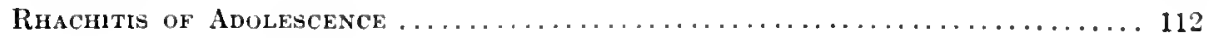

\section{DIVISION Y.}

DISEASES OF THE HEAD AND SPINE.

Supernumerary Teeth; Non-tubercular Infections of Spine; Osteomyelitis of Spine; Tuberculosis of spine. 
DIVISION VI.

THE BIONCHIA NODES - BRONCIII - LL NGS - PLEURA - HEART - PERI-

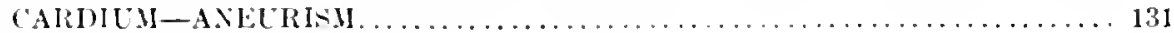

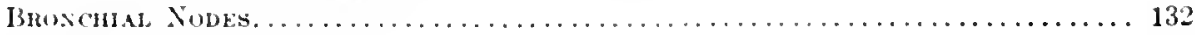

Tuberculosis.

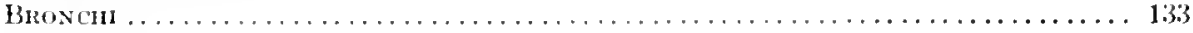

Lexes . . . . . . . . . . . . . . . . . . . . . . . . . . . . . . . . . 133

Atelectasis; Implyysema: (iangrene; 'Tuherculosis; Hydropneumothorax; Pneumothorax; Bronchopneumonia; Pneumonia.

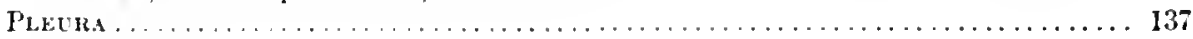

Empyema.

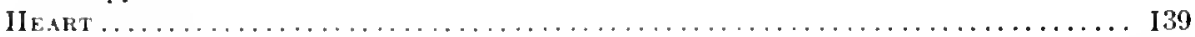

Perreardita. . . . . . . . . . . . . . . . . . . . . . . . . . . . . . . 139

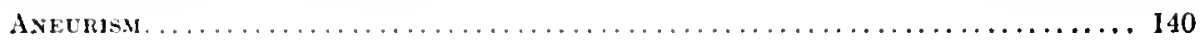

DIVISION TII.

THE ARDOMEN . . . . . . . . . . . . . . . . . . . . . . . . . . . . . 141

Ilour-glass ('ontraetion of Stomaeh; Enlarged Mesenteric Nodes.

DIVISION VIII.

FOREIGN BODIES. ..................................... 143

Urethra; Csophagus; Iaryux; Lung; Intestine; Hip; Knee-joint; Foot; Toe.

\section{DIVISION IX.}

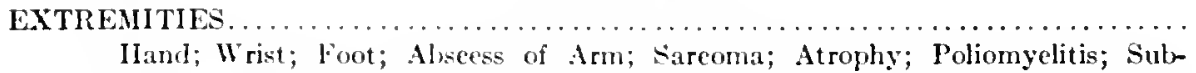

periosteal Hemorrhage; Exostoses; Callus; Flat-foot; Traumatism; Fractures; Dislocations.

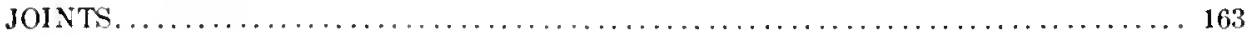

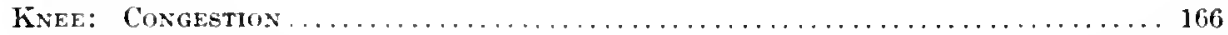

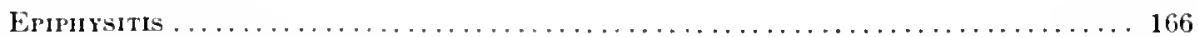

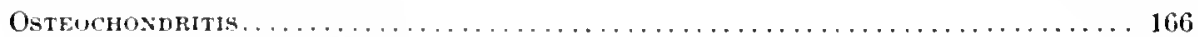

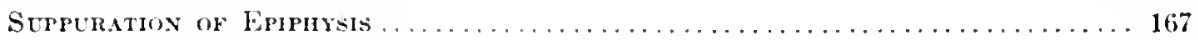

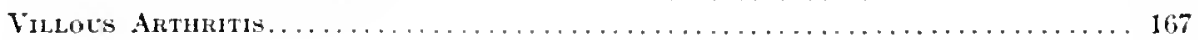

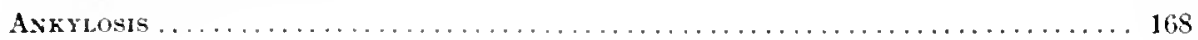

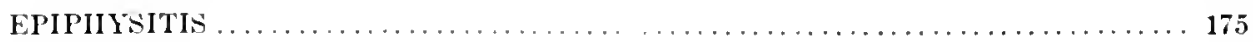

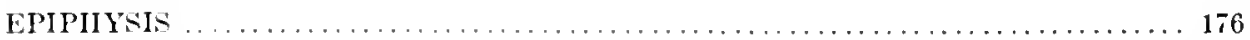

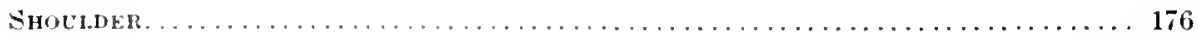

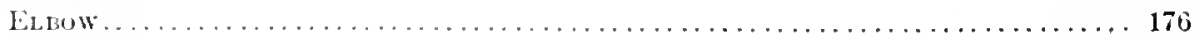

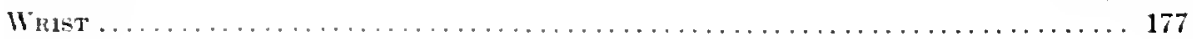

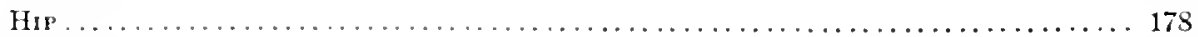

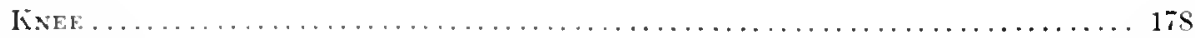

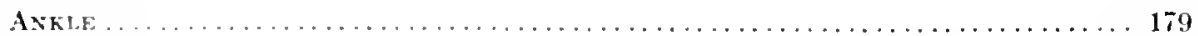

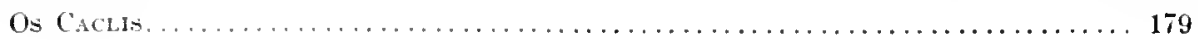


TABLE OF CONTENTS. $\quad$ xi

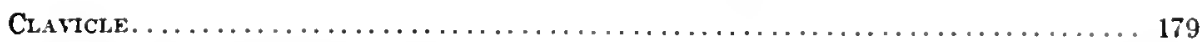

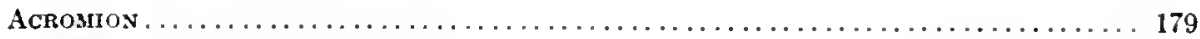

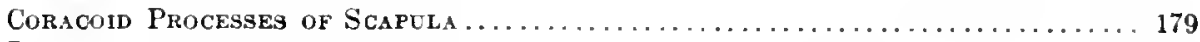

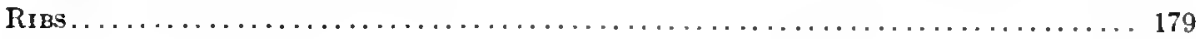

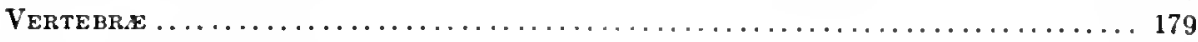

Bones of Pelvis .............................................. 179

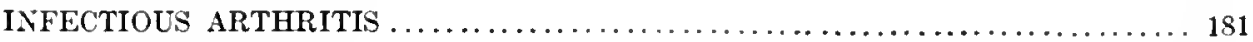

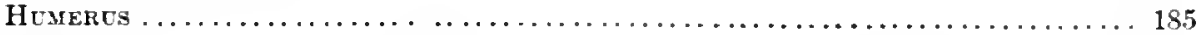

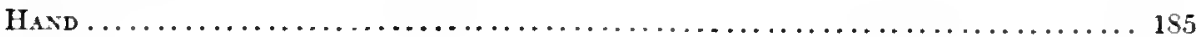

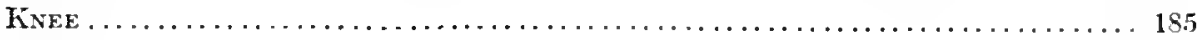

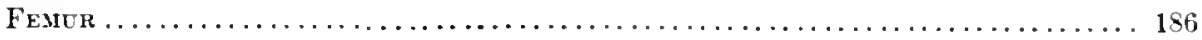

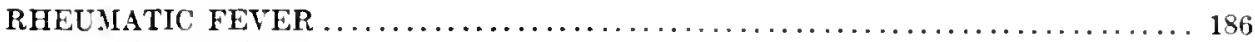

Knee; Ankle.

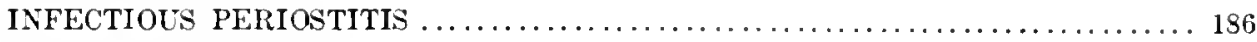

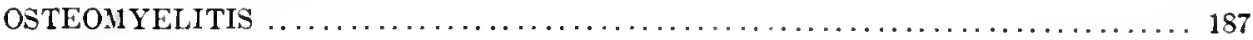

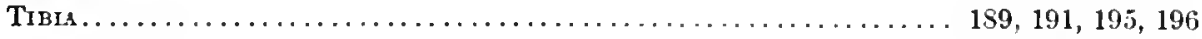

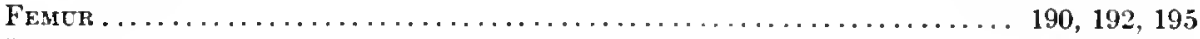

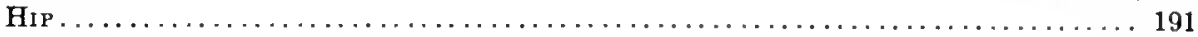

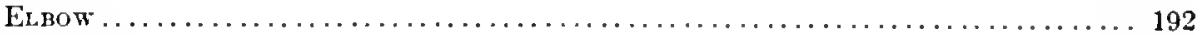

Humerus . . . . . . . . . . . . . . . . 193

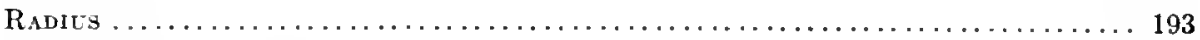

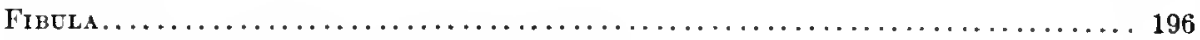

HYPERTROPHY AND ATROPHY OF JOINTS $\ldots \ldots \ldots \ldots \ldots \ldots \ldots \ldots \ldots \ldots \ldots \ldots \ldots$

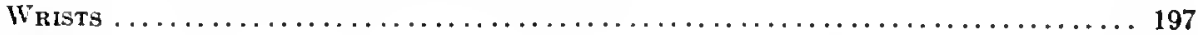

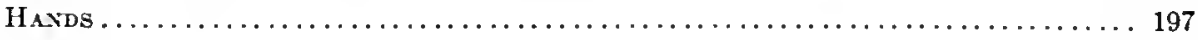

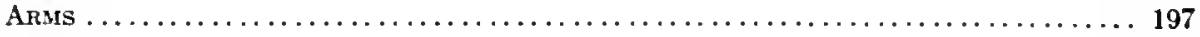

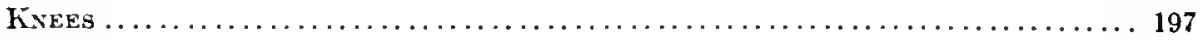

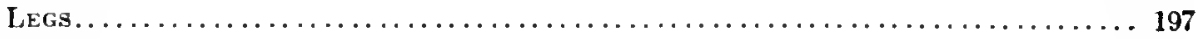

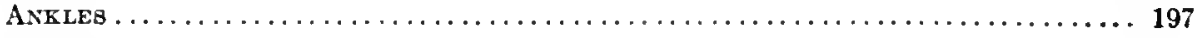

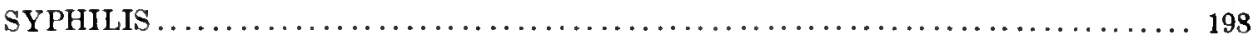

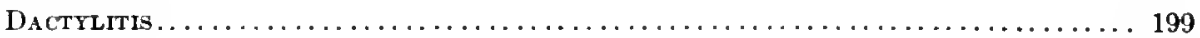

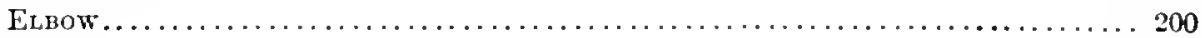

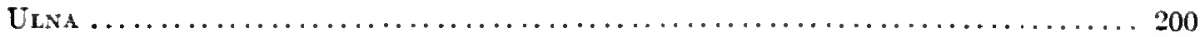

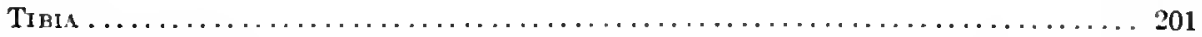

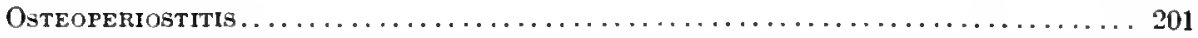

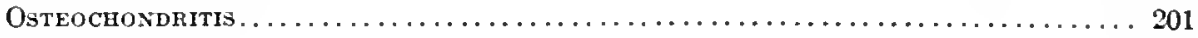

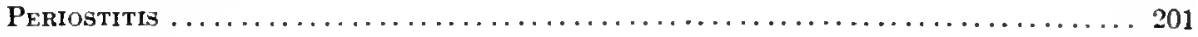

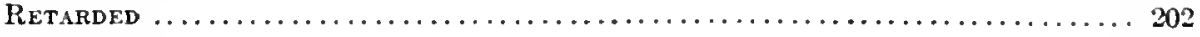

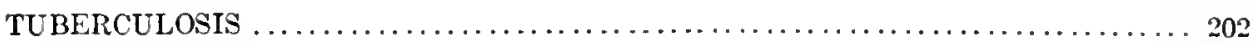

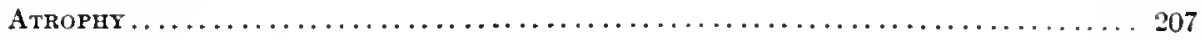

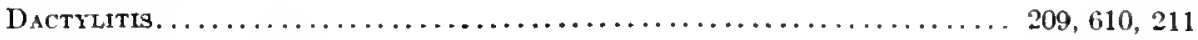

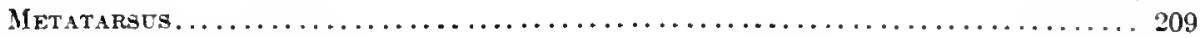

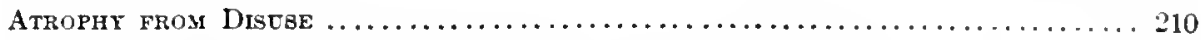

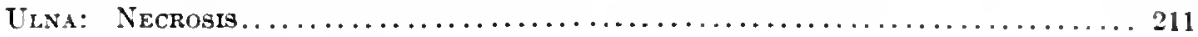

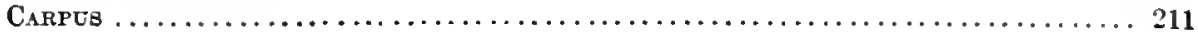




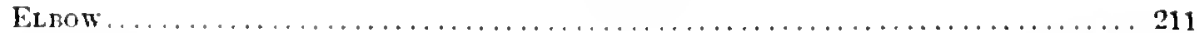

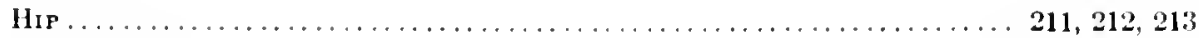

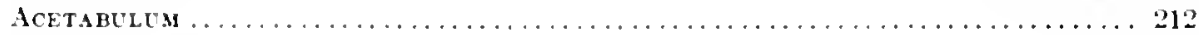

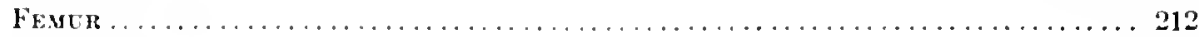

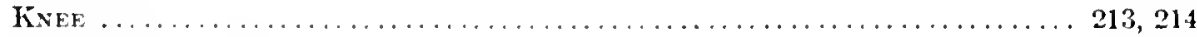

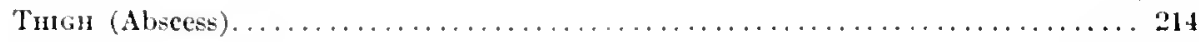

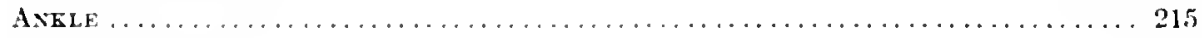

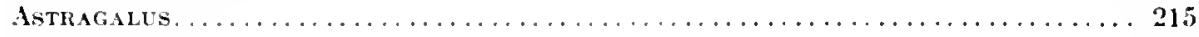

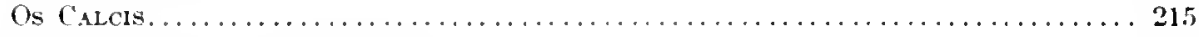

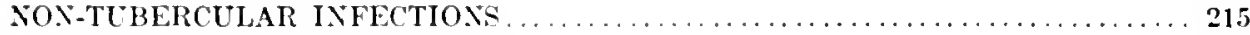

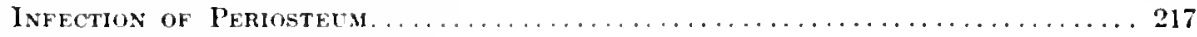

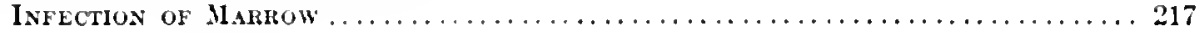




\section{ILLUSTRATIONS}

(PLATES ARE GROUPED AT THE END OF THE DIVISION THEY ILLUSTRATE.)

PLATE

1. Examples of Comparative Density

Drv.

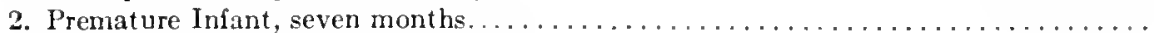

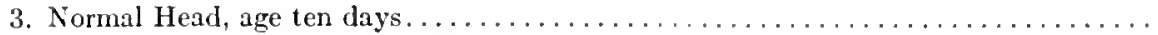

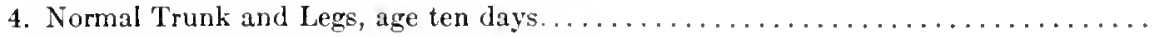

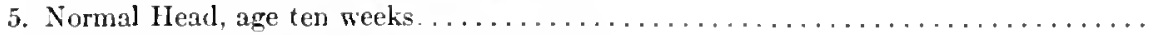

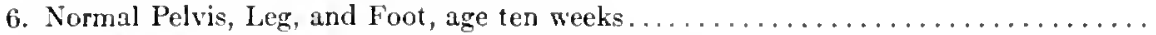

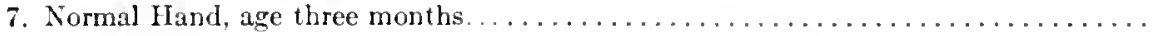

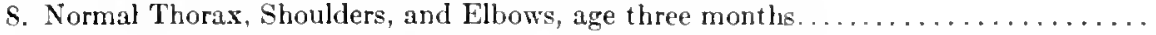

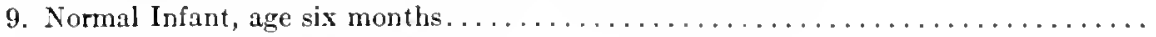

I0. Normal Thorax, age twelve months. . . . . . . . . . . . . . . . . . .

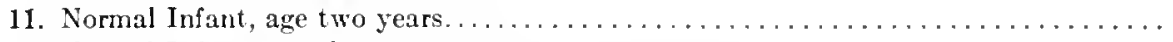

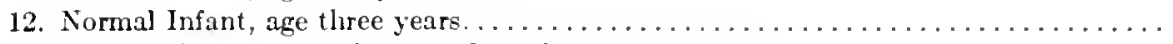

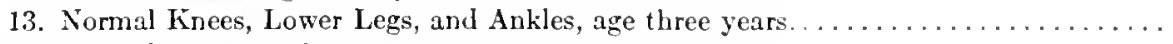

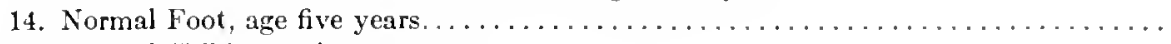

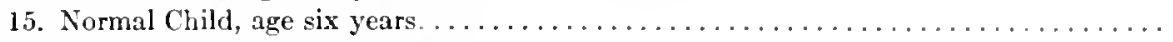

16. Normal Shoulder, age six years. . . . . . . . . . . . . . . . . . . . . . .

17. Normal Elbow, age six years. . . . . . . . . . . . . . . . . . . . . . .

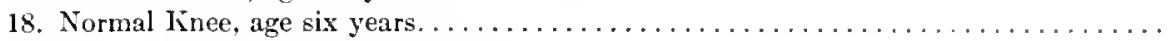

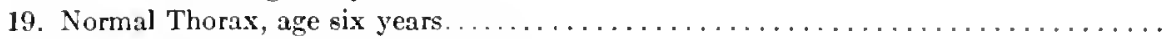

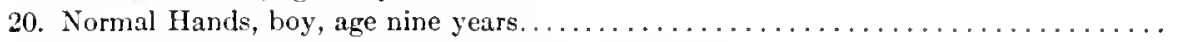

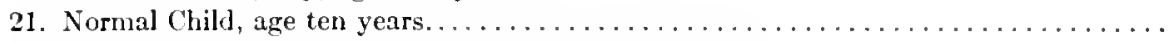

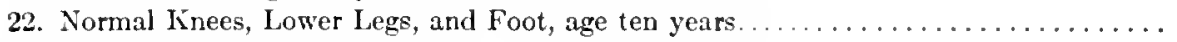

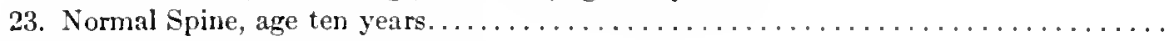

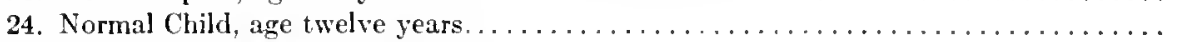

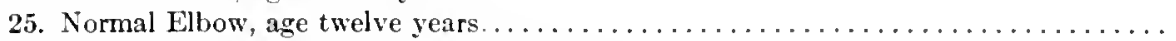

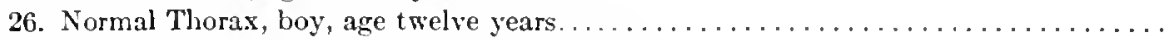

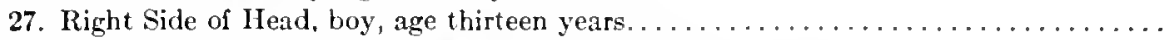

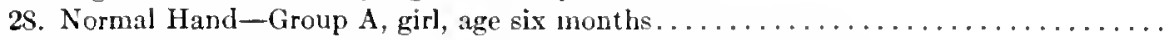

29. Normal Hand-Group B, girl, age two and three-fourths years.............. II

30. Normal Hand-Group $\mathrm{C}$, girl, age two and three-fourths years.............. II

31. Normal Hand-Group D, boy, age two and one-fourth years............. II

32. Normal Hand-Group E, girl, age three and one-half years............... II

33. Normal Hand-Group $F$, girl, age five and one-half years................ II

34. Normal Hand-Group G, girl, age six and one-half years............... II

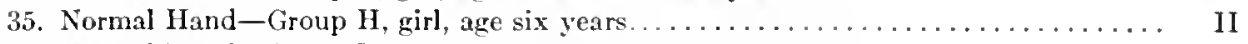

36. Normal Hand-Group I, girl, age six and three-fourths years............. II

37. Normal Hand-Group $J$, girl, age eight and one-fourth years............. II

38. Normal Hand-Group K, girl, age eleven and one-fourth years............ II

39. Normal Hand-Group L, girl, age eleven and three-fourths years............. II 
DIV

10. Normal IIand-Ciroup M, girl, age thirteen and one-half years. . . . . . . . . . . . II

4. Anomaly of leper Cervical Vertebra buy, age six years................. III

12. Spina Iifida (Goculta, girl, age three and one-half ycars, photograph............. IlI

43. Spina Jilida ()eculta, a Roentgenograph of the same subjeet as Plates 42 and $44 \ldots$ III

1.1. Spina IBificla Oeculta, a Roentgenouraph of the sane subjeet as Plates 42 and 43 ... III

15. Fusion of Ribs, Marked Seoliosis of congenital Origin, chilı, age four years. . . . . . III

46. Congenital Torticollis, boy, age six years $\ldots \ldots \ldots \ldots \ldots \ldots \ldots \ldots \ldots \ldots \ldots$ III

47. Congenital lilevation of Seapula, Left sink, boy, age six years. . . . . . . . . . . III

18. Congenital Elevation of Right Seapula, hoy, age six years . . . . . . . . . . . . III

49. Congenital klevition of Right Seapula, infant, age six months ............ III

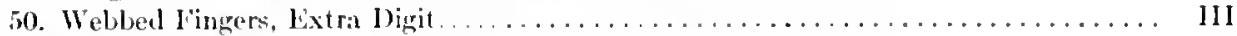

5I. Congenital Deformity of IInuds and Arms, hoy, age ten years . . . . . . . . . . . . . . III

52. Malformation of the Ratins and Tha, infint, age eight months. . . . . . . . . . . III

53. Congenital Distoeation of the Radius and Llua, bos. age ten years. . . . . . . . . . . III

54. Congenital Doformity of Foot, boy. age eight gears . . . . . . . . . . . . . . . . III

55. Roentgenograph of a Congenital Deformity of the foot................. II

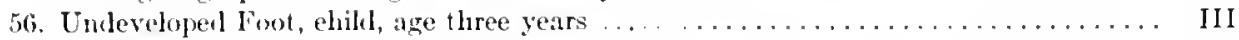

57. Congenital Delayed Development of the Right Leg, infant, age six months. (MIorse). III

58. The leet of the same subjeet as l'ate $57 \ldots \ldots \ldots \ldots \ldots \ldots \ldots \ldots \ldots \ldots \ldots$ II

59. Double Congenital Dislocation of the llip, girl, age trelve and one-half years....... III

60. Congenital Dislocation of the left Femur, hoy, age ten years. . . . . . . . . . . . . III

61. Atroply in Size of Both lemora due to Paralysis of the Legs, infant, age two years ... III

62. An Anomalous Conlition of the Second Aletaearpal Bone................ III

63. Retarded Development of Iland Corresponding with Retarded Development of Brain,

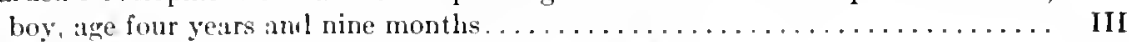

(i.t. Iyxodema-Retirded Development, girl, age eight years.............. III

65. Irregular Develofment, girl, age twenty-seven month. . . . . . . . . . . . . . III

66. Chondrodystrophia Fotalis, girl, age five and one-half years, and loy, age thirteen and one-half years. ............................... III

67. Retarded Development of the Pisiform Bone and General Anomalous Condition. . . . III

68. Osteogenesis Imperfecta, Iland and Forearm, girl, age two years.............. III

69. Osteogenesis Imperfect:a, Leg-fractures, girl, age two years. . . . . . . . . . . . . . III

70. Osteogenesis Imperfecta, Ieg-fraetures, girl, age twent $y$-five months . . . . . . . . . III

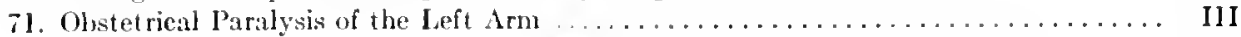

72. Obstetrical Paralysis of the Left Arm, Marked Atrophy, boy, age six years........ III

73. Obstetrieal Paralysis of the Right Shoulder, girl, age four montls.............. III

74. Osteomalacia, girl, age seven years . . . . . . . . . . . . . . . . IV

75. Infantile Atrophy, infant, age twelve months $\ldots \ldots \ldots \ldots \ldots \ldots \ldots \ldots \ldots \ldots \ldots$

76. Infantile Scorbutus, Thickened Cortex and Periosteum, girl, age eleven months..... IV

77. Infantile Seorbutus, Subperiosteal, girl, age eleven months. . . . . . . . . . . . IV

7S. Infantile Scorhutus, Organizing Clot and IIxmatoma of the Iuscles, infant, age six

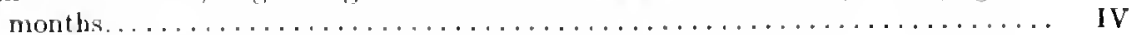

79. Infantile Scorbutus, sane subject as Plate $79 \ldots \ldots \ldots \ldots \ldots \ldots \ldots \ldots \ldots$ IV

80. Infantile Scorlutus-Infiltrated Museles, infant, age two months. ........... IV

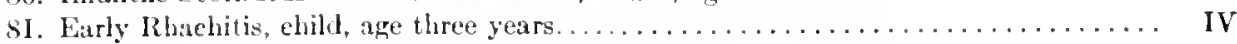

82. Early Rhachitis, ehild, arge two years. . . . . . . . . . . . . . . . . . . IV

8.3. Rhachitis, boy, age seven years. Photographs .................... IV

s.t. Early Rhachitis-Thickened Cortex-Wolff's Law, boy, age seven years.......... IV

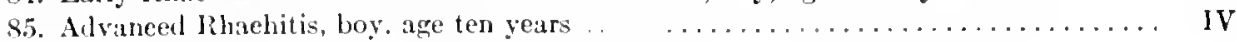

S6. Marked Rhachitis, boy, age seven years....................... IV 
S7. Protuberant Abdomen, Knoek-knee, and Flat-foot, boy, age three years.......... IV

88. Rhachitis of Adoleseenee-Normal and Rhaehitie Hands, boys, age thirtcen years... IV

59. Rhachitis of Adolescenee-Hand, child, age twelve years................. IV

90. Fracture of Skull, boy, age thirteen years. . . . . . . . . . . . . . . . . .

91. Ethmoiditis, child, age three years. ........................

92. Osteomyelitis of Lower $\mathrm{J}_{\mathrm{i} w}$, colored boy, age twelve years................. V

93. Amomalous Bieuspids-Left Side of Head, boy, age thirteen years. . . . . . . . . . . V V

94. Anomalous Lower Biscupid-Right Side of Head, boy, age thirteen years......... V V

95. Unerupted Permanent Teeth-Right Sicle of Hearl, boy, age eight years.......... V V

96. Supernumerary Tootl_-Left Side of Head, boy, age fifteen years.............. V

97. Supernumerary Tooth-Right Side of Head, girl, age fourteen years............ V V

98. Supernumerary Tooth-Left Side of Head, girl, age fourteen years. . . . . . . . . . . V V

99. Various Anomalous Conditions Connected with the Teeth. . . . . . . . . . . . . V

100. Rhaclitis of Spine, colored boy, age six years . . . . . . . . . . . . . . .

101. Osteomyelit is of Vertebra, girl, age four years . . . . . . . . . . . . . . . . . .

102. Tuberculosis of the Spine, Ilium, and Left Ilip.................... . . . .

103. Tuberculosis of the Ilium. . . . . . . . . . . . . . . . . . . . . . . . .

104. Tubereular Abseess of the Spine............................

105. Tubereulosis of the Spine, ehild, age four years . . . . . . . . . . . . . . . . . V

106. Tubereular Spine, the same subject as Plate 105 , lateral view. . . . . . . . . . . . V V

107. Normal Thorax, girl, age seven years. Long exposure. (Brown) .............. VI

108. Normal Thorax, girl, age seven years. Short exposure. (Brown) ................ VI

109. Enlarged Bronehial Nodes, girl, age twelve years. . . . . . . . . . . . . . . . . . VI

110. Transposition of Organs-Tuberculosis of the Lungs and Bronchial Nodes......... VI

111. Pnewmoeoecus Lobar Preumonia . . . . . . . . . . . . . . . . . . . . . . . II

112. Double Pneumococcus Lobar Pneumonia, boy, age twelve years............... VI

113. Lobar Pneumonia, ehild, age ten years. . . . . . . . . . . . . . . . . . . .

114. Unresolved Lobar Pnewmonia, girl, age four years. . . . . . . . . . . . . . . . . VI

115. Lobar Pneumonia, girl, age twenty-seven months. Same subjeet as Plates 65,116 ,

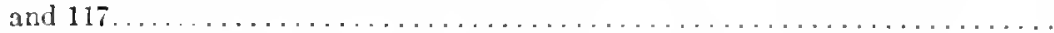

116. Consolidation of Right Lung-Mongolian Idiot . . . . . . . . . . . . . . . . VI

117. Lobar Pneumonia. Same subject as Plates 65,115 , and $116 \ldots \ldots \ldots$ VI

118. Bronchopneumonia, elild, age four years . . . . . . . . . . . . . . . . . .

119. Pneumonia-Inhalation of China Doll's Arm. Same subjeet as Plates 148, 149,

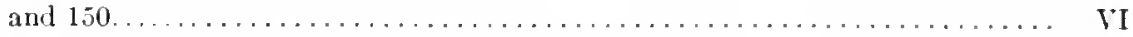

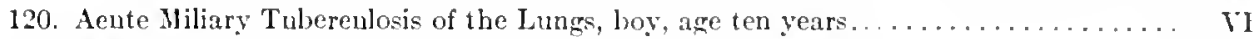

121. Early Miliary Tubereulosis of the Lungs, child, age three years................. . . I

122. Probable Old Tubereular Proeess of Lung-Caleifieation. ................. . . . . I

123. Emphysema, Gangrene, and Tubereulosis oi the Left Lung, girl, age three years..... VI

124. Aente Miliary Tubereulosis of Both Lungs, girl, age twelve years. . . . . . . . . . . . . VI

125. Hydropnenmothorax, boy age seven years. Same subjeet as Plates 126 and 127... VI

126. Hydropneumothorax. Same subject as Plates 125 and $127 \ldots \ldots \ldots \ldots \ldots \ldots$. . . . . . .

127. Pneumothorax. Same subjeet as Plates 125 and $126 \ldots \ldots \ldots \ldots \ldots \ldots \ldots$

128. Thickened Plewra, boy, age six years . . . . . . . . . . . . . . . . . . . . VI

129. Pleurisy with Effusion, ehild, age eight years.................... VI

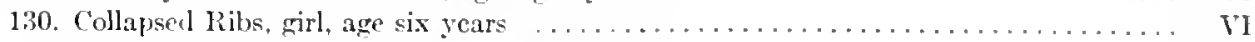

131. Eneapsulated Empyena boy, age ten years . . . . . . . . . . . . . . . . . . vi

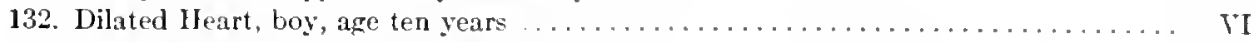

133. Perieardial Effusion, ehild, age twelve years .................... . . . . .

134. Enlarged Ileart with Pericardial Effusion, ehild, age twelve years............... TI 
135. Pericardial Effusion and Obliteration of the Cardiohepatie Angle............. VI

136. Aneurism, boy, age twelve years $\ldots \ldots \ldots \ldots \ldots \ldots \ldots \ldots \ldots \ldots \ldots \ldots$

137. Normal Abdomen, boy, age nine years. Short exposure. (1irown)............ VII

135. Cormal Ablomen, boy, age nine years. Long exposure. (I3rown). Same subject as Plate 137

139. IIour-glass Contraetion of Stomach, infant, age five weeks

140. Hour-glass Contraction of Stomach-Tube in Stomach. Same subject as Plate 139

141. Abdominal Ascites, girl, age twelve years. . . . . . . . . . . . . . . . . . . .

142. Caleified Mesenteric Torles

143. Stone in Urethra, Encapsulating a Pin, girl, age thirteen years. . . . . . . . . . . . VIII

14.4. Foreign Body in Intestine, boy, are five years. . . . . . . . . . . . . . . VIII

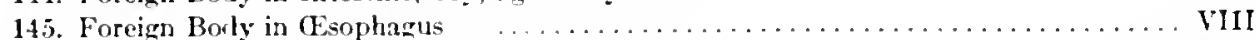

146. Ilook in the Larynx, child, age four years. ...................... VIII

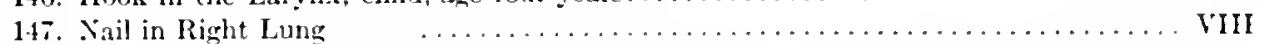

145. Doll's China Arm in Lung, girl, age four years. Same subject as Plates 119, 149, and 150

149. Doll": China Arm in Lung. Same subjeet as Plates 119,148 , and 150 , taken face down.

150. Doll's China Arm in Lung. Same subject as Plates 119,148 , and $149 \ldots \ldots$. . . VIII

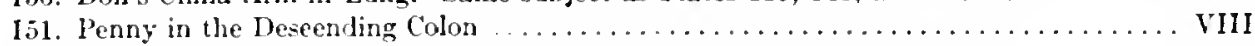

152. Veedle in the Knee-joint. Same subjeet as Plate $153 \ldots \ldots \ldots \ldots \ldots \ldots \ldots \ldots$. . . . . . . . . .

153. Seedle in the Knee-joint. Same subject as Plate 152, different position......... VIII

154. Feedle in Foot. Same subjeet as Plate $155 \ldots \ldots \ldots \ldots \ldots \ldots \ldots \ldots \ldots \ldots$. . . . . VII

155. Feedle in Foot. Same subjeet as Plate 154, different position. . . . . . . . . . . . VIII

15j. Seedle in the Tissues around the Phalanx of the Little Toe............... VIII

157. Retarded Development of Hand, boy, age eight years................. IX

155. Premature Ossifieation of the Lower Epiphysis of the Radius-Sesamoid Bone, ehild, age thirteen years. . . . . . . . . . . . . . . . . . . . . . . . . . . . .

159. Delayer Development of the Seaphoill, boy, age $\operatorname{six}$ years. . . . . . . . . . . . . . .

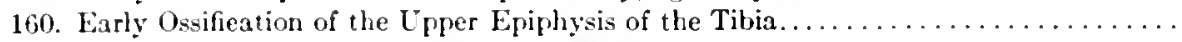

161. Cellulitis of Tissues of Left $\mathrm{Arm}$, boy, age nine years. . . . . . . . . . . . . . .

162. IIxmatoma of Heel, chill, age $t$ welve vears. . . . . . . . . . . . . . . . . . .

163. Giant-celled sarcoma of the Thigh, loy, age four and one-half years. . . . . . . . . .

164. Nedullary sareoma of the Lower Part of the Femur, boy, age twelve years........

16.5. Periosteal Sareoma-Lower Enil of Femur, boy, age ten years. . . . . . . . . . . . . .

166. Extreme Atrophy, boy, age thirteen years. .

167. Anterior Poliomvelitis of the Right IIanl anel Wrist, infant, age eighteen months ...

165. Anterior Poliomyelitis of the Left Shouder, boy, age twelve months............

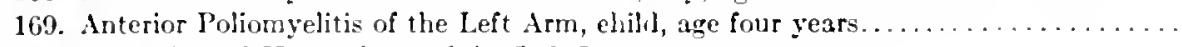

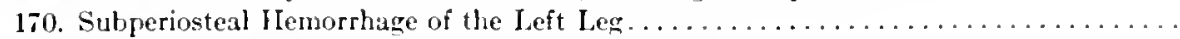

17. Exostosis of Astragalus, boy, age twelve years. . . . . . . . . . . . . . . . .

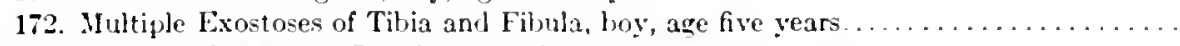

173. Sultiple Exostoses-Leg. boy, age five years. . . . . . . . . . . . . . . . .

174. Exostosis of the Lower Part of the Femur . . . . . . . . . . . . . . . . . . .

175. Exostosis of the Tibia, boy, age twebe years . . . . . . . . . . . . . . . . .

176. Calluses of Feet, girl, age ten years. . . . . . . . . . . . . . . . . . . . .

177. Alnormally ligh Areh of Foot, girl, age ten years. . . . . . . . . . . . . . . . .

175. Moderate Flat-foot, girl, age thirteen years $\ldots \ldots \ldots \ldots \ldots \ldots \ldots \ldots \ldots \ldots \ldots \ldots \ldots$

179. Backward Displacement of the Inner Condyle of the Femur, boy, age twelve years. .

190. Fracture and Displaeement of the IIeal of the Humerus

IX

IX

IX

IX

IX

IX

IX

IX

IX

IX

IX

IX

IX

IX

IX

IX

IX

IX

IX

IX

IX

IX

1S1. Disloeation and Fracture of the Anatomic Ilead of the Humerus, boy, age eleven years

IX 


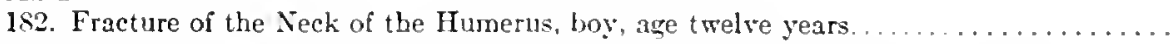

1S3. Impaeted Fracture of the Surgieal Neck of the IIumerus, boy, age four years...... IX

184. Dislocation of the Epiphysis of the Femur, boy, age five years ............ IX

185. Untreated but United Green-stiek Fracture of the Tibia, boy, age eight years..... IX

1S6. Intracapsular Fraeture of the Femur, boy, age ten years.................. IX

187. Dislocation of the Lower End of the Femur, boy, age ten years ............. IX

185. Fraeture of the Lower End of the Humerus with Inward Displacement of the Lower

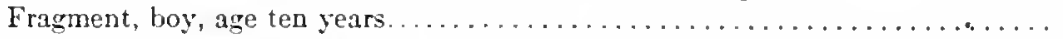

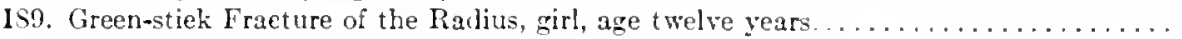

190. Complete Fraeture of the Lower Third of the Radius and Una with Impaction, child, age three to four years.

IX

191. Complete Fracture of the Radius and Lina, ehild, age three years...............

192. Incomplete Fracture of the Lower End of the Tibia, infant, age twelve months . ....

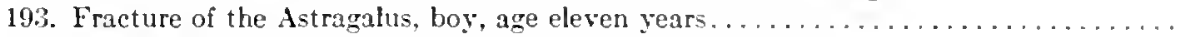

194. Old Fracture of Tibia and Fibula, with Conneeting Bridge . . . . . . . . . . . .

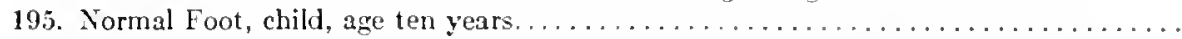

196. Fraeture of Tibia with Compensatory Growth of Fibula, girl, age twelve years.......

197. Congestion of the Inee in a Case of Chronie Arthritis, ehilk, age six years..........

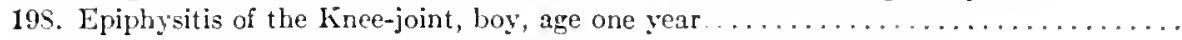

199. Epiphysitis of the Upper Epiphysis of the Tibia, girl, age ten years..............

200. Infectious Arthritis-Atrophie $T_{y}$ pe, girl, age three years $\ldots \ldots \ldots \ldots \ldots \ldots \ldots$

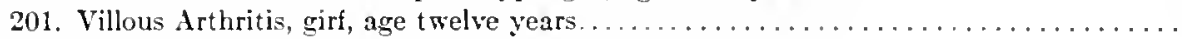

202. Ankylosis of Iñnee-joint, boy, age thirteen years . . . . . . . . . . . . . . .

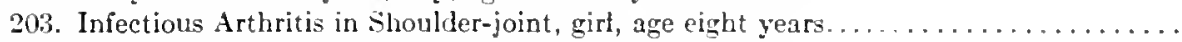

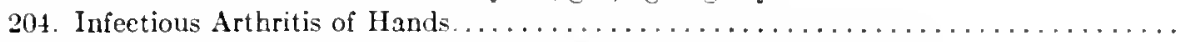

205. Infectious Arthritis of the Hand, boy, age four and one-half years . . . . . . . . . .

201. Effusion in the linee-joint, boy, age ten years . . . . . . . . . . . . . . . . .

207. Aeute Arthritis of Right Hip, ehild, age eight months....................

208. Rheumatie Fever-Knee-joint. . . . . . . . . . . . . . . . . . . . . . .

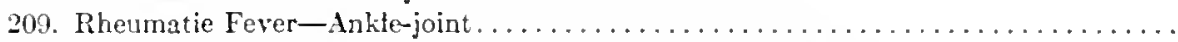

210. Osteomyelitis of the Shaft of the Tibia, ehild, age nine years................

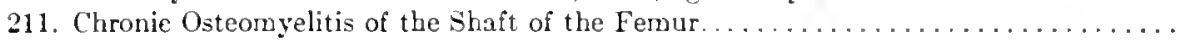

212. The Results of an Acute Destructive Process in the IIipk, chilu, age five years .....

213. Osteomyetitis of the Upper Shaft of the Tibia and of the Epiphysis, boy, age eight years

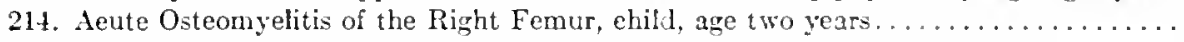

215. Chronie Osteomyelitis-Great Destruetion of the Femur, ehiłd, age two years.......

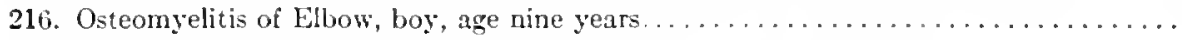

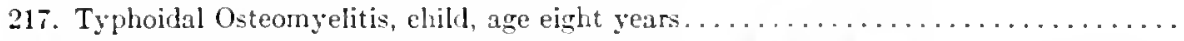

219. Osteomyelitis of the Lower End of the Radius, boy, age six years..............

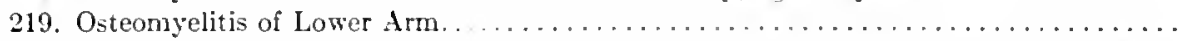

220. Osteomyelitis of the Acetabulum with Sequestrum, child, age two and one-half years. .

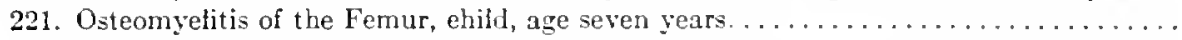

222. Osteomyelitis near Hip-joint-Pneumococcus Infeetion, ehild, age three and one-half

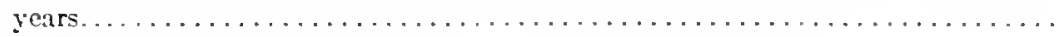

223. Osteomyelitis near Hip-joint, ehild, age four am one-half years . . . . . . . . . . .

224. Mixed Infeetion of Hip-joint-Probable Osteomyelitis, girl, age six years...........

225. Osteomyelitis of Lower End of Tibia, boy, age twelve years ..................

226. Osteomyelitis of the Lower End of the Right Fenur, boy, age seven years.........

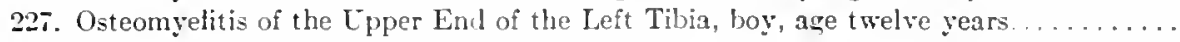

22S. Early Stage of Osteonyelitis of the Lower Extrenity of the Tibia, boy, age twelve years

II

IX

IX

IX

IX

IX

IX

IX

IX

IX

IX

II

IX

IX

IX

II

II

IX

IX

II

I.I

IX

IX

IX

IX

IX

IX

IX

IX

IX

IX

I.

I.I

II

IX

IX

IX

IX

IX 
229. Osteomyelitis of Fibula, girl, age eleven years. .

230. Undetermined Infection of the Lower Epiphysis of the Tibia, boy, age ten years.....

23I. Chronie Infeetious Osteomyelitis of the Upper Ench of the Tibia, boy, age six years...

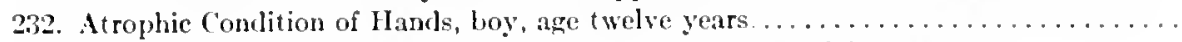

233. Chronic Atrophic Condition of the Knec-joints, boy, age eight years............

234. An lnfectious Arthritis of the Knee-joint - Chronic Atrophic Condition............

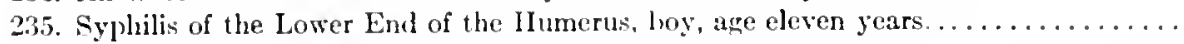

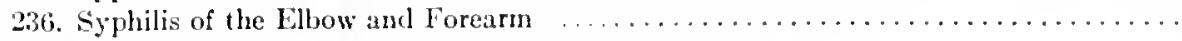

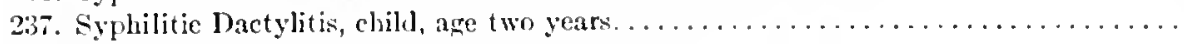

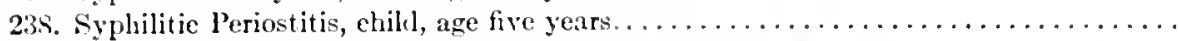

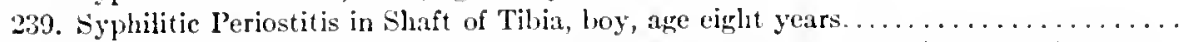

240. Syphilitic Osteoperiostitis and Osteoehondritis, child, age six weeks. (La Vétra)....

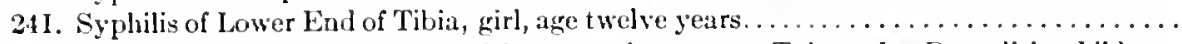

242. Infection of Metatarsal Bone, girl, age twelve years. Tubereular Dactylitis, child, age

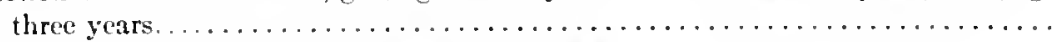

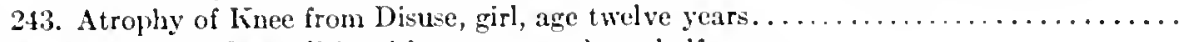

24. Tubereular Daetylitis, girl, age two and one-half years. . . . . . . . . . . . . .

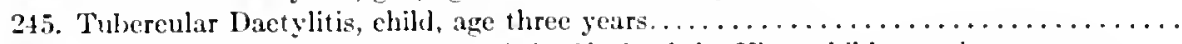

246. Tubereulosis of the Lower Part of the Shaft of the Utna, child, age three years......

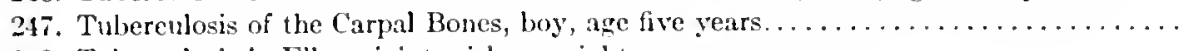

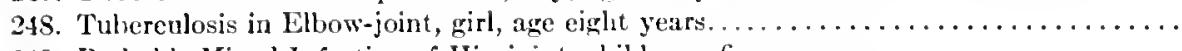

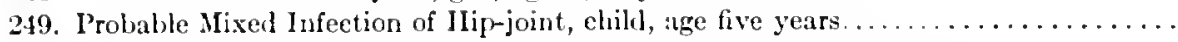

250. Tuberculosis of the Femur and Acetabulum, boy, age twelve years. . . . . . . . . .

25I. Mixed Infection of the Hip-joint and $\Lambda$ cetabulum, elnild, age five years..........

252. The Fnd Result of an Old Tubercular P'roeess, elild, age fourteen years............

253. Probable Tubercular Infection of the Epiphyses, child, age eight years............

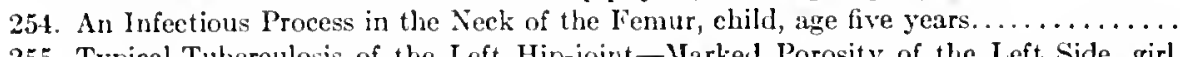

255. Typical Tubereulosis of the Left Hip-joint-Marked Porosity of the Left Side, girl,

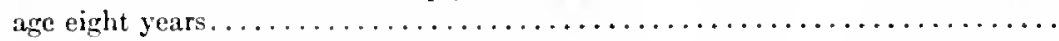

256. Questionable Infection of the Knee-joint (probably Tuberculosis)-Infiltration of the

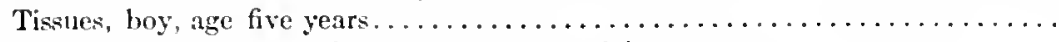

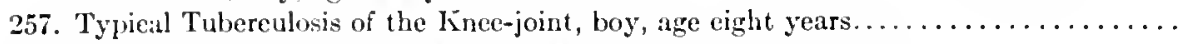

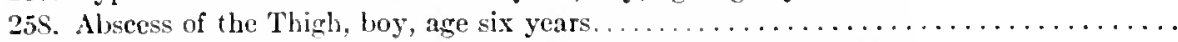

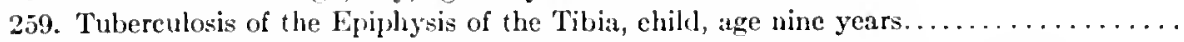

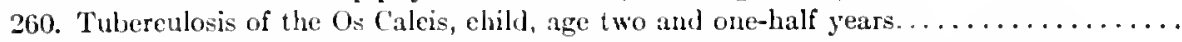

26I. Tubercular Infeetion of the Epiphysis and Astragalus, child, age four years........

262. Tubereulosis of the Epiphysis of the Tibia, child, age five years..............

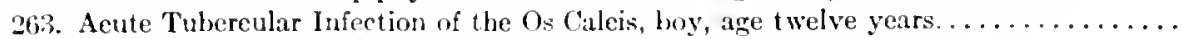

264. Non-tubercular Infeetion about the Neck of the Femur, child, age five years........ 


\title{
ILLUSTRATIONS
}

\author{
(ARRANGED ACCORDING TO THE AGE OF THE SUBJECT.)
}

\section{FIRST YEAR.}

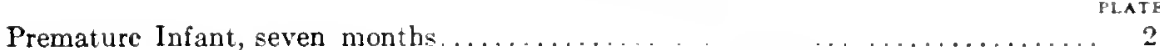

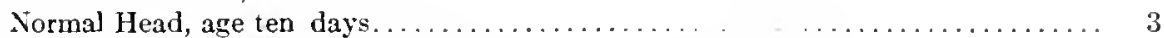

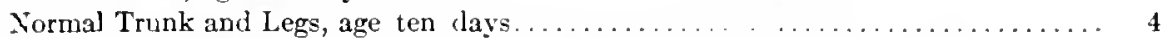

Hour-glass Contraction of Stomach, infant, age five weeks. . . . . . . . . . . . 139

Hour-glass Contraction of Stomach-Tube in Stomach, infant, age five weeks . . . . 140

Syphilitic Osteoperiostitis and Ostcochondritis, child, age six meeks (La Fetra).... 240

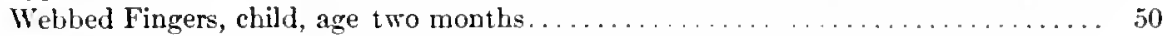

Infantile Scorbutus, infant, age two months.................... \$ 0

Normal Head, infant, age ten weeks......................... 5

Normal Pelvis, Leg and Foot, iniant, age ten weeks.................. 6

Normal Hand, infant, age three months.........................

Normal Thorax, Shoulders and Elbows, infant, age three months............. S

Obstetrical Paralysis of Right Shoulder, girl, age four months. ............. 73

Normal Infant, age $\operatorname{six}$ months. . . . . . . . . . . . . . . . . . . . . . 9

Normal Hand, Group A, girl, age six months. . . . . . . . . . . . . . . . . 28

Congenital Elevation of Right Scapula, infant, age $\operatorname{six}$ months . . . . . . . . . . . 49

Congenital Delayed Development of Right Leg, infant, age six months (Morse) .... . 5i

The feet of the same subject as Plate 57 , an infant, age six months. ........ 55

Infantile Scorbutus, Organizing Clot and Hæmatona of the Museles, infant, age six months ...................................

Iniantile Scorbutus, age $\operatorname{six}$ months. . . . . . . . . . . . . . . . . . 79

Malformation of the Radius and Ulna, infant, age eight months. . . . . . . . 52

Acute Arthrit is of Right Hip, child, age eight months . . . . . . . . . . . . 20

Infantile Scorbut us, Thickened Cortex and Periosteum, girl, age eleven months.... 76

Infantile Scorbutus, Subperiosteal, girl, age eleven months . . . . . . . . . . T

Normal Thorax, child, age twelve months. . . . . . . . . . . . . . . . . . . . 10

Anterior Poliomyelitis of the Left Shoulder, boy, age twelve months. . . . . . . . . 168

Infantile Atrophy, infant, age twelve months. . . . . . . . . . . . . . . . . .

Incomplete Fracture of the Lower End of the Tibia, infant, age twelve months . . . 192

DIv.

I

1

I

VII

VII

IX

III

IV

I

I

I

I

III

I

II

III

III

III

IV

IV

III

IX

IV

IV

III

IV

IX

\section{SECOND YEAR.}

Epiplaysitis of the Inee-joint, bov, age one year. $\quad \ldots \ldots \ldots \ldots \ldots \ldots \ldots \ldots \ldots$

Extra Digit, ehild, age one and one-half years .................... 50

IX

Anterior Poliomyelit is of the Right IIand and Wrist, infant, age eighteen months... 16i

III

An Anomalous Condition of the Second Metaearpal Bone, infant, age twent $y$-three

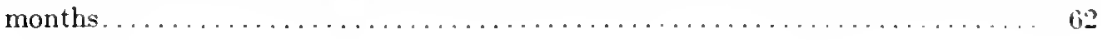


Normal Intant, age two years

PLATE

11

Atrophy in size of both Femora he to Paralysis of the Legs, infant, ase two years ................................... 6 I

Osteogenesis hnperfecta, lland aml forearm, girl, age two years ............ $6 \mathrm{~S}$

Osteogrenesis Imperfecta, Leg-fractures, girl, age two years ................ 69

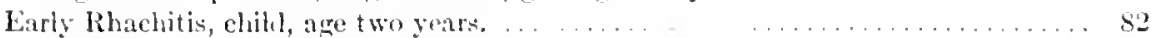

Acute Osteomyelitis of the Right Femur, child, age two years............... 21.

Chronic Osteomyelitis-Gireat Destruction of the Femur, child, age two years . . . . 215

Syphilitic bactylit is, chih, age two years. . .... . . . . . . . . . . 237

Osteorenesis Imperfecta, Leg-fractures, child, age twenty-five months. . . . . . . . . 70

Irregular Development, girl, age twenty-seven months. . . . . . . . . . . . 65

Lobar Pneumonia, girt, twenty-seven months . . . . . . . . . . . . . . . . . . . . 115

Consolidation of Right Lung-Mongolian idiot, girl, age twenty-seven monthe. . . . 116

Lohar Pneumonia, gid, age twenty-seven months-Sane subject as Plates 6.5, 115,

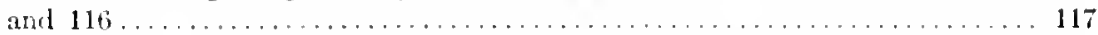

Normal Iland, Group D, boy, age two and one-fourth years ............... 31

Osteonyelitis of the Acetabulum with Sequestrum, chill, age two and one-half

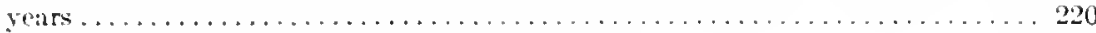

Syphitis of Elbow, girt, age two and one-half years ................... 236

Tubereular Dactylitis, crirl, age two and one-half years.................. 2.14

Tuberculosis of the $\mathrm{Os}$ Calcis, chilt, age $t$ wo and one-hatf years . . . . . . . . . 260

Normal IIand, Group B, girl, age two and three-fourths years. . . . . . . . . . . . . 29

Normal IIand, Group (c, girl, age two and three-fourths years............... 30

\section{FOURTI YEAR.}

Normal Infant, age three years........................... 12

Normal Lnees, Lower Legs and Ankles, chilıl, age three years . . . . . . . . . . . . . . 13

Undeveloped Foot, chiht, age three years. . . . . . . . . . . . . . . . . . . . . 56

Early Rhachitis, chihl, age three years.......................... 81

Protuberant Abetomen, Knock-Iinee, and Flat-Foot, boy, age three years........ 87

Ethmoiditis, child, age three years...... . . . . . . . . . . . . . . . 91

Pneumococcus 1.obar l'neumonia, girl, age three years.................. 111

Early Wiliary Tulsereulosis of the Lungs, chith, age three years ............. 121

Emphysema, Gangrene, and Tuberculosis of the Left Lung, child, age three years. 123

Complete Fracture of the Radius and Una, child, age three years. . . . . . . . . 191

Infectious Arthritis-Atrophic Type, girl, age three years . . . . . . . . . . . 200

Tubercular Dactylit is, child, age three years ........................ 242

Tubercular Dact ylitis, child, age three years..................... 245

Tuberculosis of the Lower Part of the shaft of the Clnal, chitd, age three years.... 246

Osteomyetitis near Ihip-joint-P'neumococcus Infection, child, age three and one-half

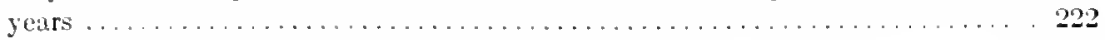

Normal IIand, Group E, girl, age three and one-half years............. 32

Spina Bifila Occulta, giri, age three and one-half years. Photograph........ 42

Spina Bifida Occult a, a Roentgenugraph of the same subject as Plates 12 and $41 \ldots$ 43

Spina Bifida Occulta, a Roentgenograph of the same subject as Plates 42 and $43 . \ldots$ il

Complete fracture of the Lower Thirl of the Rathus and L'na with Impaction, child,

age three to four years. . . . . . . . . . . . . . . . . . . . . . . . 190
DIV.

IX

IX

IX

IX

II

II 
FIFTH YEAR.

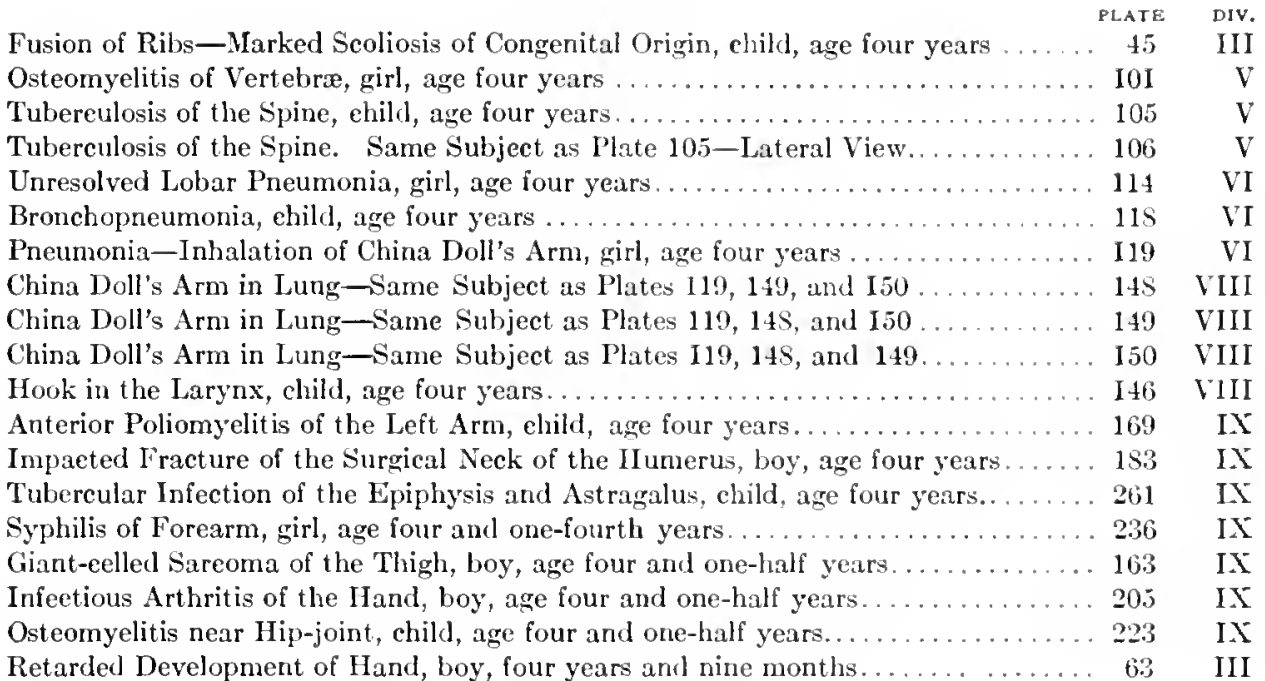

\section{SIXTH YEAR.}

Normal Foot, child, age five years............................ It

Foreign Body in Intestine, boy, age five years....................... 144

Multiple Exostoses of Tibia and Fibula, boy, age five years $\ldots \ldots \ldots \ldots \ldots \ldots \ldots \ldots$

Multiple Exostoses-Leg, boy, age five years ..................... 173

Dislocation of the Epiphysis of the Fenur, boy, age five years.............. 184

The Results of an Acute Destructive Process in the Hips, child, age five years..... 212

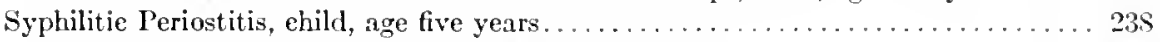

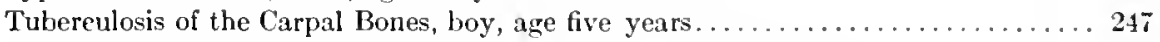

Probable Mixed Infection of the Hip-joint, child, age five years............. 249

Mixed Infection of the Hip-joint and Acetabulum, child, age five years.......... 251

An Infectious Process in the Neck of the Femur, child, age five years............ 254

Questionable Infeetion of the Knee-joint (probably tuberculosis), boy, age five years 256

Tubereulosis of the Epiphysis of the Tibia, ehild, age five years............. 262

Non-tubereular Infeetion about the Neck of the Femur, ehild, age five years ...... 264

Chondrodystrophia Fotalis, girl, age five and one-half years............... 66

Normal Hand, Group F, girl, age five and one-half years............... 33

$I$

VIII

IX

IX

IX

IX

IS

IX

IX

IX

IX

IX

IX

IX

III

II

SEVENTII YEAR.

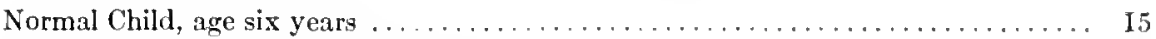

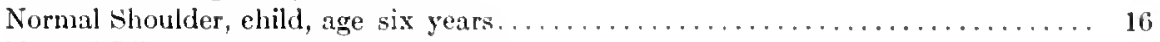

Normal Elbow, ehild, age six years........................... 1 i

Normal Knee, child, age six years............................ is

Normal Thorax, ehild, age six years............................ 19

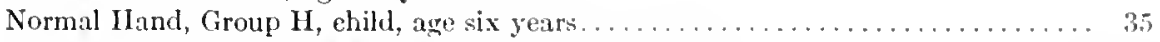

Anomaly of Upper Cervical Vertebra, boy, age six years................. 41

Congenital Torticollis, boy, age six years......................... 46 


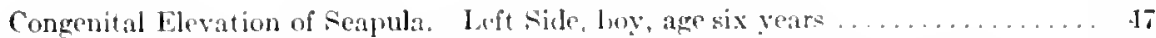

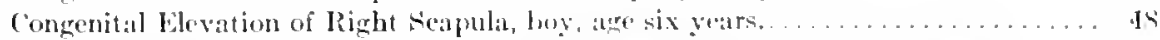

Ohstetrical Paralysis of the left Arm- Markol Atrophy, low, age six years...... i2

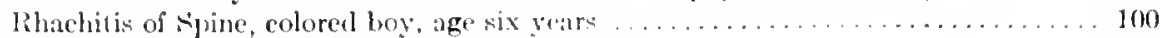

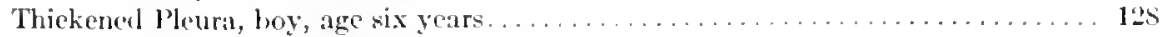

Collapsed Rils, girl, age six years. . . . . . . . . . . . . . . . . . . . 130

Delayed Development of the Seaphoid, loy, age six years ............... 159

Congestion of the fince in at tise of Chronic Arthritis, child, age six years........ 197

Osteomgelitis of the hower Lnd of the Ratlius, foy, age six years............ 215

Mixed Infection of the IIip-joint, I'rolnalis Osteomyclitis, girl, age six years...... 224

(hronie Infectious Osteomyelitis of the L puer Lud of the Tihia, boy, age six years. 231

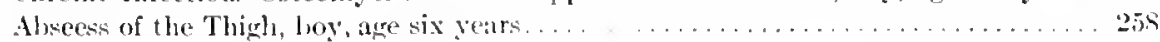

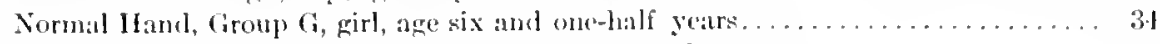

Nomal IIand, Group I, girl, age wix and threefourthe years............ 36

EITIITH YEAR

Ostcomalaeia, girl, age seven years...... $\quad \ldots \ldots \ldots \ldots \ldots \ldots \ldots \ldots$ it

Rhachitis, boy, age seven years. Photograpls.. .................. s;

Early Rhaehitis-Thichenerl Cortex-Wolf's law-hoy, age seven years......... \$I

Marked thachitis, hoy, age seven years........................ si;

Normal Thorax, girl, are seven yors-Long Exposure. (Brown)............ 107

Normal Thorax, girl, age seven years-Short Lxposure. (Brown)............ los

Iyclropneumothorax, hoy, age seven years . . . . . . . . . . . . . . . 125

IIydropneumothorax, boy, age seven years, the same Subject as Plates 125 and 127126

Ostemyelit is of the Femur, child, age seven years.................. 221

Osteomyelitis of the Lower End of the Right Hommr, hoy, age seven years....... 226

\section{NINTII YEAR.}

Congenital Deformity of lioot, hog, age eight yeirs.................. 54

Myxodema-Retarided Development-girl, age ejght years............. 6.1

Olustetricil Paralyis of the Left Arm, hoy, age night yeirs.............

Enerupted I'ermanent Tectl-Right Silc of Ilead, boy, age eight years........ 95

Pnemothorax, boy, agro cight years. Same subject as l'lates 125 and $126 \ldots \ldots \ldots 127$

Plcurisy with Effusion, ehilk, age eight years..................... 129

Retarderl bevelopment of 1 and, boy, age eight years . . . . . . . . . . . . . 15 i

Untreated lut [ nitcl Green-stiek l'racture of the Tibia, by age eight years...... 185

Infeetious Arthritis of the shoulder-joint, girl, igre eight years. . . . . . . . . 203

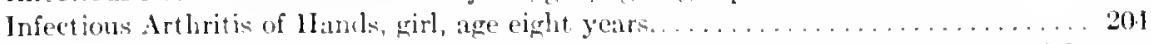

Osteomyelitis of the Cppershaft of the Tihia and of the Lpiphysis, boy, age eight

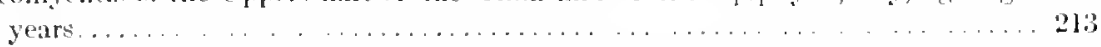

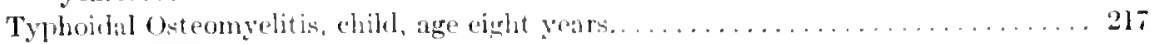

Chronie Atrophic Condition of the Inec-jonts, bey, age eight vears . . . . . . . 233

An Infections Arthritis of the lince-joint, boy, age eight years. . . . . . . . . . 234

Syphilitic Priostitis of the shaft of the Tibia, hoy, age eight years . . . . . . . 239

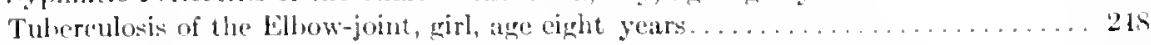

Probible Tubercular Infection of the Epiphyses, child, age eight gears.......... 25.3

Typical Tubereulosis of the Left Hipr-joint, girl, age cight years . . . . . . . . 255

Typieal Tuhrerculosis of the hinee-joint, hoy, age eight years. . . . . . . . . . 258

Cormal IIand, (iroup J, girl, age eight and one-fourth years . ............. 37 
Normal Hands, boy, age nine years .......................... 20

DIV.

Normal Abdomen, boy, age nine years. Short Exposure. (Brown) ........... 137

Normal Abdomen, boy, age nine years. Long Exposure. (Brown) ........... 138

Cellulitis of Tissues of Left $\mathrm{Arm}$, boy, age nine years .................. 161

Osteomyelitis of the Shaft of the Tibia, child, age nine years ............. 210

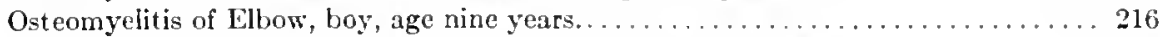

Tuberculosis of the Epiphysis of the Tibia, child, age nine years............ 259

\section{ELEVENTII YEAR.}

Normal Child, age ten years............................... 21

Normal Knees, Lower Legs and Foot, child, age ten years............... 22

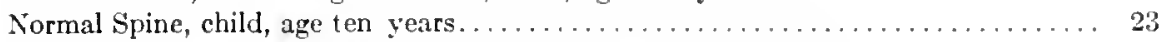

Congenital Deformity of Hands and Arms, boy, age ten years.............. 51

Congenital Dislocation of the Radius and Ulna, boy, age ten years........... 53

Congenital Dislocation of the Left Femur, boy, age ten years............... 60

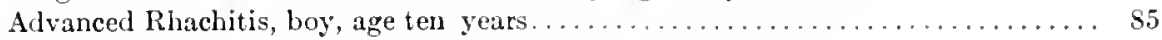

Lobar Pneumonia, boy, age ten years............................... 113

Acute Miliary Tuberculosis of the Lungs, boy, age ten years.............. 120

Encapsulated Empyema, boy, age ten years........................ 131

Dilated Heart, boy, age ten years............................. 132

Periosteal Sarcoma-Lower End of Femur, boy, age ten years............... 165

Calluscs of Feet, girl, age ten years . . . . . . . . . . . . . . . . . . 176

Abnormally High Arch of Foot, girl, age ten years...................... 177

Intracapsular Fracture of the Femur, boy, age ten ycars . . . . . . . . . . . 186

Dislocation of the Lower End of the Femur, boy, age ten years............ 187

Fracture of the Lower End of the Humerus with Inward Displacement of the Lower

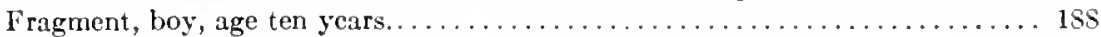

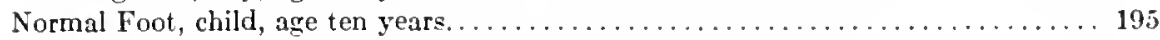

Epiphysitis of the Upper Epiphysis of the Tibia, girl, age ten years........... 199

Effusion of the Knee-joint, boy, age ten years........................ 206

Chronic Osteomyelitis of the Shaft of the Femur, child, age ten years........... 211

Undetermined Infection of the Lower Epiphysis of the Tibia, boy, age ten years... 230

IX

TWELFTH YEAR.

Dislocation and Fracture of the Anatomic Head of the Humerus, boy, age cleven years 181

Fracture of the Astragalus, boy, age eleven years..................... 193

Osteomyelitis of Fibula, girl, age eleven years...................... 229

Syphilis of the Lower End of the Humerus, boy, age cleven years . . . . . . . . . . 235

Normal Hand, Group $\mathbf{K}$, girl, age eleven and one-fourth years . . . . . . . . . . . 35

Normal Hand, Group L, girl, age eleven and thrce-fourth years.............. 39

IX

LI

IX

IX

II

II

\section{THIRTEENTII YEAR.}

Normal Child, age twelve years......... . . . . . . . . . . . . . . 24

Normal Elbow, child, age twelve years........................... 25

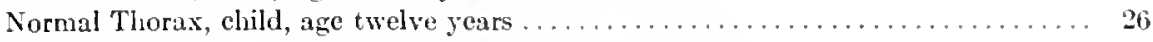

Rhachitis of Adolesccnee-Hand, child, age twelve years ............... 89

Osteomyelitis of Lower Jaw, colored boy, age twelve years................ 92

Enlarged Bronchial Xodes, girl, age twelve ycars.................... 109 
Doulde Pneumocoecus Loluar Pneumonia, boy, age twelve years . . . . . . . . . . . . 112

Probable Old Tutereular l'rocess of the Lung, child, age twelve years. . . . . . . . . 122

Acute Niliary Tuberculosis of Both Lungs, girl, age twelve years. . . . . . . . . . 124

Pericardial Effusion, child, age twelve years . . . . . . . . . . . . . . . . . . . 133

Enlarged Ifeart with Perieardial Effusion, child, age twelve yoars. . . . . . . . . . . . 134

Aneurism, boy, age twelve years.............................. 136

Abdominal Ascites, girl, age twelve years......................... 141

Nail in Right lung, boy, age twelve years....................... 147

Hamatoma of IIcel, ehild, age twelve years ...................... 162

Nedullary Sareoma of the Lower P':

Exostosis of Astragalus, boy, age twelve years . . . . . . . . . . . . . . . . 17

Exostosis of the Tibia, hoy, age twelve years...................... 175

Backward Displacement of the Inner Contyle of the Iemur, boy, age twelve years . 179

Fracture of the Neck of the IImerus, boy, age trelve years.............. 182

Green-stick Fracture of the Radius, girl, age twelve years ............... 159

Fracture of Tibia with Compensatory Growth of Fibula, girl, age twelve years . . . 196

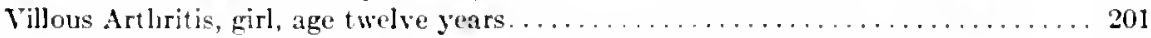

Osteomyelitis of Iower Lind of Tibia, boy, age twelve years............... 225

Osteomyelit is of the Lpper End of the Left Tibia, boy, age twelve years . . . . . . . 227

Early Stage of Osteomyelitis of the Lower Extremity of the Tibia, boy, age twelve

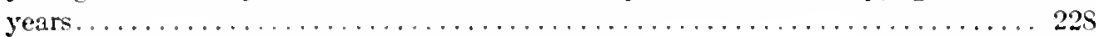

Atrophic Condition of Ilands, boy, age twelve years.................... 232

Syphilis of Lower End of Tibia, girl, age twelve years ................... 241

Infection of Netatarsal Jone, girl, age twelve years . . . . . . . . . . . . . 242

Atrophy of Knee from Disuse, girl, age twelve years. . . . . . . . . . . . . . . . 243

Tuberculosis of the Femur and Acetabulum, boy, age twelve years........... 250

Acute Tubercular Infection of the $\mathrm{Os}$ Caleis, boy, age twelve years . . . . . . . . . 263

Double Congenital Dislocation of the Hip, girl, age twelve and one-half years..... 59

\section{FOURTEENTH YEAR.}

Right Side of IIead, boy, age thirteen years...................... 27

Retarded Development of the Pisiform Bone and General Anomalous Condition,

child, age thirteen years...................................... 67

Rhachit is of Adoleseence, Normal and Rhachitic Hands, boys, age thirteen years... Ss

Fracture of Skull, boy, age thirteen years ........................... 90

Anomalous Bicuspids, Left Side of llead, boy, age thirteen years............ 93

Anomalous Lower Bicuspid, Right Side of Ilead, boy, age thirteen years........ 94

Stone in Urethra, Encapsulating a Pin, girl, age thirteen years............... 143

Premature Ossification of the Lower Epiphysis of the Radius, ehild, age thirteen years 158

Extreme Atrophy, boy, age thirteen years ........................ 166

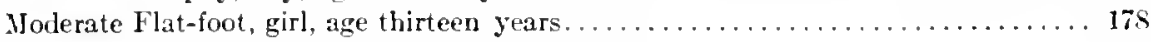

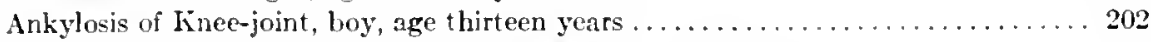

Normal JIand, Group M. girl, age thirteen and one-half years . . . . . . . . . . . 40

III

Chondrodystrophia Fotalis, boy, age thirteen and one-half years ............ 66

\section{FIFTEENTII YEAR.}

Supernumerary Tooth, Right Side of Ilead, girl, age fourteen years.......... 97

Supernumerary Tooth, Left Side of Ilead, girl, age fourteen years............. 95

The End Result of an Old Tubercular I'rocess, child, age fourteen years ........ 252 


\section{EXACT AGES UNINOWN.}

Examples of Comparative Density INTRONUCTION

Roentgenograph of a Congenital Deformity of the Foot................. 55

Various Anomalous Conditions Connected with the Teeth (some of the ages are given

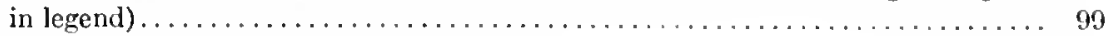

Tuberculosis of the Spine, Ilium and Left Ilip.......................... 102

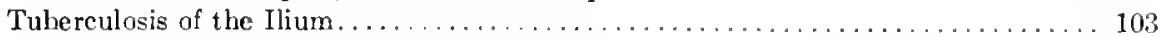

Tubercular Abscess of the Spine ................................. 104

Transposition of Organs-Tuberculosis of the Lungs and Bronchial Nodes . . . . . . 110

Pericardial Effusion and Obliteration of the Cardiohepatic Angle............... 135

Calcified Mesenteric Nodes. . . . . . . . . . . . . . . . . . . . . . . . . . . . . . . . . 142

Foreign Body in Esophagus .................................... 145

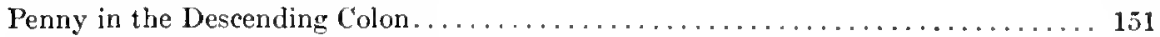

Needle in the Knee-joint . .................................. 152

Needle in the Finee-joint. Same subject as Plate 152, different position........ 153

Needle in the Foot ........................................... 154

Needle in the Foot. Same subject as Plate 154, different position. ............. 155

Needle in the Tissues Around the Phalanx of the Little Toe................ 156

Early Ossification of the Upper Epiphysis of the Tibia..................... 160

Subperiosteal Hemorrhage of the Left Leg............................. 170

Exostosis of the Lower Part of the Femur.......................... 174

Fracture and Displacement of the Head of the Humerus.................... 180

Old Fracture of Tibia and Fibula with Connecting Bridge................. 194

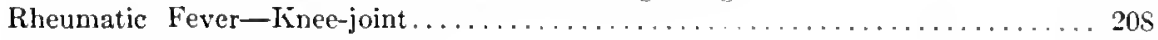

Rheumatic Fever-Ankle-joint .............................. 209

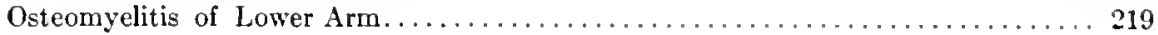





\section{LIVING ANATOMY AND \\ PATHOLOGY}

THE DIAGNOSIS OF DISEASES

IN

EARLY LIFE

BY THE

ROENTGEN METHOD

\section{Introduction}

Is a clinical text-book very little space should be given to descriptions of apparatus and to technic. These details should be acquired from books written for this purpose. The general principles should of course be understood, just as are the physics of auscultation and percussion or the principles of the ophthalmoscope and of the electric battery. In using these methods of investigation we do not attempt to remember all the mechanical details, but begin at once to obtain clinical information from them. To read intelligently and understand what is shown to us by a Roentgenograph it is important that we should first have mastered a knowledge of the appearance of the gross anatomic and pathologic conditions as shown by post-mortem examinations. We can then use the knowledge acquired in this way to compare it with the same conditions as shown by the Roentgen method in the living subject. The purpose of this book is to teach exactly what is seen by the student when he examines a Roentgen plate or a print developed from it, and to depend on his own eye to interpret the conditions presented to him. In reading through another's eye the effort for original observation is diminished and the value of the knowledge thus obtained is reduced to the level of a diagram, being of about as much value for teaching as is a diagram in a book in comparison with what is taught to the student 
by the use of the stethoscope. The difference in the methods of aequiring information on the one hand from hearing and touch and on the other from sight is that in the former case the expert instructor stands beside the student and educates his ear by telling him what he hears. On the contrary, by the Roentgen method the expert interpreter need not necessurily be present, but can be replaced by an expert explanation in a book, provided that such explanation describes accurately what the Roentgenograph shows. The personal element should in fact be eliminated as much as possible, because we all know how mistaken we may be and what different opinions are given in regard to what is heard and felt by different individuals. On the contrary, the eye does not err to the same extent. A number of pathologists do not differ so much in an opinion as to what they actually see in a liver at the postmortem as do a number of elinical experts as to what they hear in the lung or feel in the abdomen during life. This is because our senses of hearing and of touch are not constant, while, on the contrary, a photograph of the object is constant and gives reliable information. The Roentgenograph gives an actual picture of the lung, heart, and other organs ante mortem, just as the photograph of the liver, lung, or heart gives such accurate information post mortem. In either case the personal element is eliminated and the text can describe what a number of expert Roentgenologists can commonly agree to be present in the picture, just as a number of pathologists can agree in examining an organ at the autopsy. As to how much error occurs in and what can or cannot be seen in a special Roentgenograph will be explained later. It is fair to assume, however, always allowing that Roentgenology is in its infancy, and also that we cannot at present always read what is seen in the plate, that the picture as shown is true. Misconceptions may arise from a distortion of the angle at which the picture is taken, or from some faulty technic, or from lack of training in the interpre- 
tation of what the eye is actually looking at. All these obstacies to a correct diagnosis will in the future gradually disappear as progress is made in perfecting the electrical machine and when practitioners become as expert in the use of the plate or Roentgenograph as they are now in the use of the laryngoscope and of the ophthalmoscope. In order to obtain the most exact knowledge of disease, every practitioner of medicine and surgery should be taught what the Roentgen method is, and should have at his command pictures which can be readily explained. These explanations can be accomplished by a description in the text of the illustrations, aided by leaders designating the important details. These if closely studied will make the diagnosis for the observer and will be of incalculable aid in treatment.

Our knowledge of both the normal and pathologic anatomy of human beings has heretofore depended almost entirely on the postmortem findings. These findings, although of great use, especially under abnormal conditions, have never been so complete as is desirable when the different stages of development are under consideration. Still greater difficulties arise when we attempt to determine the primary pathology of the various diseases. In many instances we are dealing only with results which may represent the terminal lesions of a number of diseases, and yet fail to give us the early and characteristic lesions of the especial disease. In other words, it is important to obtain an exact knowledge of the living pathology of the part affected from the time of the earliest deviation from the normal which it is possible to recognize, as the dead pathologic conditions may only give end results, and may be end results common to a number of different infections. Any means by which we may recognize a disease in its early stages, whether it be an effusion in the pericardium or in the pleura, or obscure centres of pulmonary solidification, or an osteomyelitis, or a clin- 
ieally hidden fracture, is of extreme value, and indeed a necessity according to our modern ideas of diagnosis for treatment. This means, more or less perfected, has been given to us by the Roentgen method, and the knowledge derived from this method, teaching us the actual living conditions, is becoming more and more extensive and exact. The constant study of ehildren in health and in disease by the use of the Roentgen method will give us information of a large part of living normal and pathologic anatomy. This will enable us to judge of a diseased lung or other organ ante mortem, while prior to the discovery of the Roentgen ray we could only obtain our information from the findings of a post-mortem examination. It is therefore important that the results of the studies which have been made of a great variety of pathologic conditions by means of the Roentgen ray should be recorded and reported to the student of medicine in conjunction with his other medical studies. The Roentgen method in the practice of medicine assists rather than replaces any of the known means of diagnosis. It has opened up a new field in the study of health and of disease in both normal and pathologic conditions. It is not confined to the bones, but is applicable to all conditions of the tissnes which from their histologic nature may be differentiated by the Roentgen ray.

As is well known, there are two methods of obtaining information concerning the living normal and living pathologic conditions by means of the Roentgen ray. One is by the use of the fluoroscope and the other by the Roentgen plate. Each has its own advantages for diagnostic purposes. It is as useless, impractical, and narrow to praise one at the expense of the other, as it would be to discuss the individual merits of auscultation and percussion from the point of view that one should be used rather than the other. In one case, as in the other, it is the use of both methods which alone can give all the information ascertainable in health and in disease. There 
is no doubt that the fluoroscope in the hands of an expert will give especially valuable information of the part examined. The expansion and contraction of the lungs, the rise and fall of the diaphragm, and the expansion and contraction of the muscles of the heart can by this method be actually seen and studied, as can also the peristalsis of the stomach and of the intestines. By this method we can determine the normal excursions of the diaphragm as differing on one side from the other, and the greater or less distention of the lungs. We can thus also establish a standard which can be accepted as within the limits of normal variation according to the stage of development of the individual. In this way the trained eye is enabled to recognize the normal, to detect the abnormal, and to see the living rhythmic movements of the heart, the lungs, and the diaphragm. Bearing the normal standard in mind, and recognizing a greater or less excursion of the diaphragm, according to the greater or less expansion of the lung, we can decide whether we are looking at a normal or abnormal diaphragmatic excursion. If abnormal, and knowing that the lungs when over-distended show a greater radiability, and that when the pulmonary tissue is denser, or when the alveoli contain an exudate, they show a lessened radiability, we can infer that areas of disease are present. Also that in over-expansion we should suspect emphysma, while in deficient expansion we should infer a condition of atelectasis or of solidification. In this connection the use of the fluoroscope is of the greatest importance in the diagnosis of such conditions, as for instance an early tuberculosis. The other side of the question in regard to the fluoroscopic method is that the sense of sight may vary, although not so much as does that of touch and of sound. Even experts may differ in an opinion as to what they see, just as experts in the use of the stethoscope and pleximeter may differ as to what they hear or feel. It is also true that a good Roentgen plate tells the truth and records it 
accurately. The plate may be poor and diffieult to read, but if we grant a good fluoroscopic expert we must atso grant an excellent plate. Such an expert, howerer, with the ever moving image of the picture, which he can simply deseribe and not record exeept in tracings made by his own hand, orthodiagraphic or otherwise, is invaluable on account of his skilled observation. The Roentgen plate and the record which it makes is absolute and ean be referred to, studied, and discussed by any number of observers. There is no error in the plate due to a personal equation or to temperament. It can be studied as are photographs of the moon, which are far more valuable for exact rudimentary teaching than what can be seen through the teleseope. When, moreover, the question arises of illustration in a book for the purpose of teaching the Roentgen method, for the elueidation of diseases of all parts and organs, it is evident that the plate should be used.

To take a Roentgenograph successfully a number of points are essential. A great deal depends upon the personality and knowledge of the operator and interpreter, and in the ease of children on his patience and personal influenee. The operator should have meehanieal skill and great intelligence in making use of that skill. As an interpreter he should be as little as possible influenced by individual personality. He should also have an intimate knowledge of the pathologic conditions which have been studied and recognized as representing the various diseases as they appear post mortem. It is of course presupposed that he has also a thorough and detailed knowledge of the conditions which represent living normal anatomy at different periods of its development. In faet he should be both an anatomist and a pathologist. The degree to which pathologic conditions can be recognized in the Roentgenograph depends upon what ean be clemonstrated on the Roentgen plate. It is the grosser pathology which can be best recognized in the interpretation, 
since the finer pathologic conditions shown by a microscope will not produce effects which can be differentiated by the eye. The Roentgen picture is obtained by the greater or less atomic weight of the tissue which the ray penetrates, as shown by the greater or less obstruction of the ray. For instance, if the radiability is great, as in the case of normal lung tissue, the dark surface of the plate is shown, while the neighboring vertebræ and ribs, owing to their increased density, obstruct the passage of the ray and are shown on the plate as white. When a print is taken from such a plate the reverse of this result takes place, the lung becoming light and the bone dark, and the right in the plate becoming the left in the print. What the student must remember, therefore, in considering the pictures on the plate, is that the greater the atomic weight of the object the greater is the density and the less the radiability; also that the nearer the object is brought to the plate the more precise and less distorted is the picture. Thus, to take the two extremes, if a cavity is filled with air the effect produced on the plate is practically nil, while if the cavity is filled with water the resulting picture is lighter according to the greater depth of the cavity. The student should therefore remember what radiability means, and appreciate that innumerable degrees of light or dark effects can be left on the plate corresponding to the innumerable degrees of density resulting from a difference in the atomic weights. When the student has mastered this idea he will learn to recognize first the radiability of normal tissue, for instance, what the picture of the normal lung with its great radiability should be, and then what is seen in a diseased lung when the radiability is decreased. Again, in reading the picture produced on the plate in abnormal conditions of the abdominal cavity, he must bear in mind that the greater the amount of fluid the less is the radiability and the lighter the resulting effect. He must also note the greatly lessened radiabil- 
ity in the region of the liver, spleen, or kidney, and the abnormal size and density of the picture produced by an enlarged liver, spleen, kidney, or abnormal object such as a gall-stone, a renal caleulus, or a foreign body. It should of course be understood that the picture on the plate may not necessarily be uniformly clear. On the other hand, a clear picture of a part may be obtained by skilfully concentrating the ray on that part. It should also be understood that the print from the plate is never so clearly defined as is the original pieture on the plate itself, even in the hands of one whose technic is excellent. It, therefore, becomes merely a question of improved technic and perfected reproduction. What we wish to teach the student is to eultivate his powers of observation and to recognize what the illustration shows. This can be done by means of leaders indicating the especial parts or details of the parts explained in the text. It is evident that a very close and extended study of Roentgen plates in general should be made, in order to recognize the innumerable differences presented to the eye by a Roentgen illustration.

The different tissues of the body are made up of different elements, such as nitrogen, oxygen, calcium, phosphorus, and many others, each of which has its own atomic weight. We can therefore anticipate what the radiability of a given tissue, such as fat, musele, and bone, will be, according as we know of what elements it is conposed. Knowing the atomic weights of these elements we can then deduce that there will be a greater or less radiability. Thus where ealcium is present, as in the bones, its high atomic weight (40) would produce a lessened radiability, while water, with its low atomic weight of hydrogen and oxygen, would allow of a high degree of radiability. Again, where much air is present in the medium through which the ray has to penetrate, the obstruction is almost nil. The radiability of air is so great that in the Roentgen plate there is a 

PI.AT: 1.

LXAMPLE' (H (OMI'ARATIVE DENSITY

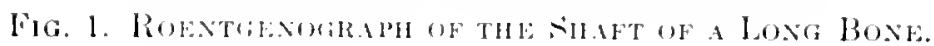

1. Cintex.

13. Mrilutiat.

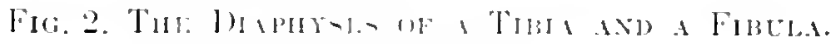

-1. Cortex uf the tibiat.

b. Cortex of the fibulat.

c. Irea of increatal density from one bone overlatping the other.

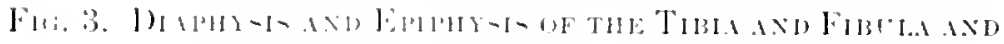

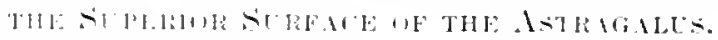

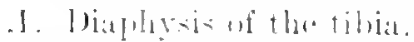

13. Miaghyeis of the fihula.

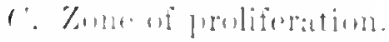

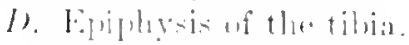

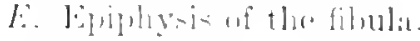

F. A-tritatur.

11. Coutilage.

1. Hark atrat fom orerlaphing of the diaplyeses of the tibiat : the of the fibulat.

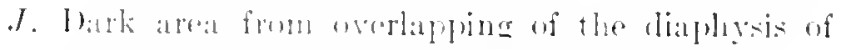
the fibulat and the epriphyers of the tibiat.

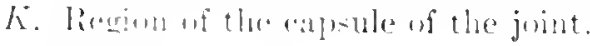

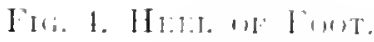

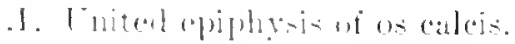

li. Fitt.

$\because$ Spare uncupied by artilage between os calcis and rubuil.

1). Plantar farciat.

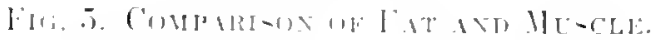

.1. liat.

li. M M11:r.t.

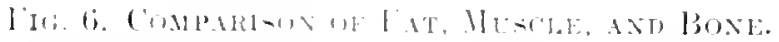

.1. Fitt.

Ii. Huscle.

'. Outline of bune.

(All these figures atre taken from different plates.) 
FIG. 1

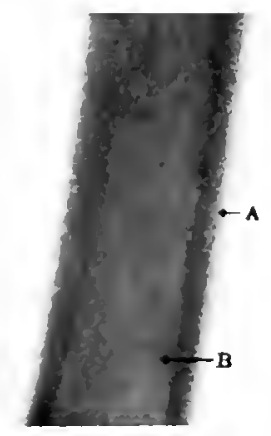

FIG. 2.

FIG. 5 .

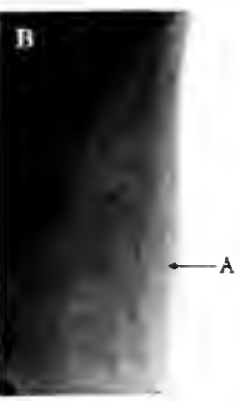

FIG. 3.
Pldate 1

FIG. 6 .

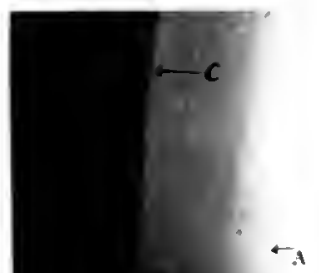

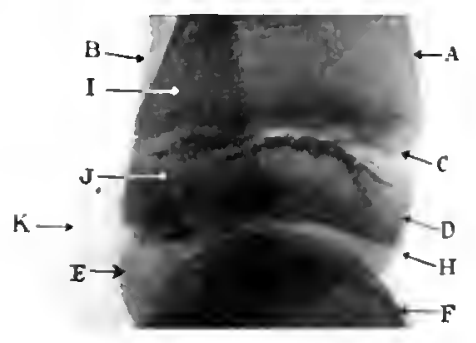

FIG. 4 .

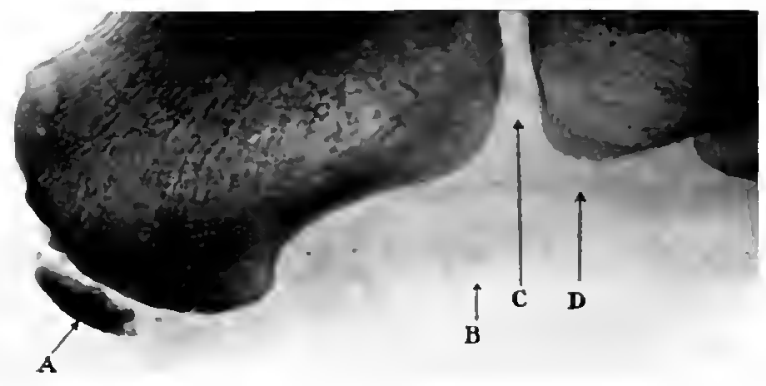



very marked difference in the effeet produced between, for instance, an emphysematous lung and an abdominal ascites. Of course, when tissues through which the ray passes have a low atomic weight, the radiability differs aecording to the number of layers of such tissues, or in other words the denser the tissue the less the radiability. The atomic weight of blood and that of water being so nearly alike, there is no great difference in their radiability. In order to explain diagrammatieally what has just been said, I have shown in Plate 1 the different degrees of radiability of different tissues and of the same tissue.

Plate No. 9 (Div. I) shows the comparative radiability of the lung, heart, spine, liver, intestine, and stomach.

The illustrations in Plates 1 and 9 emphasize that no one definite picture can be taken as a standard of one especial tissue, since the pictures of any of the tissues may differ considerably. This is shown in the difference of the radiability of the cancellous tissue in Plate 1, Figs. 2 and 3, leader A, and the musele in Figs. 5 and 6, leader B. All the reproductions in Plate 1 are taken from different prints.

It must be understood therefore that the greater or less apparent radiability of an organ on one plate, for instance, of the effect produced by a heart, may differ greatly from the apparent radiability of a heart on another plate, since the degree of radiability is determined by comparing it with the liver, lung, bones, and tissues on its own plate. Following from this the student must understand that there is no one espeeial degree of density which he is to expect to find in an individual plate. The radiability as shown in Plate 1 represents what ean be seen in a Roentgenograph, and in no way presupposes that the degree of density shown in these examples will always necessarily appear when the student is examining a plate for diagnosis. In other words, all the knowledge which is derived from 
a Roentgen plate rests on a basis of compirison. The student must also understand when he is examining a print from a Roentgen plate that he should consider on which side of the part taken is the plate and that he is supposed to be looking through the plate at the part. He nust, for instance, remember when he is looking at the trunk that he is looking at the individual from behind when the individual has his back on the plate. He must also remember, from our knowledge of the chemistry of the reproductive process, that, while the greater radiability of the lung tissue shows dark on the plate, it will show light on the print. In like manner, therefore, all light effeets in the plate, such as of bone, heart, or liver, will show dark in the print.

Like many other means for diagnosis where the teehnic is difficult, diseredit has been thrown upon the value of diagnosis by the Roentgen method. This diseredit comes from a number of causes. First, the technic is diffieult to aequire, and often has to be aceomplished by means of imperfect apparatus. Again, a systematic method of studying the plate, and thus interpreting what such a plate should show, is usually not employed. We must also understand that we often eannot expect to have as clear a pieture in disease as in health. Given the same apparatus and the same skill in taking the pictures, the rery indefiniteness of surface and of outline makes us suspect a cliseased condition on comparing the pieture with the same parts in their normal condition. In disease the greater or less atomic weight of the lungs and of fluids in the pericardium or pleura may throw a haziness over the whole pieture and may obseure anatomie details which under normal eonditions are very erident. This very haziness to the eye of the skilled interpreter becomes a definite entity and determines the diagnosis.

Different Roentgenologists prefer different positions of the subject to be taken. The plate placed against the back or front 
naturally produces pictures which, according to whether the parts are nearer or farther away from the plate, show more or less distinctly. In taking the illustrations used in this book we have chosen for our routine position the subject's back directly against the plate. In this position we do not get the picture of the sternum and front of the ribs; these we take with the subject on the side, since pictures of the sternum taken with the subject on its face are unsatisfactory owing to the interposition of the vertebra. Pictures which are taken with the back on the plate show what would be seen with the front part of the thorax removed, that is, the sternum, ribs, and cartilage about as far as the anterior axillary lines.

It is the faulty technic in taking a Roentgenograph which invalidates accuracy, and a satisfactory interpretation is often lost by not approaching the subject in a systematic manner, such as we would use in making a physical examination of a patient. Before describing a uniform method for examining and interpreting the plate it is necessary to understand the difference between a photograph and a Roentgenograph. The photograph of an object shows in its negative only its exterior, which is reflected on the samera and on the eye, giving the outline, the shape, and the surface markings. In the Roentgenograph, the plate of which is positive, we do not see the exterior of the object, with the exception of its outline, shape, and prominences, but we get the details of its internal or general structure. Therefore in order to interpret Roentgenographs correctlyit is necessary that we should not only be familiar with the external anatomy of the part to be examined but also with the interior structure. In studying a given plate certain points must be considered and the same method followed as employed in the clinical diagnosis of the lungs, pleura, and heart. In the examination of a Roentgenograph in reference to an extremity or to an organ, the general location, size, shape, and position should always be considered in 
comparison with what is known of such part or parts. For instance, in an examination of the thorax a variation from the normal, such as a deficiency in the number of the ribs, or a variation in the number on one side in comparison with the other, should be noted. In examining an extremity the general outline and the density of the part in question should be considered. Then we should study the picture as though a vertieal seetion had been made through its tissues, and we should compare such section with the section of what would be normal at the same stage of development. For example, in examining the Roentgenograph of a leg we should first compare the appearance of the fat tissue with what would normally be expected in the individual. We should next consider the muscle as to its atrophy or hypertrophy in comparison with normal muscle. Next we should observe whether the periosteum can be seen or not, knowing that under normal conditions it is not seen, and that if abnormal it may come into view. We next consider the cortical substance of the bonc as to whether in comparison with the normal conditions of the cortex at the same period of development it is narrower, indicating atrophy, or broader, indicating hypertrophy. We should also note whether it shows increased radiability, indicating absorption of the lime salts, or decreased radiability, showing thickening and greater density. Next we observe the medullary canal as to whether it is broader or narrower than under normal eonditions, and also noticing whether it is replacing the cortex of the bone or, if it looks smaller, whether it is being encroached upon by an internal thickening of the surrounding cortcx. All this is of importance in recognizing abnormal conditions, such as contusions, abscesses, atrophy due to nerve lesions or to general abnornal changes, such as growths or the presence of foreign bodics.

A short résumé of what has been stated already in regara to the detailed examination of a Roentgenograph may be of use to the student. 
1. We should always compare the part of the individual under observation with the corresponding part of the same individual. This is often of great value where there may be only a slight deviation from the normal, as for instance in an arm from the normal arm on the other side.

2. We should notice not only any change in the size but in the density, and any abnormal shape which may be present.

3. Subcutaneous Tissue.-In examining the subcutaneous tissue we should note, in comparison with the corresponding opposite side, its density, its thickness, and its shape.

4. Muscle.-In examining a muscle we should note any abnormality of outline, density, thickness, or shape, as denoting inflammation, abscess, foreign bodies, or new growths.

5. Bone.-In examining a bone we should note the general outline, comparing the age of the individual under examination with what is known anatomically of a given part in regard to its age and size. We should also note whether the periosteum is visible or not, and whether there are any irregularities such as might be caused not only by changes in the periosteum but also by exostoses. By observation and experience, therefore,

$A$. We should set a standard for ourselves as to the normal densities of the bones and of the tissues;

$B$. We should then recognize a condition of atrophy, distinguishing

a. Atrophy from disease;

b. Atrophy from disuse;

c. Atrophy from a combination of both.

$C$. The size of the cortex and of the medullary canal and their relations to each other should be considered.

Certain groups of diseases in early life, such as diseases of nutrition, are characterized by a diminution or an increase in surface and outline, and by a greater or less density of the bone. 
In examining for abnomal conditions of the cortex we should note its density and its size as compared with the medulla. The examination of a normal bone shows a uniform definition of the cortex, varying according to the density and size of the special part of the bone, but this again varies in different bones. For instance, the cortex of the femur at the upper end is thinner than the cortex at the middle of the shaft. A knowledge of the different variations in the size of the cortex is important. It will not vary, however, to any great extent in different individuals. In certain diseases, such as rhachitis, osteomalacia, and osteogenesis imperfectal, the density of the cortex will vary from that of the normal to a greater or less extent. Thus from our knowledge of the normal cortex we will be able to tell from the plate that there is a change in or a disease of the bone. It is important to compare the cortex with the medullary canal, since in certain conditions it will be found that the medulla increases at the expense of the cortex, or that the cortex increases at the expense of the medulla.

In examining the medullary canal we should note its density and compare it with the cortex, bearing in mind what should be normal in the individual stage of development present. The medullary canal can be demonstrated very definitely by the Roentgen method. It varies in density and in width in the different bones and parts of bones, and here again is impressed upon us the importance of knowing how each bone of the skeleton looks at different periods of development. This leads to the differentiation between atrophy of size of the bone and atrophy from a change in its quality or from osteoporosis.

We should also examine the structure of the bone, observing whether there is anything abnormal in comnection with its cancellous tissue or with its trabecule. It is in the differentiation of the different structures above mentioned that the Roentgen ray is able to 
detect disease. A knowledge of the structure of a given bone of the skeleton at different stages of its development will, in connection with the other points just mentioned, lead to an understanding of the early changes in a part or in the whole of the bone. In certain infectious processes of the bone-marrow, where there is an infiltration or an abscess, the normal structure of the bone will be seen to be destroyed or to be replaced by foreign material. Again in diseases such as rhachitis the structure of the bones will be seen to adapt itself to abnormal changes so as to compensate for the superincumbent weight.

6. Periosteum.-The periosteum in a normal bone is not seen, and it becomes apparent only when there is an exudate beneath it, or when it is inflamed or thickened.

7. Epiphyscal Line or Zone of Proliferation.-We should examine the epiphyseal line or zone of proliferation with great care, because it is by a knowledge of its normal appearance that slight changes, such as from infection, from disturbance of nutrition, from trauma, from epiphyseal dislocations, from fractures, and from many changes that may affect its uniformity are revealed by the Roentgen method. Fractures should be looked for, whether partial or complete. The attachment of the tendons, as represented by depressions or prominences, should be noticed. Spur formation or hypertrophic osteophytes should be recognized. No two bones of the same part will show the same outline, but it is the knowledge of the normal and the variation from the normal which makes the interpretation of a slight degree of irregularity from disease possible.

S. Epiphyses.-It should be noted whether the epiphyses of the various bones have appeared, their size, and their condition.

9. The centres of ossification should be carejully noted.

We should always bear in mind that what would be normal for one period of development may be abnormal for another. Therefore 
a knowledge of the different stages of development is of the greatest importance in determining whether disease is present or not. We must admit that we are studying histology and pathology in its most important aspect, that is, under living conditions, and that a knowledge of these conditions is exceedingly valuable not only for diagnosis but for treatment. We must remember that although the knowledge we obtain from the microseope is of very great value, yet at times macroscopic are as useful as microscopic pictures. A magnifying glass is of great use in studying reproductions from Roentgenographs. 


\section{Division I}

\section{LIVING NORMAL ANATOMY}

A THORough knowledge of living normal anatomic conditions should be acquired before we can understand what we see in living pathologic conditions and recognize the lesions of the various diseases during life. This would be comparatively simple if we could merely study the normal living anatomy of the fully developed adult, and of course such study is necessary and is very important. This living, fully developed anatomy of adults, however, is only one link of the much more extensive and complicated chain of evidence which enables us to diagnosticate normal anatomic conditions through all their stages of development in early life. During early life manifold changes take place continuously, and the younger the individual the more rapid are these changes. Therefore a knowledge of the living anatomy of each period of growth is of the utmost importance for the recognition of anomalies, of congenital conditions, and of disease. The study and use of the Roentgen method gives us a large part of the living anatomy and pathology of either an early or late period of disease, and thus enables us to decide upon the nature of the disease long before the findings of the post-mortem table can be utilized. Even anatomists know very little regarding the changes which take place in connection with the appearance and development of the centres of ossification at different ages, yet without this knowledge we cannot intelligently treat a number of diseases.

During the development of the bones the epiphyseal line illustrates the relationship of the diaphysis and epiphysis to each other and to their surrounding structures. From this it will be seen that the epiphyses present a very definite anatomic appearance at differ- 
ent periods. This not only oeeurs in the epiphyses of a speeial joint, such as the wrist, but in the epiphyses of the different joints as compared with each other. Thus the long bones have a large epiphysis at each end, while the clavicle, the metatarsal bones, and the phalanges of the foot and hand have only one. It is important to appreciate that the various epiphyses gradually appear and grow larger and larger according to the stage of development of the individual. An exact knowledge of their comparative development is therefore of great use. We know that height depends upon the development of the epiphyses. Suppose we are examining a child twelve years old who has the height of a child of six years, and we wish to determine what the chances are for his future inerease in height; that is, whether he will eventually be a dwarf. On examining the epiphyses, if we find that they correspond to the normal assification of a child of twelve years, the probability is that the child will grow very little more in height. If, on the contrary, we find that the degree of ossification corresponds to the normal epiphyseal development of six years, there is a chance that the growth in height may begin again. It is manifest, therefore, that unless we have this exact knowledge of the changes in even so small a part of the entire skeleton as the epiphyses, absolutely normal conditions may be mistaken for abnormal. This all the more emphasizes the importance of beginning our study of Roentgenology by acquiring a thorough knowledge of the normal living anatomy of the various stages of development.

To obtain correct data of these normal anatomic conditions is not a simple question, since comparatively little aid is obtained from the many anatomists who have written on dead anatomic conditions. The fact is that the knowledge of normal living anatomy up to the present time has mostly depended upon what has been derived from dead anatomy. In addition to this the anatomist has relied 
greatly on the chronologic age of the individual whose dead anatomy he has investigated, and has stated from the results of his tabulations that a certain development takes place at a given age. We are now led to believe that this chronologic dead anatomy is far from eorrect, and that it does not approach the constant standard which we need in making an exact diagnosis of anatomic conditions in health and in disease. We are also more and more impressed, when our anatomic studies are carried out on the living subject by means of the Roentgen method, that the different stages of development must be readjusted and made to eorrespond to some other condition more constant than the chronologic one of age in months and years. By taking a Roentgen ray of a large number of apparently normal children in different stages of development, it has been shown that ehronologic growth is far from exact for purposes of classifying children for school and athletics in comparison with the anatomic changes which take place from birth to pubescence. Crampton's work on the pubie hair in the latter period shows that there is a decided variation in the development of individuals of the same age. From some extensive work on this subject in which I have been engaged in the past two years, I have come to the conclusion that when we wish to determine the normal conditions of the various stages of life, some standard index, such as the changes in the epiphyses, is more reliable than the usually aceepted standard of months and years. This work is fully described in Division II. I have deduced that, given an individual supposedly normal child, the wrist and hand are the most practical anatomic parts to use as a general standard index on which to establish a elassification based on growth. From this reasoning I have concluded that if this hand and wrist standard of anatomie age proves to be superior in exactness to the ehronologic age usually accepted, it will be of the greatest use in school life. In this way also a proper adjustment of athletic 
contestants ean be made by grouping the corresponding stages of development together anatomically rather than chronologically, thus avoiding overstrain at a period of life when it is of the utmost importanee to do so. As much more work will be needed to definitely determine the shorter intervals of normal living development, I have provisionally grouped a number of ehronologie periods together on an anatomic hasis. The average periods of development determined in the past by chronologic age, weight, or height, are manifestly fallacious. We must make many observations based on a new system deduced from anatomic standards before healthy children can be clissified according to what they, as individuals, need at certain periods of development rather than because they are of the same chronologic age. The exact meaning of this is that a group of children of the same chronologic age in months and years may represent perhaps four or five anatomic ages.

In order to establish certain standards by which we can provisionally determine anatomic age in early life I have studied Roentgenographs of healthy children representing groups of chronologic age corresponding to anatomic conditions. To interpret intelligently living anatomic conditions as shown by the Roentgen ray it is necessary to be conversant with the anatomic conditions which have been revealed after death. The most important of these conditions is found in that part of the anatomy of the bones which shows the development of the epiphyses and of the diaphyses. Much valuable knowledge can be acquired also from a careful comparative study of the organs and tissues, which is of great use in the differential diagnosis of various diseases by the ray. It is certainly very important that our knowledge of the centres of ossification of the bones at different periods of their development should be perfected. This can be accomplished best by studying the living normal anatomy of the bones by means of the Roentgen ray during life. In the series 
of normal anatomic pictures which I have introduced at the end of this division it should be noted that the centres of ossification are absent at first, then gradually appear as small plates, and finally assume their normal size. This serics of cases starts with premature life and is carried on to adolescence. The determination of abnormal conditions of the lung and pleura, the degree of cardiac enlargement, and the question of pericardial effusion in the less obvious cases must also of ten rest on the evidence obtained by the Roentgen ray.

This grouping by anatomic age is of importance surgically, because the surgeon when operating on an individual can by it often learn in what stage of development is the especial part on which he is operating. It is also of importance in medical cases to take into consideration the relation of the heart to the lungs, the relative size of each, and the different degrees of the cardiohepatic angle in its various stages of derelopment. The practical use of the pictures of these groups of ages in surgery and in medicine is obvious. A surgeon is about to operate on an injury to a joint, or a physician wishes to determine where he shall operate in a pericardial effusion. Their procedure naturally would be to have a Roentgenograph taken of the especial case before them. They will then turn to the group of chronologic age or rather to the actual anatomic division of age which corresponds to the anatomic age of the individual whom they are about to treat. The Roentgenograph corresponding to the anatomic age of the especial child with which they are dealing will then show them whether at that especial period of life the appearance which they interpret from the special Roentgenograph of their patient corresponds to the general Roentgenograph of my normal anatomic series. If it does correspond they will decide that the picture of their case is that of a normal condition, and therefore that they should search further in order to find the real diseased condition. If, on the contrary, the picture of their 
especial ease does not correspond to such normal Roentgenograph, they will then judge that they are dealing either with a pathologic process or with an anomaly and that the case should be treated accordingly. In like manner in looking at the Roentgenograph which represents the heart it will be seen that the cardiohepatic angle is quite distinet, and that by the recognition of this angle on the right in a doubtful case of suspected pericardial effusion, the Roentgen ray would aid us very greatly in differentiating a normal or an enlarged heart from an effusion.

Roentgenographs of the living normal development from the seventh to the ninth month of intra-uterine life show that there is comparatively little difference between these ages, with the exception that the upper epiphysis of the tibia and the lower epiphysis of the femur appear at the latter date

We can therefore adopt provisionally the period of growth from the seventh month of fetal life to birth as a group of anatomic growth, and call it Group 1 (Plate 2). This leads us to observe how long these early anatomic conditions continue, and thus for how long a period each group of normal anatomic conditions lasts.

By following out this method of determination we can represent roughly different groups of anatomic age. Thus the second chronologie group can be represented by a period of life lasting from birth to the time when the first bones of the wrist appear in their cartilaginous surroundings. In like manner other groups may be classified until the whole period of childhood has been gone over up to the thirteenth or fourteenth year. There is much to be said in regard to these intervening periods of growth, and much practical use to which they may be put. I have thought, however, that it would be best first to show a number of illustrations of the child's development in different chronologic periods, then, in a division of the book by itself (Division II), to show to what 
practical use this anatomic knowledge can be put in the everyday life of childhood. I have represented, therefore, in Division I in a general way certain periods of development of various parts of the entire body. I have begun with the premature infant (Plate 2 ), and ended with Plates 26 and 27, which include about the twelf th, thirteenth, and fourteenth years, more or less.

It is to be noticed that in a number of plates which represent otherwise the entire skeleton the heads do not appear. The reason for this is that it is extremely diffieult to obtain a good Roentgenograph of the head of a young baby unless it is etherized. Under normal eonditions it is seldom the case that the parents will allow the infant to be etherized for the purpose of scientific illustration. On the other hand, it is not difficult to obtain a Roentgenograph of the premature infant, since its breathing is very shallow and it often does not move its head for many minutes.

The acute angle made by the right side of the heart with the upper surface of the liver is ealled the cardiohepatic angle. This is well shown in Plate 9. It is of great importance to observe this angle carefully, as later it will be of much value in differentiating between an enlarged heart and a pericardial effusion. We should also note the areas occupied by the heart, the liver, and the abdominal organs.

After this preliminary outline, we can now appreciate why we should make a careful study of living normal anatomy first, and later acquire a knowledge of liring abnormal anatomy. Having once mastered the details of these living normal conditions through all their changes of development from the very beginning of life, the diagnosis of disease beeomes greatly simplified.

In order to appreeiate why the various parts of the bone differ in their radiability according to the constituents of which they are composed, I shall describe briefly the structure of the bones and their development. 


\section{BONE}

Bone is the hardest structure of the human body, but also possesses a certain degree of toughness and elasticity. Its color in a fresh state is pinkish-white externally and red within. On examination of a section of bone we find two linds of tissue, one of which forms the dense hard external covering and is called the cortex; the other, which forms the interior of the bone and consists of fibres and of lamelle which join and form a reticular structure, is called the cancellous. It is to the variations in the relative quantities of these tissues that the difference in weight and size of the different bones, and also of the different parts of the same bone, is due. The bones are permeated by blood-vessels and are surrounded by a fibrons membrane called the periosteum, by which the blood supply is carried to the cortex. The long bones have a cavity filled with marrow, and are lined with a vascular strueture called the medullary membrane.

Periosteum.-The periosteum normally adheres to the bone in nearly all parts, excepting its extremities, which in carly life are cartilaginous. It is thick and vascular, and is incorporated at either end of the bone with the epiphyseal cartilage.

The constituents of bone, according to Gray, are as follows:

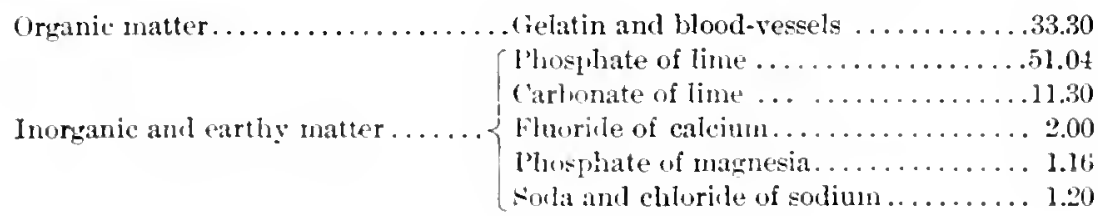

It is by the predominanee of the inorganic constituents of the bone with their varying atomic weights that we are able by means of the Roentgen method to differentiate the vilrious parts of the bone clearly.

As it is easier and more practical to study a Roentgenograph 
as a whole, and in order to appreciate all the advantages of this method, we should learn to look at the living anatomy of either head, thorax, abdomen, or limbs as a whole, and thus determine the dependence of one part on the other as representing the interdependence of all the abnormal conditions present. Having once mastered the picture in broad perspective, the especial disease for which we are looking can in this way be better differentiated and intelligently studied. When we have done this it is evident that, in such abnormal conditions as can be portrayed by the Roentgen ray, the Roentgen method is complete, rational, and satisfactory, and is therefore very important for the purpose of differential diagnosis.

Diaphyses and Epiphyses.-The long bones have an epiphysis at each end, with the exception of the clavicle, metatarsal and metacarpal bones, and the phalanges of the hand and foot, which have only one. In a premature infant of seven months the long bones have no ossific centres representing the epiphyses (see Plate 2 ). The epiphyses are the most important centres of the skeleton, for on them depends its future development, especially in infaney and childhood, and when these centres are impaired there result far-reaching influences in later life. A thorough knowledge of the different changes which take place in even these small areas is very important, for these centres are where disease may begin, from which disease may disseminate, and where it may lead to disastrous consequences by leaving its permanent marks.

The following table gives the time of the appearance of the ossific centres of the epiphyses, and represents the results of our study at the Children's Hospital of a large number of children.

It must, however, be remembered that most of these estimated figures depend upon dissections and in some cases are erroneous. Many of them have, however, been verified or corrected by our observations on living subjects. 
TABL: 1.-TME OF THE APPEARACE OF THE EPIPUYSE.

Clavicle

18 years.

I'IPEL EXTREMITY.

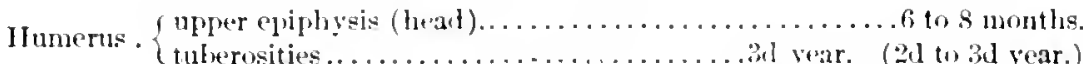

(a)pitallum ......... yt year. (20) to 3rl year.)

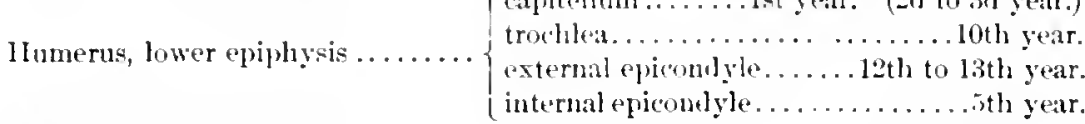

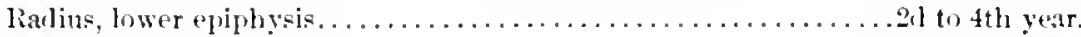

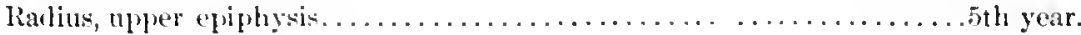

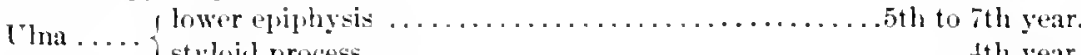

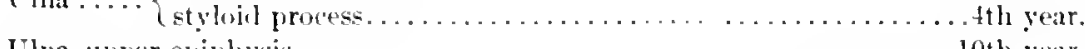

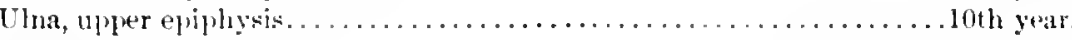

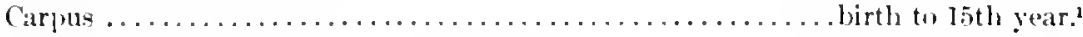

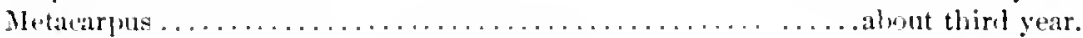

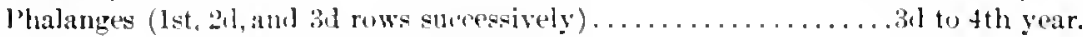

LOWER EXTLEMITY

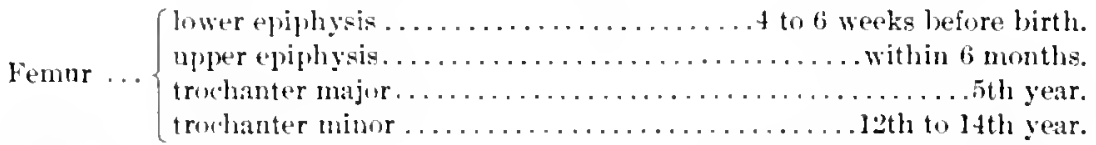

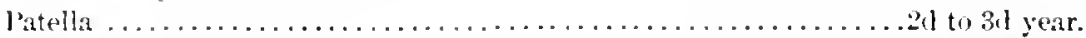

Tibia.... fupper epiphysis ................ sth to 9th month of fetal hife. $\{$ lower ejiphysia...............6 or l: nonths to 2 years.

fibula $\{$ upper epiphysis...........................

lower epiphysis ........................

Tarsus . .....................th month of fotal life to 4 th year. ${ }^{1}$

Metatarsus . . . . . . . . . . . . . . . . . . . . . . . . . . to sth year.

Phalanges ...........................th to th year (page 31 ).

Os innominatum ................ Sth to 19 th week of fetal life (page 3:).

1 For development of indivilual bones see pases $27-32$.

Development of Bone.-In the fetus up to about the fourth month the osseous sistem represented by the long bones is more or less cartilatginous, while that part which is represented by the cranial bones is intramembranous. At the third or fourth month of fetal life the shafts of the bones show a cortex and a medullary cavity, but at birth no epiphyses excepting those atbove mentioned, such as of the femur and of the tibia, are present.

There is also a third kind of ossification which is ealled subperiosteal. 
As the long bones are the most important in the osseous system and are better understood than the others, I shall mention in brief the changes which they undergo in their development. During the first three months of intra-uterine life (this varying in the individual fetus) the osseous system is practically cartilaginous. Soon after this a process begins in the centre and extends towards the extremities. Subsequently a process begins here and there in the end of the bone and gradually extends. The epiphyses, however, do not join the shaft until growth has ceased, and they remain separated by a layer of cartilaginous tissue called the epiphyseal cartilage or zone of proliferation. Early in the process of ossification the cartilage cells of the centres of ossification enlarge and arrange themselves in rows, which gradually are separated by an increase in the matrix in which they are imbedcled. A calcareous deposit now takes place in the matrix between the cells, which become further separated by columns of longitudinal calcified matrix, which gives a granular opaque appearance. Some of the matrix becomes calcified into transverse columns extending from one longitudinal column to another. In this way cartilaginous cells are enclosed in carities of an oblong shape with walls of calcified matrix. These cavities are called primary areolex.

Humerus.-The humerus usually develops by seven centres, but sometimes by eight:

One for the shaft.

One for the capitellum.

One for the head. One for the trochlea.

One for the tuberosities. One for each condyle.

At the eighth week in fetal life the nucleus of the shaft appears, and at birth the whole shaft is practically ossified. The extremities, however, remain cartilaginous. The ossific centre first to appear after birth (normal) is the capitellum at about the first year. At the sixth month an ossific centre of the upper epiphysis of the hu- 
merus appears at about the sume time as the head of the femur. At the beginning of the third year a centre for the tuberosities appears. This ossification is sometimes, but rarely, by two centres. During the middle of the fifth year or later the centres for the head and tuberosity have united to form a single large epiphysis. The eartilage, however, may persist and on examination with the Roentgen ray be confused with a fracture. The lower end of the humerus beside the capitellum has the ossific centre for the internal condyle at the fifth year. The external condyle appears about the twelf th to the thirteenth year.

Radius.-The radius develops by three centres:

One for the shaft (eighth to ninth week of fetal life).

One for each extremity.

At birth the bone is well ossified, but at the beginning of the third year the ossific centre for the lower epiphysis may appear, and between the fifth and sixth year the ossific eentres for the upper epiphysis appear.

Ulna.-The ulna develops by three centres:

One for the shaft (eighth week of fetal life).

One for the lower epiphysis.

One for the olecranon.

At birth both extremities are cartilaginous. The lower epiphysis appears at about the fifth to the seventh year. Sometimes this epiphysis appears as two centres, later umiting to form one. At the tenth year, sometimes a little later, the epiphysis of the olecranon process (upper epiphysis of ulna) appears.

Carpus.-The carpal bones derelop commonly from a single centre, and are all cartilaginous at birth. The ossific eentres under normal conditions usually appear in the following order:

The os magnum and the unciform appear very soon after birth, the os magnum usually appearing first. 
The cuneiform, about the second or third year.

The semilunar, about the fourth or fifth year.

The trapezium, about the fifth year.

The scaphoid, about the fifth to sixth year.

The trapezoid, about the sixth to eighth year.

The pisiform, about the twelfth year.

According to Pryor the appearance of the trapezium, scaphoid, and trapezoid varies, and in his cases the trapezoid precedes the trapezium, but the scaphoid precedes the trapezoid.

He has also found that at the same chronologic age the carpal bones of girls are in advance of those of boys, especially during the stage of pubescence.

These observations of Pryor, however, do not invalidate the deductions on which my principle of developmental strength is based; for, as I look at the question, it is the number of carpal bones, rather than the order in which they come, which makes this strength.

Metacarpus.-The metacarpal bones are developed from two centres:

One for the shaft (sixth week of fetal life).

One for the epiphyses.

The ossific centre of the epiphyses for the metacarpal bones appears about the third year, usually a little earlier.

Phalanges.-The phalanges develop by two centres:

One for the shaft (sixth week of fetal life).

One for the proximal ends.

The ossific centres of the proximal ends appear at about the third year.

Femur.-The femur is developed by five centres:

One for the shaft.

One for cach extrenity.

One for the trochanters (greater and lesser). 
The ossific centre of the lower end of the shaft may appear about the end of the eighth month of fetal life, but usually in the early part of the ninth month. An infant at full term shows a well developed lower epiphysis. The lower epiphysis appears at about the ninth month of fetal life. The ossific centre for the head of the femur (upper epiphysis) appears usually within the first six months. The great trochanter is not seen definitely until the fifth year and joins the shaft at about the eigluteenth year. The lesser trochanter appears from the twelfth to the fourteenth year and joins the shaft at the eighteenth year. It is to be noticed that the shaft and the diaphyses are rounded and smooth (Plate 18) presenting no sharp or ragged edges. This is an important point, as an early infection or a mutritional disturbance is first manifested by a ragged and irregular diaphysis.

Patella.-The patella develops by a single centre. It has a density more definite than cartilage as a rule, but not so definite as bone. The tendon of the quadriceps extensor passing over the patella is continuous below with the fibres of the ligamentum patellæ. The centre of ossifieation of the patella appears between the second and third year. The development of the patella is completed at about the thirteenth or fourteenth year. The bone sometimes, though rarely, develops from two centres placed side by side. It sometimes remains cartilaginous as late as the sixth year.

Tibia.-The tibia develops by three centres, one for the shaft and one for each extremity. The ossific centre for the upper epiphysis of the tibia appears before birth, at the same relative time as the lower extremity of the femur, that is, at about the last of the eighth month or the first of the ninth month. The lower epiphysis appears soon after birth, usually about the middle to the last of the first year.

Fibula.-The fibula is developed by three centres, one for the shaft (eightl week of fetal life?), and one for each extremity. The 
lower epiphysis appears first between the second and third year, and the upper epiphysis appears about the fourth year.

Tarsus. - The tarsal bones develop by a single centre; they appear approximately as follows:

Os calcis (sixth month of fetal life). This bone shows an irregular development from sometimes two or three centres of ossification. This normal condition must be carefully eonsidered when some abnormality is being differentiated from a fracture.

Astragalus (seventh month of fetal life).

Cuboid (ninth month of fetal life).

External cuneiform (first year).

Internal cuneiform (third year).

Middle cuneiform (fourth year).

Scaphoid (fourth year).

The ossific centre for the epiphysis of the os caleis appears at the ninth year, and sometimes unites before puberty, but usually soon after. It at times develops from two centres.

Metatarsus.-The metatarsal bones are developed from two centres:

One for the shaft (ninth week of fetal life).

One for the distal extremities of the four outer metatarsal bones.

One for the proximal end of the metatarsal bone of the great toe.

The ossific centre of the proximal end of the epiphysis of the first metatarsal bone appears about the third year, while the centres of the distal epiphyses of the others appear about the fifth to eighth year.

Phalanges.-The phalanges develop by two centres;

One for the shaft (eighth week of fetal life).

One for the proximal extremity. 
The ossific centre of the first row of phalanges appears at the fourth year, of the second row from the sixth to the seventh year, and of the third row in the ninth year.

Os Innominatum.-The os innominatum develops by eight centres, three primary and five secondary:

Primary $\begin{cases}\text { Ilium. } & \text { Secondary (each } \\ \text { Ischium. } & \text { with one centre) } \\ \text { Os pubis. } & \end{cases}$
Crest of ilium.

Anterior inferior spinous process (more common in the male).

Tuberosity of the ischium. Symphysis pubis (more common in the female).

Acetabuhum.

These ossific centres appear in the following order:

The ilium above the sciatic notch (eighth week of fetal life). The body of the ischium (twelf th week of fetal life).

The body of the os pubis (sixteenth to nineteenth week of fetal life).

At birth the ilium, ischium, and os pubis are separated, the crests and the bottom of the acetabulum being cartilaginous. At the fourth year the rami of the ischium and pubis begin to grow toward each other, thus completing the obturator foramen. This occurs between the sixth, seventh, and eighth years. The cartilage of the acetabulum becomes ossified by the thirteenth year. The ischium and ilium unite at the age of puberty.

\section{TEETH}

The rôle which at the present time the teeth of children play in practical medicine is so great that a description of the normal teeth as preparatory to the study of abnormal conditions is quite necessary. 
Since the use of the Roentgen ray in dentistry has been established this rôle has become more and more extensive and important. No dental surgeon or odontologist is fully equipped unless he avails himself of the valuable addition to the instruments for diagnostic precision which we possess in this wonderfully accurate discovery of modern science.

It is not my intention to go deeply into the subject of the teeth, or of the anomalies found in and about the jaws. I shall simply show that many conditions that heretofore have been seen only in the beautifully prepared specimens of the museums can now by means of the Roentgen method be studied during life.

Enamel is formed from the epiblastic layer. In the same manner are formed the skin, the epithelium of the mouth,- - except the tongue and the back part of the floor of the mouth,- and the appendages of the skin, such as the hair, nails, epithelium, glands, and nervous system. Dentine and cementum are formed from the mesoblastic layer. In this way also are formed the skeleton, muscular tissue, and connective tissue. Enamel is a substance composed of lime salts deposited by organic tissues which disappear during its formation. The entire surface of the enamel is finely striated, the striæ being transverse to the long axis of the crown. In addition to this fine striation there may be a few deeper and more pronounced grooves or pits which are pathologic and are marks of a check in development more or less complete. Two views have been held as to the formation of enamel. One is that it is formed by the actual conversion of the cells of the enamel organ into enamel. The other is that it is in some sense secreted or shed by these cells. In support of this latter theory there is the authority of well-known investigators, but some of the grounds on which their decisions are based are appearances which are open to a different interpretation.

The deformities in the human teeth are found in the enamel 3 
and are due to checks in the development of or destruction of the ameloblasts. It becomes of great importance, therefore, that the causes of this check or destruction be anticipated as much as possible.

Disturbances in the tissues formed from the epiblastic layer of cells in the embryo will in the new-born and up to the third or fourth year be likely to show their effects in the formation of the enamel of the permanent teeth. Such diseases as syphilis, tuberculosis, rhachitis, measles, and scarlet fever have long been considered as causes of these deformities, although it has been impossible in many cases where deformities exist to find any history of these diseases.

The diseases of nutrition, while they may not cause deformities in the enamel, can so weaken the structure of the entire tooth that it may be impossible to preserve it. For this reason it should be understood how important it is to protect young children during the period of the first dentition from these infectious diseases and from the so-called diseases of nutrition.

While we recognize the serious results which arise from disturbances of nutrition, those which arise from infectious diseases are not so commonly understood and accepted.

For various reasons the laity are apt to say and to believe that it is well to allow the other children in a family where one child is infected, to be exposed to the disease. It is the duty of the physician under these circumstances to impress upon the parents that such grave secondary conditions as have just been described should be aroided. This is, of course, only one of the reasons for protesting against allowing a young child to be exposed unnecessarily to an infections disease.

In Table 2 and Table 3 I have indicated in a general way when the different groups of temporary and permanent teeth respectively are to be expected to erupt. 
There may be a variation of a number of weeks in the eruption of the various groups given in Table 2.

Table 2.-Temporary Teeth, First Dentition, 20 in Number.

Dental Periods. Eruption of Groups of Teeth. Beginning of Caleification.

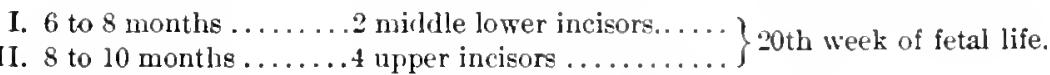

III. 12 to 14 months ....... 2 lateral lower incisors......

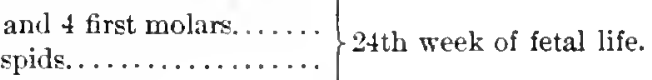

IV. 18 to 20 months .4 cuspids.

V. 24 to 30 months

.4 second molars

There may be a variation of a number of years in the eruption of the various groups given in Table 3 .

Table 3.-Permanext Teeth, Second Dentition, 32 in Number.

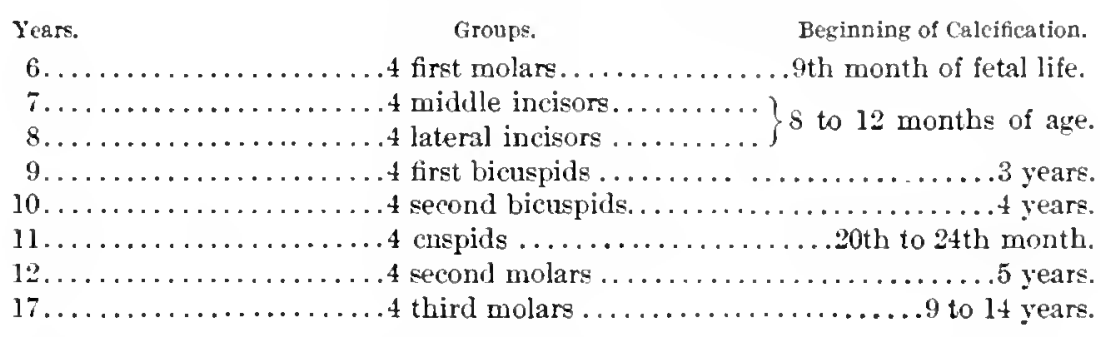

At the time of birth calcification of the entire crowns of the temporary central incisors and lateral incisors, and of about onethird of the cuspid teeth, usually takes place. About one-half of the first temporary molars, a little less than one-half of the second temporary molars, and the tips of the cusps of the first permanent molars also appear at this time (see Plate 3 and Plate 5). The calcification of the roots progresses until at about the eighteenth month when the apices of the temporary superior central incisors should be completely calcified. At the sixteenth month the apices of the temporary lateral incisors become calcified, and at about the twentietl month those of the first temporary molars. At the thirtieth month the apices of the temporary cuspids and those of the second temporary molars become calcified. These teeth 
begin to erupt when about one-half of the root has become calcified. Tomes gives as stages of caleification at birth: "A full half of the length of the crown of the central incisor, about half that of the laterals, and the tip only of the cuspids. At birth the first temporary molars are eomplete as to their masticating surfaces. The second temporary molars have their cusps more or less irregularly united. in many eases the four cusps being united into a ring of dentine, the dentine in the central depression of the crown not being yet formed."

Cases of deformity of the jaws or threatened deformity should be placed under treatment at an early age, in most instances before the temporary teeth have given place to the permanent ones. Room can thus be made for the misplaced or unerupted tooth to come into position where it will stay without long retention. If the Roentgen examination is resorted to at an early period much trouble will be saved for the child as well as for the orthodontist.

(Plate 91, Division V, shows the normal temporary teeth in a child three years old.)

During the period between the fourth and sixth years separation takes place between the temporary teeth anterior to the first temporary molar. This is due to the increased outward and forward development of the jaws, and also to the advancing permanent teeth which lie in the lingual aspect of the temporary cuspids and incisors. The bicuspids, which take the place of the temporary molars, lie directly above and below the latter, their crowns when in normal position being surrounded by the temporary molar roots. In many cases the second temporary molar is retained for quite a period of time, occupying more space than will be needed for the second bicuspid. Under certain conditions it may be necessary to extract, although the temporary molars are firm. Owing to the relation of the crowns of the bicuspids to the temporary molar roots, 
these partly developed permanent teeth may be sacrificed by the extraction of the temporary molars. This depends, however, upon whether the roots of the latter have reached a stage in absorption which will render them powerless to disturb the position of the underlying teeth. The Roentgen examination is invaluable in these cases, for the unnecessary loss of one or more bicuspids may cause serious deformities. The calcification of the first permanent molar starts at the ninth month of fetal life (see Plate 3 ).

On an average it is found that at the first year after birth the calcification of the tips of the central and lateral permanent incisors has taken place. In the second year one-sixth of the crowns of these teeth has been completed, and the tip of the cuspids. At the third year the tip of the first bicuspid has begun to calcify. At the fourth year about one-half of the central and lateral crowns, one-third of the cuspid crowns, and the tips of the sccond bicuspids have become calcified. Between the seventh and eighth year calcification has taken place in about one-half of the root of the central and lateral incisors, has just begun in the cuspid roots, and has just finished in the crowns of the bicuspids. The first permanent molar has kept just a little ahead of the central incisors in calcification, and at about the fourth year, when one-half of the central crown has become calcified, two-thirds of the crown of the first molar has been completed. In the fifth year we find the crown of the first. molar fully calcified, the central incisors almost so, and the tips of the cusps of the second molar just beginning. Plate 91 shows the stage of development of the first permanent molar in a child three years old. The average time for the development of this tooth is during the fifth year.

At a given stage in the development of a tooth it is due to erupt, and if the question arises regarding its eruption, the Roentgen examination will determine whether from its development it is due 
or overduc. If the picture indicates an abnormality, steps should be taken to correct it. If we are expecting a tooth to erupt, say the cuspid, any time between the ninth and fourteenth year, there are five intervening years in which serious trouble may occur. For instance, the tooth was due to erupt at nine years. From uncertainty the case might be allowed to progress with the result that through further development of the root and lack of space, it might be deflected from its course and later would have to be forced into its place. It is possible, also, that one or both lateral incisors may be missing. If these teeth do not erupt at the proper time it is wise to resort to the Roentgen examination in order to determine their position or absence.

Plate 27 shows the normal development of the permanent teeth and the Roentgen method of determining whether a doubtful tooth belongs to the temporary or permanent groups. This plate shows very plainly all of the permanent teeth that should have erupted at this age in the upper jaw posterior to the cuspids.

\section{CHRONOLOGIC EXAMPLES OF NORMAL LIVING ANATOMY}

After these preliminary remarks on anatomic development, it will be useful to look at a number of plates of normal living anatomy in early life which I have arranged chronologically. The degrees of development shown, however, do not necessarily correspond in sequence to the chronologic ages. A careful inspection of these plates will demonstrate to the student the significance of the distinction between chronologic age and anatomic growth which I shall fully explain in Dirision II, and the results of which seem to show that the child's well-being, in determining rules for its life, is best protected by relying on anatomic rather than on chronologie age.

When the details of these normal living conditions through all their stages of development from the rery beginning of life are 
mastered, the diagnosis of disease will be greatly simplified. We shall then be able to recognize in our search for a diseased condition whether it is a stage of normal rather than abnormal development in the special individual whom we have before us.

In the third month of intra-uterine life the skeleton of the fetus is practically completed.

Plate 2 is that of a premature infant seren months old. The head of this infant, which was under my care at the Children's Hospital, shows quite a broad zone of increased radiability in both the frontal and upper parietal regions and in the occipital and postparietal, where there is an almost purely cartilaginous condition. The cranial bones at this stage of development show the condition of intramembranous ossification. All the sinuses are absent except the orbit, which is quite distinct, as is also the nasal cavity. Both the upper and lower jaws show indistinct pictures of the teeth in their cartilaginous surroundings, but the density is not sufficient to differentiate them. At the base of the skull is to be noticed the rudimentary atlas and axis and then the vertebral column through its whole length. Especially marked is the extreme radiability of the intrarertebral disks in comparison with the lessened radiability of the bodies of the vertebre, which, however, also show a cartilaginous condition of the transverse processes throughout the whole length of the spine. Starting from above it should be noted in comparison with the radiability in the cervical region the decreased radiability in the cardiac region, where the density of the heart is added to that of the spine. Between the ribs is seen the great radiability of the lung. The greater density of the liver is seen just below the lung, and below this again round light areas representing parts of the intestine. The pelvis is in a semi-cartilaginous condition. To be noted is the entire absence of bony structure in the front of 
the pelvis, where the ilium, ischium, and os pubis are widely separated. All the long bones are developed, but no epiphyses are present in any of the joints. The clavicles are ossified. The humerus, the radius, the ulna, the femur, the tibia, and the fibula are represented entirely by their diaphyses, which, however, are somewhat cartilaginous towards the ends at this age. No carpal bones are present at this stage of development, and no tarsal bones except the os calcis and the astragalus. To be noted also is the greater radiability of the central part of the tibia which shows the marrow light in comparison with the dark edges representing the cortex. The small size of the entire skeleton will be appreciated if we observe that the trunk in comparison with a moderate-sized safety-pin is only four times the length of the pin.

Plate 3 represents the normal head of an infant aged ten days. The proportion of the face to the head at this age is very small. (For a further description of the face and cranium see "Pediatries," fifth edition, page 26.) The antrum shows no signs of development and does not appear until the eighth or the tenth month, except that it has gradually become a marked depression in the wall of the masal cavity just at the time of the eruption of the first temporary teeth. The first temporary teeth are seen in their crypts partly calcified.

In the upper jaw the incisors are indistinetly seen.

The cusps of the temporary molars show the calcification which corresponds to a development of ten days.

Plate 4 represents the thorax and upper legs of an infant ten days old. There is nothing especially noticeable about the thorax and abdomen to distinguish it from that of the fetus of nine months.

The ilium, ischium, and os pubis are still ununited, and their cartilaginous separation still covers quite a broad area.

While in the premature infant the lower epiphysis of the femur 
as seen in Plate 2 has not appeared, it has beeome quite distinct in this infant of ten days.

Also to be noted in this infant, which is the same subject whose head has just been deseribed, is the great radiability at the head of the femur, and that the cardiohepatic angle is elearly defined.

To be noted in eonnection with these earlier stages of development is the pieture of a normal stomach artificially dilated in an infant five weeks old (Division VII, Plate 140).

Plate 5 shows the head of an infant ten weeks old. The first and second temporary molars show the degree of caleification which has taken place in their erowns. Anterior to the first temporary molar the teeth are indistinet and the picture is confused.

Plate 6 shows a lower extremity of the same infant ten weeks old. To be espeeially noticed is the still undeveloped eondition of the bones of the pelvis. The lower epiphysis of the femur has become quite prominent, and this is also the case with the upper epiphysis of the tibia. The other epiphyses are not yet seen. The bones of the foot have increased in size and lessened in radiability in comparison with the same bones shown in Plate 2. They are the os ealeis, the astragalus, and lower down the cuboid.

Plate 7 shows the hand of an infant three months old. No epiphyses are present, and the only earpal bones that have appeared are the os magnum and the unciform.

Plate 8 shows the normal thorax, humeri, and elbows of an infant from two to three months old. The only epiphysis present is the upper epiphysis of the humerus.

Plate 9 shows the entire skeleton, excepting the head, of a girl six months old. To be especially noted are the cardiohepatic angle on the infant's right, the clearly defined outline of the stomach, and the presence of the lower epiphysis of the femur and the upper epiphysis of the tibia. There is no especial change in the spinal 
column, except that the radiability in comparison with that in the plate of the earlier age just shown has gradually decreased. The outline of the stomach is very clear at both the cardiac and pylorie extremities. The front of the pelvis is still in a cartilaginous condition and is widely open. The shafts of the long bones are clearly marked. The cortex of the femur shows its lessened radiability in comparison with the radiability of the marrow. It is possible that the area marked $B$ in the cardiac end of the stomich may represent the left kidney. The upper epiphysis of the humerus has appeared. No epiphysis has yet appeared at the lower end of the humerus. There are no epiphyses seen at the upper or at the lower ends of the radius and ulua. The bones of the carpus are very indistinet if seen at all, the os magnum and cunciform bones possibly being shown in the midst of the irregular area to the left of the left wrist. Neither the upper nor the lower epiphysis of the fibula has yet appeared. The upper epiphysis of the tibia is present. On examining the tarsal bones it will be seen that not only the os calcis and the astragalus are present, but that the cuboid also seems to be present, while in the picture of the premature infant (Plate 2) the cuboid has not yet appeared.

Plate 10 shows the normal trunk of a girl twelve months old. The different organs as described in the legend belonging to this plate are seen plainly.

Plate 11 shows the skeleton of a girl about twenty-four months old. In this plate the transverse processes of the cervical vertebre show a decreased radiability and are more ossified. The ribs show a greater density, and the stomach is not so clearly defined as in Plate 9. The front of the pelvis is not so widely apart, ossification having apparently taken place in the ischium and ilium. The sacrum has become still less cartilaginous as shown by its lessened radiability. The shafts of the bones still show great radiability at 
their distal ends, but their eortex is distinct. The epiphysis of the upper end of the humerus has increased in size and shows lessened radiability as compared with the infant of six months. There is no epiphysis at the lower part of the humerus. A small epiphysis of considerable radiability is seen at the lower end of the radius. No epiphysis is seen at the lower end of the ulna. The carpal bones have now become much more distinct. The os magnum and the unciform are plainly in sight with their rapidly increasing density. Just behind the unciform bone is seen a minute highly radiable carpal bone, probably the euneiform, although this bone is usually not supposed to appear until the third year. The metacarpal bones show the epiphyses. The epiphyses of the phalanges of the fingers are present. The epiphysis of the upper end of the femur has become much larger than seen in Plate 9 and shows decreased radiability. The epiphysis of the lower end of the femur has inereased much in size, and the epiphyseal line is much decreased, its boundaries being smooth and regular. It is to be noticed that the trochanter major and trochanter minor have not yet appeared. The epiphysis of the upper end of the tibia has come plainly into view. The epiphysis of the upper end of the fibula is not yet seen, but is present at the lower end. The tarsal bones have much increased in size and show decreased radiability, the cuboid and external euneiform apparently being absent. The metatarsal bones and the phalanges of the foot are too much massed to be differentiated.

Plate 12 shows the skeleton of an infant about three and a half years old. In this plate there is no especial change in the density of the cervical vertebræ. The lumbar vertebræ are, however, unusually distinct and show decidedly lessened radiability in their bodies. There is somewhat greater radiability than is seen in the younger subjects already described. There is still no epiphysis at the upper end of the ulna or of the radius, but the epiphysis at the lower 
end of the radius has increased in size. There is no epiphysis seen at the lower end of the ulna. The carpal bones have become more distinct and increased in size, being represented by the os magnum, the unciform and the cunciform bones. The epiplrysis of the upper end of the humerus las increased in size. The capitellum is shown at the lower end of the humerus. The heads of the metacarpal bones, the epiphysis of the metacarpal bone of the thumb, and the epiphyses of the proximal ends of the first phalanges are coming into view. The epiphysis of the upper end of the femur has beeome larger and the epiphyseal line narrower. The epiphyses of the lower end of the femur and of the upper end of the tibia have increased in size. The epiphysis of the lower end of the tibia shows increase in size and the epiphyseal line is narrower. The epiphysis of the lower end of the fibula is seen just behind the astragalus. The euboid, external euneiform, and internal cuneiform bones have become more prominent. No especial change is noticed in the metatarsal bones nor in the phalanges.

Plate 13 shows the knees and lower limbs of a child three years old.

Plate 14 shows the normal foot of a child five years old, which should be compared with the abnormal foot of a child three years old in Division III, Plate 56.

Plate 15 shows the skeleton of a child about six years old. There is no especial ehange to be noted in the thorax of this ehild. The bodies of the transverse processes have become much more ossified and show greater density and lessened radiability. The ilium and ischium are closely approaching each other and are gradually completing the acetabulum. The processes of the ischium and of the os pubis have almost elosed in so as to form the obturator foramen. The front of the pelvis is elosing. The lower epiphysis of the ulna has begun to appear and is represented by two fine points. 
The lower epiphysis of the radius is gradually approaching its lower diaphysis. Starting with the os magnum, we see to the right the unciform and next to this the cuneiform, then the semilunar in the centre and just below the epiphysis of the radius, then the scaphoid, the trapezoid, and the trapezium. The four metacarpal bones show their epiphyses plainly. The first metacarpal bone (thumb) shows its epiphysis. The phalanges show their epiphyses plainly. The upper epiphysis of the femur is inereasing in size. The greater trochanter appears very plainly and also the lesser trochanter. The lower epiphysis of the femur has become much more developed. The tarsal and metatarsal bones have beeome larger, but excepting the os ealcis and the astragalus they are not clearly differentiated in the plate on account of a side view laring been taken.

Plate 16 shows the shoulder of a ehild six years old.

Plate 17 shows the elbow of a child six years old. This is the same subject as Plate 16 and Plate 18.

Plate 18 represents the knee of a child six years old, and is the same subject as Plate 16 and Plate 17 .

Plate 19 shows the normal thorax of a child six years old.

Plate 20 shows the normal hands of a boy nine years old.

Plate 21 shows the normal thorax, arm and hand, pelvis, and upper legs of a child ten years old. In this case the ray has been especially directed on the pelvis and hips, there being nothing special to be noted in the picture of the thorax. The sacrum is seen very plainly in the brim of the pelvis. The epiphysis of the upper end of the humerus is definitely developed, as are the external and internal eondyles of the humerus, the epiphysis of the scapula, and the glenoid cavity. The upper epiphyses of the radius and of the ulna show plainly. The rami of the ischium and the os pubis have joined so as to form the obturator foramen. The ilium and the ischium have not yet joined. The epiphyseal line of the upper 
epiphysis of the femur has become quite narrow and is smooth and even. Especially to be noted is the epiphysis of the greater trochanter. The epiphyseal line of the lower end of the radius is quite narrow, and the epiphysis of the lower end of the ulna is distinet.

Plate 22 shows the knees, lower legs, and foot of the same subject. Especially to be noted are the well-developed epiphyses of the lower end of the femur and the upper end of the tibia with their narrow, clearly defined epiphyseal lines. The lower epiphysis of the tibia is present, and the upper and lower epiphyses of the fibula also are present. All the bones of the tarsus appear in this picture. Especially to be noted is the small pin-head development of the middle euneiform and of the scaphoid: the latter is seen to have two centres of ossification.

Plate 23 shows the normal spine of a child ten years old.

Plate 24 shows the skeleton of a ehild twelve years old. All the epiphyses are well developed, and all the bones of the wrist are present. Note especially the pisiform bone overlying the unciform bone. The ilium and ischium have practically joined, forming the acetabulum. The os pubis is still open. The epiphyseal line of the greater troehanter has almost disappeared. The epiphysis of the os calcis with its narrow epiphyseal line is evident. All the carpal and tarsal bones are present.

Plate 25 shows the normal elbow of a child of twelve years. The details of this plate are described in the legend.

Plate 26 shows the normal thorax of a boy twelve years old. Especially to be noted is the epiphysis of the humerus, which is elearly defined and shows its mion with the diaphysis well adranced. (The complete union occurs from the eighteenth to the twentieth year.) Sote the decidedly lessened radiability of the heart and the sharply defined cardiohepatic angle. 
Plate 27 shows the head of a boy thirteen years old with a normal permanent set of teeth. This plate shows very plainly all of the permanent teeth that should have erupted at this age. In the upper jaw posterior to the cuspid the bicuspids are seen. The root of the seeond bicuspid and a small cavity ean be seen in the floor of the antrum immediately above it. The first molar is in similar relation to the antrum, and small eavities can be seen in its floor over the anterior and posterior buccal roots. The second molar roots do not show so clearly. The third molar, only partly formed, can be plainly seen in its crypt. In the lower jaw the third molar can be seen with the distinet outline of its erypt and some calcification of its crown. The roots of the second molar are not yet formed. The erown of the first molar has been extensively filled, which accounts for the change in the density. The roots appear normal. The anterior root is not clearly shown, nor are the roots of the teeth anterior to the first molar, on aceount of the interposition of the teeth on the opposite side of the jaw. The object of this illustrative examination was to determine the nature of a tooth in the position of the first bicuspid. Here it is seen that the crown is much deformed, due to earies, in this case making it impossible to determine whether it was the first bicuspid or the first temporary molar, other indications pointing to the latter. The plate has not given us any assistance on this point, however, for a tooth extensively reconstructed on the other side of the jaw lies in the same field, destroying all faint outlines by its greater density.

The doubtful tooth in question was again Roentgenographed by using a film inside the mouth, and the result is shown in the picture at the bottom of the plate, so that it could be determined that it was the first bicuspid instead of a temporary molar. If it had been the first inferior temporary molar, it would have had four roots and would have shown two in the picture. The second root shown in the pieture, however, declares the tooth's identity. 
PIATE 2.

PREMATURE INFANT.

7 montls. (Reduced 55\%.)

A. Frontal and upper partictal region.

B. Occipital and postparictal region.

C. Orbit.

D. Axis.

X. Nasal cavity.

$E$. Shows an anterior view of the third intervertebral cartilage.

$F$. The arrow points towarl the sixth vertebra.

$G$. Heart.

H. Lung.

1. Coils of intestine.

$J$. Cardiae end of stomach.

$K$. Liver.

L. Ramus of os pubis.

M. Clavicles.

$N$. Shaft of humerus.

$O$. Shaft of radius.

$P$. Shaft of ulna.

$Q$. Shaft of femur.

$R$. Shaft of tibia.

$S$. Shaft of fibula.

$T$. Os calcis.

$U$. Astragalus.

$V$. Phalanges of hand. 
l'JA'TE:

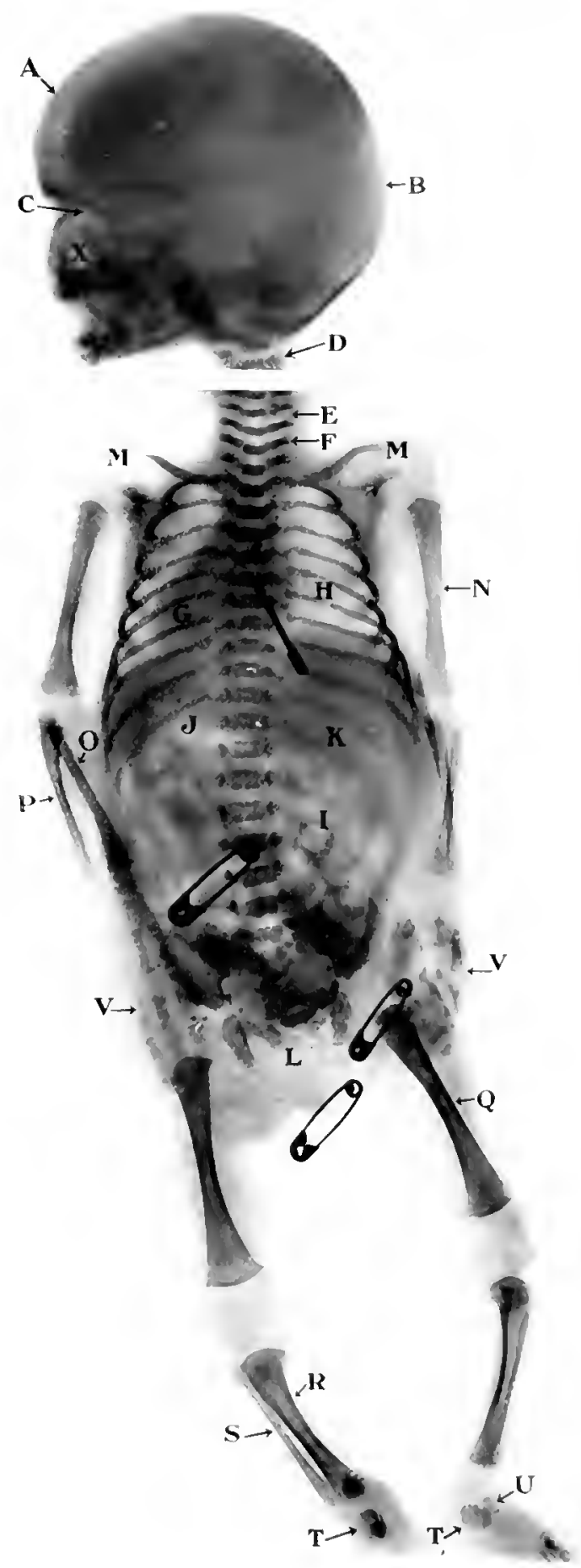


PI.ITE:?

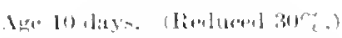

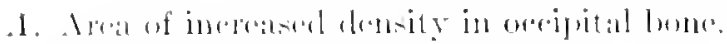

1). Antritur fontinulle.

C. Lime of parictal and frontal sutures.

1). P'onteriol fortancelte.

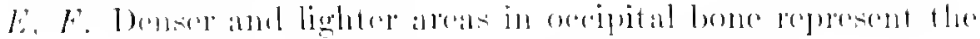
tillolin.

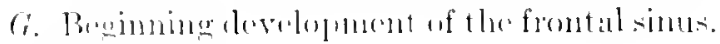

II. OHhit.

I. Supring maxilla.

I. Ethmuilal arello.

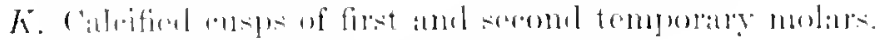

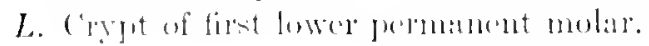


PIATE:3

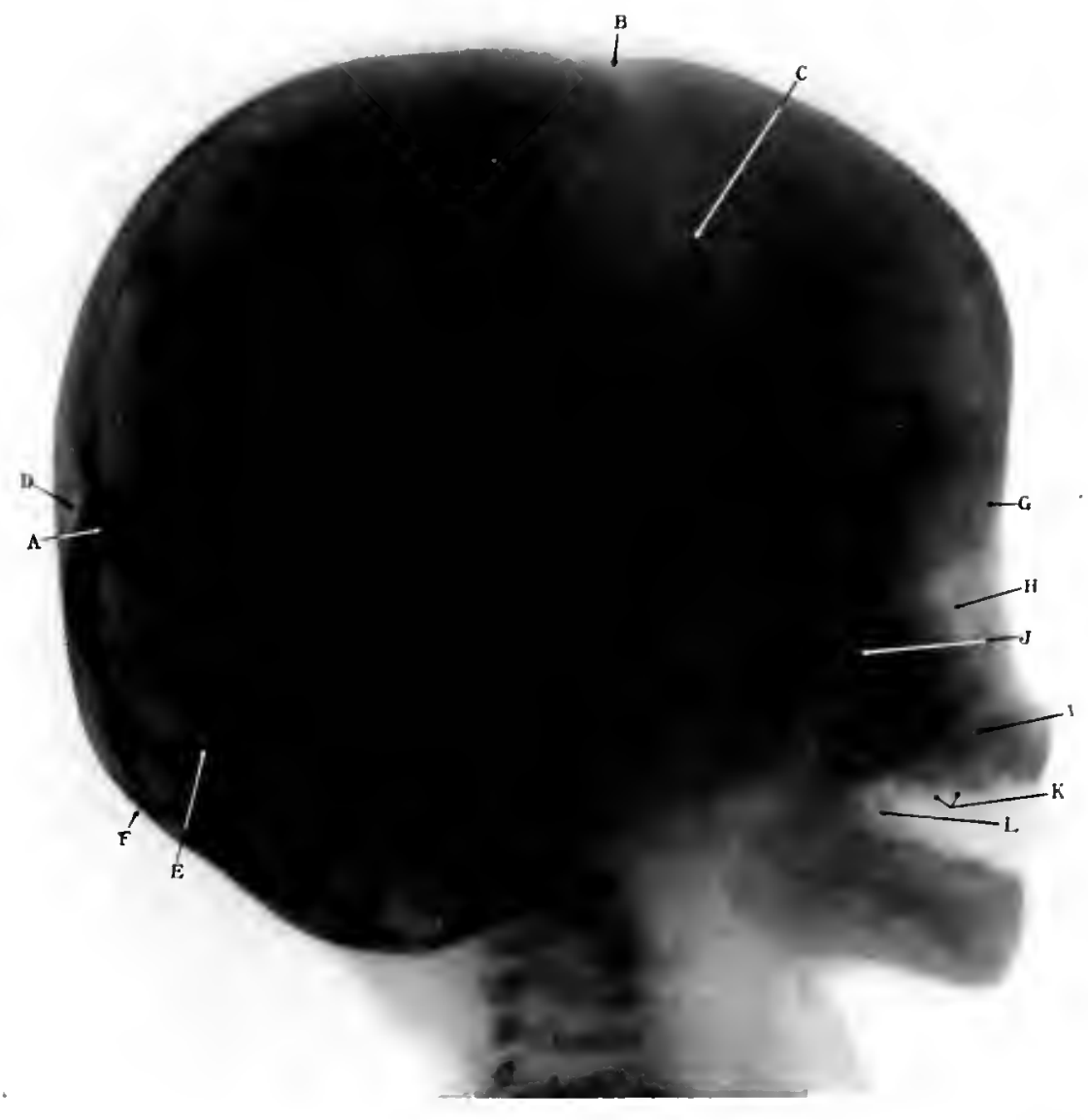


1'1.1T1: 1.

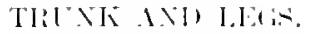

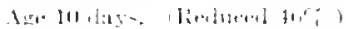

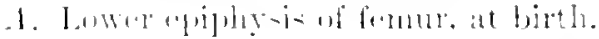




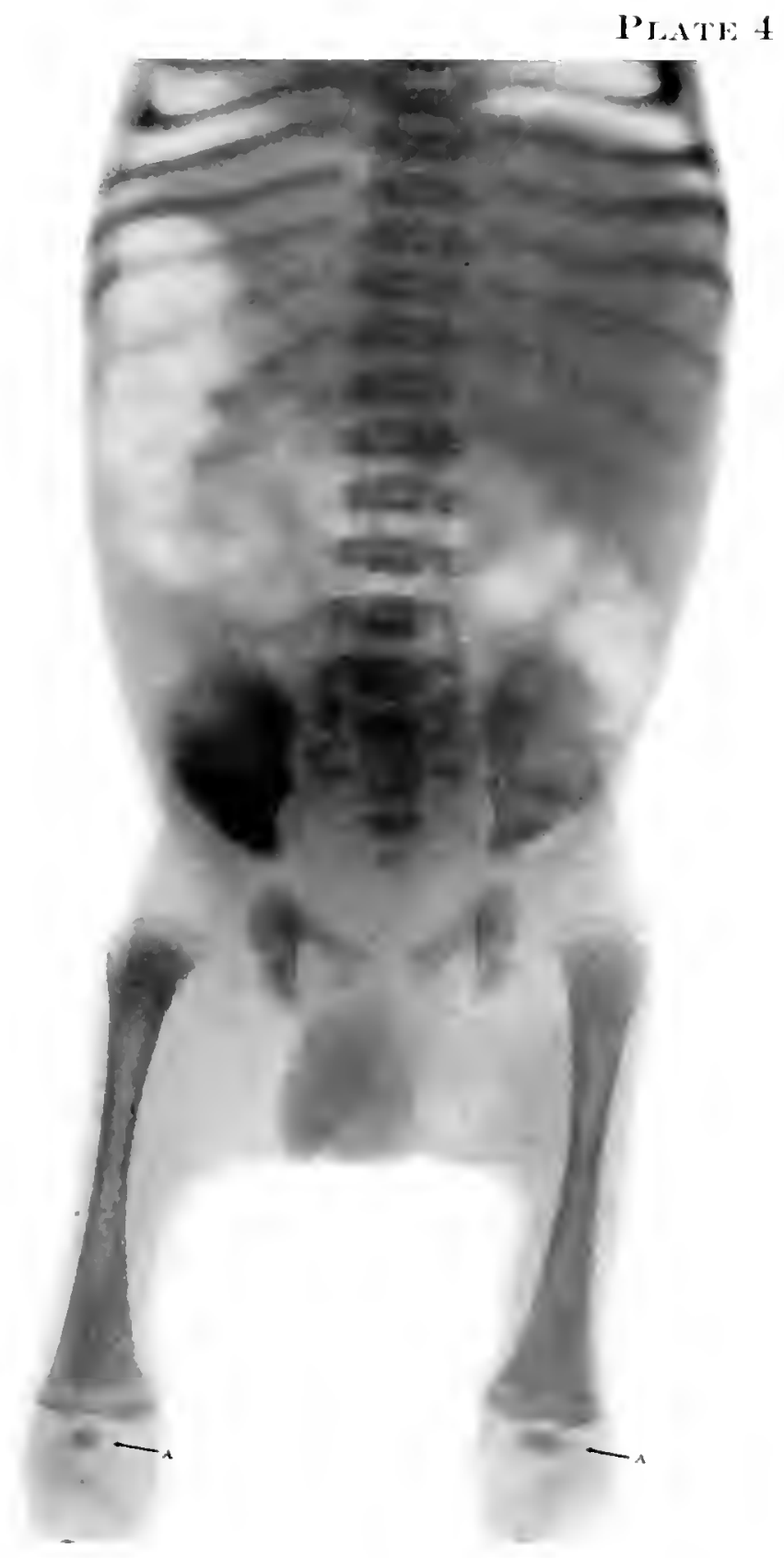


PI.ITE i.

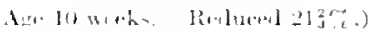

1. Interion fontanclle.

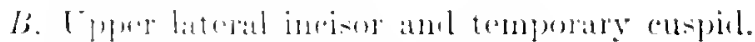

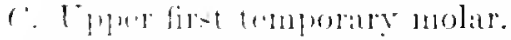

1). Crypt of unger left first permanent molar.

E. Cryet of lower left first permanent molar. 
Prate j

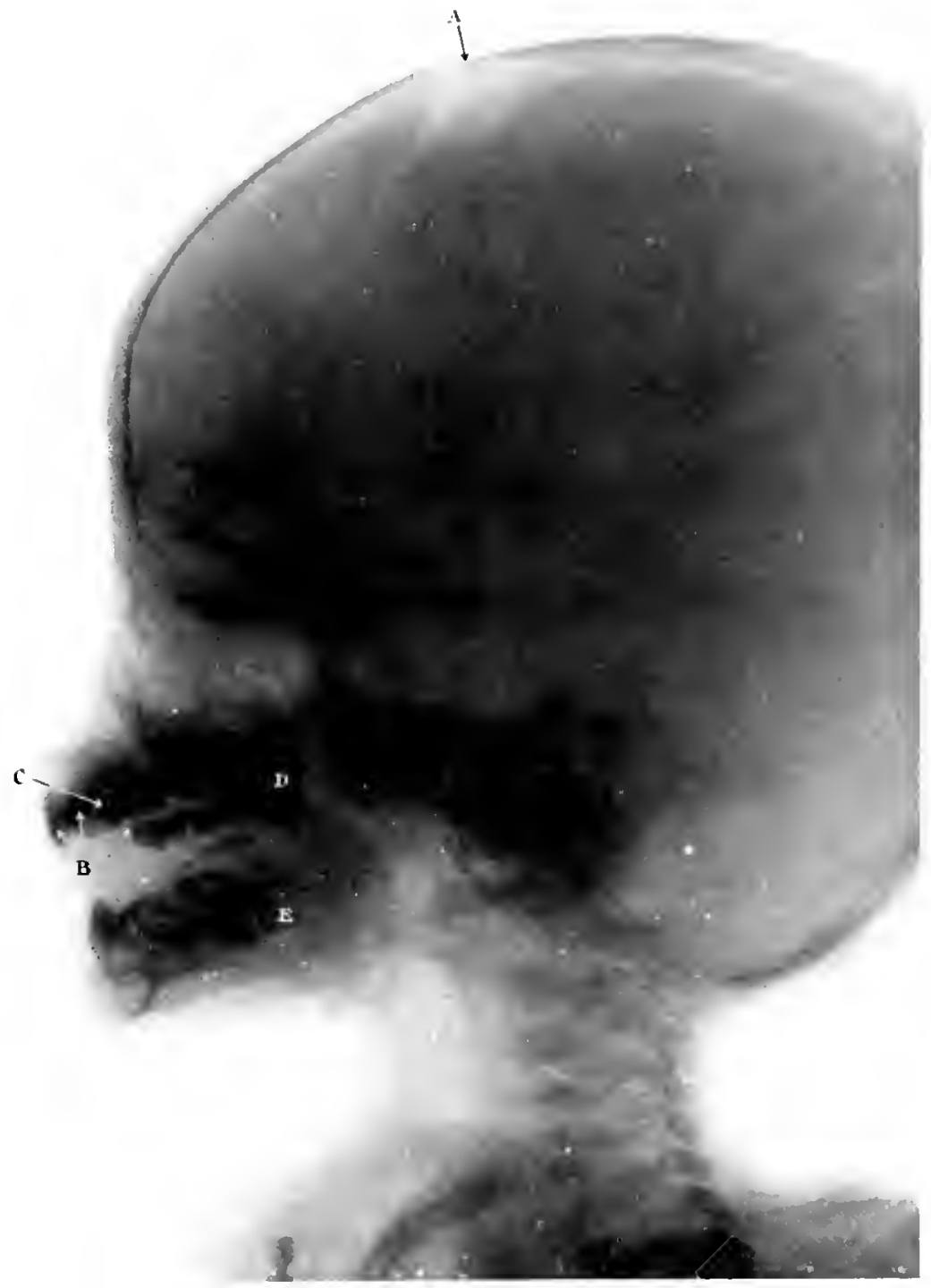


PIATE 6.

POATE-LET-BUT.

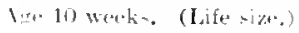

Xommal devedopment. 
PLATE (;

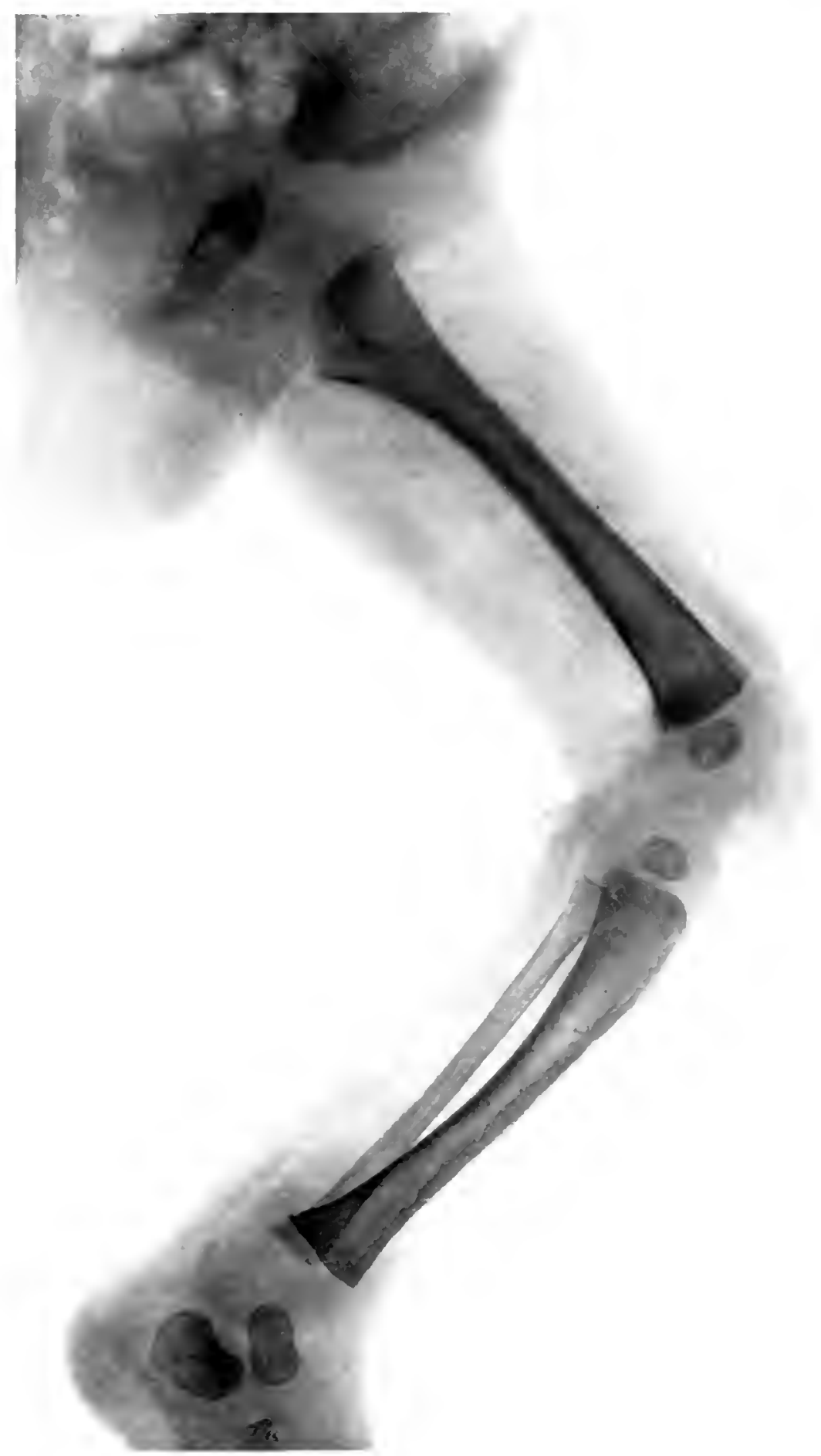


Pl. ITI: 7 .

NoMUMII IIXI)

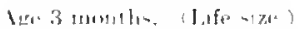

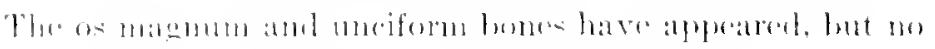

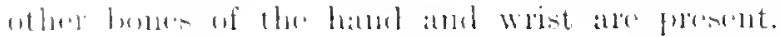


PIATE

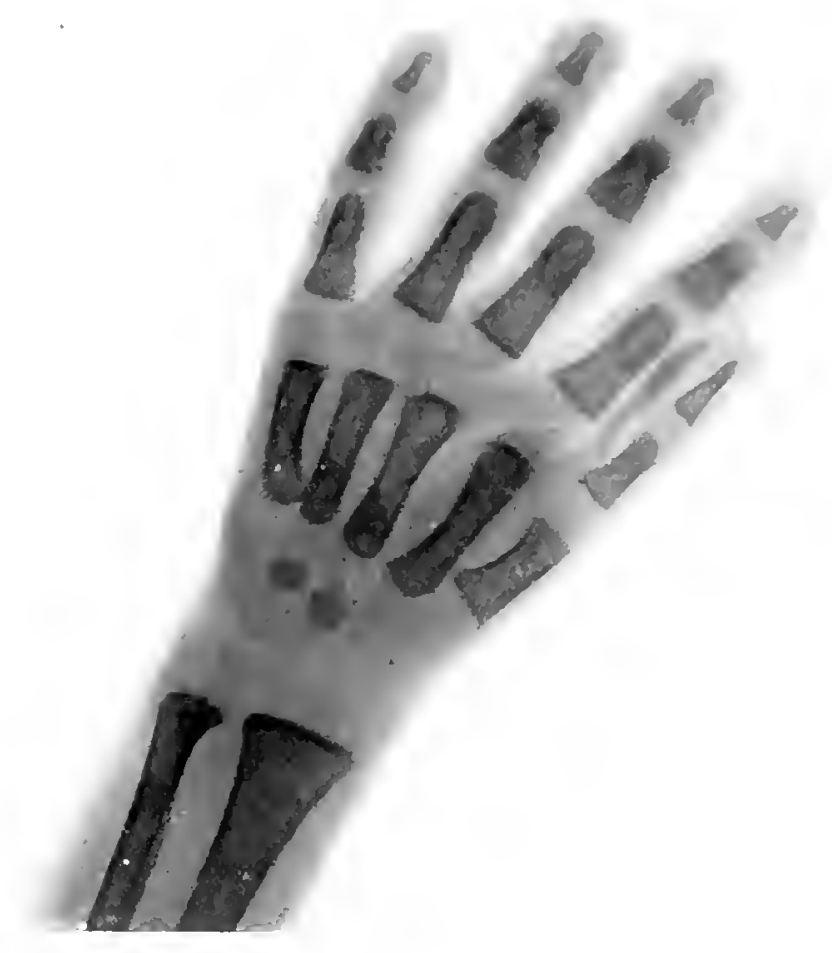


P'IATTS.

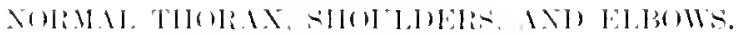

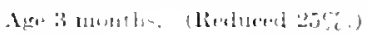


PLATE S

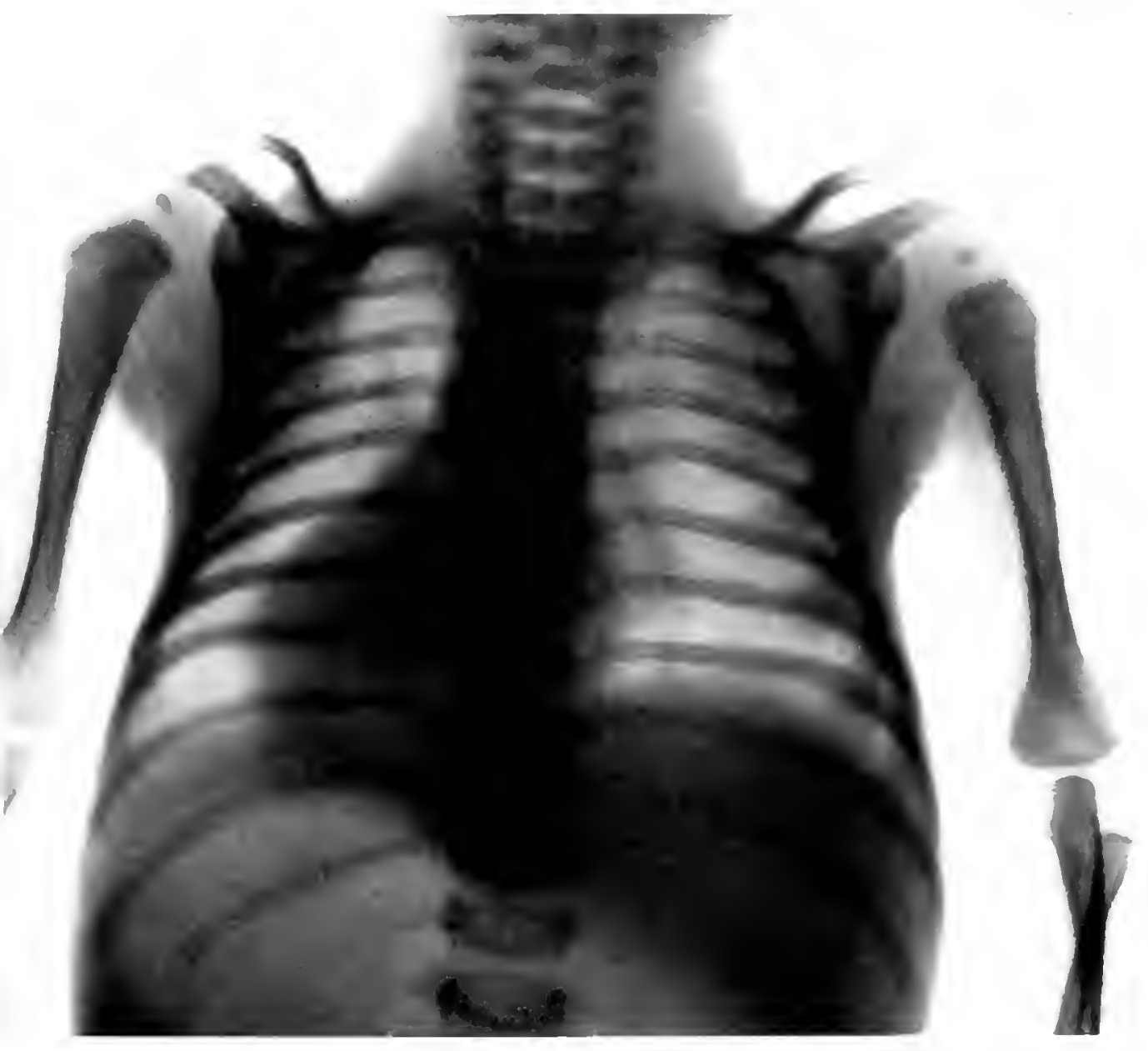


Pl.TPl:

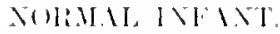

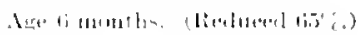

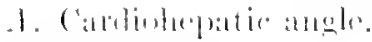

li. Sinumath.

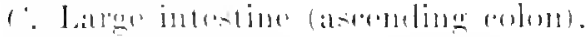

1). Corter al fomml.

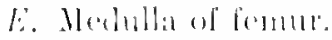

F. Ephphysis of humerts.

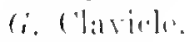

H. ('alpus.

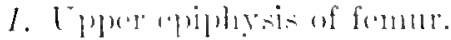

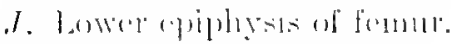

K. Lpure "piphysis of tibia.

L. Tillints.

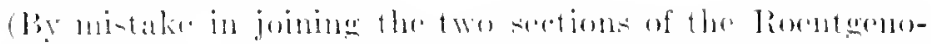

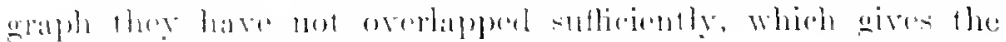
intant seren hunbal verteblat insteat of live.) 
P'IAF!

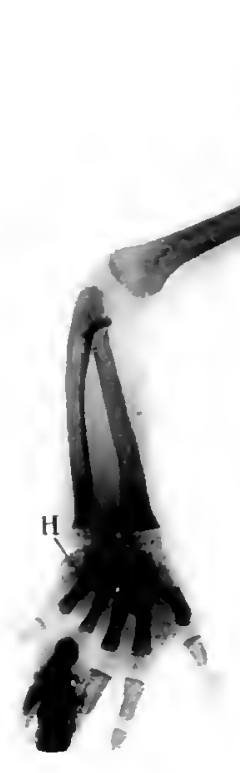

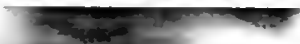

c:

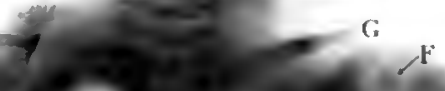

,

M
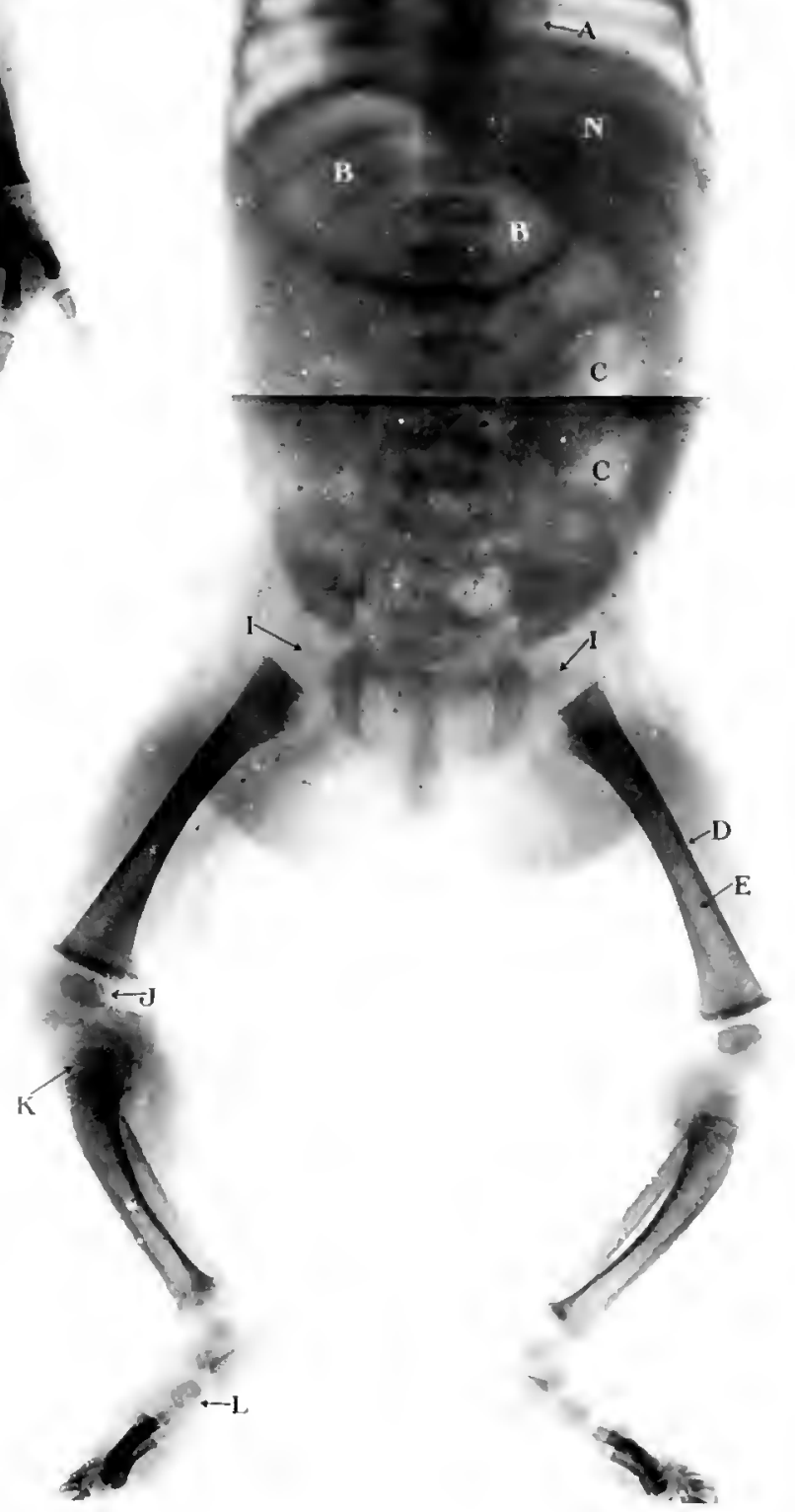
PLATE: 10.

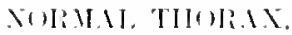

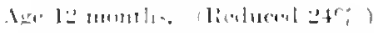

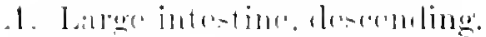

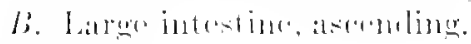

(1) Liver.

1). i.Anter.

l. Ilart anul spine.

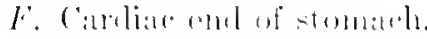

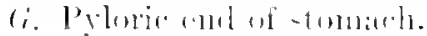


P'IATE 10

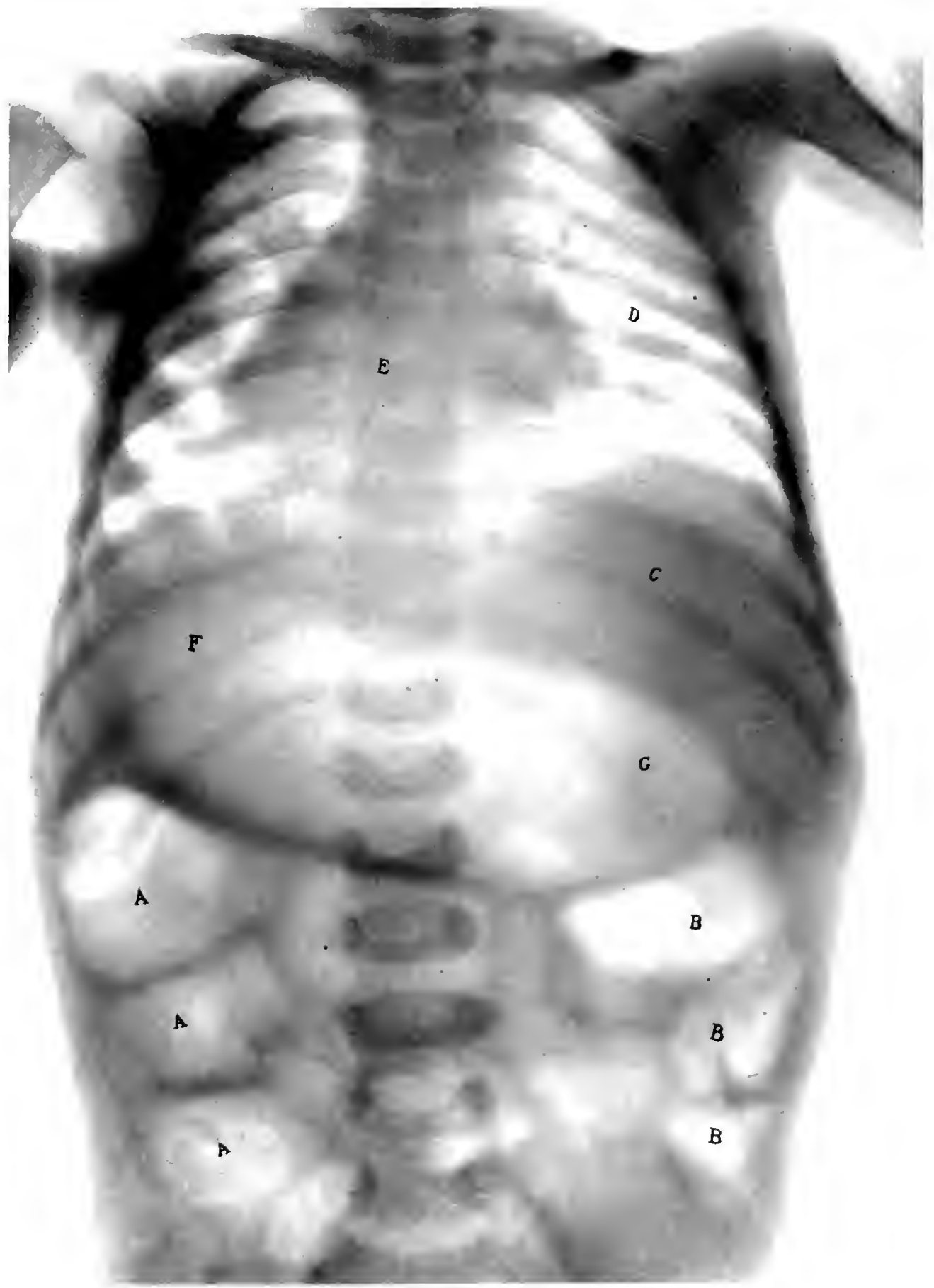




\section{P..Tl: 11.}

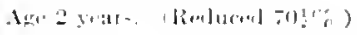

1. Cpres apphysis of humerus.

li. Cilpitrllum.

(․ lower (")

l). (unciform.

E. Epipheric of heat of fomur.

F. Epiphese of tibial and fihulit.

The lower epiphysis of the fomme and the upper "piphysis of the tibita are atos shown. 
PLAT 11
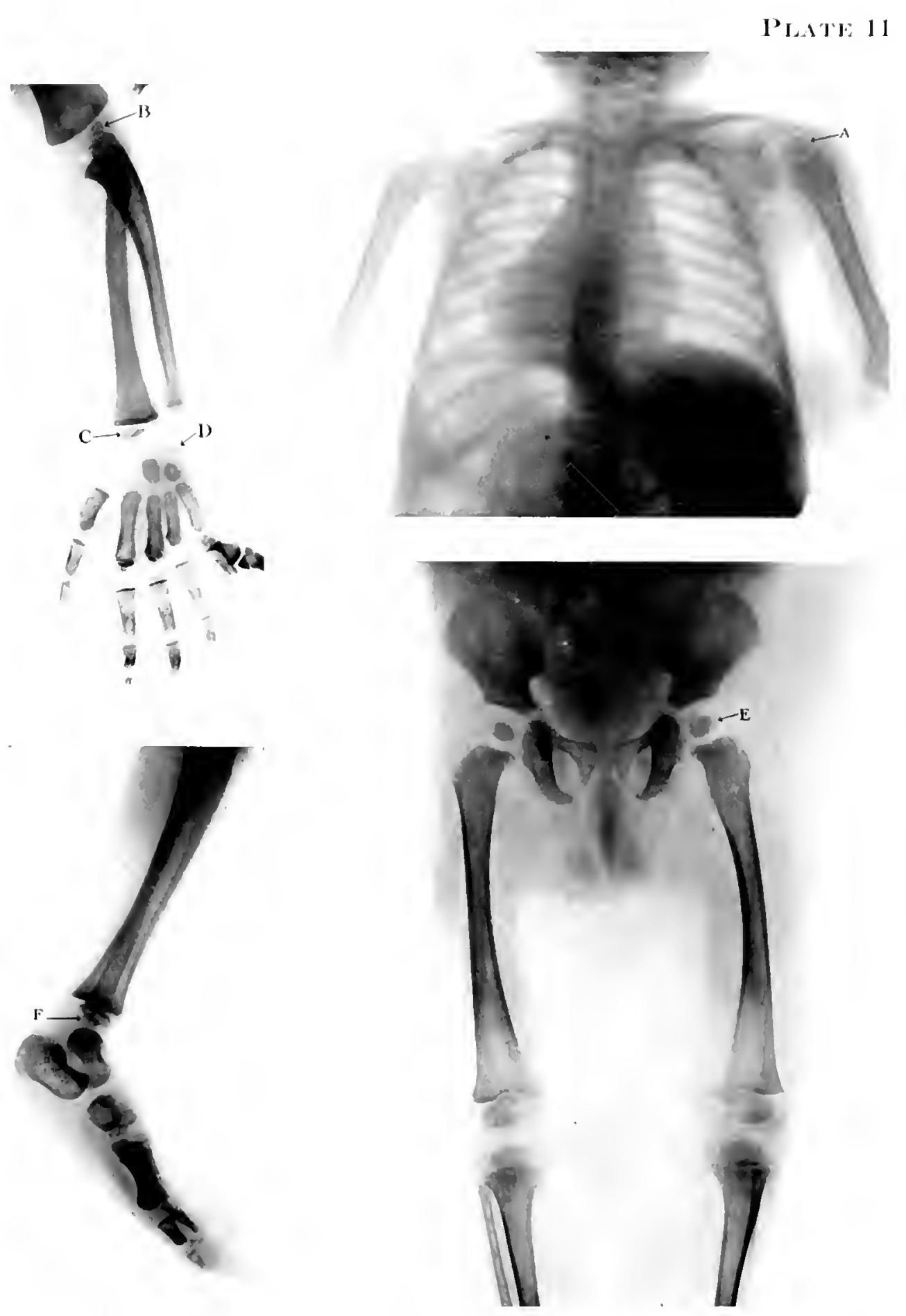
Pl.ITl: 1?.

1. Ilium.

$1+2 \times 3 \times 3 \times 4$

li. Isilium,

( $:$ (1) pulsis.

I). ()htmatur for:month.

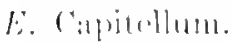

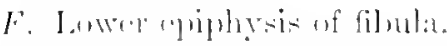

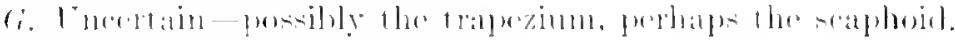

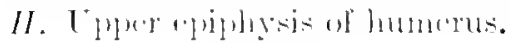


PIATE IE
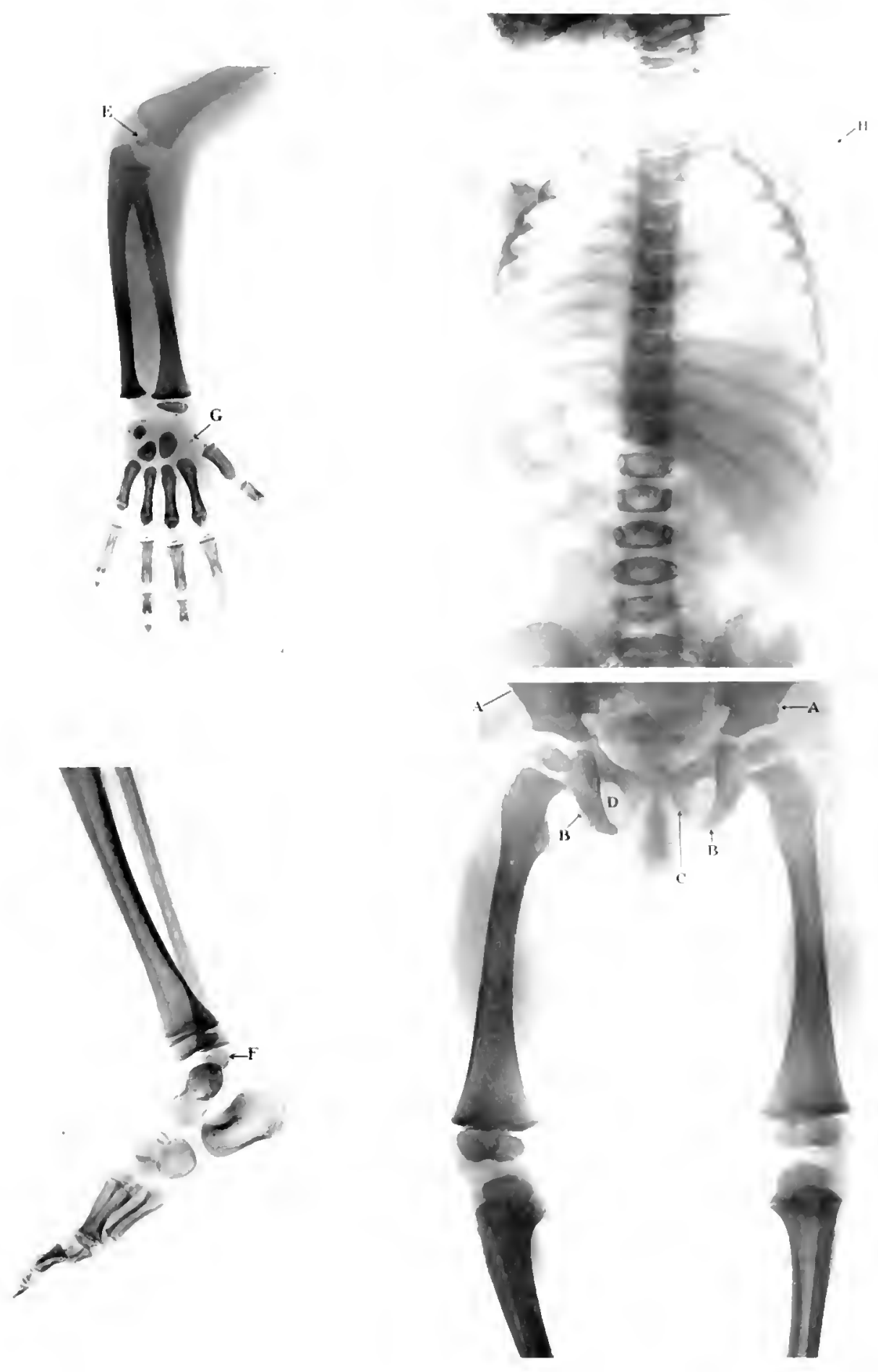
I'1.1'T1: 1:3.

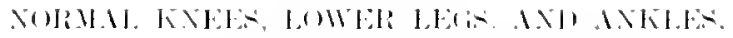

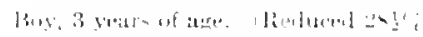

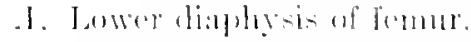

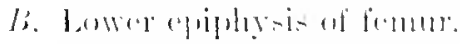

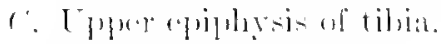

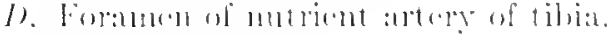

E. Cortex of liti:t.

f. Iower "pliphysis of tibia.

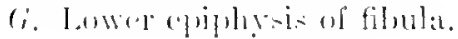


PrATE I:3
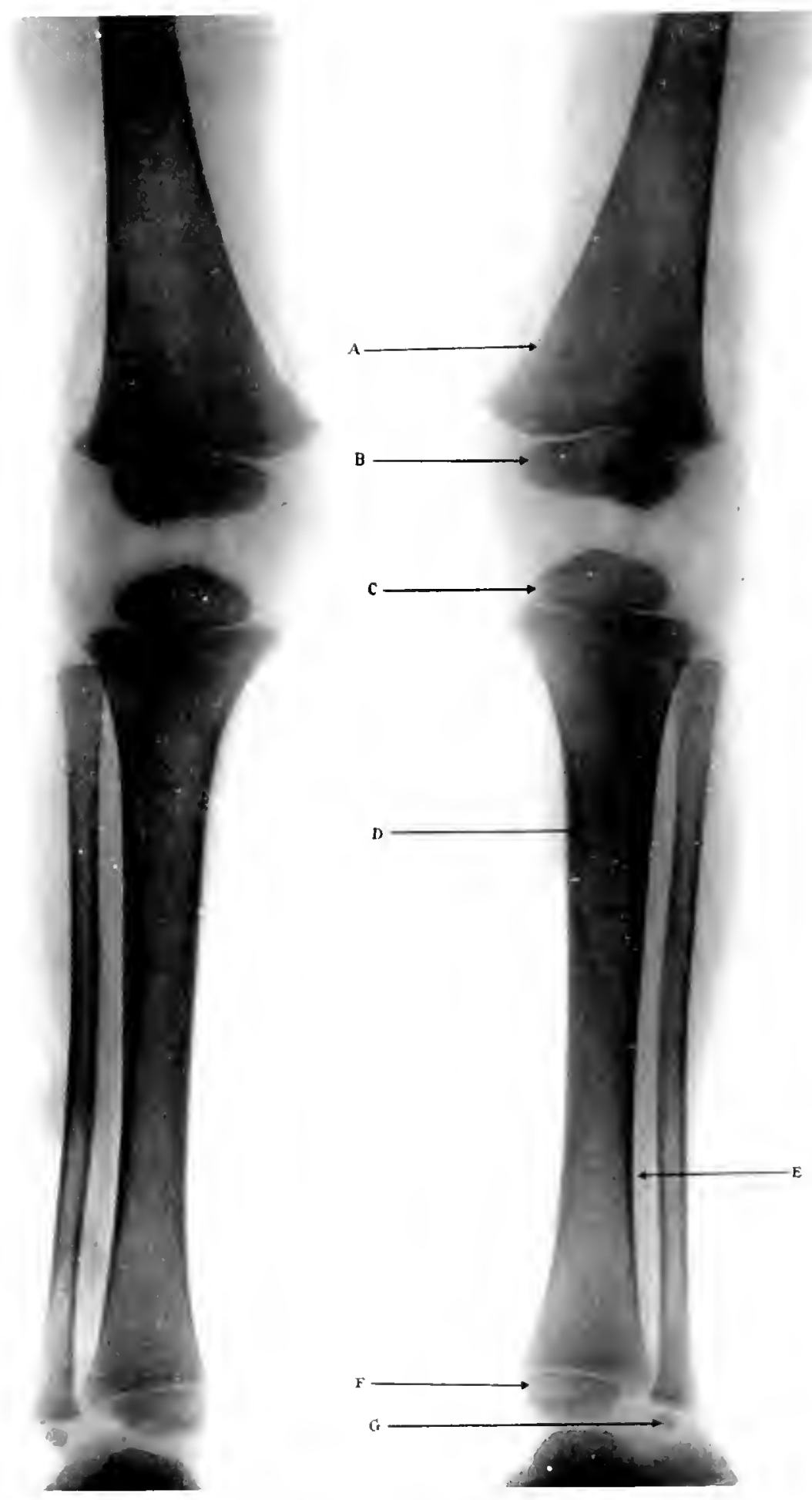
PIATE: 11.

NilliliL FoOT.

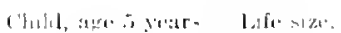

The strueture of the bome is momal.

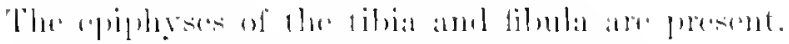

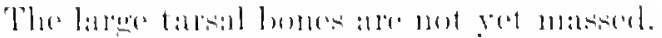

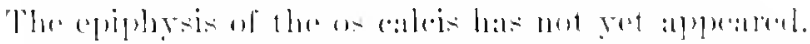

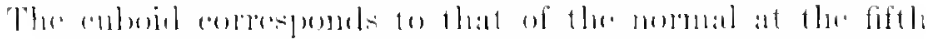

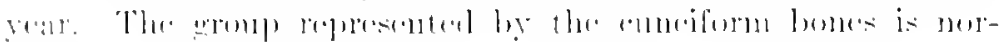

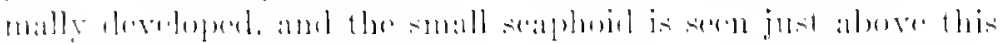

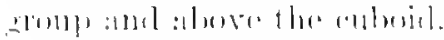

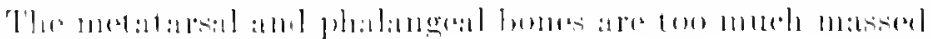
1.1 be difterentiatul. 


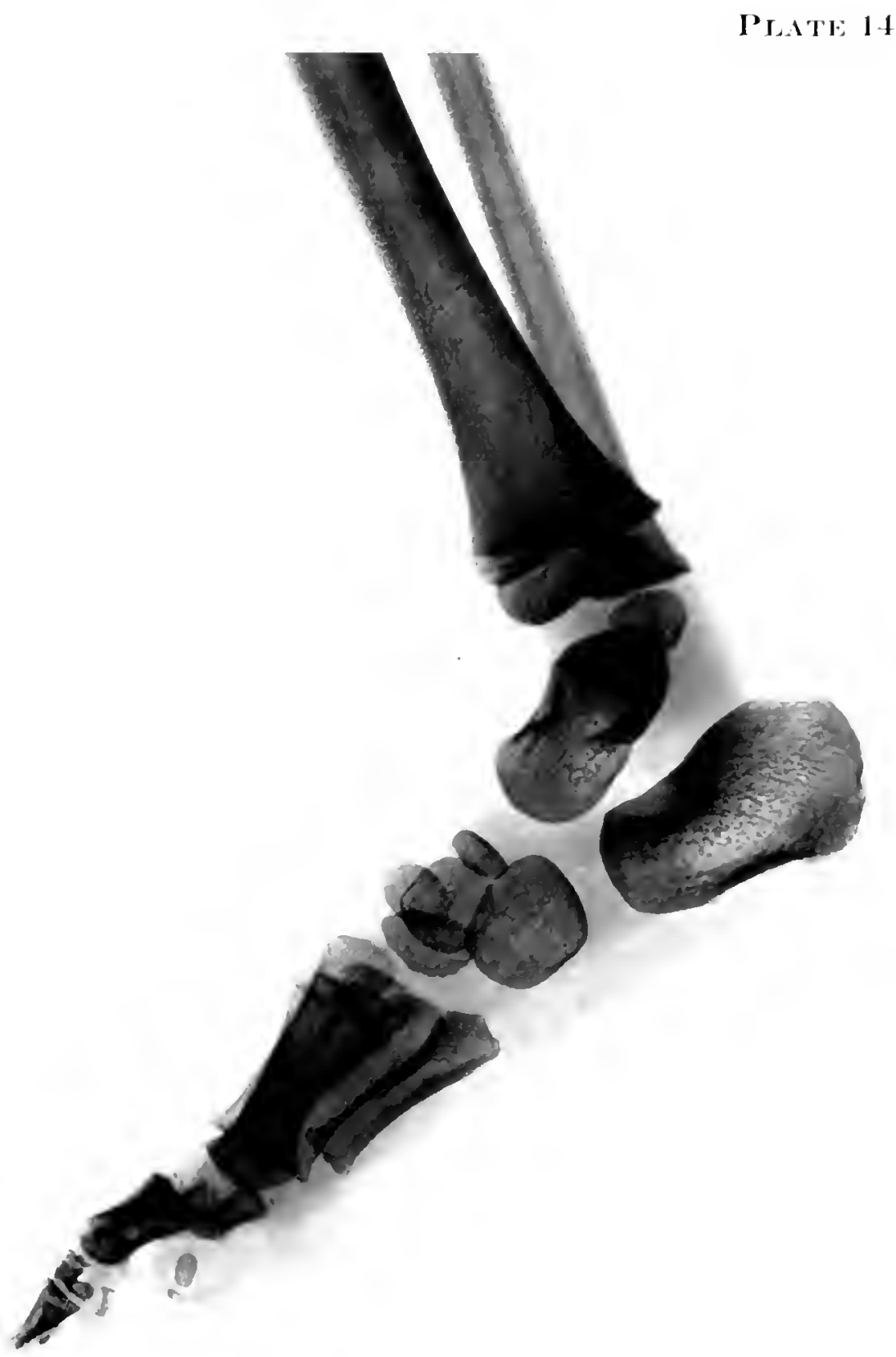


Pl.tTl: 1.5 .

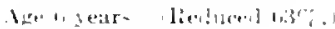

.1. Ohturator forment

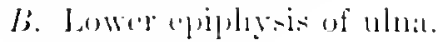

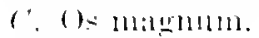

l). ('un iform.

l. Jrapezuil.

F. lienion of ereater trochanter.

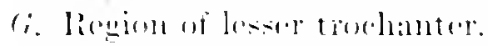

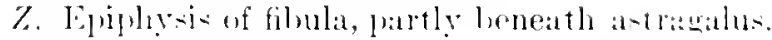

1. 1-trat gallus.

2. (1): (a) 1 (cis. 
PIATE 1.j
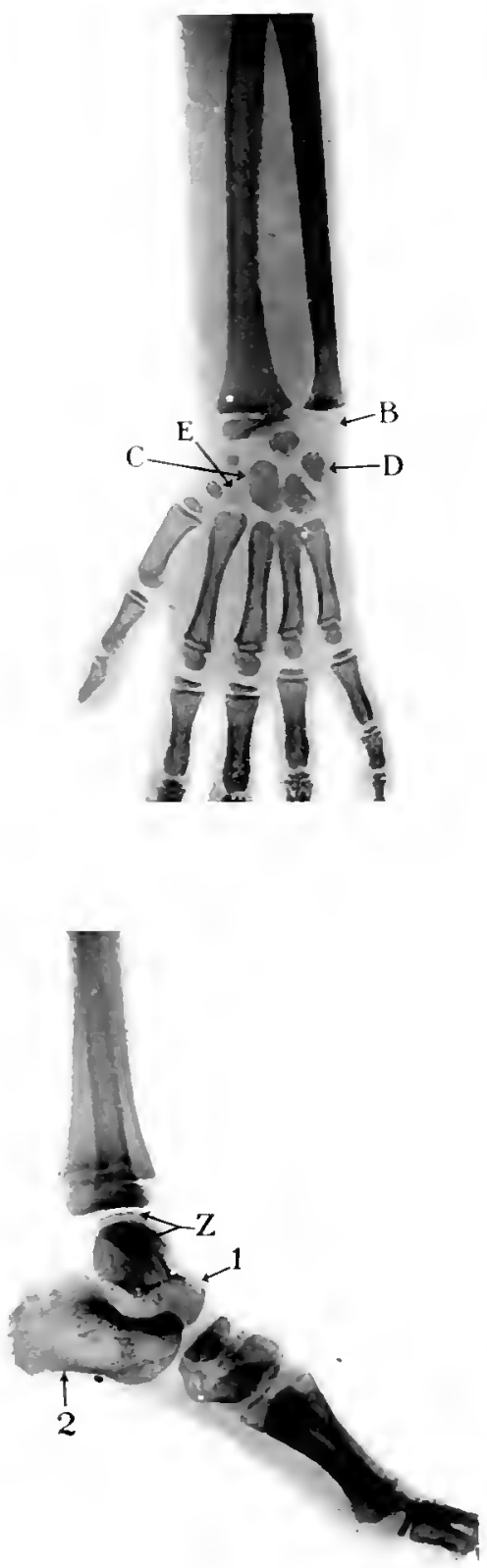

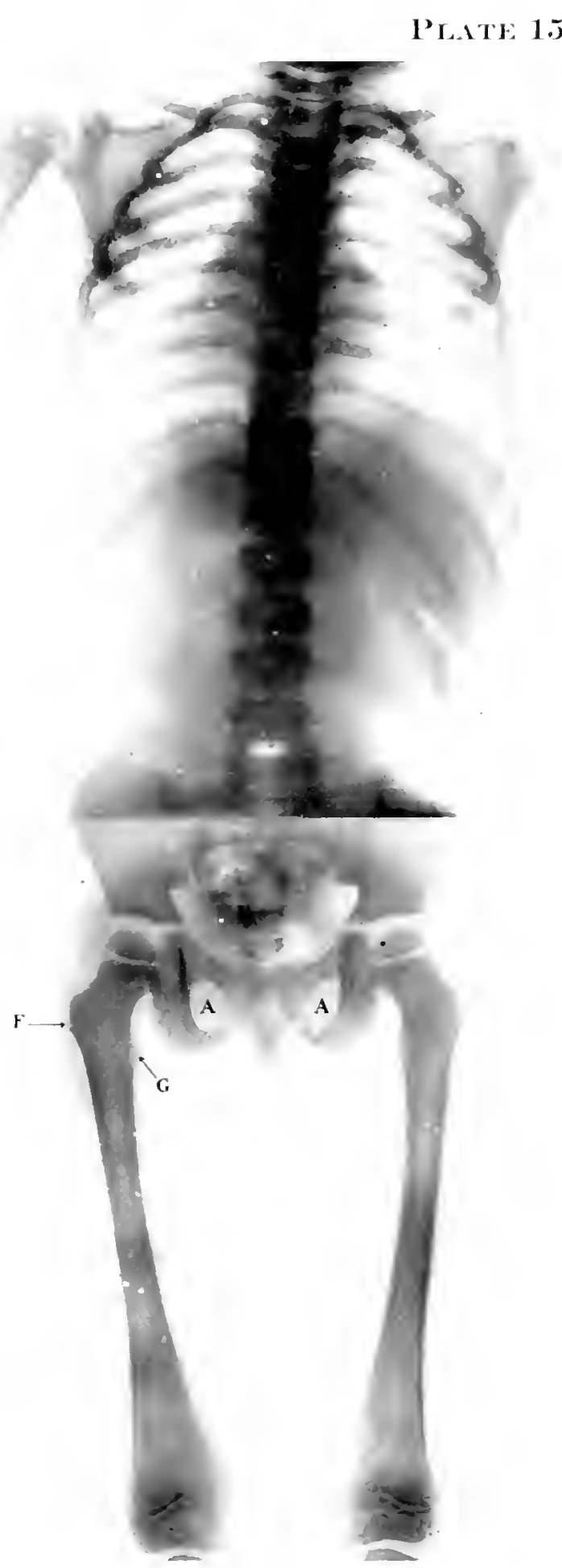


I'LATE 11:

NORMAL SHOLLIOER.

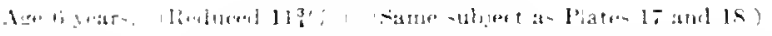

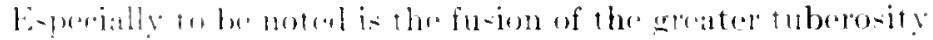
with the heal of the hinntrus. 
PJATE 16;

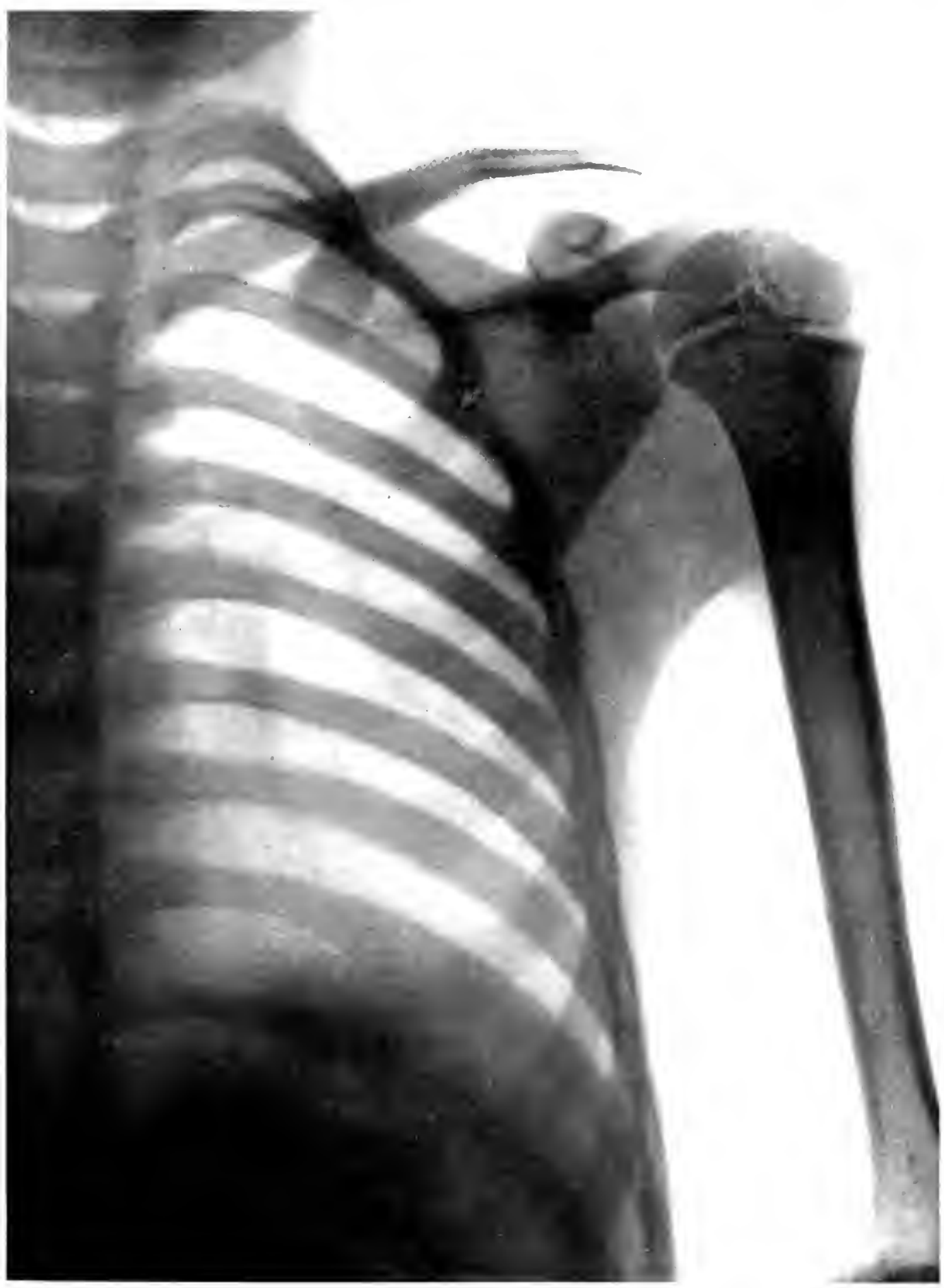


I'HATI: 17.

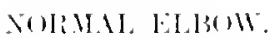

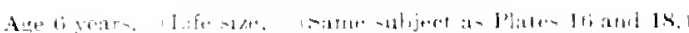

1. SHow- the catpitchum of the humerts. 


\section{Plate 17}

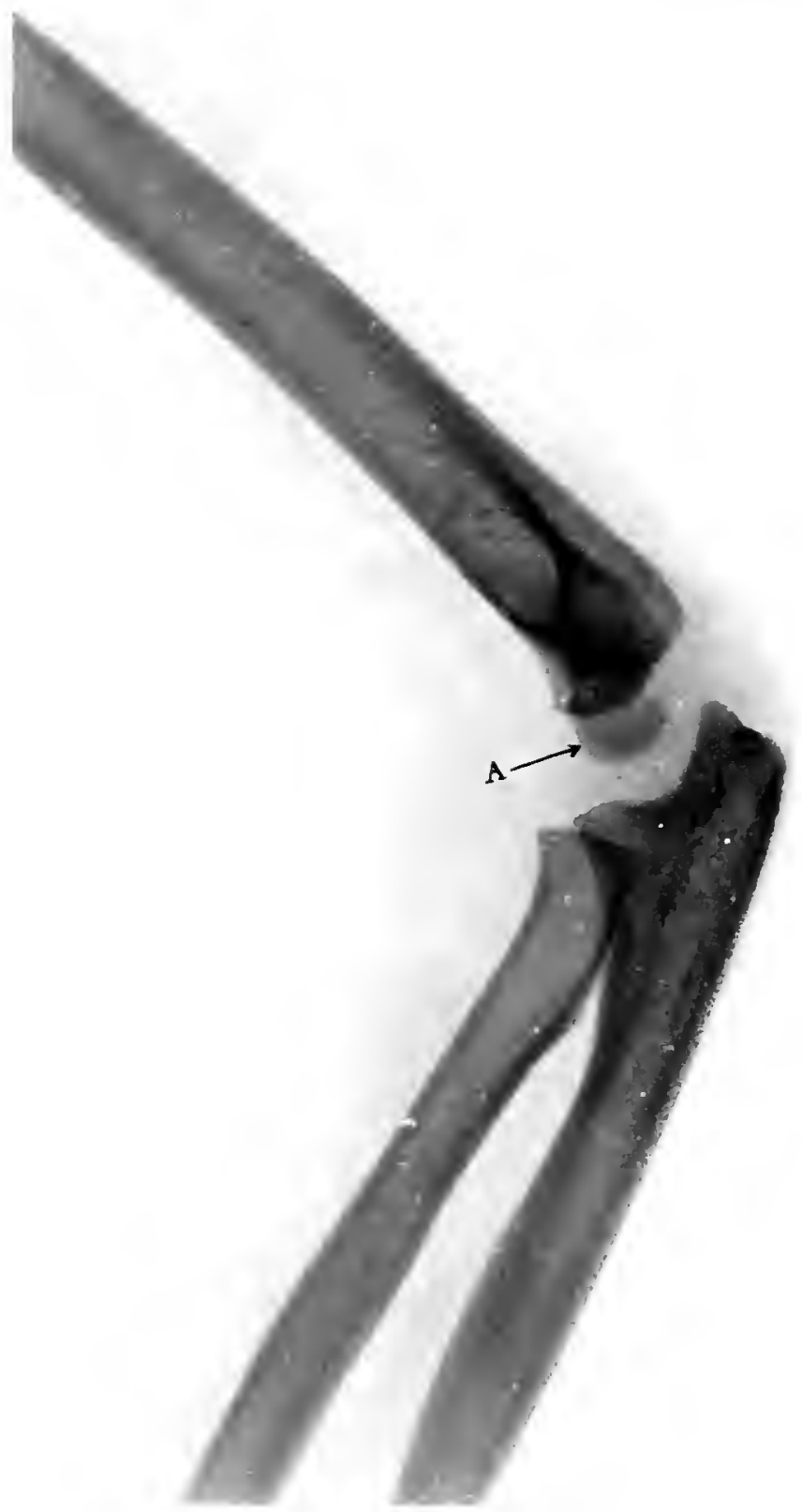


PL.TTE I

NolRMIL, K. LEL.

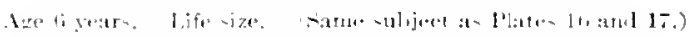

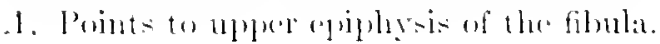




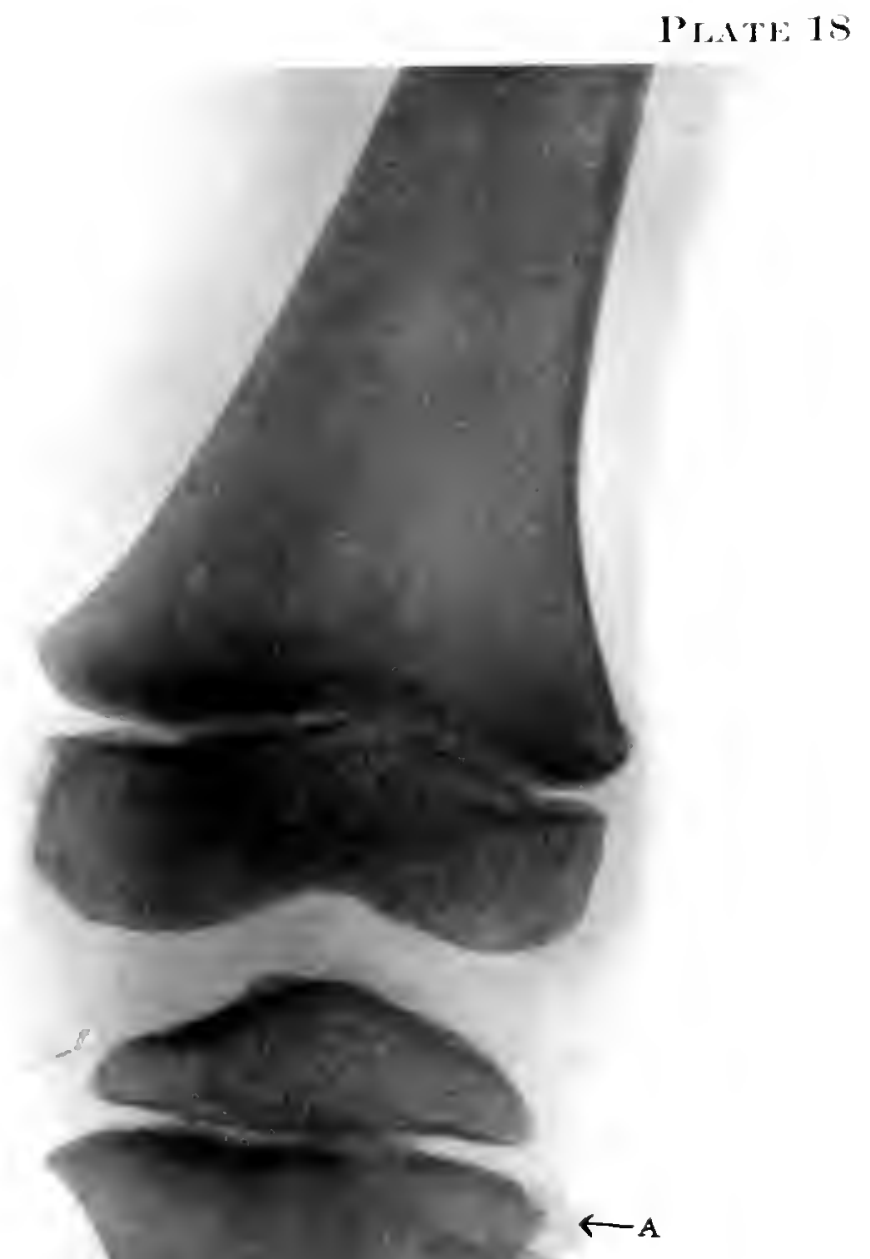


I'L.TTE 19

NMIIII THORAX.

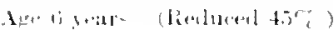


PL.TT: 19

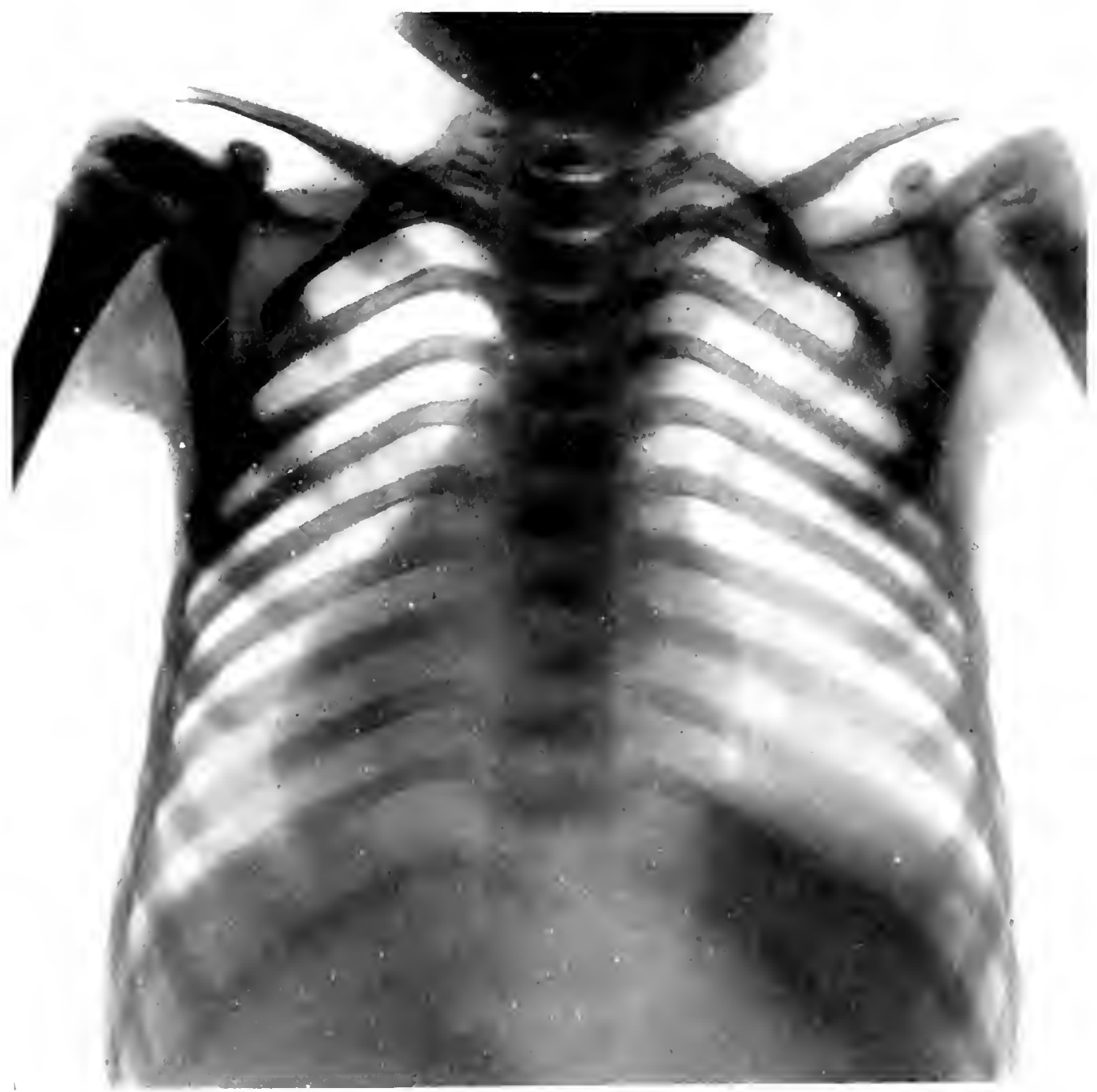


PI.TTE: 20.

voling HANH.

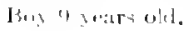


PILite 20
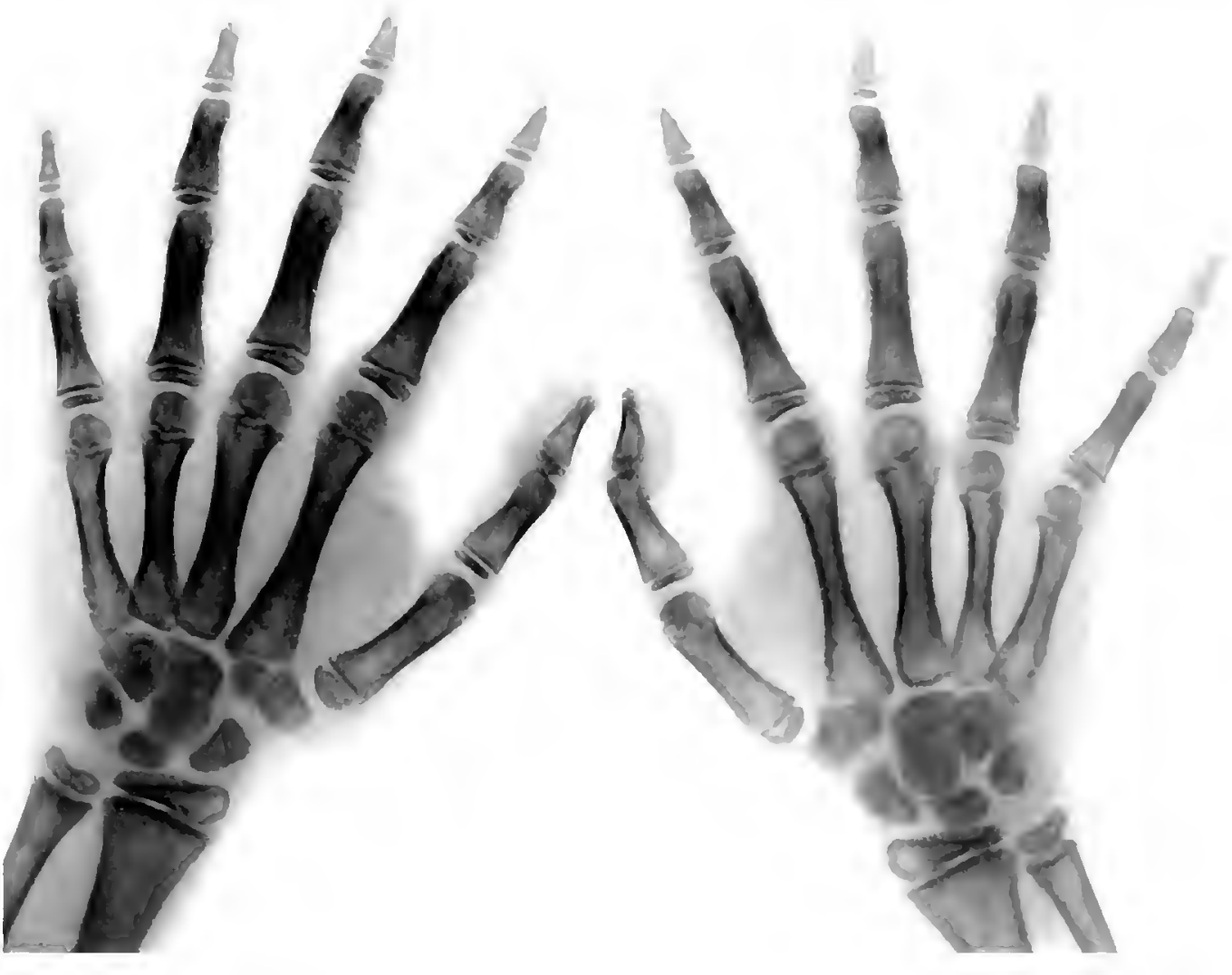
I'L.1TE:21.

NORMAL (HILI).

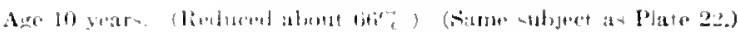

1. Chosed mami of pubes and i-rhium.

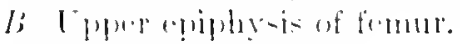

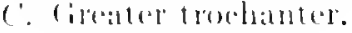


PL.八T: $\because 1$
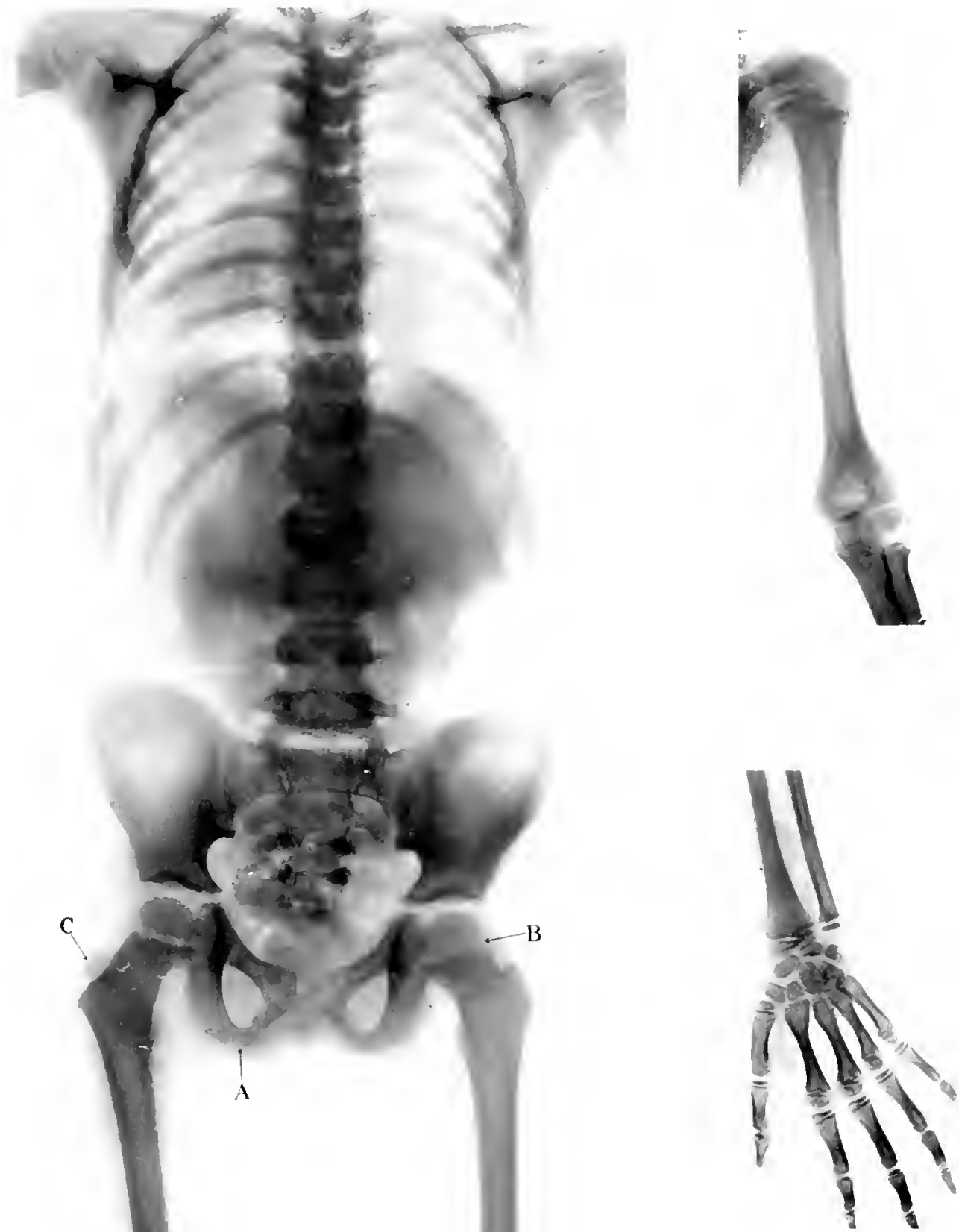
IIATI: 2:2.

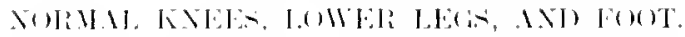

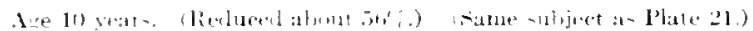

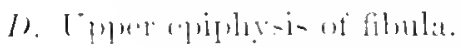

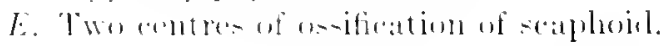




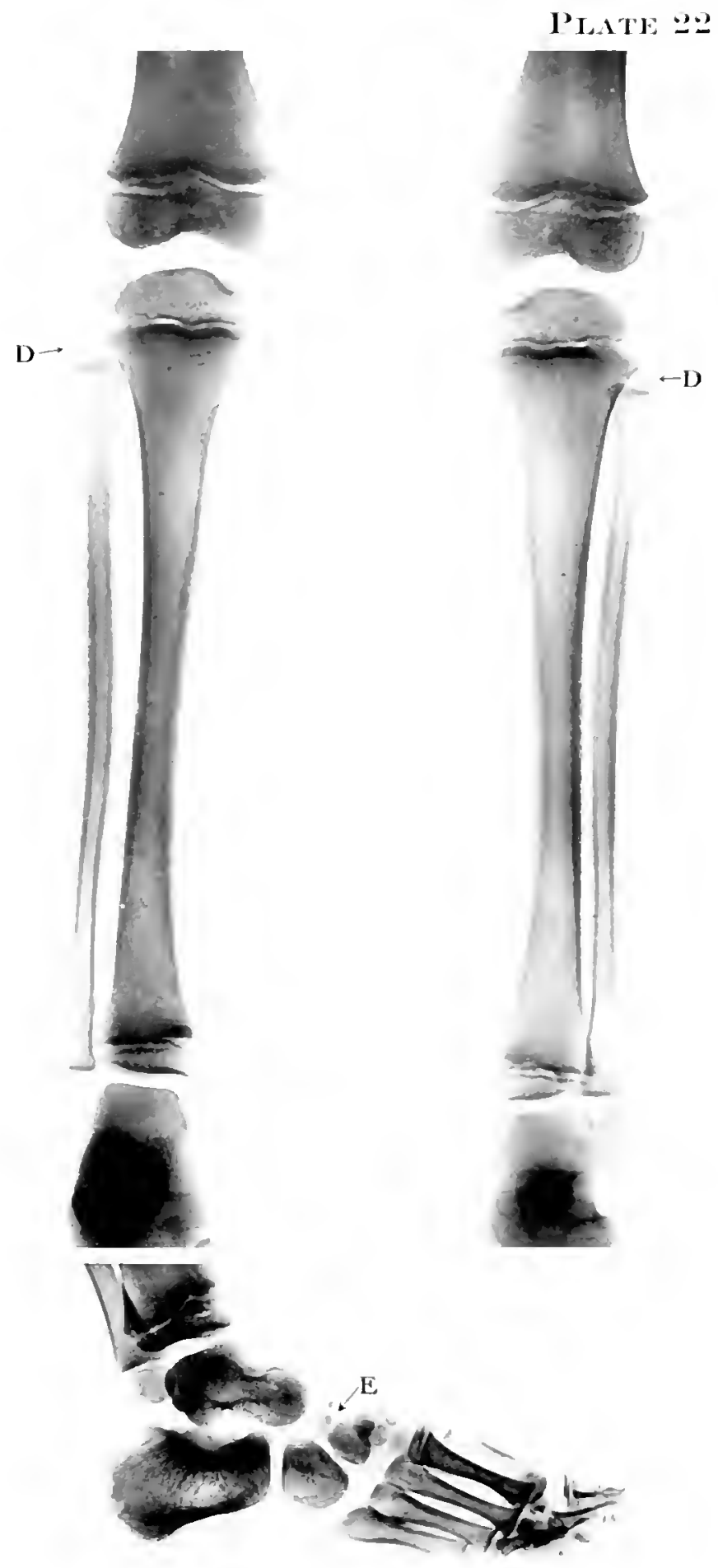


PIATE

NOLMAL SIINE.

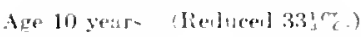

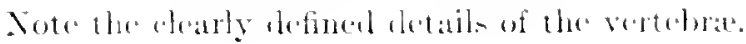




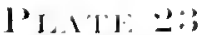

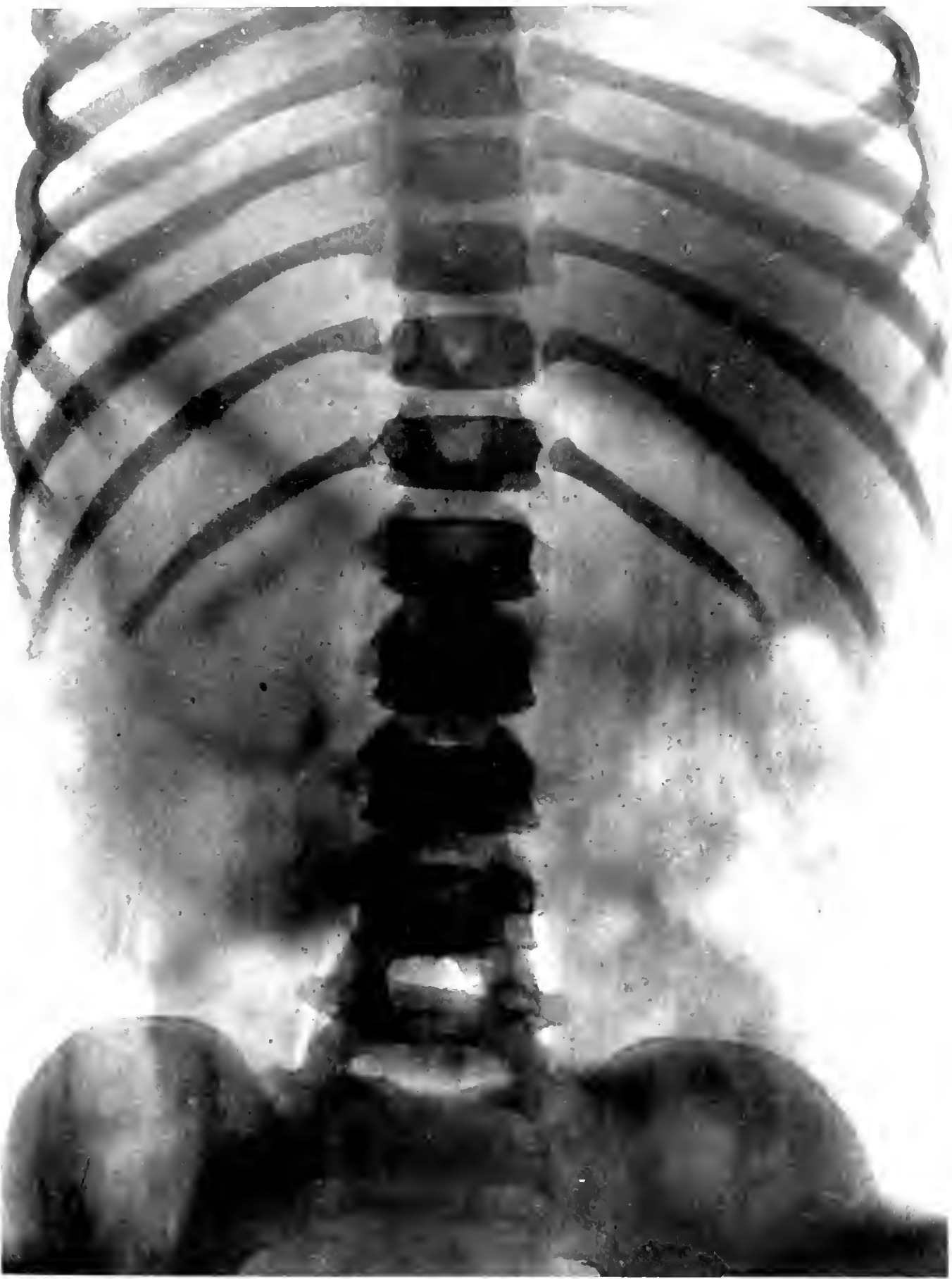


PLITI: 24.

12u 123 stit

1. Priform bone.

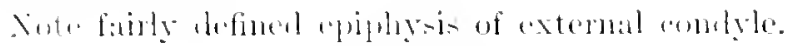


PIATE 24
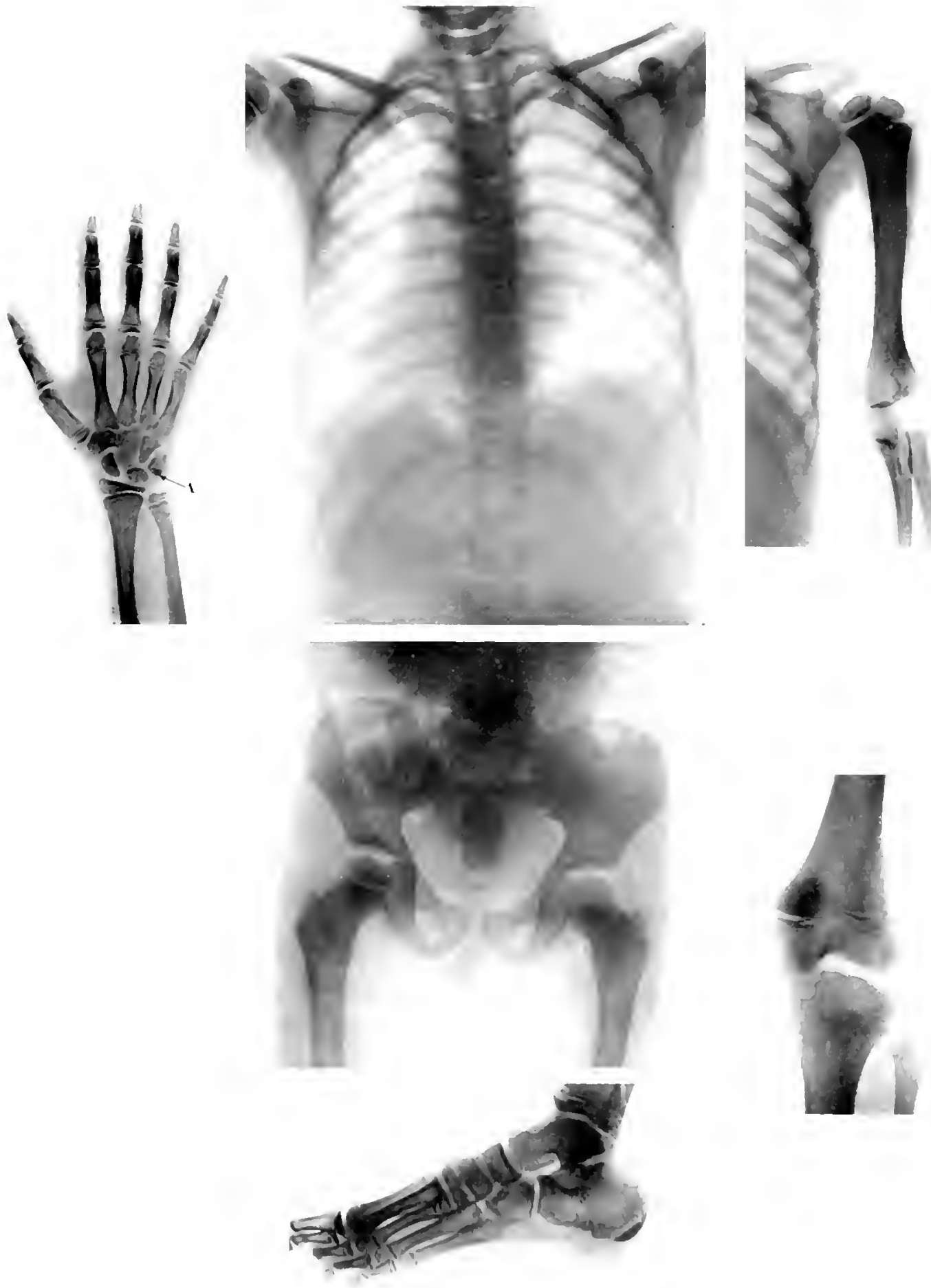
PLATE 2.:

SORMAL ELBWIT.

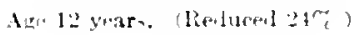

1. Citpitrllum.

b. lutremal rontyle.

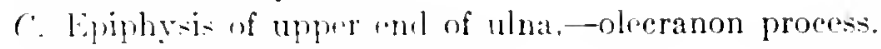

I. Cuper epiphysis of ratius. 


$$
\text { PI.ATE: }
$$

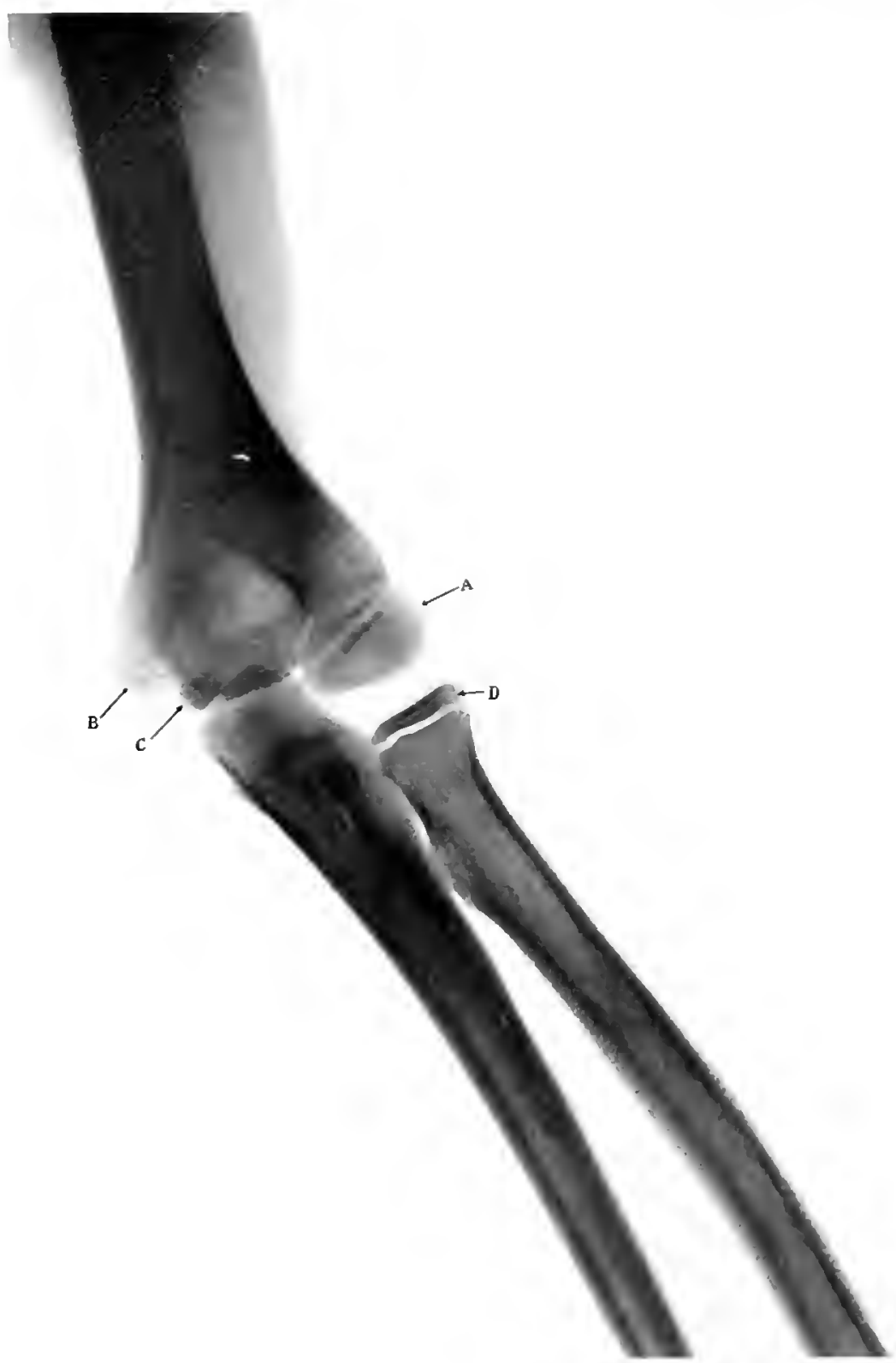


PHAT: 26.

Noling THOPAX.

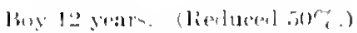

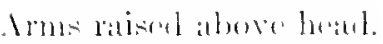

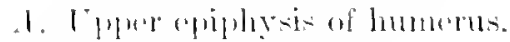

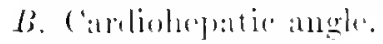




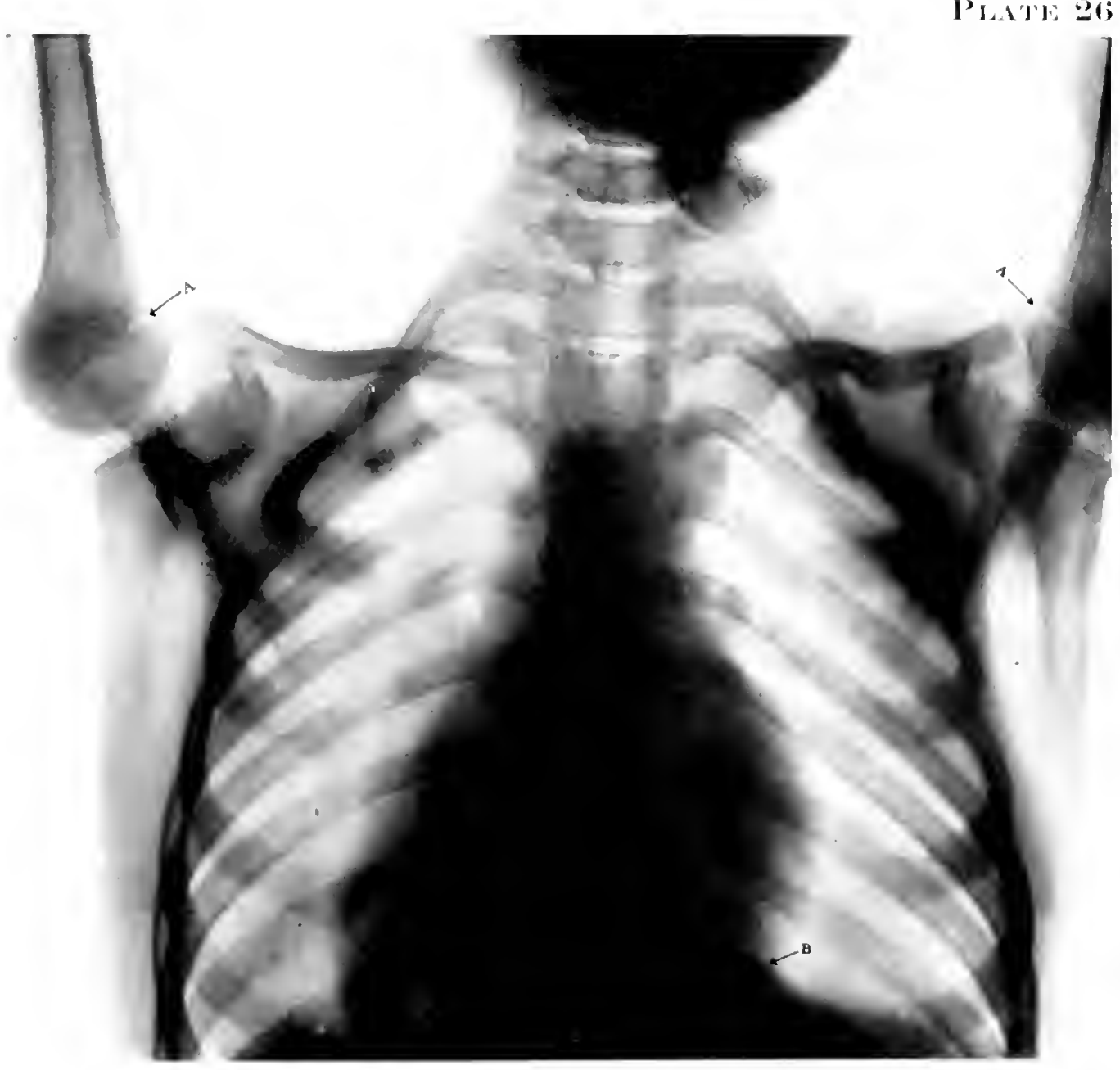


1'1.176:

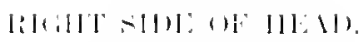

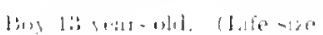

1. Imontal -inms.

15. (1)tit.

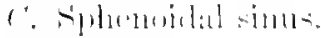

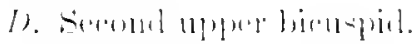

E. Antmun.

F. Worst numer molats.

(i. Thind nuner molut.

It. Thind lonel molits.

1. First lomel birn-bind.

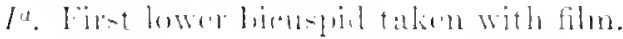


I'I.กT:

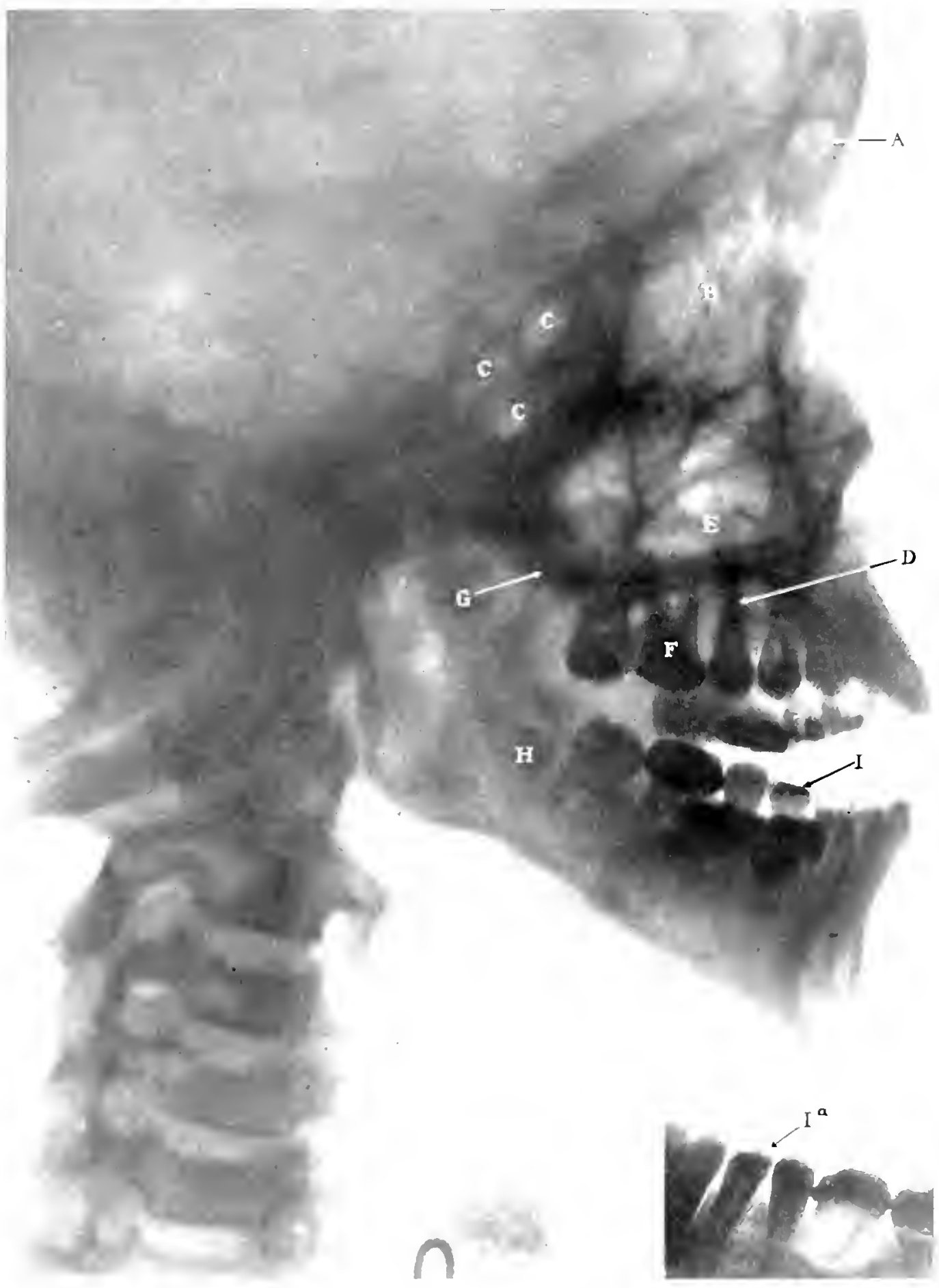





\section{Division II}

\section{ILLUSTRATIVE USE OF LIVING NORMAL ANATOMY}

THE consideration of chronologic and anatomic age in early life has become very important, since the work of various investigators, especially of Crampton, has shown the great discrepancy which exists between them. 'This discrepancy is not merely of scientific interest; on the contrary, it opens up a vast field for the study of the management of child life and for the change from existing customs in connection with it.

Up to the present time the age of an individual has been computed by years, and this rule will probably always hold good from a legal point of view in all civilized communities. The question whether chronologic age is a wise division during the formative period of early life when brought to bear on our school systems, whether in classifying and grading children as to their studies, or pitting them against each other in athletic sports, becomes a very serious one. Again, when the important question of child-labor is brought before us and we have to determine at what age a child should be allowed to work, we can at once see that a chronologic division for this purpose is not only insufficiont but clearly pernicious. It behooves us then to look at the question of age in early life: (1) from a chronologic point of view,-manifestly legal,-(2) as regards athletics,--manifestly anatomic,-(3) educational, as regards school grades,- - manifestly a combination of chronologic, plyysiologic, and anatomic conditions.

1. Legal chronologic age does not enter into this discussion.

2. Anatomic age should first be carefully studied before school and child-labor can be dealt with intelligently. From a medical point of view the physiologic and anatomic conditions can for the 
present be considered as one, since at this point of the discussion we can consider the normal physiologic development of the various functions to correspond to and keep pace with the normal anatomic development. I wish it to be understood, however, that this supposition is merely preliminary to some work which I expect to carry out later on this very subject, namely, whether it is true that deductions made from a physiologic standpoint can be properly used when compared with anatonic conditions. This will be a much more difficult investigation and probably will merely show that there is such correspondence between the physiology and the anatomy of human beings. This, however, should by no means be taken for granted and is still sub judice. The great importance of an exact knowledge of the anatomic development as expressed by the bones and joints to avoid overstrain at a time of life when this avoidance is of prime necessity should be impressed forcibly upon the public in general. This knowledge should be acquired especially by those who have charge of the athletic grading of boys and girls, whether in football or basketball or general gymnastics. Having determined what the anatomic growth is at different stages of development during the growing period, we can then apply this knowledge to the broad question of education and of child-labor. The problem at once presents itself as to the best method for determining the normal anatomic development from birth to perhaps fourteen years, and how to express it in as small intervening periods as is possible and practical. This anatomic knowledge, however, should represent the different stages of living anatomy rather than depend on the results obtained from studying dead anatomic conditions. As our knowledge advances the anatomic intervals will become shorter and shorter. There is no doubt that from a somewhat extended study of the subject which I have made during the last two years a much closer and more reliable estimation of age from an anatomic 
point of view can be made than could possibly be acquired from a chronologic. My belief is that in the future we shall determine age, whether for gymnastics, athletic sports, kindergarten, school, or child-labor, by means of anatomic conditions rather than by chronologic periods. It has been shown by a number of writers that growth is influenced by race and heredity and by social and hygienic conditions. Thus, for example, the Scandinavians, Scotch, and lighthaired Germans are tall, while the Italians and French are short. The offspring of a mixed anatomic parentage is more like the large parent. The upper middle social stratum is most favorable for large growth, as is the upper middle hygienic stratum. It is known that at the period of pubescence the girl increases in growth more rapidly than does the boy, and that at or about the period of puberty the bones increase rapidly in length and in girth. As an instance of the unreliability of chronologic age can be cited the term puberty, a physiologic condition which is supposed to occur at a certain chronologic age. According to Crampton's admirable work on this subject, the age at which puberty in a group of children of the same chronologic age and of either sex is attained as expressed by years is fallacious, and indeed practically so variable as to amount to nothing. In a large number of healthy boys varying from ten to fifteen years the time of pubescence showed such a variation that Crampton claims that the growth of pubic hair indicates pubescence much more exactly and with far less differences of time than has been supposed to have been shown by stated years. In like manner Crampton has shown that the menstrual function of girls varies to a far greater extent in years than it does when reckoned by his physiologic sign of pubescence. Crampton also shows that such indications of growth as height, weight, appearance of teeth, and strength seem to correlate quite closely with the physiologic signs of pubescence. These indications, as individual factors of growth, 
however, vary in the individual to such an extent that data taken from them for classifying children by years proves to be inadequate and indeed incorrect. Groups of boys of equal age and in the same grade in school, their grades being determined by their ehronologie age, were found to vary perhaps by a year or a year and a half, not only in their height, weight, strength, and in the eruption of their permanent cuspids and second molars, but also in their being bright, moderately bright, or dull. With these results of Crampton before us, it is manifestly absurd to place children of the same ehronologic age necessarily in the same grade at school. Therefore some more practical and closer data for classification should be sought for if the children are to be intelligently dealt with during their school years. Crampton's work and his deduction that the test of the growth of the pubic hair should be made use of in determining the real age of the child are based upon certain results which he elaims show a much greater uniformity than does the division which has previously been made on the basis of years. Some of these results are as follows:

Post-pubeseents were found to average from 24 per cent. to 33 per cent. heavier than pre-pubescents of the same age. Post-pubeseents averaged as much as 11 per cent. taller than pre-pubeseents of the same age. Post-pubescents averaged about 33 per cent. stronger than pre-pubescents. The higher the grade the fewer the pre-pubescents for age, hence post-pubescents are better in scholarship than pre-pubescents, and the more advanced a group is in pubescence the better will be the scholarship. Crampton also concluded that by far the greater part of the correlation of weight, height, and strength as found in scholarship was due to the fact that they were all correlated in common with the pubescent factor. Crampton has also concluded that earlier pubescence favors good scholarship, later pubescence poor scholarship. In a study in regard 
to the eruption of the teeth in about one thousand elementary school boys from ten to fifteen years of age it was found that the appcarance of the teeth had but a slight relation to the actual chronologic age. It was also found that those boys who had a full set of permanent cuspids averaged from five to seventeen pounds heavier, and from one inch to three inches taller than those with none. A preliminary study of weight, height, and strength showed that menstruées are taller, heavier, and stronger than non-menstruées, and that weight showed the closest correlation. These investigations of Crampton's have been based upon a period of years from nine to sixteen or seventeen, and on such physiologic data as could be deduced from observations during the pubescent period. Although certain investigators (Narro, "La Puberté") had already suggested that the growth of the axillary and of the pubic hair was significant as showing physiologic periods, yet such extensive investigations as Crampton's had not been carried out previous to his publications, and he was the first one to present the thesis that all our treatment of the child, whether educational, medical, or sociologic, should be based upon physiologic age rather than upon chronologic age. Deductions made from the observations of such external appearances as the pubic hair must necessarily not represent very short, and in that sense practical, intervals for the purposes of classification of which I have just spoken. It has therefore seemed to me, allowing for the present that a correlation of physiologic and anatomic conditions may be taken for granted, that a study of the anatomic conditions present from birth to thirteen or fourteen years may be of still greater value than the physiologic data based on the observation of the pubic hair. If such an anatomic classification can be made it will make no difference in its practical application whether the individual child is healthy or unhealthy, normal or abnormal, or is of one race or of another. It may be of a parentage differing in physical 
development, excepting in so far as boys and girls can be separated normally in their anatomic development. That is, with such an anatomic classification we ean praetically work out our problems of ehild life irrespective of whether the individuals are boys or girls, and whether the girls menstruate early or late. This anatomie classification also is valuable for the purpose of adapting children from birth to adolescence to their proper healthy surroundings and to a life suited to their individual strength and capacity. In order to prepare for and clear the ground for the study of this question, namely, how to determine the normal condition of young human beings from birth to thirteen or fourteen years of age, certain preliminary investigations are necessary.

With this end in riew I have had over two hundred ehildren, who according to our previous ideas should be considered normal in the different years of life, examined carefully. In order to obtain the required data the hygienic surroundings were investigated. It was noted whether the child was bright or dull mentally, what its general condition was with regard to its appearance, its various functions, and its appetite, and what diseases, if any, it had had previous to the time of its examination. Its age was noted; at what age it sat, stood, and walked alone, and a record of its weight and height made. If a girl, whether the eatamenia had appeared; at what age, at what intervals, and with what regularity; also at what age the mamma began to develop and their present condition. The circumference of the head was noted, and also any information which could be obtained as to the closure of the fontanelle, and at what time the first dentition began; whether the temporary teeth had appeared at regular intervals; when the second dentition began, and whether it was being earried on regularly. A Roentgenograph was then taken of the carpal bones and the child was classified and placed in the division to which the bones of its wrist corresponded. Roent- 
genographs were taken of those children only who were shown by examination to be presumably normal in their general development.

This mass of data can be used for still further study of the correlation of weight, height, teeth, chronologic age, physiologie age, and various external evidenees of normal development as shown in the reeords. It is my intention in the future to continue the study of early life on these lines in order to determine what correlation there is between all these different factors of the problem and the results which I have obtained from my investigations of anatomie age. As there can be but little doubt that normal physiologic age corresponds elosely to normal anatomic age, it seems to me safe and warranted to assume that Crampton's physiologic investigations correlate closely with anatomic normal development.

In undertaking to classify the different periods of early life on an anatomic basis, which will be of practical use in athletics, in school, and for questions relating to child-labor, it is evident that some reliable anatomic standard representing the entire physical development should be found. It is also important that sueh an anatomic standard should be readily obtained, and should be determined in the living child with the least degree of complexity and in the most simple form possible. In the study of over a thousand cases of healthy children at the Children's Hospital by means of the Roentgen method our Roentgenologist, Dr. A. W. George, found that the most practical and reliable index of development was represented by the hand and wrist. It is true that various changes which progressively take place in the development of the shoulder, elbow, knee, and ankle are possibly somewhat finer than those of the wrist. For the purpose, however, of obtaining a practical and rapid knowledge of the progressive normal changes which take place at different periods of ehild life, the parts just enumerated do not compare with what can be aceurately acquired in a few 
seconds from the wrist and hand. It has oceured to me therefore that a careful study of the normal anatomic conditions present in the bones of the hand and wrist during different stages of their development rould best aid us in classifying the different stages of development, and having formulated such a classification we conld then apply it practically to physieal and edueational problems. During the last thrce years I have therefore had made careful studies of a large number of apparently healthy infants and children of different ehronologic ages. A record bas been kept of each of these children, not only as to their age, weight, height, teeth, general physiologic and anatomic condition, and social surroundings, but also a special reeord of the development of their hands, by means of the Roentgen method. As a result of these investigations it has been made very evident how unreliable are ehronologic records in regard to weight, height, and teeth, the teeth especially showing the greatest variations. I shall not here, except in a general way, refer to the information which I have obtained regarding, for instance, the weight and height in reference to the chronologie age, since the unreliability of such correlation has often been shown by other investigators. I have, however, ehosen for my classification only those individuals who by careful examination could be presumably considered normal. It must be understood that I am no longer dealing with stages of development as represented by years, but that my divisions are placed under letters. Having taken perhaps ten or a dozen children of each age chronologically I have then placed them under their respective anatomic stages of derelopment, designating these stages by $A, B, C, D$, and so forth. I have then used these different stages for purposes of grading for kindergarten, for school, for athleties, and for child-labor. In a general way in making up these alphabetical groups I have arbitrarily assumed that the appearance of the carpa! bones and of the lower epiphyses 
of the radius and ulna should, according as they developed early or late, represent each alphabetical division. As none of these bones are normally present at birth, and as the os magnum and uneiform bones appear in the first year, I hare placed this group of eases under $A$. Under $B$ I have grouped those hands which show the presence, in addition to Class $A$, of the lower epiphysis of the radius. Again under $C$, those which in common with $A$ and $B$ show the presence of the euneiform bone. The heads of the metacarpal bones and the epiphyses of the first phalanges at this period can be used as controls of this latter group $C$. Still under these arbitrary divisions I have continued to place groups which suceessively show the presence of the semilunar and trapezium bones with the second and fourth phalangeal epiphyses as controls of this group. I have then introduced as additional groups those which show the scaphoid, the trapezoid, the lower epiphysis of the ulna, and the pisiform bones. These primary divisions are shown in Table 4 and this table can be used as a key by which we can show under which alphabetieal division an especial case should be placed. This is accomplished by Roentgenographs showing a picture of such individual carpal bones and epiphyses. It is fortunate that the carpal bones and the lower epiphyses of the radius and of the ulna furnish a progressive series of anatomic development year by year, so that only in unusual eases will it be necessary to refer to the other epiphyses of the skeleton for the purpose of corroboration.

Table 4.-Key to Index Development. Represents in Alphabetical Divisioss the Developmextal Stages of the. First If Years of Life.

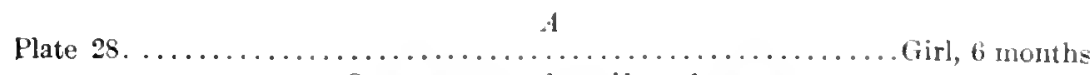

Os magnum and unciform bones.

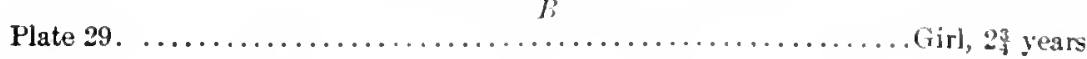

Os magnum, unciform, and lower epiphysis of radius 
$r^{\prime}$

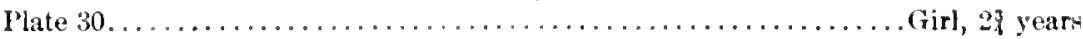

Os magnum, uneiform, lower epiphysis of rallis, cuneiform.

l)

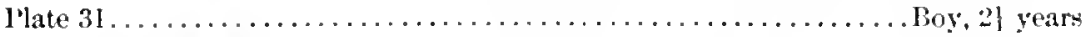

Os magnum, nuciform, lower epiphysis of ratius, aluneiform, semilnnar. $\mathrm{E}$

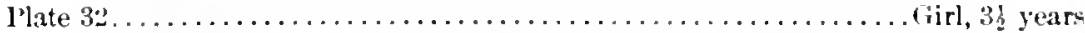

(s magnum, anciform, lower epiphysis of radius, enneiform, semilunar, trapezium. (According to l'ryor, scaphoil shonled come after semilunar.) $F$

l'late 33.

$$
\text { F }
$$

(iirl, 51 years

Os mannum, unciform, lower "piphysis of ratius, cuneiform, semihmar, trapezimu, seaphoid. (According to I'ryor, trapezoil should come after seaphoid.)

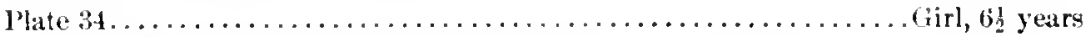

Os macmum, unciform, lower epiphysis of radius, cunciform, semilunar, trapezium, seaphoid, trapezoid. (Accorling to Pryor, tr"ipezinm should come after trapezoid.)

II

Plate 35

Os marnum, unciform, fower "piphysis of rafius, cuneiform, semilunar, trapezium, scaphoid, trapezoidl, hwer epiphysis of ulna. Girl, ti years

\section{$I$}

l'late $36 \ldots \ldots \ldots \ldots \ldots \ldots \ldots \ldots \ldots \ldots \ldots \ldots \ldots \ldots \ldots \ldots \ldots \ldots \ldots$
Same number of bones as $I I$ but more developed.

. Girl, 6a years

$$
J
$$

Pliate $37 \ldots \ldots \ldots \ldots \ldots \ldots \ldots \ldots \ldots \ldots \ldots \ldots \ldots \ldots \ldots \ldots$
Same number of bones as $I$ but more developet 1.

$\kappa$

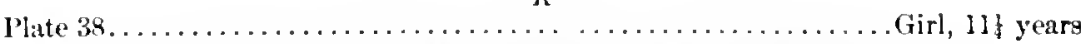
.Girl, 81 years

Same number of bones as $J$, except that possilly the pisiform is beginning to show through the cuneiform. The rarpal bones also are massing together eloser than in $J$, whieh is significant of a more alvanced development.

Plate 39.

L.

Os marnum, meiform, lower epiphysis of radius, euneiform, semilunar, trapezium, scaphoid, trapezoid, lower epiphysis of una. I'isiform seen eoming ont from under the cuneiform.

II

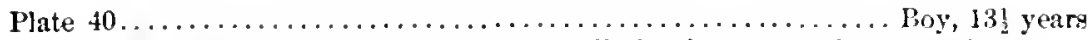

Same number of bones as $L$, but all the bones are larger and] closer together, and the entire hand shows a much greater and later development than any of the other indices. Pisiform bone almost as large ats cuneiform.

In pursuing this investigation I have allowed for what appeared to be marked rariations from the normal development. I have made my divisions irrespective of deviations, by grouping those 
cases together which were manifestly in about the same stage of development in the large majority of cases. I am aware that the divisions which I have made may have to be subsequently changed according as further investigations are carried out in this line of study. In order, however, to formulate and start what I think in the future will be found to be of great aid in dealing with the early years of life, both anatomic and physiologic, it was necessary to begin by arbitrarily suggesting groups of classifications as they appeared to my eye to show uniformity. Some rather interesting observations in this connection have already come under my notice. For instance, hands may be long or short, broad or narrow, and yet the Roentgenograph will show that they may have the same degree of anatomic development, showing also that they should be classed together in one group. In making up such a group, however, we cannot be guided except to a limited degree by the external appearances of the hands. I have also noted in my study of these two hundred hands that although there may be a large hand, with large bones, as shown by the Roentgen picture, yet this does not necessarily imply advanced development, for the development of these large bones need not necessarily be great. I have noted also that the normal appearances shown in Roentgenographs of the hands of boys and girls do not materially differ, and that the left hand does not show any difference in development from the corresponding right hand. In all probability it will be found that many of the statements which have been made in the different anatomies, which are the results of investigations on dead subjects, will be proved eventually to be incorrect when submitted to the more exact observations which can be carried out on the living subject. Notably one of these discrepancies is that in a number of anatomies, English, French, and German, the lower epiphysis of the ulna has been placed as early as two and a half 
years, three years, four years, and five years. The appearance of this epiphysis in my cases, however, was found to be very rare before the sixth year, and it usually began to appear in division $I I$, where the children were from six to seven years old. It is also to be noted that while the pisiform bone has been found as early as the tenth year, and according to Porier, the eighth year, in none of my cases was it found before the twelfth year, excepting once as an anomaly in the eighth year. I have also noted that the heads of the metacarpal bones are not especially satisfactory, so far as classification is concerned, and should therefore be used more as controls in cases where the development of the wrist is not sufficiently conclusive.

In order to represent this elassification practically we should have pietures which represent the development of the special group before us, and using these for comparison determine an individual child's anatomic age. These pictures I have prepared, choosing them as representative of their especial group. It is very evident, if this system of grading ehildren in the future proves to be correct and useful, that, except legally, we should no longer think in years and months, but in normal anatomic groups $A, B, C$, and so on. We should remember also that all our physiologie knowledge should be brought to bear on this subject, using it in conjunction with, and as a control on, our anatomic knowledge; also that in doubtful cases the development of other parts of the skeleton, such as the shoulder, elbow, hip, knee, ankle, and foot, should be used as controls. We might indeed say that it is the sum total of all our physiologic knowledge, together with that of the development of the entire skeleton and the different organs, which tells us exactly how to solve the many problems of early life. For preliminary information, however, on these points we should first turn to what seems to be the most reliable index, namely, the wrist. These groups of course 
are manifestly somewhat arbitrary, and depend upon my observation of such cases as my experience has shown to represent the majority of normal conditions rather than variations. Further study may prove that it will be more exact to adopt certain other divisions and possibly to merge some of these present divisions into one. This, however, can easily be done if we are sure that the divisions are improved and made more practical in this way. What I wish to state, however, is that I believe we are working with the right principle, and that practical results in the line of perfecting the development of children and guarding them during the process of their development from overstrain both mentally and physically can thus be accomplished. It may be stated as a rule that, while in all probability weight and height correlate with a stated stage of normal anatomic development, that is, greater or less according to the anatomic development, yet this is true only where a group of individuals of the same anatomic development are concerned, rather than where the height and weight are compared with an individual of a stated chronologic age. It is very evident, as shown by those who have investigated the eruption of the teeth, that such eruption is entirely unreliable for purposes of classification. The sigma (range of variation) is very great as regards the eruption of the teeth and the height and the weight in comparison with the chronologic age, which in its turn shows a corresponding sigma as regards the anatomic conditions.

The regulation of questions concerning the physical and mental care of the early years of life should be much more under the control of the medical profession than is now the case. An intimate knomledge of medical subjects and advanced medical training are needed to grasp these questions fully and to treat them intelligently. Surely it is one of the gravest duties of physicians to turn their attention to all subjects connected with children, and not to leare them to 
be worked out by the non-professional laity. Educators dealing with these questions cannot accomplish what they are not by long years of physiologic and anatomic study fitted for. The educator in the public schools in grappling with the difficult questions of physical training should work hand in hand with the medical profession, for thus only can the great end in view, of producing not only highly educated but healthy and rigorous citizens, be attained. The papers read in 1908 at the Delaware Water Gap by Abt, of Chicago, and by Kerley, of New York, on the vital questions connected with kindergarten and school life show the necessity for physicians, and especially for those who are interested in pediatries, to take at once active measures to change the unwarrantable situation which confronts us in our schools and factories. This situation, with its irrational conditions and baneful results, is threatening to destroy the heaithy bodies and minds of a large majority of our future citizens at the very beginning of their careers. It is giving them as preparatory for their race in life the handicap of a weak and easily strained physique and an overtaxed brain, at the very period of development when it tells most severely. I say this with a full understanding of how great is the recuperative power of the young. There are certain overstrains, however, which can never be eradicated, notwithstanding this high grade of recuperative power. At any rate we who have the knowledge and wisdom of adults have no right to subject millions of ignorant and helpless young human beings to a system of living which is disastrous to the majority. We also have no right in connection with this phase of life to trust to the survival of the fittest. On the contrary all these young lives should if possible be made to be the fittest. Such being our manifest duty, any means by which we can judge in the readiest way of the physical and mental conditions of the individual, and in this sense to care for and treat him personally according to his indi- 
vidual needs, should be sought for. If it is decided that by the Roentgen method applied to the hand, young human beings can be classified as individuals, it becomes a simple matter for the State or city by means of a Roentgen ray apparatus to rapidly make preliminary examinations. They can then classify physical conditions and the usually correlating brain vigor, for this can easily be done in an individual in less than a minute. In regard to the classification of young children for kindergarten, it is well known that in Massachusetts children may enter the kindergarten at from three and a half to five years of age and remain there for two years. They are then advanced to the first school grade, and thence through the successive grades to the ninth grade, from which they are graduated to the high school. Special localities have variations from this rule. In Melrose, for instance, children must be five ycars old before they are allowed to enter school. In the first year of their teaching they take partly kindergarten work and partly work of the first grade. The next year they take the work of the first and second grades, so that when they enter the third grade they have an equal standing with those of other third-year grades. By classifying young children according to their anatomic rather than to their chronologic age, we do away with the necessity for diverse rules. By grouping them according to their physical conditions we avoid the overstrain of both body and mind which arises from making them compete mentally with their stronger and more vigorous companions of equal chronologic age. This same method and this same idea should be carried out in the various school grades when a preliminary sifting and placing of the children is undertaken. To those who in reply to this would ask what is to be done with the precocious and bright children who are intellectually superior to their companions of equal chronologic age, I would say that I do not belicve that bright children are dealt with as they should be or as is manifestly safe 
for their future welfare. Both parents and teachers wish to stimulate these bright children in their studies and have them graded with groups of children who are older and are better fitted physically to undergo school life withont overstrain. According to the physician's point of riew these children when they are found to be behindhand in anatomic growth slould not be allowed to be advaneed on account of their mental brightness. They should be kept out of doors is much as possible, and special attention should be paid to their physical development until such development has shown the normal conditions requisite for health. As to the comparatively stupid children who present a normal or even more than normal physical development, as shown by the Roentgen examination, they should be allowed to find their level in the lower grades and, indeed, no great anxiety in regard to them is ealled for. In regard to gymmasties and athletics many a boy or girl could by eareful anatomic grading be saved from the reactive debility which we so often see arise from an unwise overstrain of a physical handicap. Supposing we take twenty boys or girls at random, except that they are all ten years old chronologieally. If we pit ten of these individuals against the other ten, either in football or in basketball, we are ruming the risk of pitting in a contest of strength ten individuals who may be only eight and a half or nine years old against ten who may be eleven or eleven and a half years old. Such a variation in chronologic age is found when we elassify chronologically instead of using the anatomic standard which wonld pit ten against the other ten in such a way that the contestants would be of equal physique, which means equal anatomic arge and the least likelilood of overstrain.

Finally, in regard to the question of ehild-labor, it is eertainly the duty of physieians to join hand in hand with edueational, State, and city authorities to prevent children from being allowed to work, excepting under certain physical conditions. 
I have already shown that a child may be, so far as chronologic age is concerned, of the age demanded by the law for child-labor, especially in factories. These laws of chronologic age differ in different States, and in some of the States there is not much to be found fault with in respect to the law. In others, however, the law permits children to begin work in the factories at entirely too early an age. I shall not discuss this question of child-labor very much in detail, as it is too broad a subject. There are, however, certain facts connected with the determination of the child's physical condition which should manifestly be referred to here. In the first place, we know that a child may be only eleven years old and yet look as though it were twelve or thirteen. In like manner, in my investigations, I have found that a child may be thirteen years old and yet have only the normal anatomic development of eight years. This means that although it has the age of a child which would, according to the laws of certain States, allow it to work in the factories, yet, really, it is absolutely unfitted to do so.

It may be said, of course, that these cases are rare. It is, however, manifestly important to detect them when they present themselves. That is, the individual should be examined to see whether physically and physiologically he or she is fitted for work in the factories or elsewhere, and not trust to chronologic age, which may differ by a year or a year and a half from anatomic age.

It is not always the fault of those who employ children in the factories or elsewhere, as it often rests in the hands of the parents themselves. For instance, if a girl is tall and looks older than she really is, the parents are very apt to wish to make her eam wages for the support of the general household. Under these circumstances, over and over again, they will say that the child is as old as she looks, and not tell the truth about her actual age. Again, often among the poorer elasses in the South, the parents do not 
always know exactly how old the children are, as they may have forgotten. This not infrequently happens. How is the State law to be carried ont justly when it is so handieapped by the ignorance and the lack of veracity of the parents, confirmed, under certain circumstances, by the outward appearance of the individual child? Manifestly, we should have some standard which does not depend upon veracity, intelligence, memory, greed, or external appearances. This ean be accomplished by means of the Roentgen method, which always tells the truth, and is an open book to those who have learned to read its language. The simplicity of the method, merely to have a child for a second put its hand on a table and have it pictured by the Roentgen ray on a plate, without harm and without the slightest danger, must recommend it to all intelligent educators and to State officials. Physicians well know how important an aid to our medical armamentarium is the Roentgen method of examination.

In the following table, No. 5, I have brought together some of the chronologie ages which I have extracted from the report of the Industrial Commission on Labor Legislation, Vol. V, Boston Public Library, 9331.073.

Table 5.-State Lawa Regarding Childolabor.

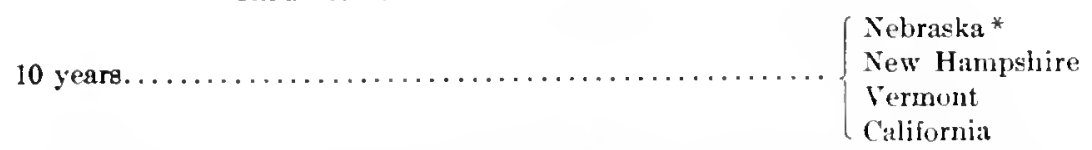

In South Carolina if the parents are dependent children are allowed to work in the mills when they are 10 years old.

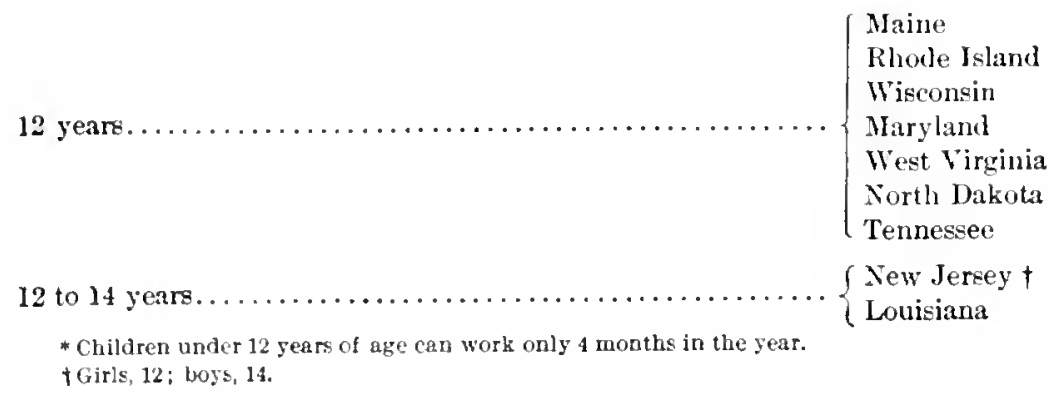




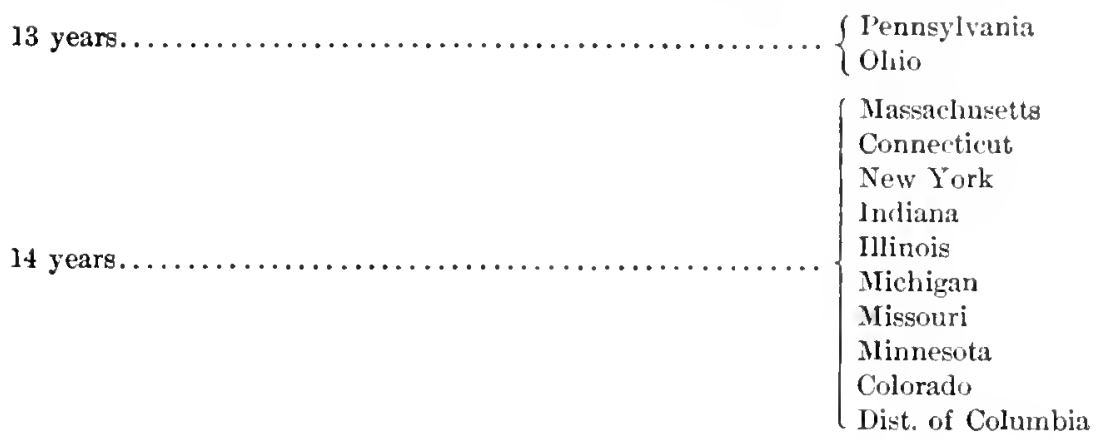

The employment of ehildren under a certain age is absolutely forbidden in factories. The law also limits their employment in various forms of labor, with a view to their education in the public schools. It accomplishes this by restricting it unless they have certain educational qualifications, or by requiring licenses from the truant officers, factory inspectors, boards of health, or other persons before they can be employed in factories at all.

Looking at this list of those who can endorse child-labor, we are at once struck with what an immense number of loop-holes may be present by which the law of the various States can be evaded.

It is significant, however, in how many States the law is placed at twelve years, and still more in those which place it at ten. Probably in some of the Southern States children are allowed to labor in the mills at a still earlier chronologic age.

It would seem that the influence of the medical profession should be brought to bear more than it ever has before on this subject. As a safeguard to the health of the children throughout the States, commissioners should be appointed who, by their training as physicians and caretakers of early life, can inform the State at what period of development it is safe to allow child-labor to begin.

Of course a much further study of the carpal bones and of the epiphyses of the radius and of the ulna must be made before we 
conclude what are to be considered and can be recognized as variations or anomalies. This, however, I believe is possible if a number of Roentgenologists and physicians throughout the country pay attention to this anatomic question. Of course such physiologic observations as Crampton has placed before us will aid very much in the determination of anatomic age by means of the anatomic development of the wrist.

In bringing before the medical profession this idea of using the Roentgen method for physical and mental classification, I wish it to be understood that I am merely asking it to endorse, and educators to make trial of, what seems a probable means of doing justice to young human beings.

I have had to make arbitrary divisions, and these divisions should be studied and careful work of verification or refutation carried out on these lines. Such work, however, I believe to be worthy of trial, as if successful it simplifies the whole question of the guidance of child life, and wards off the dangers resulting from our present ill-advised management of the whole subject. 

l'I.ITl: 24.

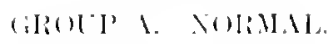

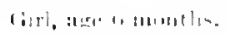

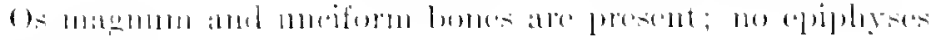
haverevelopel. 


$$
\text { Prate } 28
$$

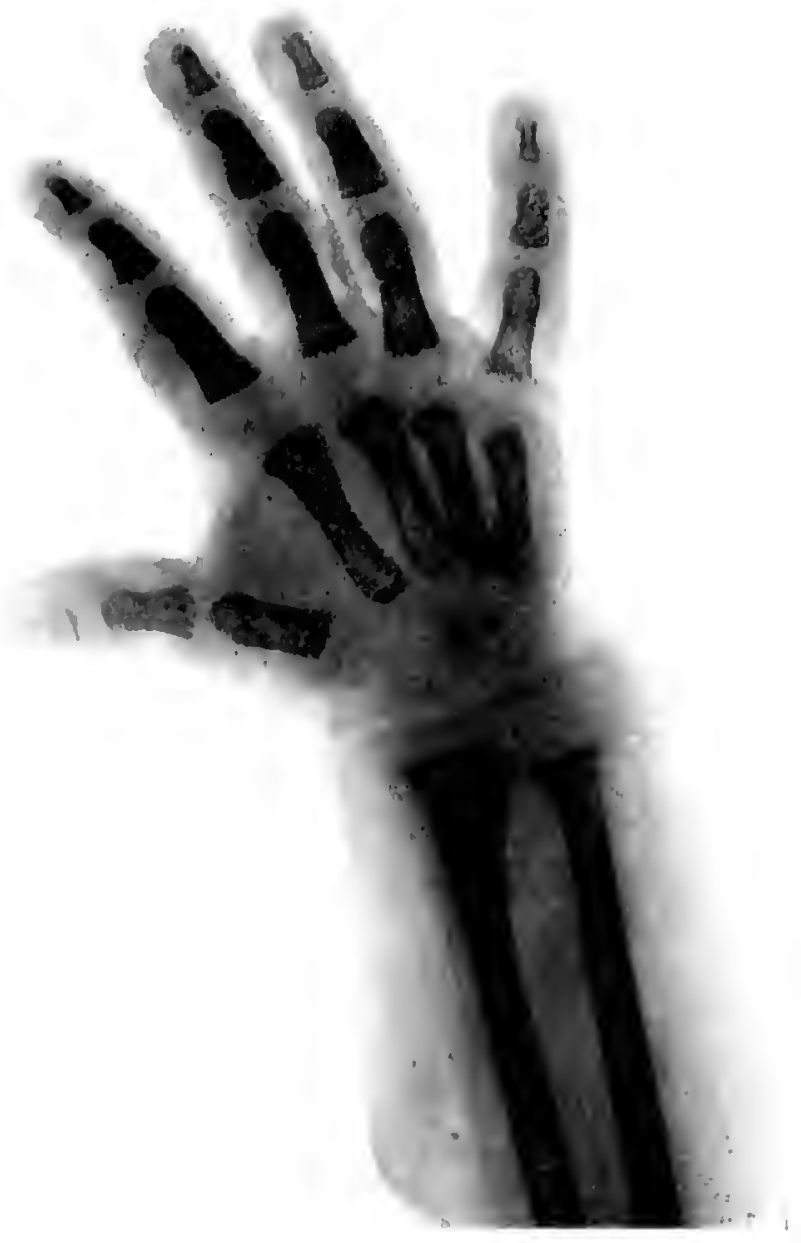




\section{P'L.1TE: 29.}

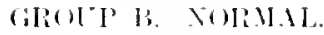

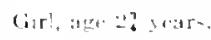

Os magnum, unceform, and lower epiphysis of ratios.

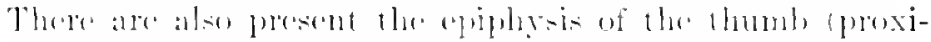

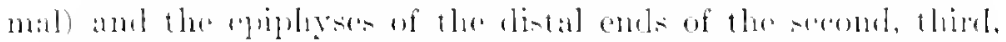

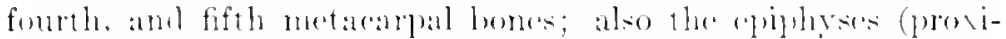

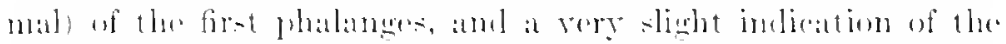
phiphy:i- of the distal phalanx of the thumb, and also a faint

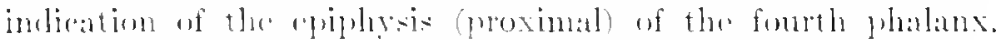

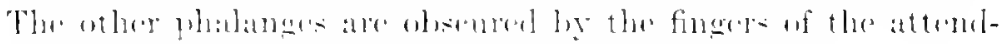
ant : ham which was restratime the infant 's hand. 


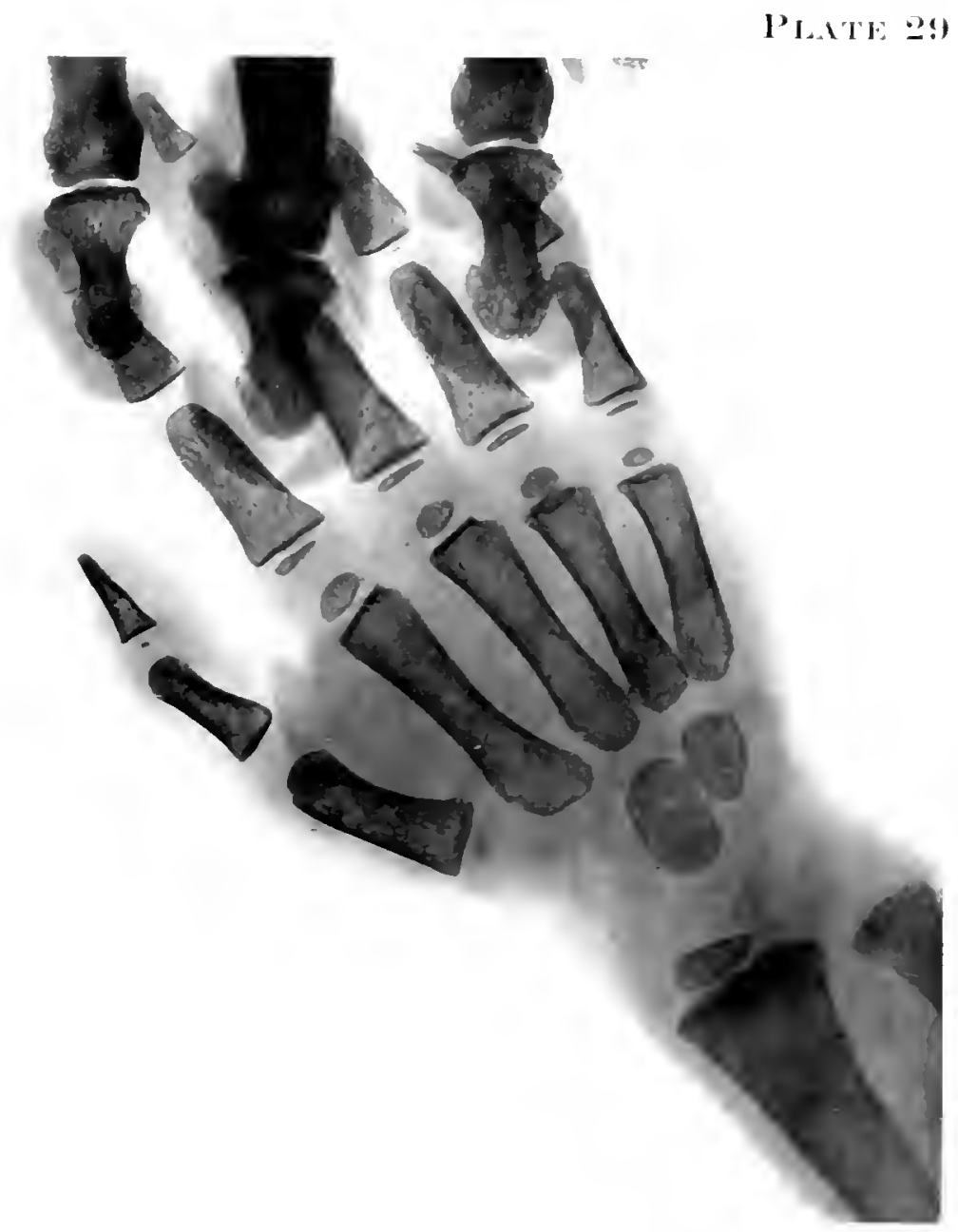


I'I.1T1: :30.

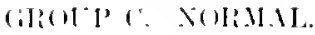

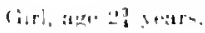

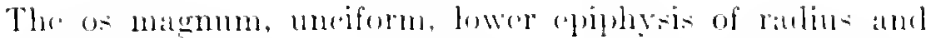
runciform :tre present.

The "piphyes of all the metacarpal bones and of the phat-

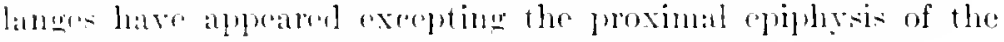
listal phalanx of the first finger and of the fifth. 


$$
\text { PIATE: :O }
$$

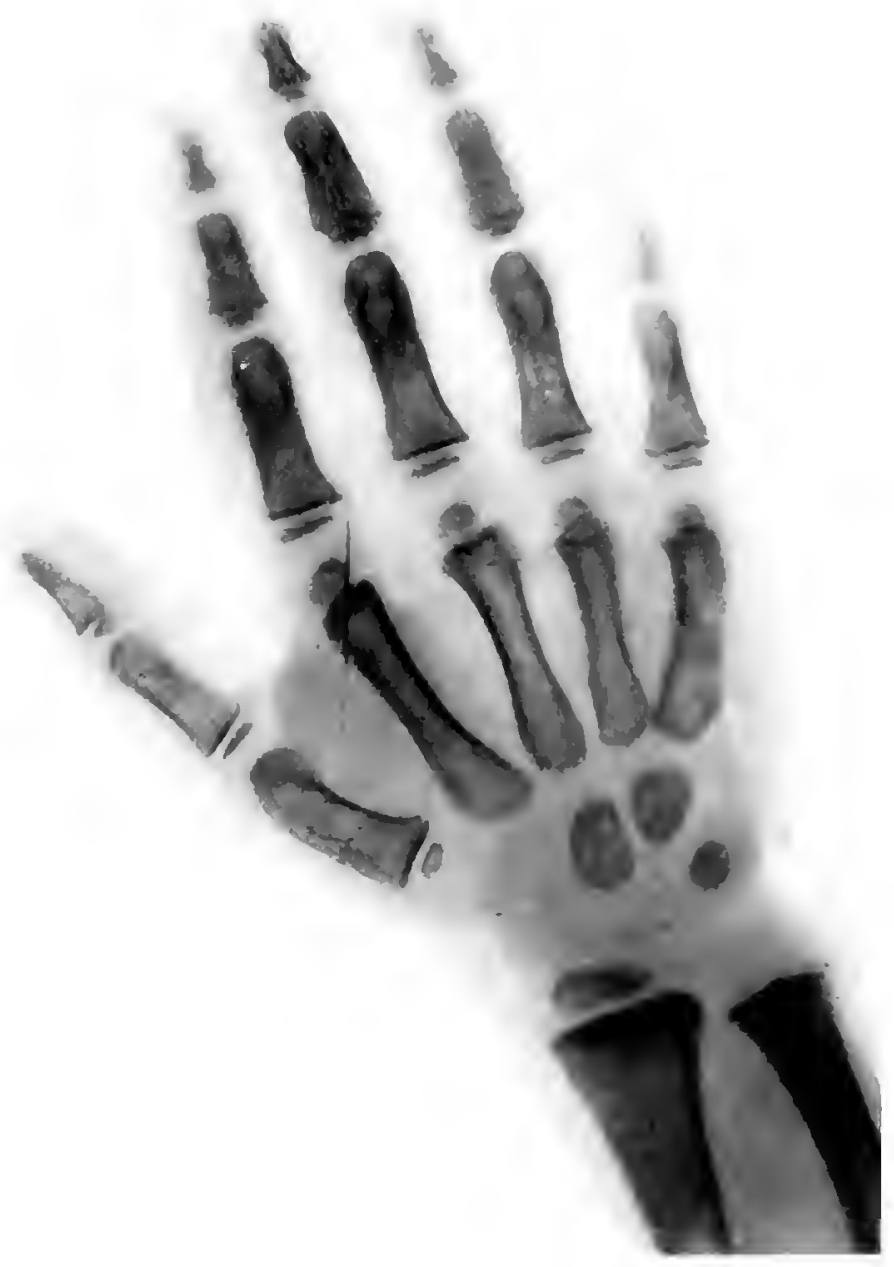


PLATE:31.

(IRUIP J). NORMAL.

13isy atye $2 \frac{1}{4} \mathrm{y}+\mathrm{ar}$

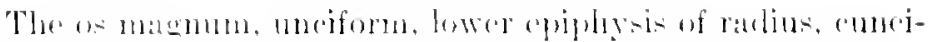
forme, :11n] armilunar ance presont.

.11 the epiphyses of the metalearpal bones ame of the phat-

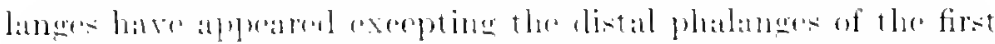

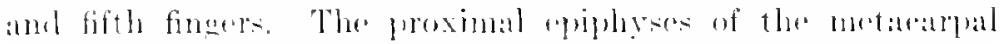

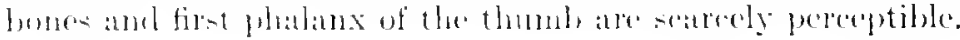


PJATI: :

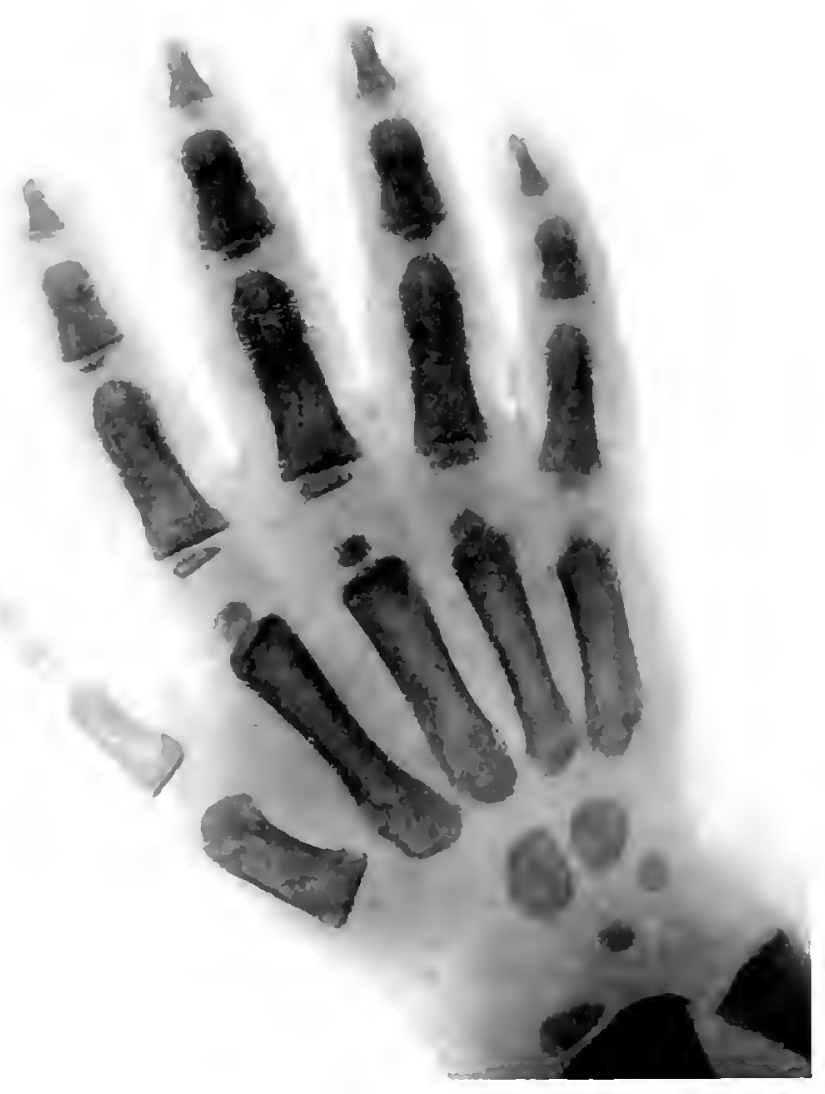


I'LATY: :3.

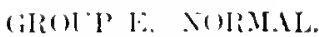

Girl, :Le 3: 3tar

The of manmm, moriform, hower ppiphysis of the radius,

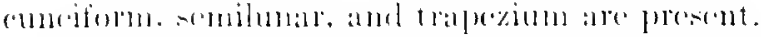

All the epiphyses of the metacearpal homes and of the phat

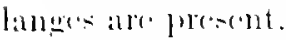

The arrowheal points at what is prosumaliy the trapezium

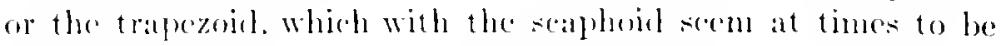
interchanewable. 
P1.1TI: :32

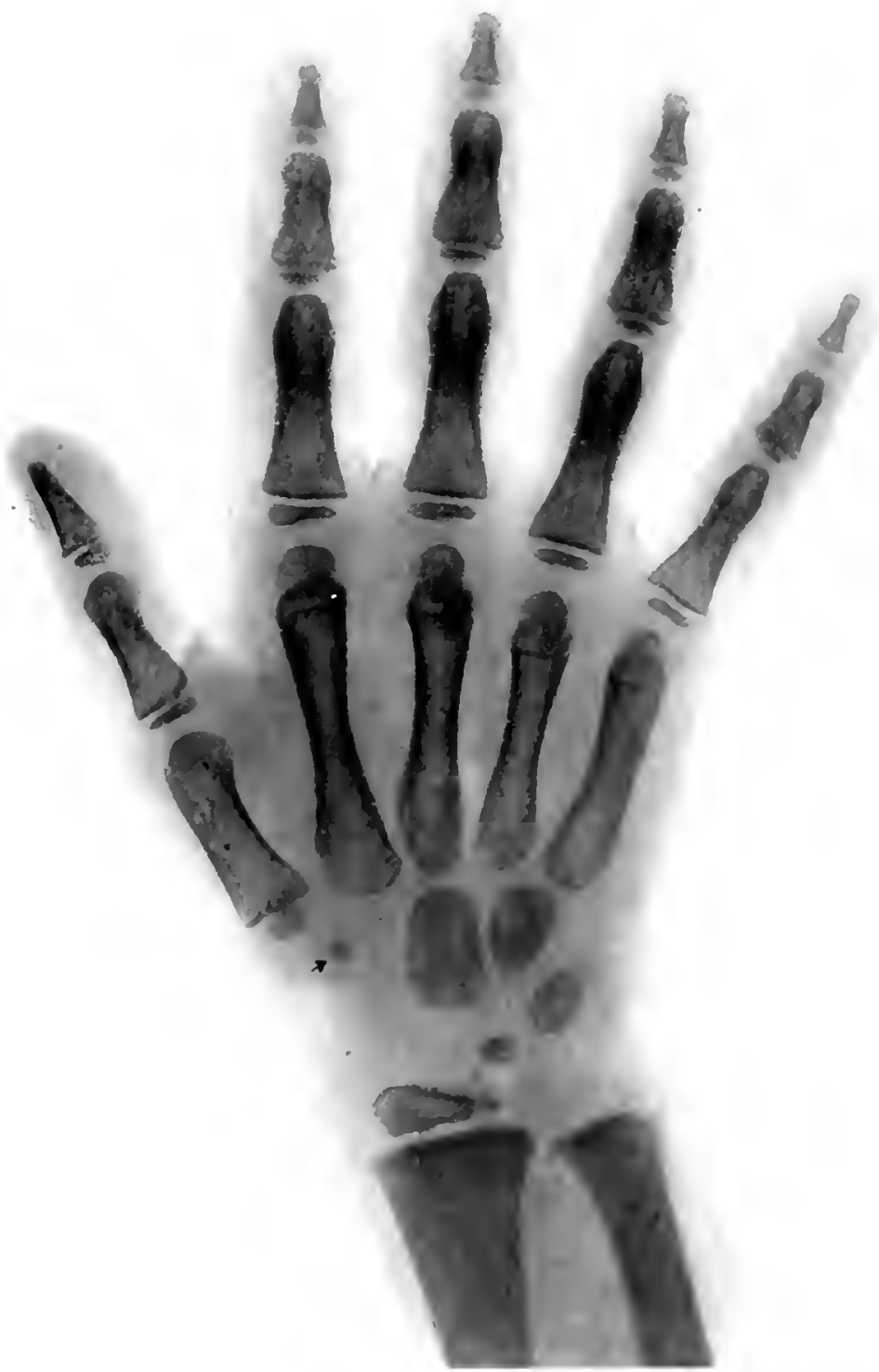


PL.ITE:33.

GiRute l: NoRMAL.

Girl, age is year.

The as matmm, unciform, lower epiphysis of the radius,

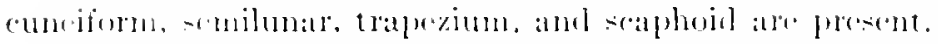

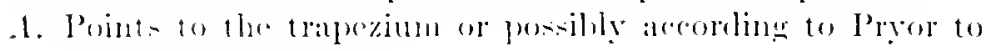
the trat)x:oill.

I. Points to the seaphoist.

All the epiphyeses of the metalcarpal bones and of the phatlanges atre proment. 
Plate:3:3

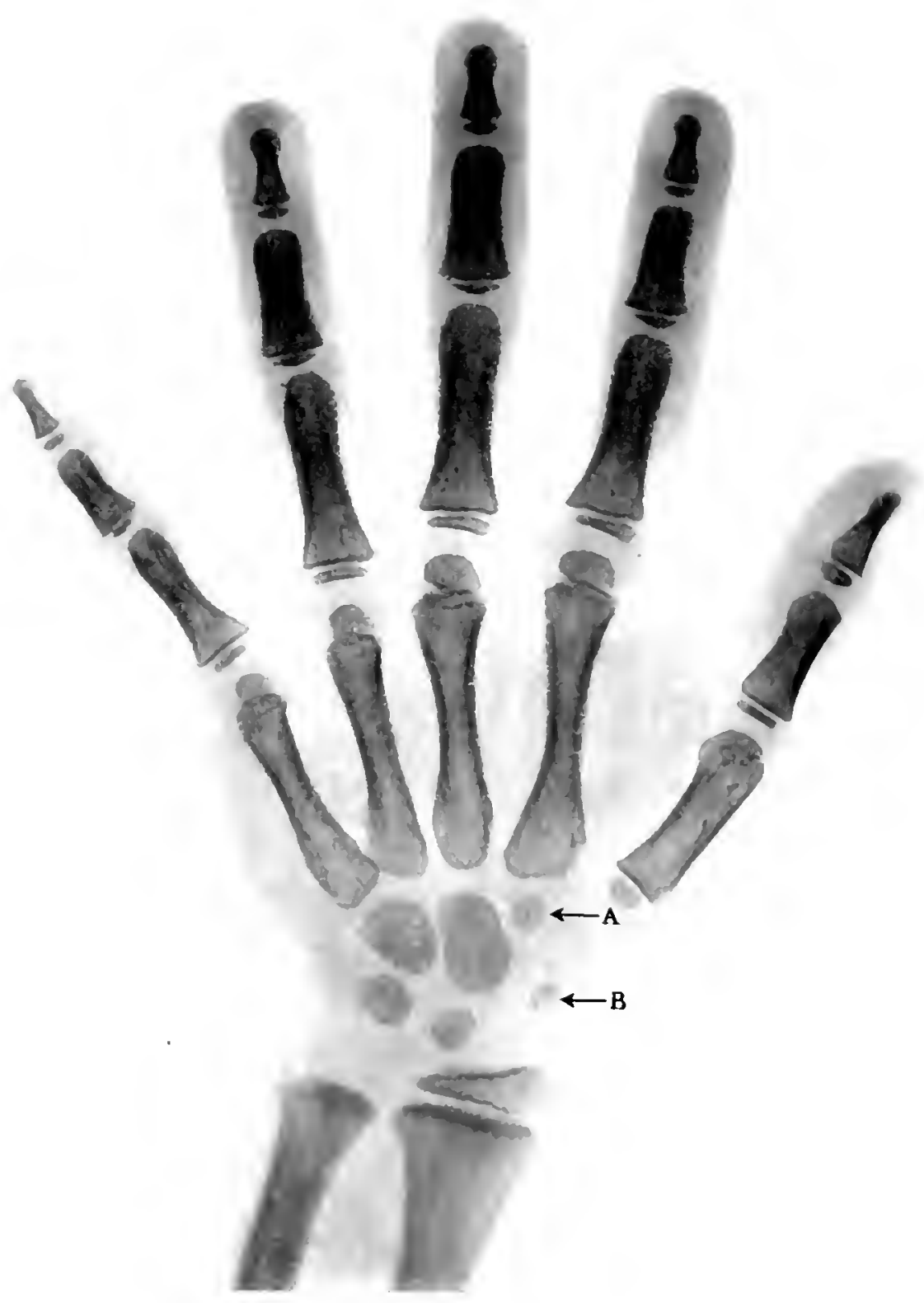


PI.ATE:31.

GROLP G. NORMAL.

Girl, :age tis: $31: a r-$

The os matrmum, uneifom, lower epiphysis of the radiun,

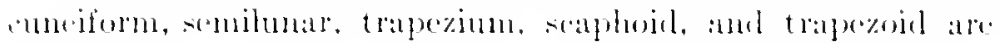
present.

All the epiphyses of the motaranpal bones and of the phat langes are present. 
PLATE: 34

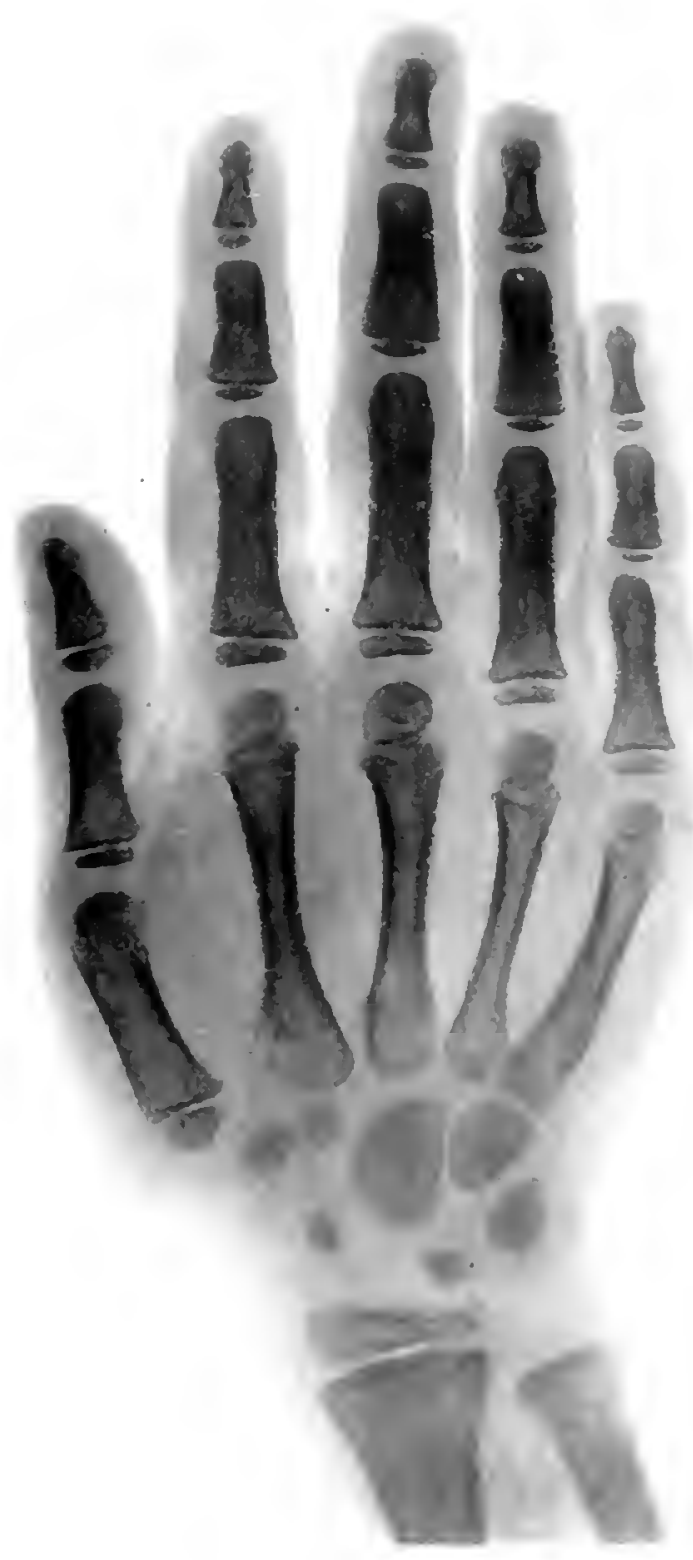


I'I.AT': :3.5.

GRoT'H. NORMAL

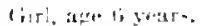

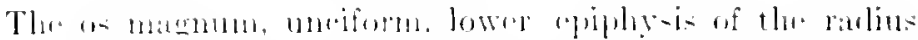

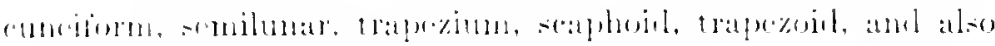

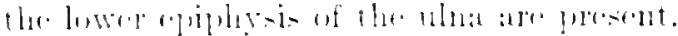

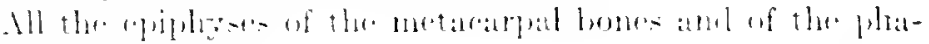

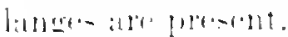

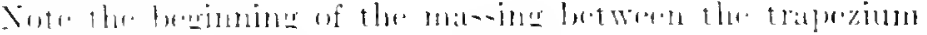

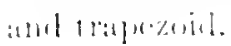


PIATE: :S

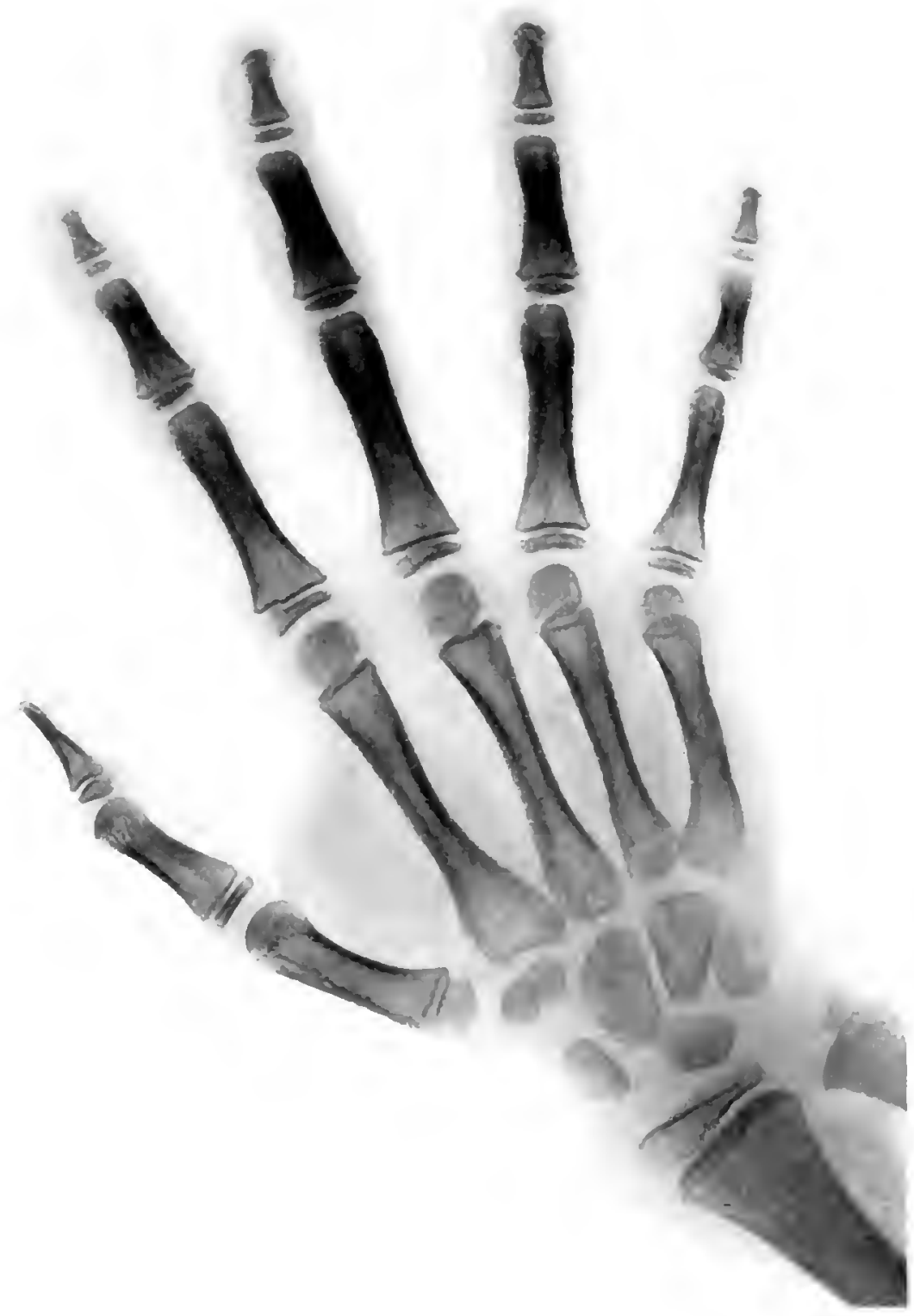


Platti:3i.

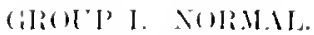

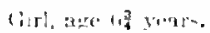

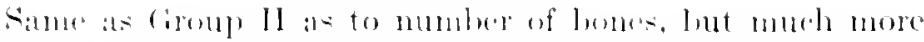

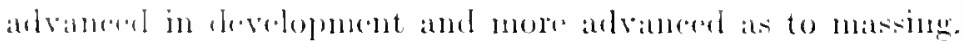


P'LATU: :36

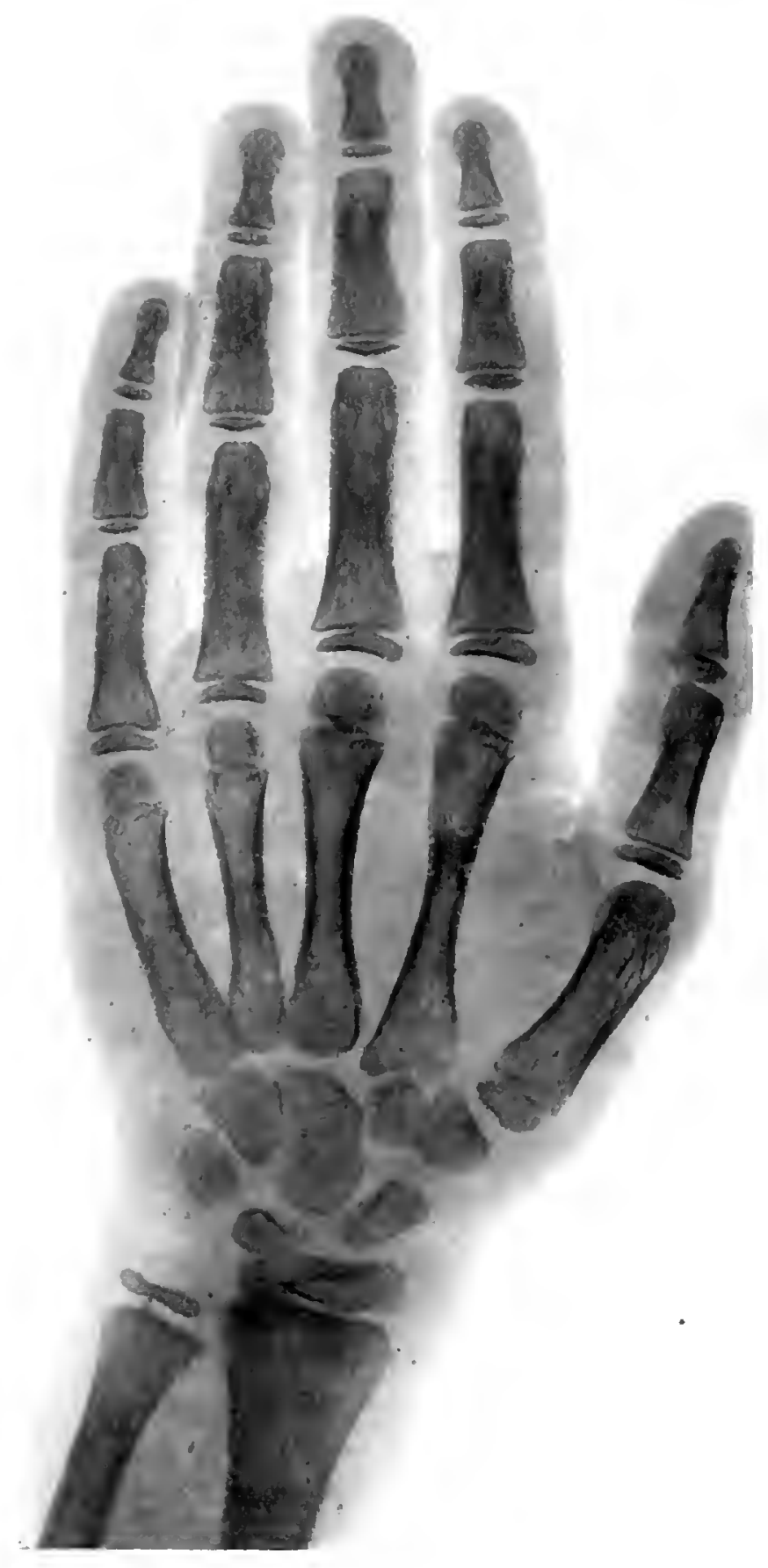


PLATL: :T.

GROEl J. NORIAL.

Gitis

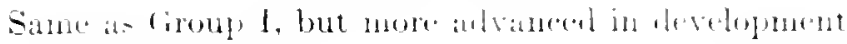


Plate:37
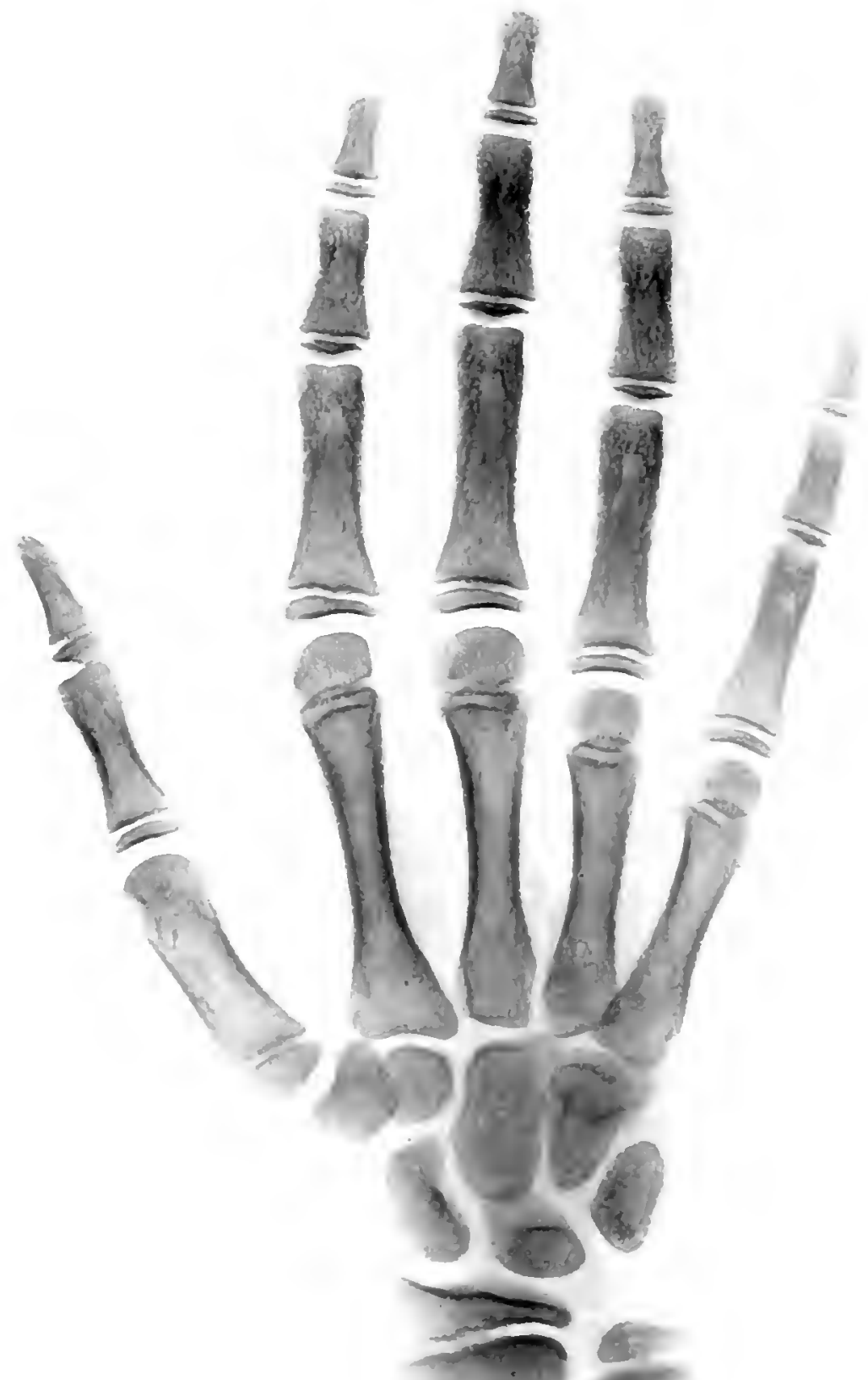
PITI:3S.

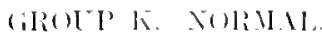

diml ane 11 sears.

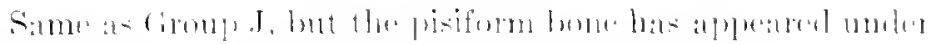

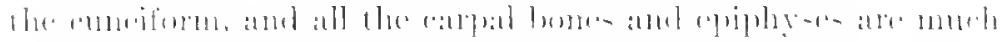

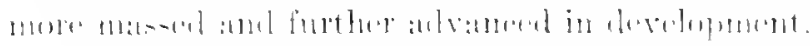

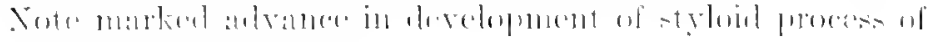
lowe rophysio of the uhat. 


$$
\text { Plate:3 }
$$

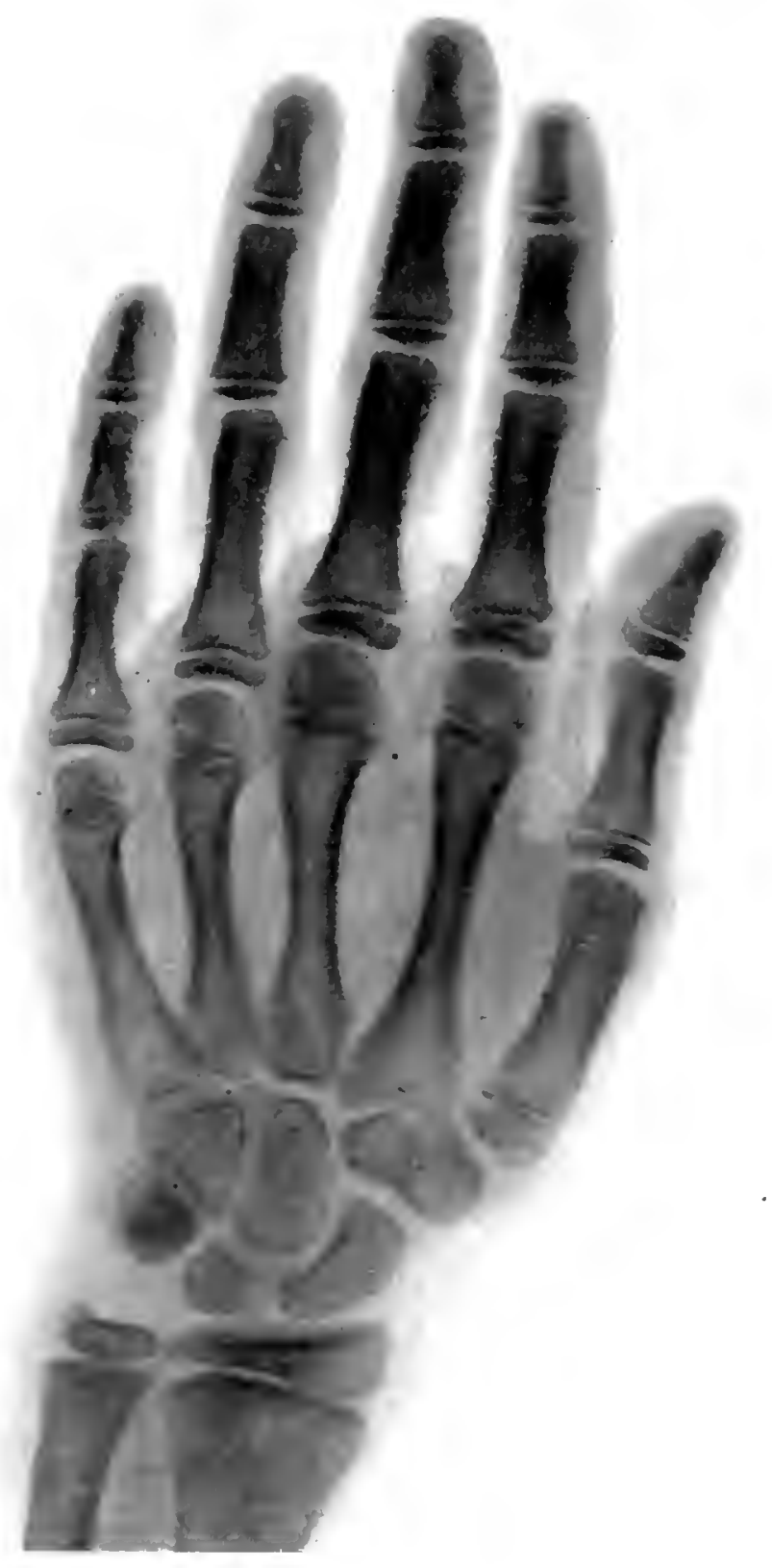


Pl.tTl: :39.

Git)l' L. NORMAL.

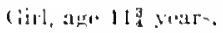

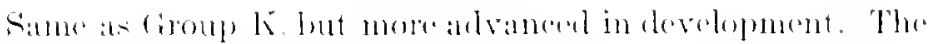

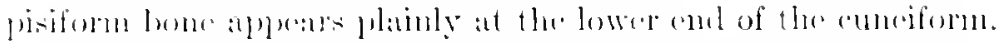

The : arow point: to the pisiform bune.

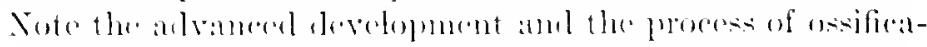

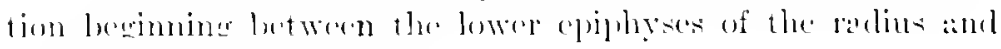
whe with their diaphyses. 
P'LATE: ;:9

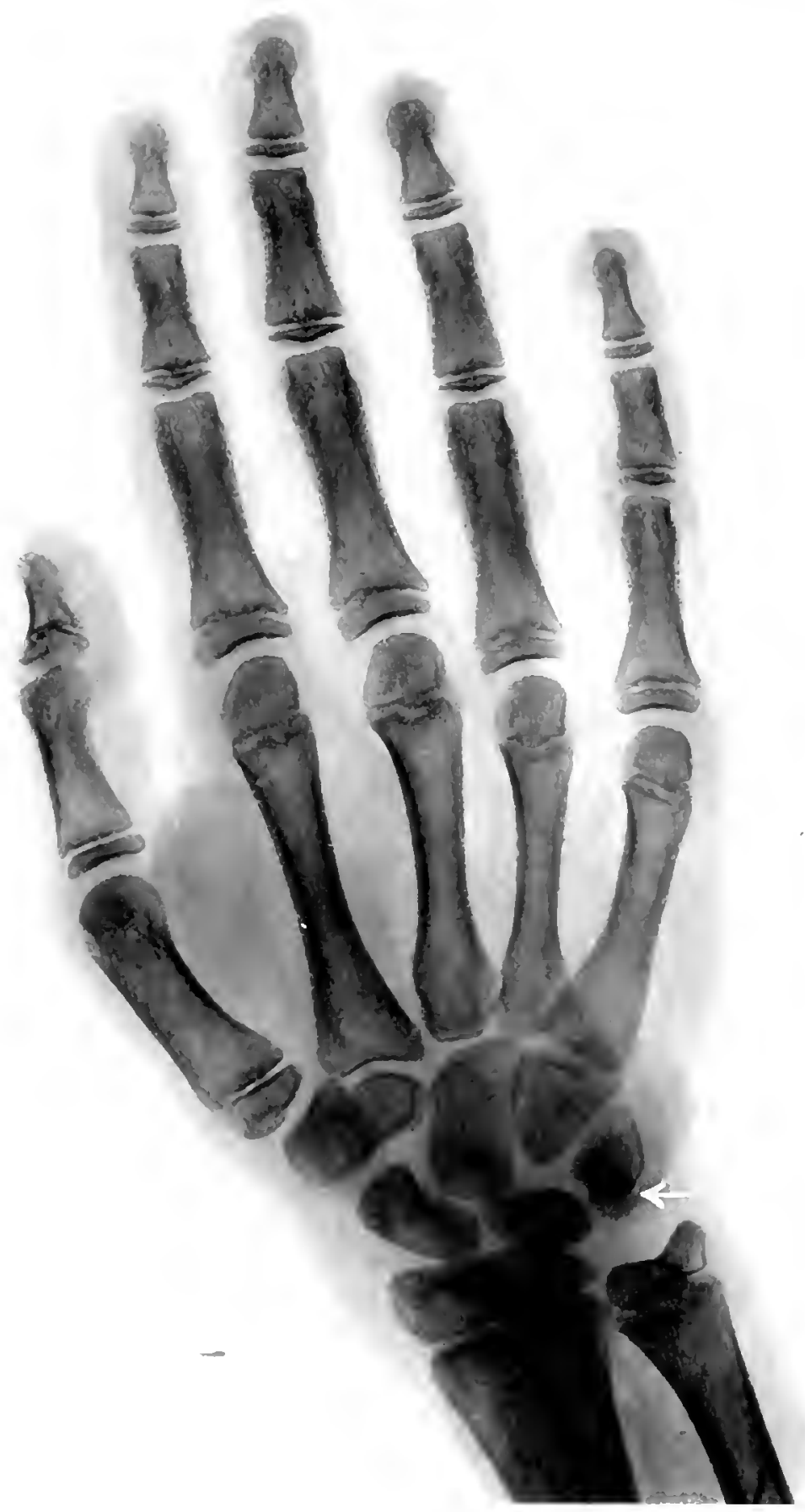


PI.ITF: I0.

\section{GRUTP M. NORMAL.}

Buy, :ate $12:$ yeat 2

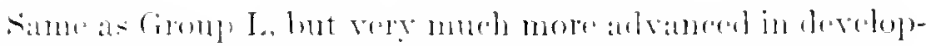

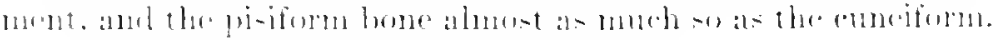

The arrow puint- to the piriform bome. 
P'LATE 40

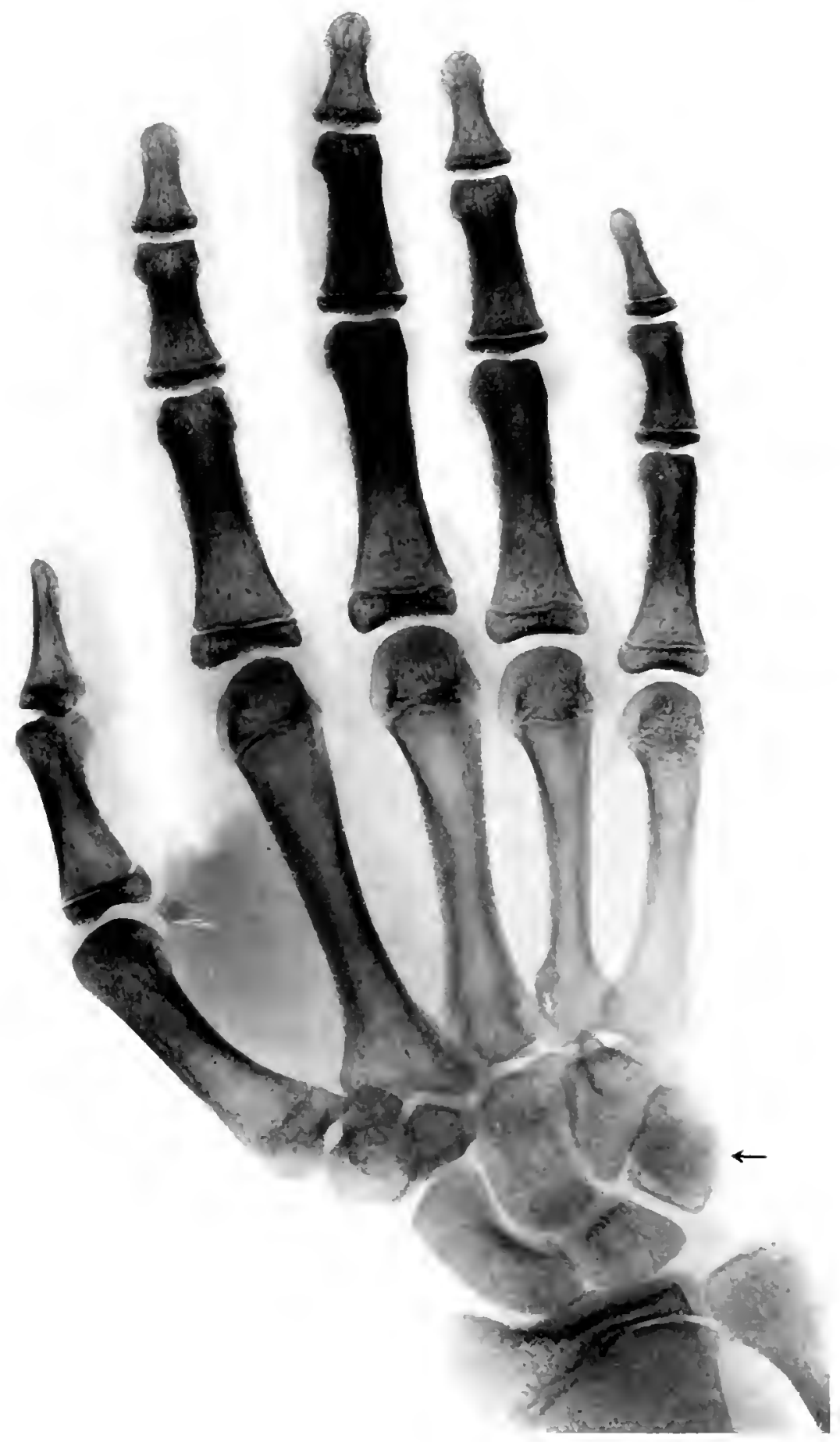





\section{Division III}

\section{DISEASES OF THE NEW-BORN}

$\mathrm{Br}$ diseases of the new-born we mean such abnormal conditions as have occurred in intra-uterine life and are present at birth and in the early days of life. All these diseases, whether acquired in intra-uterine life or at the time of birth, depend in most cases on the great rôle which is played by development in its various stages. It is this developmental class which almost entirely represents the diseases of the new-born which can be shown by the Roentgen method of examination. It is well known that the growing tissues are more vulnerable and their power of resistance less when their complete development has not taken place. The tissues of early life are open not only to specific infections, but to numberless as yet undifferentiated varieties of pathogenic microörganisms which are but little understood. The effects, however, which these organisms have upon the normal growth of the various tissues and the abnormalities which may result from delayed or perverted normal anatomic conditions are recognized, and these conditions are shown in what are called malformations. The Roentgen examination of certain malformations of the mouth, nose, and extremities is of especial significance from a surgieal point of view. Those of especial interest medically are malformations connected with the development of the teeth, heart, ribs, stomach, intestines, and liver. Under this class come also such conditions of arrested development as are represented in the bones where the transformation from cartilage to bone has either been arrested entirely or retarded to such an extent that marked shortening will always be present. The importance of the study of diseases of the new-born has become more marked since the discovery of the Roentgen ray. In almost every 
abnormal eondition of this class we can by the Roentgen method determine the exaet arrangement of the organs and bones, and in this way the underlying conditions of a given deformity can be made evident at once. By means of the Roentgen method of examination the question of corrective measures and the prognosis in regard to future use are made possible. For purposes of simplicity these abnormal eonditions can be elassified into:

Anomalies of the head, spine, and ribs.

Intrathoracie and intra-abdominal diseases.

Diseases of the extremities and pelvis.

Chondrodystrophia fœtalis.

Osteogenesis imperfeeta.

Fetal rhachitis.

Obstetrical paralysis.

While the number of malformations in each of these divisions is large and represents definite abnormal conditions, yet those which can be portrayed, explained, and diagnosticated by the Roentgen method are comparatively small. New and heretofore unseen anomalies are met with from time to time, and to coilect all the anomalous conditions which have been seen up to the present time would require a speeial work on this subject. I shall, therefore, only speak in a general way of diseases of the new-born in order to illustrate what great aid the Roentgen method of examination gives us by diselosing what the anomalous condition may be. If the disease is surgically eurable the pieture shows us where and how best to operate. If it be of the class of cases which are represented by transposition of the organs, such as the heart in the thorax (see Division VI, Plate 110), or the liver and spleen in the abdomen, the knowledge of these conditions is of infinite aid. As the child grows older and its development increases, the Roentgen method assists in 
the diagnosis of these diseases and proves that the condition is anomalous and does not represent acquired disease. It is a fact that it is rare to find two identical anomalies in two individuals. It is to be clearly understood that the classification which I have just suggested is not based on any systematic division etiologically, pathologically, or symptomatically, but is merely an attempt to bring before the student the pictures of the various parts in such a way that he can readily grasp their meaning. In studying a Roentgenograph of the entire skeleton we would naturally begin by examining the head, and then logically follow the picture down through the spine, thorax, and upper extremities, abdomen, pelvis, and lower extremities.

\section{ANOMALIES OF THE HEAD, SPINE, AND RIBS}

\section{HEAD}

The anomalies of the head which can be distinguished at birth by the Roentgen ray and classified under diseases of the new-born are comparatively few in number. The variations and anomalies of the teeth would naturally be spoken of at a later period of development when the teeth, whether unerupted or erupted, play a more prominent rôle than is the case at birth. They will therefore be described in Division $\mathrm{V}$ under diseases of the head.

\section{SPINE}

Anomalous conditions of the vertebre may occur in any part of the spine, as is seen in Plate 41, which shows the head and neck of a boy six years old with a congenital anomalous condition in the upper cervical region. There appears to be a fusion of the atlas with the base of the skull. The axis also shows marked deformity as compared with the normal upper cervical vertebra shown in Plate 27. The remainder of the vertebræ seem to be normal. The child in this case shows a quite distinct normal orbit. The frontal 
sinus does not show definitely. The antrum, as would be expected at this age, is small and ill-defined. In the upper jaw, beginning in front, we find all of the temporary teeth in place normally, but not elearly defined by individual markings up to the first temporary molar. This is due to the angle at which they were taken and to the consequent interference of the pietures of the teeth on the opposite side of the jaw. The first temporary molar and also the second temporary molar show the development of the erown and root plainly. Immediately above the first temporary molar in the alveolus can be seen the tip of the first bieuspid becoming ealeified. The second temporary molar shows plainly the eharacteristic large pulp chamber and the delicately peneilled buceal roots. In the light area above the second temporary molar is the erypt of the second bicuspid, but no signs of ealcification are seen. Immediately back of the sccond temporary molar is the first permanent molar with only its erown caleified. There is no appearanee of the second permanent molar or of the third permanent molar.

In the lower jaw beginning behind the erypt of the second permanent molar are shown the buecal cusps completely caleified. Directly in front of the second permanent molar is the crypt and ealcified crown of the first temporary molar, with its light area below, in which lies the formative organ (dental pulp or bud). Anterior to this sccond temporary molar the pietures are indistinet, but probably represent normal temporary teeth.

The spinal column may show an absence of one or more vertebræ, or an additional vertebra, or lordosis or kyphosis. The main object in view, however, is that having seen one or two illustrative cases we may thus be able to recognize other anomalous conditions of this nature. The condition of rhachischisis is one of the principal forms of congenital defects of the spine, but though a Roentgenograph of this condition might be interesting from an embryologic 
point of view, the malformation is so evident at birth that the Roentgen ray for diagnosis would be unnecessary. It is a disease of pathologic rather than of clinical interest, for not only is there a deficiency of the vertebral arches, but the cord itself is rudimentary. Comparatively few anomalies of the vertebre have been seen which can be classed under diseases of the new-born and are of interest from a Roentgen-ray point of view. In the study of each case the vertebra should be counted, and we should note whether the intervertebral discs are distinct, or are compressed, fused, or destroyed. The number of vertebræ may be increased or diminished, a deficiency in one region usually being replaced by an additional vertebra in another. It is to be noted, however, that the number of bones in the cervical region is rarely increased or diminished. An additional or lessened number of vertebre in the lumbar region is of especial interest in comection with the technic of lumbar puncture.

Spina Bifida.-The most common disease of the spine which occurs during intra-uterine life is spina bifida. This condition is getting to be of additional importance, since by means of the Roentgen method considerable advances have been made in the treatment of these cases through the more precise diagnosis which can be attained by detecting the exact type, locality, and extent of the lesion before operation. Spina bifida is a condition in which there is a failure of closure of the laminæ of the vertebre. At certain intra-uterine periods this anatomic condition is normal, but when it is found at full term it becomes abnormal from a derelopmental point of view and represents a distinct malformation. The laminæ close normally at the beginning of the fourth month of fetal life, and fusion takes place at the base of the spinous processes from above downward. As the lumbar vertebre are the last to unite, spina bifida is most common in the lumbar region. A true spina bifida commonly appears as a tumor in the lower part of the spine or in the sacrum, 
and is corered with either healthy or diseased tissue, which is usually very thin and transparent. At times the tumor is solid, containing only fibrous and fatty tissue, with no comnection with the spinal cord or canal other than a dense fibrous pediele with no nerve tissue or filaments included. At other times it may be represented simply by a defect in the skin covered usually by a tuft of coarse hair; in this latter case it is called spina bifida ocenlta. The condition of pylo-nido-sinus must of course be thought of differentially, but would usually be readily distinguished from spina bifida.

Plate 42 represents the photograph of a case of spina bifida occulta occurring in a girl three and a half years old. The museles of the right thigh were atrophied.

Plate 43 shows a Roentgenograph of the same case. The vertebra above the saeral region do not show any numerical variations, and not being well developed are not easily distinguishable. There is a definite change not only in structure but in size of the third lumbar vertebra. The third intervertebral dise is narrowed. The fourth and fifth lumbar vertebra are larger and narrower from side to side with very small and irregularly shaped spinous processes. There is a partial sacralization of the transverse processes of the fifth lumbar vertebra. The sacrum presents a eleft to the left of the median line. A dislocation of the right femur is shown to be present, and is probably congenital in origin. A rudimentary acetabulum and a delayed development of the right upper femoral epiphysis are shown.

Plate 44 represents the lumbar vertebræ, pelvis, and thighs of the same ease. The spinous processes of the third and fourth lumbar vertebre are bifid. The child was operated on, and the plate shows the abnormal conditions five months later, and represents an apparently satisfactory reduction of the femur. The dislocation of the femur was not reeognized clinically. 


\section{RIBS}

A precise knowledge of an anomalous condition of the ribs, such as fusion of the ribs, an extra rib, or any malformation of the ribs, is often of great value when the physician wishes to determine the best point of entrance when performing thoracentesis.

Plate 45 illustrates this fact. It is that of a child who showed a congenital fusion of the sixth, seventh, and eighth ribs on the left side. Especially evident are the greatly deformed fourth, fifth, sixth, and seventh dorsal vertebræ, and a decided scoliosis to the right. To be noted also in the lower lumbar region was a marked spina bifida (not shown in the plate). If in a case of this kind there happened to be a pleuritic or pericardial effusion the information gained from the Roentgenograph would be of infinite aid and almost absolutely necessary in determining the position of the heart and at what point the needle should be introduced.

In cases of torticollis it is often very difficult to determine the cause of the condition without having recourse to the Roentgen method of examination. This is especially evident where the clinical symptoms arise from a malformation of the vertebræ or ribs. Plate 46 explains the cause of the condition where the clinical picture was that of torticollis to the right in a child six years old in whom the Roentgenograph showed that there was a congenital cervical rib attached to the seventh cervical vertebra on the left side, while on the right side of the same vertebra there was none. On the side of the malformation the child will be seen to have thirteen ribs, instead of the twelve which normally appear on the right side.

In connection with the ribs and spine a malformation of the scapula is found at times. Although this condition can usually be detected by the usual external physical examination, yet it is far more satisfactorily explained by means of the Roentgen method.

Plate 47 shows the photograph of a boy six years old with an 
elevation of the scapula on the left side. He also shows a marked scoliosis to the left.

Plate 48 shows a Roentgenograph of the same deformity. In this picture is shown the scapula in the region of the muscles of the neek on the right side, while the clavicle on the opposite side is in its normal position. The remainder of the picture is normal.

Plate 49 shows the same condition in an infant six months old. In this case the elevation of the seapula reached high up into the neck. There is also a left dorsal curve of the spinal column. The first dorsal vertebra also shows some deformity. Otherwise the pieture is normal for the age.

\section{INTRA-THORACIC AND INTRA-ABDOMINAL ANOMALIES}

Although the deteetion of the various transpositions of organs, as the heart in the thorax and the liver in the abdomen, is comparatively simple, yet there are eases in which it is almost impossible to determine whether the transposition of a heart is due to a congenital deformity or to adhesions holding it out of position. In such eases as these we have to depend upon the transposition of other organs, such as the liver or spleen or stomach, as shown by the ray. Plate 110 , Division VI, is an illustration of this difficulty.

\section{ANOMALIES OF THE EXTREMITIES}

In connection with the study of the bones, especially of the extremities, it is well to understand that all congenital deformities in connection with the bones show their changes in the epiphyses. Among the anomalies of the new-born the malformations of the hands and feet appear to be the most common, but no part of the osseous system is exempt. Until the Roentgen ray came into general use these conditions could only be studied by what could be seen externally or at the post-mortem, although an actual and precise knowledge of the relation of the bones to each other was very neces- 
sary in order to obtain the best results from operative treatment. Club-hand and club-foot are congenital malformations which may be due to an undeveloped condition of either the bones, the ligaments, or the muscles, and the Roentgenograph is therefore of the greatest importance in determining which condition is present in the especial individual. In the more simple forms the extremity is pulled into position by the action of contracted muscles or tendons. In the more severe forms the bones may be so misshapen that the separate segments are almost unrecognizable, and therefore a picture of these segments is required for intelligent operative interference. For practical use surgically we may divide the malformations of the hands and feet into three types:

An extra fully developed separate digit.

A fully developed hand or foot with one or more digits webbed, or with a single or multiple anomalous condition of any of the carpal or tarsal bones or the phalanges, metacarpal, or metatarsal bones.

A hand or foot with a less number of fingers or toes than normal and with an imperfect development of one or more bones.

Upper Extremities.-As illustrations of the various types of deformities just spoken of are the following:

Plate 50 (Fig. 1), the hand of a child two months old, shows a webbed condition of the second and third phalanges. A number of deficiencies are also present which may be seen in the illustration. It is to be especially noted in this plate that none of the epiphyses of even the metacarpal bones are present. Otherwise the bony structure is fairly normal.

Fig. 2 shows the left hand of a child one and a half years old. The Roentgenograph shows an extra digit, and also the fusiou of the fifth and extra metacarpal bones. The Roentgenograph in 
this case was of course of the utmost aid in determining what the operative treatment should be.

Plate 51 shows a photograph (Fig. 1) of a boy ten years old with double congenital club-hands. The pieture also shows a defieiency in the number of the fingers, the thumb being absent and two of the fingers of the left hand being webbed. The Roentgenograph (Fig. 2) of this case shows a number of anomalous conditions which are described in the corresponding legend. Club-hand is of ten aecompanied by an absence of the radius, and this condition is shown in the Roentgenograph. It is to be noted that the bones in this case are smaller than normal and show a defieieney in the lime salts. The deformities in this case are so complex that it is evident that a Roentgenograph was necessary if any radieal surgical operation was to be performed. Also to be noted in this plate is the absence of the epiphysis of the lower end of the humerus, the slightly developed epiphysis of the lower end of the ulna of the right hand, the rudimentary metacarpal bone of the left hand, and the lack of development of the carpal bones.

Plate 52 shows a deformity of both ulna and radius, and a bowing of the ulna seemingly in order to compensate for the radius, which is considerably shortened.

Plate 53 shows an anomalous condition in the arm of a boy ten years old. The abnormal difference in size in the bones, and in the outline and dislocation of the radius, and also the irregular deposit of bone in the una probably arise from some unknown eongenital cause.

Lower Extremities.-Plate 54 shows the photograph of the feet, only two toes being present, of a boy eight years old. The Roentgenograph shows a fusion of the astragalus and the os ealeis. According to Dwight, this abnormal condition oecurs at the posterior end of the sustentaculum, and may be attributed to the fusion of the 
os sustentaculi with both bones. The bones may be firmly assified or they may be united by gristle, the apposed surfaces showing the characteristic irregular finish. Either of these conditions is decidedly uncommon. A Roentgenograph was manifestly very important in this case.

Plate 55 shows another somewhat similar deformity of a child's foot in which only one toe is present.

A very striking instance of how important to the surgeon may be the anomalous conditions connected with the malformations of the foot is represented in Plate 56. This is the foot of a child three years old. It shows the absence of the tarsal bones, with the exception of two which are very slightly developed. The metatarsal bones are also absent. This case was under the care of an orthopædic surgeon for some time and was considered to be connected with the malformation of club-foot. The Roentgen ray showed the anomalous condition, and the knowledge received from it, if it had been obtained earlier, would have saved the patient months of treatment. Of course to understand thoroughly such an anomalous condition as this it is necessary to compare this picture with that of the normal average development at three years.

Plate 57 shows the delayed development of the right lower extremity in a child six months old. There is an entire lack of development of both trochanters, and the upper epiphysis of the right femur is markedly smaller than the left. Evidently the cartilage has been arrested in its change into bone at an early period, while on the opposite side this process has continued normally, and the resulting shortening of the leg is shown by the ray. Plate 58 shows the lower legs and feet in the same case. On comparison with the foot of a child of the same age it shows a lack of development of the deformed limb and of the tarsal bones of the foot, the undereloped epiphysis of the tibia, and the general irregular distribution of the metatarsal bones. 


\section{ANONIALIES OF THE PELVIS}

Congenital dislocations of the hip are the most frequent of and play an important rôle in the malformations of the new-born. They depend upon a faulty development of the acetabulum and of the head of the femur. Plates 59 and 60 show instances of this nature. These Roentgenographs are of especial importance in that they make erident whether the dislocation is due to a lack of development of the acetabulum, to an absence of the head of the femur, or to some other anomalous condition which it would be impossible otherwise to recognize during life or without operation. With the aid of the Roentgenograph the operation can be determined with more exactness than by any other method.

Plate 59 shows the dislocation of both femora in a child twelve and a half years old.

Plate 60 shows a congenital dislocation of the left femur in a child ten years old.

Plate 61 represents the legs of a child where there was a congenital paralysis on both sides. The cause was not determined, but was supposed to be the result of some congenital lesion of the cord. As shown by the Roentgenograph there is an undeveloped and atrophic condition of the acetabulum and femur. The atrophy is not in quality but only in size.

\section{BACKWARD MENTAL DEVELOPMENT-MYXCEDEMA-CRETINISM}

Plate 62 shows the hand of a case of hydrocephalus, and delayed development of the carpal bones in an infant twentythree months old. In addition to the delayed development of the bones, which represent chronologically about six months of age, there is to be noted an anomalous ossific centre at the proximal end of the second metacarpal bone, which may be possibly the trapezoid, trapezium, or an anomalous epiphysis. 
Plate 63 shows the hand of a boy four years and nine months old, who was decidedly backward in his mental development, and whose hand shows that the wrist is developed about as much as would be usually found between the second and third year.

Plate 64 shows the hand of a girl eight years old, with myxœdema. Nothing especially abnormal is noticed in the bones, excepting that the wrist presents a development about two years behind what would usually be normal for eight years in a girl.

Plate 65 shows the delayed development of the carpal bones in the hand of a sporadic case of cretinism in a child twentyseven months old. It also shows the ill-clereloped bones of the leg, and the narrow zone of proliferation which is supposed to occur in cases of cretinism. Fig 3 shows this narrow zone in a section of the femur of a cretin. This specimen is in the Warren Museum of the Harvard Medical School.

\section{CHONDRODYSTROPHIA FETALIS (ACHONDROPLASIA)}

Although various names have been used to represent this condition, investigators at present as a rule prefer to limit the term to chondrodystrophia fœtalis. In order to recognize the disease from a Roentgen-ray point of view the gross anatomy and coarse histology should first be carefully studied. In this way the Roentgenograph can differentiate this disease of the new-born from such changes in the bones as take place in osteogenesis imperfecta, and those nutritive conditions acquired after birth, such as rhachitis and osteomalacia. The word achondroplasia was first used to represent this condition by Parrot in 1878; but Kaufmann, in 1892, in describing the pathology of the disease, spoke of it as a disturbance of the endochondral ossification, while the ossification on the side of the periosteum was perfectly normal. Later investigators have usually followed Kaufmann in using the name chondrodystrophia fœtalis. 
It is a clisease of the osseous system beginning in intra-uterine life between the third and sixth months and usually ending at birth. Its effects, however, persist through life. Owing to similar macroscopic appearances it is of ten erroneously classified as fetal rhachitis, but microscopically it presents marked differences. The cause is not known. It is usually accompanied by general malnutrition, and the majority of the eases die soon after birth, but the less severe cases are compatible with life. The characteristic changes in the bone result in shortened and deformed extremities, and a large square head and flattened nose, but the trunk usually remains normal. These abnormalities are brought about by a disturbance in the normal process of ossification of the primary cartilages. The flattening of the bridge of the nose is due either to primary tribasilar synostosis or a failure of development which causes the base of the skull to be shortened. In the long bones a eartilaginous ossification of the epiphyses results in an arrest of development and a corresponding shortening. The shafts consist ehiefly of periosteal bone, while the medullary canal is sometimes replaced by hard bone. The ends of the bones may be so affected by the overgrowth of periosteum as to produce deformities which elosely resemble those of rhachitis. Similarly there may be at the junction of the ribs and cartilage a flattening of the sides of the chest, and there may be also a flattening of the pelvis.

Kaufmann has divided chondrodystrophia fotalis into three types: (1) chondrodystrophia hypoplastica, in which there is a failure of the development of the eartilage; (2) chondrodystrophia malacica, in which there is an abnormal softening of the cartilage; (3) chondrodystrophia hyperplastica, in whieb there is excessive development of the eartilage in all directions. Osteoporosis and osteosclerosis may occur as complications. A tendency to deformity in these cases may be increased by fractures. Instead of the enlarge- 
ment of the ends of the long bones due to excessive enlargement of the epiphyseal cartilage, as is seen in rhachitis, chondrodystrophia shows an overgrowth of the periosteum. The zone of proliferation while it is widened in rhachitis is narrowed in chondrodystrophia, and the vascularization in the latter disease is marked. There is probably no direct relation between chondrodystrophia and cretinism. The differential diagnosis between chondrodystrophia, rhachitis, osteomalacia, and other diseases by means of the Roentgen ray is given on page 107. Since the introduction of the Roentgen method of examination, a diagnosis on the living subject of the various intra-uterine conditions connected with the bones and with their epiphyses has rapidly advanced in precision. While in the past there has been almost inextricable confusion in the differentiation of these diseases, we can now make a diagnosis with a fair chance of its being correct. Especially to be noted is the recognition as distinct diseases of the conditions of chondrodystrophia fotalis, osteogenesis imperfecta, cretinism, and possibly fetal rhachitis. $\mathrm{Up}$ to the present time, however, no case of intra-uterine rhachitis which has been studied by modern methods of examination has been published.

Plate 66 shows two cases of chondrodystrophia fœtalis. Fig. 1 is the picture of a girl five and a half years old, showing the flattened nose, comparatively short arms, and especially short legs of a case of chondrodystrophia fotalis. For the description of this case, see "Pediatrics," page 333, fifth edition.

Fig. 2 is the picture of a boy thirteen years old, showing the flattened nose, the short legs, and the noticeably short arms.

Plate 67 shows the Rocntgenograph of the hand of the same case, Fig. 2, in comparison with the normal hand (Plate 20) of a boy nine years old. A number of changes besides the broad thickened bones will be noticed. 
Durante, in 1902, stated that " the eartilage shows more vascularization than normal. It is separated from the border zone by a vascular fibrous band. This band diminishes in breadth inward and towards the centre of the epiphysis and is often represented only by an interstitial sclerosis of the layer of the cartilage cells, which are disposed parallel to the line of ossifieation. Below this the proliferation and arrangement of the cartilage cells is very defective. The cells, well separated in the midst of an interstitial substance suggesting. fibrous tissue, are, as a rule, not disposed in columns even partially. There often is no trace of cartilage columns, and the cells lie isolated and scattered without order, even up to the edge of the line of ossification. From this there results the formation of bony lamelle, small, thin, and essentially irregular, or rather a series of points of ealcified nodules irregularly disseminated in the marrow of the bone. A certain number of projections of marrow oceupy the line of ossification and extend toward this fibrous band. Below the line of ossification the medulla is represented by a very close dense areolar connective tissue with fibres decidedly coarser than normal. In short, there exists an intense sclerosis with disappearance, or at least absence, of normal medullary cells."

While the cartilaginous ossification is insufficient and defective, the periosteal ossification appears active, though equally pathologic under certain aspects. On a transverse section of the cliaphysis of the femur, we perceive the eceentric position of the medullary canal, which is diminished in size. We nowhere find compact bone. These bony partitions may run parallel to the surface of the bone, or may be perpendicular to it, and at their peripheral ends may become lost in the internal fibre-bundles (faisceaux) of the periosteum. Where the periosteal layer begins to bend inwards and to ealcify, there is a transformation of the periosteum into fibrocartilage and then into ossification. 
Résumé: There is present a sclerosis of the epiphyseal cartilage while in preparation for multiplication. A lack of serial arrangement of the cartilage, and a deficiency in cartilaginous ossification successively occurs, while processes from the marrow cross the line of ossification. Abundant periosteal ossification is produced as well by means of osteoblasts as by direct calcification of the fibrous lamellæ, but only eventuating in the formation of a spongy bone with thick resistant lamellæ. There is sclerosis of the bone-marrow. This condition is entirely distinct from that known as osteogenesis imperfecta, in which the periosteal ossification is especially defective. Mullen's case of osteogenesis imperfecta (reported in 1897) showed that the endochondral ossification was wholly normal. In this case no real periosteal bone formation was anywhere established.

The shafts of the bones often show great thickening and shortening of the cortical substance, and overgrowth of the bony epiphysis is so marked that it often appears to overlap that of the epiphyseal line.

In examining the living conditions in these cases, first the periosteum shows great thickening; second, the cortical substance is broader and thickened; and third, the medullary canal is narrowed, sometimes showing its cavity to be replaced by hard cortical tissue. It is seen that the arrest of development and consequent shortening of the long bones is due to the cartilaginous ossification of the epiphyses, and it is very evident that these abnormalities are brought about by the disturbance in the normal process of ossification in the primary cartilage. Although many of these cases are born in such a condition that they do not live, yet the milder cases recover, and it is important to differentiate by means of the Roentgen ray this class of cases from osteogenesis imperfecta, rhachitis, and various anomalies such as the shortening shown in Plate 57.

The examination by the Roentgen method of cases of chondro- 
dystrophia gives results as characteristic as the clinical examination, though varying in certain details of the bony change which takes place. A deseription of the Roentgen pietures of these cases is not definite unless we divide them into two groups and consider the age of the individual.

1. The cases which are seen up to pubescence.

2. The eases of pre-pubescence with marked deformities of the limbs, and in which a secondary lesion of the joint is found.

In the first group of cases the typical Roentgen picture of a given bone, for instance the tibia, will show a bone that is shorter and wider than the bone of a normal ehild, but which has a welldeveloped ossific centre for the given age under examination. The picture produced by the bone generally compares well with that of a normal child. Deformities of the shaft, with the exception of bowing, are rare in this group. The cortex is thicker, particularly in the middle of the shaft, showing considerable deposit of bone, but thinning out towards the diaphysis. The medulla, though small at the middle of the shaft, is increased proportionately with the thinning of the cortex. The bone structure is in some cases practically normal as compared with a normal child, but in the majority of eases is coarser and sometimes a more irregular deposit is apparent, particularly in the diaphyses. The most characteristic appearance of the long bones is that near the epiphyseal ends the diaphyses are spread like a cup (Plate 67). This produces a T-shaped outline without any disturbance of the epiphyseal line or zone of proliferation, though it may be uneven and show more or less fantastic shapes as compared with a normal individual. They are, however, not so uneven as is seen in diseases of nutrition, such as rhachitis (see Plate SS, Division IV), where there is a definite disturbance of the zone of proliferation, but where the ossific centres lave been well formed and the epiphyses are apparently fitted into the cup- 
like diaphyseal ends. In this first group of cases there is nothing seen in the Roentgen plates which could be confused with rhachitis.

The second group of cases is characterized by extremities extraordinarily shortened in comparison to the body. The upper arms and thighs are shortened in comparison to the forearms and legs, but are distinguished from the first group in that the osseous system shows more definite pathologic changes in the joints, such as scoliosis, kyphosis, lordosis, coxa vara, genu valgum or varum, with ankylosis, partial or complete, of one or more joints, either owing to a mechanical distribution or to a pathologic change. In the Roentgen examination of these cases, although the underlying appearance of the bone is that of the first group, we have added a more definite change of the articular surfaces of the joints and of the epiphyses in general.

In chondrodystrophia there is a rather narrow but fairly regular zone of proliferation, the shafts are short, not bent, and usually thick.

\section{OSTEOGENESIS IMPERFECTA}

\section{SYNONYMS : FRAGILITAS OSSIUM, IDIOPATHIC OSTEOPSATHYROSIS}

This disease is characterized by brittleness of the bones and multiple fractures. It has been shown to have a definite pathology of its own. As at birth the clinical diagnosis is often obscure and unsatisfactory, the Roentgenograph becomes of importance in differentiating between this condition and chondrodystrophia fœtalis, or possibly a rare case of infantile osteomalacia. In considering this class of congenital diseases we must remember that the skeleton at birth is still in its formative stage, and it is therefore difficult to distinguish between the effects of disease and those of malformation. The diagnosis of fetal diseases of the bone has been greatly confused, since the same name has been given to different pathologic and clinical conditions, and different names to the same disease. As is shown by Nichols, however, osteogenesis imperfecta has a distinct 
pathology of its own and should be entered under its own heading. It differs from osteomalacia, chondrodystrophia, and rhachitis. I have bcen unable to find any reliable modern report of a histologic examination made of a case of ostcomalacia occurring at birth, although of course it is possible that it may occur. Osteogenesis imperfecta may be said to be an abnormal intra-uterine condition resulting in multiple fractures during intra-utcrine life or infancy. Clinically in this disease the infants are smaller than normal and their extremities are short and usually curved. The skin is thick and cedematous, and their skulls are probably imperfectly ossified. A case of osteogenesis imperfecta was referred to me by Dr. R. W. Lovett, and I had the infant under my care until its death at the age of ten months. The case was minutely examined and studied post mortem by Dr. A. G. Nichols. According to his examination the following pathologic conditions were found:

"The new trabecula are formed by direct metaplasia of persisting cartilage cells into bone, whereas the normal development of the trabeculæ of bone is formed by apposition of bone, by osteoblasts upon a persisting cartilaginous matrix. In this condition the bone cells are large, oval, not stcllate, and show no tendency to form connecting canaliculæ. The bones in this condition are very imperfectly formed, and would suggest a general diseased condition, the character of which is not perfectly clear. The fibrous matrix is not calcified as in normal bone, and the capsules of the cartilage cells do not rupture. The periosteum does not form normal bone, is much thicker than normal, and is incomplete. The Haversian canals are supplemented by large marrow spaces and the trabecula in the marrow canal are fewer. Near the epiphyseal lines and in the marrow spaces of the cortex the marrow consists of an cedematous, myxomatous connective tissue. The whole process of the development of the bone is checked and is of an abnormal kind. Metaplasia of cartilage 
is very much greater in this condition than normal, while apposition of bone is much less. The bones show lessened density and appear thin and atrophied. The medullary cavity seems to be increased at the expense of the cartilage.

"The epiphyseal lines are sharp, but perhaps less regularly so than normal. Fractures are numerous and appear clearly in the Roentgenograph. The bones show increased radiability.

"It is improbable that this process is identical with the process which occurs in acquired fragility of the bones." (For a more complete histologic report of this condition, see British Medical Journal, October $4,1906$. )

Plate 68 shows the forearm of a girl two years old. Clinically there were no deformities, except a tendency to valgus of the left foot. The shafts of the long bones, especially of the radius, were thickened and heavy. Numerous irregularities simulating a callus were detected, and were especially well marked on the left humerus. The Roentgen examination showed that the phalanges of the fingers were regular in shape, excepting that the proximal portions were toothed and cup-shaped. There was very little cortical bone present, and the bony structure was coarse with very little evidence of the bone. The carpal bones were delayed in development. The radius showed evidence of at least two fractures, and the ulna of one or more.

The lateral view of the leg, Plate 69, of this same subject, shows a fracture of the femur without any evidence of cell formation. The outline of the bone was regular, excepting for the fracture. There was very little cortex. The medulla was increased proportionately and showed marked increase of radiability. There were coarse trabeculæ in the structure of the bone. The epiphyses were poorly defined and there was a marked increase in the zone of proliferation of all the bones. A fracture ras also apparent in the fibula. 
Plate 70 shows the leg of a girl twenty-five months old. There was no history of previous multiple fractures in the family. The legs and arms of the ehild seemed very weak at birth and they seemed to be very sensitive to touch. The right arm just below the elbow showed on palpation displacement, but the motion of the elbow was not impaired. Nothing abnormal was discovered about the joints. The Roentgenograph showed an unreduced fracture of the lower end of the humerus, and three fractures of the left arm, also a fracture of both femurs. The lateral view of the leg showed a marked overgrowth of periosteum, with evidence of fracture in the middle of the shaft. No formation of callus was present. The cortex was ill defined and in places absent. The epiphyses were fairly regular, but there was considerable disturbance of the diaphyseal portion of the bones which were toothed and eup-shaped. A fracture of the tibia and of the fibula was seen to be present.

\section{FETAL RHACHITIS}

Although it is possible that rhachitis may be of fetal origin, I have been unable to find satisfactory proof of this in any reported case where modern methods of examination have been employed, so that the existence of intra-uterine rhachitis is still sub judice.

\section{OBSTETRICAL PARALYSIS}

The most common form of paralysis of the arm occurring at birth is that of the upper arm, although that of the lower arm type occurs at times, and both the upper and lower arm type may be present together. A knowledge of the anatomical conditions, especially of the upper arm type, which represents these forms of paralysis, is important in connection with the differential diagnosis from various other forms of paralysis or pseudoparalysis, especially poliomyelitis anterior. This is especially true in eases of obstetrical paralysis where this condition first comes under the physician's 
observation in older children, especially where the whole arm is involved. There is no need of entering into the question of etiology, as this question has been fully discussed by a number of writers, as by Thomas on the lower arm type, Boston Medical and Surgical Journal, October 19, 1905, by Stransky, Centralblatt s. $d$. Grenzgcberte d. Med. u. Chi., 1902, and by Bullard, Amer. Journal Medical Sciences, July, 1907, the latter paying especial attention to the upper arm type. It is now conceded pretty well that the upper arm type at least is produced by injury due to stretching of the fifth and sixth cervical nerves in the neck, which may be more or less ravelled out or torn asunder. The external points for diagnosis, both by manual examination and electrical reaction, hold their own place in importance. In some cases when the recognition of the condition is uncertain, the Roentgen method is especially useful for completing the examination and determining the lesions which are characteristic of obstetrical paralysis pure and simple without other injuries. There may, however, be a number of other anatomic conditions, resulting from fractures of the clavicle and arm and dislocation of the shoulders, which may so complicate and obscure the original injury of the cervical nerves that a precise and definite diagnosis cannot be made without the aid of the Roentgen method. It is therefore necessary that we should be familiar not only with the normal anatomic development of the shoulder, elbow, and wrist at different stages of growth, but also should recognize the deformities of these parts which are dependent on the obstetrical paralysis itself.

The following Roentgenographic plates illustrate cases of obstetrical paralysis of the upper arm type. If the injury has existed for some time after birth we find in these cases evidence of atrophy or non-development of the bones, in addition to the muscular atrophy.

Plate 71 shows a case of obstetrical paralysis in a boy eight 
years old. Within twenty-four hours after birth, paralysis of the left upper arm was noticed. When the child was three years old the paralysis of the deltoid was still marked and some atrophy of the left shoulder. At present he can elevate his arm only to the level of the shoulder and cannot supernate the upper arm beyond the median line. Extemal rotation was also found to be impaired. There was also marked shortening of the left arm, with atrophy of both the upper and lower arm and of the seapula. A Roentgenograph of this case showed a rudimentary development of the glenoid eavity and of the head of the humerus. Ossification of the upper epiphysis of the humerus and of the epiphysis for its greater tuberosity was found to be delayed. The scapula was smaller than on the other side. The superior angle of the scapula was elevated. The acromial process was shorter and narrower than that on the opposite side and was delayed in its development. There was no apparent atrophy of the shaft of the humerus.

Plate 72 shows a boy six years old. An hour after birth it was noticed that neither of the arms could be moved. When he was three years old he could use the left upper extremity from the shoulder. He could flex his forearm, but could not completely extend it. He could not pronate or supernate, and could raise his arms only about the level of the mammx. Seven months later the left shoulder was found to be higher than the right, the head of the humerus being pushed forward to the left. The scapula were of equal size, measuring $9 \mathrm{~cm}$. in length. The atrophy of the arm and forearm was noticeable. There was a primary rotation of the arm at the shoulder, and the shoulder was elevated. The movements were for the most part free, although there were adhesions between the shoulder and the scapula. The Roentgenograph shows the left shoulder to be lower than the right, and there is considerable atrophy in size of the shaft of the humerus. The scapula was a little smaller 
on one side than on the other, but otherwise seemed to be normal. There was some delayed development of the epiphysis of the humerus.

Plate 73 shows the condition of obstetrical paralysis in a girl who on the third or fourth day after birth was noticed to be unable to move her right arm. She could move her fingers. The arm could not be moved at all, and practically there was no voluntary movement of the entire arm except in the fingers. Posterior motion of the shoulder was painful and there was a distinct click of the clavicular humeral joint, but no definite crepitus. A Roentgenograph taken four months later showed a rudimentary development of the glenoid cavity, delayed development of the epiphyses, and the scapula to be very much smaller than normal, with a prominent superior angle and a rather large acromial process. 
PLATE 41.

HEAD AND NECK.

A. Orbit.

Boy, age 6 years. (Life size.)

$B$. Sphenoidal sinus.

C. Artifact.

D. Antrum.

E. First temporary molar.

$F$. Second temporary molar.

$G$. First bicuspid.

$H$. First upper permanent molar.

I. Second lower permanent molar.

$J$. First lower permanent molar.

$R$. Points to an anomalous condition of the upper cervical vertebræ. 
I'LATE 41

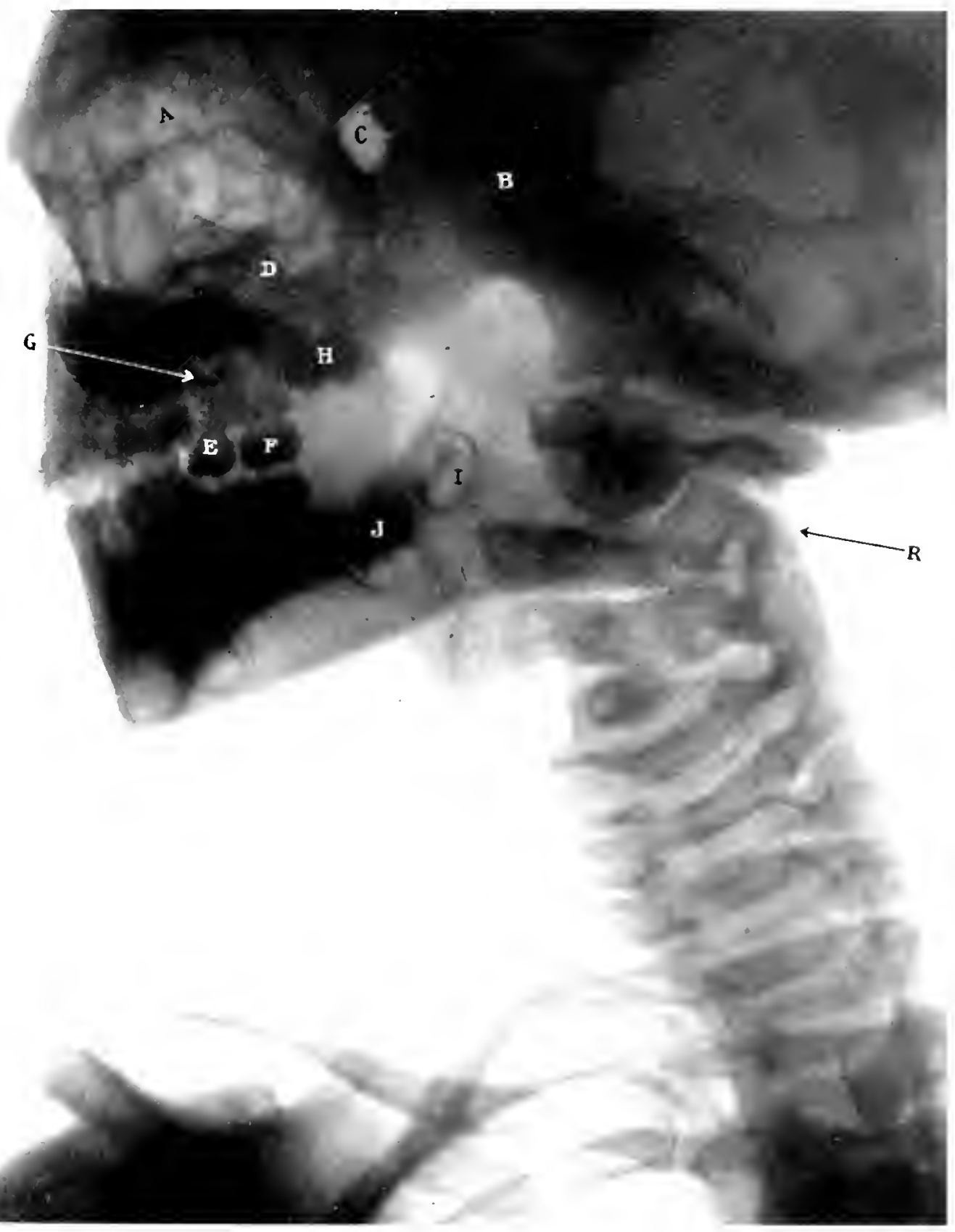


IPITI: 1.2.

SPINA BIFH METITA.

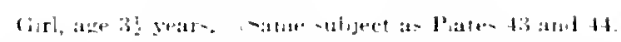

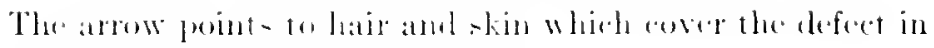
tim. verturter.

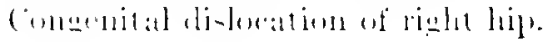




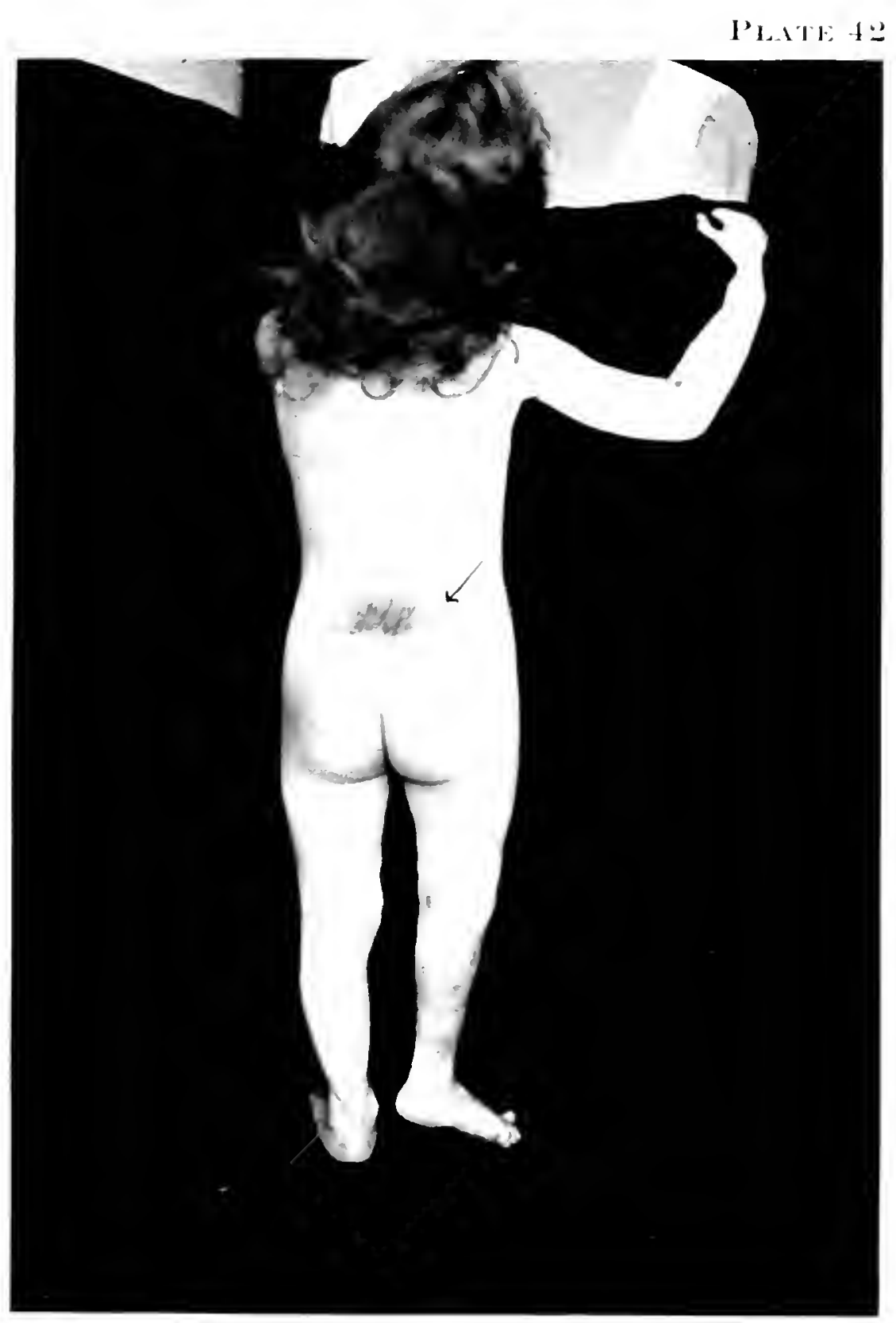


PIATE: 13.

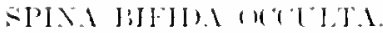

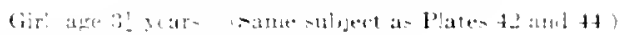

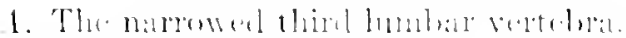

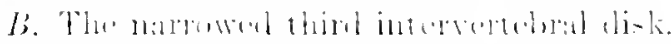

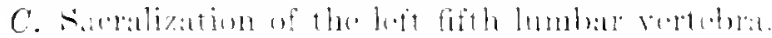

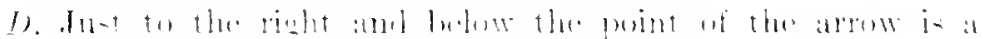

fir-itre of the firs bouly of the satrum. 
I'LATE 4:3

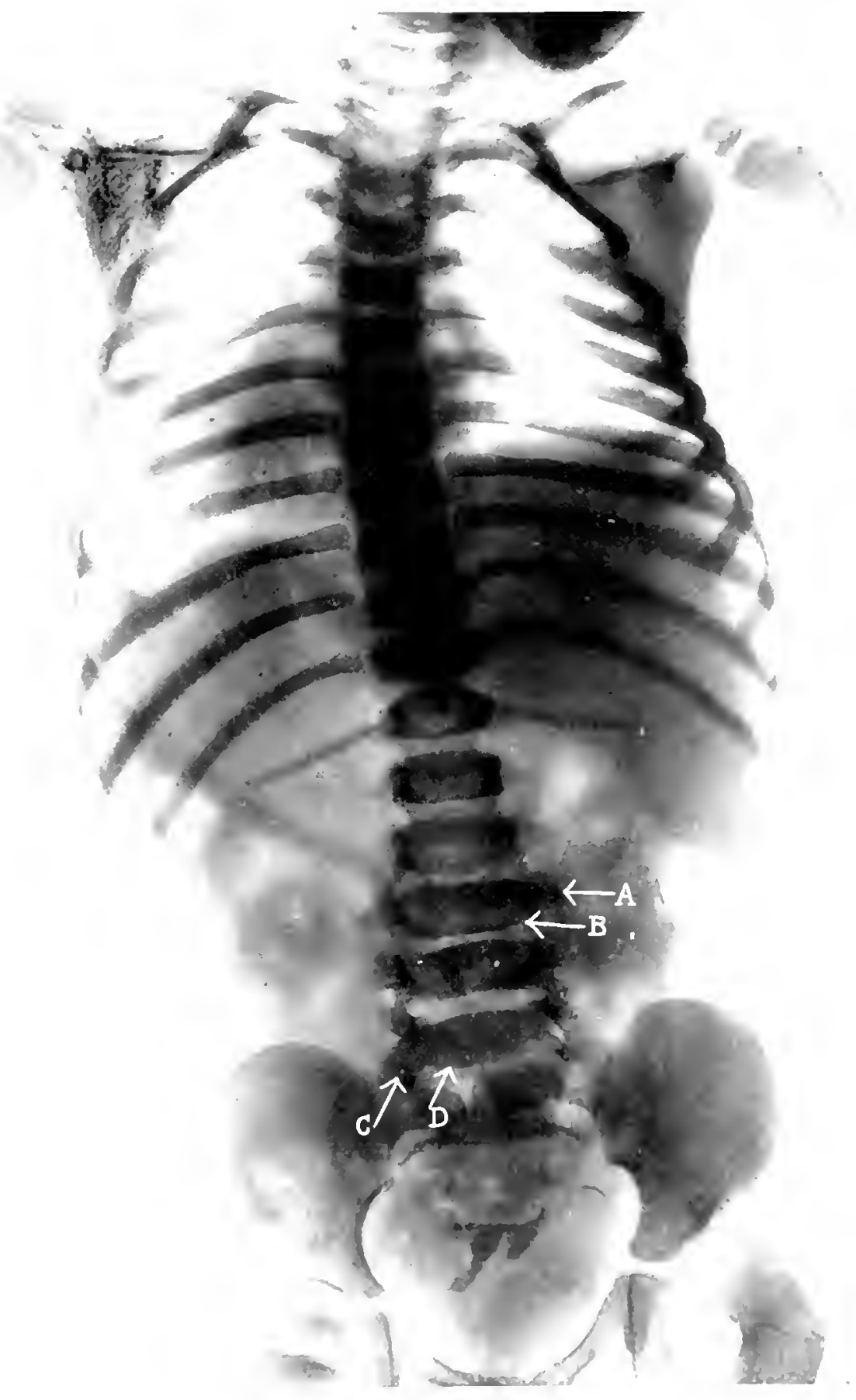


PI. TTH 41.

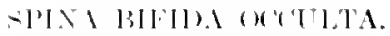

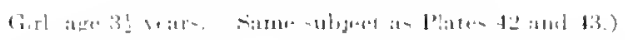

The arme prints to the right hip afteg poluetion.

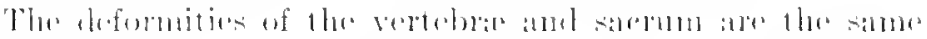
at in Plate l?.

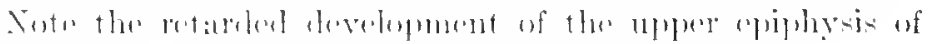
the right fomm in enmpration with that of the heft. 
PIATH +1

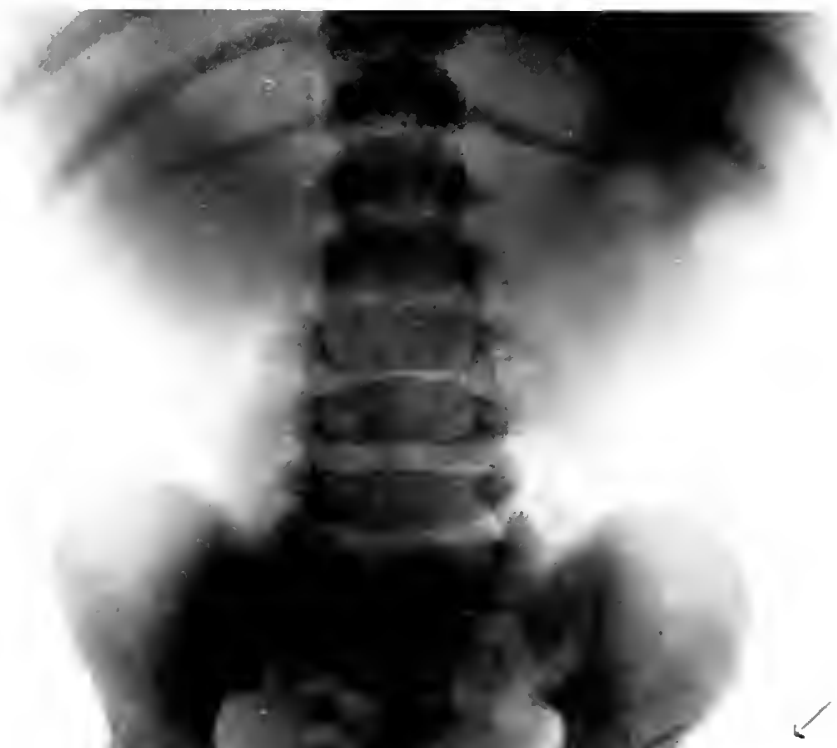


Pl. TT': 1.

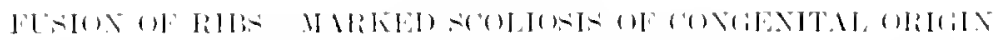

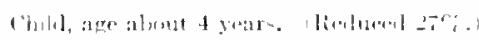

d. Ilyoill home.

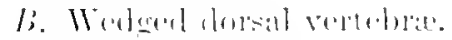

(. Firion of rils. 


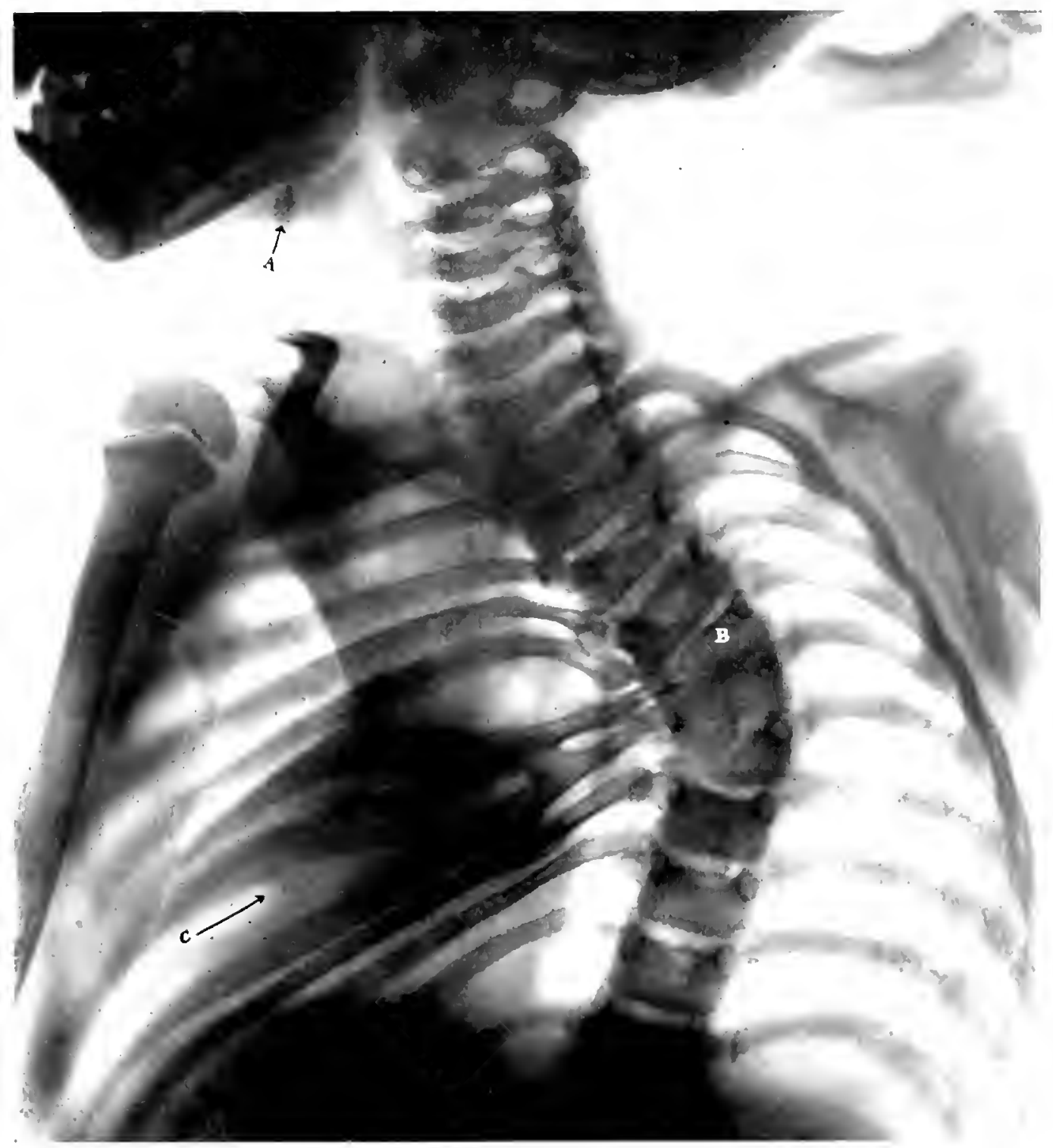


PITIE H.

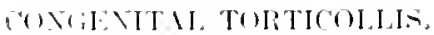

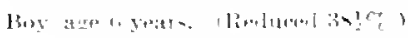

1. Fotrit rit.

l. riml rih. 
I'ITI\% 46

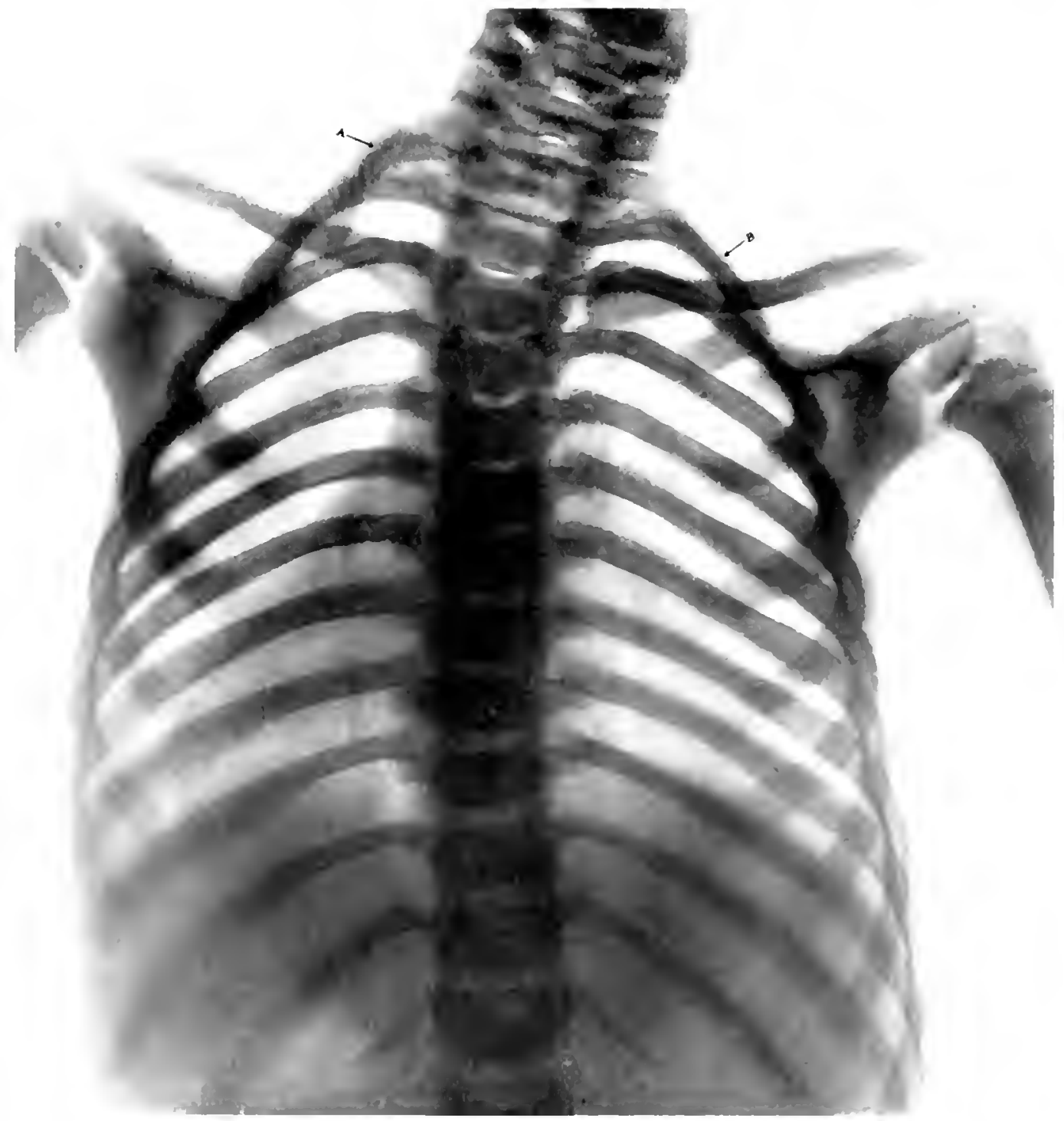


PLATE: 17.

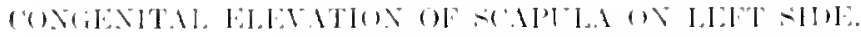

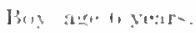

1. Finvitmi antulat.

I. Sinlisi- 
PIATE 4T

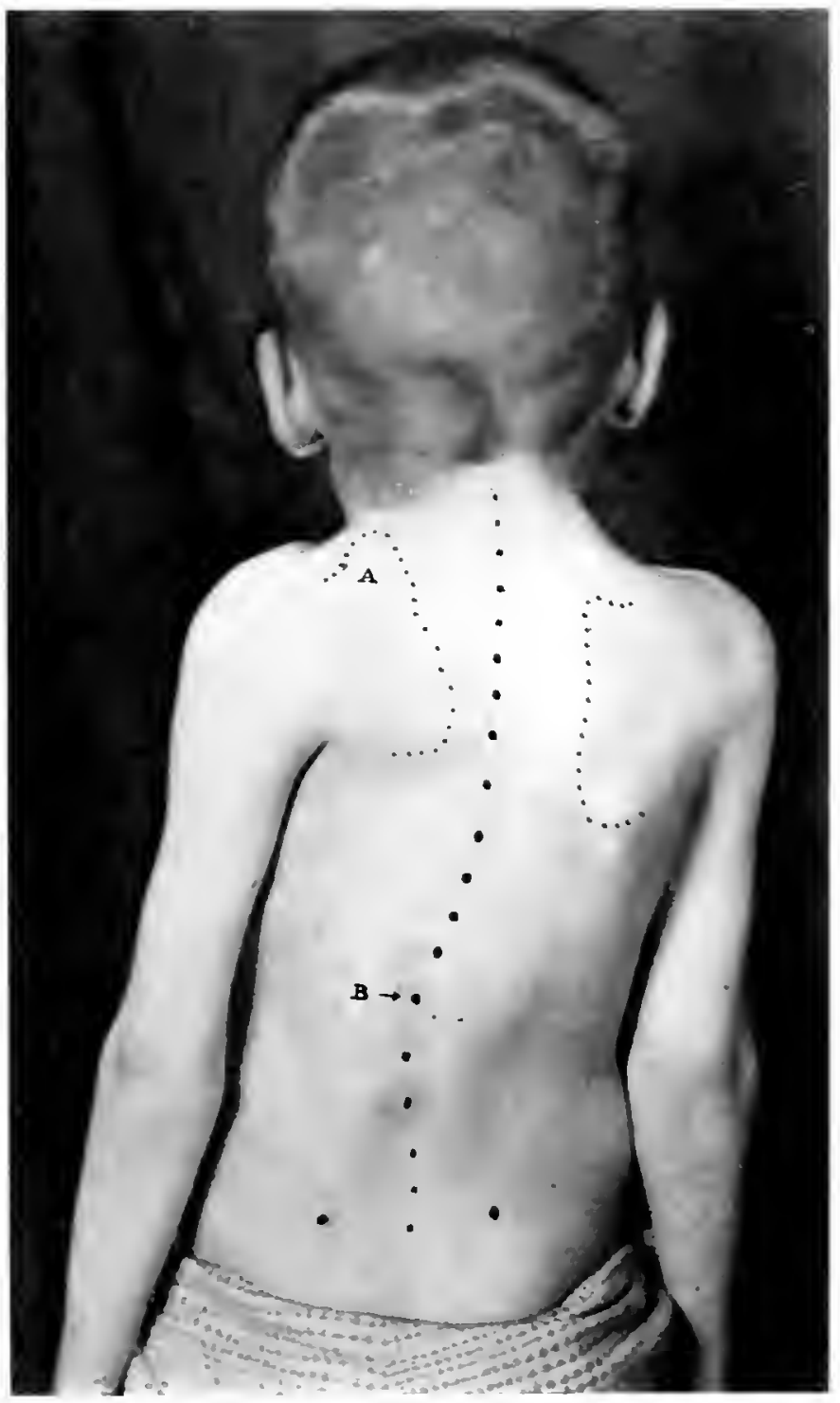




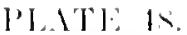

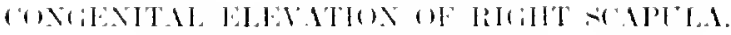

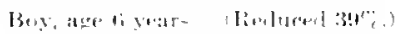

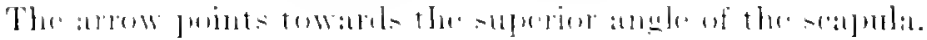


PLATE t9.

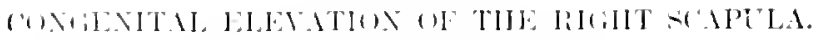

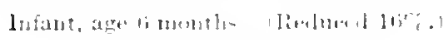

.1. Siripulat. 
T'LATE 4 !)

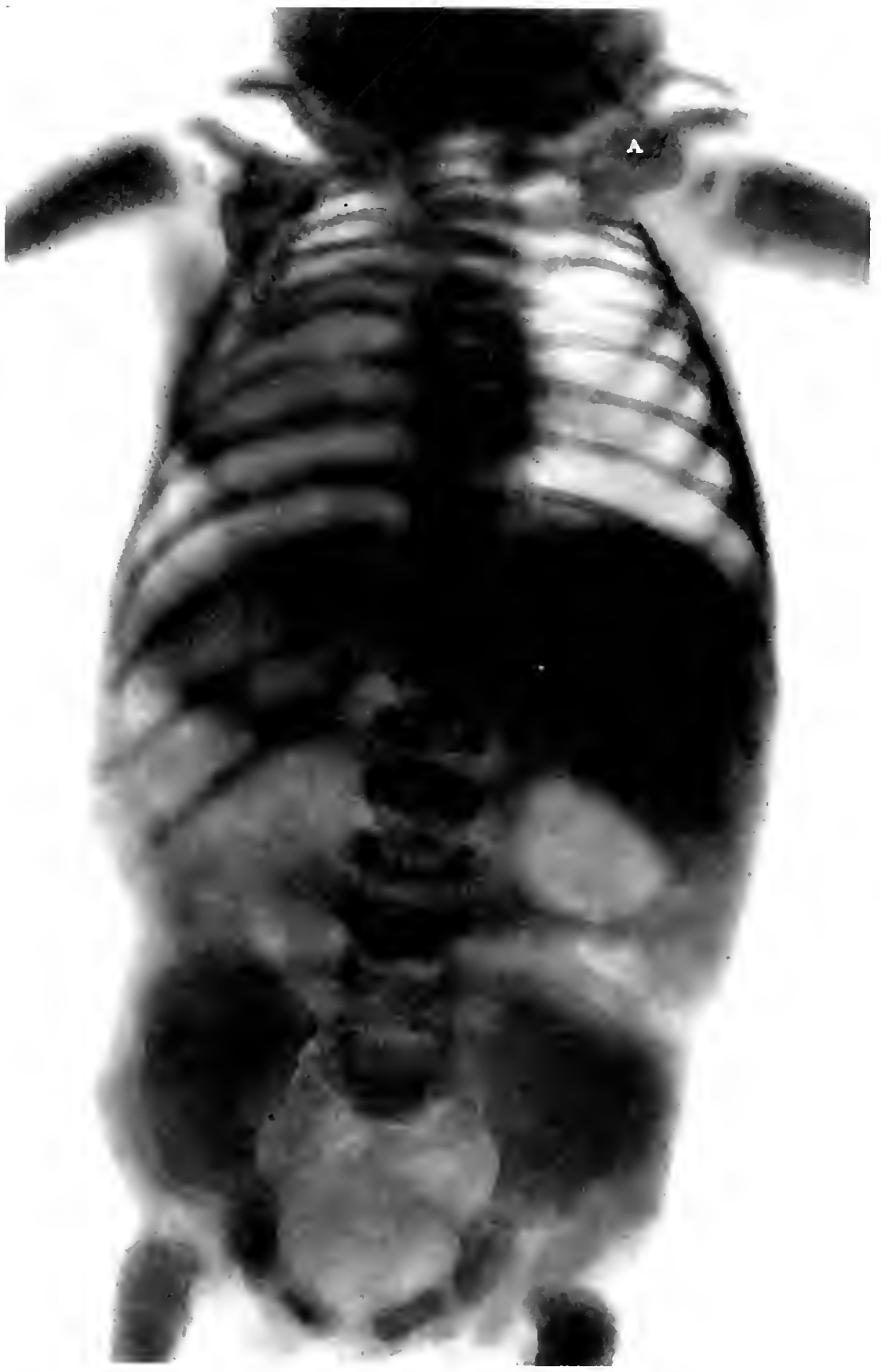


PLATL: 50.

(Lif. rim.)

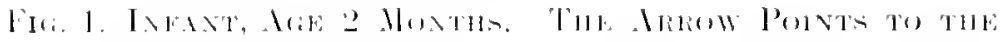
WEHBE, FINGILA

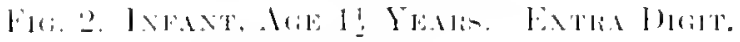

I. Extra digit.

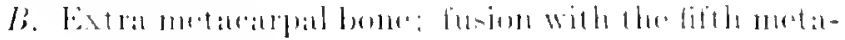
ralpial bomis. 
FIG. 1.

PIATE jo

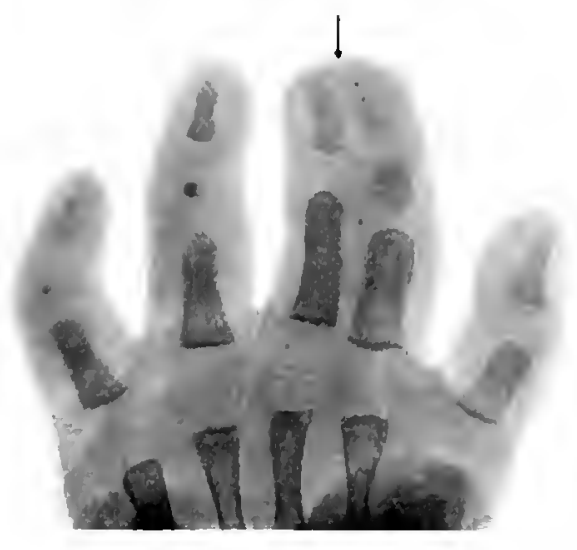

FIG. 2.

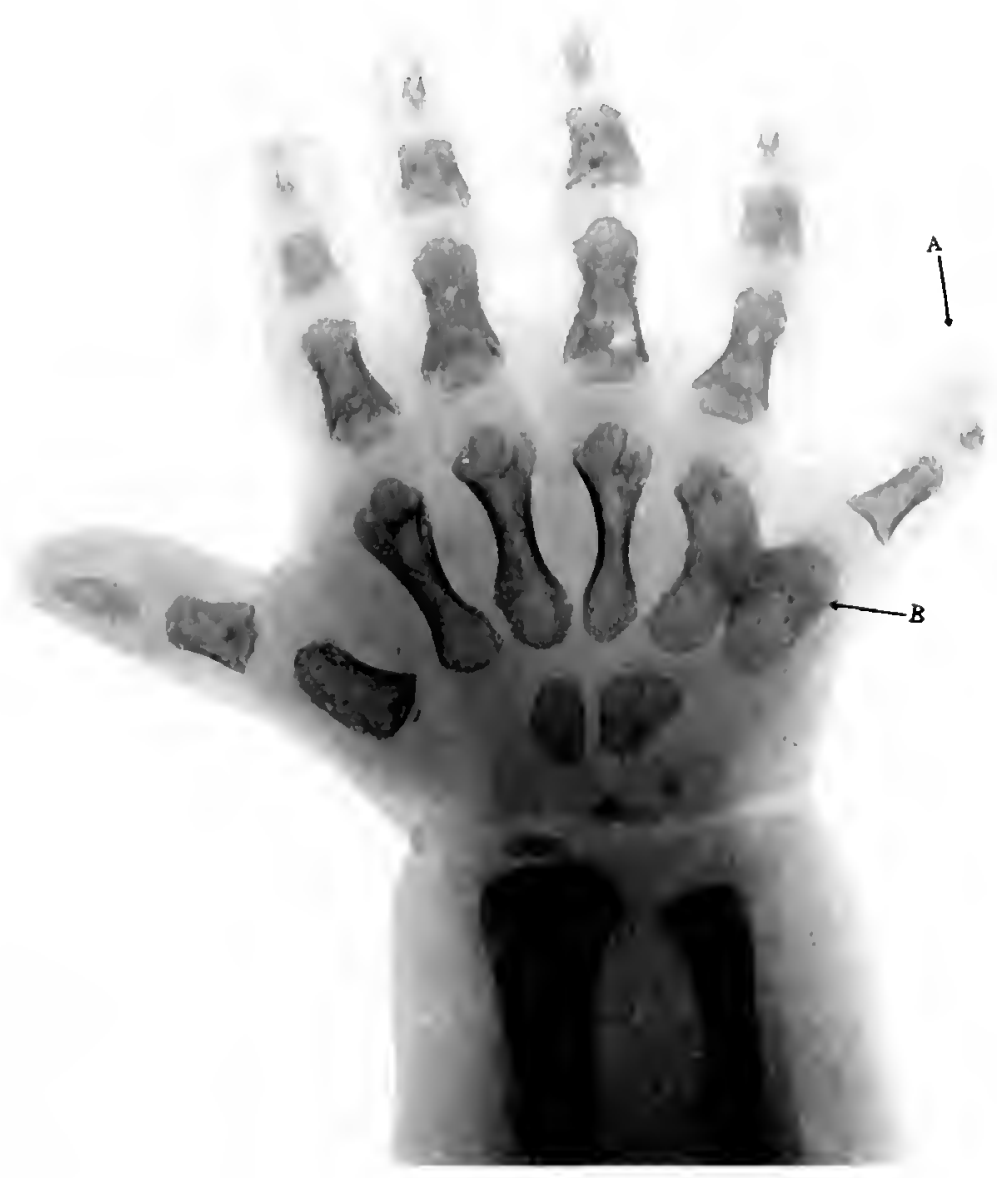


[1.1T1: il.

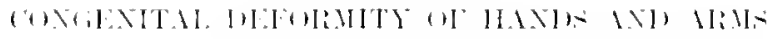

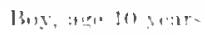

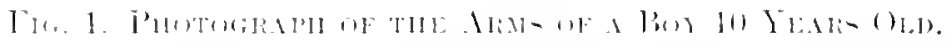

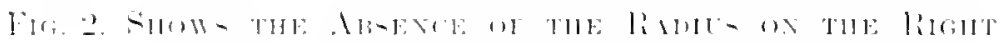

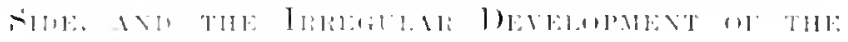

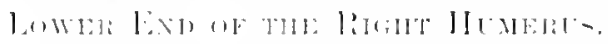

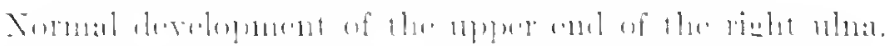

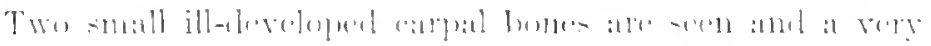

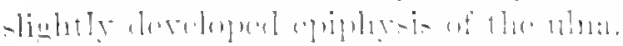

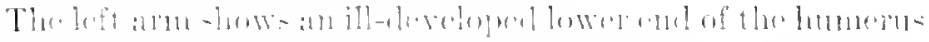

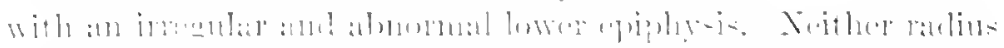

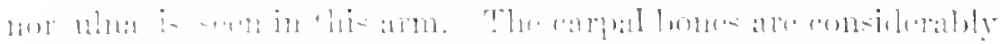

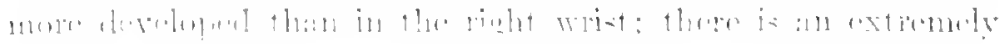

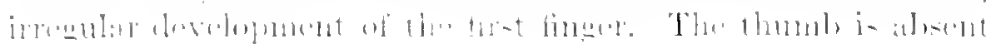


FIG. 1.

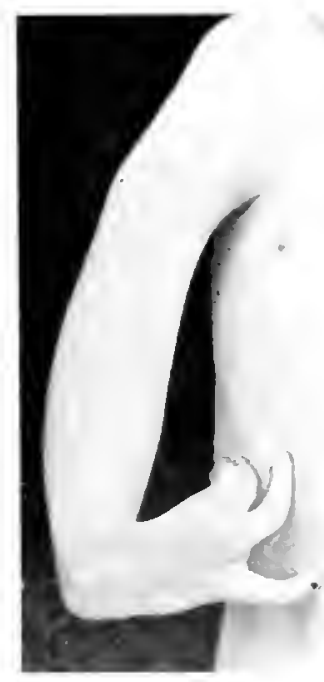

Prate. 1

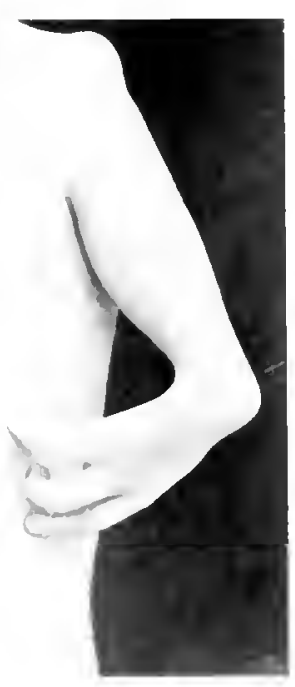

FIG. 2.

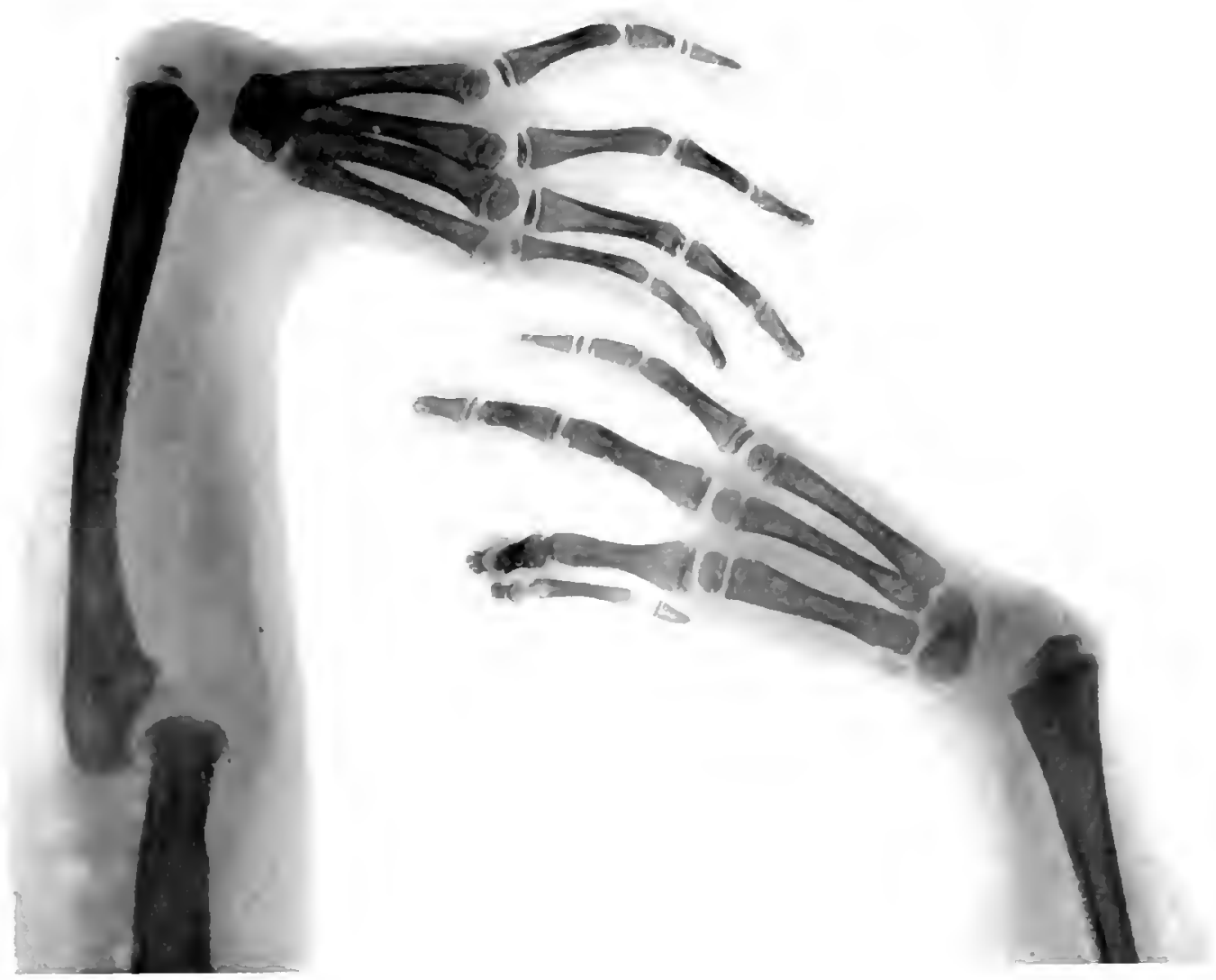


PLATE 52.

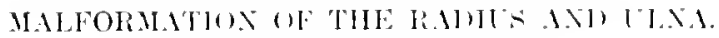

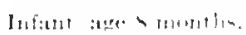

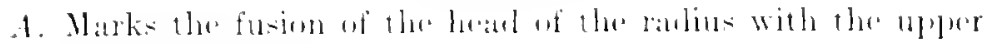
r.nel of the what.

B. ('apitrollum. 
PIATE .)

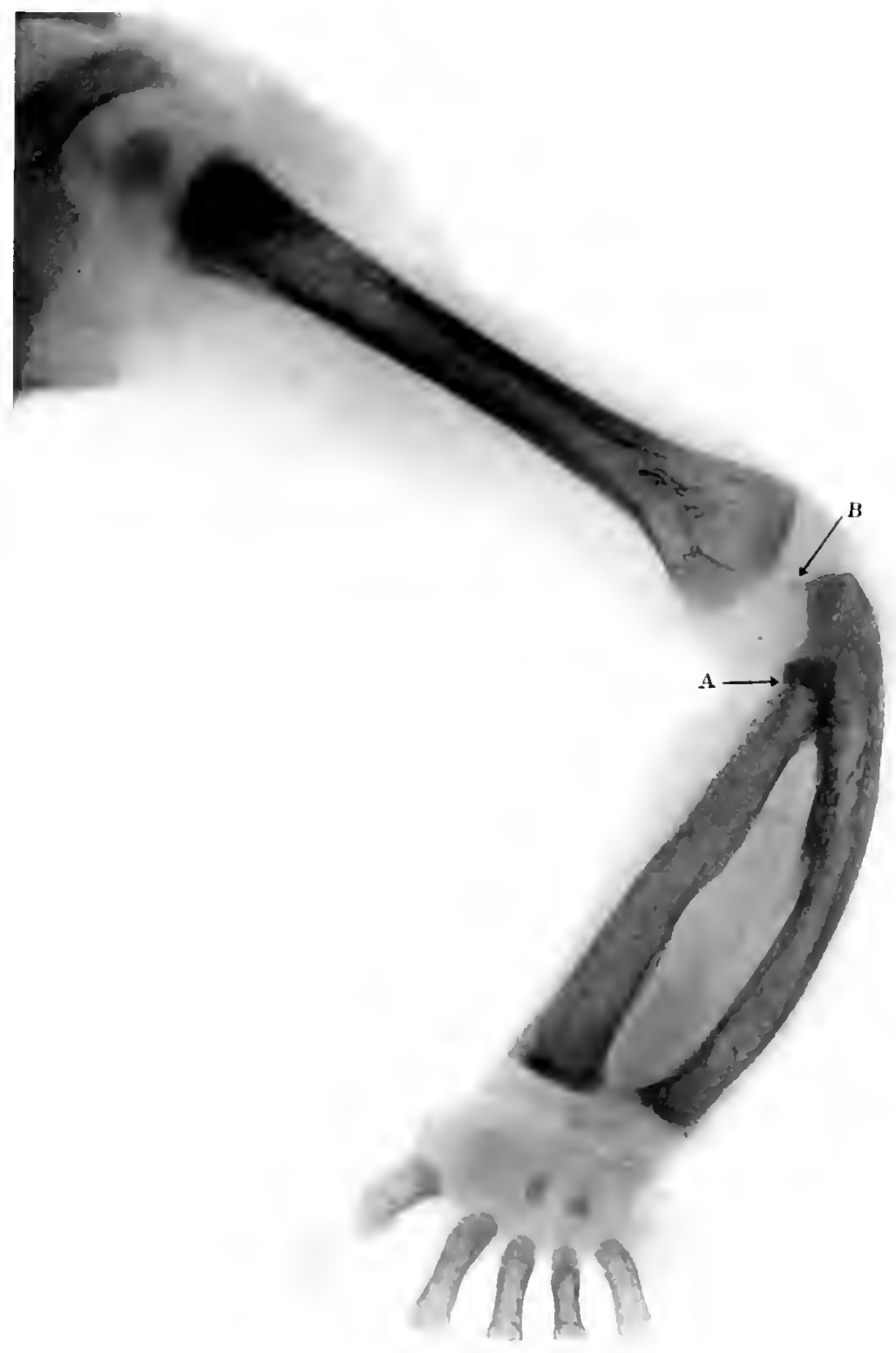


PI.ITI: i3.

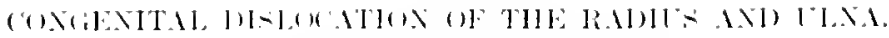

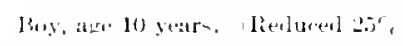

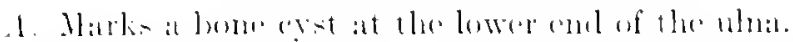

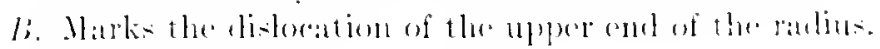

i. ('ipitellum.

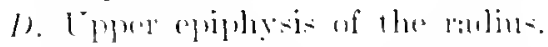

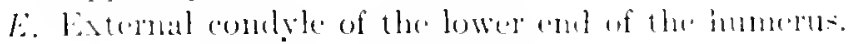


PLATE 5:;

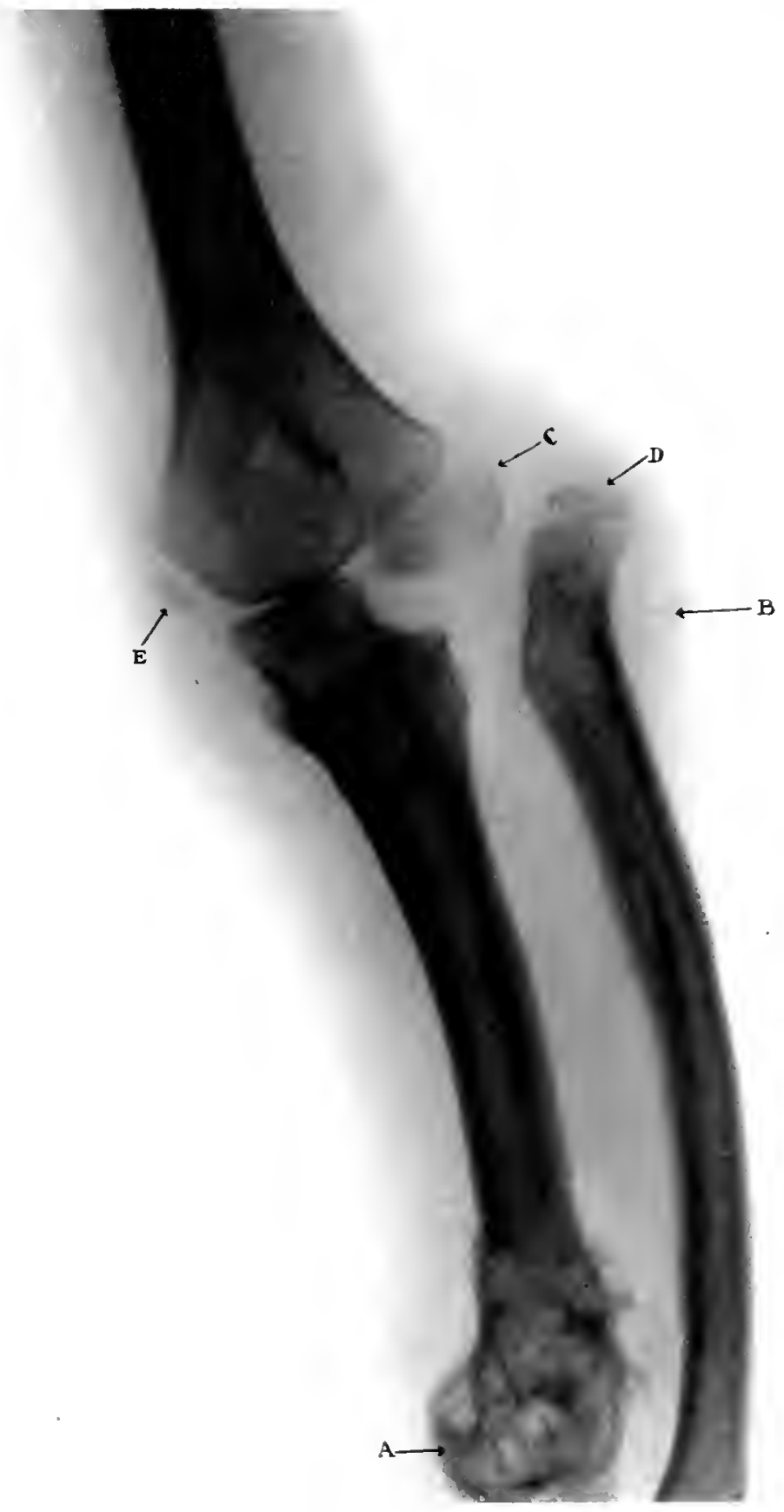




\section{I'LATL:}

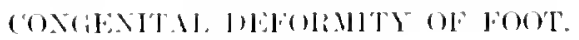

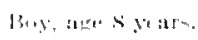

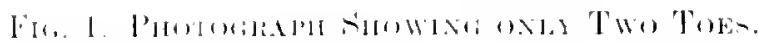

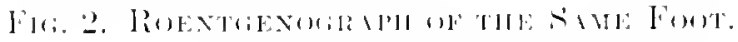

1. Marks thr furioln of the as abled with what was

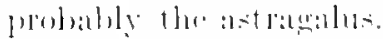

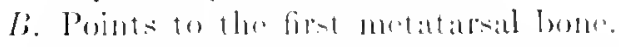

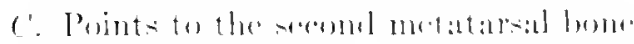

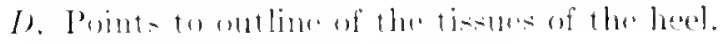

E. Points to the lomble leloilli- 
FIG. 1.

PIATE 54

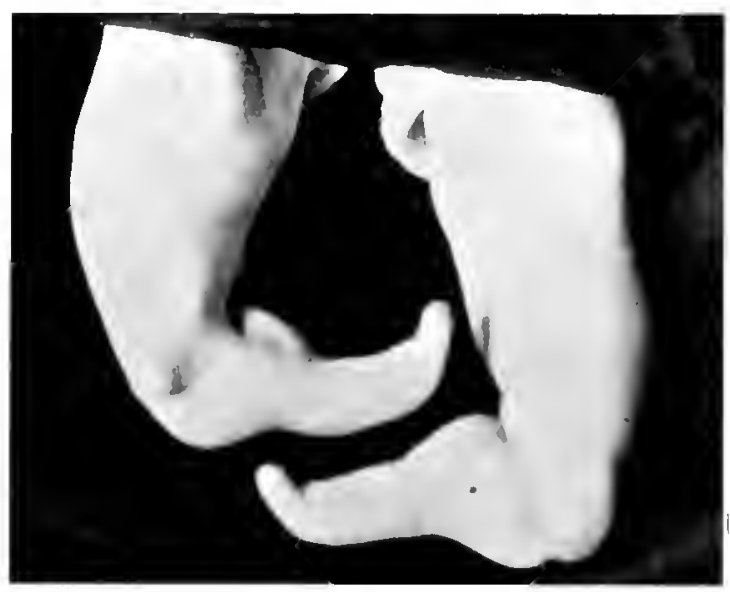

FIG. 2.

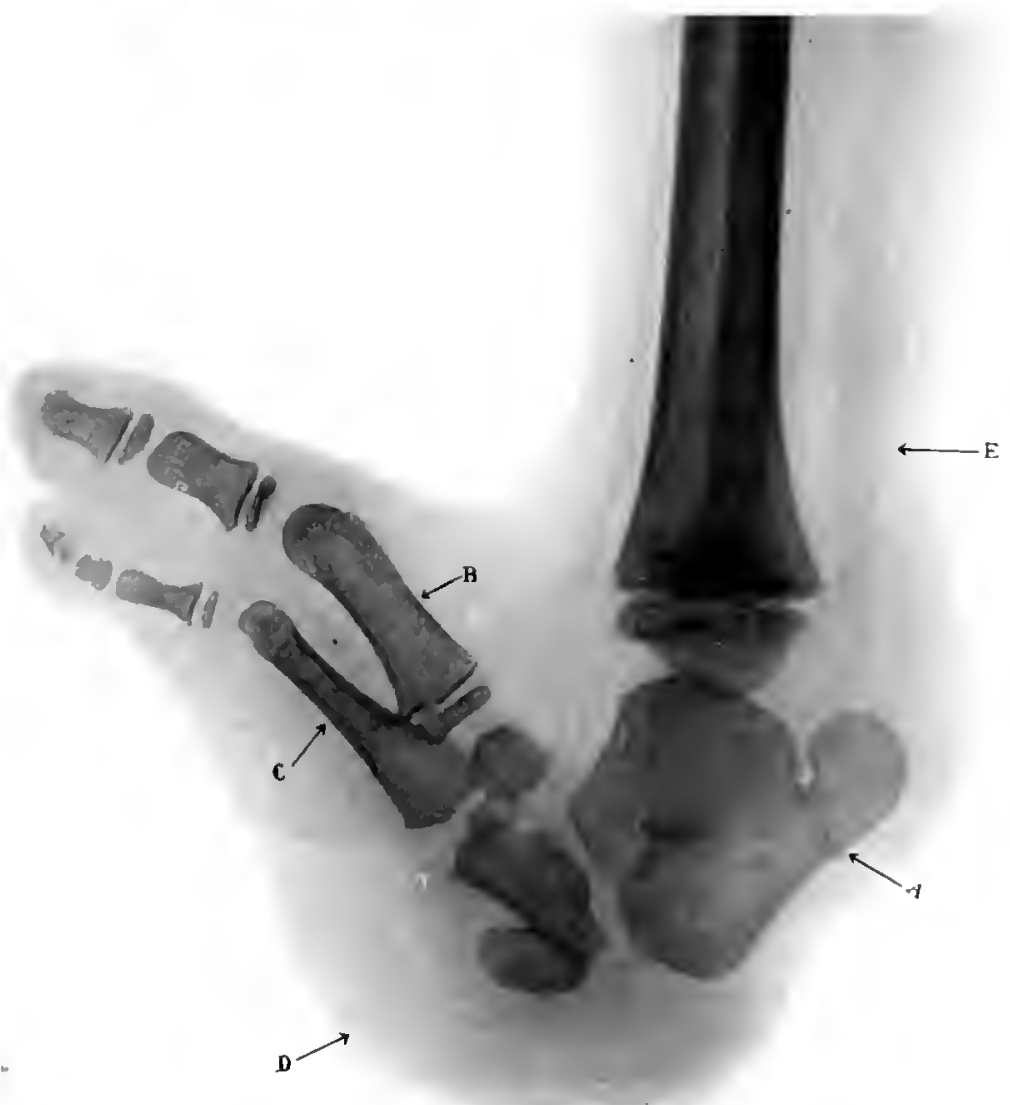


PJIT: $\therefore$.

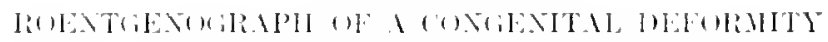
OF THE FOOT.

(L) fesize.

Slum - nhe toe and a rudimentary - newoll tue. 
PLite J.j

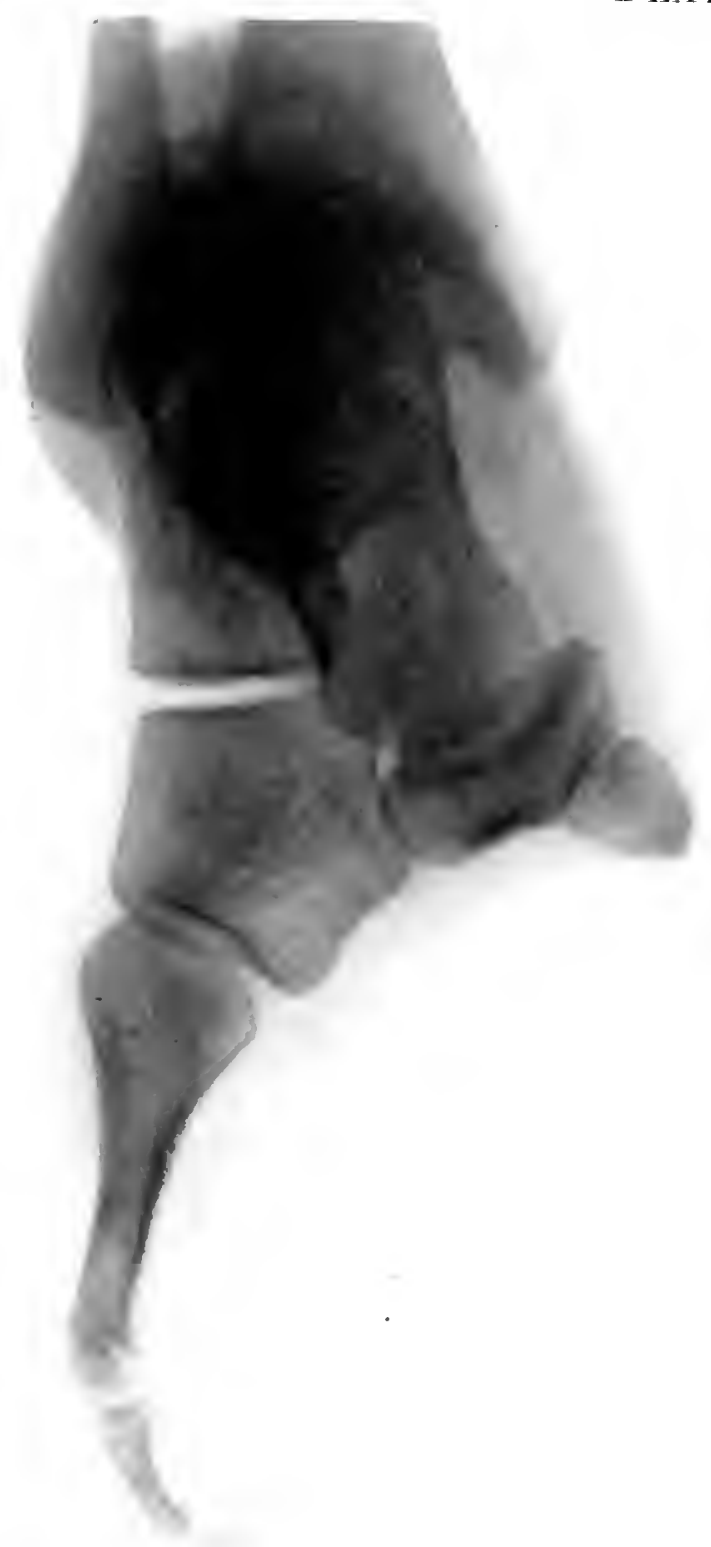


P'LATL: if.

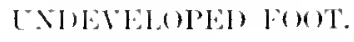

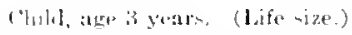

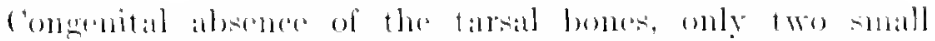

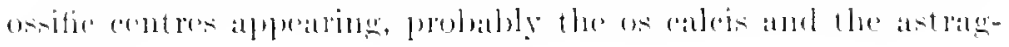
:1his.

'The lower epiphyses of the tibiat and fibulat are present.

Sote the meshes in the fat tisisure of the herel in contratst with the restilage in the tarsil region, the museles and the tencle Ichillis. 
PLATh: j;

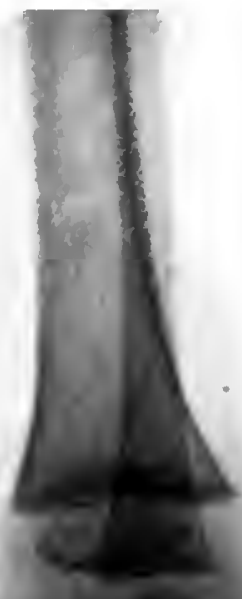


PLATE:

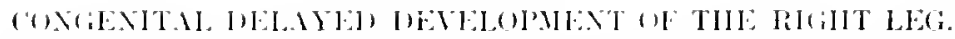

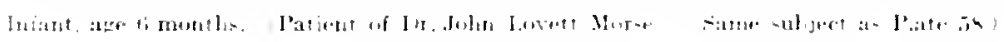

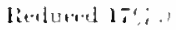

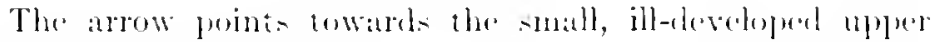
"piphysis of the right femur.

Sote the shertenel femur and the small size of the femur, tibia, and fibul: in comparion with the left lear. 


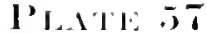

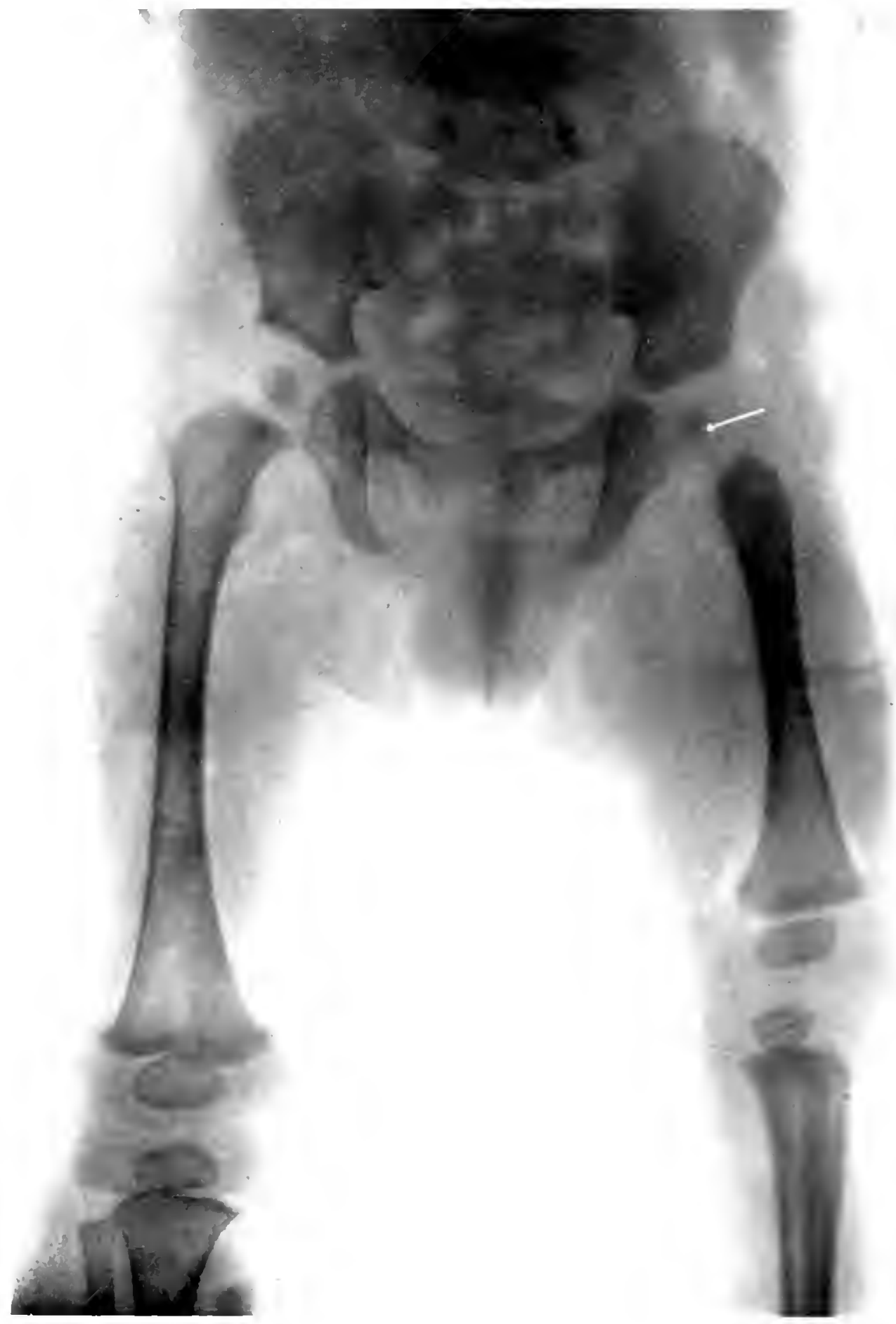


Pl.1Tl: is.

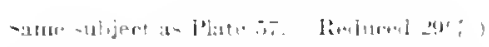

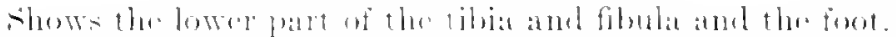

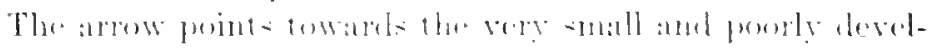

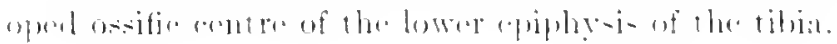


Plate jo
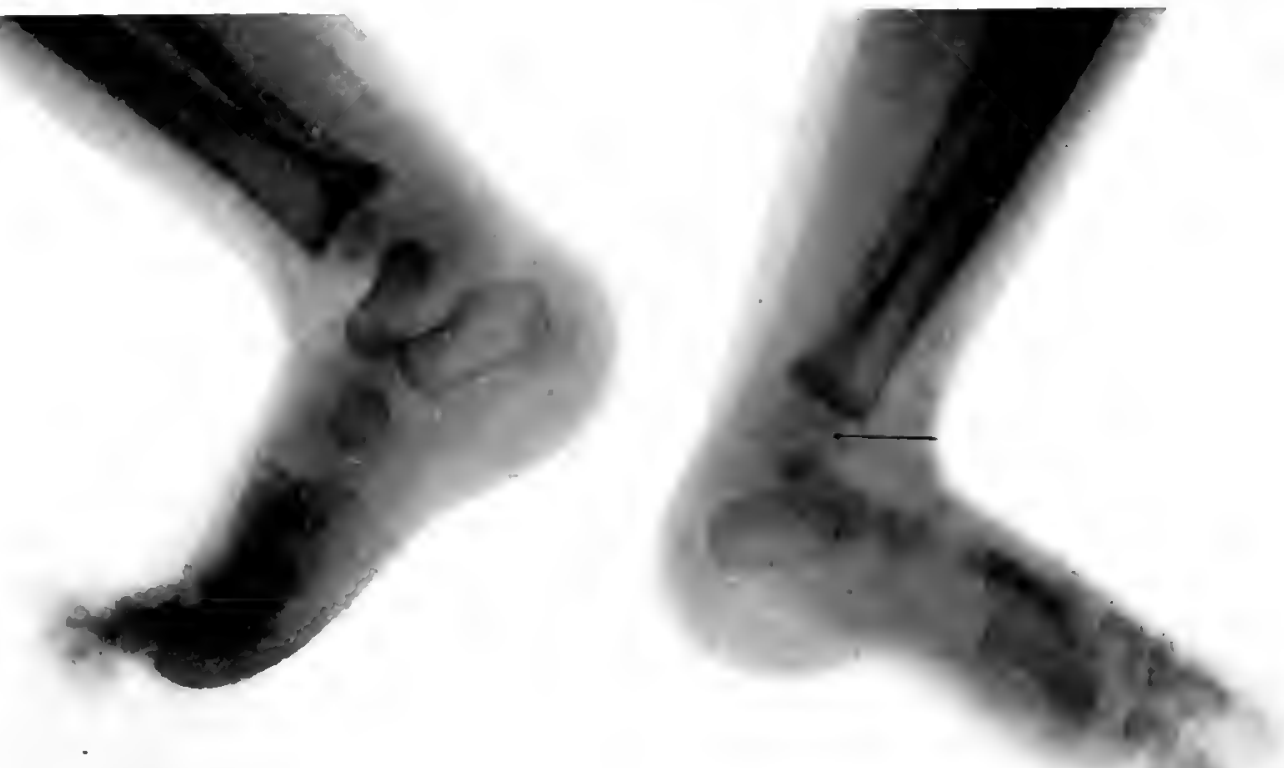


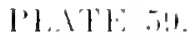

Im"13.1: (6)

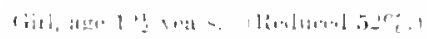

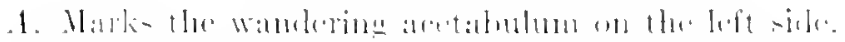

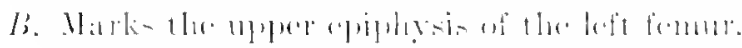

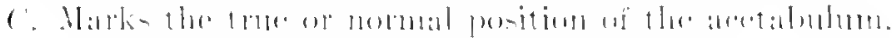

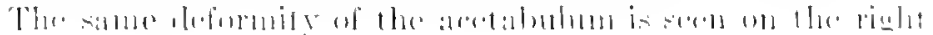
sill. 
P'LATL:

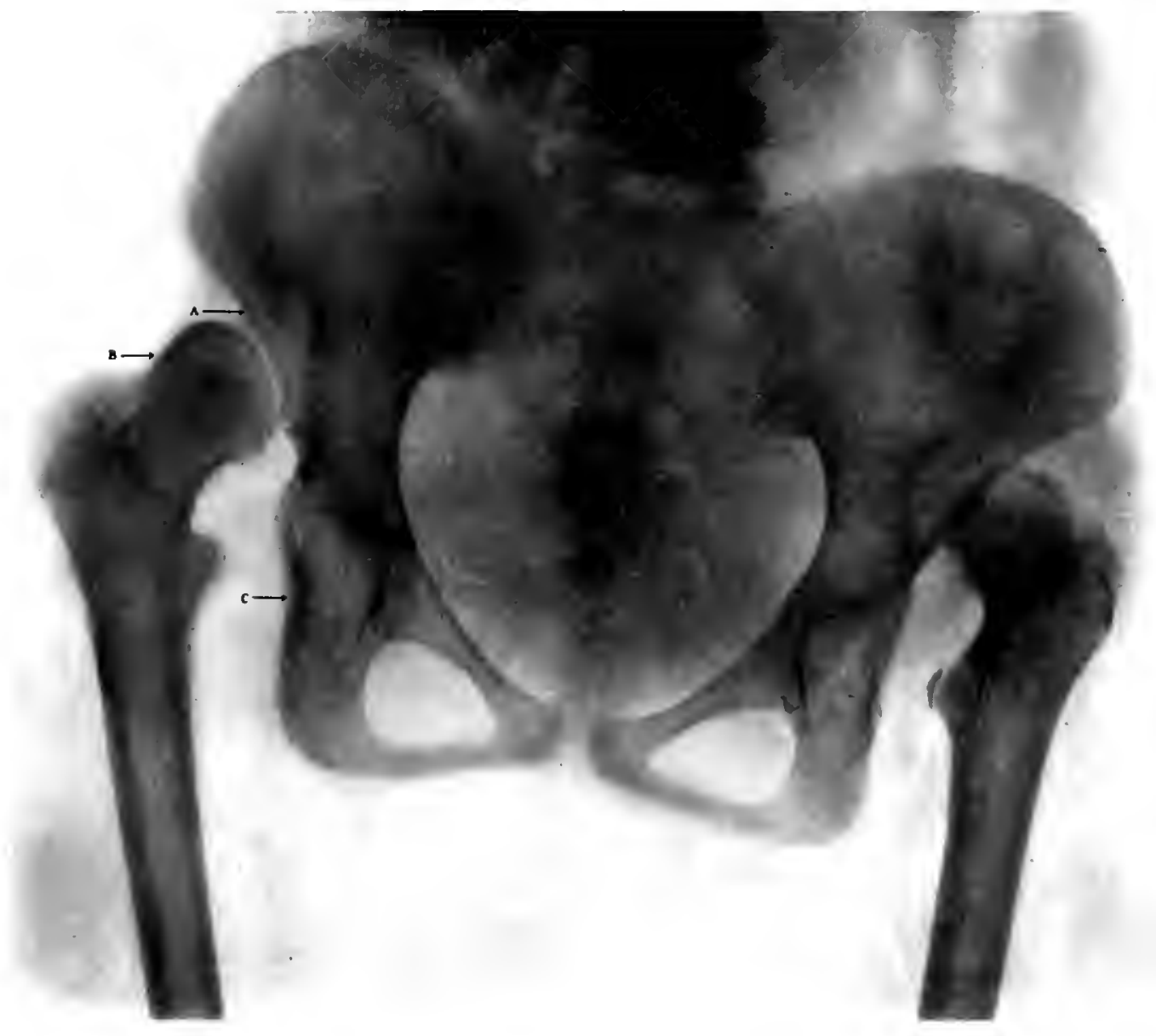


PLATH 60.

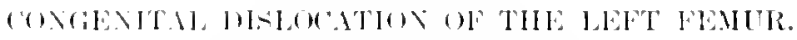

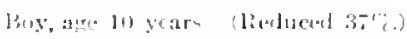

1. Shems the aldinentary isehiatie pution of the acetalulum.

li. Marke the iliare portion of the aretahulum.

Note the rudimentary comblition al the epiphysis, and of

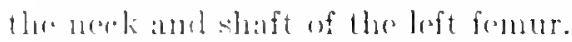

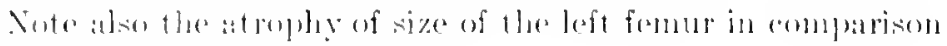
with the right formur. 
PJATH $1 ; 0$

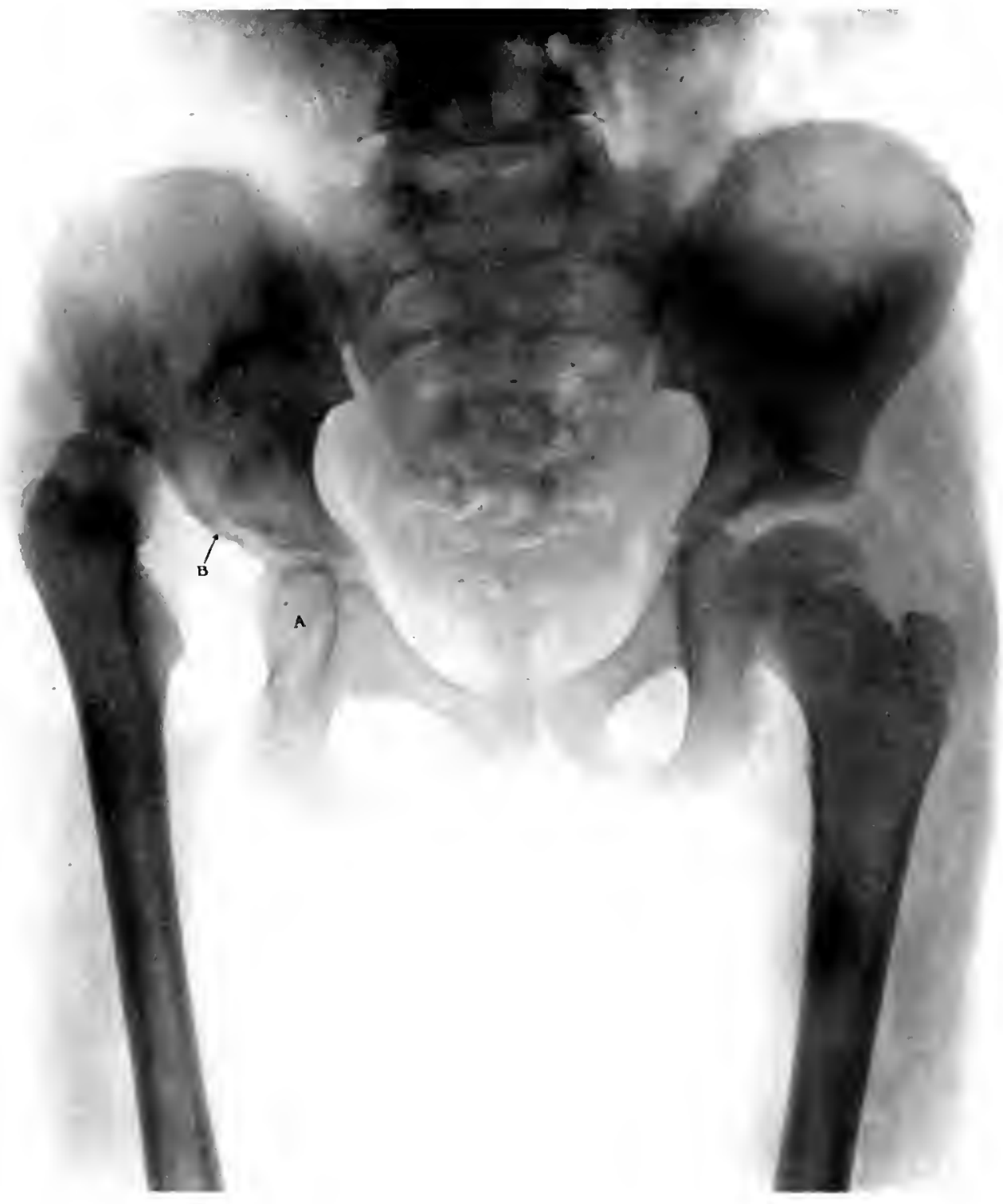


I'L.TTLitil.

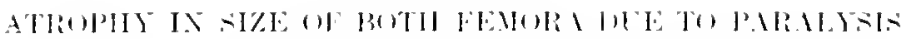
WF THE HEC:

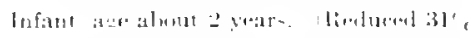

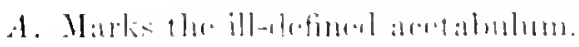

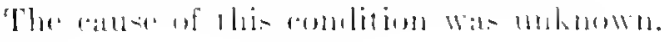


PLATE \&I

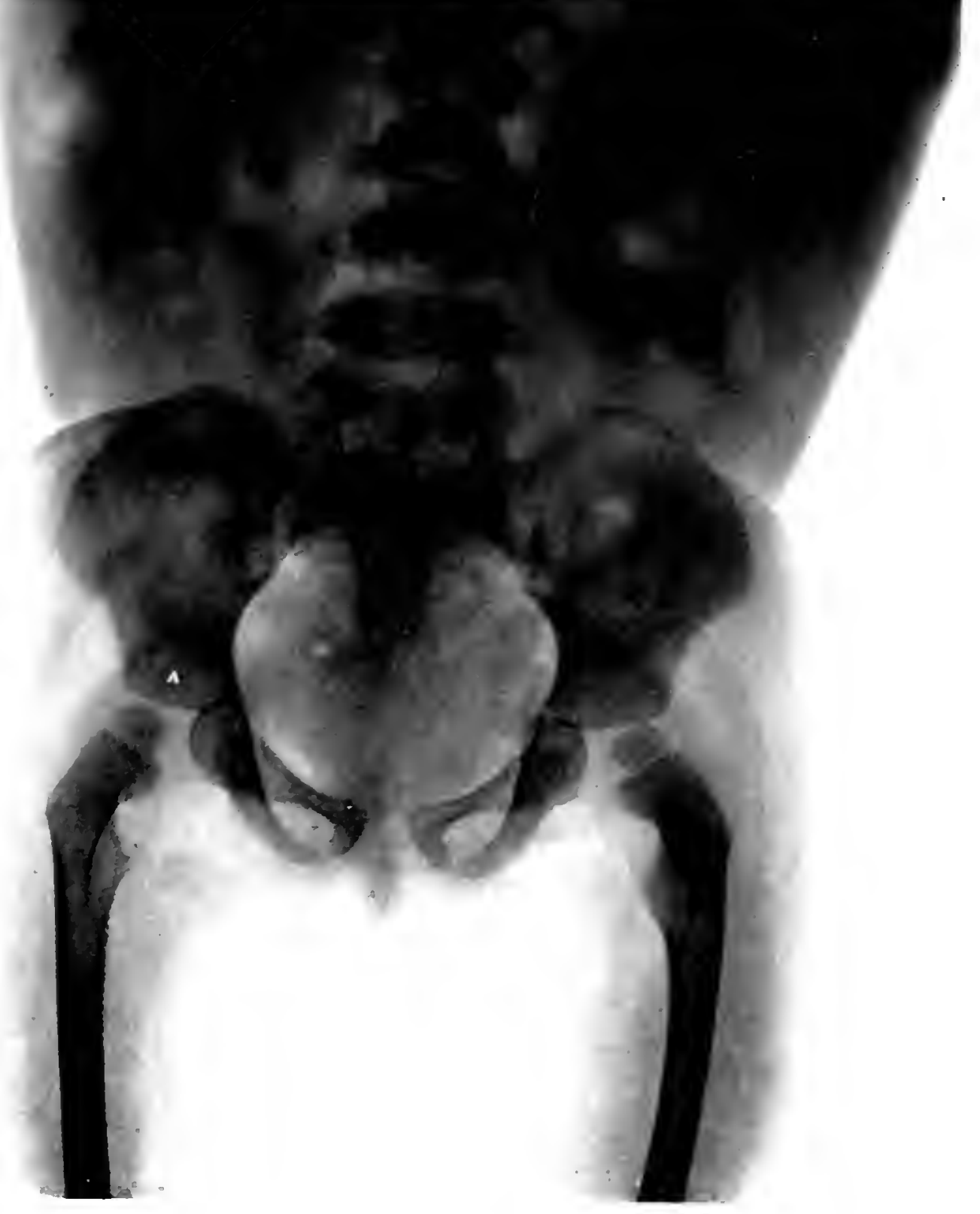


PI.ATE 6.2.

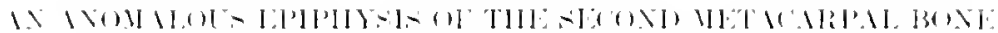

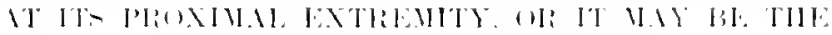

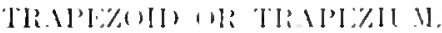

11.19 $1 .+2 * 0)$ 
PISTH AZ

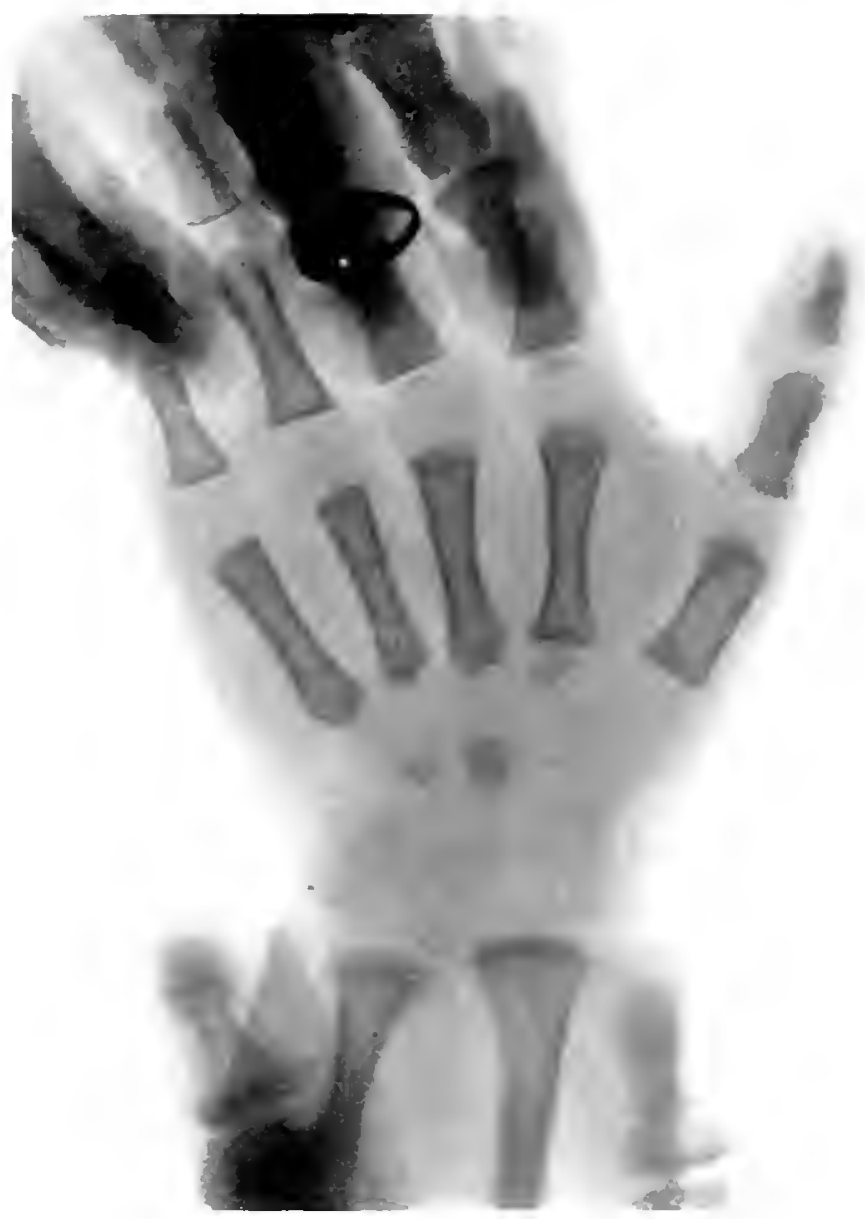


PITTE: (ii).

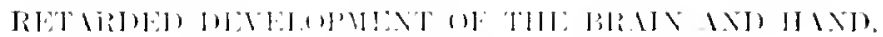

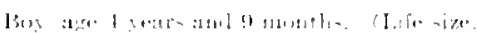

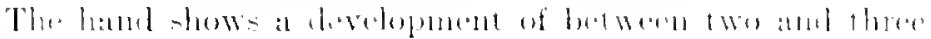
realls. 
PJATE 6:3

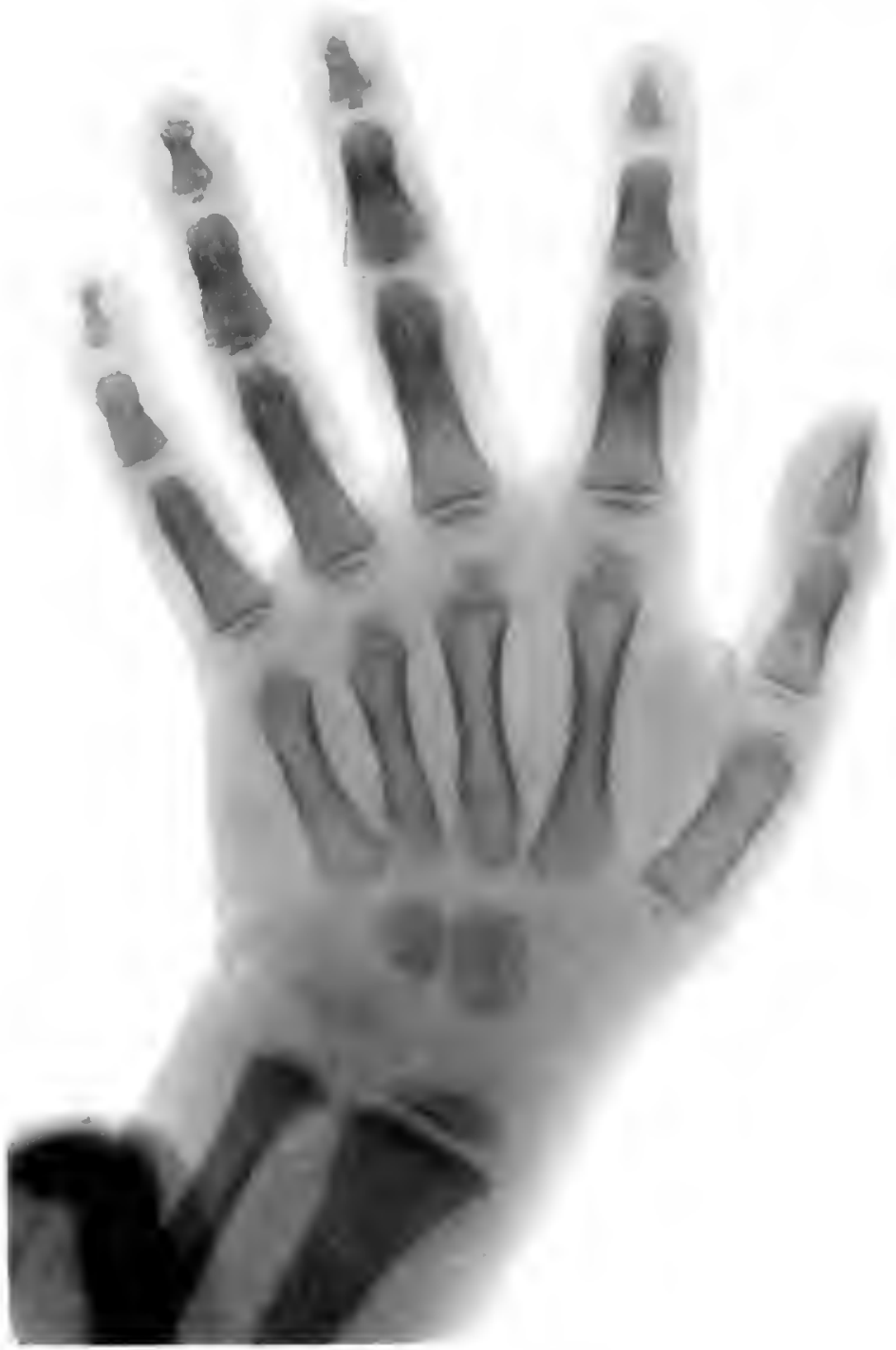


PI.ITT: tit.

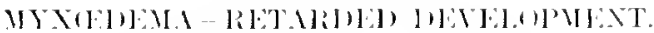

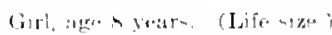

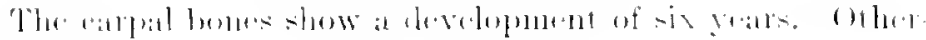
wise numal. 
PIATE: 6it

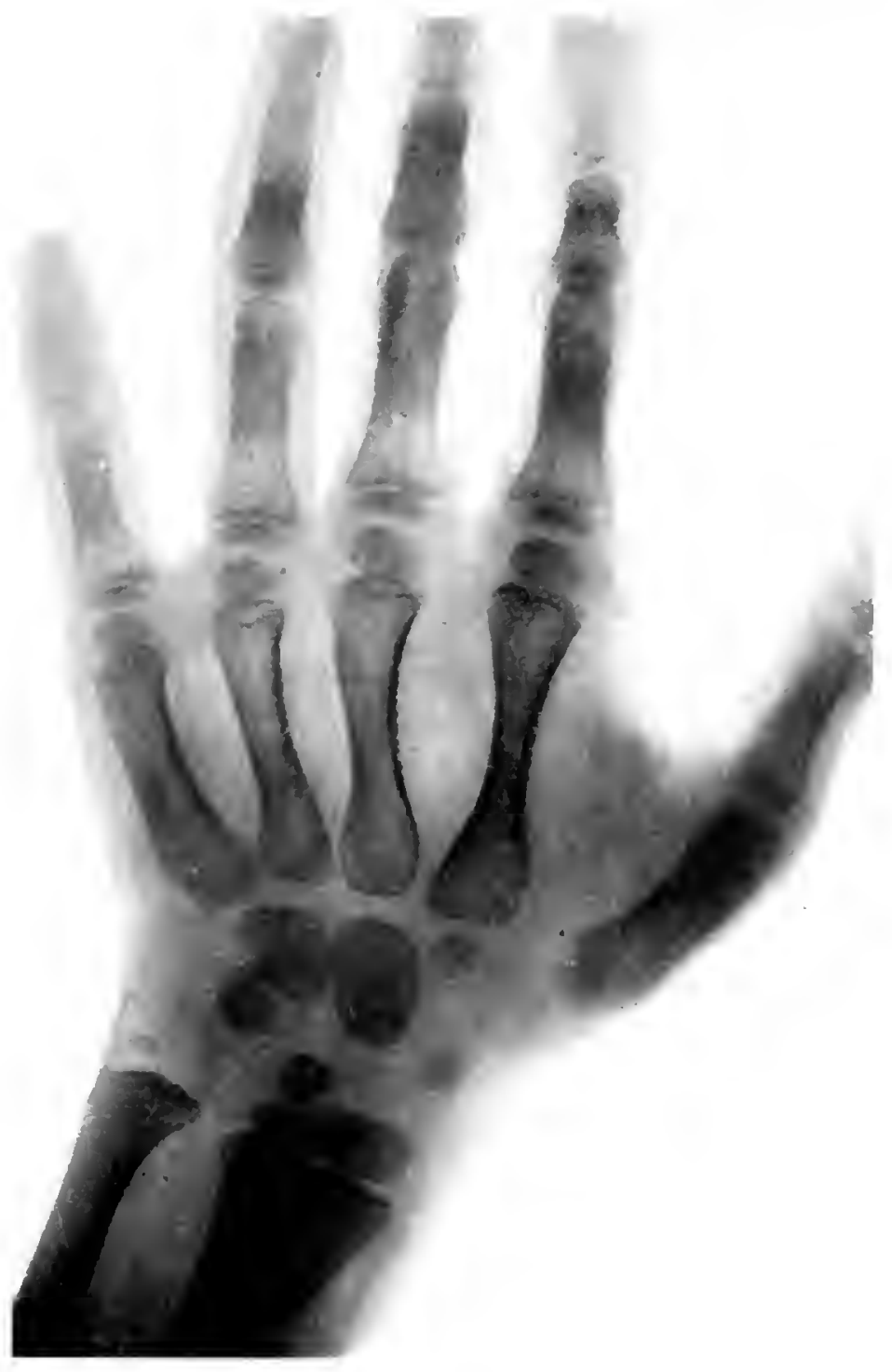


P.ATlis.

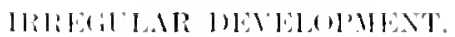

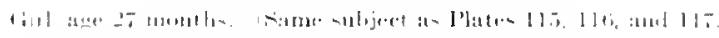

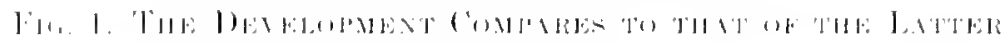

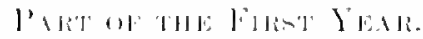

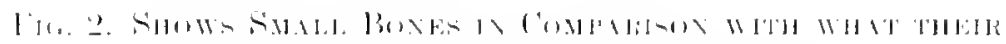
1)

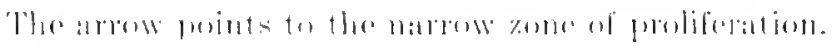

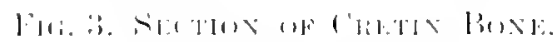

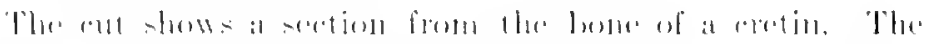

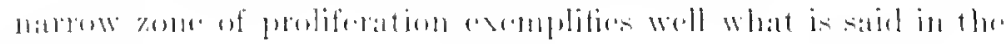
tixt. 
Prate (i.j)

FIG. 1.

FIG. 2.

FIG. 3 .
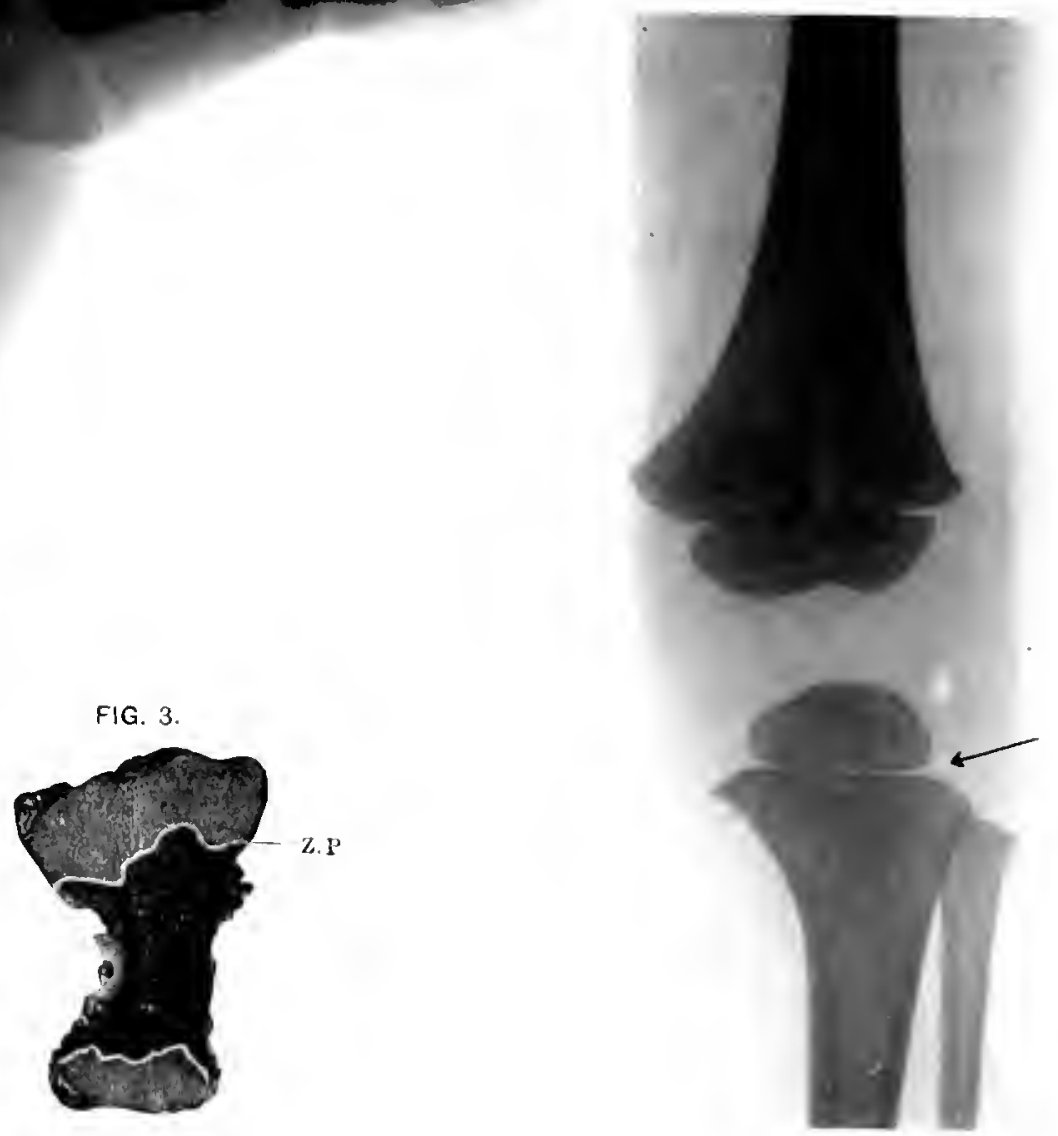
PLAT'titi.

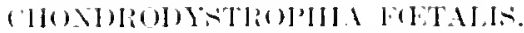

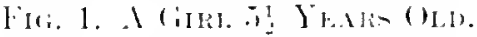

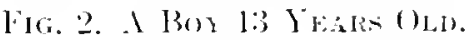


Plate 6if

FIG. 1 ,

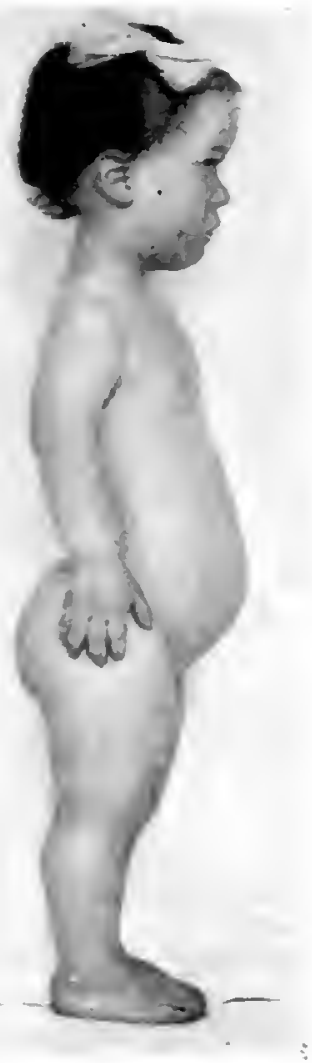

FIG. 2.

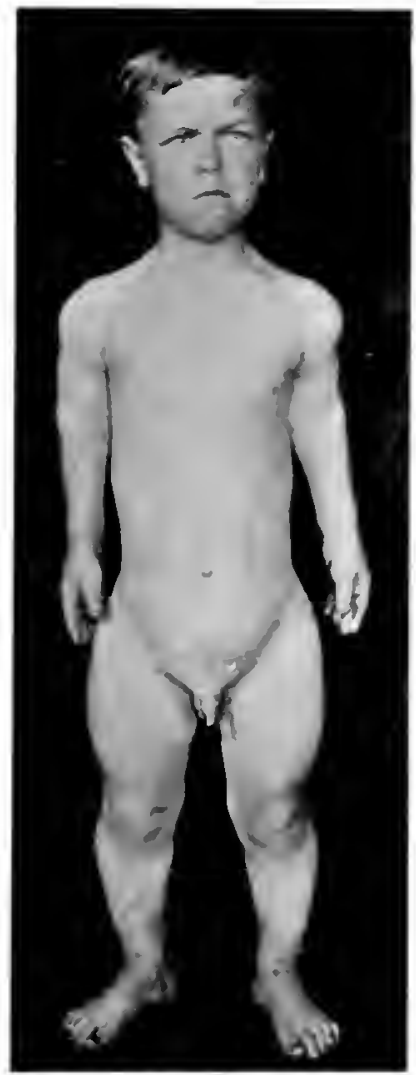




\section{I'I.ITY (i⿱⺈.}

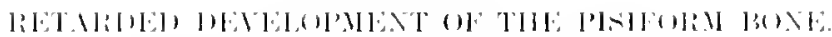

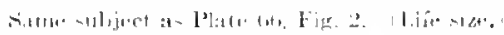

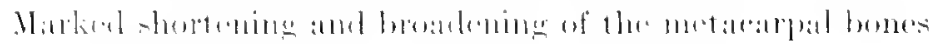

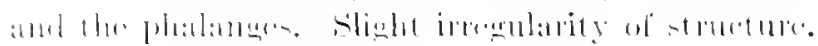


Plate:

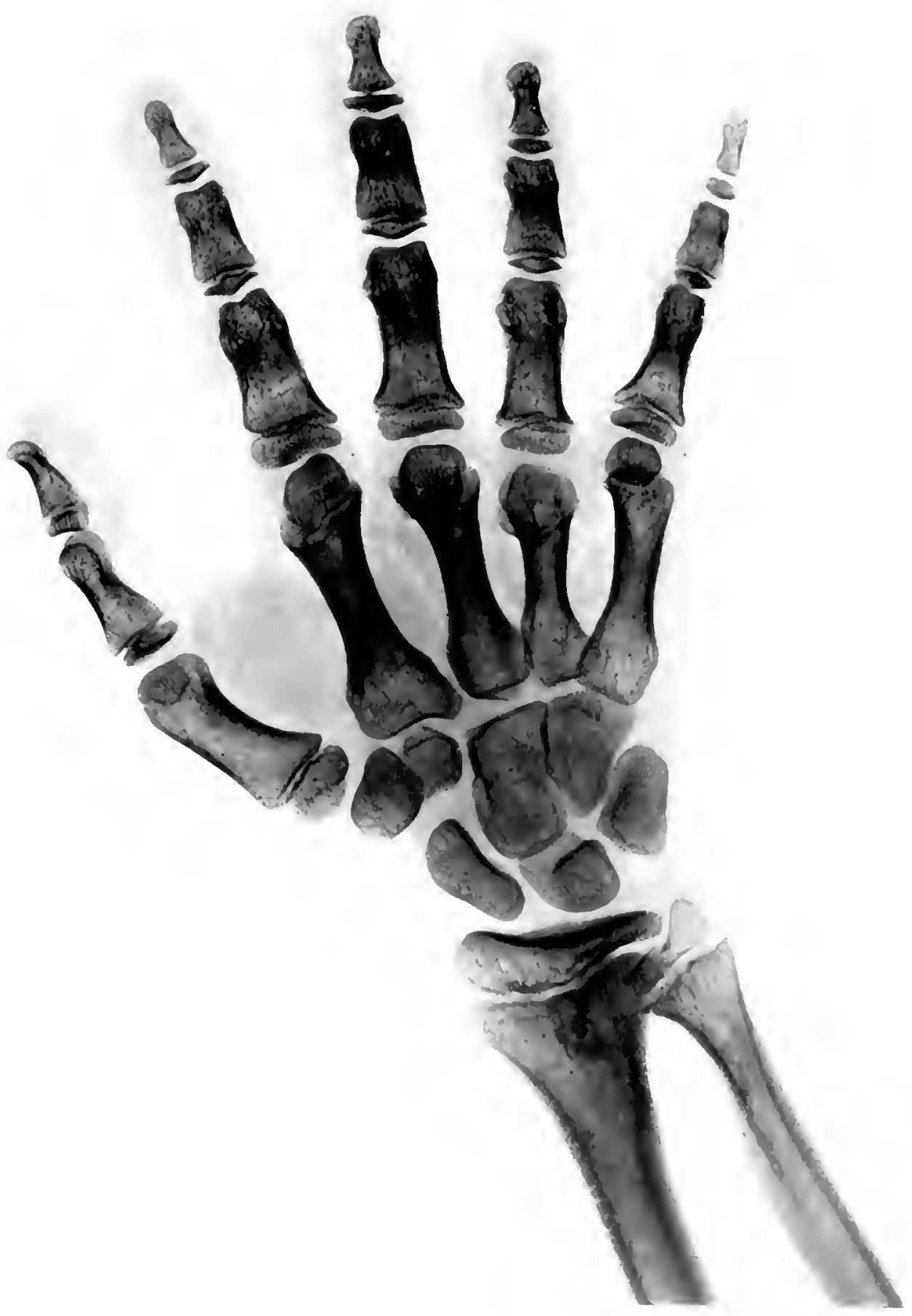


Plater:

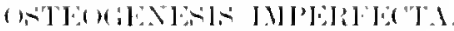

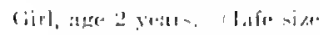

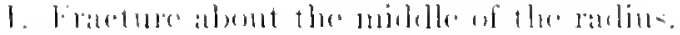

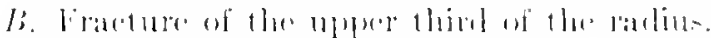

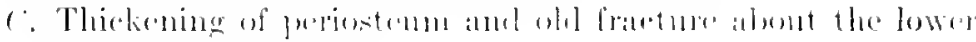
llivel of the ulnt:

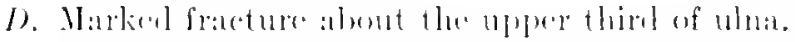

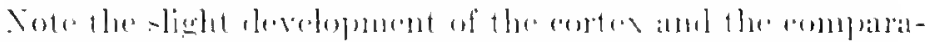

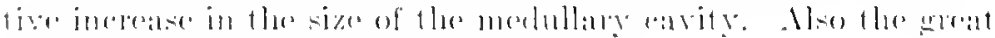
irregulanity of the outlines of the bunes. 


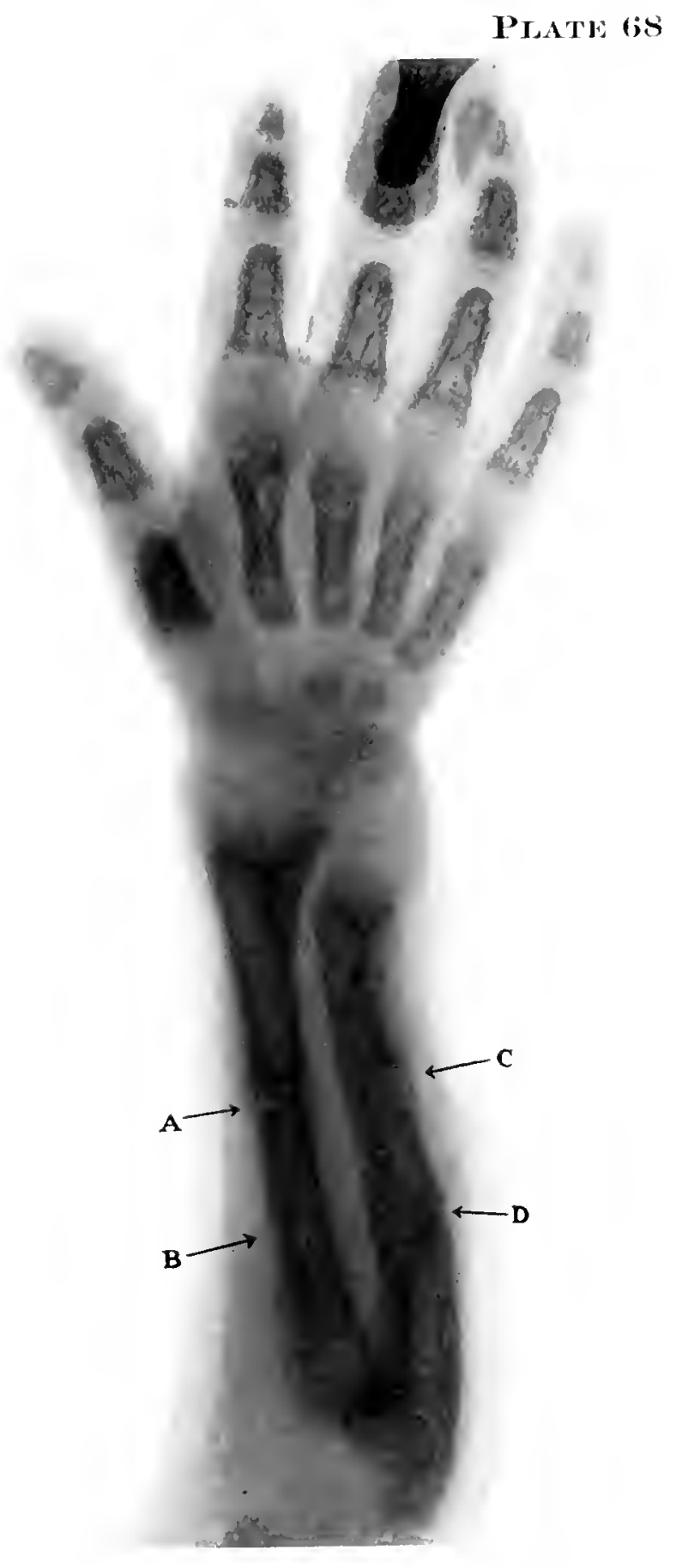


1'1..1'1\%: 1i!.

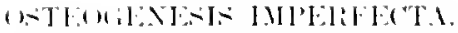

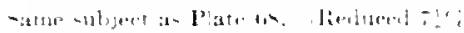

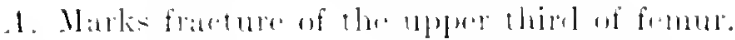

b. Narke frarture of the lewer thiml of fibulat. 
B 
PIATI: TO.

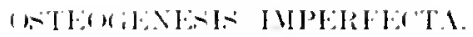

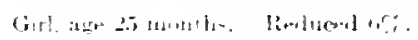

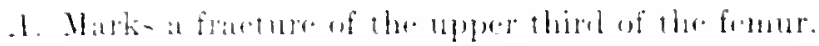

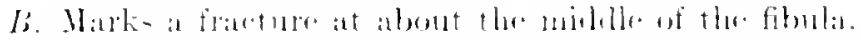

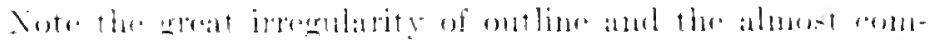

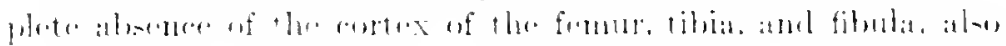

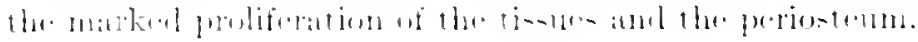


Plate 70

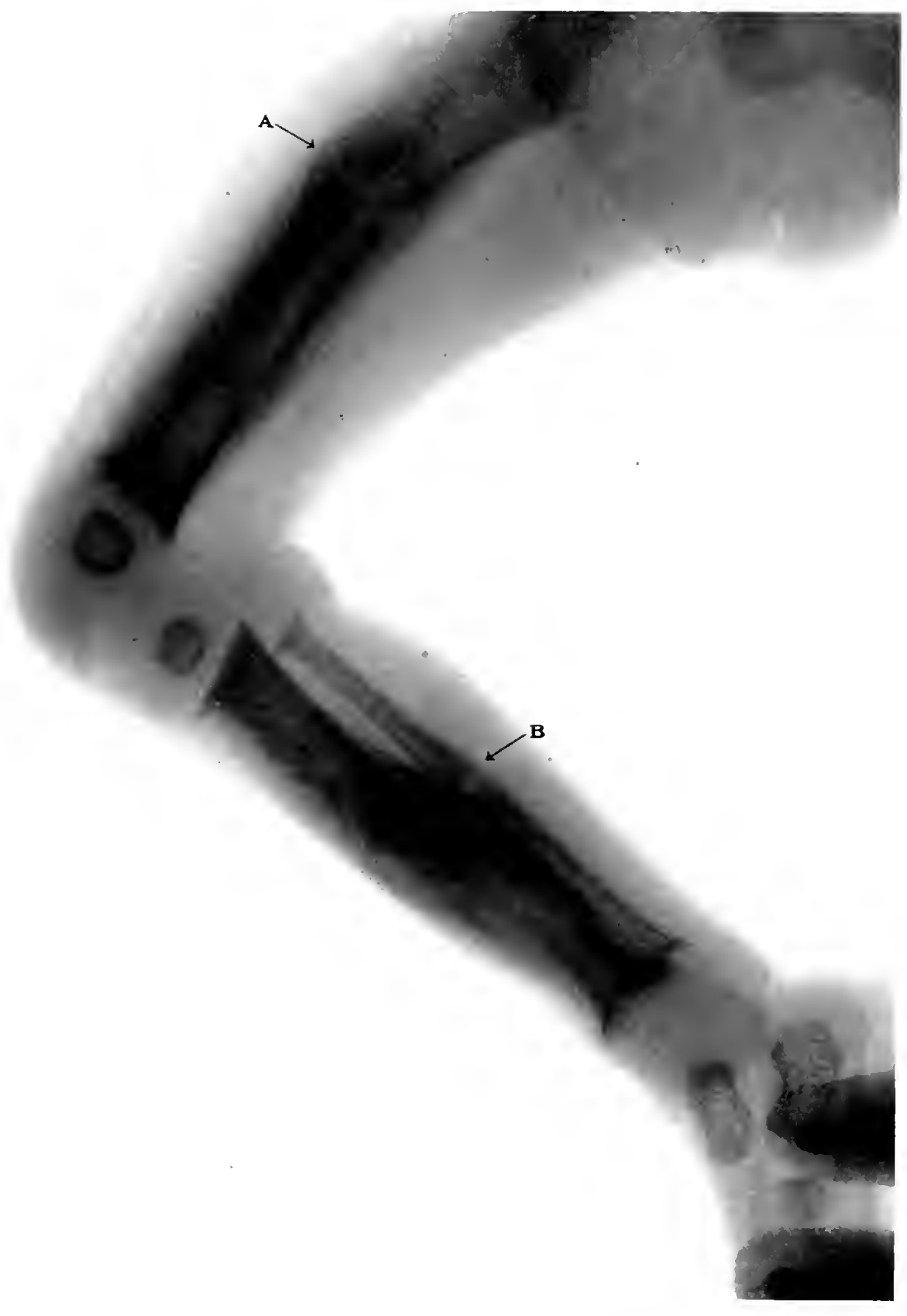




\section{PI. I'T: 71.

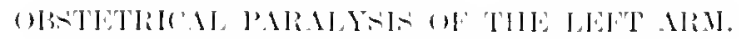 \\ (Roluent in .}

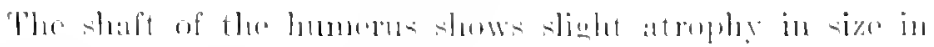

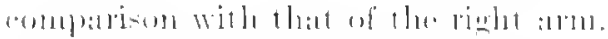

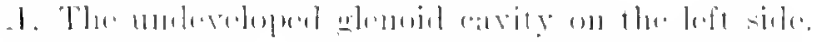

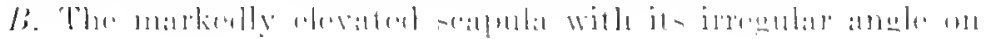
the latis - isle.

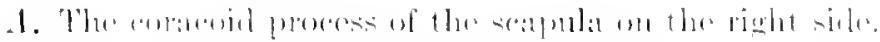

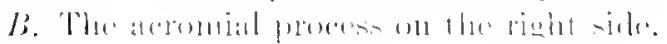


Plate 7

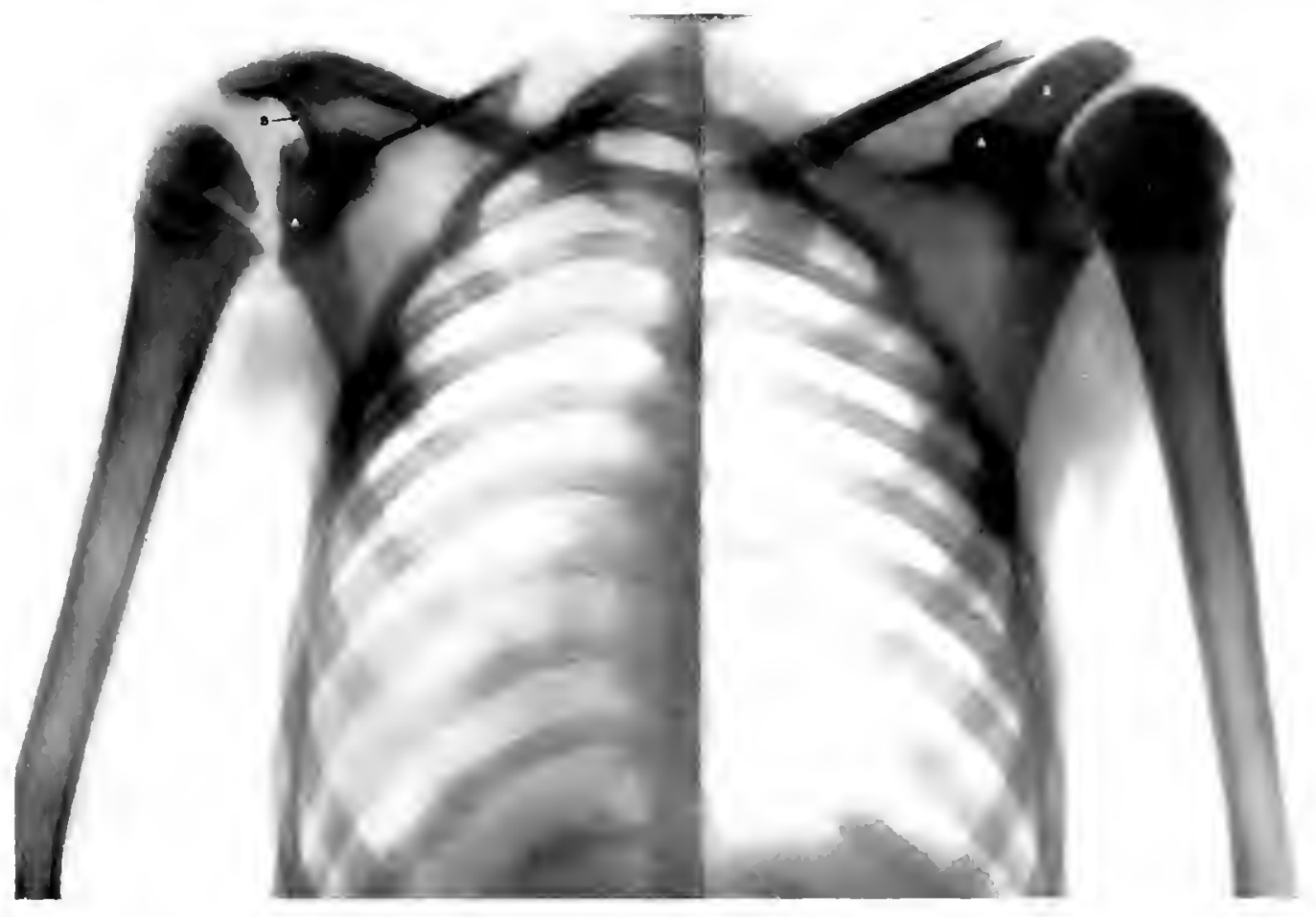


IITTE: T:

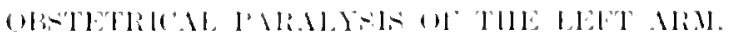

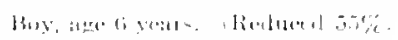

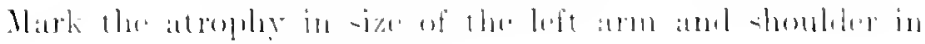
('onulation with that of the right. 
PLATE: $7:$

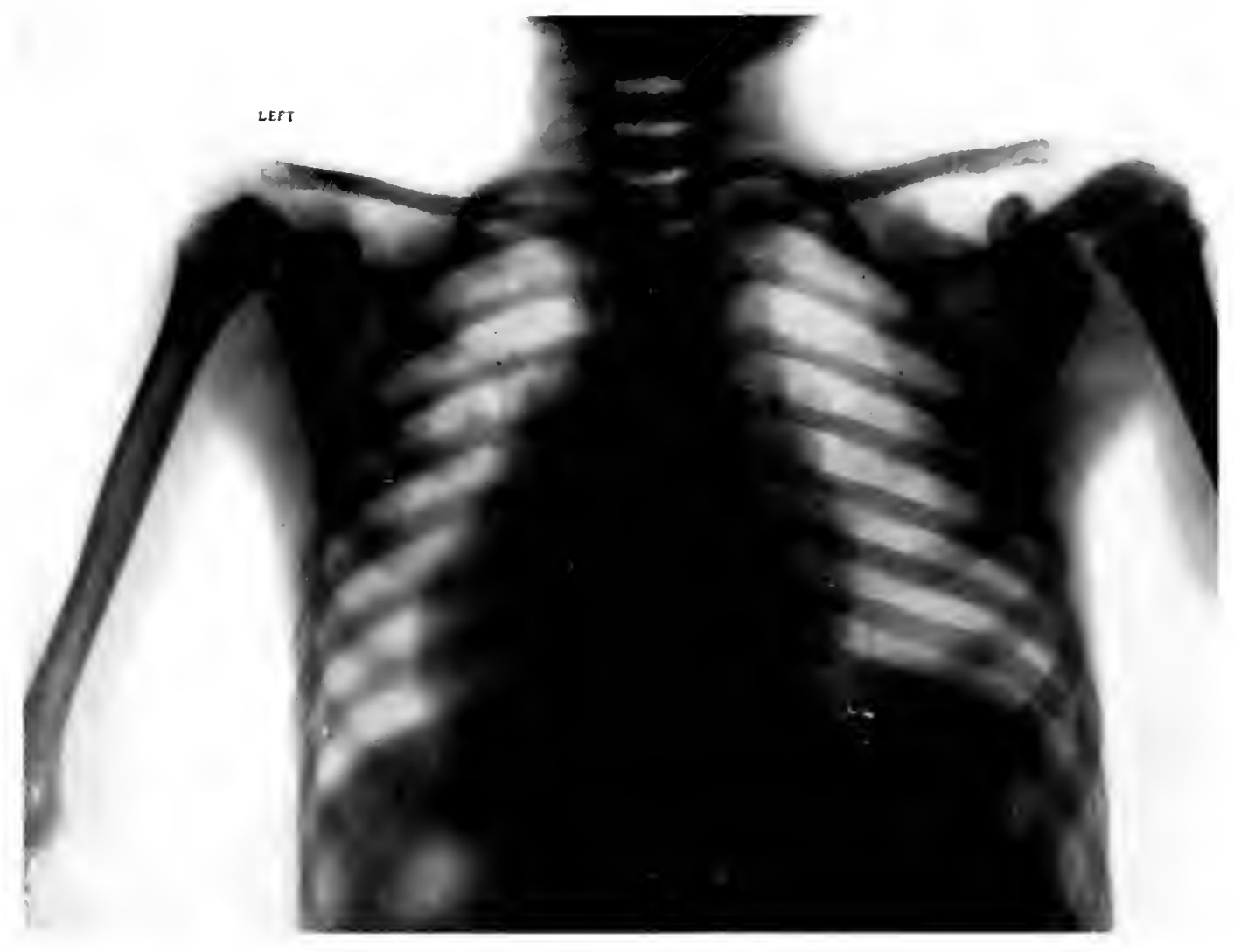


PL.ITL: T:

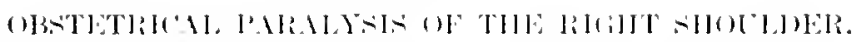

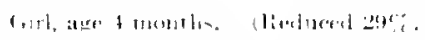

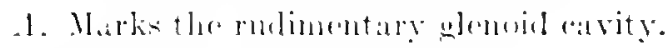

B. Markis the aromial purest. 


$$
\text { Plate t:; }
$$

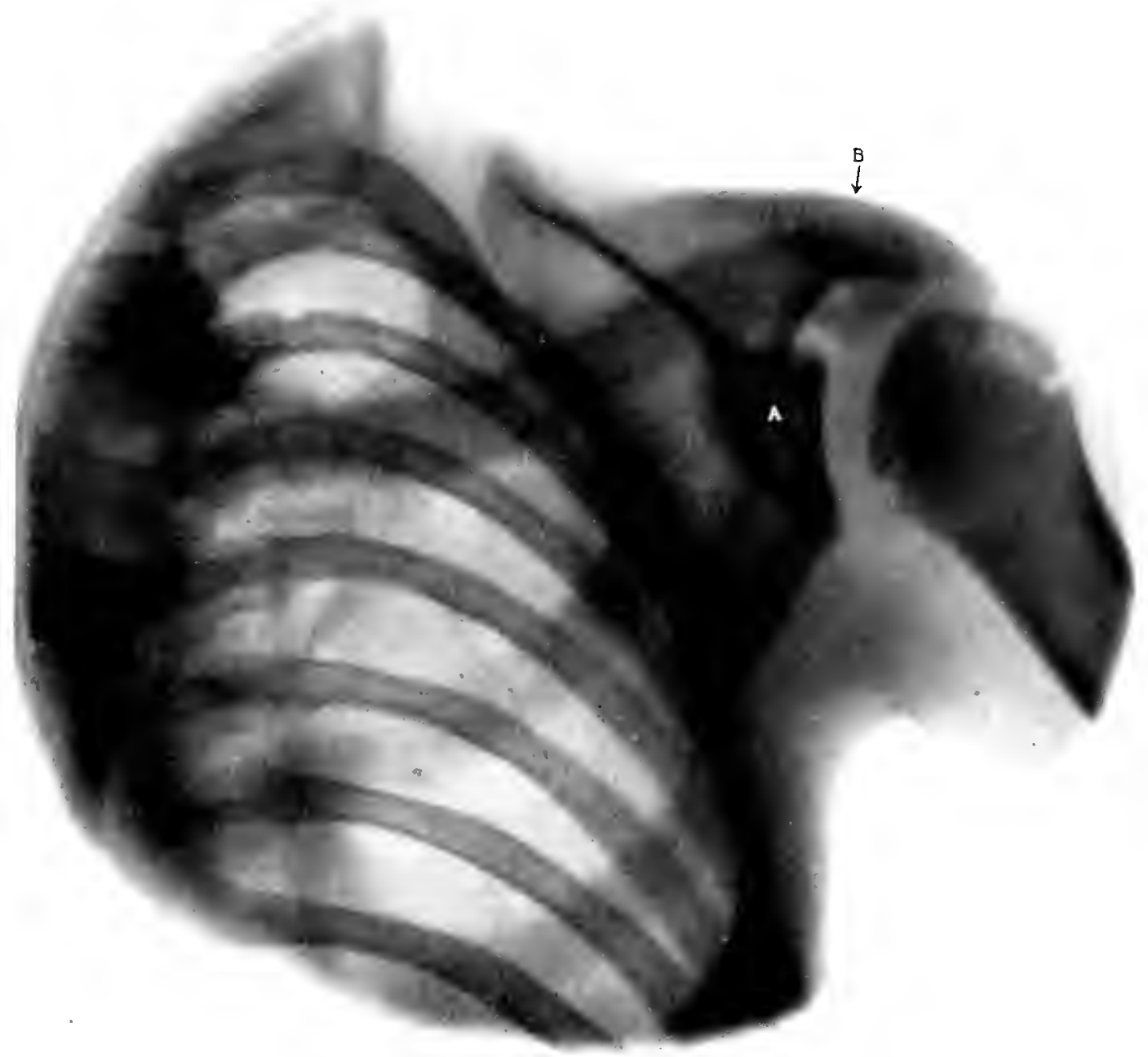





\section{Division IV}

\section{DISEASES OF NUTRITION}

IN contradistinction from those diseases which have a distinct etiology and which can be classified according to their etiological factors,. is a class in which abnormal conditions of all the tissues of the body arise seemingly from lack of proper hygienic surroundings and from poor food. This group comprises osteomalacia, infantile atrophy, scorbutus, and rhachitis. These diseases so essentially belong to the early periods of life and are so obscure in their etiology, while presenting each in its own peculiar manifestations a clear picture of a rice of nutrition, that for the present we must classify them by themselves as diseases of nutrition. The recognition of the early lesions of these diseases is important, as it is in the very beginning that the later and more serious manifestations may be obviated. Although they may be amenable to treatment, yet they render the individual infant more vulnerable to disease as it grows older, and leave its susceptible tissues in such a condition that when it is exposed to specific infections these infections are more dangerous to life on account of the lessened resistance of the tissues. The Roentgen méthod of examination is especially valuable in this class of cases, since by it alone the earliest abnormal changes which correspond to the special condition can be recognized and its treatment begun before more pronounced symptoms and graver lesions lead us to suspect that it is present. It is in this class of diseases, as well as in that which is represented by such infectious processes of the bones as osteomyelitis and tuberculosis, that it is especially necessary to first carefully study the pathologic lesions as shown by post-mortem examinations before attempting to diagnosticate them by means of the Roentgen method. These examinations and 
studies of dead histologic conditions are often invalidated because the various dead lesions are terminal processes and thus do not show the characteristic lesions which during life represent the actual pathology of the especial disease. This, however, is in so many cases only a presumable difficulty that there is no question but that if we recognize this pathologic fact we can recognize sufficiently in the Roentgen picture the typical gross lesions which will enable us to diagnosticate all the diseases just mentioned during life. That the Roentgen method of examination can accomplish this has been clearly proved, so that to-day we are in a position where an early diagnosis is almost alwars possible and where appropriate treatment can be begun at once. This group of diseases is assuming a more and more important position among the morbid conditions belonging to the early years of life. It is becoming evident that it is more far reaching in its effects and influences to a much greater degree all the diseases of early life than in former years was thought to be possible. The prognosis in those diseases which have a definitely determined etiology is markedly graver where such diseases occur in an infant who is suffering from one of the general disturbances of nutrition. In this respect especially both rhachitis and infantile atrophy have come to play a great and important rôle in our study of infantile diseases in general and in their therapeutics.

\section{OSTEOMALACIA}

Osteomalacia is a disease which occasionally occurs in children, but not so frequently as in adults. It causes softening of the bone and in this respect is somewhat similar to rhachitis. According to Ziegler, there is an absorption of lime salts, beginning first at the medullary cavity and proceeding outward. The epiphyses are not notably affected by the continuance of the absorptive process, but the cortical bone becomes spongy and decalcified, and in the 
severest cases there may remain little but marrow and periosteum. The opinion is generally held that in osteomalacia the layer of osteoid tissue results from decalcification, while in rhachitis a similar layer represents a new growth deficient in lime salts. According to Buck and Bryant osteomalacia pathologically consists principally in decalcification of the old bone with a simultaneous formation of new bone, which, however, remains imperfectly calcified. The process of decalcification begins at the periphery of the bone trabeculæ and gradually extends to the deeper parts. The line of demarcation between the normal and diseased bone is sometimes even and continuous, but may be irregular with excavations resembling Howship's lacunæ. There may be an intermediate zone in which the lime salts are not completely removed but remain in the form of a crumbling detritus. The periosteum is likely to be thickened and vascular and the medulla resembles that of infancy in its gross appearance. Spontaneous fractures and various distortions may occur in osteomalacia and the thorax is apt to be flattened laterally. Osteomalacia is recognized clinically in children as a rare condition, but it has not yet been proved that it may not occur as a disease of the new-born. No modern and exact histologic examinations, however, have been made of infants at birth which have shown the pathology of the osteomalacia of post-natal cases, and it is still uncertain as to how early this condition may appear. It has therefore been thought better to provisionally classify osteomalacia with diseases of nutrition, although such classification is manifestly empirical. Cases examined by the Roentgen method give a noticeable and characteristic picture. The general development of the skeleton is normal. The bones are generally a little longer and a little narrower than normal, with or without deformity or fractures. Usually one or more fractures of the bones, commonly the femur, are found, which fail to unite, and a deformity results from super- 
incumbent weight. At the point of fracture very little formation of new bone, represented by a callus, will be seen, though at eertain portions of the bones there will be a very dense, definite cortical bone giving the so-called peneilled outline. Marked increased radiability of eertain portions of the bones is seen. The irregular cortex, with more or less osteoid tissue, is apparent without any great change in the medulla or in the structure of the bone exeepting a lack of density. The knowledge which we have derived from studying the living pathologic anatomy in these cases is simply what we should expect from the post-mortem findings, namely, that it is a condition of osteoporosis, which accounts for the increased radiability and corresponds to the low atomic weight. Aceording to Buck and Bryant the Roentgenographie pictures present:

Cystic formation with medullary and cortical destruction.

Aeute bowing and angular bending of the bones.

Large amount of partly ealeified callus and eartilage at the seat of a spontaneous fracture.

Diminution of lime salts.

Our experience at the Children's Hospital leads us to believe that many of the details of the Roentgenographs of living eases of osteomalacia correspond to those seen in osteogenesis imperfecta, but the dead pathology shows the two diseases to be very different.

Plate 74 shows the photograph of a girl seven years old with the general elinical signs of osteomalacia. Especially to be noted in Fig. 2 is the bowing of the upper part of the left femur. Ever since the child was able to walk there had been a tendency to bending of the extremities without adequate apparent trauma. Fracture or something closely allied to it had been suspected in the right femur, but nothing definite was deteeted until the Roentgenograph diselosed the condition shown in the plate. 
Fig. 1 shows where a break in continuity was found in the upper part of the shaft of the right femur without the callus or a lesion which would be found in the fracture of a normal bone. In the same region on the left side, as is seen in the plate, there is a decided bending. A coarse arrangement of the structure of the bone, with increased absorption of lime salts, is seen in the neck of the left femur.

\section{INFANTILE ATROPHY}

A condition of the tissues called infantile atrophy, which is essentially one of infancy and early childhood, and most commonly occurs in the first six months of life, is usually classified among the diseases of nutrition. No specific pathologic lesions have been found in these cases. They merely show extreme anæmia of all the soft tissues without demonstrable disease of any of the organs. It has been thought possible that infantile atrophy is merely a form of starvation. Starvation, however, even in its most aggravated forms, in most cases quickly responds to special manipulation of the food, while infantile atrophy shows such resistance to all kinds of treatment and to all manipulation of the food that at present, although ignorant of the cause of the disease, beyond its possible connection with food, I assume it as an entity. Roentgenographs show, so far as I have been able to determine by a careful study, nothing which has not already been determined at autopsies, that is, we are still absolutely ignorant of the etiology of infantile atrophy, and know nothing of its pathology beyond a wasting of all the tissues.

Plate 75, Fig. 1, is the photograph of a girl about twelve months old. The progressive and extreme emaciation and the resistance to all of the most approved modern methods of feeding of this class of cases showed that it was not a simple case of starration. (For a discussion of this condition see "Pediatrics," 5th ed., p. 341.) 
Fig. 2 is the Roentgenograph of this same ease. The lower half of the femur, the knee, and the tarsal bones are shown. To be noted are the elear-eut, smooth epiphyseal lines of a perfectly normal condition. The cortex is possibly somewhat more dense than normal, as shown by a certain amount of deereased radiability, but there is nothing sufliciently marked to allow us with the present knowledge which we possess of interpretation to make the diagnosis of infantile atrophy from the Roentgenograph, although the elinical aspect of the case readily makes the diagnosis.

The differential diagnosis from general tubereulosis is at times exceedingly difficult, so that Roentgen evidence if present is extremely valuable.

\section{SCORBUTUS}

Scorbutus, as it manifests itself in earty life, and usually in the first year and a half, is closely related to the scorbutus of later life. It is a constitutional disease closely associated with imperfect nutrition, and plays so definite a rôle in infancy that it is usually accepted as a special disease of infancy. It is characterized by anæmia and a tendency to hemorrhage, and in most eases is accompanied by a condition of the gums which is present in stomatitis ulcerosa, the latter condition, however, only occurring when teeth are present. The tendeney to hemorrhage, although it may be from any organ, is in most eases so common in the bones that the use of the Roentgen ray in determining the disease becomes invaluable. There may be deep hemornages into the muscles and oceasionally about or into the joints, but the hemorrhage is essentially subperiosteal and confined chiefly to the long bones. The femora are most commonly affected and there is a tendency to separation of the epiphyses. It is the subperiosteal hemorhage of greater or less extent which when recognized in the Roentgenograph aids us in making a differential diagnosis from other diseases, which manifest their primary lesions in other parts of the bones. We should notice 
first whether there is a greater or less area of fatty tissue, or whether or not there is infiltration. Second, we should examine the muscles as to whether they are infiltrated, atrophied, hypertrophied, or present a clearly defined outline. Third, we should observe whether the periosteum can or cannot be seen in the picture. In the latter case we must look further for disease by examining the cortex of the bone and finally its medullary cavity. If, on the contrary, the periosteum can be seen we must determine whether we are dealing with a thickened periosteum, or with a periosteum separated from the cortex. In the latter case we should decide whether regeneration of the periosteum and bone formation has begun (as this is the process by which repair takes place), or whether it is the lessened radiability of a fresh exudation beneath the periosteum. If we find that there is a decided bulging of the periosteum, and a certain amount of infiltration of the surrounding tissues, we are led to suspect that we have a subperiosteal effusion, but whether it is blood or pus the Roentgenograph, according to our present interpretation, does not tell us. The symptoms differ greatly according as an acute painful localized condition is present, depending upon the rapid process which occurs in osteomyelitis, or whether the condition is the slow, subacute clinical sequence of an essentially chronic constitutional disease such as scorbutus. In the latter case acute pain is usually absent if the affected part is not disturbed. If the diagnosis of scorbutus has been made the Roentgenograph will when taken from time to time show whether the pathologic process is increasing or diminishing. It will also show the degree to which the periosteum has been stripped from the cortex and whether it has begun to invade a joint. All this knowledge acquired by the Roentgen ray is of the greatest importance in the treatment both medically and surgically. The recognition by the Roentgenograph of a scorbutus which shows itself under the periosteum of the hip is especially valuable, as it saves the infant from the excruciating pain which 
movement of any kind produces. It tells the surgeon that he is not dealing with a case of osteomyclitis or of tuberculosis, or possibly of a number of other infections, and enables him to avoid the necessary and painful manipulations which he would otherwise employ in determining whether the hip was affected. The same reasoning can be carried out when the other joints are affected. I would especially add that the differentiation of the enlarged wrist of scorbutus from that of rhachitis, and also from syphilis, by the Roentgen method is of the greatest diagnostic value and ean be accomplished without harm to the infant. Again, the differentiation from a possible fracture is made with certainty and without pain, or a suspected separation or break in the region of the epiphysis. In some cases most serious mistakes have been avoided by the use of the Roentgen ray. Various operative procedures, and even amputation of the thigh for sareoma, have been given up because the ray has shown that the surgeon was not dealing with a sareoma, but with a large organized subperiosteal effusion which simulated sarcoma. In some of these cases the tissues were found to be enormously infiltrated and hardened so that the tissue of the thigh assumed the appearance of a new growth. Instances of this class, which are only examples of what may occur in any part of the osseous system, have come under my notice quite a number of times, and in these cases after the differential diagnosis by the Roentgen method had been made the infants rapidly recovered under no other treatment than the prompt and free administration of orange juice.

Plate 76, Fig. 2, shows the photograph of a girl eleven months old with scorbutus. Note the enlargement of both femora, especially the upper part of the left femur. The disease had been going on for some months and the diagnosis of sarcoma was made at a consultation of surgeons. The infant was extremely pale and there was oedema of the feet and lower legs, with extreme tenderness on pressure of the hips, knees, and ankles. The upper part of the 
left thigh showed a diffuse hard mass involving all the tissues. There was no fluctuation even on deep palpation. There was a rapid recovery after the administration of orange juice.

Fig. 1 represents a Roentgenograph of this case, and should be compared with Plates 163 and 164, Division IX, sarcoma of the thigh. The swelling and lack of clear definition of the muscles of both thighs is marked, and the irregular toothed appearance of the diaphyseal and in some places epiphyseal outline is accentuated. Note the roughened appearance of the zone of proliferation in contradistinction to the smooth-edged zones in Plate 75 , infantile atrophy.

Plate 77 represents the same case in a late stage and shows the organization of the clot considerably advanced. The thickened periosteum and the line of hemorrhage can easily be distinguished in the femora and in the tibiæ. The epiphyses are irregular and the picture shows us that rhachitis is also present. Note here also the absolute dissimilarity of these epiphyses and zones of proliferation from what is seen in the case of infantile atrophy, Plate 75 .

Plate 78 represents the Roentgenograph of the leg of an infant six months old. There is considerable involvement of the soft parts of the thigh, and the subperiosteal hemorrhage is easily collowed along the whole length of the shaft of the femur. The extent of the hemorrhage along the line of the tibia is even more marked than in the femur. There is no especial change in the shaft of the bone, but the zone of proliferation is somewhat irregular.

Plate 79 represents the same subject as Plate 78 .

Plate 80 shows an infant two months old. The infant was normal at birth, and had always been fed from the breast. The parents were healthy, and the mother apparently well at the time of nursing. The swelling and tenderness first appeared in the right arm and then involved the lower extremities. The left arm mas slightly affected. The infant seemed to have considerable pain. It showed 
evidence of no other disease. There was marked thickening of the periosteum of both lower extremities; this condition is shown in the Roentgenograph.

\section{RHACHITIS}

The condition which in diseases of nutrition plays the greatest rôle, in that we find it associated with a large number of other diseases, is rhachitis. It is chiefly characterized by a local or a general disturbance of the normal process of ossifieation, but exactly how the hygienic and dietetic eauses, which are supposed to produce these osseous changes, accomplish their results is still unkmown. The uniform and definite changes in the bones in rhachitis lead us to consider it a disease by itself, and one which, although connected with nutrition, is not wholly a form of malnutrition. The pathologic lesions of rhachitis are represented chiefly in the bones and oceur during the period in which the normal processes of ossification are most active, that is, during the first year and the first part of the second year of life. The normal growth of bone depends upon three conditions: (a) Multiplication of cartilage cells in definite lines, (b) followed by calcification of the intercellular spaces for the entrance of blood-vessels with specific absorption of tissue, and (c) finally the concentrie deposition of bone within the medullary spaces. The bones grow in length by the produetion of bone-tissue in the cartilage towards the epiphysis and the diaphysis, and in thickness by the growth of bone from the inner layer of the periosteum. At the same time the medullary eanal is enlarged in proportion to the growth of the bone by the absorption of its inner layer. These processes progress in definite order and in clearly defined zones.

In rhachitis the chief microseopic features are the changes which occur in the zones of growth and the asymmetrical character of the proliferative processes. The cartilaginous and subperiosteal cellgrowth which produces ossification goes on with inereased rapidity and in an irregular manner both between the epiphysis and the 
diaphysis and beneath the periostem. If we eximine microscopically the region between the epiphysis and the diaphysis, usually called the zone of proliferation, we find that the cartilaginous cells are not regularly aranged in rows around a definite zone in adrance of the ring of ossification, as in normal tissue. On the contrary there is an irregular heaping up of cartilaginous cells, sometimes in rows, sometimes not, covering an ill-defined irregular area. This zone of proliferation also, instead of being narrow and sharply defined, is quite lacking in uniformity. It presents a broad, reddishgraty appearance, with marked thickening and hyperamia. The medullary spaces are much more viscular than normal, and are so increased in area as to extend into the zone of ealcifieation, and sometimes through it. The deposit of bone-tissue within these spaces is, however, either absent or very irregular, and is for the most part replaced by a solt, friable substance, consisting of a bone-tissue that is very lacking in lime salts, with cells of various kinds embedded in a fibrillated ground-substance. This tissue is called "osteoid," and is similar to that formed by osteoblasts.

In the region of ossification (ends of diaphyses and epiphyses) there is microscopically a pronomeed increase of blood-ressels and cartilage-cells, with lengthening of cell columms, and disturbanee of alcifieation of the intercellular substance. ('alcification, if present, may be isolated in the region of the proliferating cartilaginous cells, or may be altogether absent over considerable areas. The subperiosteal layer of cells, which is normally thin and searcely noticeable macroscopically, becomes hyperimic and thickened with an appearance similar to that of spleen-pulp. Beneath this periostem is also to be found the "osteod " tissue seen in the zones of proliferation.

The medulla of the bone is more hyperamic eren tham nomal. Its tissue is rich in cells, and, like the fetal medulla, contins dilated vessels and fat. The intercellular substance may show mucoid degeneration or even be of fluid consistency. In such a condition 


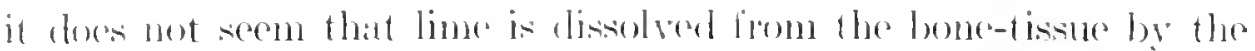

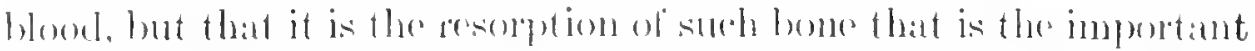
faretor in the moress. liesorption at the age at wheh rhachitis

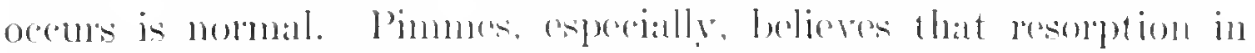

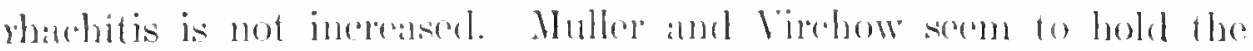

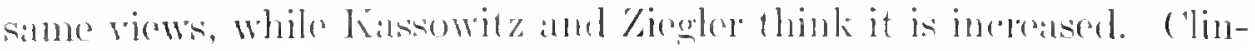

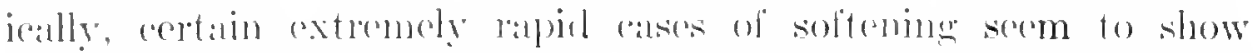

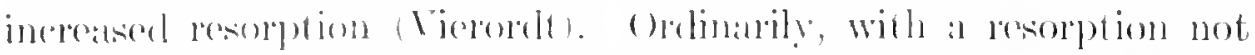

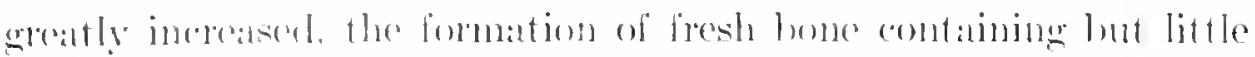
lime results in less of strength. In the skull, in somo planes, alsorp-

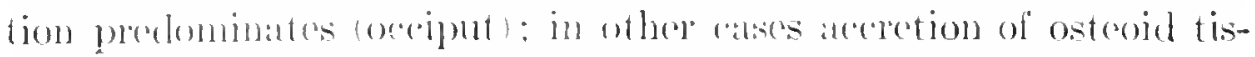

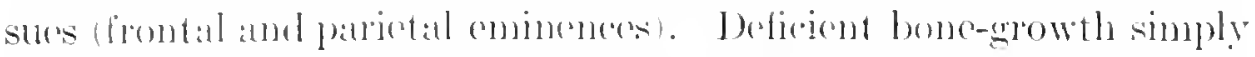
dotemines opent fontanelles. In convalesence lime is deposited in the previousty limeless asteod tisste, and the result is at the and hearte bone. In fratetures at this period callus-formation is rexersive.

In excessive probineration of retls in the inner layers of the periosteum, the irregular cateilieation which oecens about them, and the albsence of uniformity in the elaboration of the strueture of the bone, produce an irregular, sponger bontissue insteat of the compatet lamellated tissue wheh is so necessany for the miformity of the structure. The increased eoll-growth botwen the epophysis and the diaphysis prohtures the perolial knobby swellings which are chatrateristir of thathitis. It the same time the medullary carty increases rapidly in size, and the inner layers of the home beeome sponery. The result of these processes is to climinish the soliclity of the bones so that they camnot resist the strain of the museles or outside pressure. Niter at time the rharhitio process may stop and the bones maty assmone a more normal chatrater. The porous bonetissue becomes comparct, and ren mmaturally dense, so that in later childhood the rhathitic bone is musually hard, like ivory, at romdition notieed by those who have to operate on these bones.

Fig. 1 represents a section of a normal bone taken from an 
infint, and shows the normal zone of proliferation (Z. P.) Jetween the epiphysis and the diaphysis.

Fig. 2 represents a section of a rhachitir bone, and shows the broad, irregular, and abnormal zone of proliferation (Z. P.).

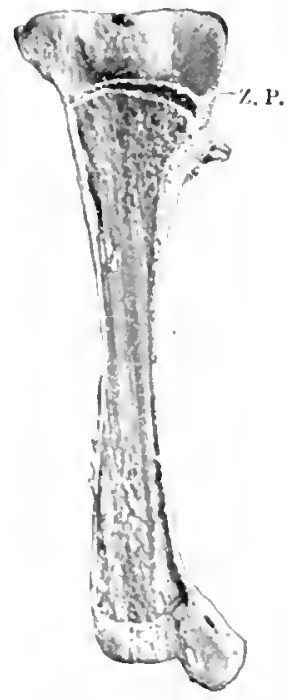

Fo. 1.- Sormal bone: Z. P'., zone of proliferation.

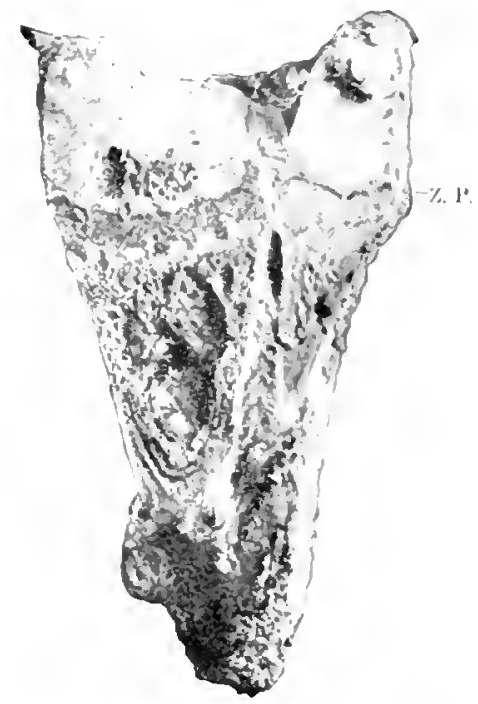

Fi:, 2.-lihachitic fune: Z, 1', zum of proliferation.

The clinical diagnosis of rharhitis from diseases which may simulate it need not be fully stated here. I few words, howerer, may aid us in making the best use of our Roentgen pieture. In chondronlystrophin fotalis the trunk is normal while the extremities are short and deformed. The entargements of the ends of the long bones are due to overgrowth of the periosteum, insteal of to changes in the epiphyseal cartilage, and the zone of proliferation is narrower than in rhachitis. In rhachitis there is an absence of the disproportion between the trunk and the limbs which is seen in chomdrodystrophia fatalis. In chondrodystrophia fatnlis also the epiphyseal lines instead of being irregular are straight, although much narrowed, and the shatits of the bones are often thick and striated. The charateristic symptoms of osteomalacia, a rery rare disease in childhood, do not 


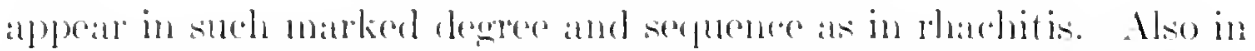

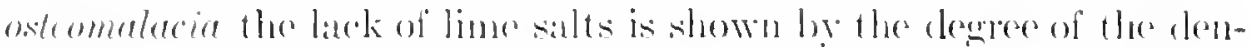
sity, and is msually well matkel, and the deformities of the shatis of

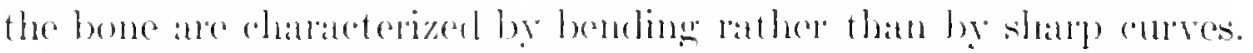

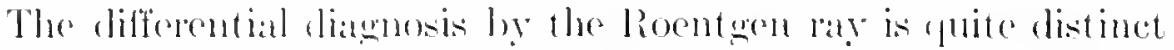

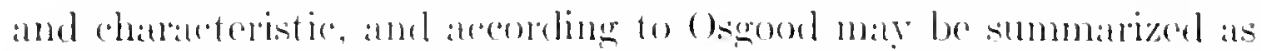
follows: In rhathitis there is ereat imecrularity of the epiphyseal lines with hyperplasia of the asteoid tisste and hypertrophy of the

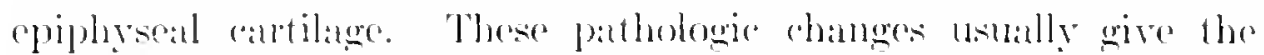
atpreatrance of a enealt disproportion betreen the bony epiphysis and the cpiphysath end of the diathysis. The amves in the shatts of the hones are often sharp. In chombodystionhia jotalis and asteomalnein the abnormal densities atre seen most often to involve the joints, atthomgh the appearances in the anljatent hones ane sometimes

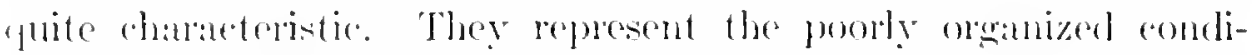
tion of ill-nomished bone with a deficiency of lime salts. If foed (alleas of definde necrosis) appear they ane prome to involve the epiphyses. In ostomyelitis the abnommal densitios atre to be seen in the diaphyeses and are generally due to imegular neerotie areas, with or without sefuestrit. There is asublly no lessening of the densities of the involved bone or smrounding bones.

Thr chamges in the osseous systen due to mathitis ane detected by the lioenteren mothod of examination before it is possible to do

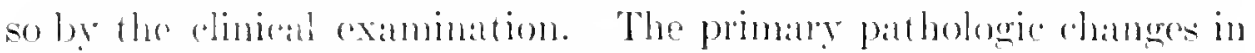
rhachlitis oeren in the parts in which new bone formation talies place, the periostemu and repiphrses, and these can be clatssifed for the purpose of liocuteren study and description als follows:

The epiphysis and zone of proliferation.

The ontline of the entex ame periostems.

The modullatry (:inatl.

The sencral structure of lhe bone. 
The changes in the bones of rhachitis are varied, although characteristic of the condition.

There are some cases in which at any age from birth to puberty marked disturbances of the zone of proliferation take place and, though the case may not be recognized pathologically, yet clinically it presents the picture of a severe degree of rhachitis. We often find by examination that the epiphyses of all the bones are delayed in ossification, that they are smaller than they should be for the given age, and that there is such a lack of inorganic material in the bone that it gives an increased radiability. The zone of proliferation is a great deal wider than normal, with irregular deposits of bone on the epiphyseal side, so that we get a "toothed appearance" of the diaphysis with a degree of radiability in the zone of proliferation which is more like that of cartilage than bone. There is usually also a definite thickening of the periosteum. The changes in the bone are a thinning of the cortex, with an irregular deposit of bone-cells of irregular outline, an increase of the medullary cavity, and a marked re-arrangement of the bone structure. These changes go on to such an extent that cystic formation of the bone at times becomes apparent. Marked deformities of the osseous system may not always be present, but sooner or later, according to the activity of the child and the progress of the disease, deformities of the bones take place, so that we get the characteristic bowing which is so marked clinically. Plate 87 illustrates what has just been said. In this plate it is to be noticed that the substance of the bone is greater on the concave than on the convex side. This is usually seen in the middle of the bone, and the medullary carity of these cases often shows areas of increased radiability and apparently of eystic formation. This appearance is caused by areas filled with cartilage or osteoid tissue which has not calcified.

Fracture, partial or complete, may be seen in any of the bones, and very little tendency towards callus formation, although appar- 
ently enough to make the bone more or less solid (Plate \$2). At points where the strain becomes great, as the deformity increases, an effort is male on the part of nature to prevent the bone giving way at this point by the laying down of new bone-cells, which are readily recognized by a more definite re-arrangement of the structure of the bone (Wolff's Law, Plate S4). This is common in the lower third of the femur and the tibia in eases where there is elinically knock-knee or bow-leg.

There are other eases which show a delayed ossification, a marked inerease of the bones in size with no great ehange in the zone of proliferation, and in addition to this marked deformity. Clinically these cases show an enlargement of the epiphyses and of the diaphyses, but the bones generally show a more definite change in their structure. The outline may be irregular, very seldom the periosteum is thickened, and the cortex is thin or very little laid down. There is a marked increase of the medullary canal, with a definite change of the structure of the bone and a coarse re-arrangement of bone trabecula. The epiphyses in these cases are characterized by symmetrical enlargement of the diaphyses and with no apparent changes in the zone of proliferation.

According to George these types of rhachitis from a clinical point of view are not distinguishable, but from a Roentgen point of view are quite evident.

Plate $\$ 1$ shows a very early stage of rhachitis in a child three years old. The shafts of the femora are comparatively normal in structure and in size. If, however, we compare this picture with the anatomic conditions seen in Plate 13, a normal child of three years, it will be noticed that the zone of proliferation is beginning to show a disturbance by its toothed and irregular appearance. The epiphyseal cartilage is also seen to be broader than normal, and this is seen to be still more evident if we compare this zone of proliferation with that of the ease of infantile atrophy shown in Plate 75. 
Plate 82 shows the characteristics of a typical case of rhachitis in the leg of a child two years old. The periosteum is thickened in several places. There is an irregular deposit of the cortex of the bone, especially at the upper ends of the tibia and fibula. The normal striation of the substance of the bone has been replaced by an irregular formation of the bone, showing itself by the jagged edges of the distal ends of the femur and tibia and of the epiphyseal line. There is a spontaneous fracture in the lower part of the femur. There is a deficiency of lime salts shown in both shafts and epiphyses, the latter being surrounded by normal epiphyseal cartilage.

Plate 83 shows a boy seven years old with typical rhachitis. Fig. 1 represents the typical rhachitic position in sitting, and the prominent abdomen, enlarged wrists, knees, and ankles so common in rhachitis.

Fig. 2 is a front view and Fig. 3 a side view of the same case.

Plate 84 represents the Roentgenograph of the same subject shown in Plate 83 . This plate shows some of the earlier manifestations of rhachitis. There is a somewhat more marked bowing of the tibia and fibula, due to superincumbent weight, than would be expected from the slight alteration of structure presented in the bones. The diaphyses of both tibia and fibula are enlarged as compared with their shafts. The cortical bone is thicker on the concave side of the shaft at the part where the deformity is greatest, which is in accordance with Wolff's law. The structure of the bone is considerably coarser than normal, especially in the diaphyses. The epiphyses are all fairly well defined. There is very little disturbance of the zone of proliferation, which shows a lessened deposit of lime and increased radiability.

Plate $\$ 5$ represents a typical case of advanced rhachitis in a boy ten years old. The bones are increased in size and in thickness. The large amount of newly-formed bone and the decreased radia- 
bility, especially at the ends of the bones, are in striking contrast with the lessened radiability of the shafts with their areas of irregular formation of bone and their lack of lime salts. Note the areas of thickened periosteum on both tibia, and the decided thickening of the cortex on the curved sides of the bones in comparison with the convex sides.

Plate S6, Figr. 2, represents the photograph of a boy seven years old, showing especially an extreme bowing of the lower part of the tibia and fibula.

The Roentgenograph, Fig. 1, shows a marked coxa vara with deformity of the pelvis. There is marked symmetrieal inerease in the size of the bones. The upper parts of the bones show a heavy deposit of eortical bone, especially on the concave side, and are also marked by increased radiability. There is a marked irregularity and an indefinite deposit of the lime salts, a condition which frequently oecurs in rhachitis and which results in an increase of radiability in certain areas, especially in the lower parts of the femora and in the marrow. There is some disturbance of the zone of proliferation shown by the cortical bone being laid down in a somewhat irregular mamner. The leader points to a needle which was accidentally discovered in the soft parts.

Plate 87, Fig. 1, shows the photograph of a boy three years old with a protuberant abdomen, knock-knee, and enlargement of the ankle-joints.

The Roentgenograph, Fig. 2, of the same case shows a thinning of the cortex with increase of the medullary canal, a lack of lime salts and a consequent increased radiability.

Rhachitis of Adolescence. - At about the age of puberty in certain individuals the physical signs of rhachitis, with the exeeption of a lesser degree of epiphyseal enlargement, oceur, and in these cases albuminuria is usually present. Cases of this class are desig- 
nated as the rhachitis of adolescence or late rhachitis. The disease is a rather common one and is characterized clinically by genu valgum or varum, scoliosis, or nothing more marked than enlargement of the epiphyses and of the diaphyses. Some authorities claim that this condition is the forerunner of osteomalacia. The characteristics of the disease are:

The outline of the bone is usually normal.

The size of the bone is usually normal.

The epiphyses are well developed.

The zone of proliferation presents more of a change than is present in normal epiphyseal cartilage.

The diaphyses are toothed as well as the epiphyses and show an irregular deposit of bone-cells.

Plate SS shows the hands of two boys, each thirteen years old. Fig. 2 is normal for the given age while Fig. 1 shows the condition of the rhachitis of adolescence. By comparison with the normal hand, Fig. 2, it is noticeable that there is a characteristic increased radiability. The medulla is apparently normal, excepting for its increased radiability. The structure of the bone is coarser, but no more regularly deposited than that seen in the rhachitis of an earlier period, having generally coarser yet regular bone trabeculæ.

Plate 89 shows the hand of a boy twelve years old with the rhachitis of adolescence. The bones show a thickening of the periosteum along the phalanges, and a slightly greater toothed and ragged appearance of the zone of proliferation than in the case of the same disease just spoken of (Plate 88). It also shows more cortical substance than in the previous case.

Intra-uterine Rhachitis (Fetal Rhachitis).-- $\mathrm{MI}$ opinion in regard to this condition has been stated on page 90, Division III. 


\section{PLATE 74.}

OSTEOMALACIA.

Girl, age 7 years. (Reduced 52t\%.)

Fig. 1. A Roentgenograph of the Pelvis and Legs.

$A$. Points to the upper third of the right femur.

$B$. Points to the coarse bone structure in the upper part of the left femur. Just below this is a marked bowing with evidence of a previous fracture, now united. The bones in general show an irregular deposit of cortical bone.

Fig. 2. A Photographl of the Same Subject.

Showing considerable bowing of the upper third of the left femur. 
FIG. 1

PLITE 74

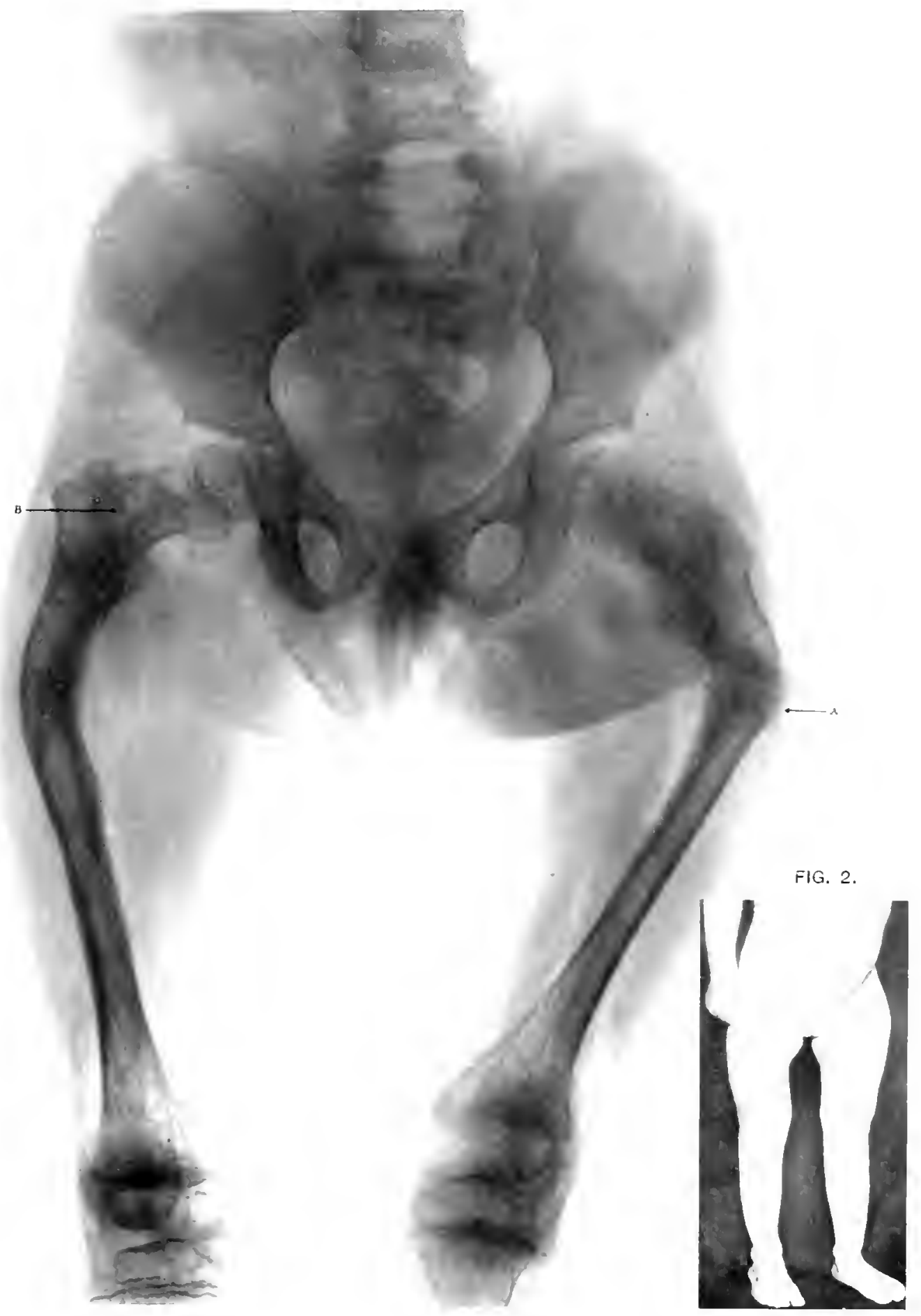


PIATE T-

INFATTIL ATIMIIY.

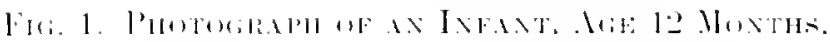

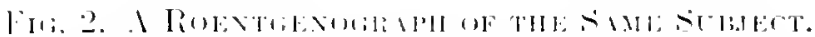

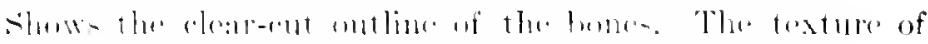

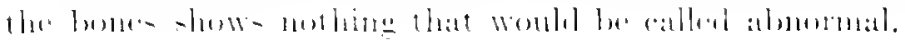


FIG. 2. PIATE: T.J

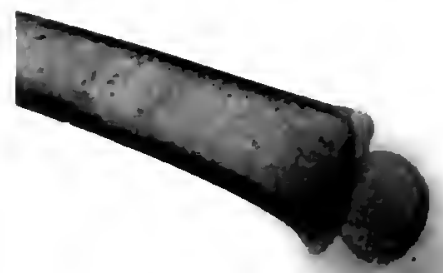

FIG. 1.
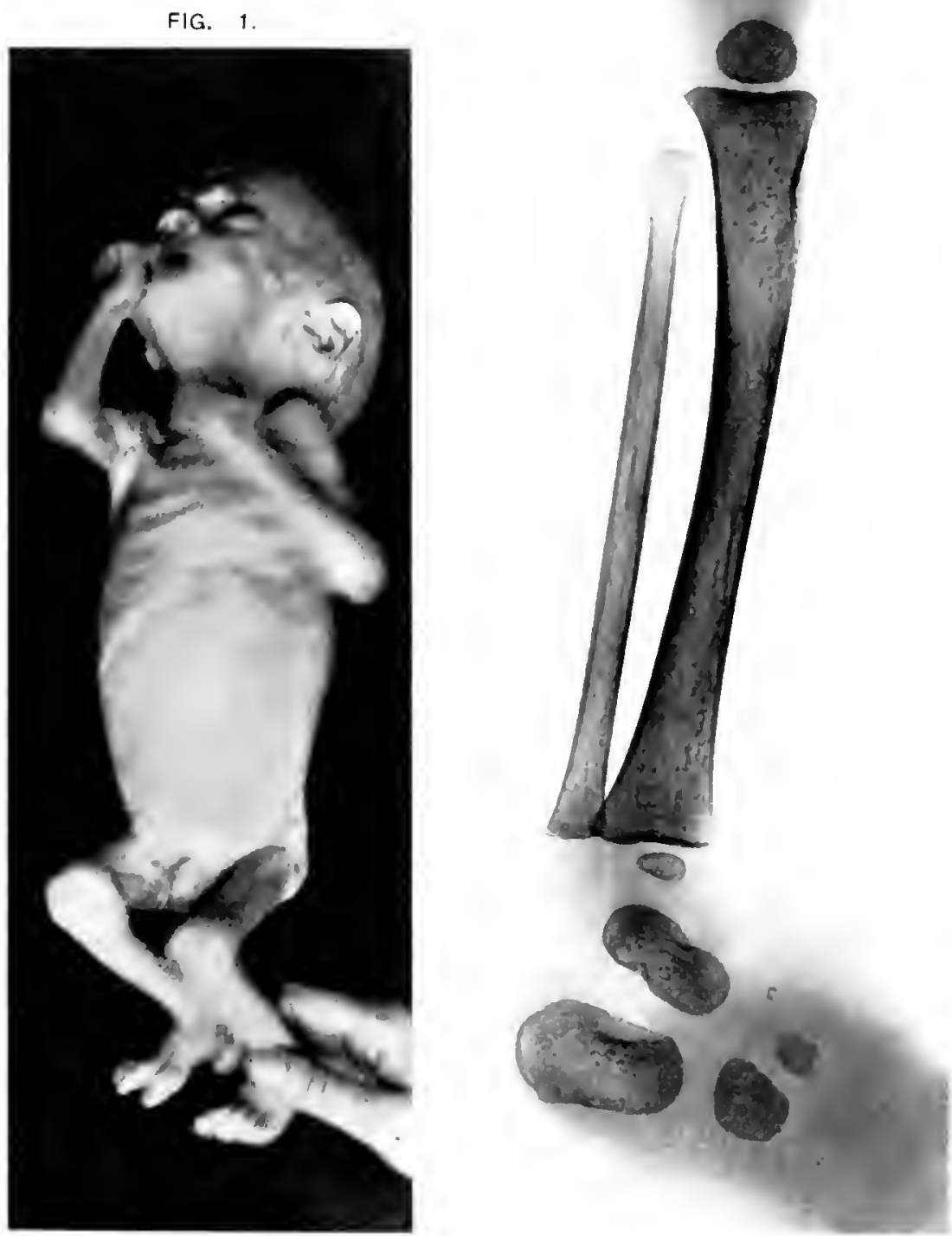
PLITE: 76.

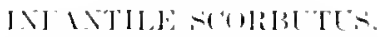

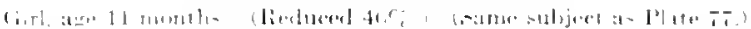

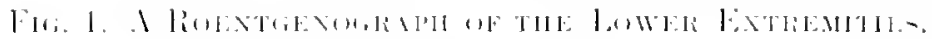

1. Slightly thisknent onter.

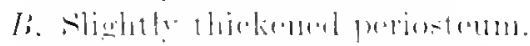

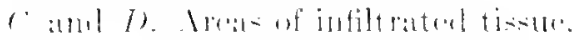

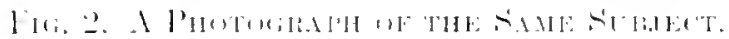


FIG. 1.

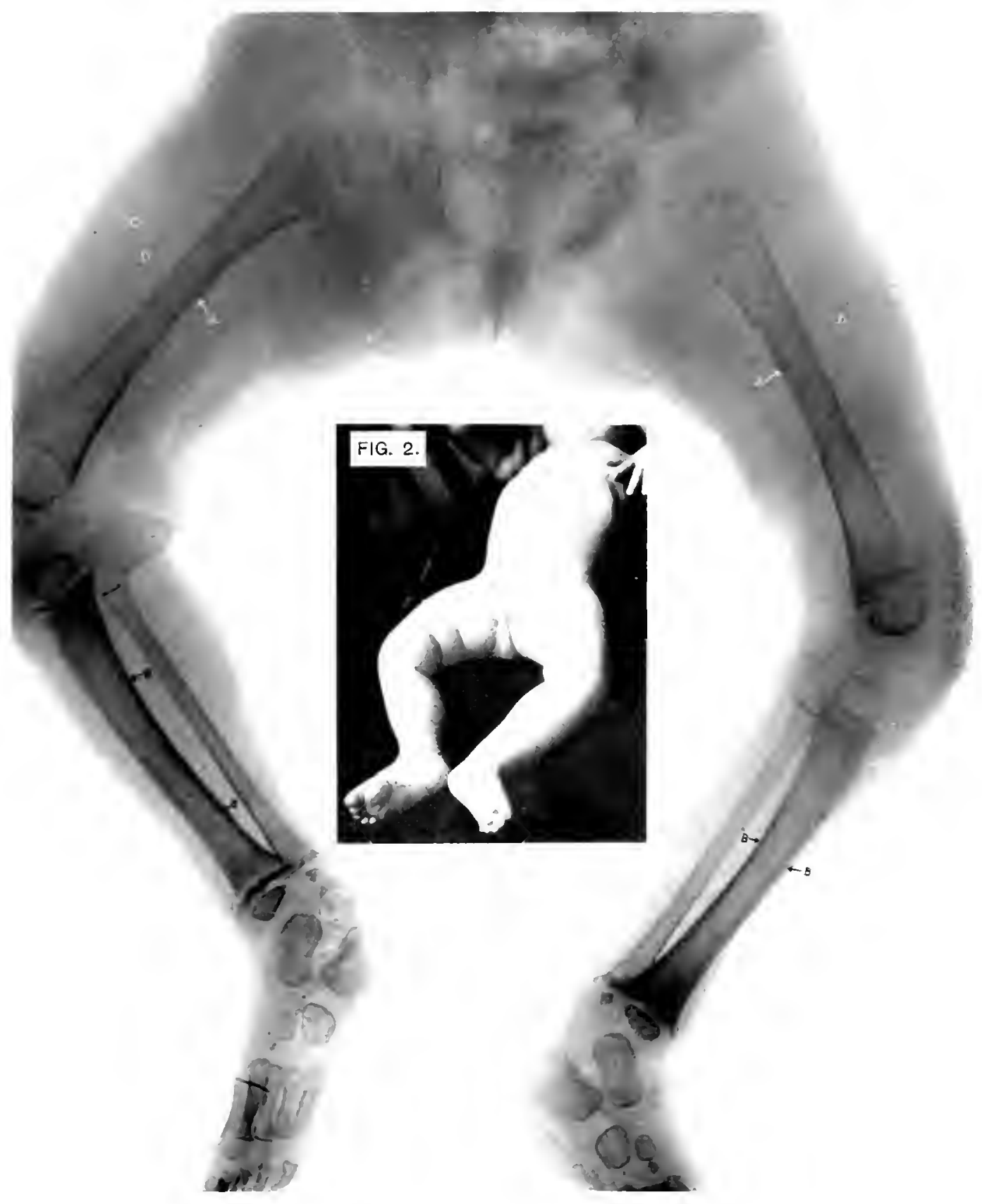


I'LAT:

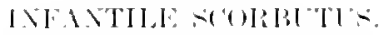

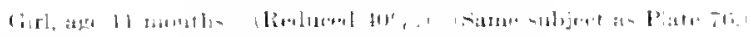

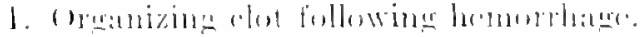

I. Thickomed puriustrum.

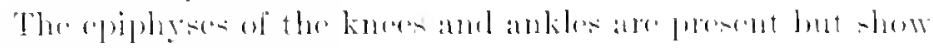
lese lensity thatu nomal.

\%ono of proliferation imentar. 
PIANE T

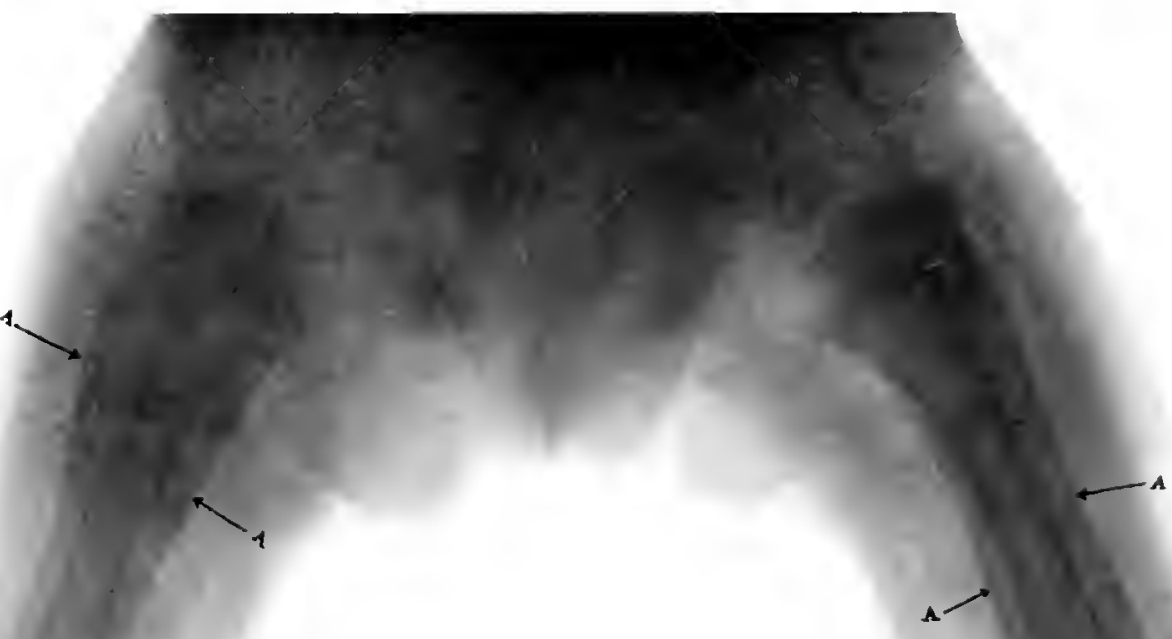


PI.1TEー゙

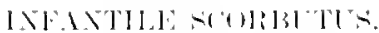

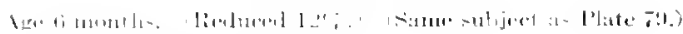

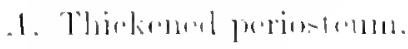

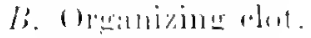

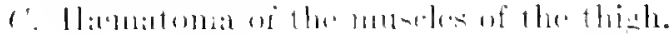




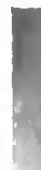

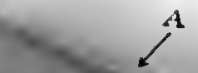

C

\section{B}

$-\mathrm{B}$ 
PI.ITL: 7!

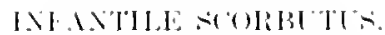

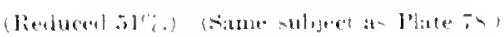

1. Thirkened periestrunt.

l). OMatniziner len.

$\therefore$ Imenular zome nif proliferation.

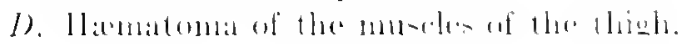


PIATE: T!

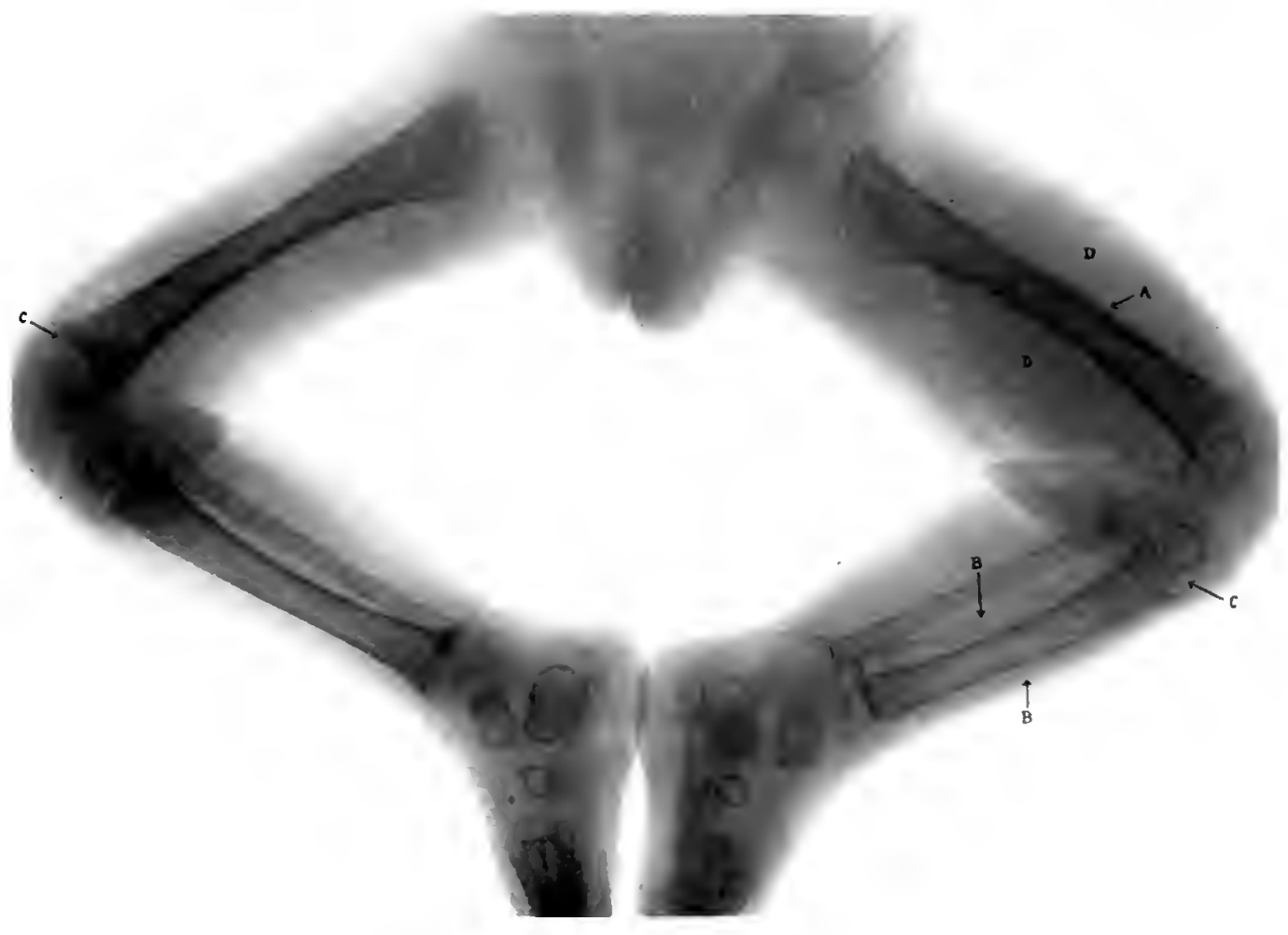


PlAT't: 40.

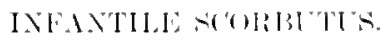

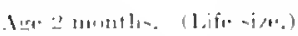

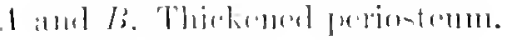

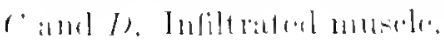




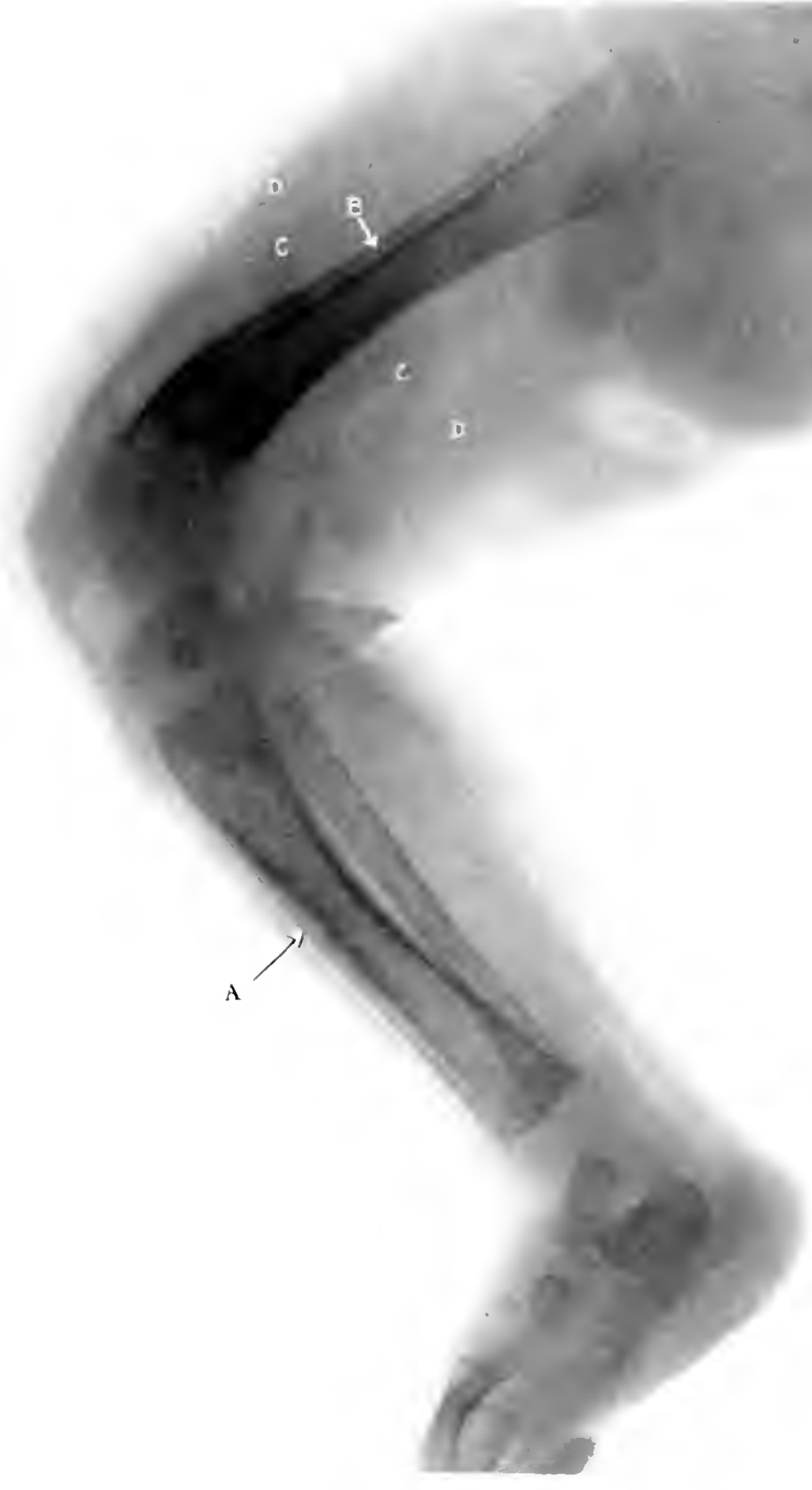


H.ATH 1.

WART RHACHTIS

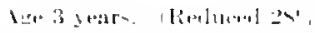

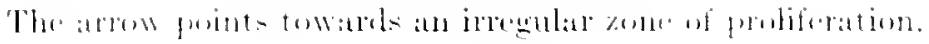


I'J.ITH:SI

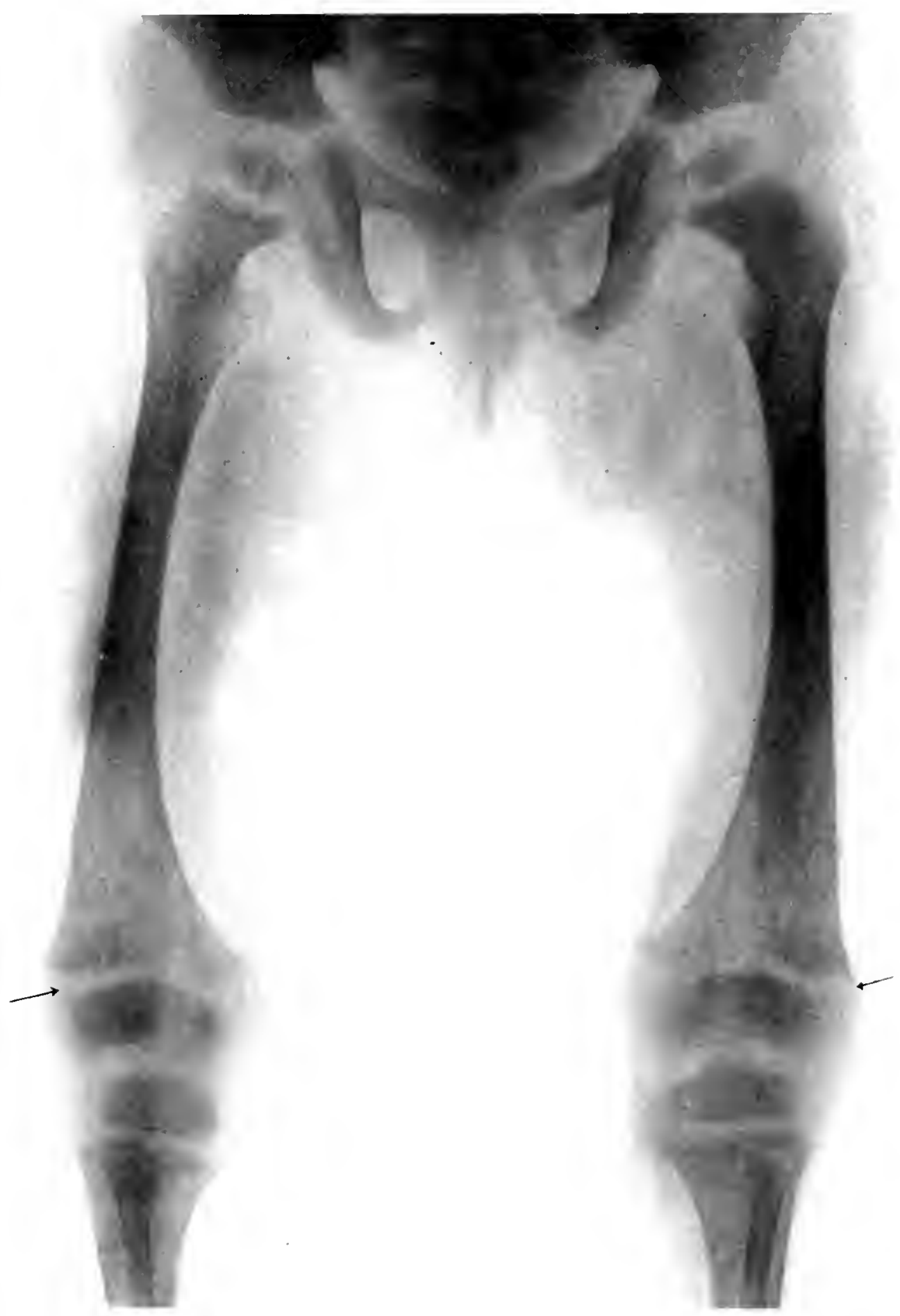




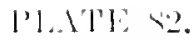

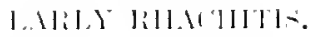

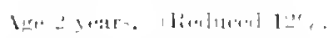

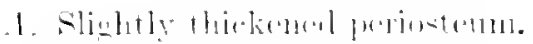

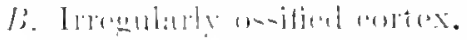

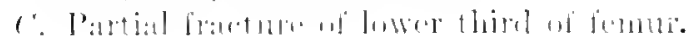


PILATE SQ

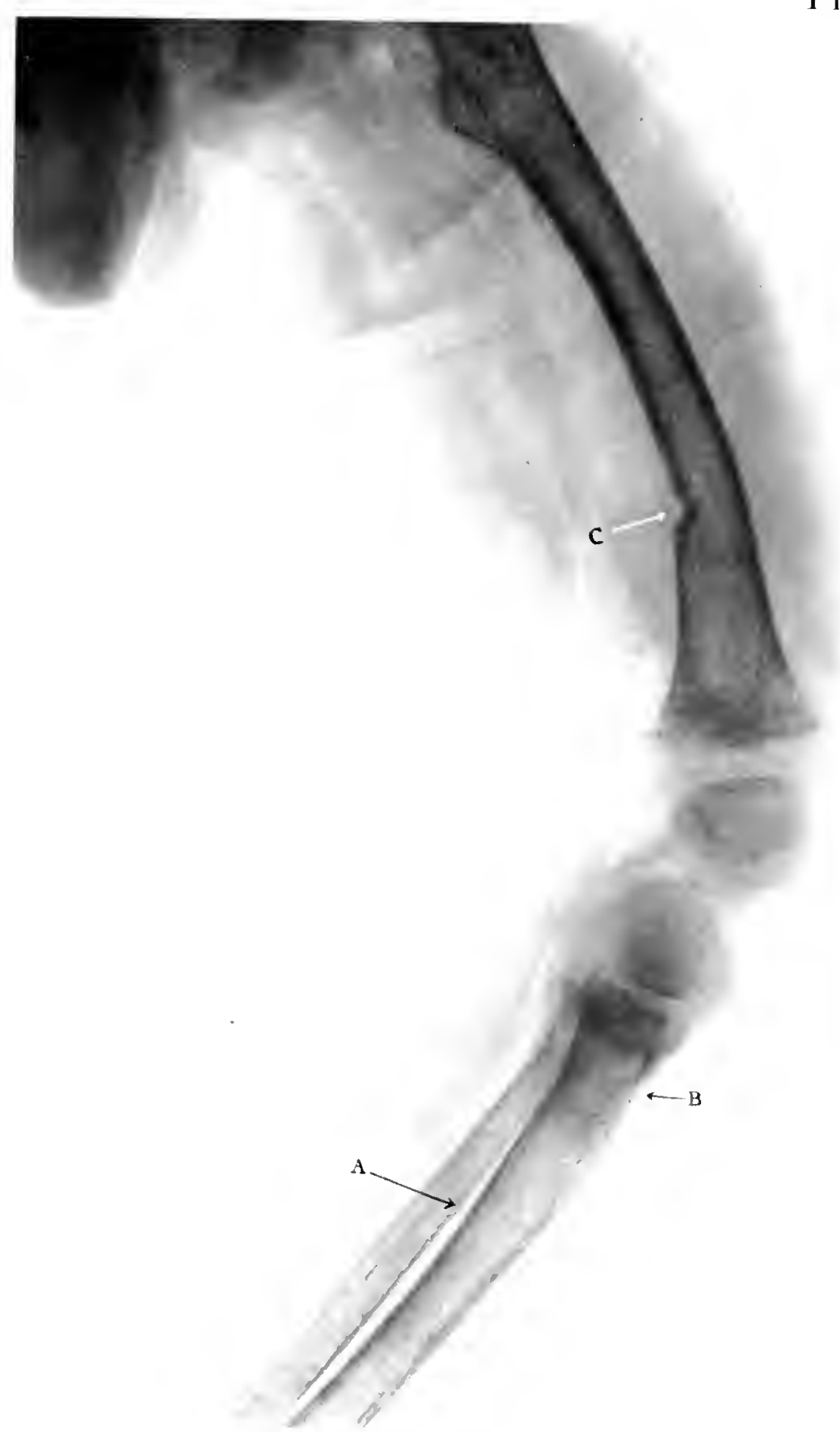




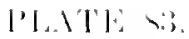

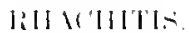

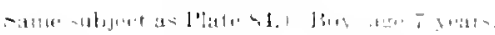

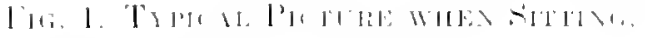

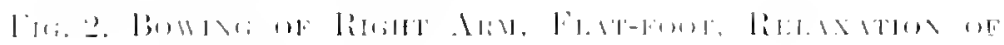

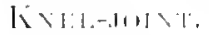

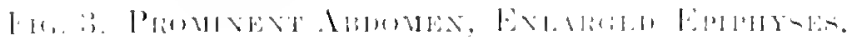


FIG. 1.

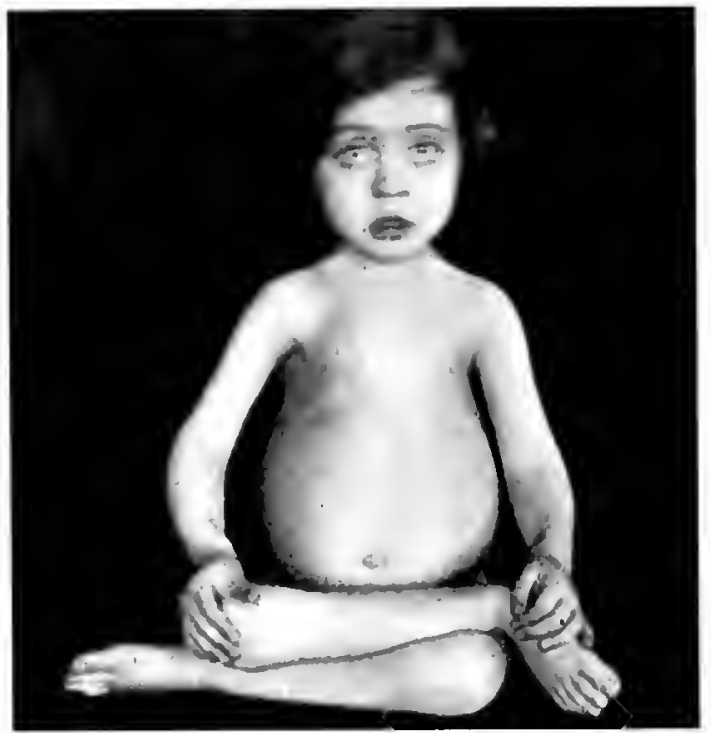

PLATI: S:

FIG. 2.

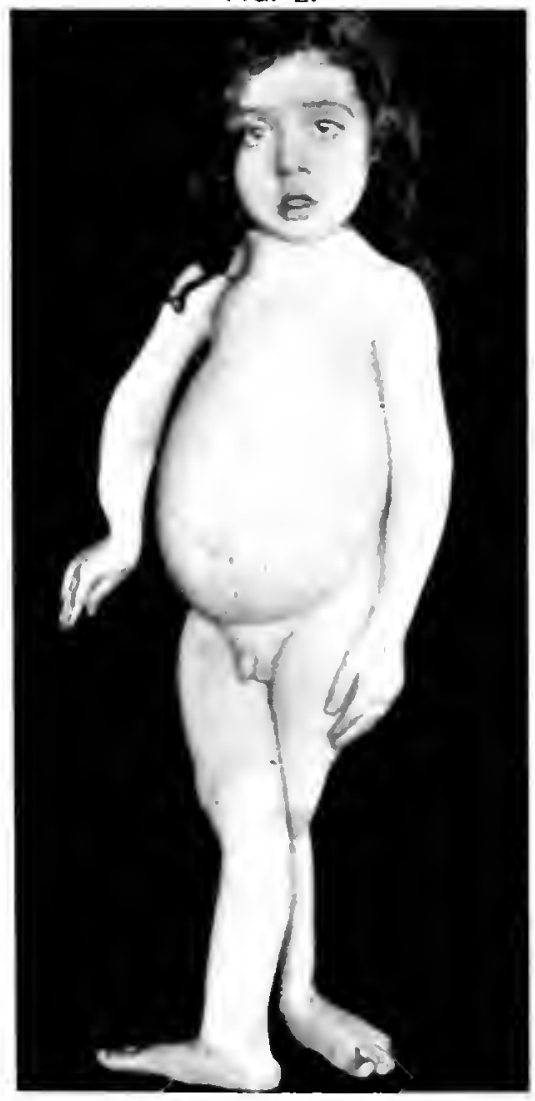

FIG 3.

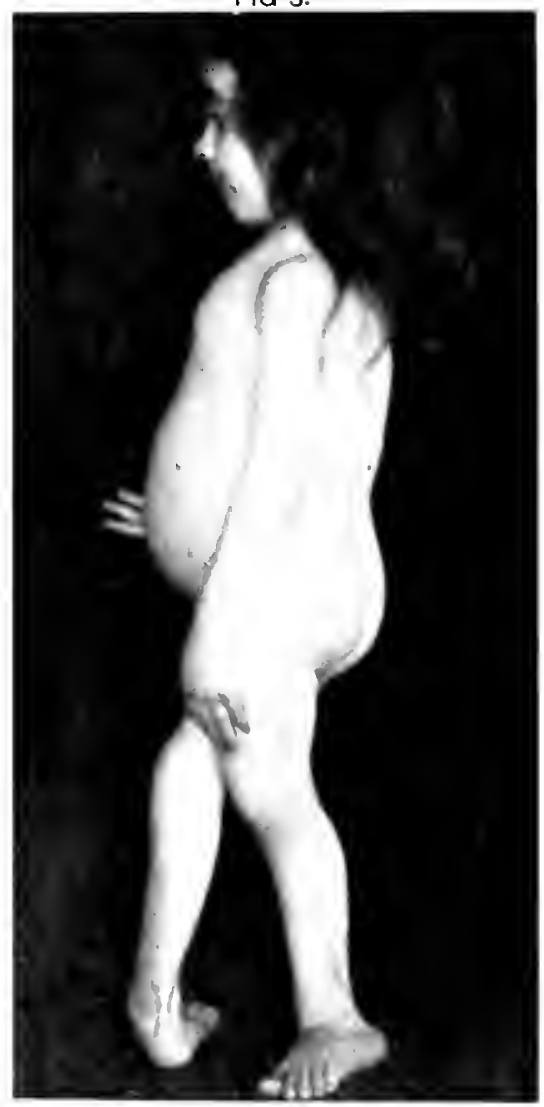




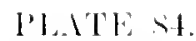

EARLY RHATHTLS.

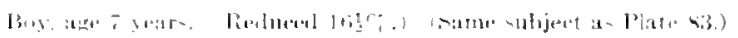

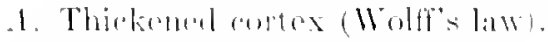

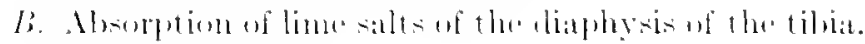

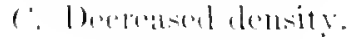


Plate st 
PLATE S.5.

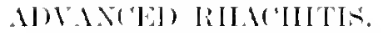

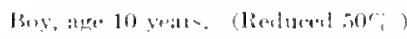

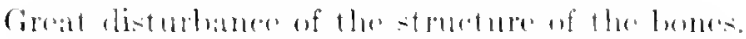

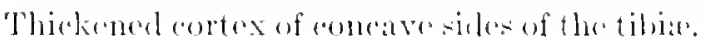


PIATE S.J

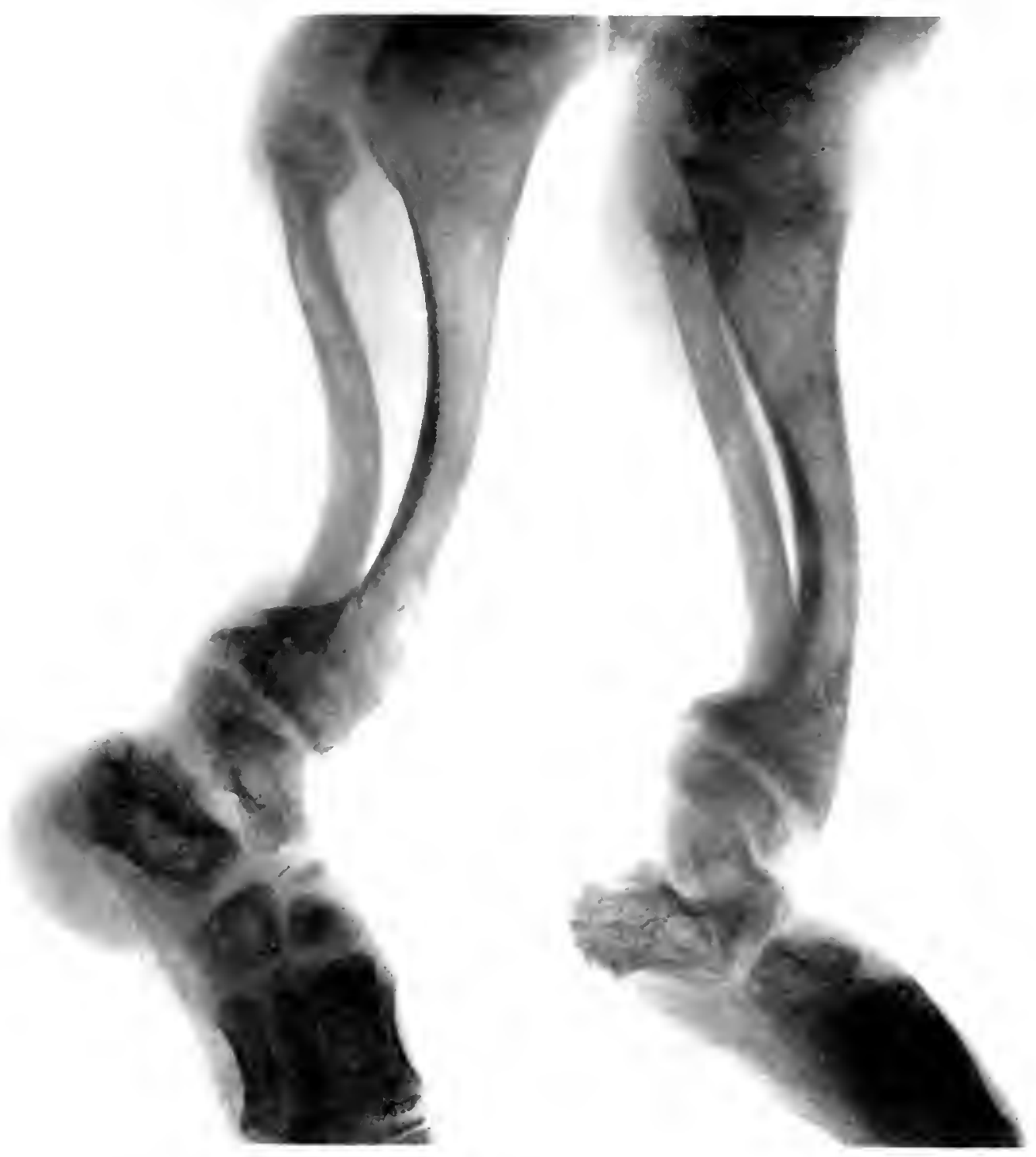


1'1.1T1: 48.

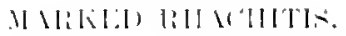

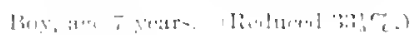

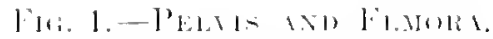

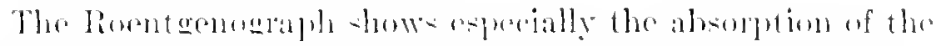

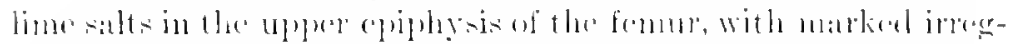

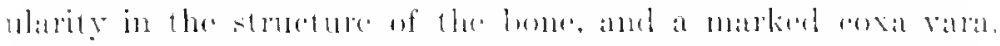

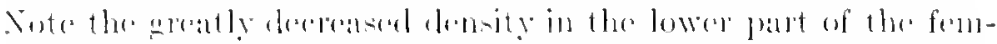

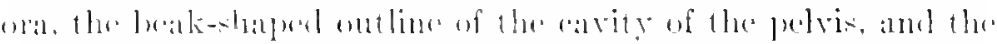

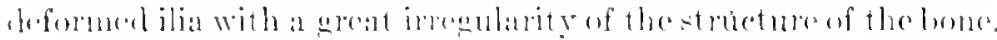

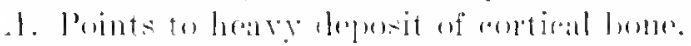

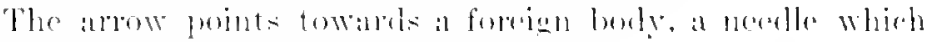
was acrobentally fomm al the ramination.

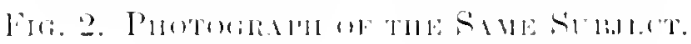

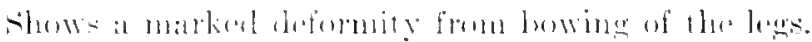


FIG. 1 .

I'IATTE SU;

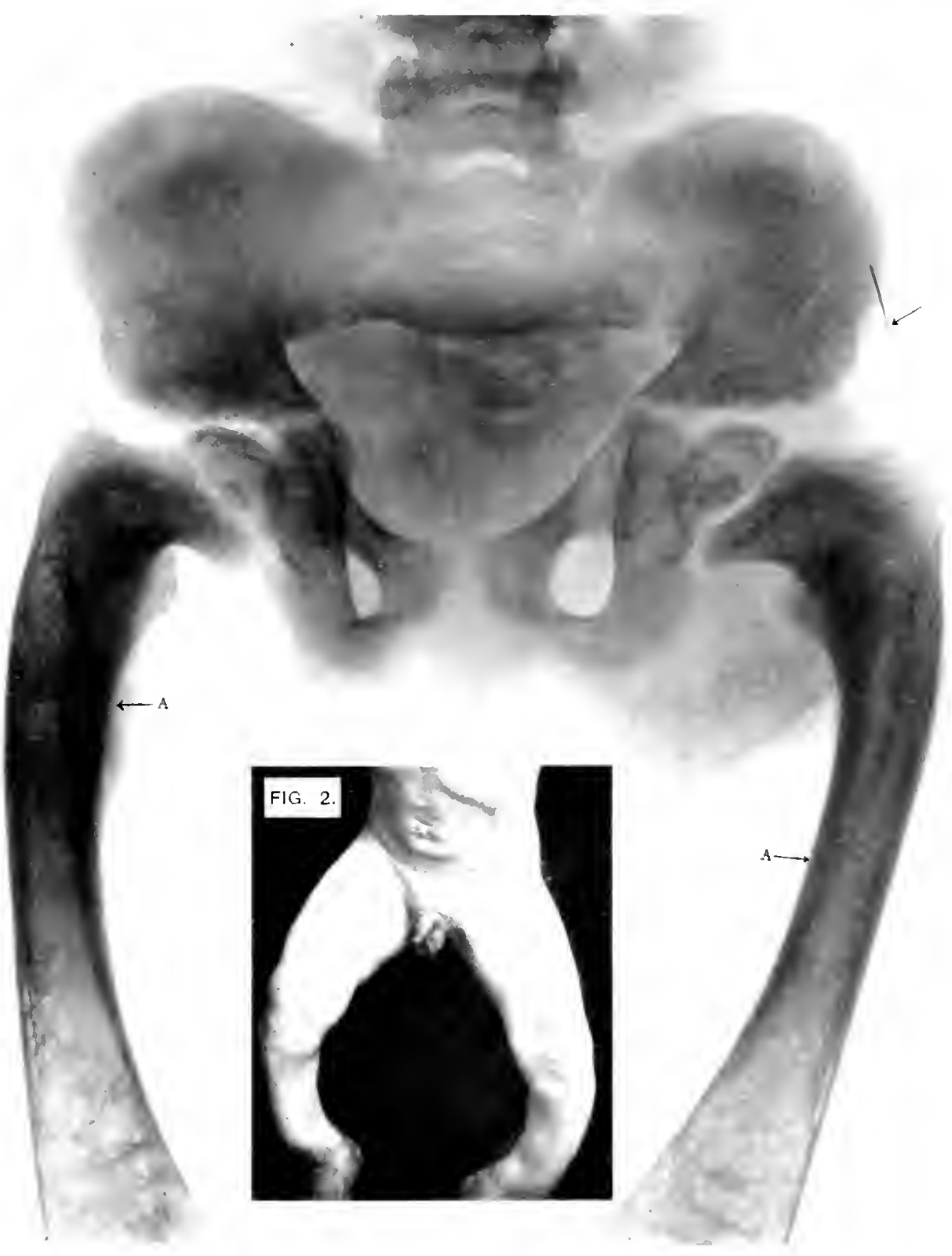




\section{PI.ITI: - :}

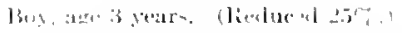

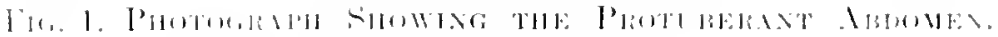

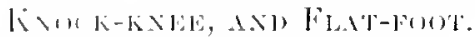

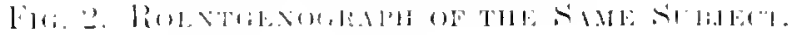

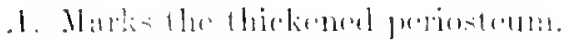

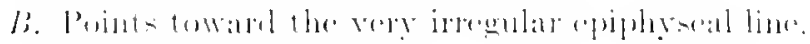
('. Thinkmed priosteum. 
PLATE カ
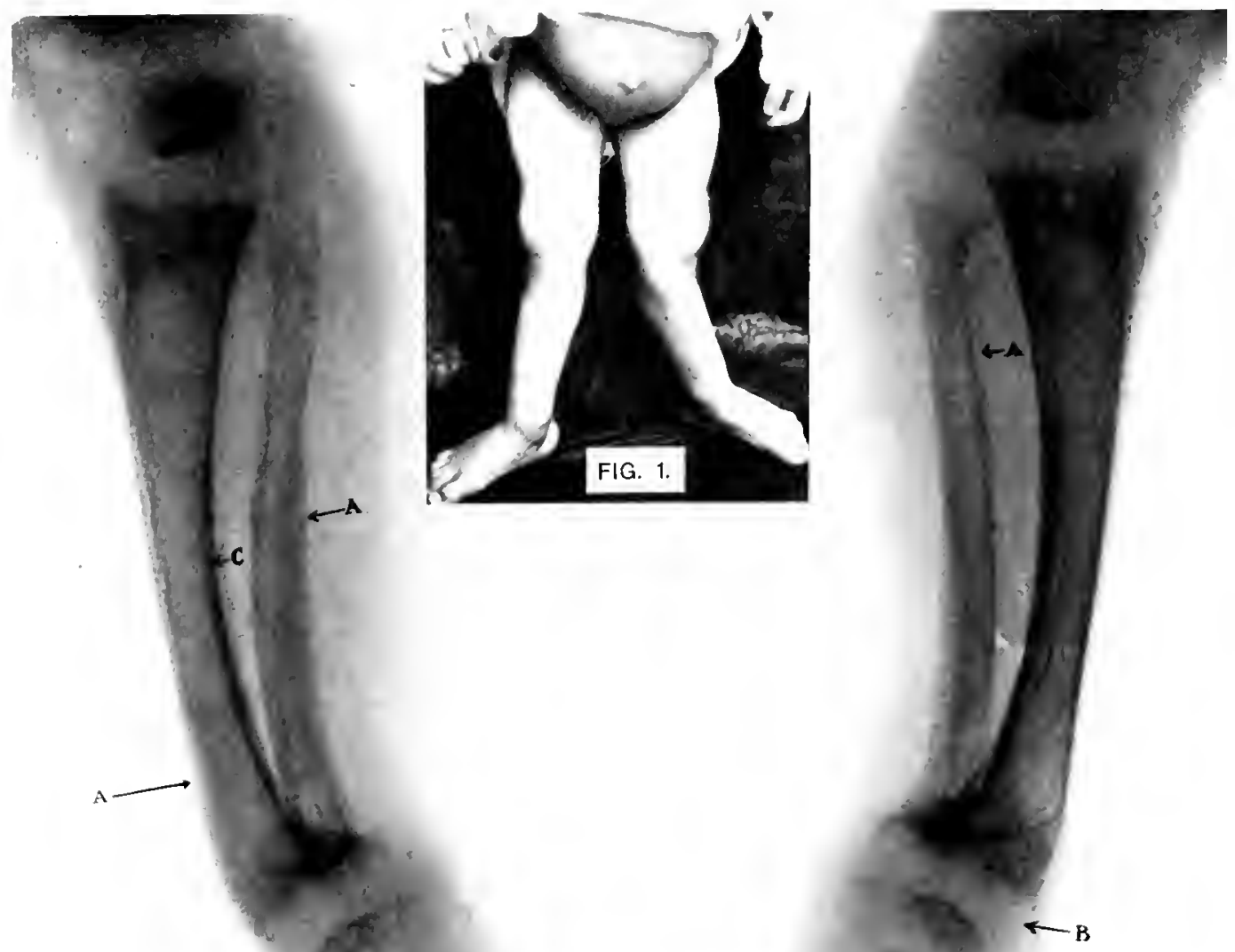

$\leftarrow$

-c
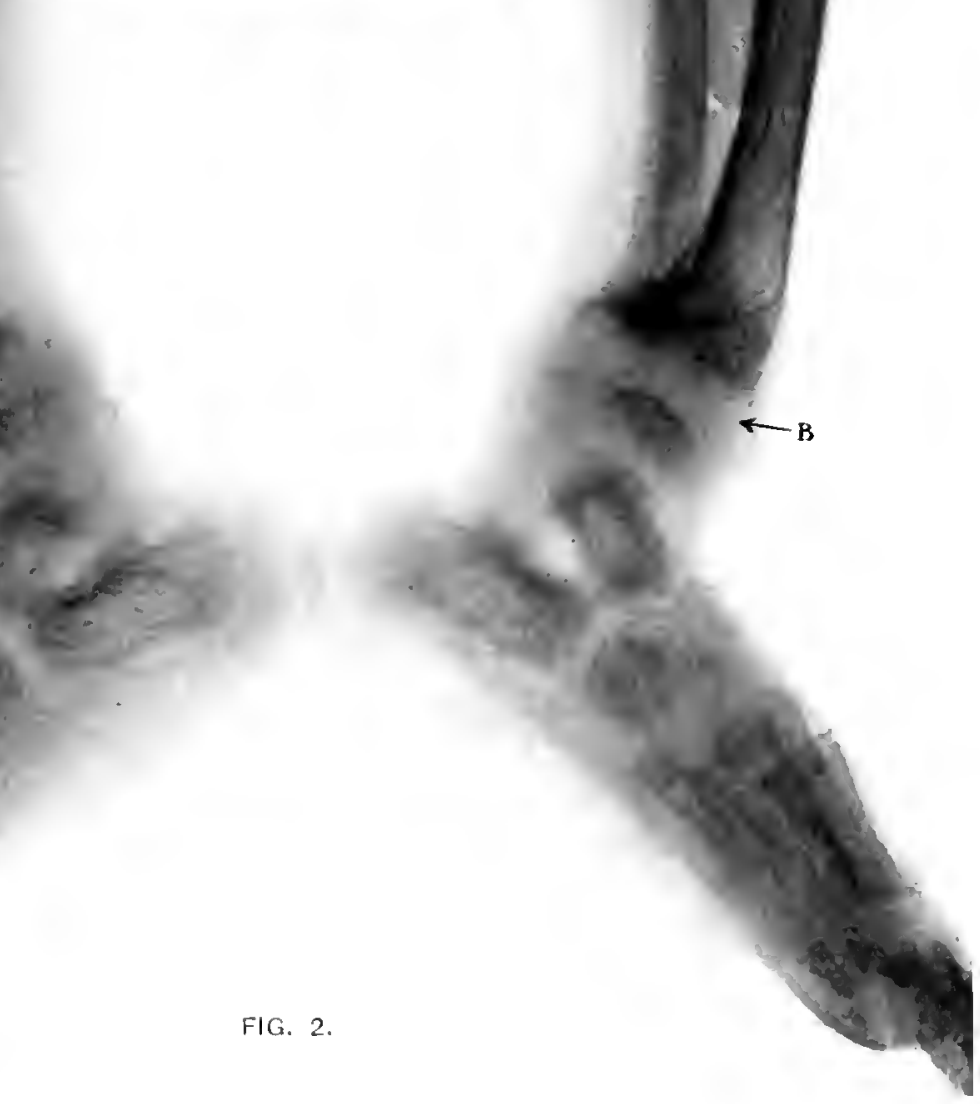


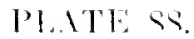

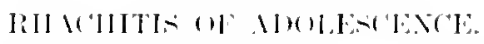

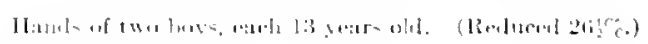

Fir. 1. Rin

The

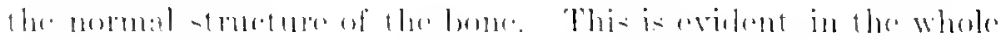
li:mil.

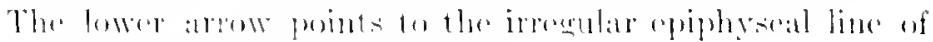
the lomer epiphysis of the rablins.

Fui, 2, Xmana, I1,kn. 


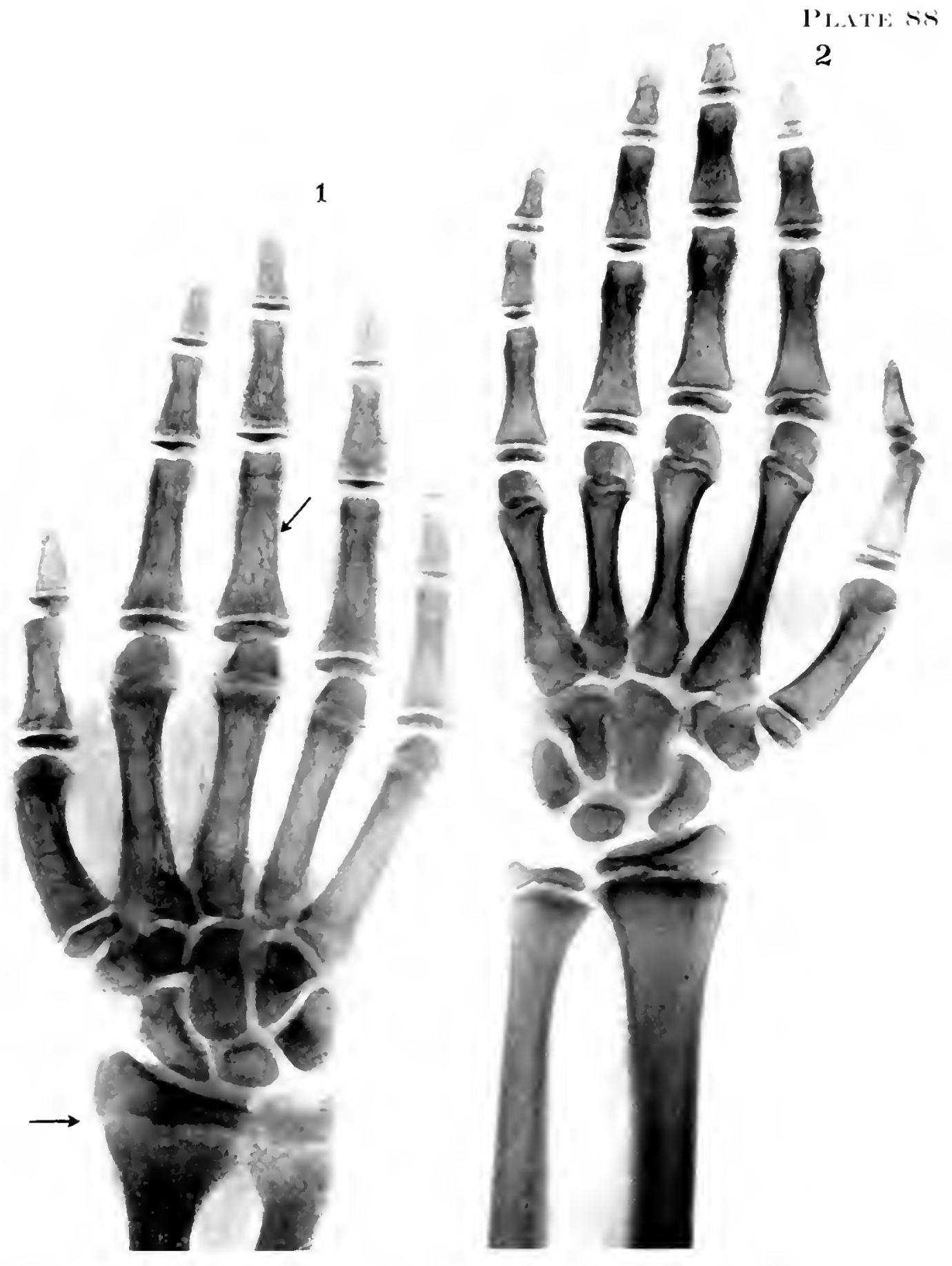


I'I. IT'L: S.

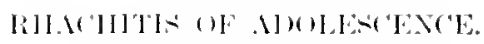

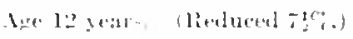

1. Shom: laserned dem-ity uf andtex.

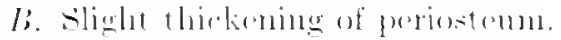

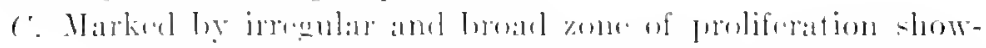
ing the lack of bone-forming colls and an increatse of the ('artilatere reolls.

D. Sismonoil Ione. 
PI,ATH:S!)

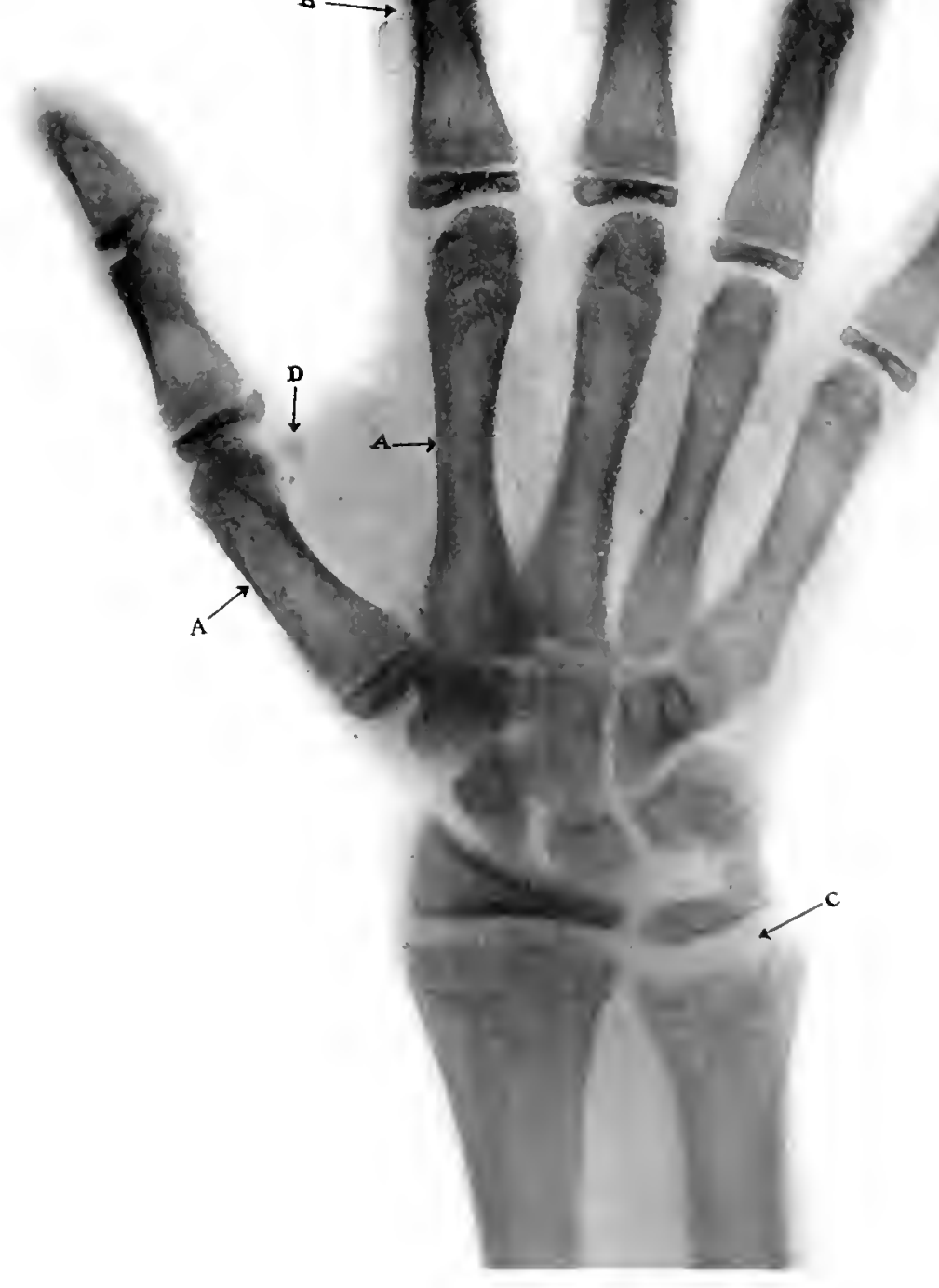





\section{Division $\mathrm{V}$}

\section{DISEASES OF THE HEAD AND SPINE}

\section{HEAD}

There are comparatively few abnormal conditions of the head which can be satisfactorily recognized by the Roentgen method. In tumors of the brain, where there is a reconstruction of the tissues by new tissues being added, or where there is less tissue than normal, a picture representing the tumor can at times be demonstrated. Where, however, there is the same amount of tissue as is in the normal brain, a very small amount of information can be obtained by means of the Roentgen ray. We at times have been able to detect a tumor of the brain in early life, and have had under our care at the Children's Hospital a case of this kind in which the tumor was located by means of the Roentgen ray. Certain results of traumatism, such as fractures, play an important rôle, and certain infections of the jaws and surrounding tissues can often be diagnosticated definitely only by means of the Roentgen ray. Of great and increasing value is the aid given by Roentgen films of the teeth. By their assistance in the diagnosis of either variations or anomalies of the teeth, the orthodontist is enabled to carry out his work with a precision which before the Roentgen ray could be applied to this class of cases was unknown. As in the study of other parts of the body, so it is with the head, that to understand diseased conditions and to intelligently diagnosticate and treat them, a knowledge of the normal conditions is essential. We must be able to say whether the different sinuses, frontal, ethmoidal or sphenoidal, are in a normal or abnormal condition. A suspected fracture of the skull can be often diagnosticated only by means of the Roentgen method.

As an example of this, Plate 90 shows a fracture of the skull 
in a boy thirteen years old. This fracture was not recognized elinieally until a Roentgenograph was taken.

Plate 91 shows an inflummatory process indicating ethmoiditis in a child three years old. This plate also shows a full set of normal temporary teeth.

Plate 92 shows how important it is to have the temporary teeth eared for. In this case a lack of care of the mouth and teeth resulted in caries and suppuration of the roots of the teeth and finally an osteonyelitis. This class of cases arises usually from negleet, and oftener from this cause than from traumatism. The legend of this plate shows what extensive lesions may result from osteomyelitis of the jaw.

The anomalous conditions which may arise in connection with the teeth in early life are very numerous. A few of these, however, will be sufficient to show how important it is to recognize such conditions when present, and how impossible it is to definitely localize, diagnosticate and make use of this exaet knowledge without the aid of the Roentgen ray.

Plates 93 and 94 slow the sides of the head in a boy thirteen years old. The value of obtaining an exact knowledge of the existence and position of an unerupted tooth by means of the Roentgen method is obrious. These plates show the seeond lower bicuspids wedged between the first and second molars. Under intelligent treatment by mechanical means, which eould be carried out when once the abnormal position of the teeth was determined, the abnormality was corrected.

Plate 93 shows the left side of the skull. In the upper jaw the teeth are very well defined. The central and lateral incisors can readily be seen. The permanent euspid does not show very clearly but its eruption at a later period proved its existence. The unerupted first and second bicuspids partly calcified can be seen 
directly above the temporary molar, the roots of which have been absorbed, leaving them about to be exfoliated. The first permanent molar can be seen completely formed, while the roots of the second molar, unerupted, are but partly calcified. The third molar does not show much evidence of being present, although there seems to be a slight outlining of the crypt in which its formative organ lies and the beginning of calcification of the tips of the cusps.

In the lower jaw the crypt of the third molar is fairly well defined and four points of calcification can be seen. The second molar shows clearly. The roots are partly formed with wide openings, large pulp canals and large pulp chambers. A comparatively small amount of caries at this period of development might have involved the pulp, and its loss through irritation would be more serious to the future usefulness of the tooth than after the entire calcification of the tooth had been completed. The first molar shows the complete calcification of the roots with much smaller pulp canals and pulp chambers. This same decrease of size of canals and pulp chambers will take place in the second molar during its continued calcification.

Anterior to the first molar can be seen the reason for the Roentgen examination. Here lies the unerupted second bicuspid tooth, held in this position by the forward growth of the first and second molars, due to the premature loss of the second temporary molar, leaving a space of one-eighth of an inch between the crown of the first permanent molar and that of the first bicuspid. The root is but partly formed and the tooth would readily erupt if it had sufficient space. This proved to be true later when space had been made by lengthening and widening the lower arch. The same condition existed on the right side of the jaw, which is shown in Plate 94.

Anterior to this first bicuspid is a cuspid which is just erupting. Anterior to this tooth the teeth are indistinct. A Roentgenograph 
of this size is not satisfaetory for determining the position or condition of the individual teeth anterior to the second bicuspids. A small film placed inside the mouth gives a much elearer view of the point of interest, and by this means we are not confused by the tceth on the other side of the jaw.

Plate 95 shows the erupted first permanent molars and some of the unerupted teeth in a boy eight years of age.

In the upper jaw the teeth are shown to be in an abnormal eondition of caleifieation and eruption. The picture shows the right eentral ineisor unerupted and the root partly calcified. The permanent lateral incisor has just appeared through the gum and its root is also partly ealeified. The permanent cuspid lies posterior to the lateral incisor, which has just appeared through the gum, and its root is only partly calcified. The first bicuspid is only partly ealcified and its root not entirely formed. The space between this tooth and the lateral incisor is not sufficient to allow the cuspid to erupt in a normal position. Posterior to the first bicuspid root lies the seeond bicuspid, with only its erown calcified. Below are the roots partly absorbed and what is left of the carious crown of the second temporary molar. The first permanent molar lies posterior to this tooth and appears well formed and fully erupted. Posterior to the roots of this tooth lies the second permanent molar, with only its crown formed. There are no signs whatever of the third molars in either the upper or lower jaw.

In the lower jaw from behind forward is the second molar in its crypt with its crown caleified. Next in front is the first molar fully erupted, with the apices of its roots not as yet caleified. Next in front of this is the second bicuspid in its crypt with the ealcification of its root not begun. Above this is the carious crown of the second temporary molar. The next anterior tooth is the first bicuspid just showing its crown through the gum. Its root is but partly formed. 
Immediately above are the outlines of the teeth in the opposite side of the upper jaw, and they are not considered in this description. Next can be seen the form of the unerupted and partly formed cuspid, the crown of the temporary cuspid lying above and a little in front. Its root is nearly absorbed.

The inferior incisors have all erupted but are in malposition. This is not shown very clearly from this view.

Supernumerary teeth play quite an important rôle in dentistry, since, although comparatively rare, they may occupy space needed for the proper position of the other teeth.

Plates 96,97 , and 98 are examples of this kind and show the great value of Roentgen examination.

Plate 96 shows the left side of the skull of a boy fifteen years old. The sinuses are well developed. The teeth are very well shown in the upper jaw, but in the lower jaw they are somewhat confused on account of the presence of the teeth on the opposite side.

In the upper jaw the central and lateral incisors are fully erupted and the cuspid almost in position. Just above the lateral incisor is a supernumerary tooth unerupted. The first and second bicuspids are normal and fully erupted. The first and second molars are clearly defined. The third molar shows beautifully in its crypt, with only the crown calcified in accordance with the boy's age. The lower third molar shows a similar condition. The second and first molar roots do not show clearly, nor do those of the second and first bicuspids, although the crowns of the latter are well marked and the pulp canals and chambers can be traced. Anterior to the first bicuspid the teeth are not distinct enough to allow of any accurate description.

Plates 97 and 98 show the right and left sides of the skull of a girl fourteen years old. The sinuses are normal and very large in both plates. 
The temporal bones and the glenoid fossæ, in which lie the condyles of the inferior maxilla, are clearly defined.

The right side of the head shows the superior cuspid retained in its alveolus in a flat position (this is shown better in the film print below). In the film print (looking out from the inside of the mouth) the superior right cuspid lies in the centre. Back and to the right of the cuspid and partly obscuring it is the first bicuspid. Then come the second bicuspid and the first molar, the roots of the latter not being so elearly defined as those of the cuspid. To the left of the cuspid can be seen three incisor teeth. The one to the extreme left is the right central incisor, and next to the right of this tooth is a supernumerary tooth, in the palatal aspect of the central and lateral incisors the next tooth to the right.

There is nothing of interest in the plate until we come to the region of the superior seeond molar. This tooth is indicated by the great density of the first molar and the tooth which is a supernumerary in the palatal aspect. Back and above the second molar is the partly caleified and unerupted third molar.

In the lower jaw from back to front the teeth are normal.

Plate 98 shows the left side of the head. The superior cuspid is seen in a somewhat crowded position. The film below shows the tooth much more plainly. The left side of the mouth has the same anomalies as the right, with the exception of the lack of a supernumerary tooth in the second molar region. In the film the tooth, only part of which shows in the lower right-hand eorner, is the left central incisor. The next tooth to the left is a supernumerary tooth partly obscured by the left lateral ineisor. The partly formed third molar is unerupted, as is the case on the right side.

Plate 99 illustrates the value of the Roentgen method of examination in a number of cases.

Figs. 1 and 2 represent the mouth of a ehild fourteen years 
of age. Fig. 1 (the left side) shows the absence of the second bicuspid $(A)$. The accompanying Fig. 2 shows the opposite side of the mouth which also lacks the second bicuspid. The crown of the second temporary molar is still in place, although the absorption of the greater part of the root has taken place without the formation of the permanent teeth underneath it.

Fig. 3 shows the mouth of a child thirteen years old with the absence of the second bicuspid. The temporary molar has remained in the mouth with no absorption of its roots.

Fig. 4 shows an interesting picture with possibly a supernumerary tooth. It will be seen that the cuspid $(B)$ lies in the upper left-hand portion of the picture; immediately beneath it is the lateral incisor $(C)$, and immediately beneath the lateral incisor and a little in front of it is the crown of the temporary lateral incisor or possibly a supernumerary tooth $(D)$. Lying directly back of the central tooth, which is the lowest tooth on the plate, lies a temporary cuspid $(E)$, and immediately above it is the permanent first bicuspid $(F)$. Back of this temporary cuspid root lies the crown of the first temporary molar. It will be seen that the permanent central incisors are in malposition, as are also the lateral.

Fig. 5 shows the superior cuspids lying in the palate directly behind the central incisors. It was found that the apices of their roots were in normal position. These teeth are now being moved into place.

Fig. 6 shows the upper jaw of a girl nine years of age. The plate is read from right to left. In the right upper corner is seen the tip of the right permanent cuspid, and after this come the permanent central incisors. There is an entire absence of permanent lateral incisors in this jaw. The temporary left lateral incisor, next to the left central incisor, has its root almost entirely absorbed, 
and above it is the permanent cuspid $(H)$ somewhat rotated. Behind the temporary lateral incisor is the temporary cuspid $(I)$, and behind this tooth are the first and second temporary molars. The angle at which the film was exposed does not allow the amount of absorption of the roots of these teeth to be shown, but the crowns of the first and second bicuspids ( $J$ and $K$ ) can be seen directly above them.

Fig. 7 shows the mouth of a child eight years of age with the temporary cuspid and the first and second molars in place. Above these are the permanent teeth developing and erupting. This plate shows very clearly the absorption of the temporary teeth.

Fig. 8 represents the mouth of a girl between eight and nine years of age. The picture shows the teeth of the left side of the upper jaw with the permanent central ineisor well developed and the lateral incisor presenting an interesting feature. There seems to be a thickness of its roots about one-sixteenth of an inch from its apex, and also an apparent constrietion of the root eanal $(L)$. The crown also seems to be misshapen, but this is due to a slight torsion or rotation of its root. The temporary euspid $(M)$ is still in place with but little absorption of its root. Above this tooth and in front of it can be seen the crown of the permanent cuspid with its root partly formed and with a wide apical opening and a large root canal. Behind the erown of the first bicuspid below this tooth is the crown of the first temporary molar.

Fig. 9 shows the mouth of a child ten years of age. The sccond temporary molar is still in place and the second bicuspid $(N)$ is directly above it. This plate shows the undeveloped root of the first bicuspid, also the crown of the upper temporary cuspid (O) tilted back, and the permanent euspid almost erupted.

Fig. 10 shows the right side of the upper jaw of a girl between seven and cight years of age. The central incisor is well developed 
and the temporary lateral incisor is still in place, not absorbed but deflected forward by the erupting permanent cuspid $(P)$. The temporary cuspid $(Q)$ shows its root partly absorbed. The first and second temporary molars have not been lost, but their roots are completely absorbed. The first and second bicuspids can be seen directly above them. The development of the roots of these teeth does not appear.

Fig. 11 shows that with the exception of the tooth at the extreme left, which is the right upper central incisor, all of the other areas of density represent the temporary teeth. The areas above represent the permanent teeth, that is, the right upper cuspid $(R)$ and the first and second bicuspids which are in the process of eruption.

\section{SPINE}

The spine is best described separately from the rest of the skeleton, as its position behind the sternum and the various organs not only interferes with a clear picture of other parts but also is liable to affect its own definition. It is, however, my intention to describe the spine and its diseases in a general way and only with the purpose of indicating what especial conditions can be practically shown by the Roentgen method. According to our experience in the various clinics of the Children's Hospital, where a very large number of Roentgenographs are taken every year, the information obtained by the clinical examination of the spine rarely compares favorably with the condition disclosed by the Roentgen ray. At present, owing to the difficulty of the technic, it is not possible to take satisfactory Roentgenographs of the lateral views of the spine. Almost without exception our hospital subjects are taken with the back on the plate, since this is the position in which the pictures of the vertebræ are least interfered with by those of other parts of the body. It is important that the entire vertebral column should 
in each case be looked over carefully, for in this way most valuable information can be obtained in regard to practical treatment. What we should determine in abnormal conditions of the spine is the differentiation of abnormalities which have occurred before birth during the process of development from post-natal traumatism and infection. The former elass of cases, the prenatal, has already been described in Division III, Plate 41 (anomalous atlas and axis), Plates 42, 43 and 44 (spina bifida). I shall not attempt to describe the tranmatic class of cases, as they are so closely connected with the work of the orthopedist, and comprise in themselves such a wide field of study, that they would require a special treatise to do them justice. The various degrees of so-called functional, better designated as inorganic, abnomalities of the spine, such as lordosis, kyphosis and scoliosis, arising from postural deformities, are readily diagnosticated by means of the Roentgen ray. Although the external clinieal evidenee of these conditions is of the greatest value in the hands of an expert in this branch of medicine, yet the readily obtained Roentgen pieture is so valuable, instructive, and important, especially in complieated cases, that it is safer to make use of it, if for no other reason than that some unsuspected abnormality may be brought to light. It is worth while, therefore, to become familiar with the normal conditions of the spine, and to study its position, the texture of the rertebra, the radiability indicating breaking down of one or more bodies, the condition of the intervertebral discs, and the relation of the transverse processes to the lines of the entire column. In this way also we can detect various degrees of curvature and rotation and recognize not only postural defects but actual lesions of the bone and cartilage. Keeping this idea in view I will refer you to Plate 23, which shows the normal spine at ten years.

It cannot be too strongly emphasized that a recognition of 
abnormal conditions of the spine, as shown in the Roentgenograph of the vertebræ and cartilaginous dises, depends upon a preeise knowledge of the various details shown in the pictures of such normal conditions. The finer diagnoses of diseased conditions are attained by the power to interpret the normal conditions in their various stages of development, as well as by the skilful differentiation of the densities where they are interfered with by the thoracic and abdominal organs.

The diseases which can be diagnosticated in the spines of ehildren are not very numerous. The various degrees of thickening and rigidity of the vertebræ, caused by hypertrophic and atrophic conditions, are as uncertain in their etiology as are the same conditions in the joints. They are indeed so rare in early life that they should not be dwelt upon to any great extent. Rhachitic eonditions not uncommonly show themselves in the spine. There are a few specific infections which attack the spine, oceasionally an osteomyelitis, but this is rare. The organism which plays the greatest rôle in the spinal infections of childhood is the bacillus of tubercle. The result of this infection in its chronic form is what is usually, though not advisedly, spoken of as Pott's disease.

A few rules in connection with what we may expect to see and what we are to look for in the Roentgen examination of the spine may be of value to the student and to the general practitioner. Functional or inorganic lateral eurvature is charaeterized by a single or double curve of comparatively slight degree and usually to the left in about 90 per cent. of all eases. In such eases the left shoulder is higher than the right. There is usually no rotation of the transverse processes which are seen equally well on either side of the spine. There are also transitional cases which are produced by such causes as congenital absence of the ribs, causing scoliosis. Again in this set of eases there are curvatures resulting from operations on the 
thorax, notably for cmpycma. Plate 130, Division VI, is an example of this condition. In structural or organic curvatures the following conditions may be looked for: single curves, representing either kyphosis, scoliosis, or lordosis; and compound curves consisting of one or more curves in different parts of the spinal column with rotation in the opposite direction. There are also to be noted changes in the intervertebral discs and in the bodies of the vertebrac showing disturbance of normal structure and abnormal radiability. The pathologic condition may be a periostitis, osteitis or osteomyelitis, with the end results of breaking down of onc or more bodies of the vertebra. The non-tubercular infections are much more rare than the tubercular, which are very common, and of the tubcrcular class the chronic form is more common than the acute. Other conditions, such as osteomalacia, osteogenesis imperjecta, chondrodystrophia foctalis, and malignant growths, may sometimes also be present. Having once detected and located the part of the spine affected, note should be made of the amount of deformity or curves of the whole spine, the number of vertebra diseased, the amount of bone involved, and the condition of the intervertebral dises. The interrertebral dises when diseased usually show atrophy and they may seem to be nearer together than normal.

Plate 100 shows the spine of a colored boy six years of age with rhachitis. The rhachitic process is in this instance in an carly stage of change from the normal, and is especially seen in the transverse processes of the third lumbar vertebra, which are slightly increased in size and show, as does the ilium, a coarse arrangement of the structure of the bone. The radiability is increased throughout the lumbar vertebræ. There is no particular disturbance in the zone of proliferation.

The non-tubercular infections of the spine, although rare, yet must be recognized and separated from the common tubcreular 
infections. The most common form of the non-tubercular affections which can be studied by the Roentgen ray is osteomyelitis. An early diagnosis of osteomyelitis of the spine is difficult. In contradistinction from tubercular there is a more localized infection of one body or partial destruction of the vertebræ. There is more proliferation of tissue about the body of the vertebra and usually very little deviation of the spine. There is usually the history of an acute infection.

Plate 101 shows the lesions of osteomyelitis of the spine in a girl four and a half years old. There is a destructive process with an effort at reconstruction of the second, third, and part of the fourth lumbar vertebræ. The body of the third lumbar vertebra is almost destroyed, especially on the right side.

Tuberculosis of the spine locates itself almost exclusively in the bodies of the vertebræ, attacking primarily only the interior of the bodies and rarely their surface. This tubercular osteitis, when it has progressed sufficiently, causes collapse of the vertebral columns. The intervertebral cartilage is also often destroyed by the process, but the arches of the vertebræ are only in rare instances affected. Abscesses may form later in the midst of the broken-down tubercular bodies of the vertebræ and may be of varying size after they have broken through into the surrounding soft parts. Where the diseased condition is at all advanced the diagnosis by means of the Roentgen ray is easily made, but it is much more difficult in the very early or hyperæmic stage. As the process goes on, however, a larger area is involved and this will be seen in the bodies of the vertebræ, particularly in the spongy portion, as an area more or less definite and showing an increase of radiability. This means that the normal structure of the bone is changed, and that the ray passes more easily through the bone in this, the diseased portion, than through the other portions. If the process continues the area of discase becomes 
larger, destruction of the bone takes place, and finally cheesy degeneration of its centre goes on with still greater increase of radiability. Possibly a localized abscess of the bone may result. This too may be readily detected by its increased density. If the disease progresses we find sof tening, greater density of one or more bones, and a crushing togrether of the bodies. At this time in the history of the case the intervertebral cartilage has disintegrated and disappears. In regard to the formation of an abscess, the abscess may be first exterual, and second, internal, as a mediastinal abscess. Almost every case of tubercular infection of the spine will be seen to have an abscess. 'This condition can readily be differentiated by the ray. To be interpreted from the plate are:

1. The external area of disease, shown in the plate by decreased radiability, usually limited to the diseased portions.

2. The mediastinal as a definite area of increased density ballooning out and around the diseased portion.

Plate 102 shows an absorption and fusion of the intervertebral cartilages of the third and fourth lumbar vertebra. The destruction of a part of the left ilinm is seen just above and outside of the acetabulum. The third and fourth vertebra rive evidence of necrosis, showing more destruction on the right side than on the left. There is an evident necrosis of the bone of the ilium, with abscess about the left hip-joint. There is decreased radiability of the femur on the left side.

Plate 103 shows a tubercular process of the sacro-iliac joint, causing deformity of the pelvic line of the left side. There is also a marked increase in the radiability of the ilium, except where a dark rim denoting the boundary of the destructive process shows new bone formation and consequent decreased radiability. There is more or less destruction of the sacrum on the left side, and marked deformity of the pelvis resulting from the actual destruction of the 
sacro-iliac joint. There is also an atrophy of quality of the acetabulum, as well as of the whole femur on the left side, as shown by the increased radiability, although there is no change in the size of the shaft.

Plate 104 shows in the tenth clorsal vertebra the beginning of an absorption and a destruction with fusion of the body with the ninth dorsal vertebra. At about this point thickening of the tissue and the formation of an abscess are apparent in the Roentgenograph.

Plate 105 is that of a child four years old and shows tubercular lesions of the spine. There is an absorption of the tenth, eleventh, and twelfth dorsal intervertebral cartilages to the right, with an apparent fusion of these bodies. At the tenth dorsal vertebra is seen the beginning of an absorption and a destruetion with fusion of the body with the ninth dorsal vertebra on the left side. At this point also there is either breaking down of the neighboring tissues or the formation of an abscess, as is seen to the right in the Roentgenograph.

Plate 106 gives a lateral view of the same subject, and shows that there is eomplete absorption of the twelfth dorsal and partial destruction of the eleventh dorsal vertebre. It is well to compare this process, whieh is clean cut, with that shown in osteomyelitis, Plate 101. It will be noticed that the abdomen is rather prominent and that lordosis is present. 
PLATE 90.

FRACTURE OF SKULL.

Boy, age 13 years. (Reduced $39 \%$ )
A. Frontal sinus.
B. Orbit.
C. Sphenoidal sinus.
D. Region of antrum.
$E, F$. A fracture of the base of the skull. 
Piaté 90

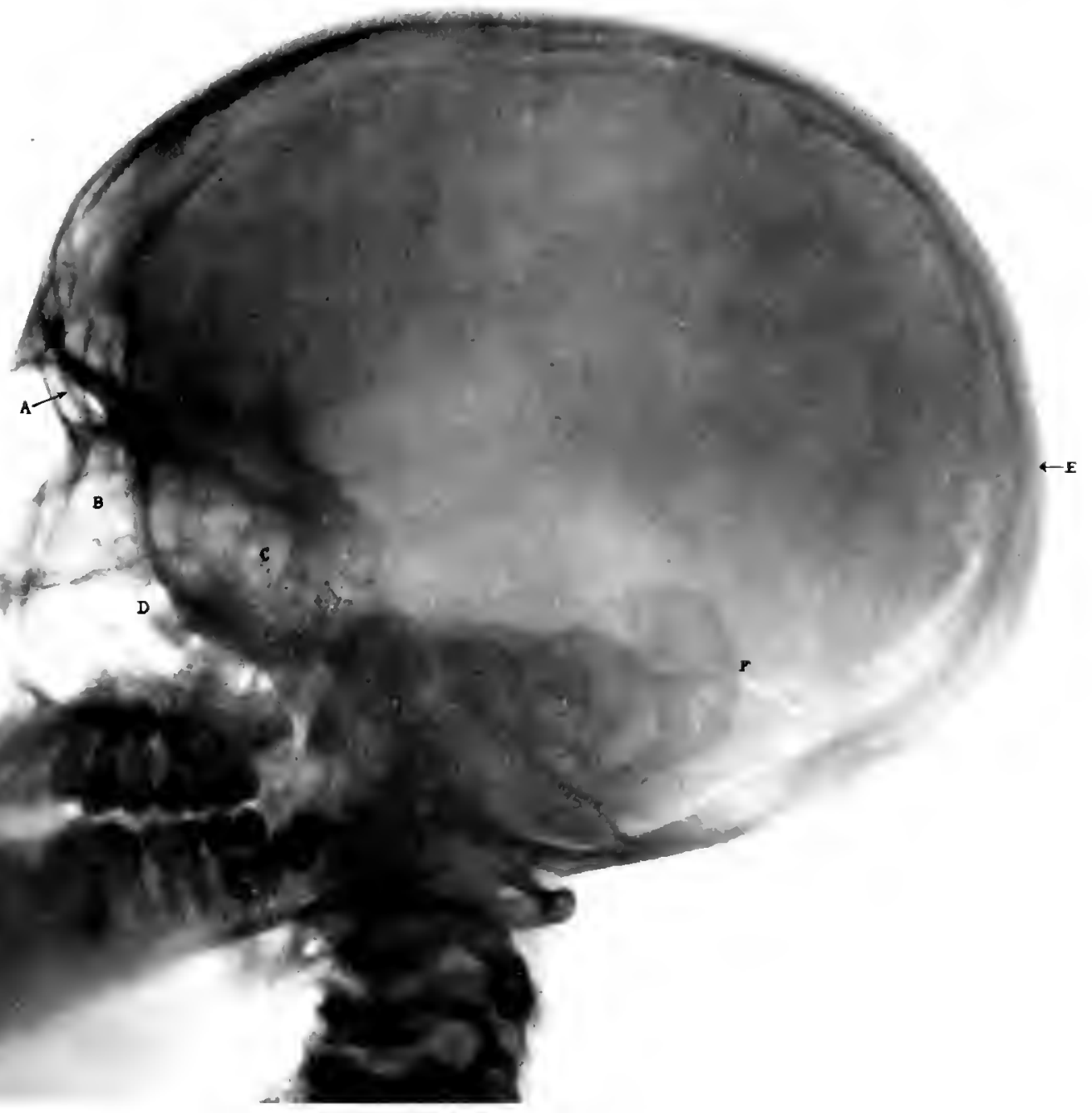


I'INT: :11.

1:THMOHITH:

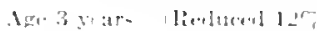

1. Frontal sinut

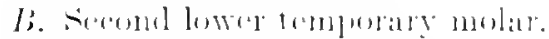

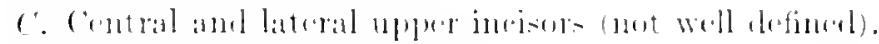

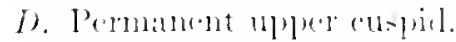

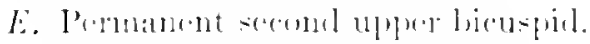

F. First permanent upper melat.

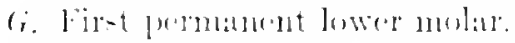

II. Inflammatery annal (nthumbir.

I. Hinit.

The increated denaty shows the prowes of inflammation in the athoneid bonc, frontal sinus, ant ant rum. 
PIATE: 11

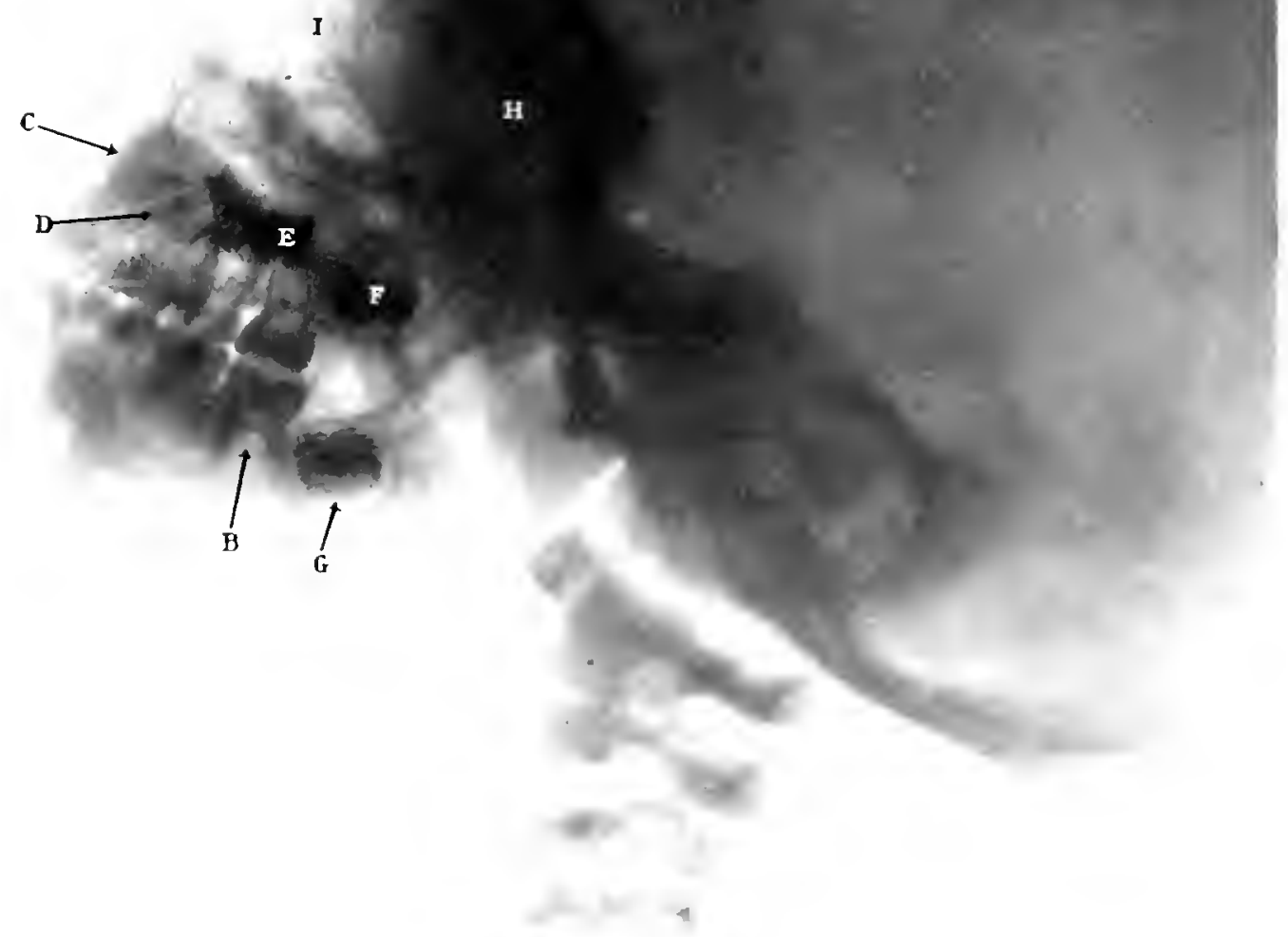


PLAT: :2.

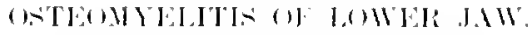

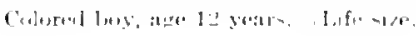

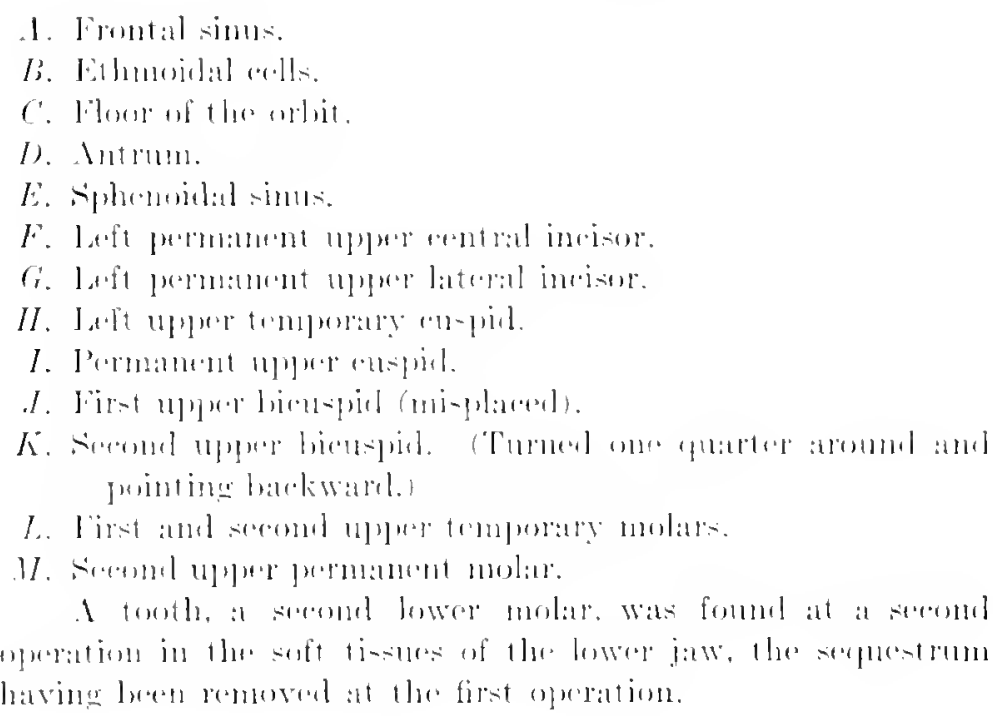


I’」.XT:

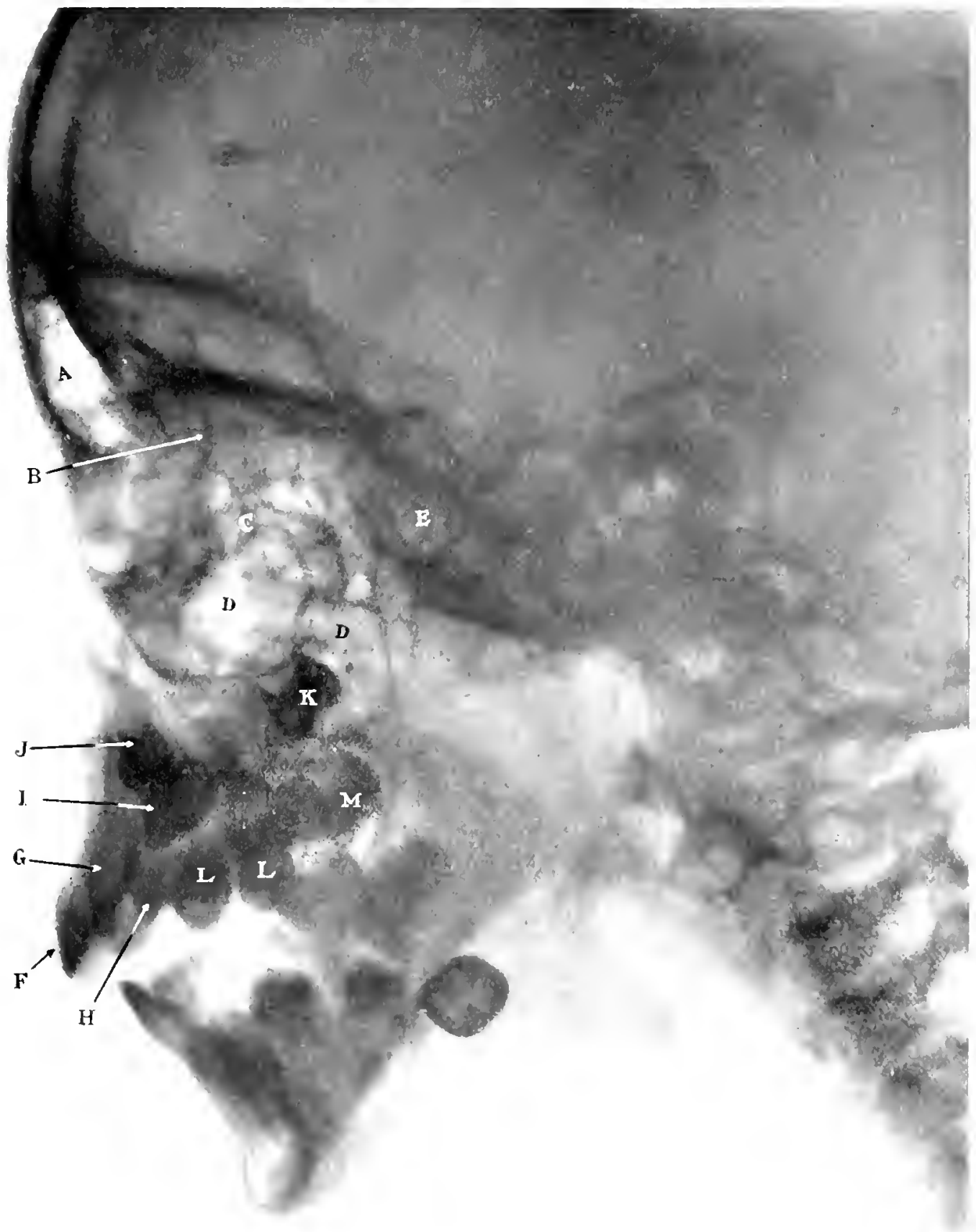




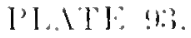

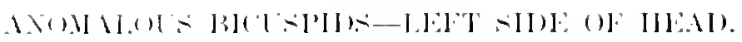

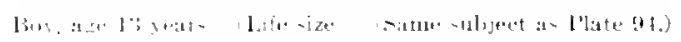

1. Inontal -imits.

13. Whil.

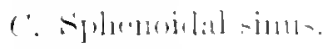

f). Intrmis.

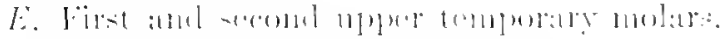

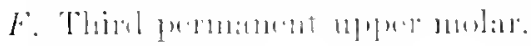

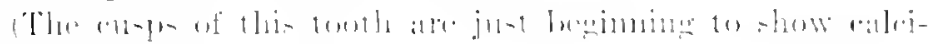
fic:ation).

r. Cly of thiml hown mular.

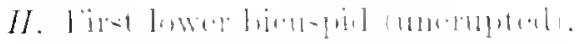

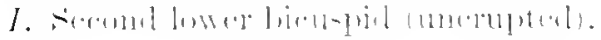


PLATL:?:;

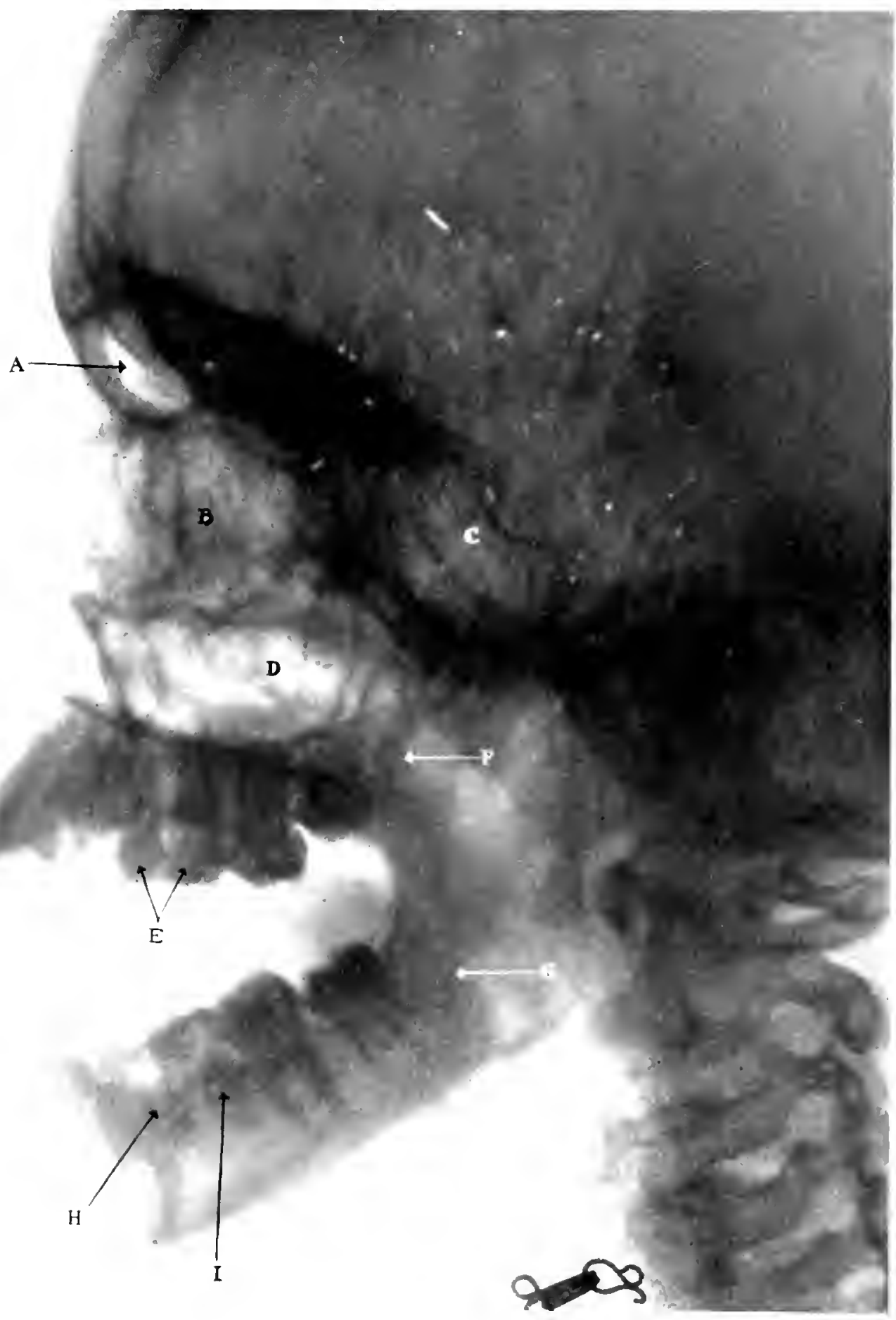


1'L. TT: :1.

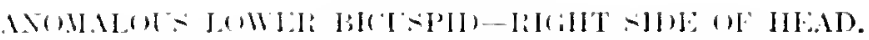

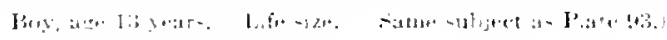

1. lonisil -inns.

IS. Hhil.

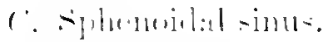

J). Intrumi.

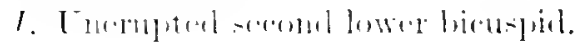


PI. 1T\%: 9.8.

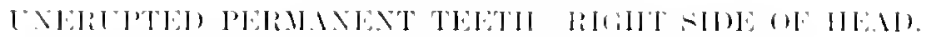

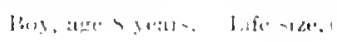

1. Findial -inus.

li. Mhil.

(. Sillemorial sintus.

1). Jithmoislal rells.

E: aml Ji, Intmint.

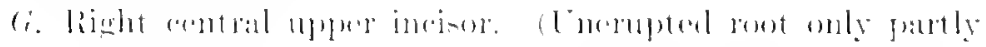
(alleifinl.)

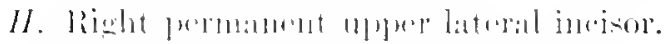

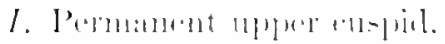

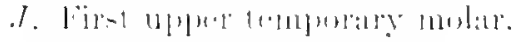

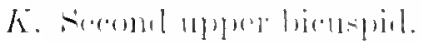

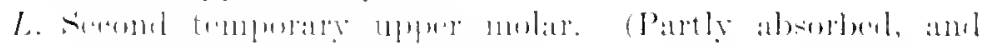

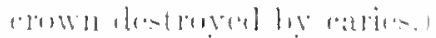

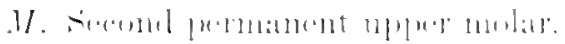

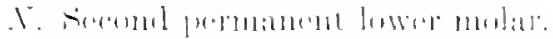

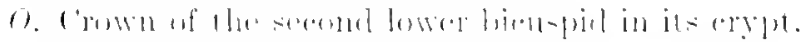

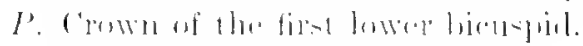

(?. Tintle on left sille of the jall. 
I'I.119:5

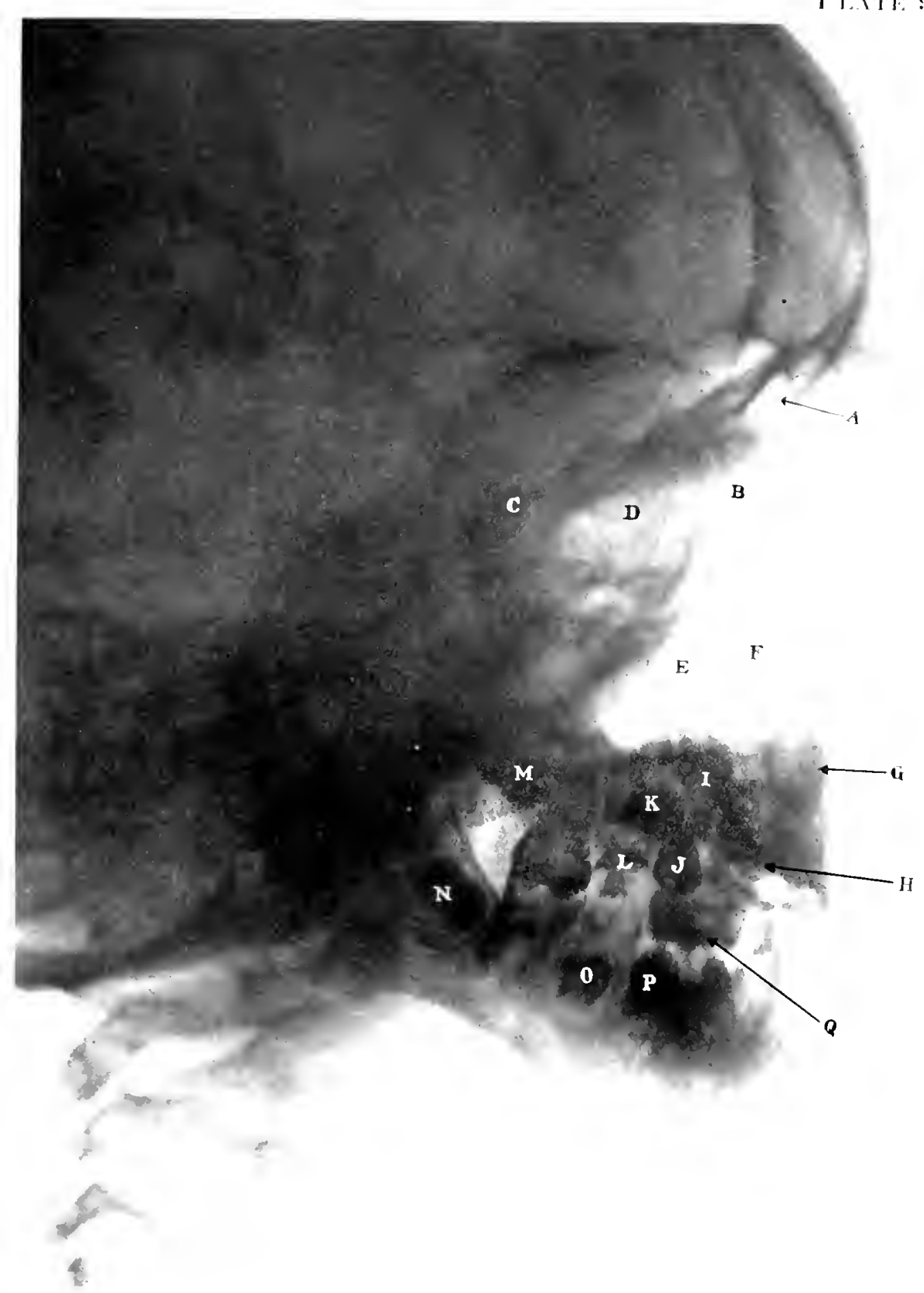


P'. ITE: 90.

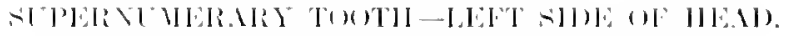

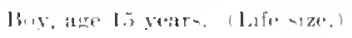

1. linolital simus.

li. lithmitial rells.

1'. Sphonomial simus.

1). Roof of arlit.

l: Intrum.

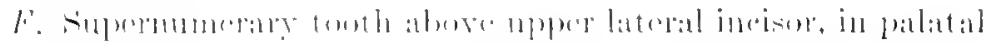
ingerent.

fi. Thinl แp

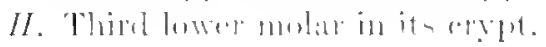




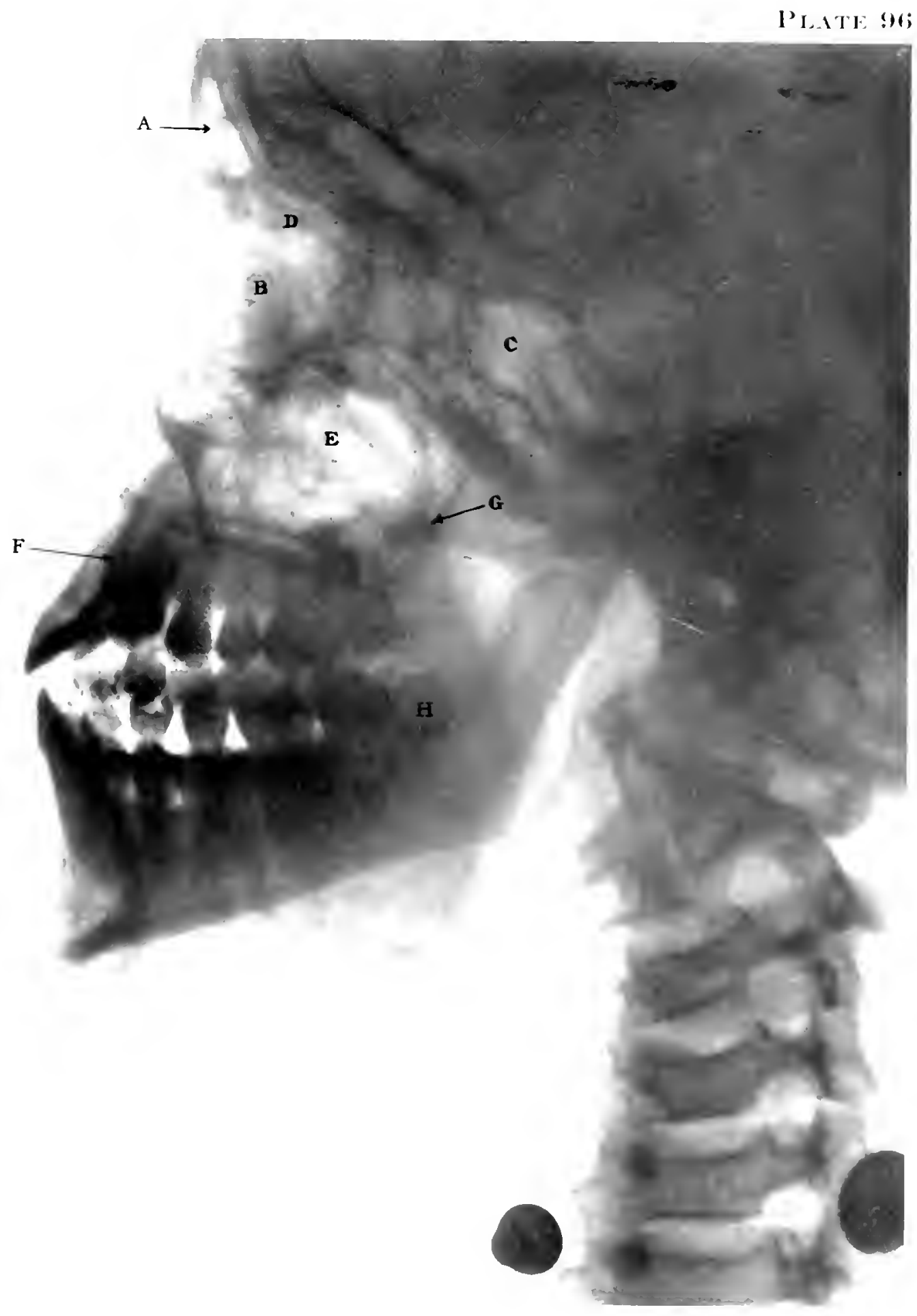


PI.ATI: $1 \%$

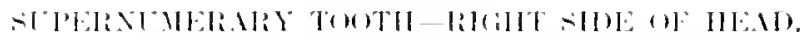

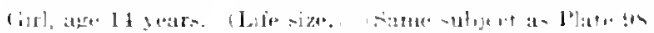

1. Fontal-inus.

IS. Ethmoiclal rells.

r. ()rbit.

E. Intrum.

F. Cilcunoid foris.

r. Comblyle of inforior masillang bome.

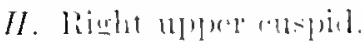

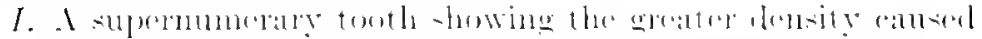

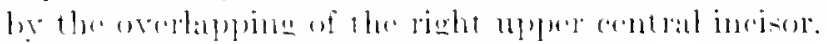

$J$. Right upper lateral incisor. 
PLATE:

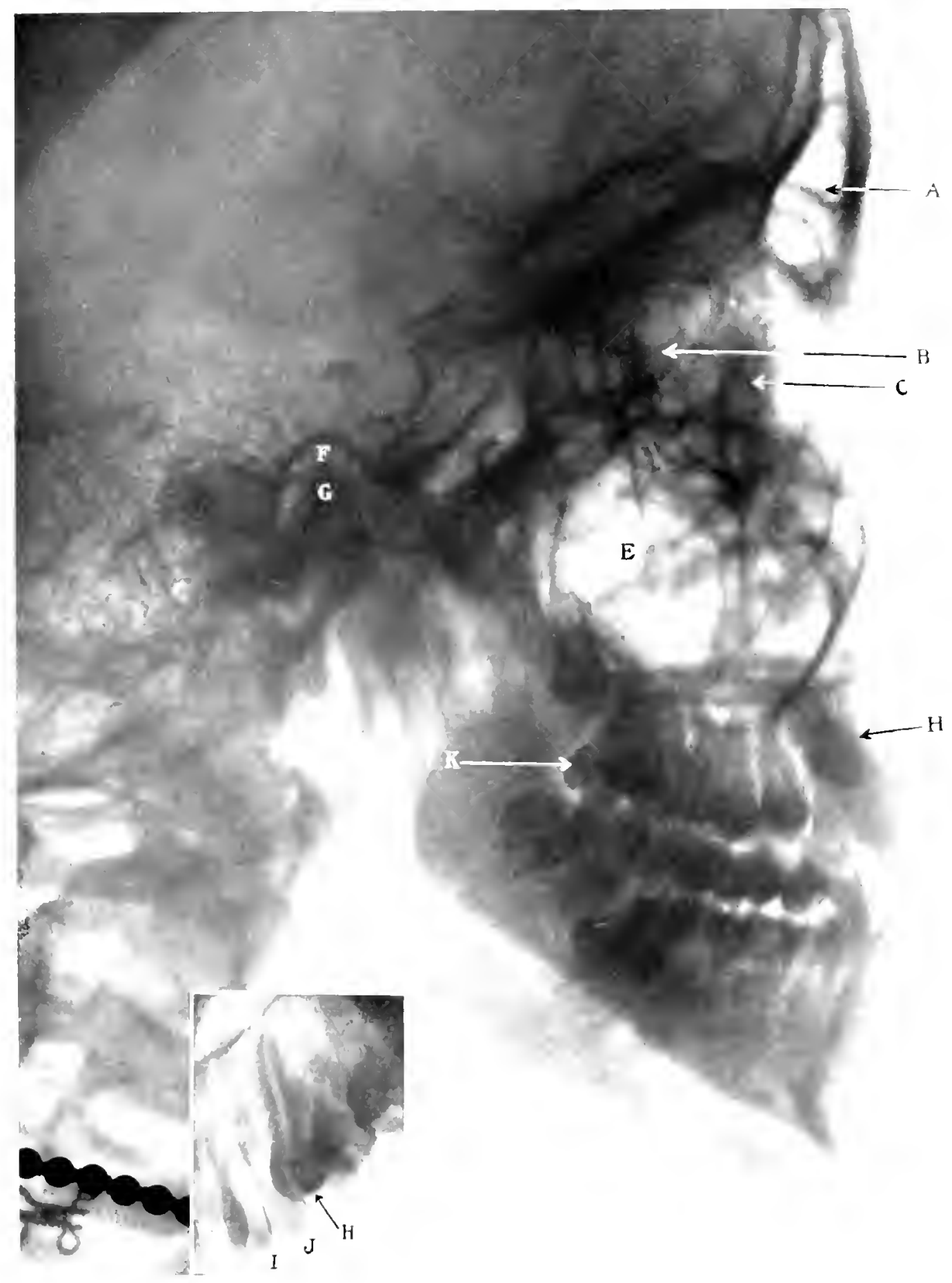


J.Al:

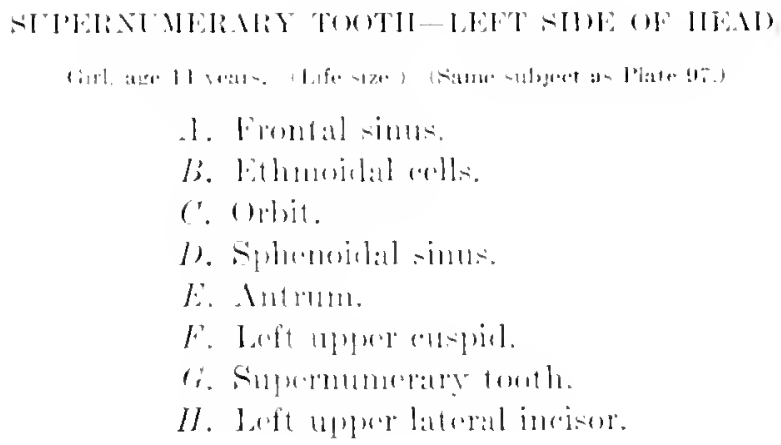


PHATL:9

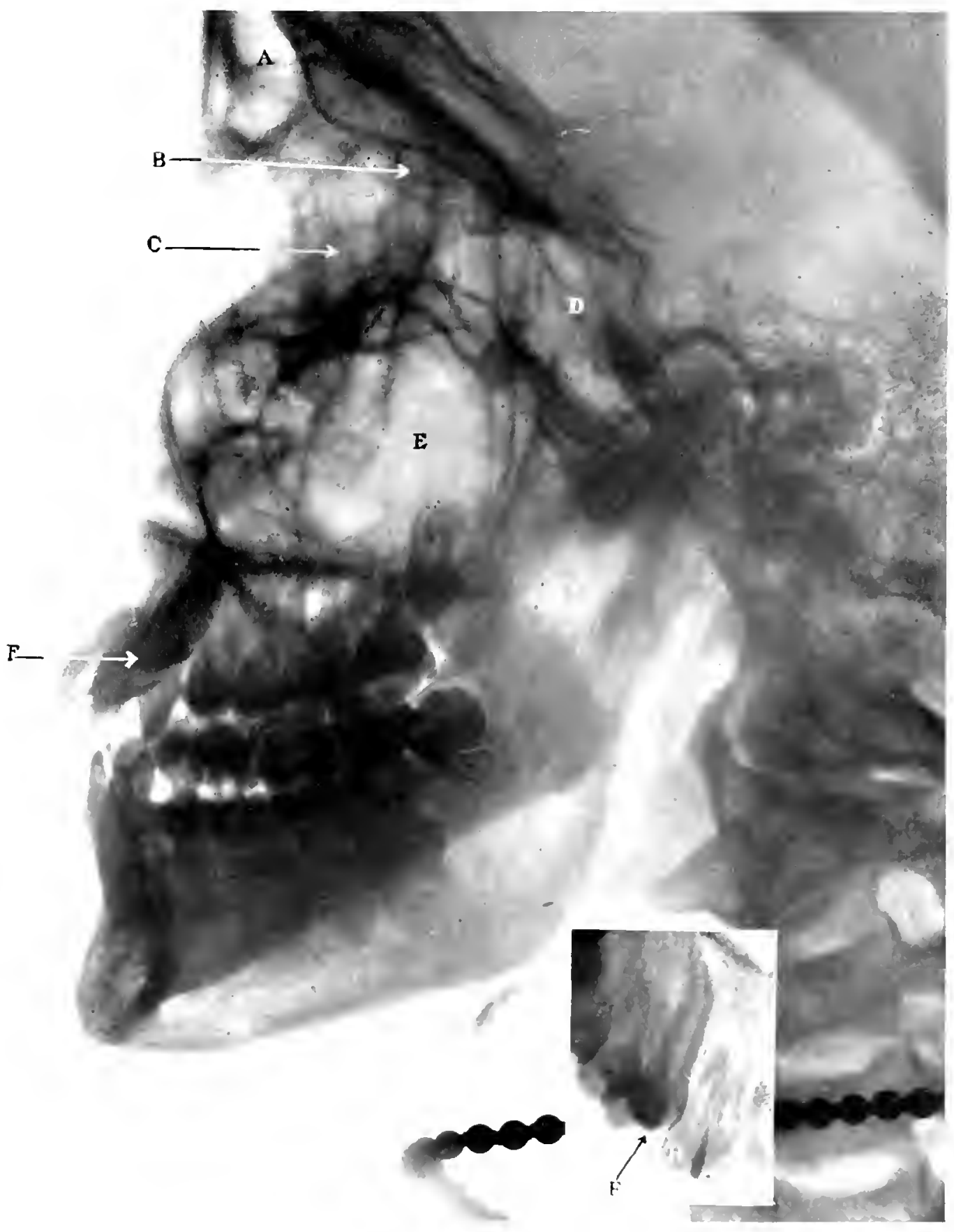


P'. $1 \mathrm{TP} 99$.

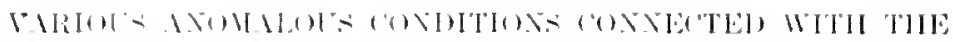
]EFTH.

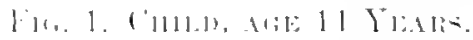

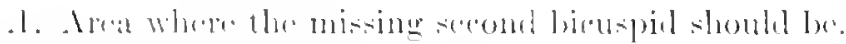

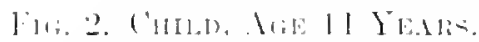

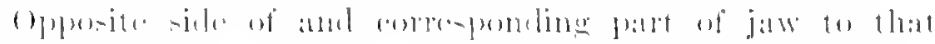

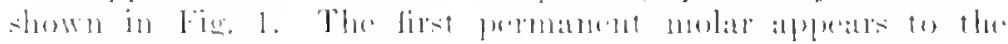
lieht in thi-lin.

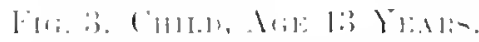

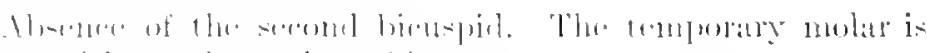

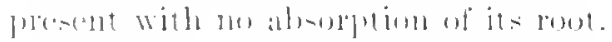

I'ic, I.

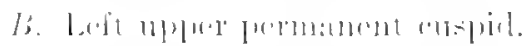

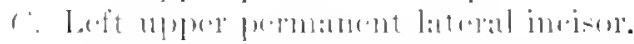

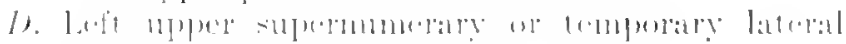
incoines.

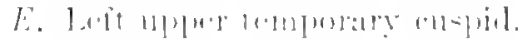

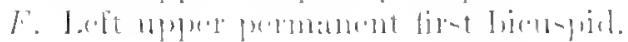

Jisi, .j.-

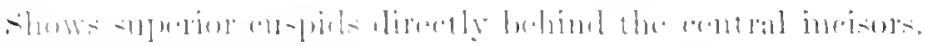

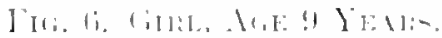

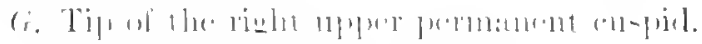

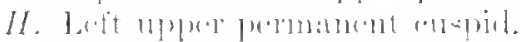

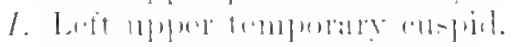

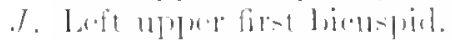

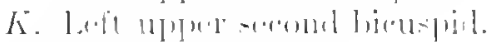

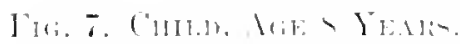

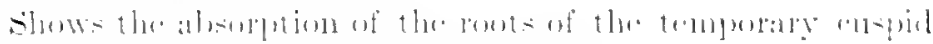

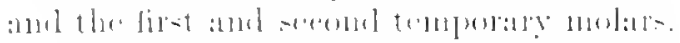

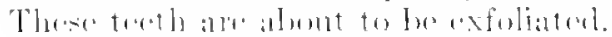

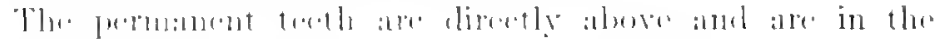

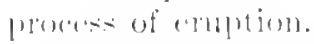

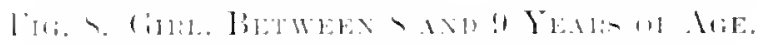

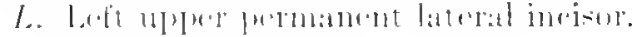

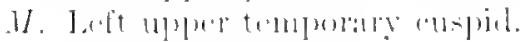

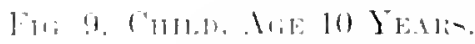

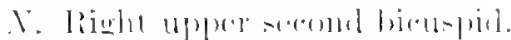

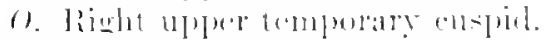

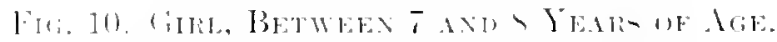

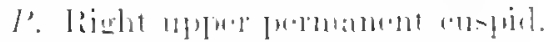

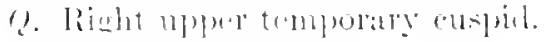

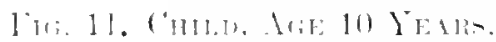

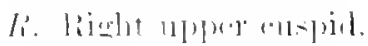


PLATE (I!)

FIG. I.

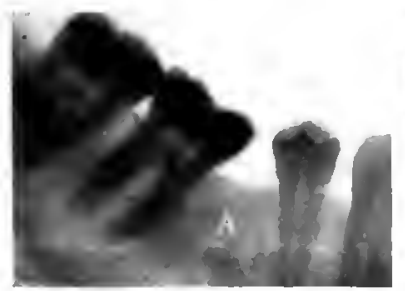

FIG. 4.

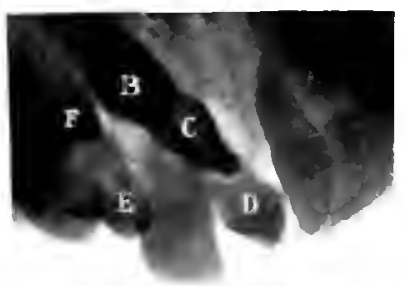

FIG. 7 .

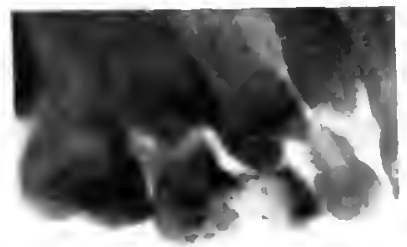

FIG 10

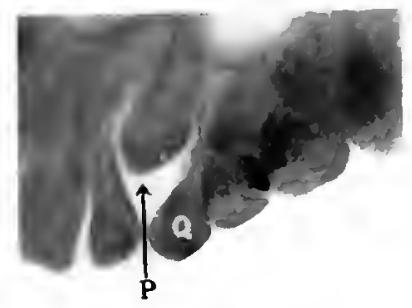

FIG. ?

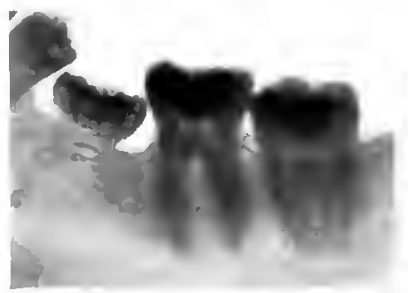

FIG. 5 .

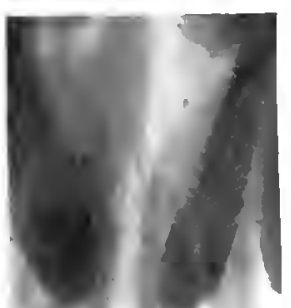

FIG 8

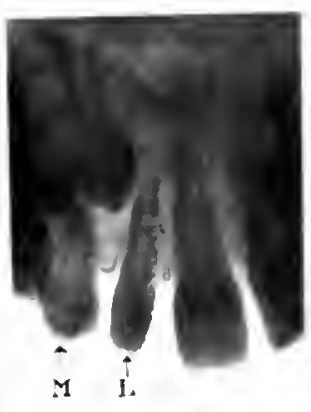

FIG. 3.

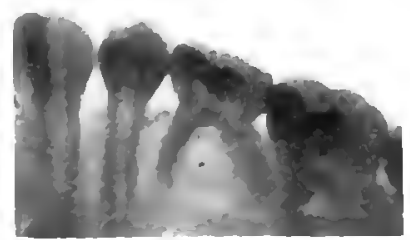

FIG. 6

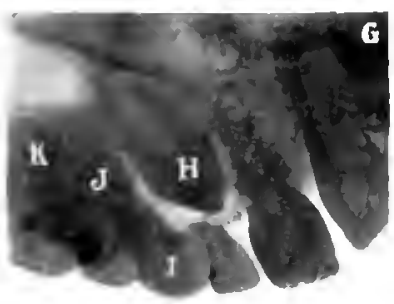

FIG. 9.

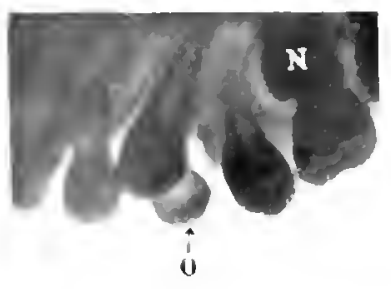

FIG. II.

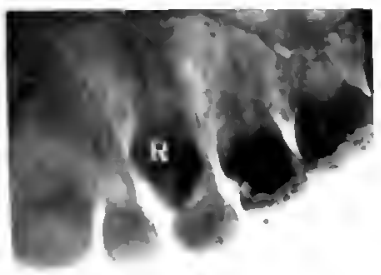


I'ISTTE: 100.

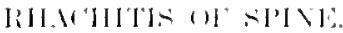

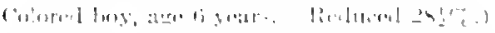

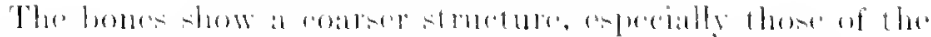

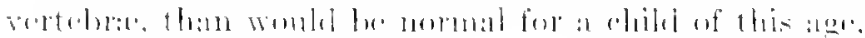

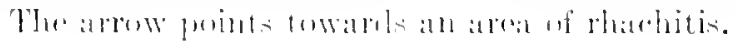


P'LATE 100

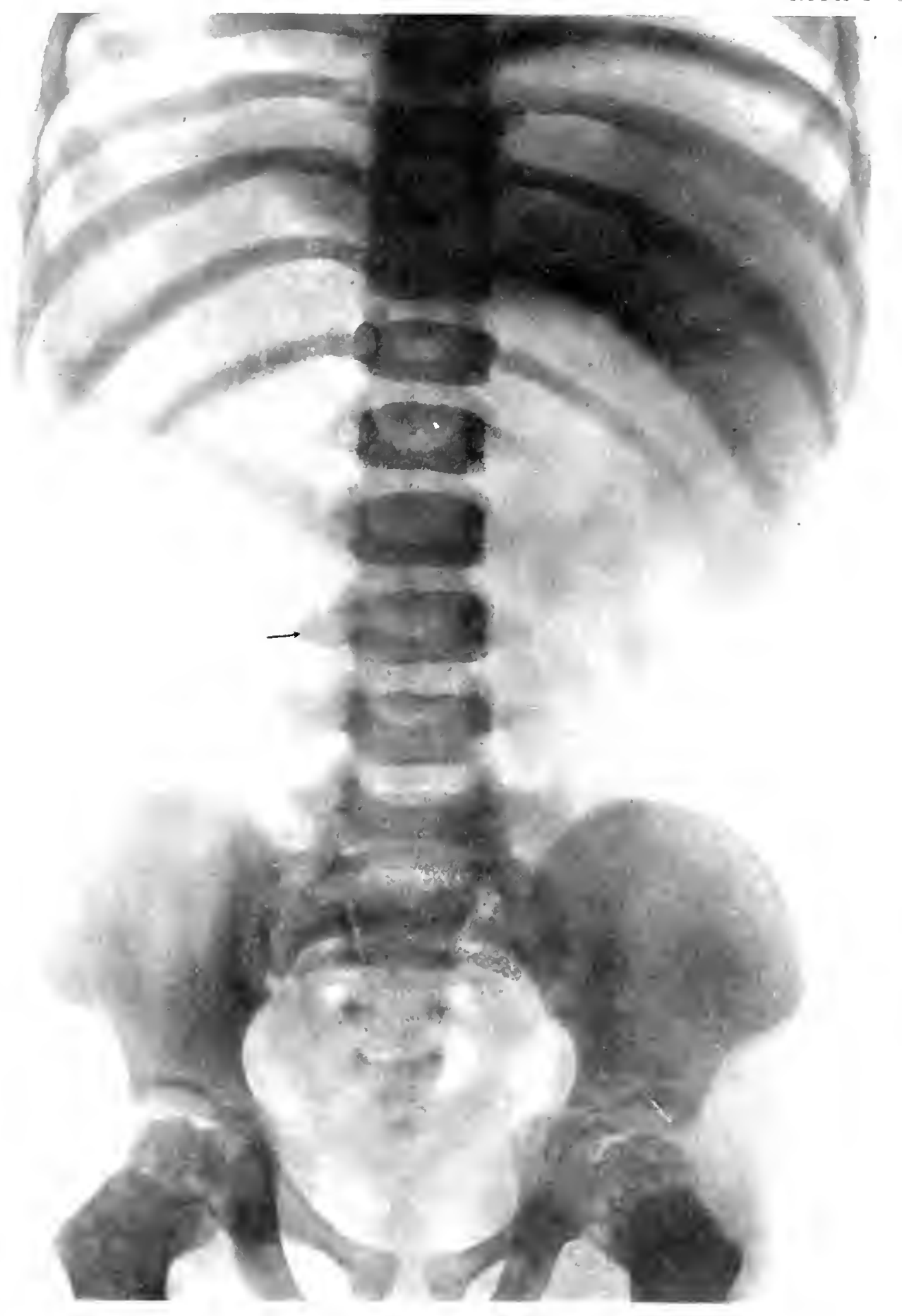


P'L.T'T: 101.

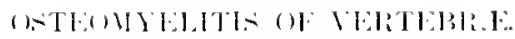

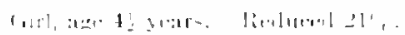

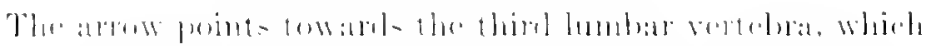

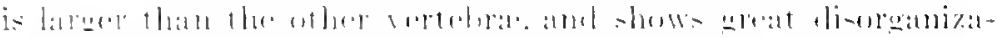

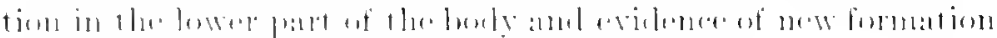

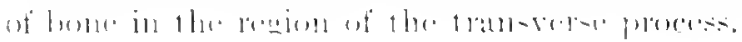


I'L. STE 10I

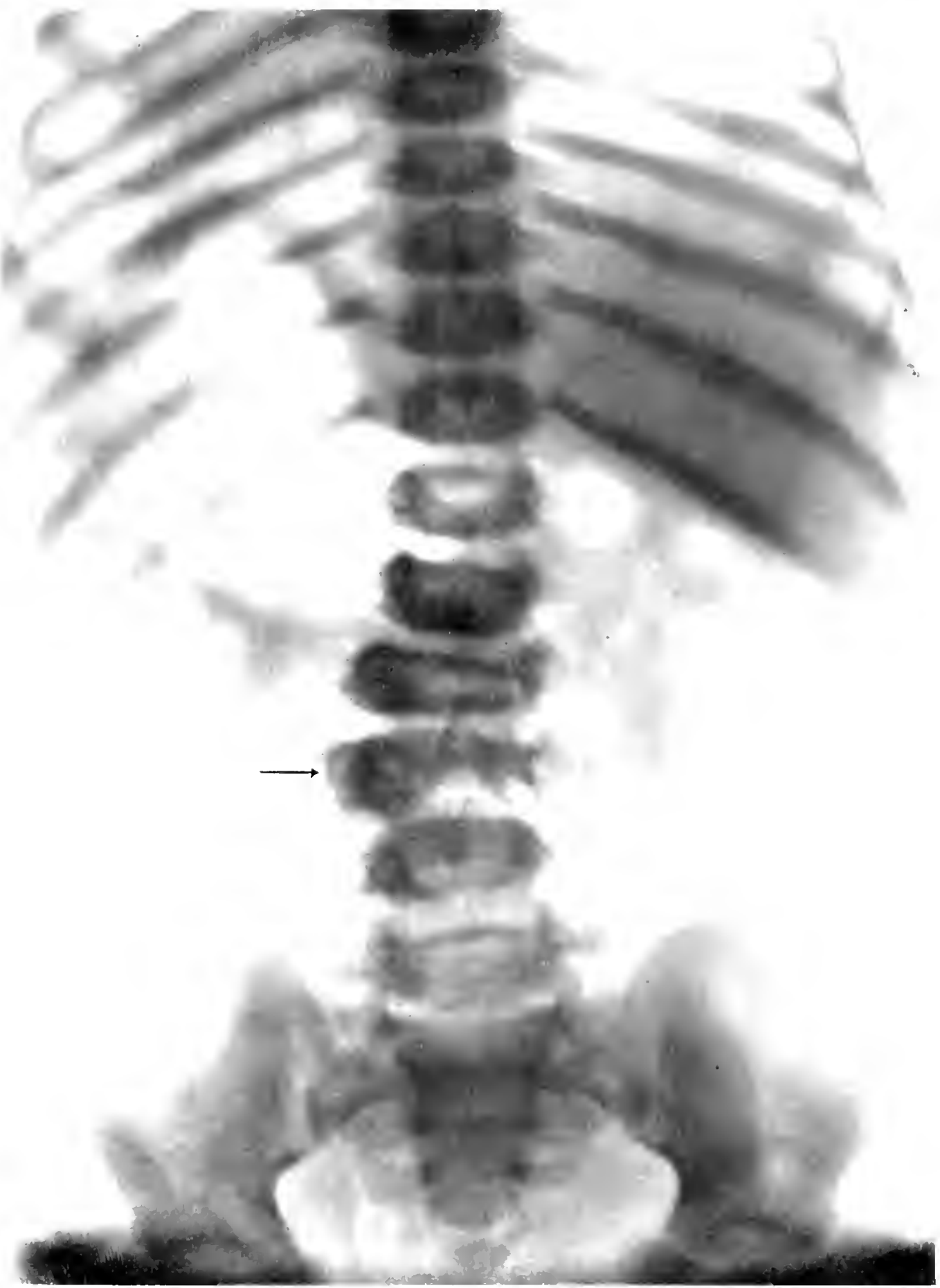




\section{I'I.ITI: 10.}

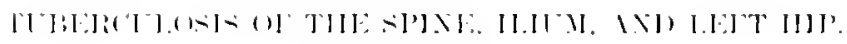

lienthered $21+2$

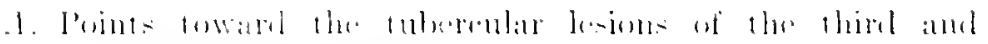
foumh lombar vertobrats.

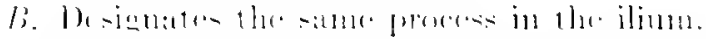

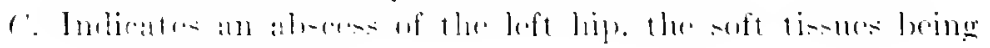

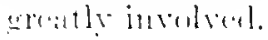


PIATE lon

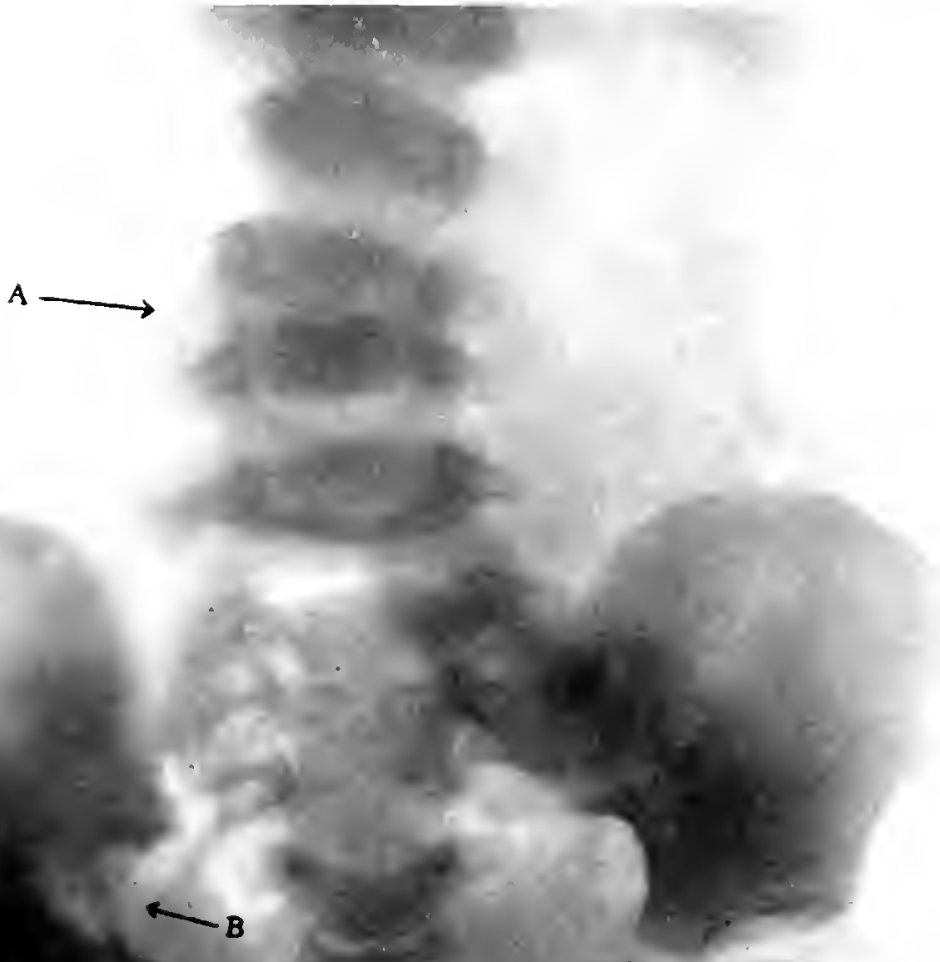

C
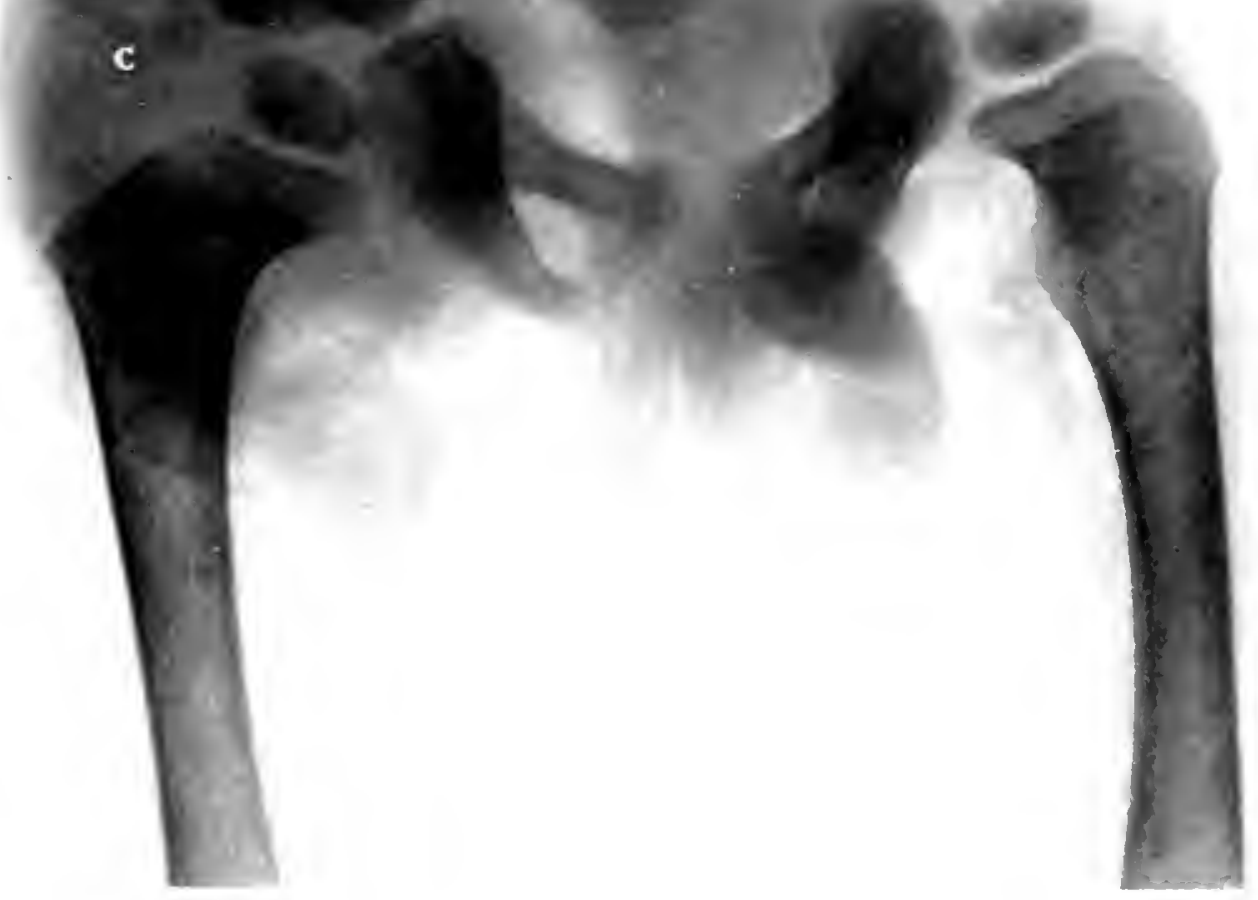
PI ITE 10:?

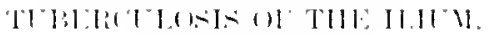

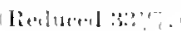

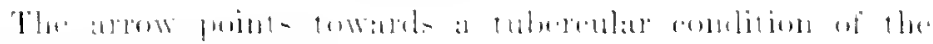

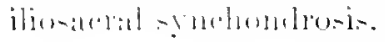


PLATL: I1:?

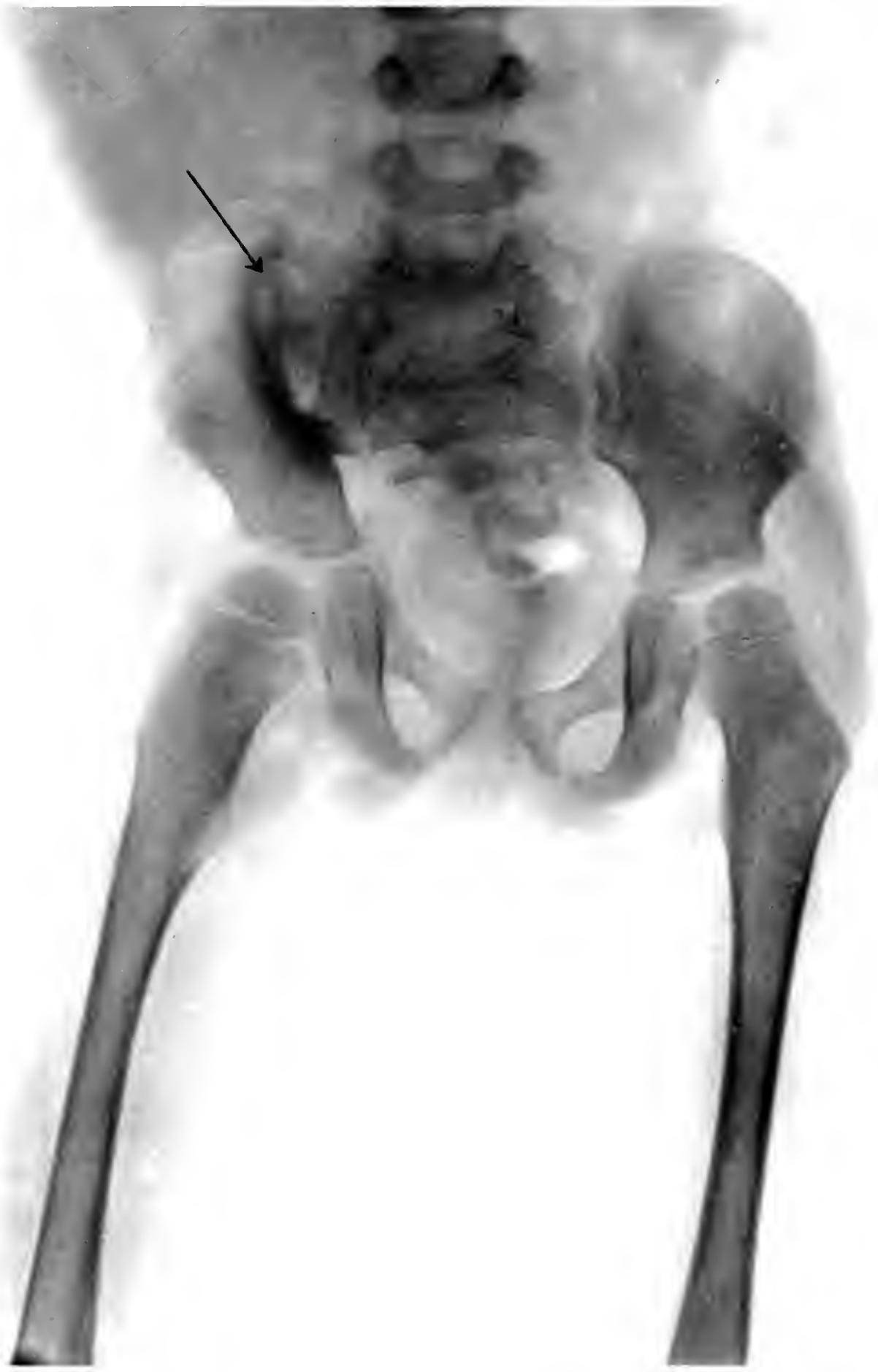


PI.TTY: 101.

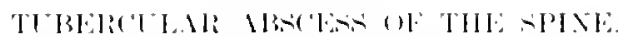

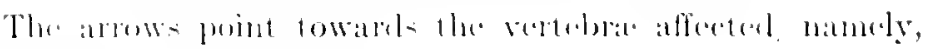
llu ninth :mol tomth dorsil. 
Plate 104

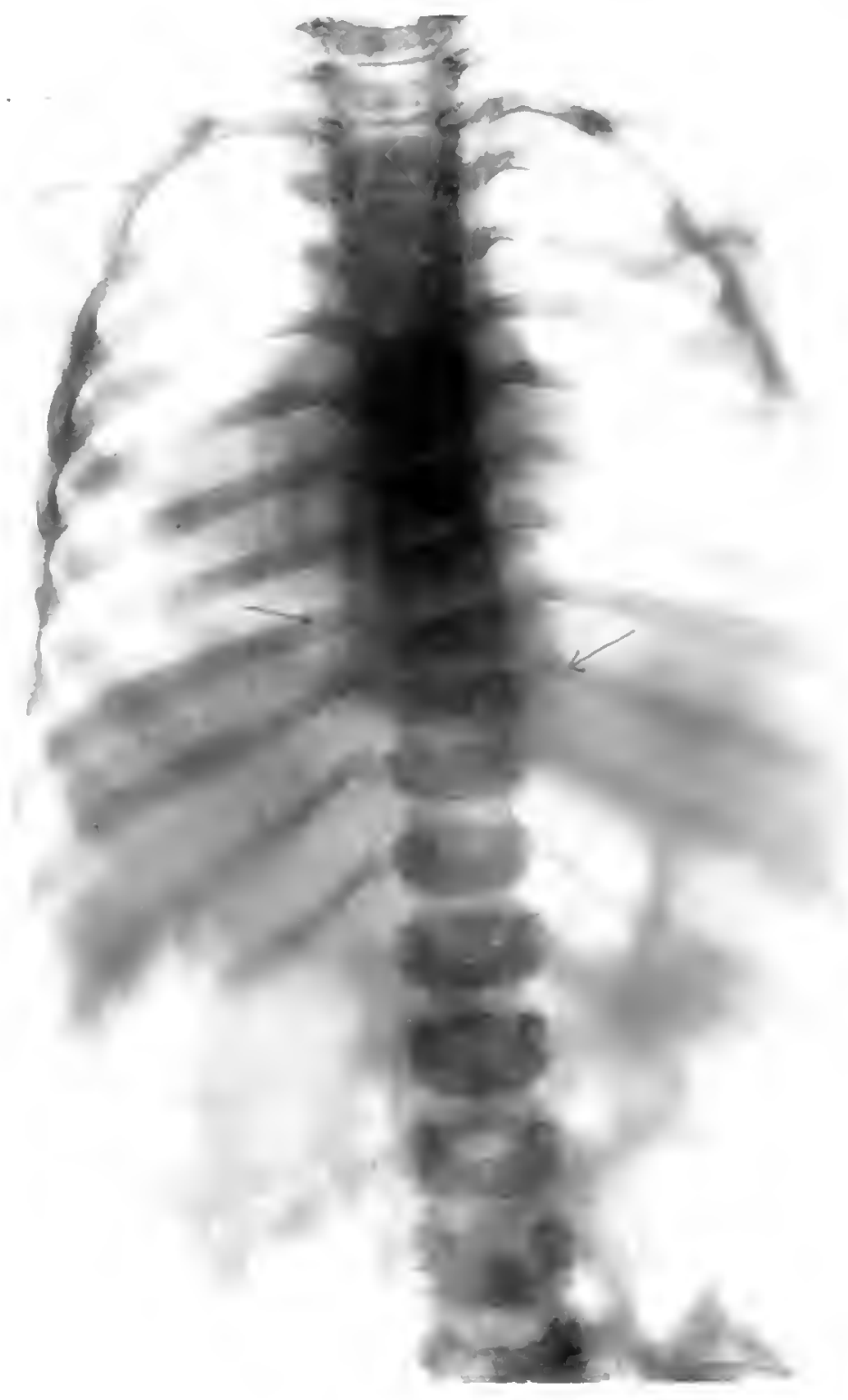


P'L IT' $10 \overline{\text { T. }}$

TIBLATIAS OH THE SPIXE.

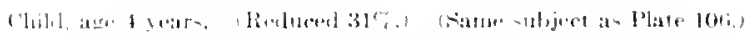

10. I a and l's indieate the breaking dewn of the interverfolmal ratriligen and the fusion of the bolies. 


\section{PIATE 10.5}

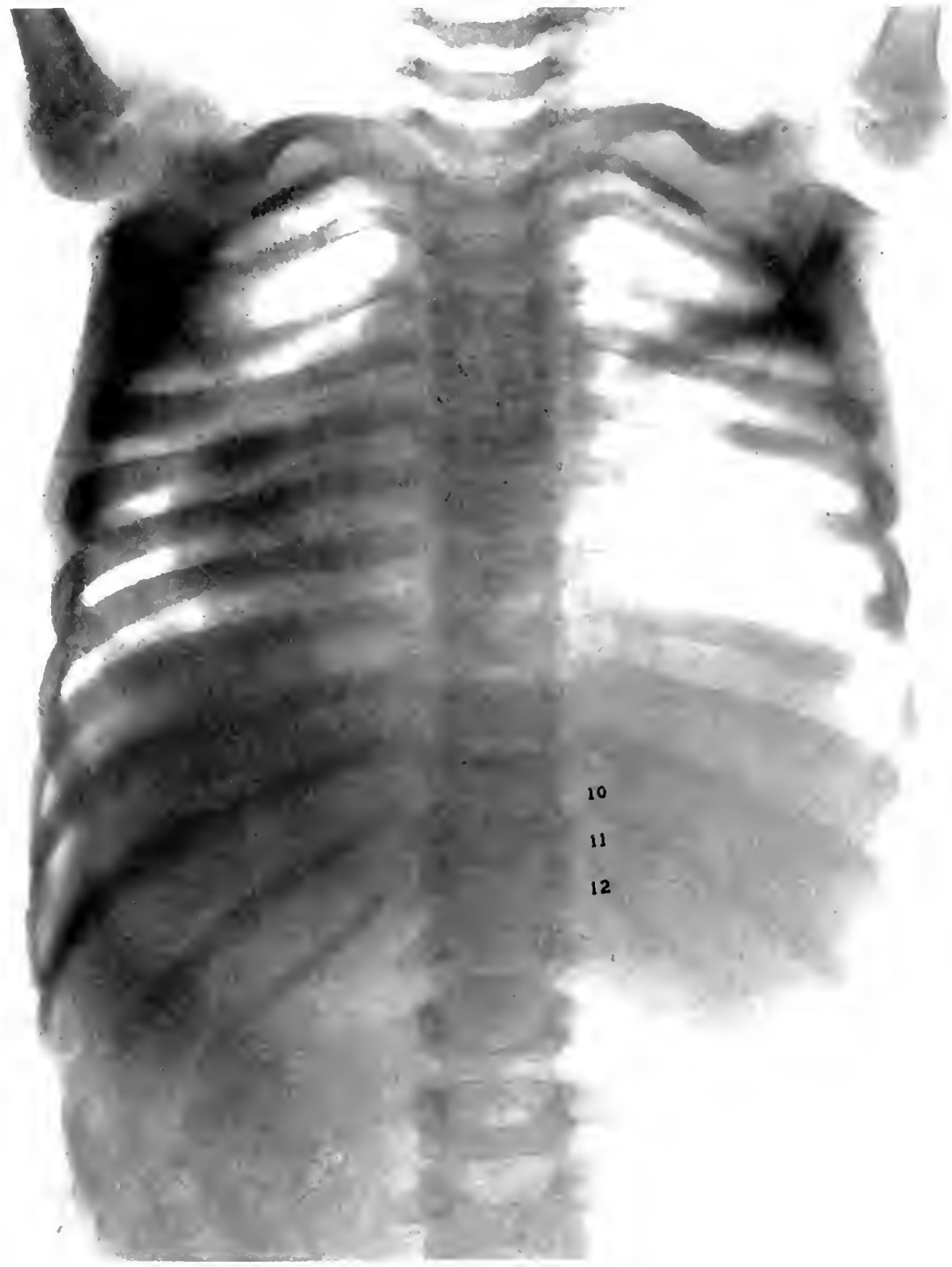




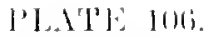

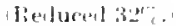

The same "ase ats Plate 10,j but prosenting a lateral vine of the shine.

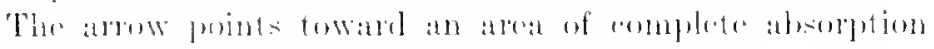

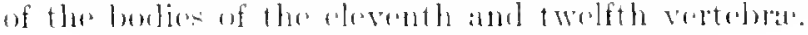

l'. The anterios atseret of the bouly. 
PIATe lo6; 



\section{Division VI}

THE BRONCHIAL NODES-BRONCHI-LUNGS-PLEURA-HEARTPERICARDIUM-ANEURISM

Anovg the more difficult interpretations which we have to make in reading a Roentgenograph are those connected with intrathoracic conditions. It is very necessary that we should first earefully study the normal conditions as seen in Plates 8 and 19, before attempting to interpret the abnormal. This is diffieult since there is no one set of pietures which we ean memorize as normal, and since the entire interpretation of an individual plate depends upon the eomparison of the different densities which happen to appear on that individual plate. These densities differ very mueh, whether of lung, heart, or liver. In a Roentgenograph of a normal thorax we can see the very slight density of the lung with its great radiability, the slightly greater densities of the bronchial nodes, the much greater densities of the heart and spine, and the still greater densities of the heart and spine where they overlap each other and show very slight radiability. To be noted earefully as an aid in detecting abnormal eonditions are the outlines of the heart and its angle with the liver, especially on the right side. This angle is very important where there is a question between a pericardial effusion and an enlarged heart. To obtain the best results in Roentgenographs of the thoracic organs certain details connected with the technic are important. Among these is the time of the exposure.

Dr. Percy Brown has shown for me the great advantage of a short over a long exposure in Plates 107 and 108. Plate 107 shows an exposure of six seconds with the child breathing normally. Plate 108 shows the thorax of the same child taken with the respiration arrested and with an exposure of one second. The latter 
picture is seen to portray the lungs and heart with far greater accuracy than the former.

The immobility of the parts taken explains these results, and naturally the immobility is greater the more the respiration is arrested.

\section{BRONCHIAL NODES}

The bronchial nodes under normal conditions are of ten not seen in the plate, but where the movement of the lung is at all restricted, as in disease, and often when the child is breathing very quietly and the expansion for half a minute is slight, the nodes when enlarged can be distinguished. They are seen especially to the right quite elose to the border of the sternum, where they are not complieated by the pieture of the heart. When enlarged they become quite distinct.

Plate 109 shows the picture of a girl twelve years old. The lungs, pleura, pericardium and heart are normal. On the right side of the thorax just outside the boundaries of the heart and extending in a narrow area from the top to the bottom of the thorax are a number of separate dark areas in each of the interspaces. They evidently represent areas of enlarged bronchial nodes, and are seen at times in a normal thorax.

Plate 110 shows a transposition of the heart and liver in a child. On the left side of the thorax there will be scen to be far less dark areas than on the right, but that whatever areas of lessened radiability there are on the left from the second to the seventh ribs are very marked. These dark areas show the characteristic appearances of an advanced tuberculosis of the bronchial nodes. The entire area to the left of the heart is mottled and heary in outline, showing that the bronchial nodes are involved, but to a lesser degree than on the right side. The lower part of the lung on the left is seemingly normal; on the right side the density is very 
great throughout, but is greatest from the fifth down to the tenth interspace. In this region and between the sixth and eighth interspaces just to the left of the spine can be seen the density of the heart. The cause of the general intensely increased density on the right is possibly thickened pleura, and except at the apex practically no lung substance can be seen. The dark area at the lower point of the thorax on the left is the picture of the transposed liver

\section{BRONCHI}

When expansion of the lung is restricted by consolidation, or when the breathing is unusually quiet, the bronchi can be distinguished quite readily as faint areas of increased density radiating from the base of the lung. This is seen in Plate 19, Division I. If this appearance were produced by a mediastinal abscess the condition would be represented by a balloon-shaped area on either side of the mediastinal line instead of the radiating pranch-like irregularities seen in the picture. The movements of the bronchi are usually very free on account of their being surrounded by normal elastic tissue, and therefore we are more apt to see them when there is a consolidation of the lung, with its consequent restriction of motion.

\section{LUNGS}

Atelectasis. - There is no especial difference which can at present be detected between the decreased radiability of an atelectasis and that of a pulmonary consolidation. When at birth, in connection with symptoms of cyanosis, rapid irregular breathing, coldness of the extremities, and a subnormal temperature, we find parts of the lung-tissue showing a decidedly decreased radiability, we can with considerable assurance make the diagnosis of atelectasis. At a later period also as a sequel to pertussis an atelectasis may occur and be detected. When this condition is seen, the differential diagnosis 
from the pictures resulting from infectious processes in the lungs is made by the absence of fever, and, if the ehild is old enough to expectorate, by the absence of organisms in the sputa. In saying this we simply acknowledge our lack of skill in the interpretation, as a different picture is evidently before us, the atomic weight being different in the two eonditions, and the radiability therefore being greater in atelectasis than in consolidation. When in the future the Roentgen apparatus has been perfected and the eye is more educated, we shall be able to accomplish far more than is now possible by means of our present art.

Plate 134 represents the condition just described as collapse of the alveoli of the lung.

The pictures which show abnormal conditions in the lungs vary according to the extent of the areas affected. Thus, when a whole lobe is involved, as in lobar pneumonia, a continuous broad tract of lessened radiability discloses such a condition, while smaller, irregularly distributed areas, usually bilateral, point towards a bronchopneumonia. Again, still smaller scattered areas over the whole lung lead us to suspect miliary tuberculosis. We must, however, merely consider these Roentgen pictures as suggestive rather than diagnostie, and only confirmatory of other signs, both rational and physical. In certain cases, especially in infants and in young children, where the physical signs from auscultation and percussion fail to detect a consolictation of the lung-tissue, the Roentgenograph shows a density which reveals the cause of the respiratory and constitutional symptoms corresponding to a consoliclation of the lung-tissue, and gives a means by which we can eliminate the presence of a foreign body or a mediastinal abscess. A mediastinal abscess is rare in early life except in connection with tuberculosis of the spine (Plate 104), where it can be detected by a picture in connection with the Roentgen examination of the spine. 
Plate 111 shows the homogeneous area of a lobar pneumonia of the left upper lobe in a girl three years old. This picture gives the characteristic appearance of an involvement of a whole lobe. The outline of the scapula can still be seen. The remainder of the lung and the density of the heart are normal.

Plate 112 shows a lobar pneumonia in a boy twelve years old. The process is seen to involve the right middle lobe and is plainly seen in the third, fourth, fifth, sixth, and seventh interspaces. A consolidation on the left is also seen at the base of the lung from the fifth interspace down. The outline of the heart is rather indistinct, but the cardiohepatic angle can still be distinguished. The upper and lower lobes on the right and the upper lobe on the left seem to be normal.

Plate 113 shows a lobar pneumonia of both lower lobes behind in a child ten years old. The right lower base shows much decreased radiability but the cardiohepatic angle is evident. The process seems to involve the entire lower lobe on the right. On the left a similar process in the lower lobe makes the outline of the heart rather indistinct. The upper lobes are practically normal, and on both sides show the faint pictures of the bronchi, especially at the right apex.

Plate 114 shows the density of what from the clinical history was a case of unresolved pneumonia in a girl four years old. The pneumonic process is shown in the upper part of the right lung. There is a dorsal curvature from position shown in the plate.

Plate 115 shows the thorax of a girl twenty-seven months old, where the clinical diagnosis of a Mongolian idiot was made. The history of this ease has already been described in Division III, Plate 65, showing the hand and knee. When the child was first seen the clinical examination showed that it had a consolidation of the upper part of the right lung. The Roentgenograph showed complete consolidation of the upper lobe. Four 
weeks later another Roentgenograph was taken and showed, in the middle of the dark area representing the solidification, a round, elearly defined area with increased radiability. This is seen in Plate 116. The tuberculin test in this case was negative, and the question was whether we were dealing with a eavity or with an area of the decreasing consolidation of a resolution in a pneumonic area. The elinical signs were those of resolution. A Rocntgenograph taken still later, Plate 117, showed that the light area was increasing.

I was unable to get a later Roentgenograph of the case, but I was notified that the temperature was normal and that the child was improving. A series of Roentgenographs of a case of this kind would be exceedingly valuable for learning to determine whether we are dealing with a eavity or with an area of resolution. Sufficient work in this direction has not yet been done to justify us in any further conclusions regarding the case at this stage. Two months later the ehild was reported to have had a hemorrhage seemingly from the lung and to have died suddenly (no autopsy).

Plate 118 shows the Roentgenograph of a child four years old. The coarse mottled appearance throughout both lungs shows general infiltration, and the definite areas of decreased radiability are what would be expected from a case of bilateral bronchopneumonia. To be noted is the still ununited upper epiphysis of the humerus, which is supposed to join at about five ycars.

Plate 119 shows a pncumonia of the right lung produced by a china doll's arm which was inspirated by a girl four years old. The progress of the case is seen in Plates 148, 149, and 150, Division VIII.

Plate 120 shows the lesions of an acute miliary tuberculosis in the lungs of a child ten years old.

Plate 121 shows the early signs of tuberculosis of the lungs which were not detected by the elinical examination until some time 
after the tuberculin test had shown the presence of tubercle bacilli. The Roentgenograph, on the other hand, at once showed that the case was one of miliary tuberculosis.

Plate 122 shows the lesions in the right lung of a child twelve years old. There is an area of calcification about one inch in diameter. There are also multiple areas of calcified material, showing the remains of an old tubercular caseous area with an active process around it.

Plate 123 shows the Roentgenograph of a girl three years old. This picture presents the lesions of emphysema, gangrene, and tuberculosis. The left lung shows the emphysematous condition expressed by extreme radiability, as compared with the picture of the right lung. At the base of the left lung a general infiltration has taken place and is represented in the plate by a mottled appearance due to a miliary tubercular lesion. The autopsy proved that the lung on the left side was completely disorganized and that the base was riddled with miliary tubercles. There was also a complete gangrenous condition of the left lung. The right lung was normal.

Plate 124 shows acute miliary tuberculosis of both lungs in a girl twelve years old.

Plate 125 shows a case of hydropneumothorax in a boy seven years old, the picture being taken in the upright position. Plate 126 shows the same boy taken when lying down.

Plate 127 shows the condition of pneumothorax in the same boy, who one year previously had had an attack of hydropneumothorax.

\section{PLEURA}

Plate 128 shows thickened pleura over the left side of the chest in a boy six years old. The left side of the thorax will be scen to be of uniform density, except for a small area in the fifth interspace just beyond the border of the heart. Thoracentesis failed to detect 
any fluid, and as the density did not change with change of position, the provisional diagnosis of a thickened pleura was made. The lung on the right side appeared to be emphysematous, the emphysema being partly caused by the forced compensatory action of the lung.

It must be remembered that in all these Roentgenographs of the lungs the cases have been carefully examined clinically and that the interpretation of the plate must be much influenced by the clinical examination. In the pneumonic cases where they were at all obscure, not only was the exploratory needle used to support the diagnosis, but the tuberculin test was given in those eases where the resolution was prolonged; that is, the Roentgen examination was in all cases additional and confirmatory to other methods of examination, and often proved to be the most valuable.

Plate 129 shows an effusion into the left pleura of a ehild eight years old. There is a uniform radiability over the entire left lung, which was found to change as the position of the child was changed. It is to be noticed that where the fluid was greatest in amount, as in the middle of the involved area, the density was greatest, while on the edges it was less and showed greater radiability. The heart was slightly displaced to the right, and the right lung showed slight compensating emphysema. The bronchi are also seen on either side of the sternum. The diagnosis was confirmed by an exploratory thoracentesis.

Plate 130 shows a collapsed condition of the upper ribs on the right side in a girl six years old, resulting from an effusion, probably an empyema. 'The heart is drawn to the right and held there by adhesions. There is still either some fluid at the light base or a greatly thickened pleura. There is a compensatory emphysema of the left lung.

Plate 131 shows an encapsulated empyema verified by an operation on the right side at the lower base in a boy ten years old. 


\section{HEART}

Plate 132 shows an extreme enlargement of the heart in a child ten years old, filling almost the entire anterior area of the thorax. The lung on the right side shows compression. The cardiohepatic angle is seen to be intact, and is extremely valuable in making the differential diagnosis from pericardial effusion, which in this case it closely simulated, since the encroachment of the dull area in the fifth right interspace was much greater than usual. This similarity of physical signs was accompanied by a similarity of rational signs, as the extreme dilatation and weakening of the heart prevented almost entirely the cardiac impulse from being felt and there was no audible murmur. There were orthopnœa, cyanosis, a weak fluttering pulse, and a picture as much of pericardial effusion as of enlarged heart, confirmatory paracentesis being considered seriously, when the ray settled the question by showing clearly the cardiohepatic angle, and the consequent contraindication for paracentesis.

\section{PERICARDIUM}

Plate 133, a child twelve years old, is an instance of what has just been stated in speaking of Plate 132. This picture shows a typical case of a heart enlarged considerably on both sides, but encroaching but little on the fifth right interspace, thus following the clinical rule as to the usual presence of resonance in that space in an enlarged heart. Around the heart is seen the greater radiability of the effusion, with its pyramidal outline below and at the sides and extending over the great vessels at the base of the heart, being indeed the typical outline of a pericardial effusion, namely a half circle with its greater radius to the left.

So far as I know this is one of the first cases where a large pericardial effusion being present, and the rational symptoms of pericardial effusion and of enlarged heart bcing similar, it was possible not only to diagnosticate the effusion by the Roentgen method. 
but to prove also that the heart was cnlarged. This was aecomplished by recognizing the difference of radiability between the heart itself and the perieardial effusion. The Roentgen examination was made in the upright position and the eardiohepatic angle as seen in the plate was obliterated.

Plate 134 is a case of pericardial cffusion in a child twelve years of age, where the outline of the heart shows it to be enlarged. The eardiohepatic angle is obliterated by the pericardial effusion. The lung shows compression of the pulmonary tissue, eorresponding to what would be expected in the Rocntgenographic picture produced by an atelcetasis.

Plate 135 is the picture of a pericardial effusion in a child. The decreased radiability extends from the sixth rib to the base on the right, and from the fourth interspace to the base on the left. The cardiohepatic angle was obliterated, as is seen on the right. The whole lung shows compression and lessened radiability. The darker area in the centre of the large and lighter area shows indistinetly the heart itself, which does not seem to be enlarged. The Roentgen ray gives us the only known method by which in most cases of large perieardial cffusion a detcrmination can be made as to whether the heart is of normal or abnormal size, suspended as it is in the pericardial sac. This is accomplished by a close observation of the dark central outlines of the heart, which we are thus able to distinguish from the surrounding outline of the cffusion and the still lighter area of the compressed lung.

\section{ANEURISM}

Plate 136 is the Roentgenorraph of a boy twelve years old in whose thorax the elinical diagnosis of ancurism was made. The picture shows an area of increased density in the region of the great vessels at the base of the heart in the fourth left interspace, near the edge of the sternum, and its interpretation was confirmatory of the clinical diagnosis. The rest of the picture was normal. 

PI.ITI: 111.

Nolimil, TIUHR.X.

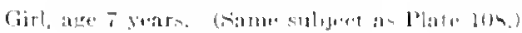

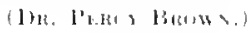

Lispresure beconds.

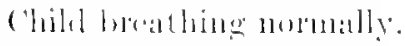

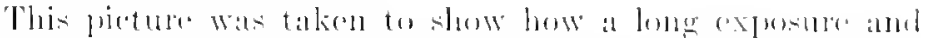

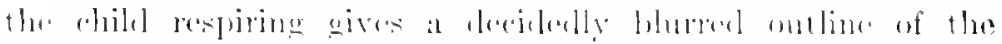

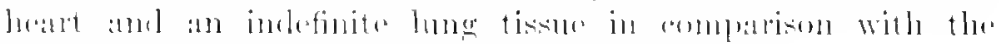
results of a short r.spensure als shown in Plate los. 
Piate 107

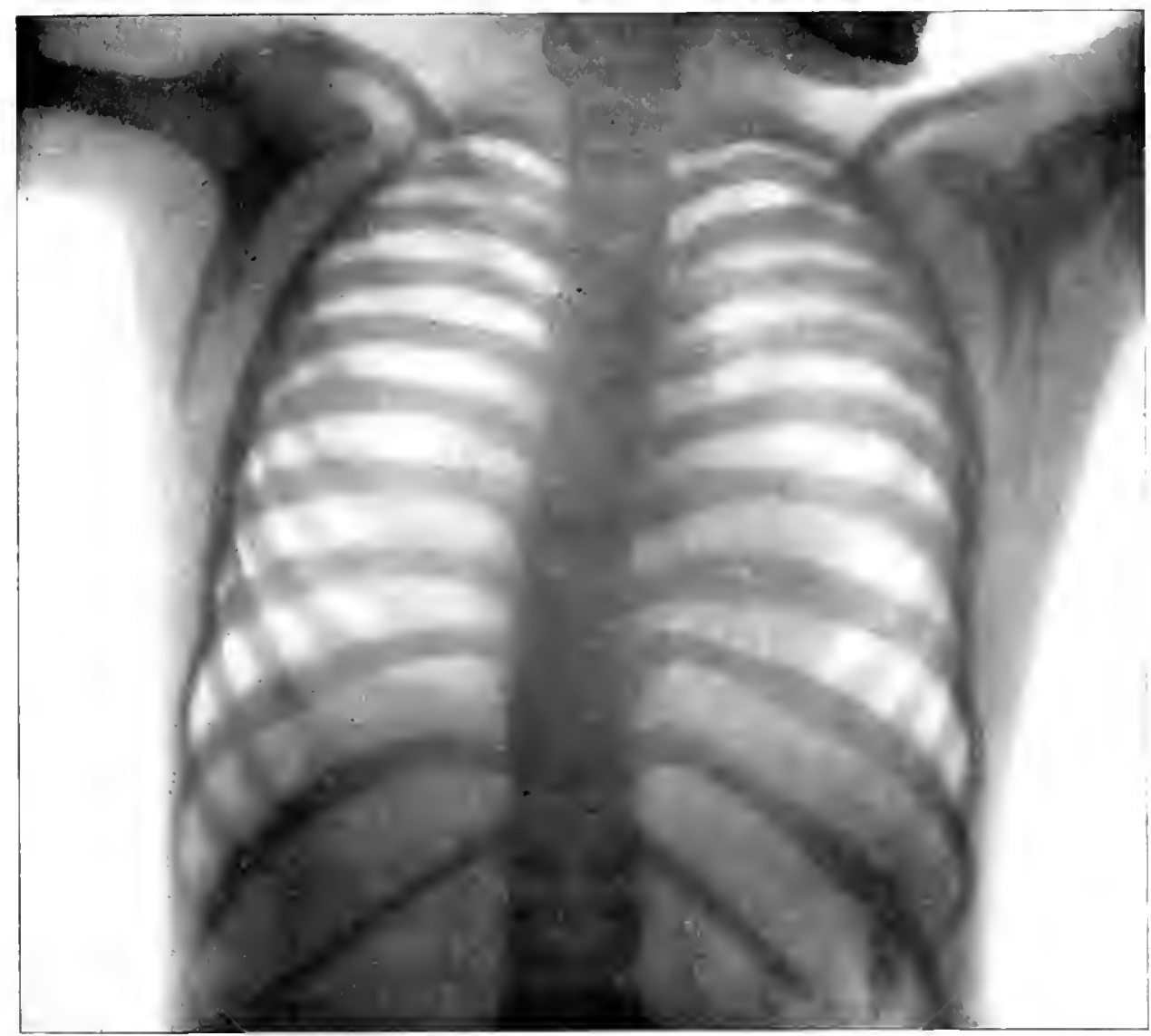


Ji.1'1: 10,

volinte THOHIX.

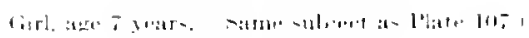

(i) I', In, Bн,

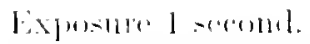

licepriattion arrented.

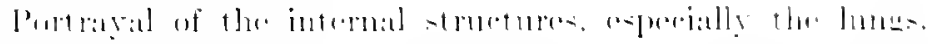

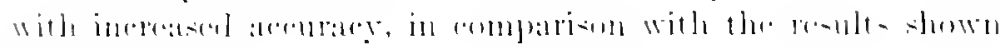
in l'late 10\%. 
Plate los

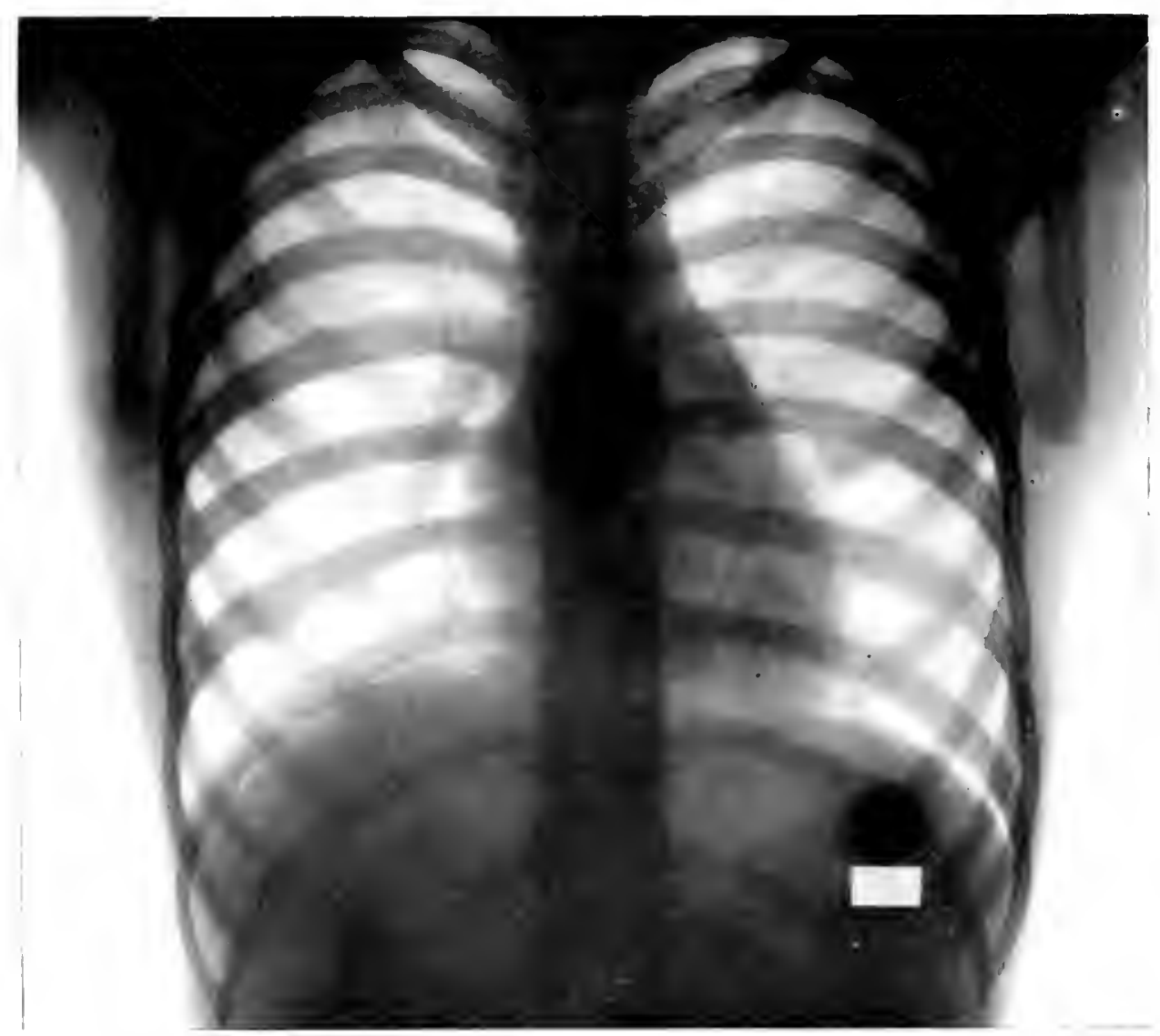


PI. ITE: 10!\%.

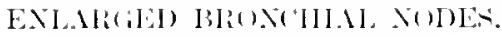

Girl, :1: 12 years.

A shertly abmomal momlitim.

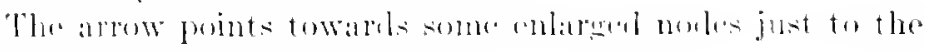
richt of the outline of the heart. 
Plate 109

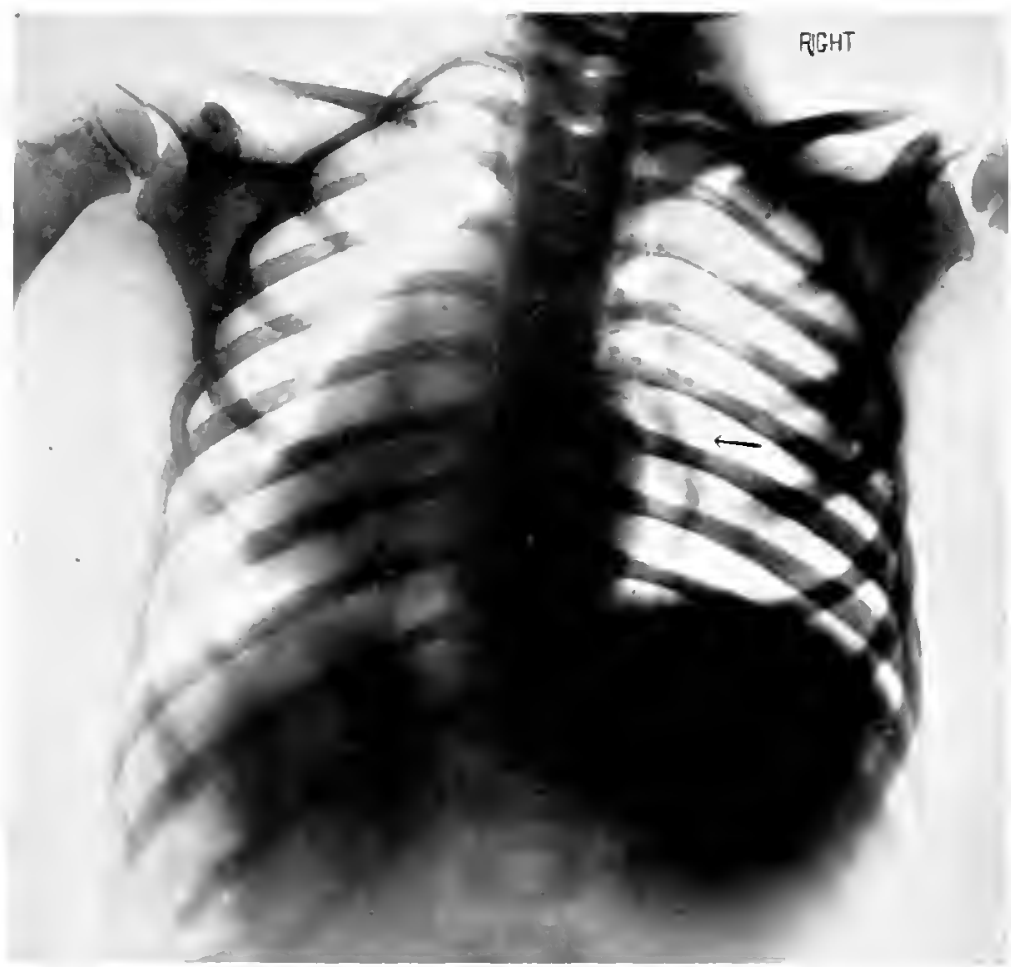


PI.1'1: 110 .

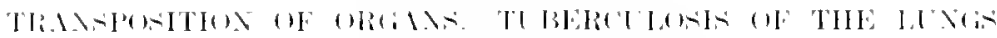

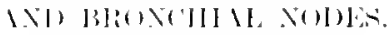

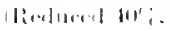

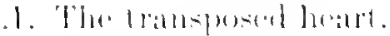

S. The livere trangmand lo the loft.

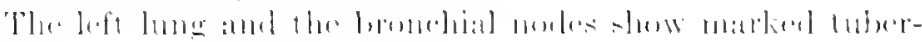
"ulu-i: 
P'IATE 110

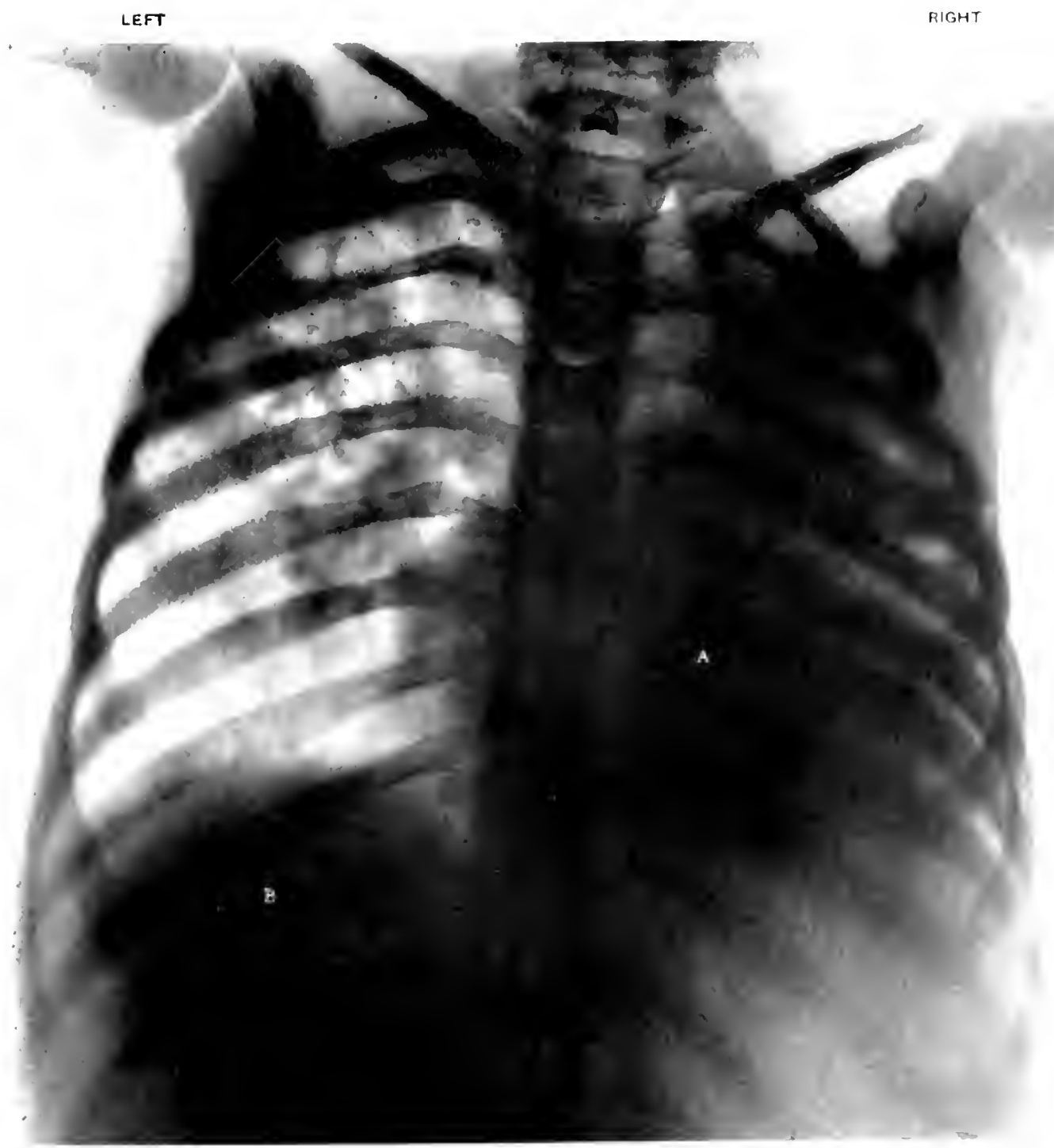


I'I.ITJ: 111.

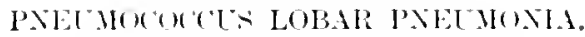

Girl, age 3 sears. (Redured 33\%.)

'The armer prints to a consolicition of the upper lobe of the left lung, at about the level of the angle of the seapulat. 
PJATE 111

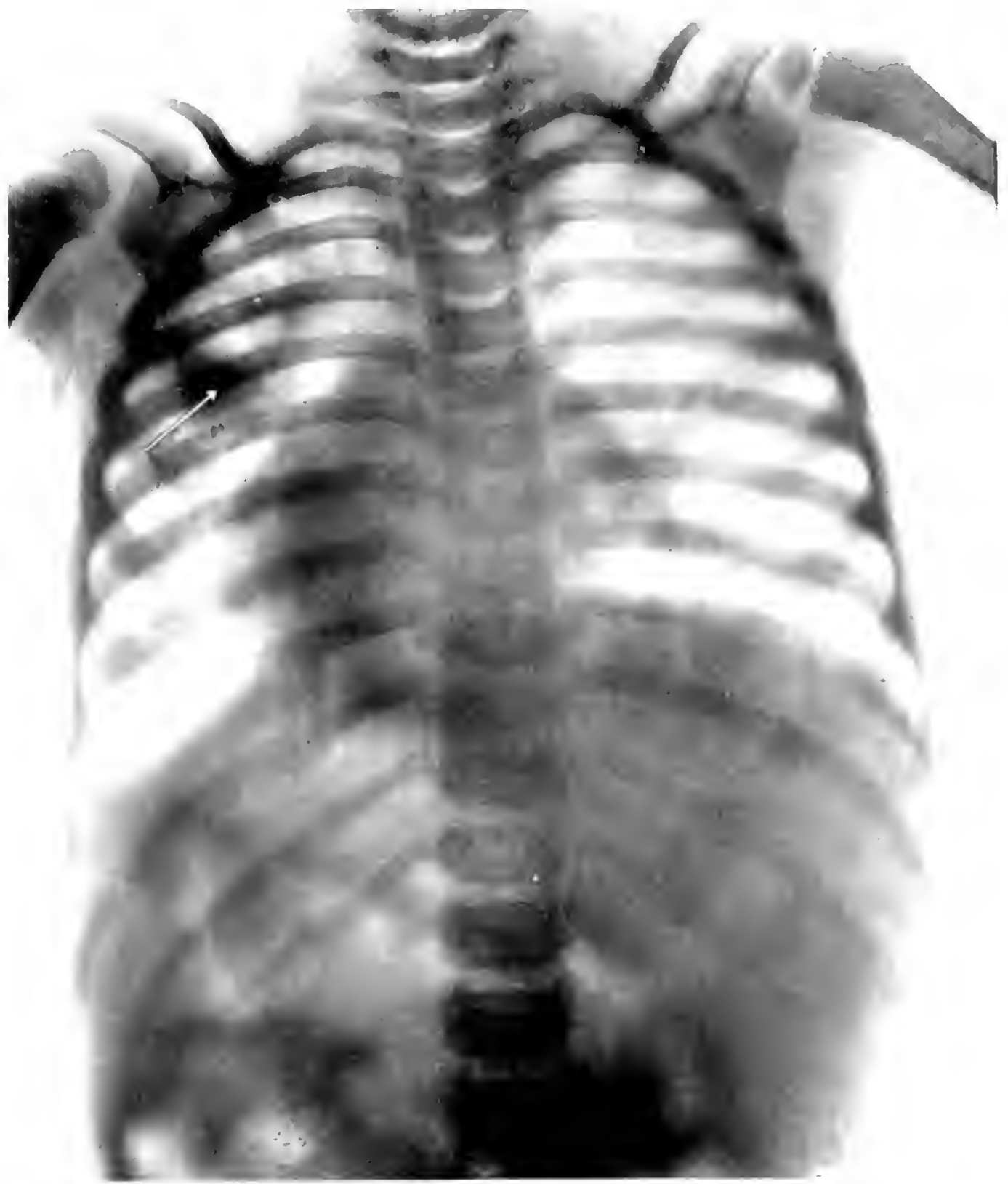


H. ITE, 112.

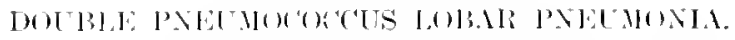

Here, ane 12year. (Rouluced tary-)

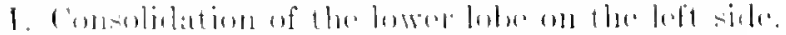

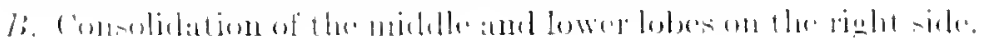


PIATE 112

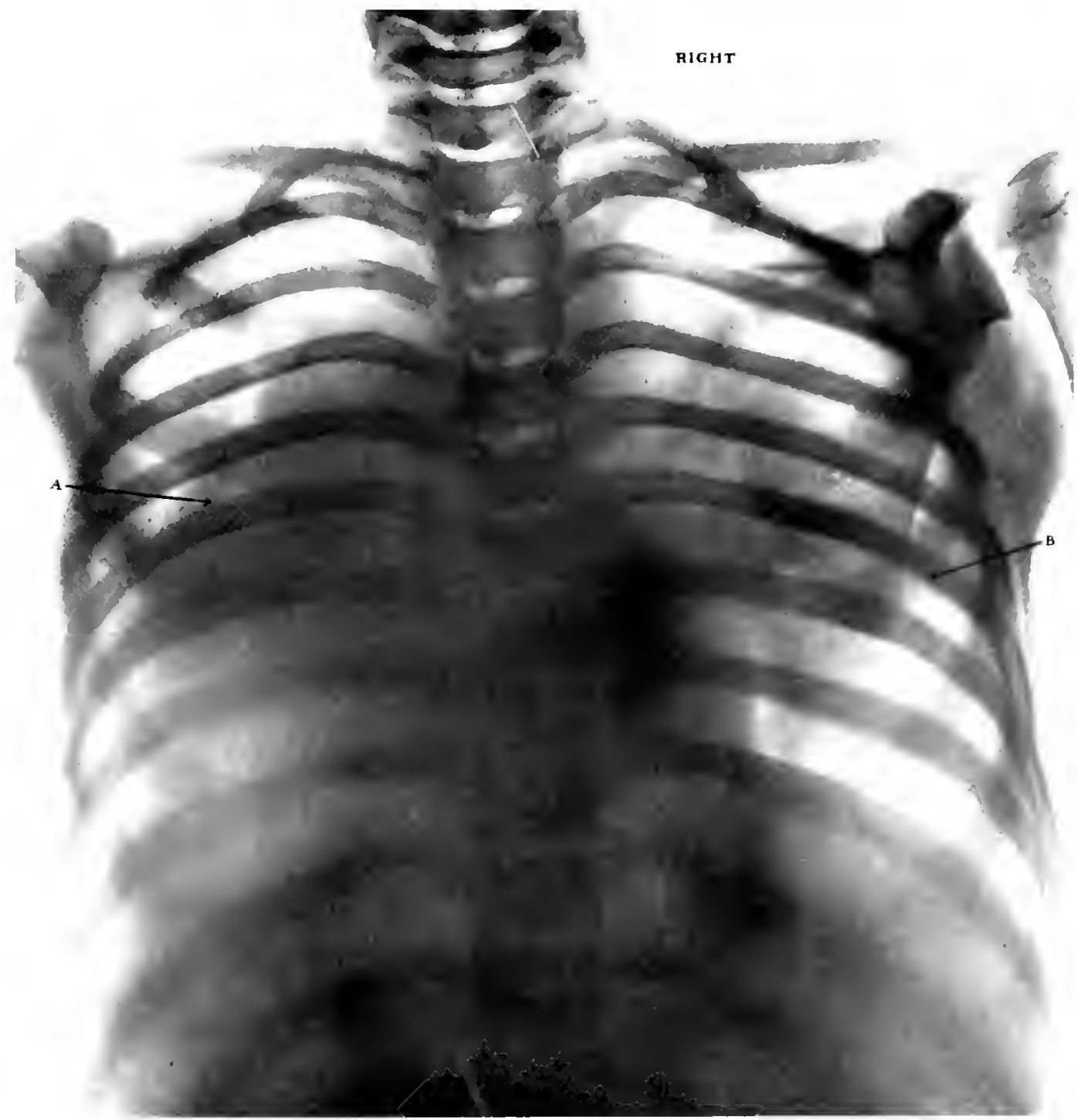


Pl..TTE 11:3.

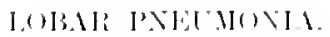

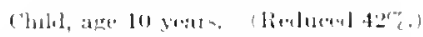

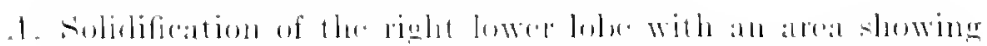

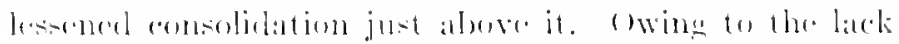

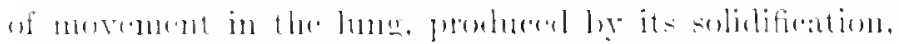

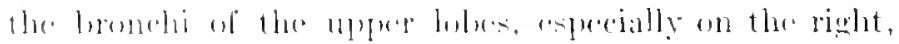

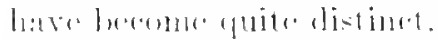

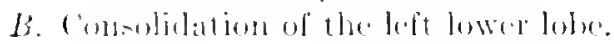


PIAT: 1]:;

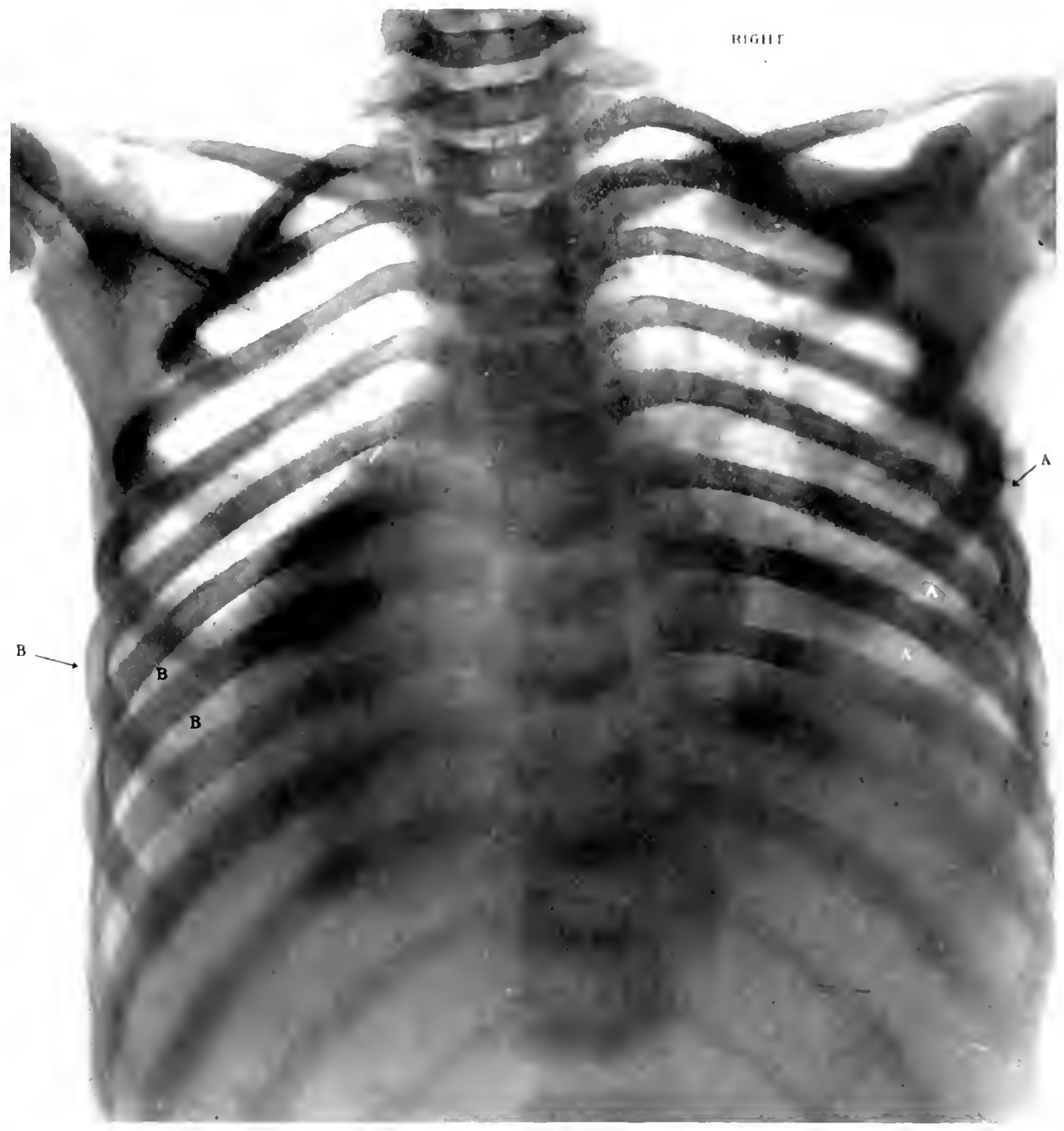


I1. $11 \%: 11 \%$

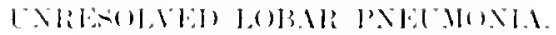

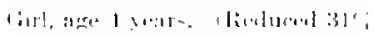

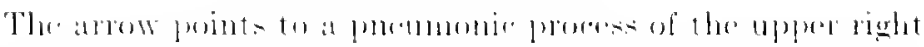

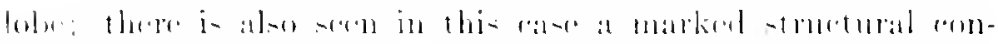

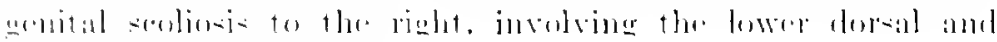

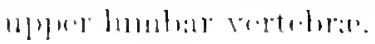


PIATE 114

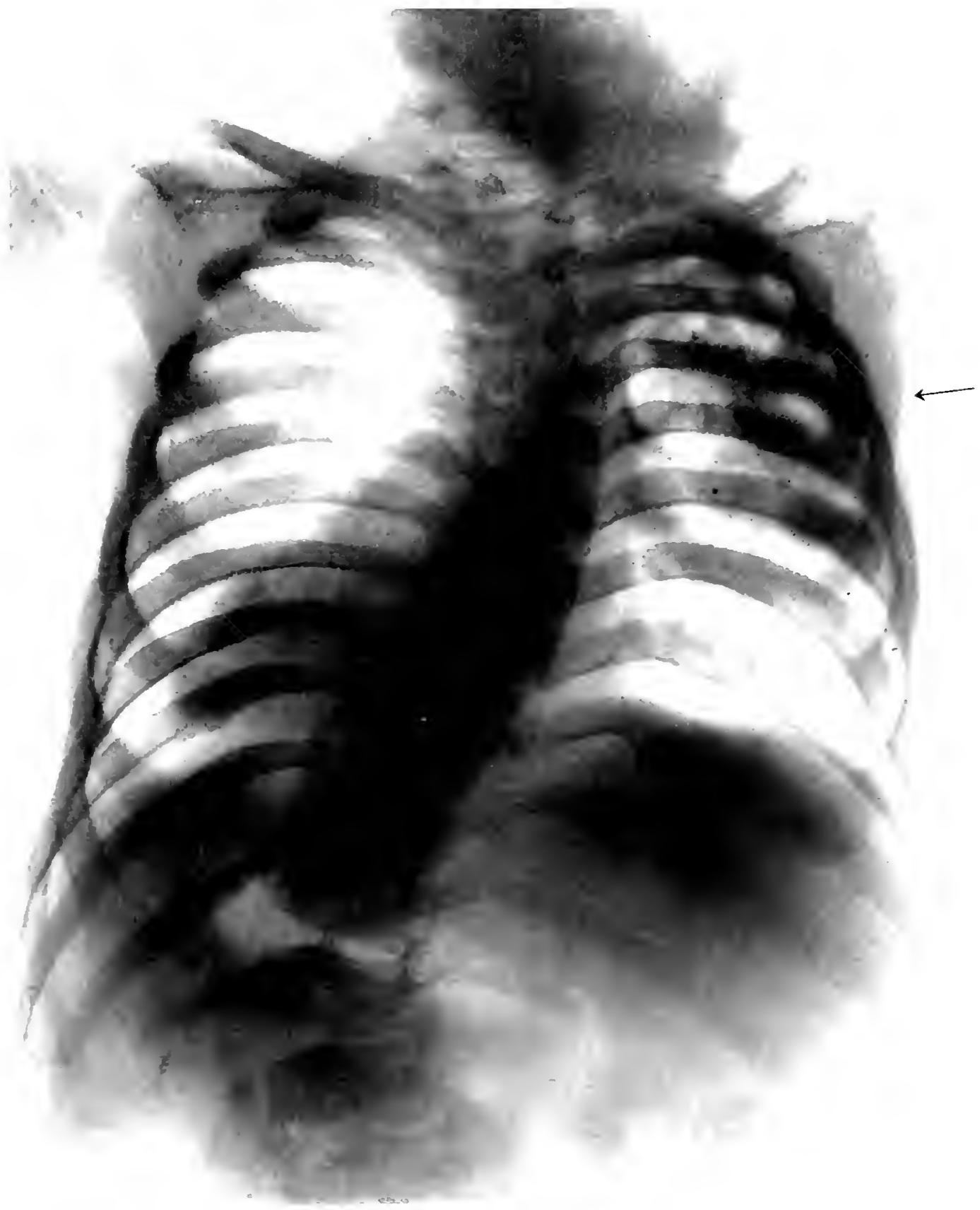


PI.ITI: 11\%

Holl: PNETHONA.

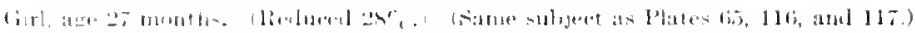

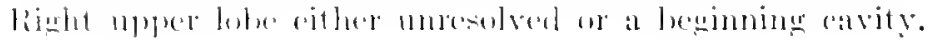


PJATE JIJ

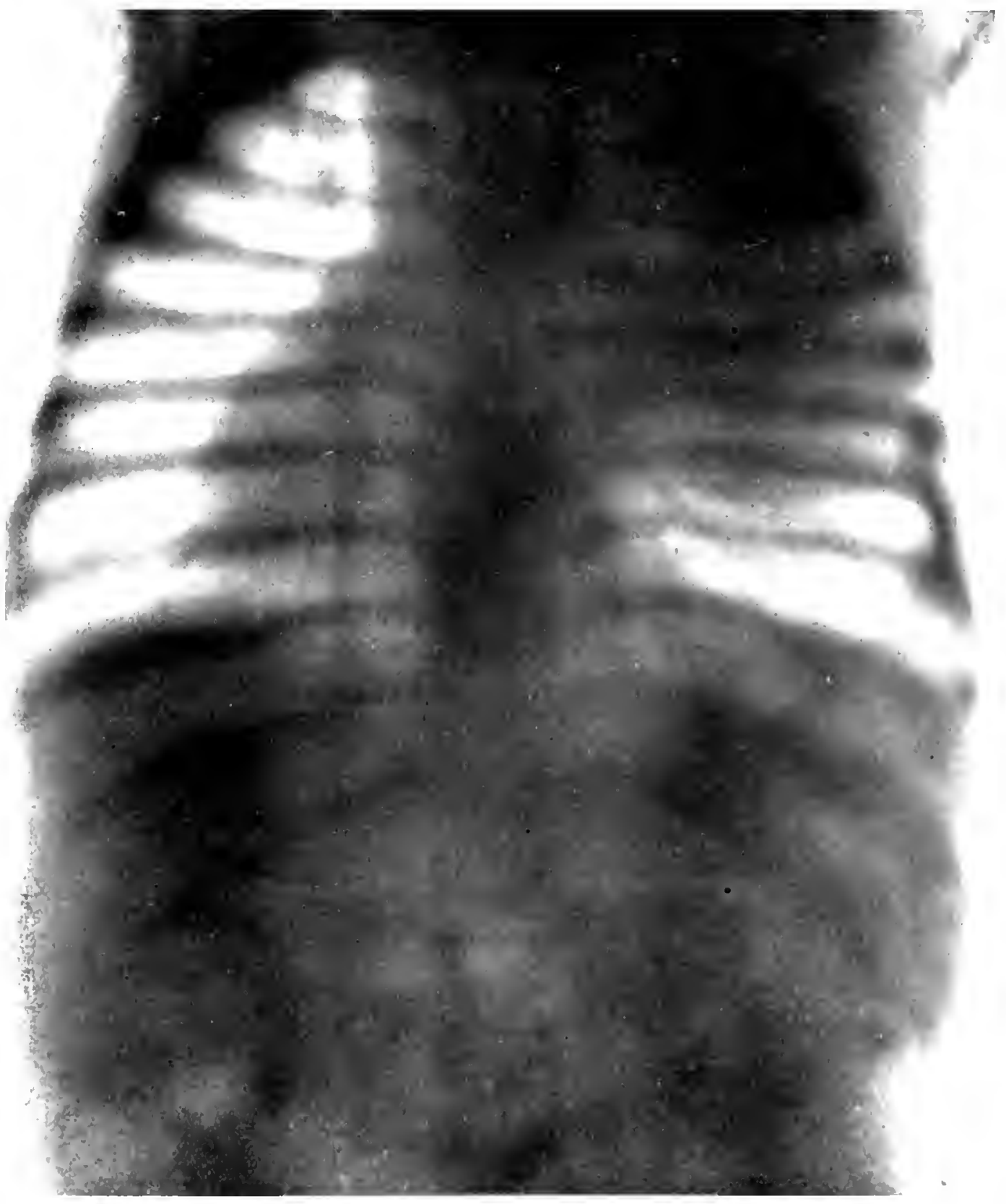


II. 1T1: $11 \%$.

(1) II)](IT.

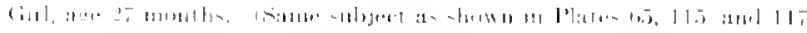

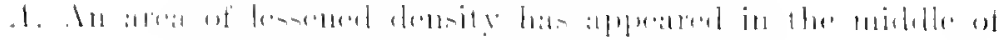

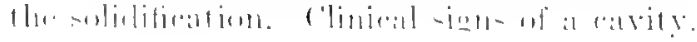


I'I ITE 116

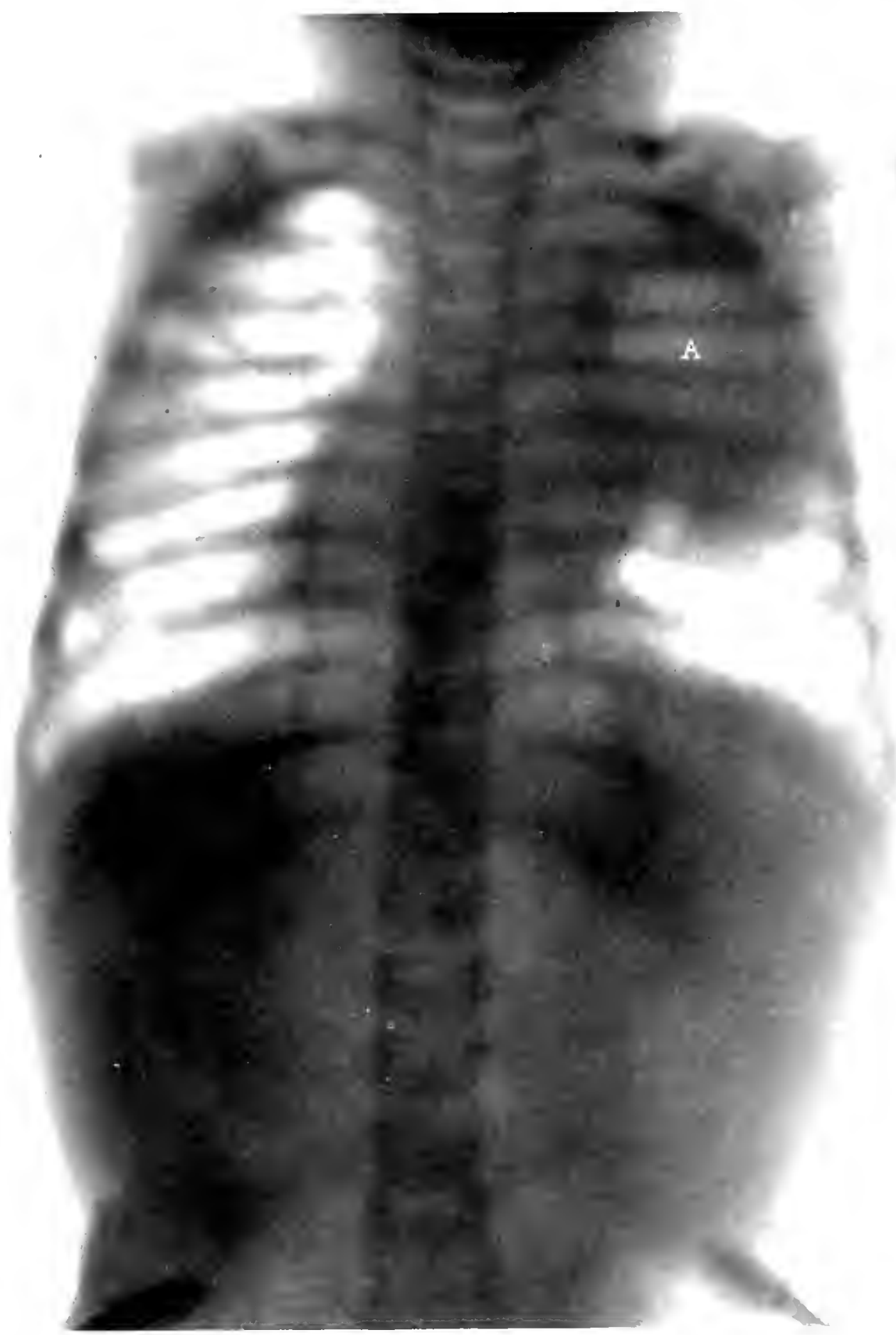




\section{PITI: 117}

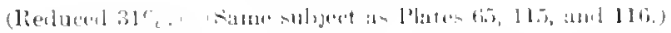

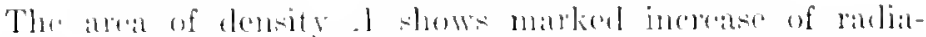
bility. In the lowe part of the lung the proceses, possildy

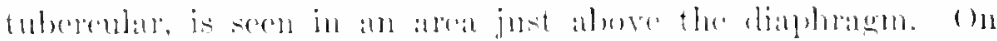

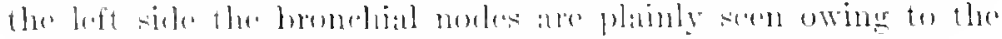

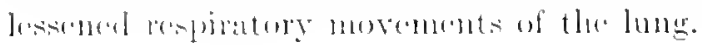


PLATE 117

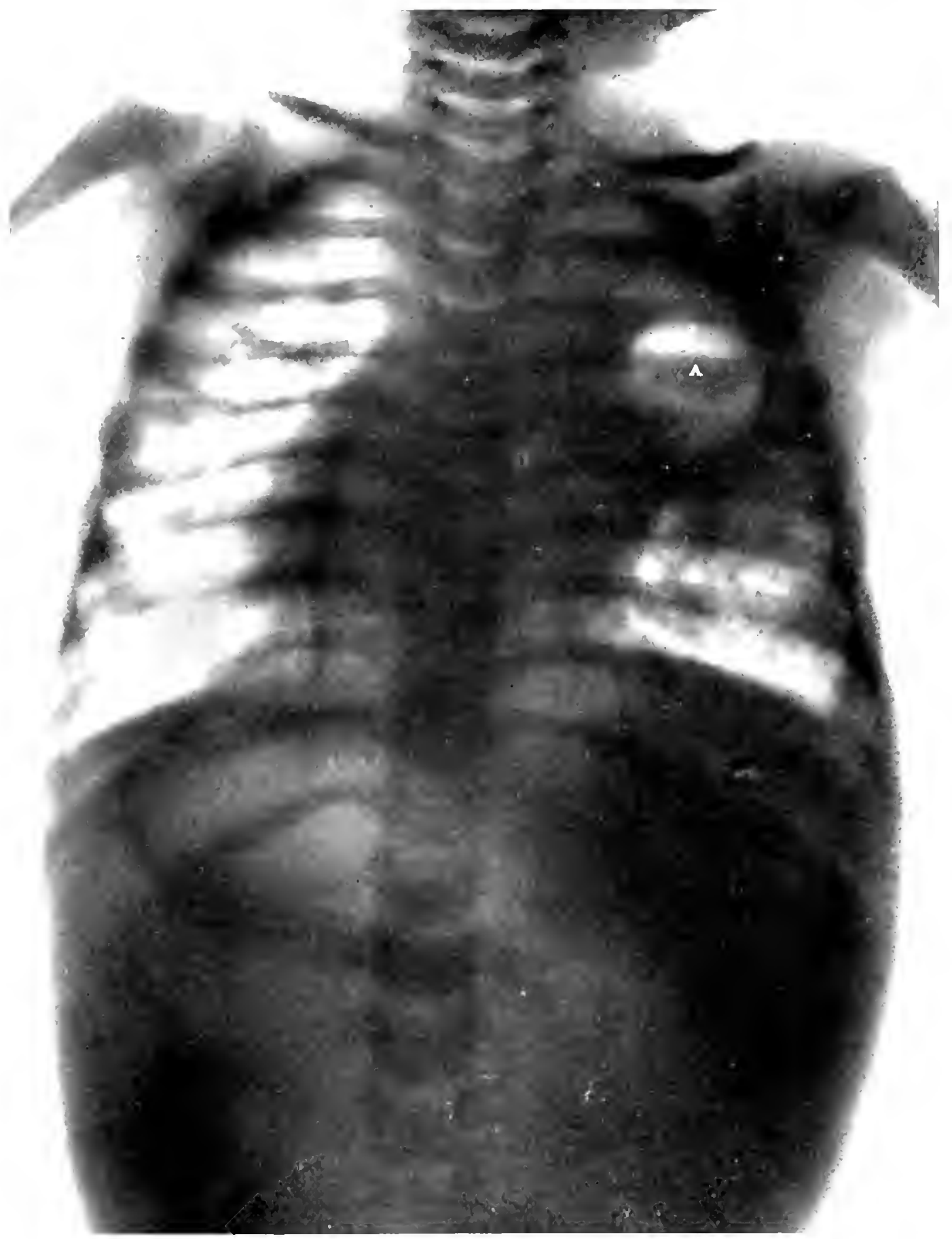


I'ITI: II.

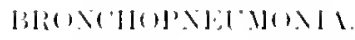

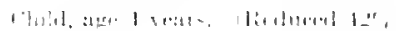

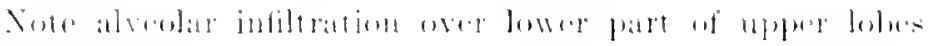
(n) luth - inler. 
P'LATH

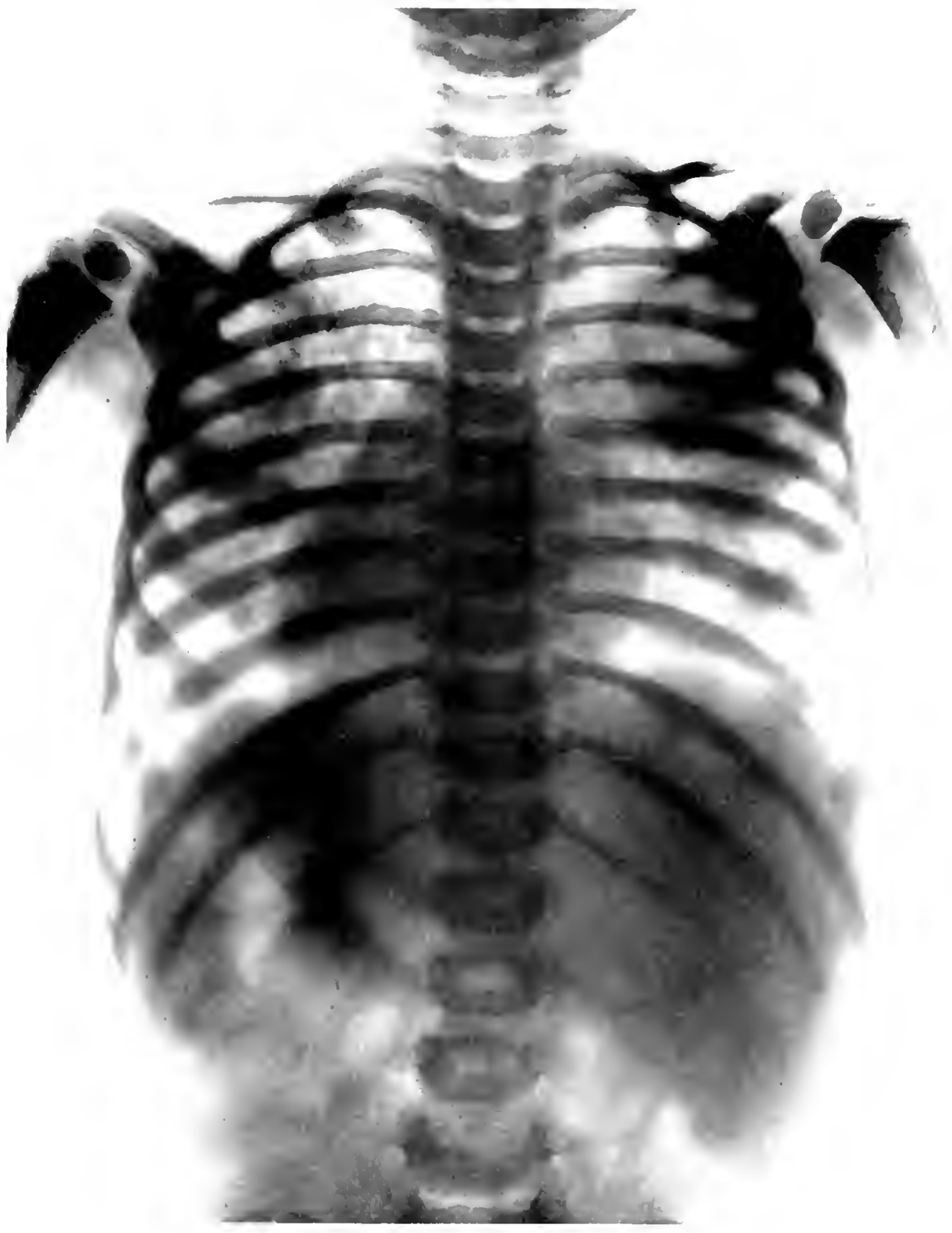


I'LTE 11 \%.

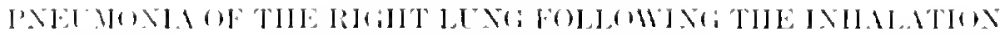

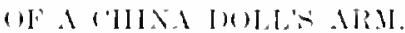

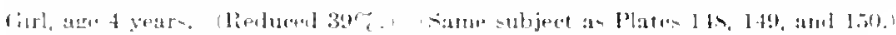

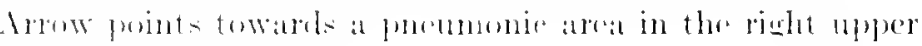
loke prestured by the arme 
PIATE 11!

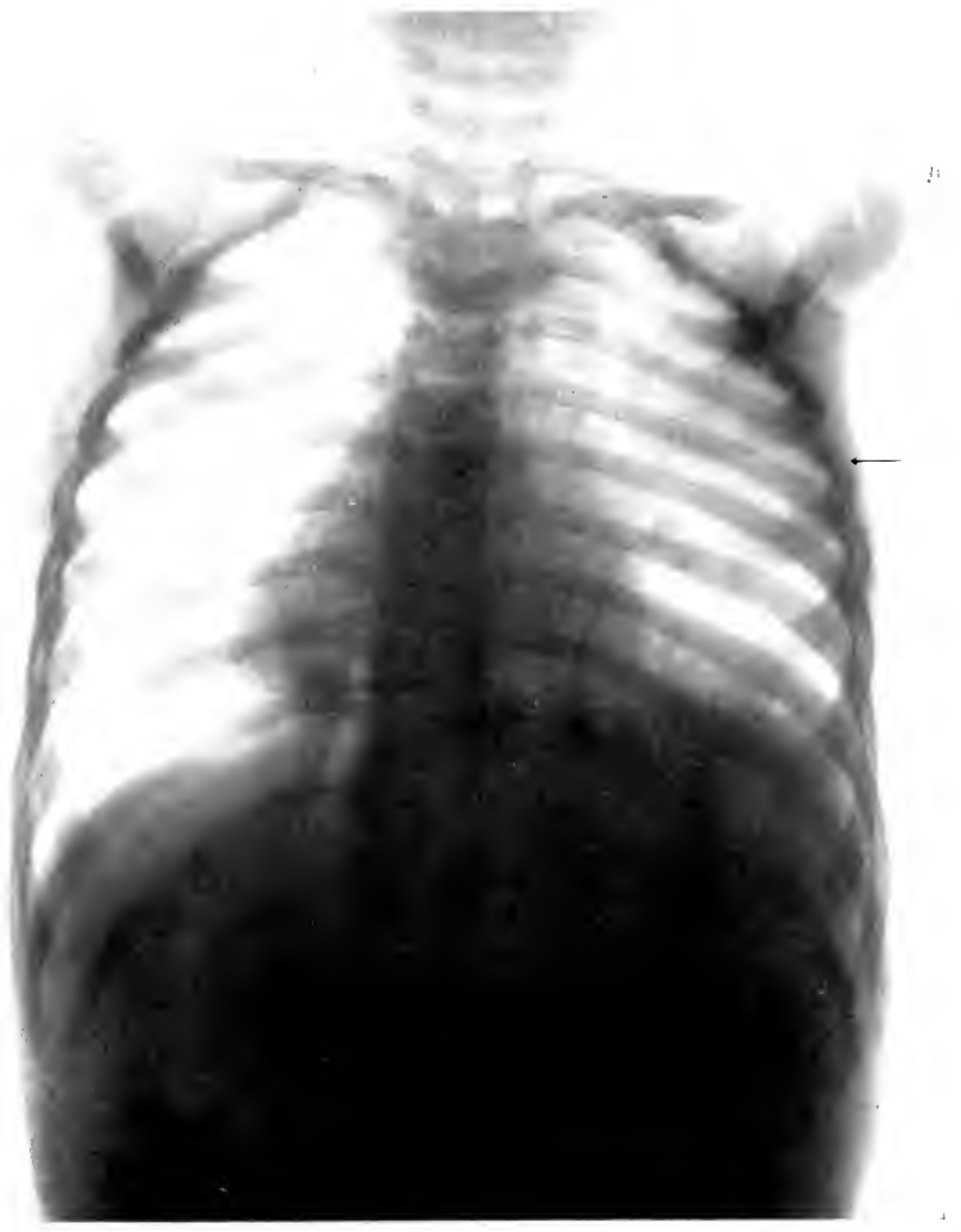


I'T.1T1: 1201\%.

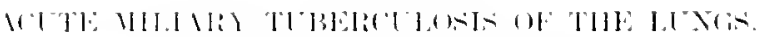

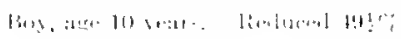

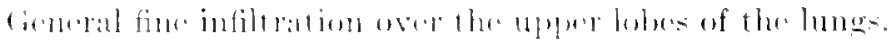

I. Mrifind. 
PLATE: 1 20

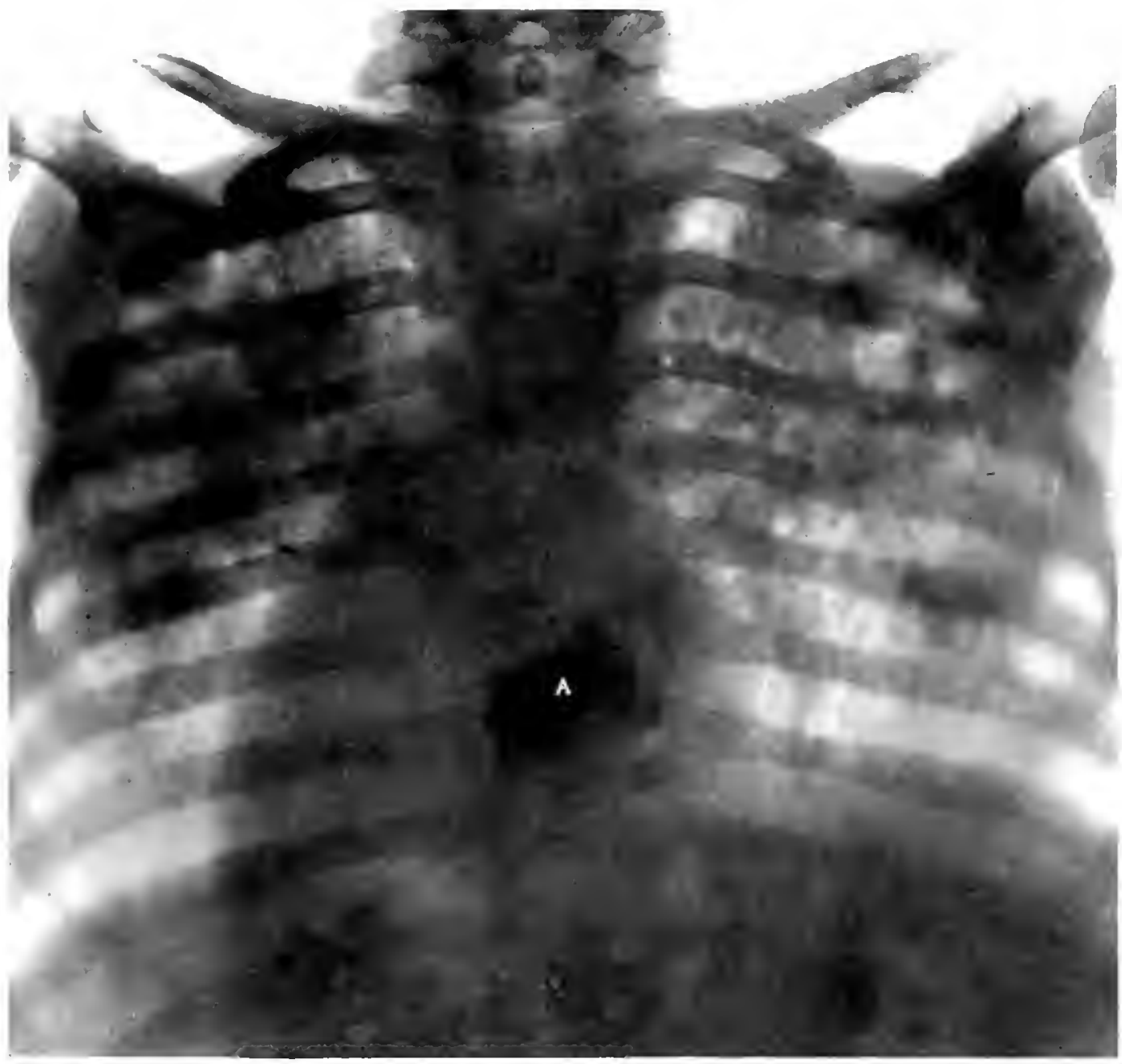


P'I.TT: 121.

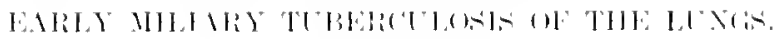

$1,0: 3: \cdots, 14$

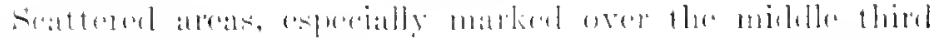
of the right lume. 
PLATE 121

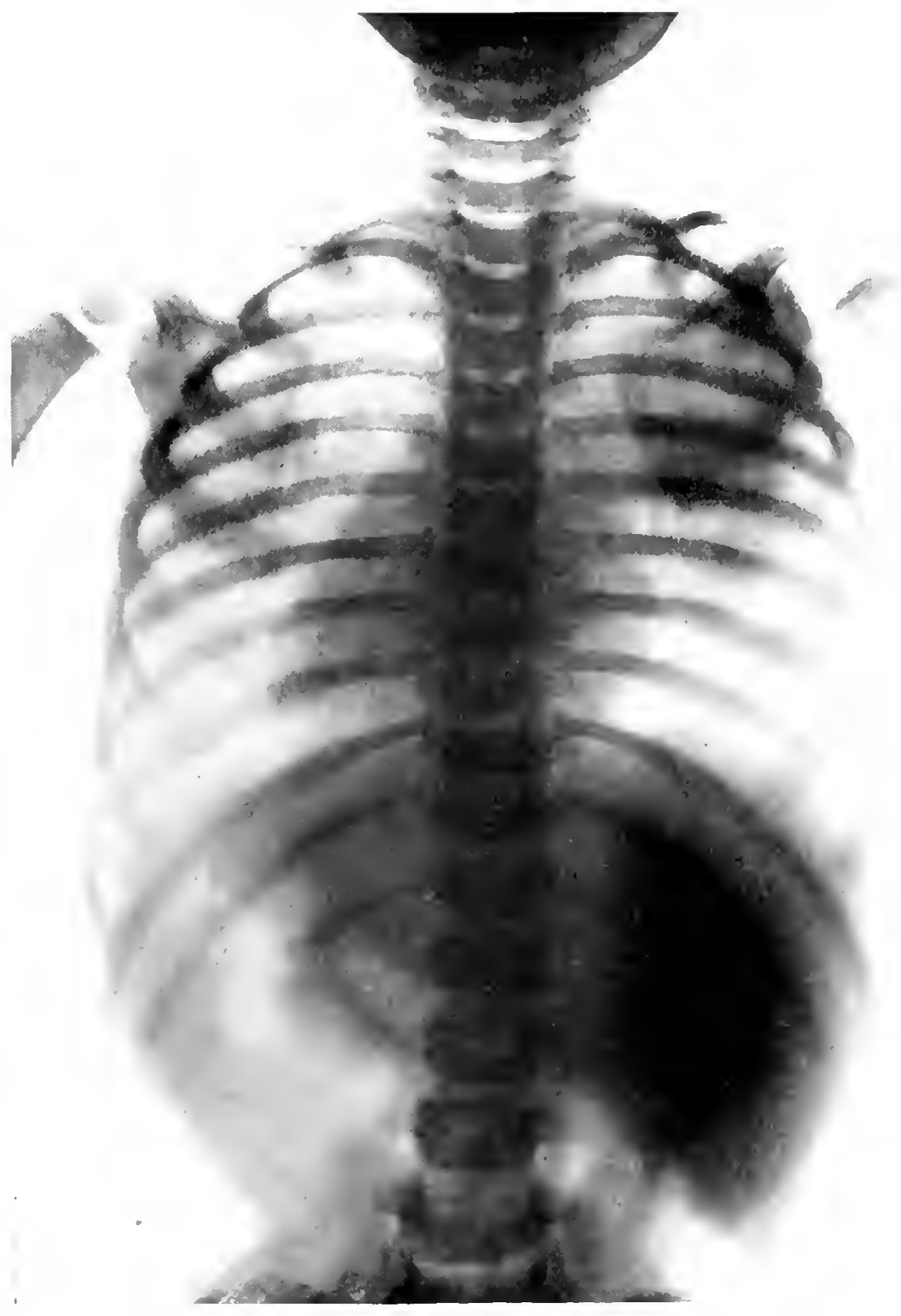


1'1.171: 1'.".

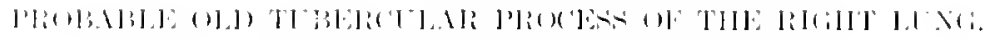

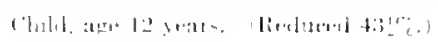

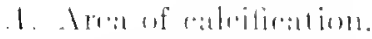

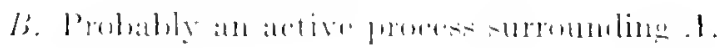


PIATE $1 \stackrel{2}{ }$

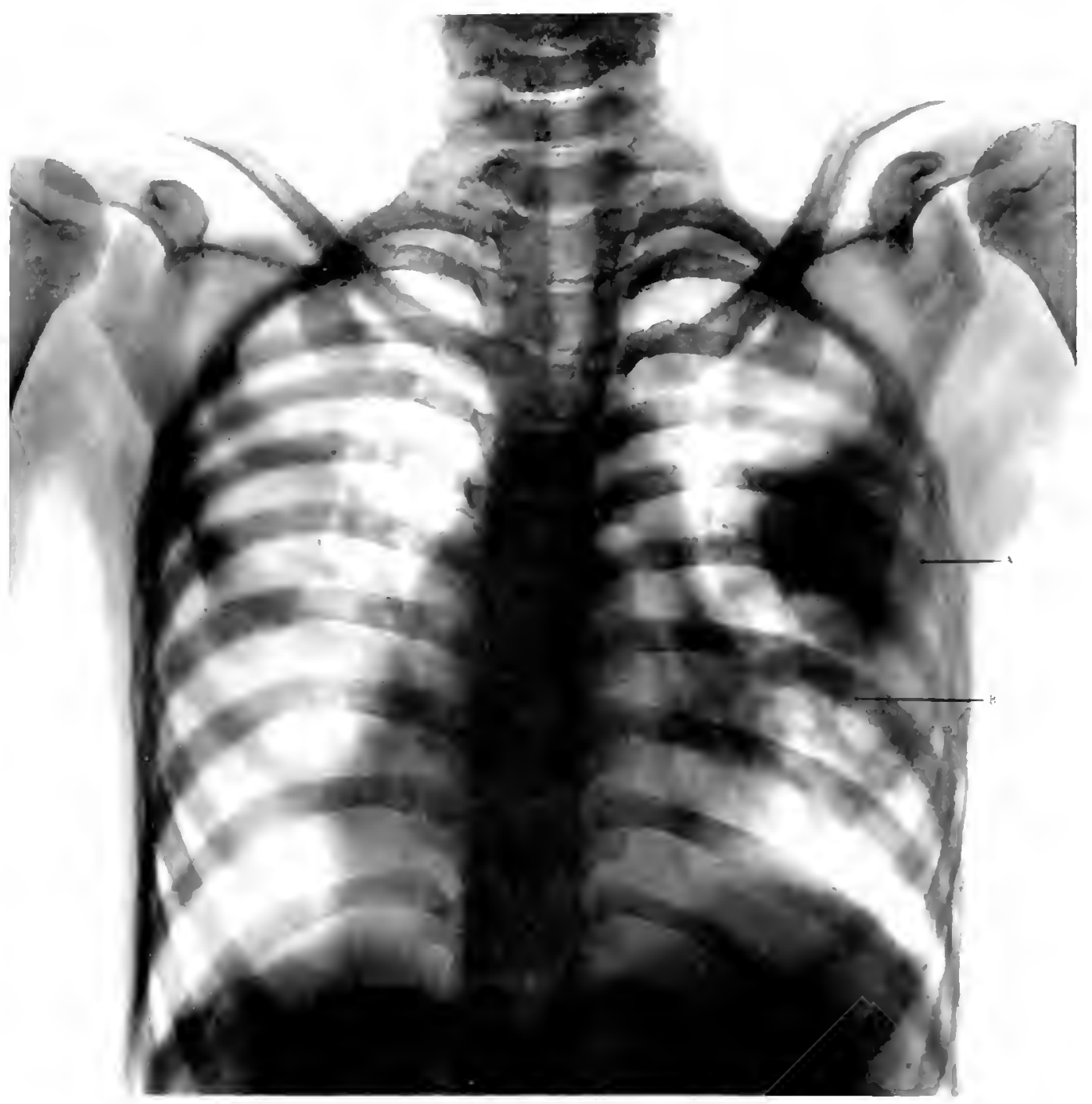


PIATI: 12:3.

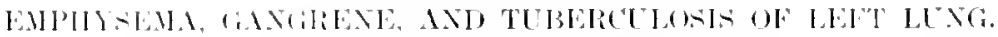
(IIRITED BY AUTOPSL.)

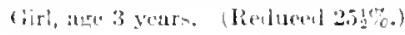

1. Lreal of cmphyistmit.

I3. Comeral infiltration of miliary tuberoulosis. Right lung normal. 


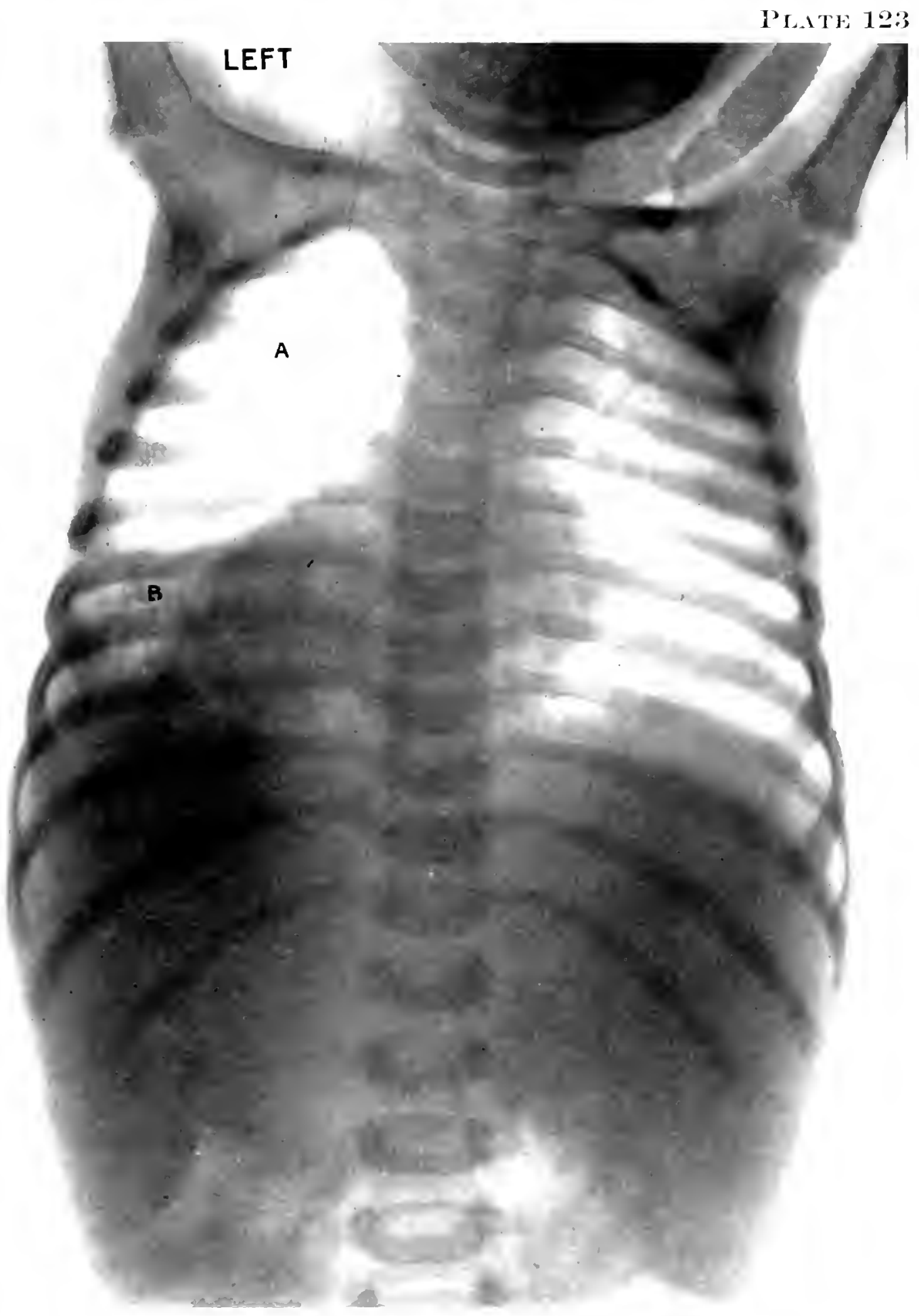


PIATI: 1:4.

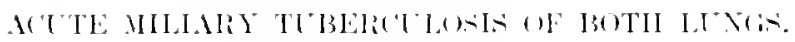
Girb, atepe ley you,

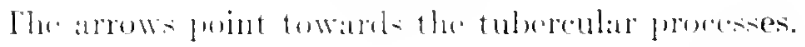




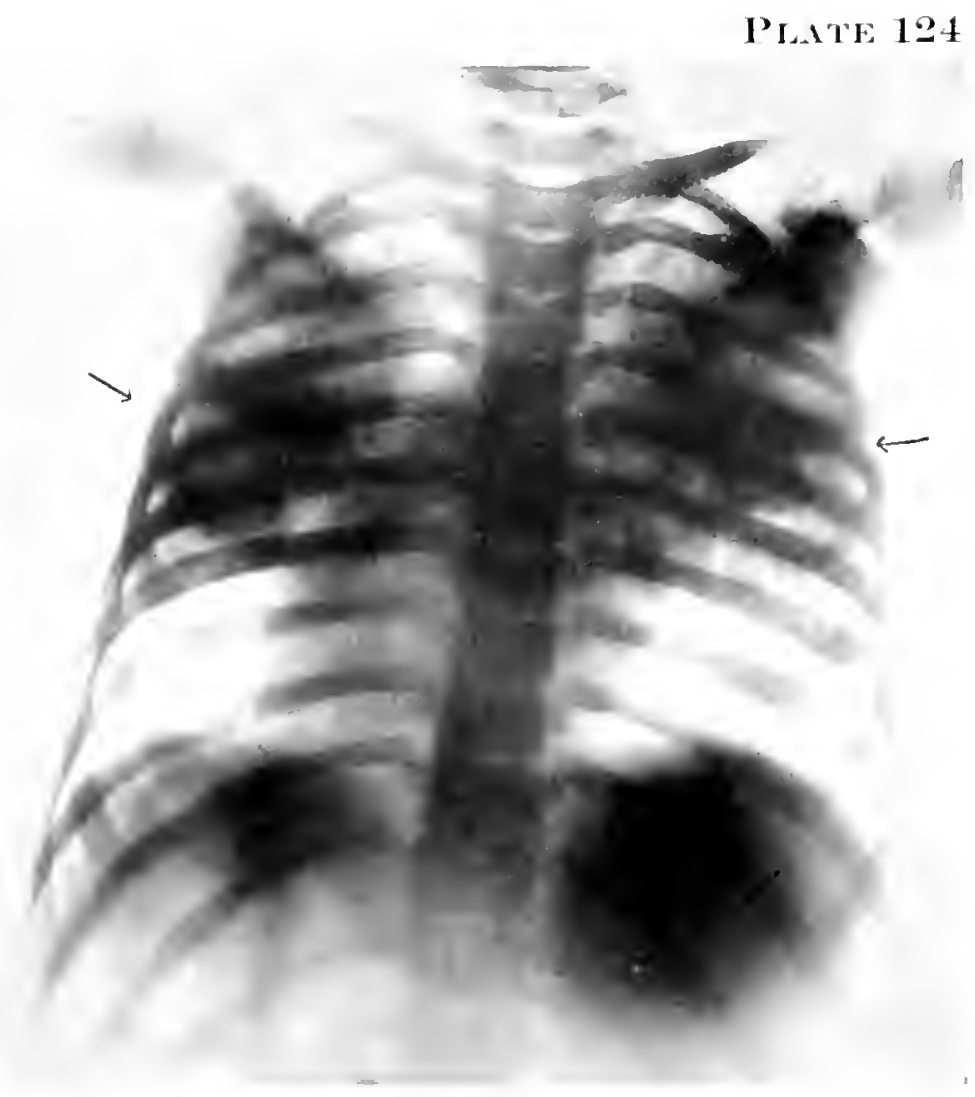


PLATE: 1‥

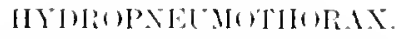

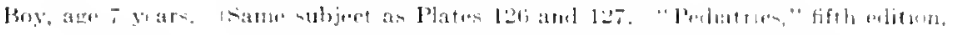
Plat, SIII)

The pirture is taken with the bey in an upright position.

The leset of the offusion on the left sille in eteaty shown, as is the matried dispharement of the herat to the right. 
PInte 125

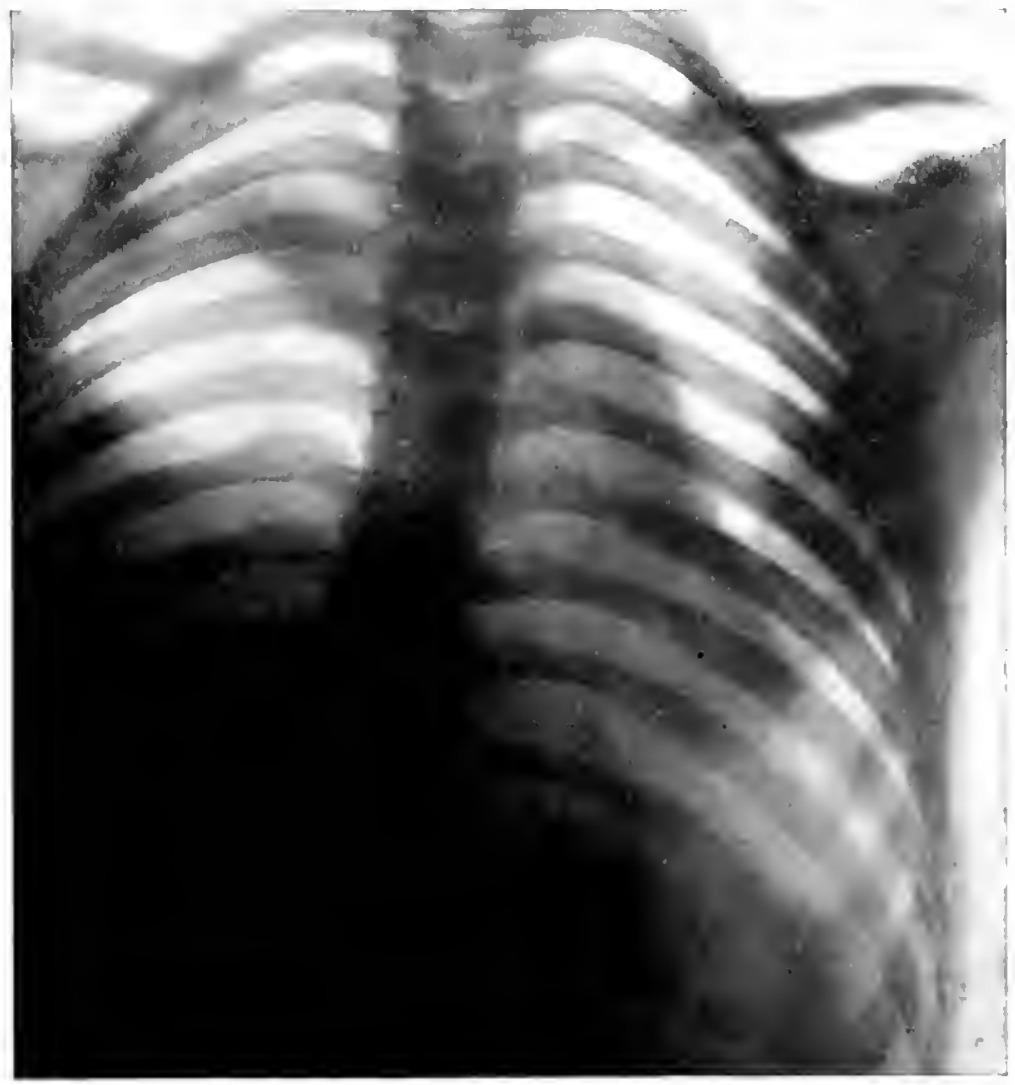




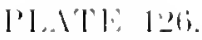

HITHOTSET MUTHORAX.

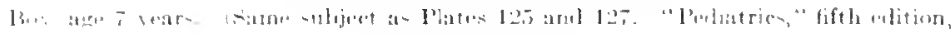
I'ia's Xll.)

The portare is takin with lhe hog lyine down.

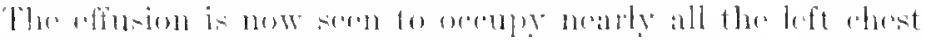

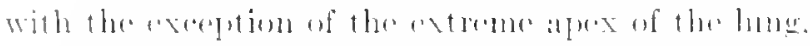

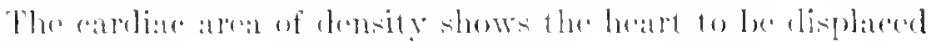
(1) the right. 
PI.nTe 126

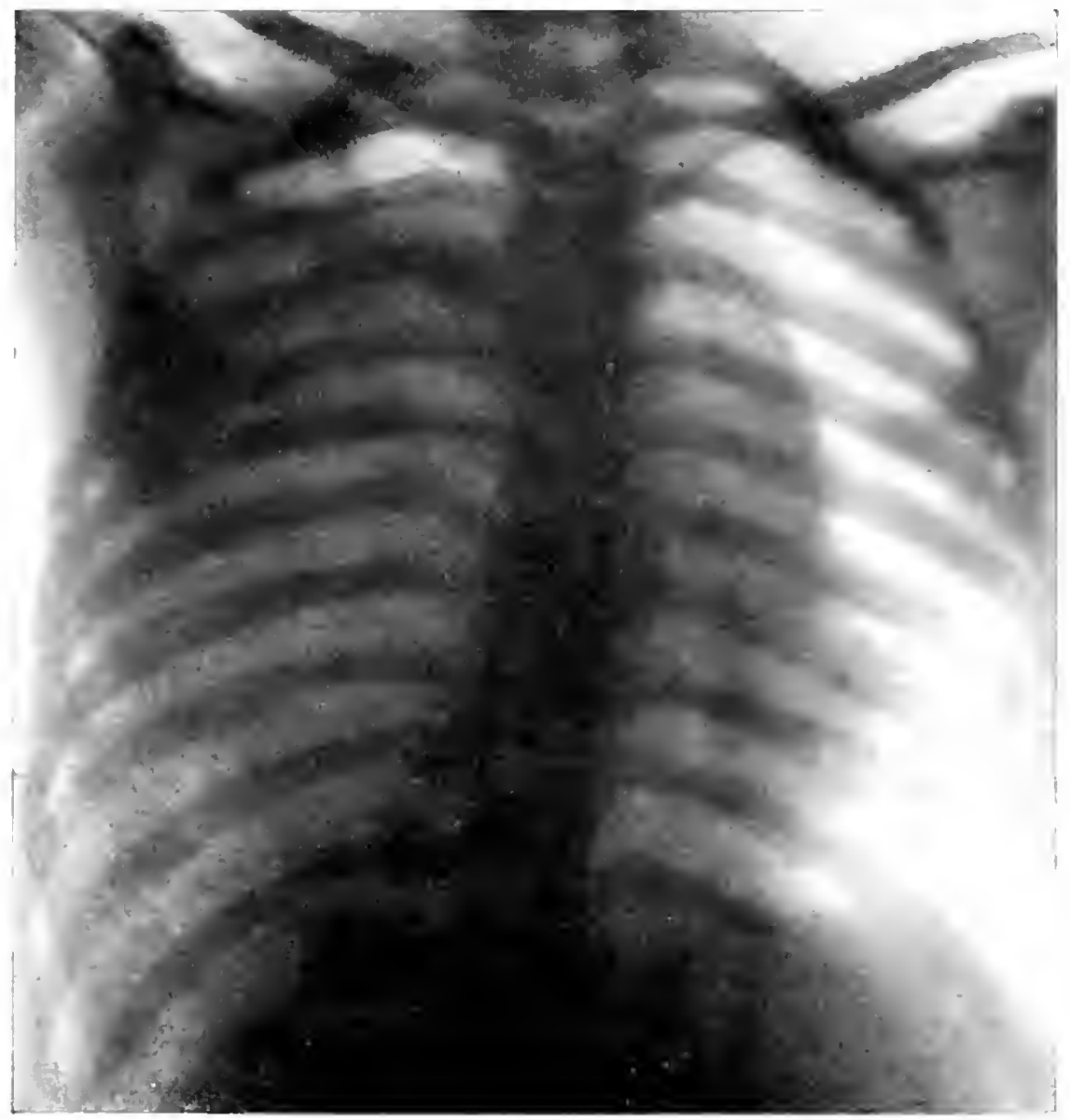


PI.АTI: 127.

\section{PNELAOTHORAX.}

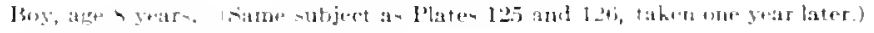

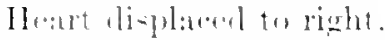

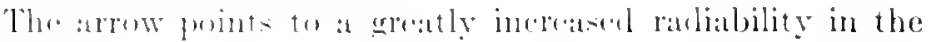
loft lunge. 
Plate 127

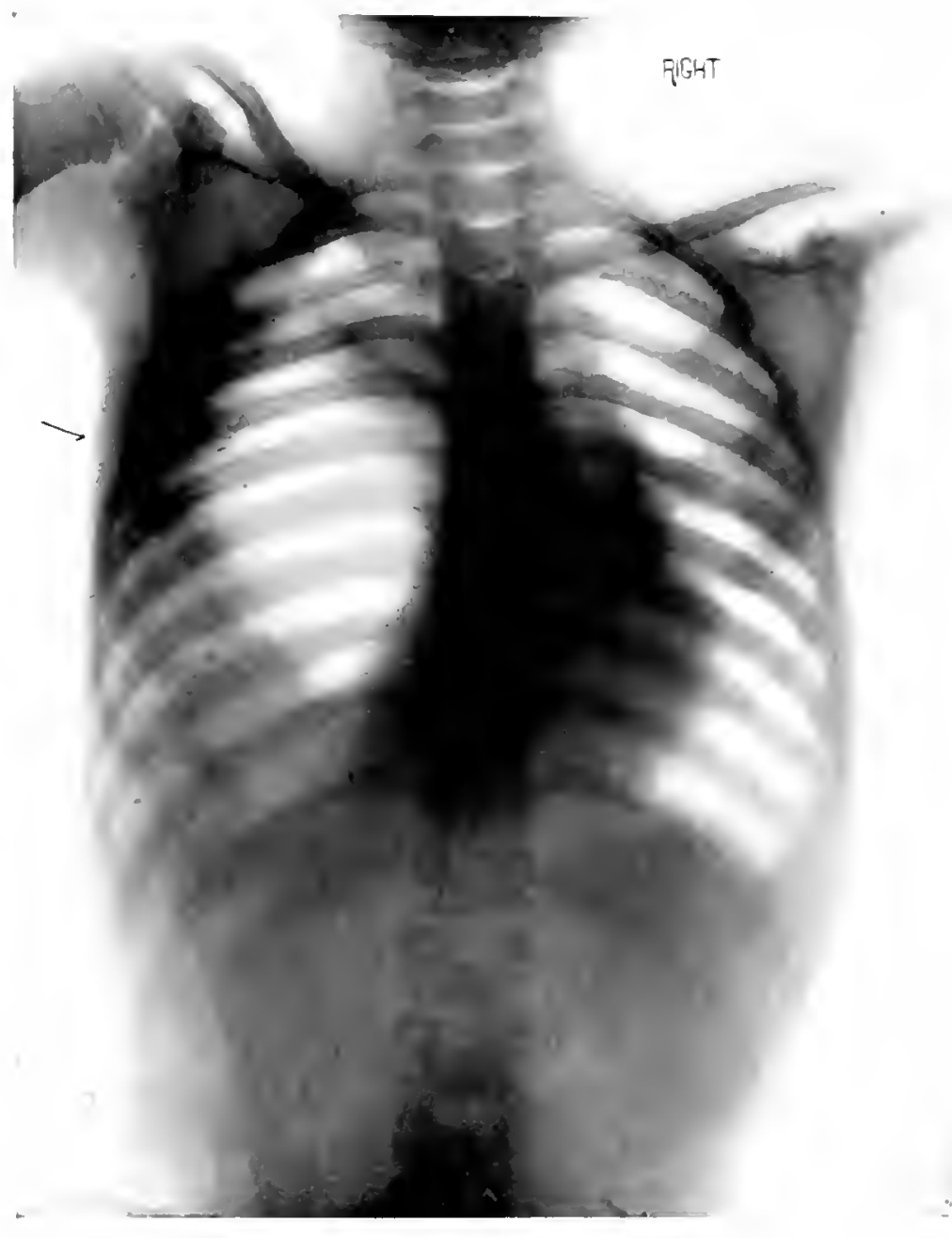


I'T.TTE: 124.

THUREXI) PLELA.

lons, ayn b yearn.

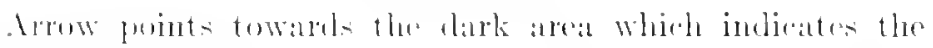

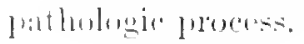




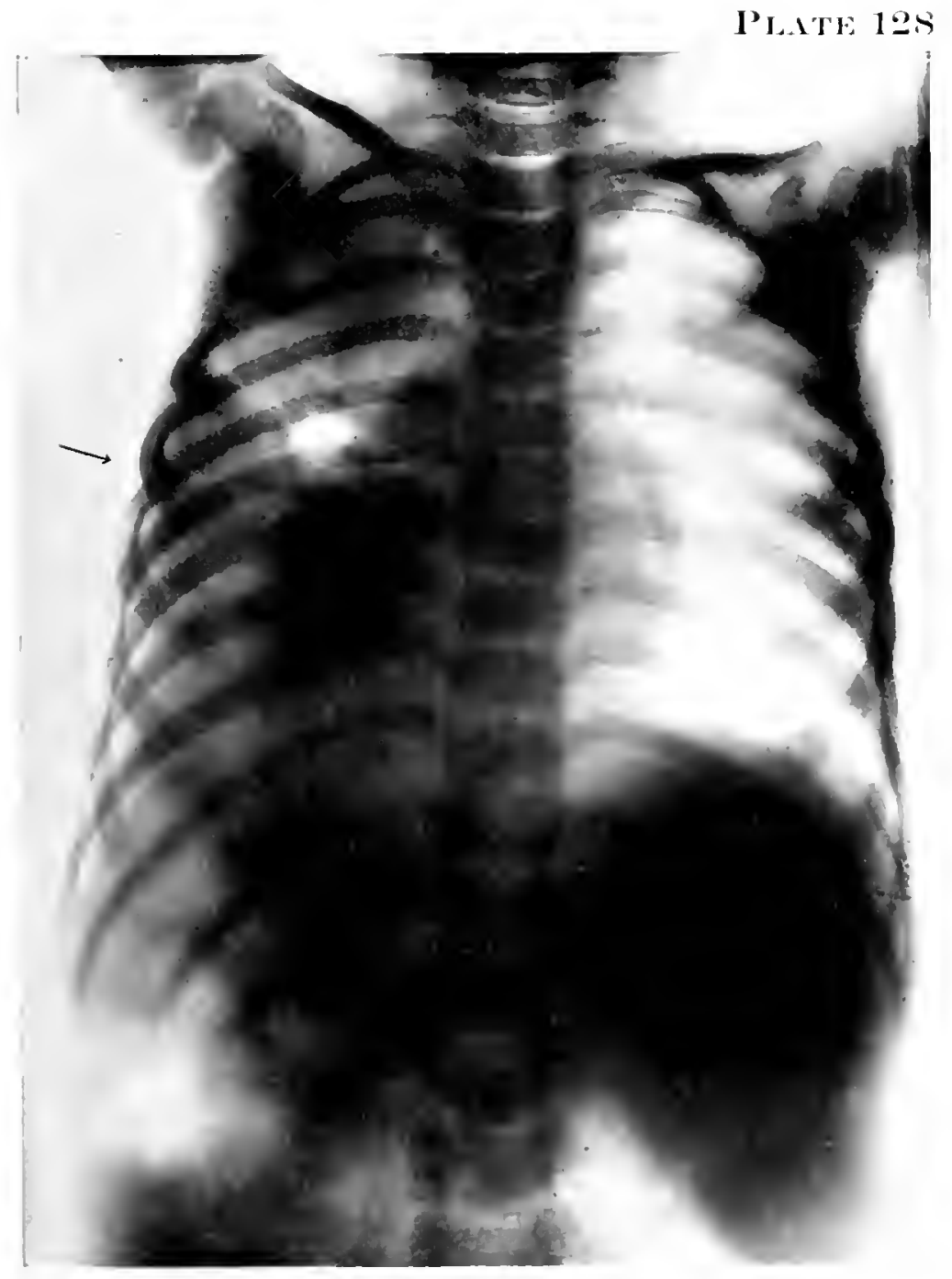


PI.IT'1: 1:9!.

PHELRIS WTH EFFESOA,

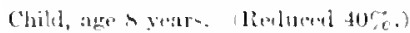

The arow points towarkis the effusion which fills the whoke

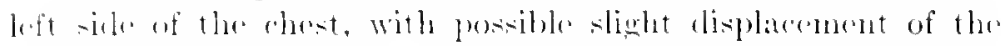
heart to the right. 
PLATE 129

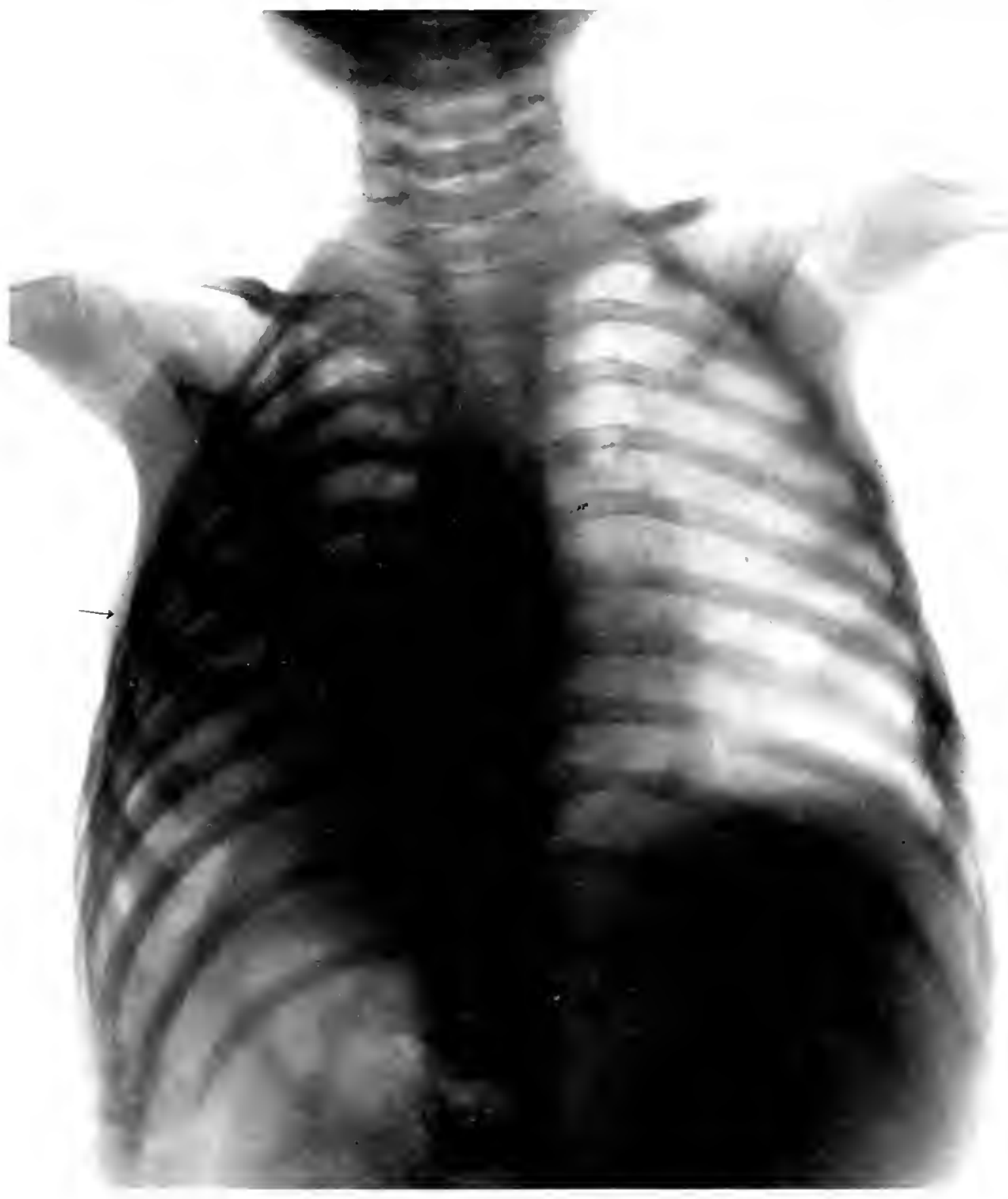


Pl. 1T1: 130.

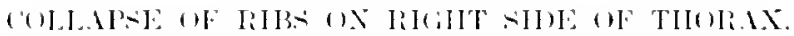

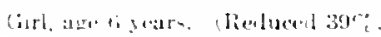

This condition followed an ohl emusenat on the right side, with revult ing :ulhw:

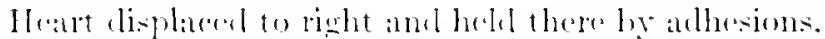

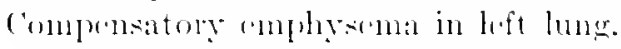

The arrow pinte to the enllapsed rils: 


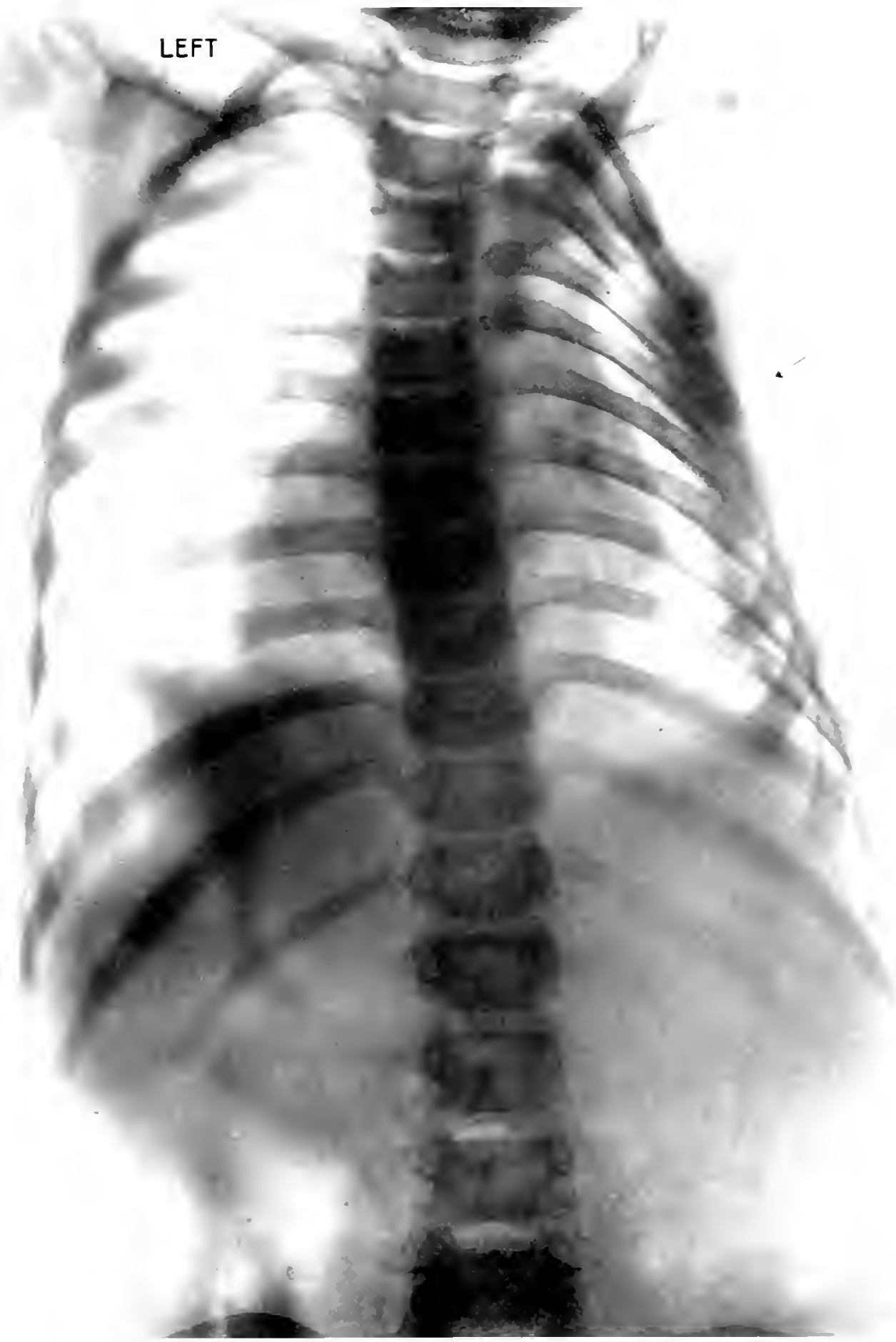


PIATE 131.

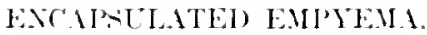

Boy, age 10 year. (Reduced 45\%

The arrow points towirds an encapulated empsema at the hatse of the right liner.

This ("at: wats operated upon and the Roentgen diagnosis: wat formel to be correct. 
PI, TE 1::1

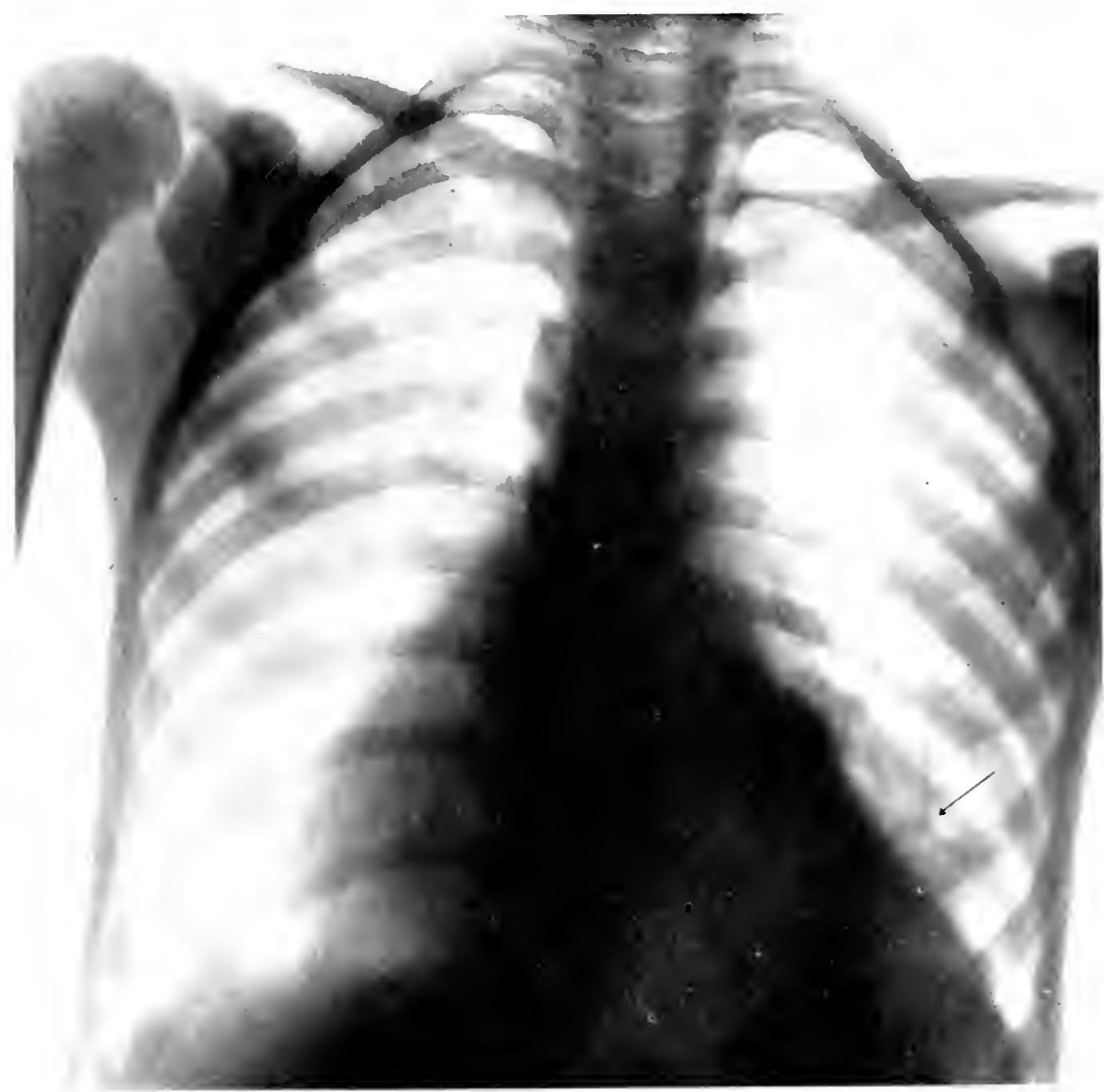


I'I.XTE 1:32.

II.ATIS) ILEART.

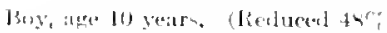

The arrow points towards a listinct carliohepatic angle. 
PIANT $1: 32$

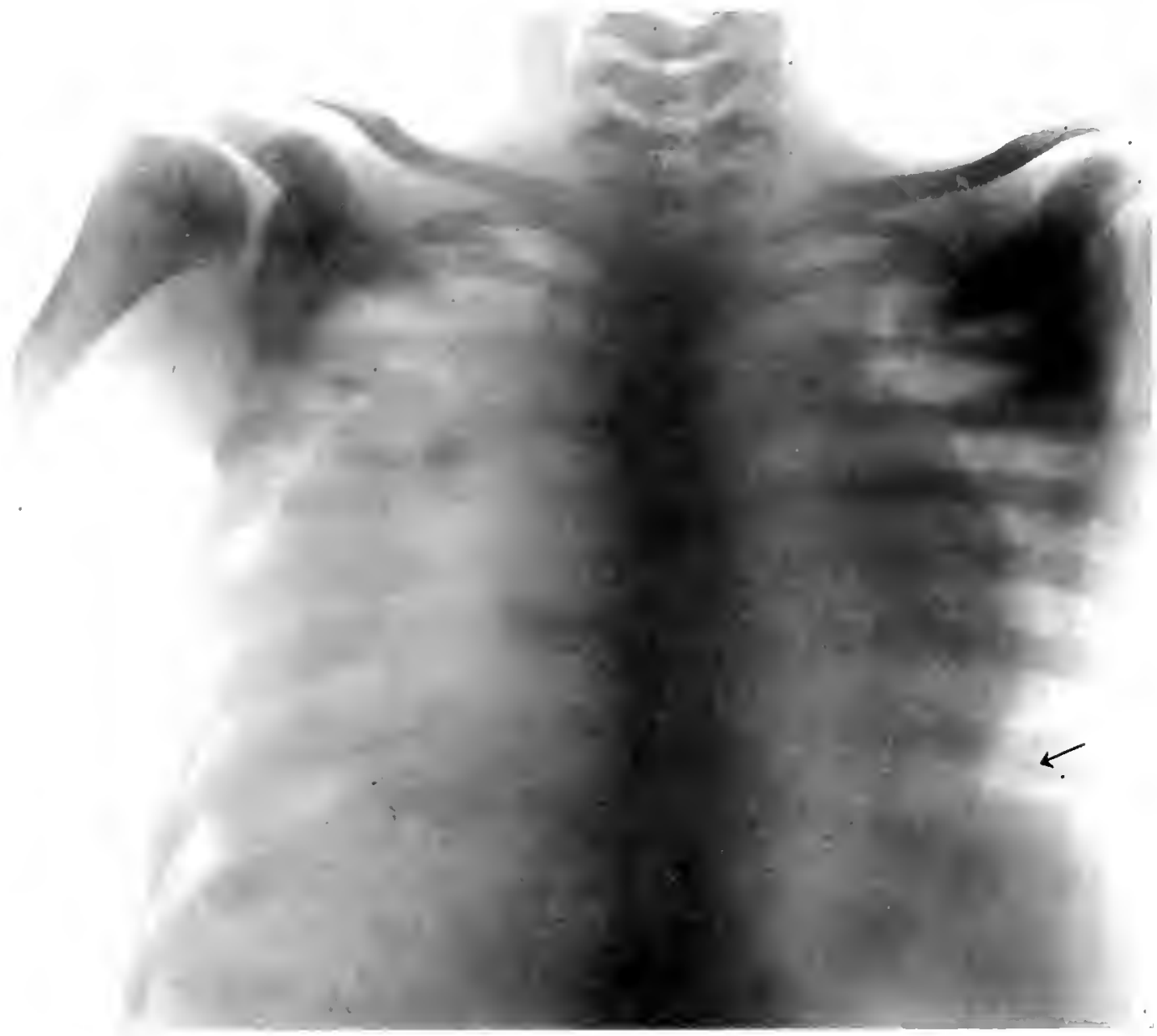


Pl. TTE 1:3.

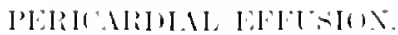

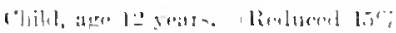

A and b. The outlines of the heart.

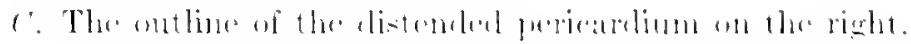

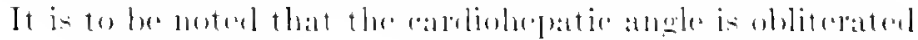


Prate 1:3:

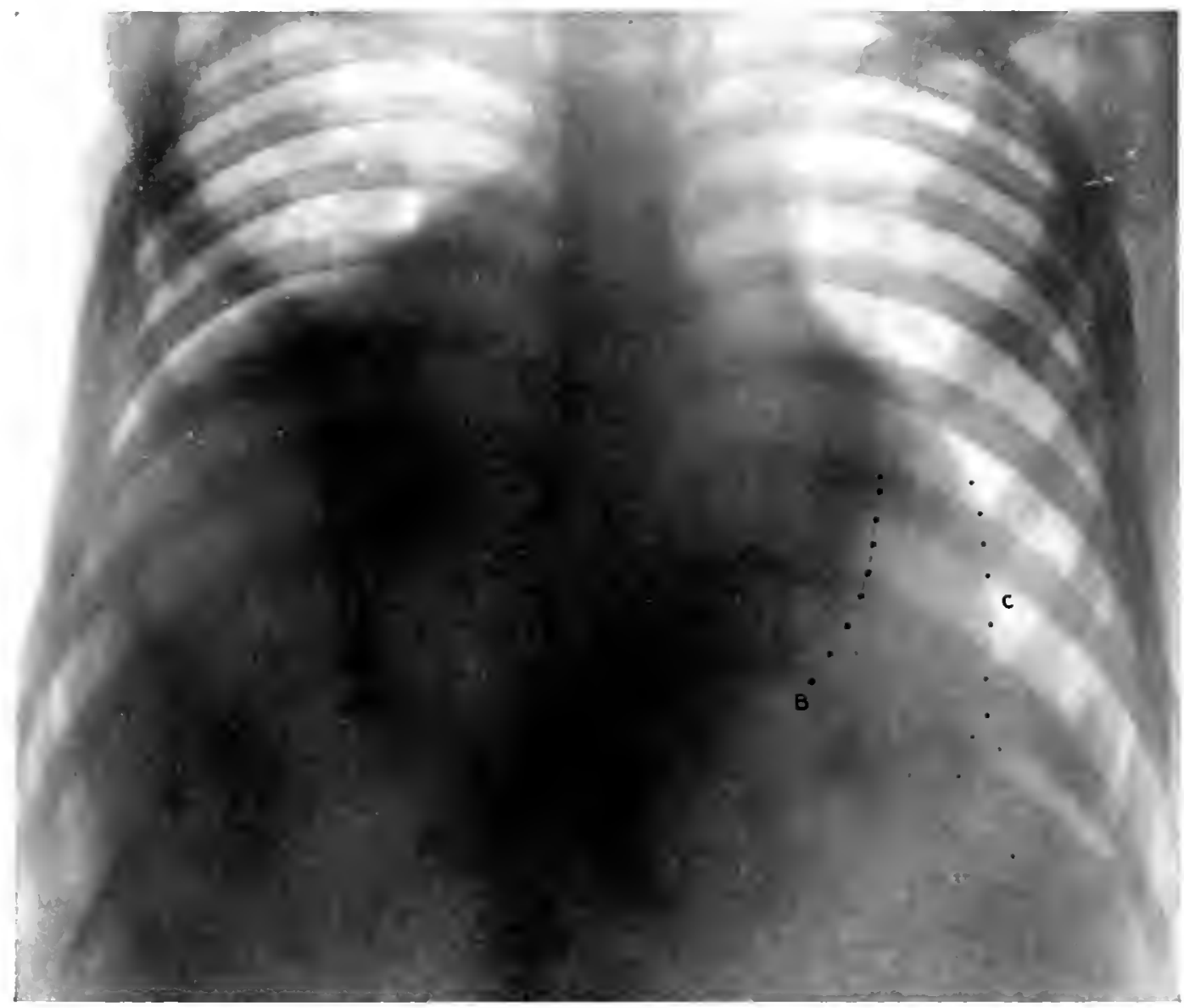


l'1.17\%: 1:31.

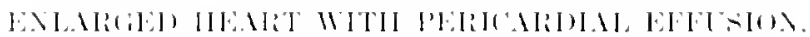

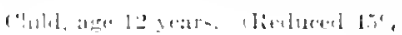

1. The likitul hente

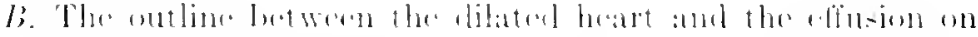
threlit.

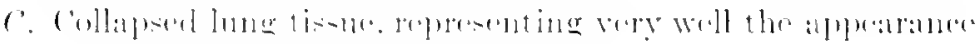
wheh wombl bo fomel in atclectasis. 
PISTE 1:; 1

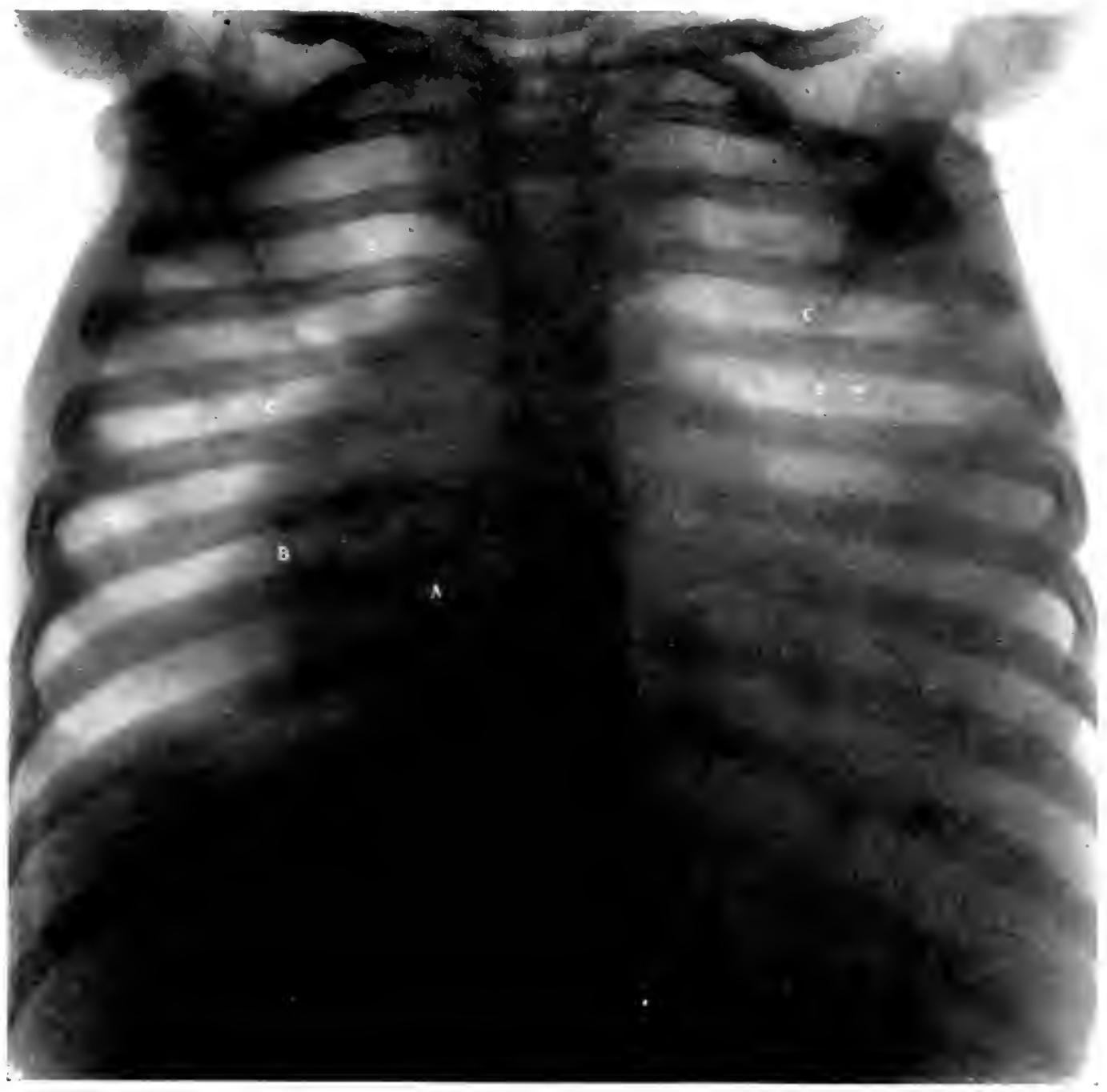


PJ.A'l: 13.i.

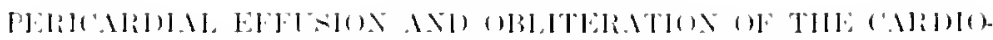
IIISTITI INGI,

\section{lind}

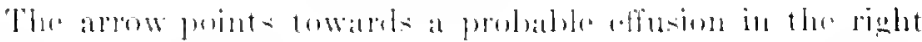
plemar: 
PIATE 1:35

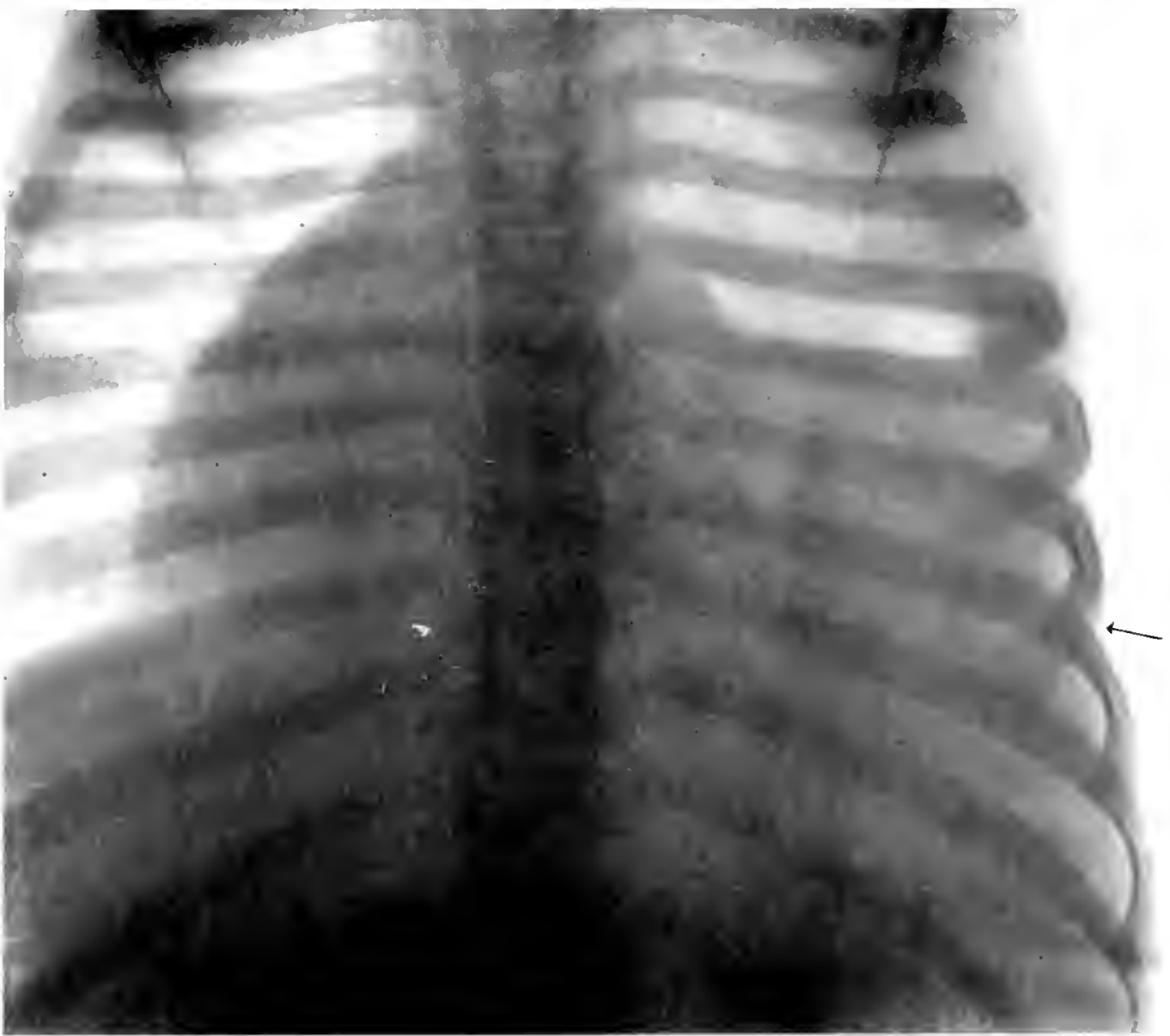




\section{Pl..1'l: 1:30;}

\section{A.TERIRI.}

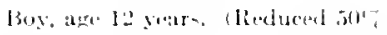

The atrow points towateds an increatsed andal of density

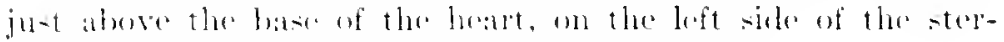
num, and present the cliniral signs of ancurism. 
Piate 1:36

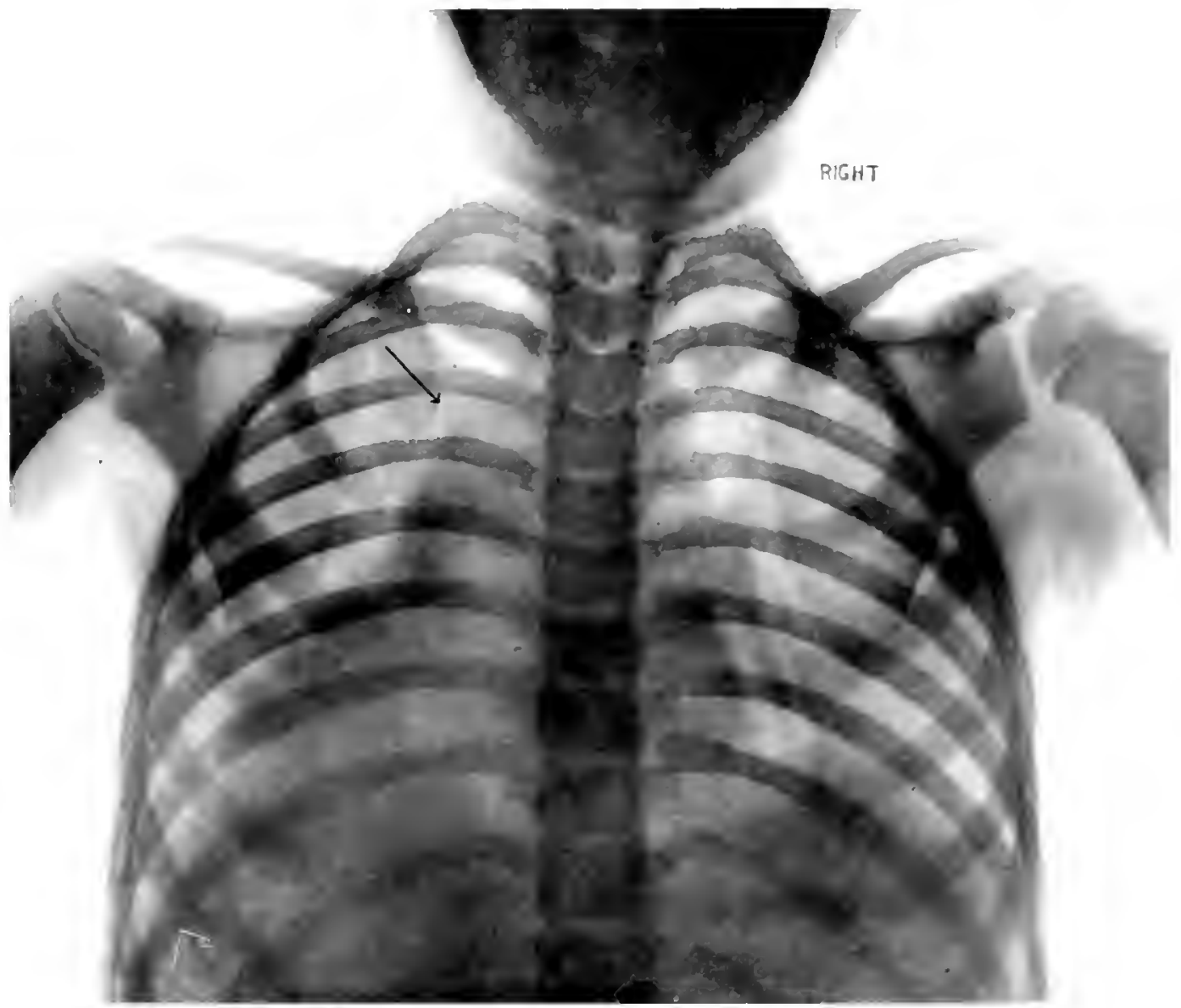





\section{Division VII}

THE ABDOMEN

Althougin it is difficult to obtain satisfactory Rocutgenographs of the abdominal organs, yet great advances have been made by using new methods. A striking illustration of this is shown in Plates 137 and 138 .

These Roentgenographs were taken by Dr. Percy Brown to show the greater accuracy of outline obtained by a short exposure, Plate 137, seven seconds, the child not breathing, over Plate 138, a longer one, thirteen seconds, taken during nomal respiration. The child had been given a large dose of bismuth and in this way the resulting great density was obtained. The plates illustrate how the comparatively dean-ent edge of the stomach shown in the short exposure is contrasted with the blurred ontline of the long exposure. There are a number of congenital malformations of the intestinc, represented by obliteration of the intestine from vilrions cunses, such as filmrous bands, yet it is rare that we cin show these conditions by Roentgenographs. The malformations of the cesophagus and stomach are also difficult to obtain a picture of, but considerable progress is being made every day in the diagnosis of gastric conditions.

Plate 139 represents the stomach of an infant five weeks old, showing the so-called hour-ylass contraction. The outline of the stomach is very distinet, especially on the left side where it is of the normal size, whereas on the right side it is very small, due to a contraetion just behind the lumbar vertebre. This gives the hourglass appearance. Otherwise the stomach appears to be normal. The outline of the intestine is distinctly marked below that of the stomatch.

Plate 140 shows the stomach of the same subject after the 
hom-glass contraction has heren removed by the inflation of the stomach with air thomely a soft mbber cathoter.

Wo must remember that as our technie is perforted and our powers of interputation increased we shall probably in the future muderstand much which we ammot now interpret, although we know it must be on the plate. Expecially in ablominal rases we are polvably looking at conditions which ane molombtedly in the plate but which we "amot resogntize.

Plate 111 shows the pirture produced by a fluid in the abdomen of a girl twolve yours old. The pirture in the midele is darkel than at the sides, wheh. alroolding to Georere, is on alerount of the ereatre depth of the fluid in the middle. Dr. Cieorere also interpreted

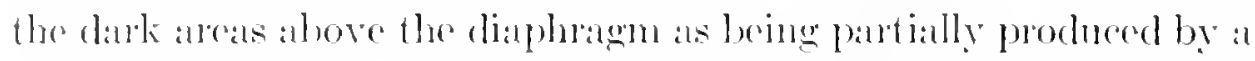
considerable amount of aedema which was present in this case and also by compression of the lung.

Plate 142 slows what appears to be a mass of enlarged mesenteric nodes in the right side of the abohominal "arity in a child ten ol eleven yaus old. By relinical axmination it was impossible to detect handened fieces or any tumor or loreinu boty. As the child diel not react to the mberenlin test, the diannosis as to whether the

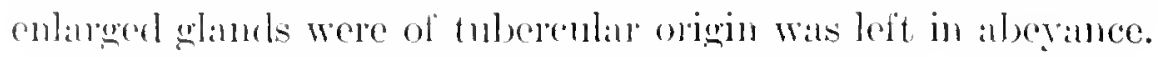



PI.ITL: 137.

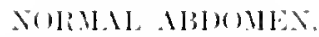

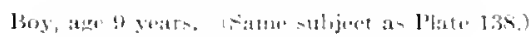

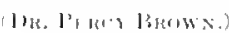

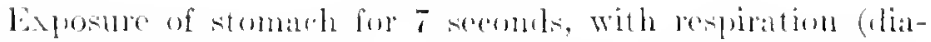

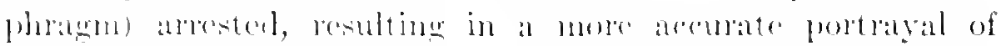
the pastrite outline.

Stomath contains abunt one ounce of bismuth.

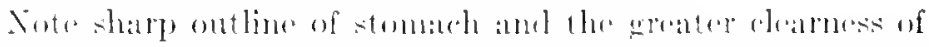
detail in complarison with the same subject under long "xpostare shown in Plate 138. 
Plate 1:37

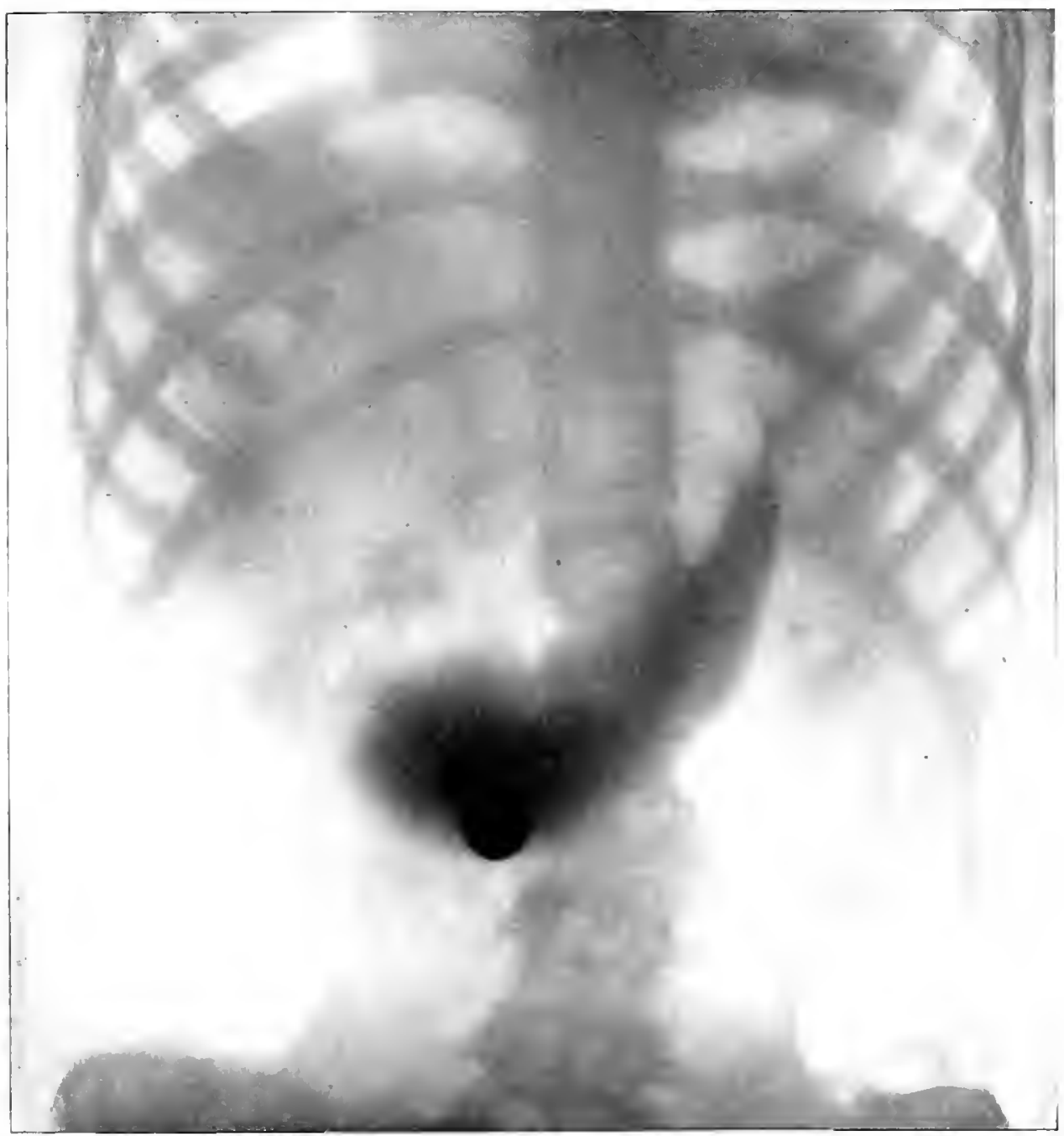


PLITl: 13S.

IORMAL ABDOUEX.

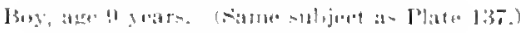

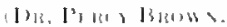

stomach contains ahout ome oumer of hismuth.

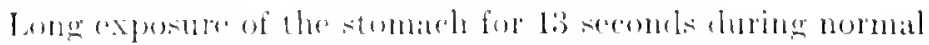
mispirition.

Note the blumerel outline of the stomath in amparison with the shapply defined outlime and eneneral pieture of the same child muler short exposure. 7 socomels, shown in Plate 137. 
PIATI: 1:3

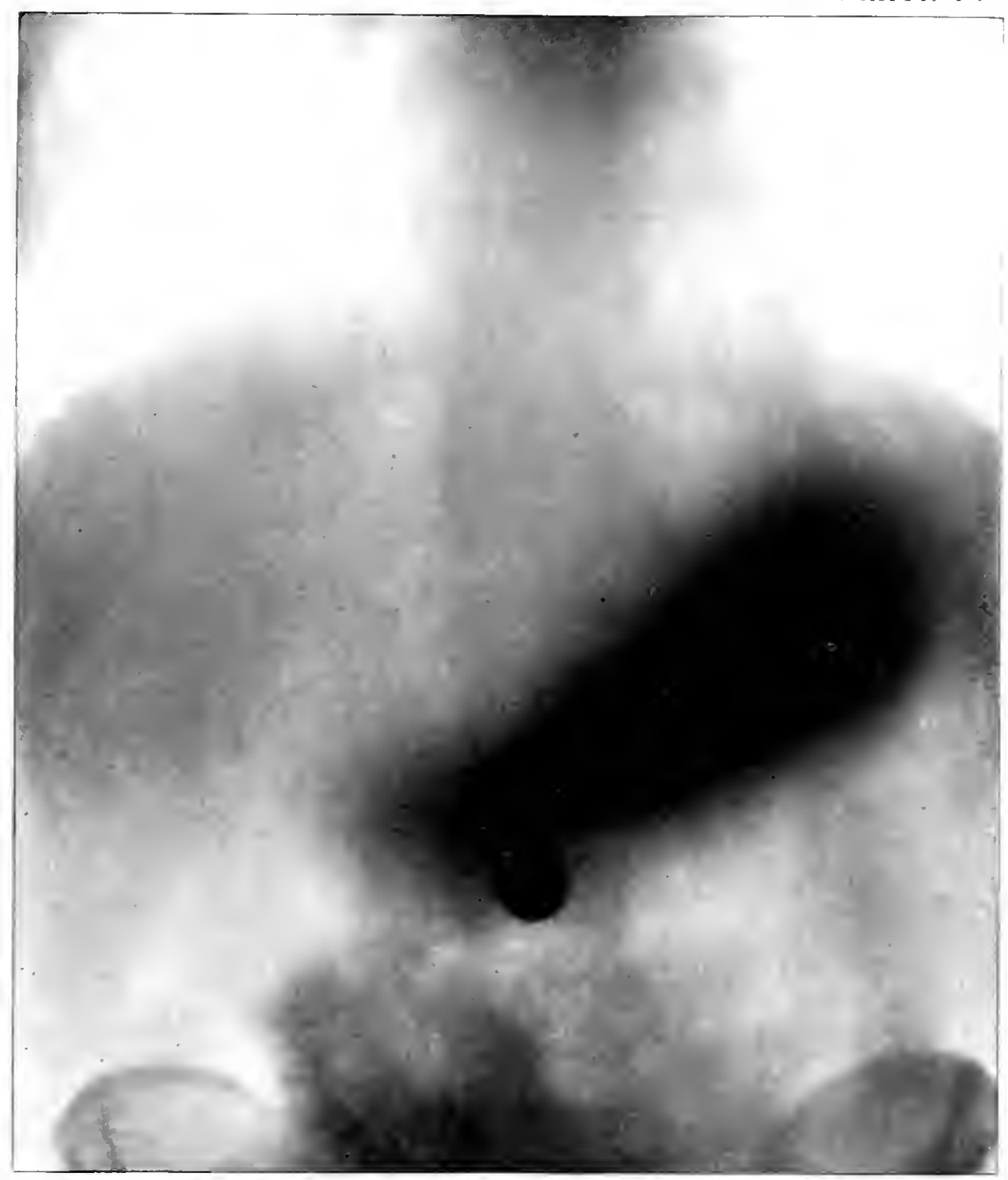


PI.TTY: 139.

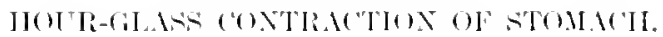

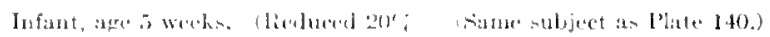

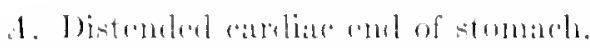

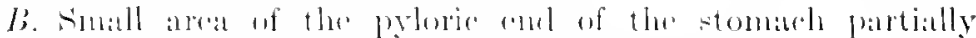
separated from cardiate cuel ly contraction. 
Plate 1:39

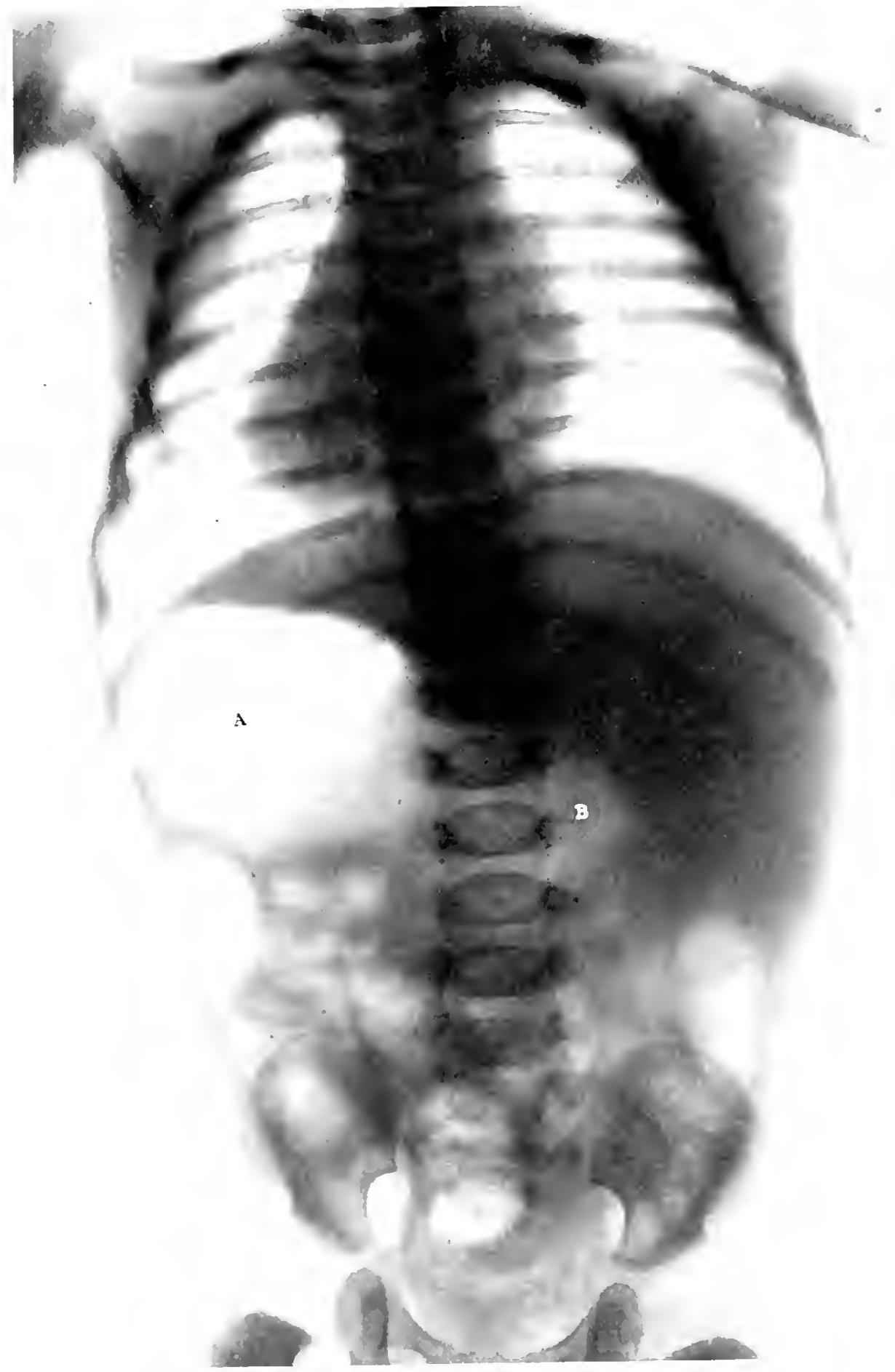


P'L 1 T'L 140.

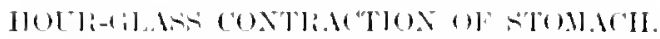

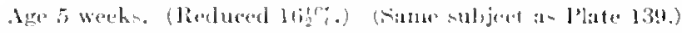

Stomareh distemeded with air by moans of a soft mubber (:athererr.

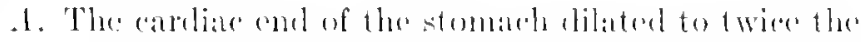
size which alpreaned when it was omly dilated by the contraction. The contration has disalypeared, and the areat reperesented ly C'still shows a slight contriation.

B. Stomach-tubu lying along the greater curvature of the stomath.

D. The distencled intustine. 
PIAT' 140

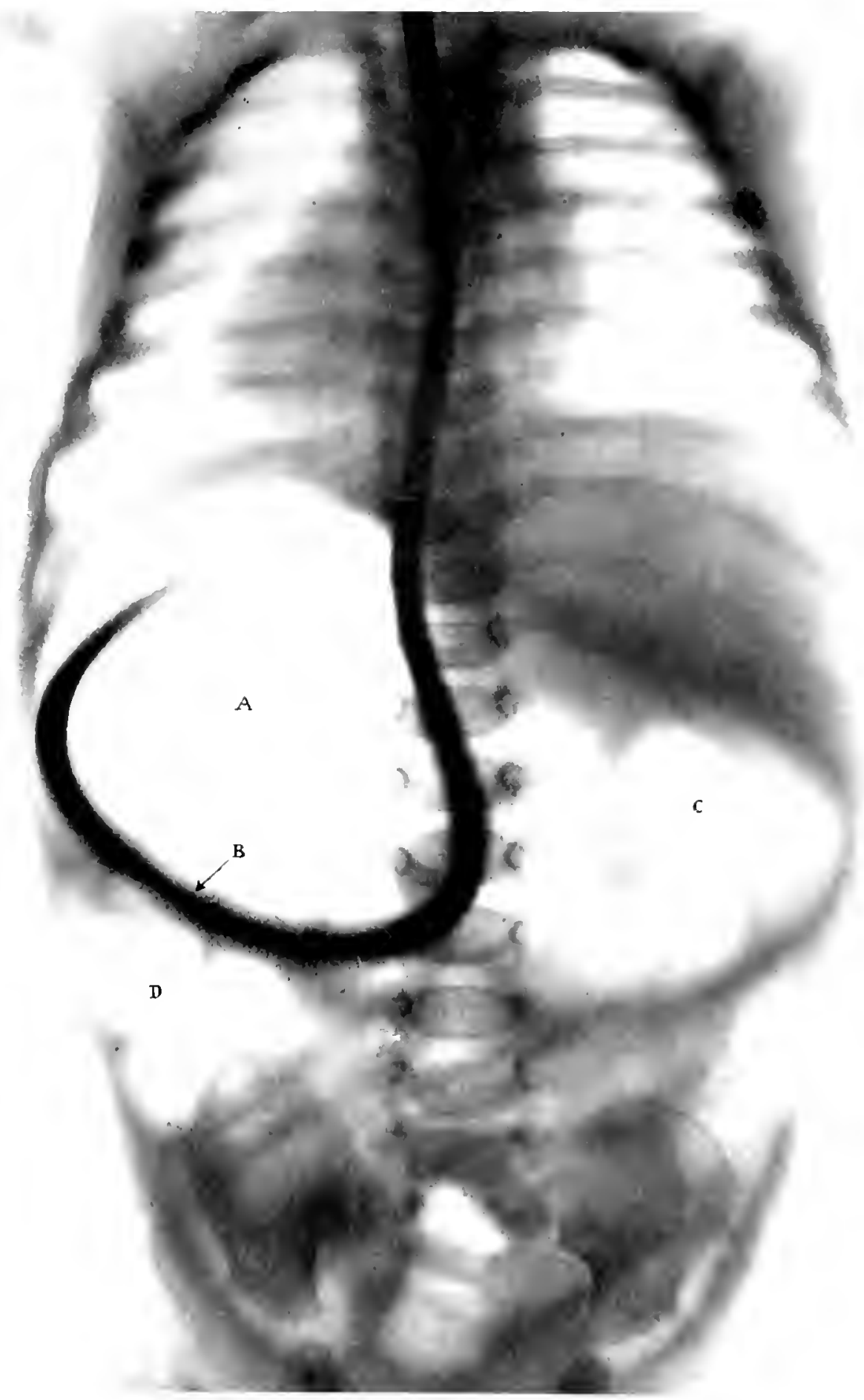


PIATL: III,

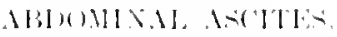

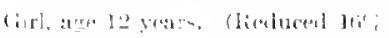

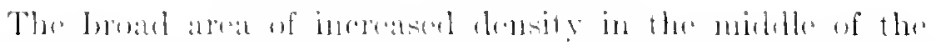

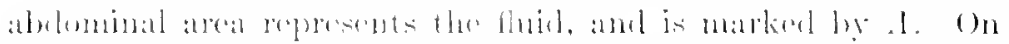

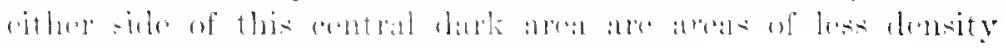
which represent at thimme layer of fluid, $B$. 
PIATf: I H

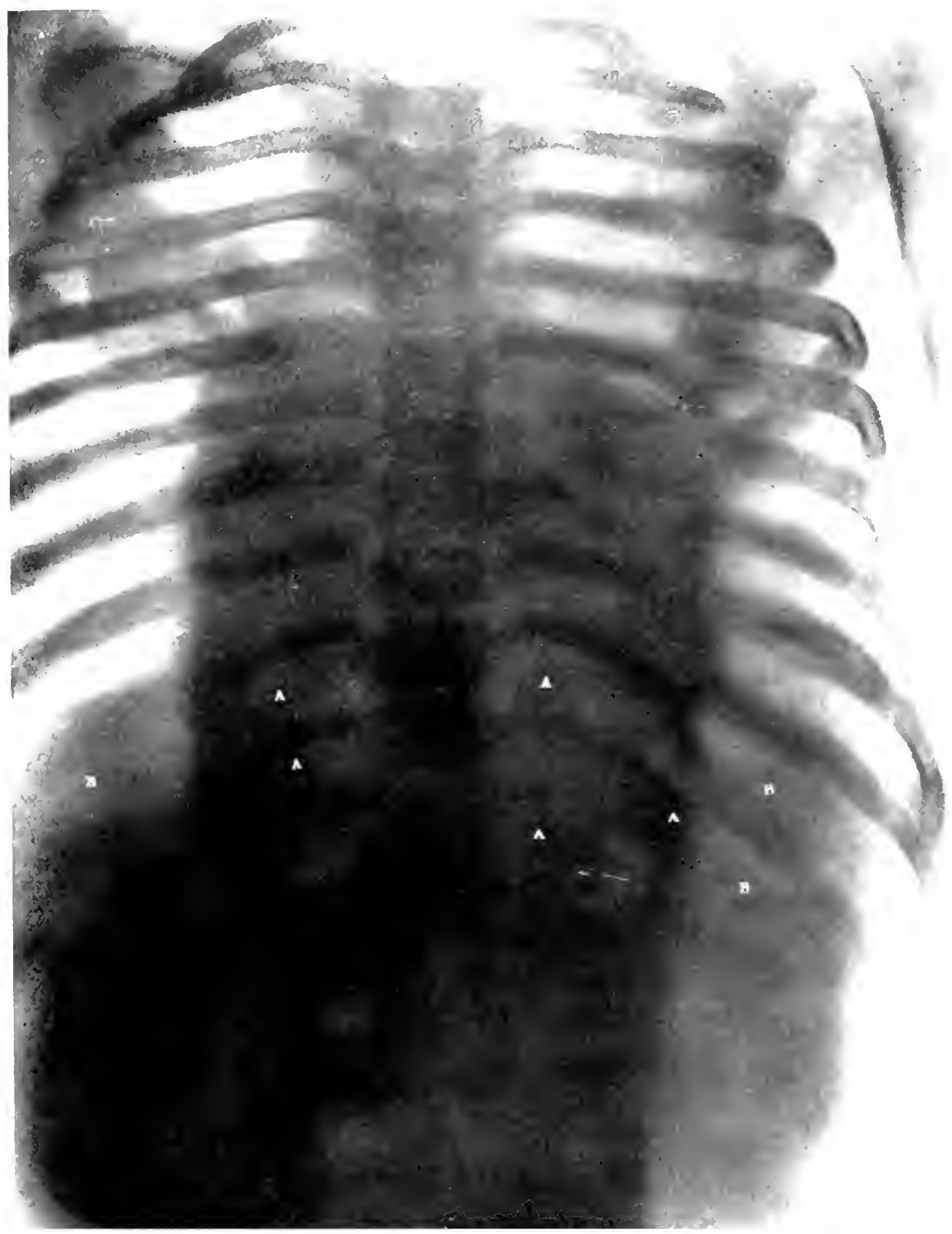


PJ.ATE 112.

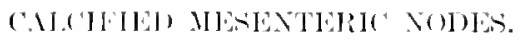

(Rerluced $35^{\circ}$.)

The arrow boints to an areal of increased density just to the right of the fourth humbar vertehrat. Clindeal differentiation

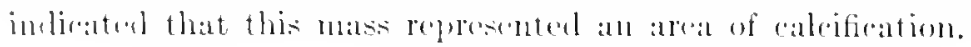


PLATL: 14Q

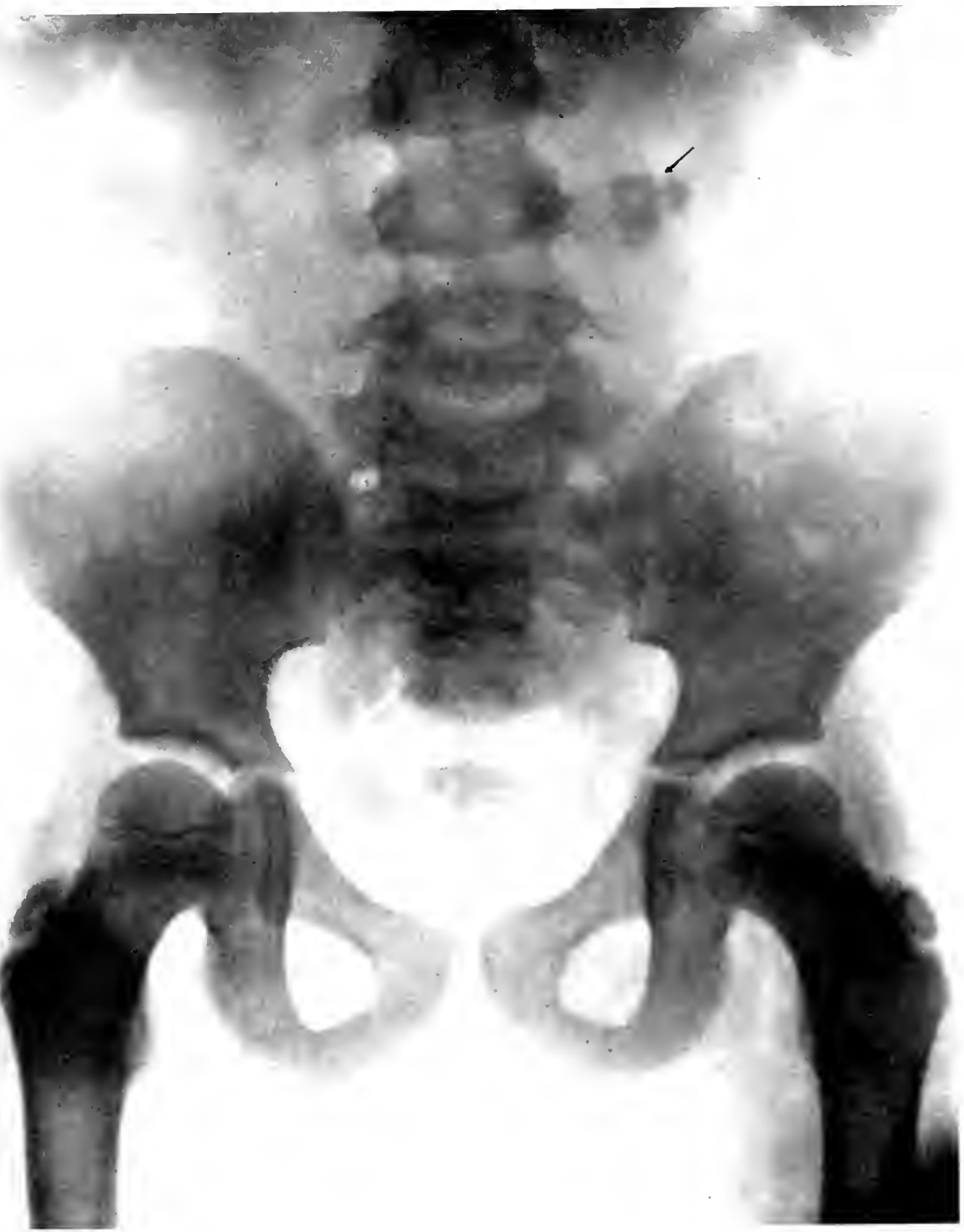





\section{Division VIII}

\section{FOREIGN BODIES}

TuE importance of the lioenteren method of examination for the detection of foreign bodies his now heen recognized for some time, and its value is increasing every day as ou linowledge of its serpe and the perfection of its trohnic increases. Even in the aldomen, which usually presents such diflicultios for obtaning a satisfactory plate, erreat progress has bern made in the line of diagnosis, and in the near future we shall probably receive much aid from the ray in differentiating a tubercular mass from at foreign body. Children are so much more apt than adults to swallow or inhale foreign bodies of all kinds, that the rôle which the Roentgen ray playss in this cliss of cases has become an importint one.

Plate 143 shows a stone in the urethra of a girl thirteen years old. In this case a erstitis had existed for ten years, and although the ehild had never been considered very strong she was bright and active. Even as carly as her third year she had complamed of painful nicturition, and the pain in the bladder was at times intense. At no time had the relief from the srmptoms been complete, relapses being frequent. The Roentgenograph, besides showing an oval stone approximately the size of a moderate-sized hen's egg, also disclosed in the centre of the stone a bent pin with a distinct head to it. The stone was removed by operation.

Plate $14 t$ is that of a boy five years old. It was known that the rhild had swallowed a nail, and the Roentgenograph shows distinetly the presence of the mail in the intestine.

Plate 145 shows a pemny which was by means of the ray detected in the asophagus. The location of the penny having been definitely made by the ray, it was easily remored with the asophageal foreeps. 


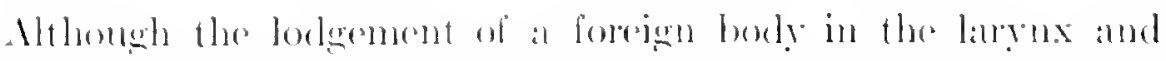

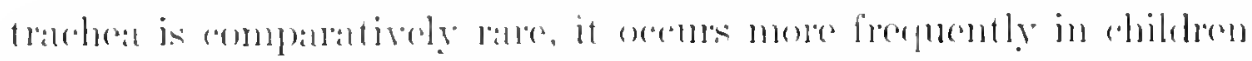

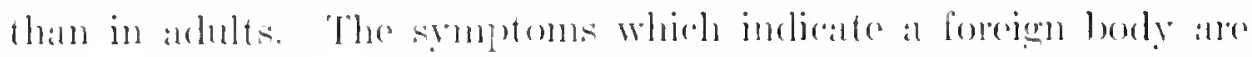

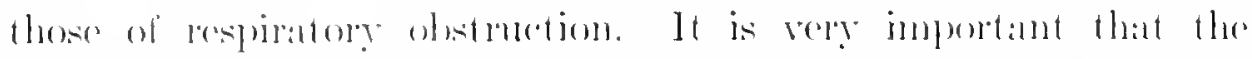

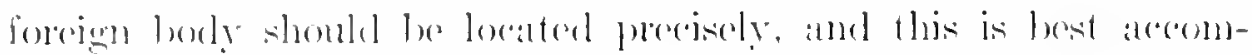

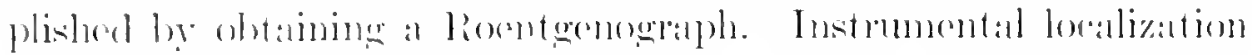

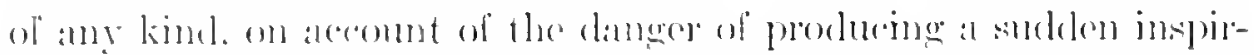

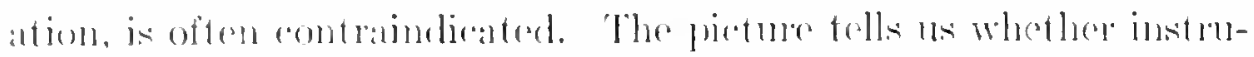
montal removal should be attempted of tratheotony be proformed at onces.

Plate 166 shows the definito lowalization made he the laty of a

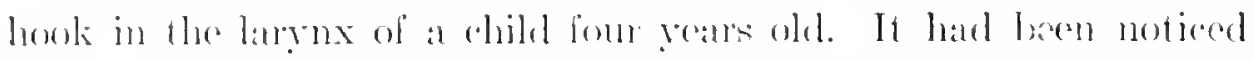
for two days that the child was mathle to rey alome and hat difliculty in beathing. The hook was located oppesite the fifth

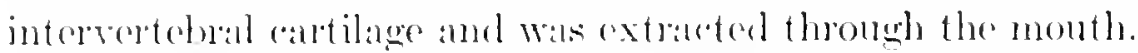

The differential diagnosis to be mate ly the ray in cases of this lind is from acute tracheal laryngitis, tramal and mombranoms baryongtis. All these, however, may he diagnosticated by other sperial symptoms directed to the larym as the souree of the irri-

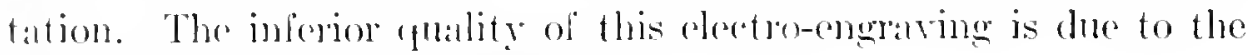
difficulty of trehnic in these cases.

Plate 1 to shows a nail wheh was deterted in the lung after it hatd beren there for five years, in a boy twolve years old. The nail is seren in the light lower lobe. It hat boen inspirated five geats before the pioture was laken, and was not suspereded at that the, although a lioenterenograph had been taken. The boy had been treated during this interval of five years for valious discases of the lungs

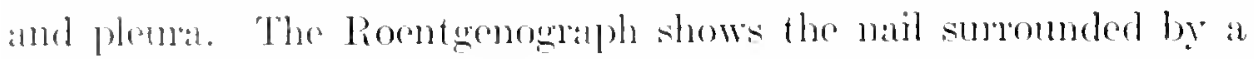
dark atrea indjeating thirekened plemas and collapsed lung. In the pirture will be notjeed at dals romel aneal in the ninth interspare at about the postrutor axillary line. This represents a picee of lead 
shot placed there as a guide at the time of the first operation, after which this picture was taken. The tenth rib just below and in a line with the shot will be seen to have been resected for about an inch. It will be noted in this picture that there is pratically no lungtissue in the right lower chest. In operation was not successful. although performed twice.

Plate 14S, the same case as Plate 119. Division VI, shows the presence of a doll's china am which was inhaled into the lung. The history of this ause is of ronsiderable interest. A girl four years old entered the hospital February 10, 1907, with pulmonary symptoms. The statement was made that she had swallowed the arm of a china doll in ()cober, 1905. At that time she became cyanotic and had a severe attack of coughing. Since that time she had had considerable dyspnow, had never seemed well, had coughed spasmodically from time to time, hut had been up and about with a good appetite, sleeping well, and with no history of any other symptoms. On entering the hospital, she did not look very well and her temperature was $10210 \mathrm{~F}$. respirations 62 , and pulse 165. She had a short irritating rough and there was a marked dilatation of the alæ nasi. There were signs of consolidation in the lower right back and the respiratory excusions were greater on the left side. In the left back there were lessened rocit resonance and tactile fremitus, and at about the middle there was hyper-resonance over an area of $5 \mathrm{~cm}$. in diameter. The rest of the phrsicul examination was negative. There was a leucocytosis of 5.5.500. The Roentgenograph showed a pneumonic process corresponding to the clinical examination of the right lung. Nothing was show by the ray in the left lung. The pneumonic condition showed well in the plate but is not so satisfartory in the electro-engraring.

Plate 149, taken February 10th, shows the presence of the foreign body (the picture is reduced 35 per cent. and the plate was talien 10 
with the child's face lying on the plate). The foreign boty was

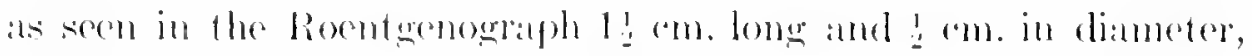
situated at the junction of the seventh rile with the left edee of the stermum. This eorrespomeded to the piece of the arm which hath been broken off from the rhikl's doll. The diannosis was mate of the inhatation of a piece of a china arm, oceduding probaldy oue of the left lonedni, and followed by puemonia of the right lume, with probable interference of expansion of the left lume, as an aspiration of the left rhest was negative.

Pate 1in), taken liebruary loth, shows the sime pieture. The ehild's symptoms inereased in sererity, but the white comt dropped to 23.000 on Fenduary loth and to 18,300 on February 13th. The

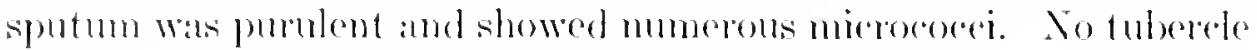
hacilli, influenza bacilli, or ponemocoeri were present. On February 20th there were a few pnemoeoced. Fobmury 2ath the white count was $16, f(0)$ and the phesical signs in the hmes were the same.

A plate taken Febmary 13th showed that the position of the arm had rhanged, it now being seen between the sixth and seventh ribs.

A plate taken February lth showed the position of the arm to be about the same, hut this picture wats taken with the ehild lying on its face on the plate. The temperature at this time was ranging from $99 !_{2}^{\circ}$ to $101^{\circ} \mathrm{F}$, with the pulse 140 and the respirations 150 to 160 .

The rhild eomghed up the ehina arm on Mareh 12th. A Roentgenograph wats immediately taken, and it was foumd that the pieture of thr atm had disappeared, but that the pucumonic process still eontinurel. The white eount was 14,700 .

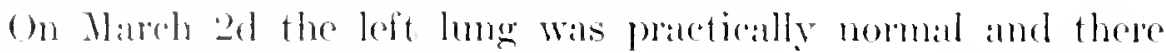
were signs of resolution in the right lung.

(I) Mareh loth the rough, dyspneat, and general symptoms had 
almost disippeared and the child was up and about and was looking well. The temperature, pulse, and respirations were practically normal by March 21st and the signs in both lungs were normal. The child was discharged well March 28th.

Although the bronchuscope hat been used successfully in a number of cases, it was not deemed allvisable after a few attempts to use it further in this calse, as the Roentgenograph showed that the arm was located too deeply in the chest.

Plate 151 shows a penny localized by means of the Roentgen ray in the left inguinal region. The pemy is seen in the descending colon, as a large round arra just above the crest of the ilimm. The outline of the intestine on the right is clearly seen.

Plate \$6, Division III, shows a needle which was by chance detected in the right hip when the child was being examined for some other trouble.

The following group of plates shows needles in virious parts of the body:

Plate 152 shows a needle in the knee-joint.

Plate 15.3 shows a needle in the linee (same case).

Plate 154 shows a needle in the dorsum of the foot.

Plate 15.5 shows a needle among the tarsil bones and in close juxtaposition to the cumeiform bone.

Plate 156 shows a needle in an area just moler the little toe. 
I'T.ATE 113

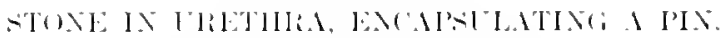

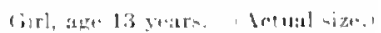

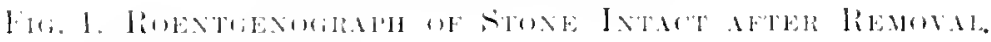

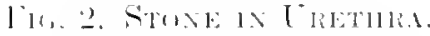

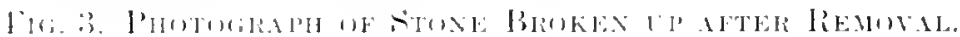


FIG. 2

FIG 3.

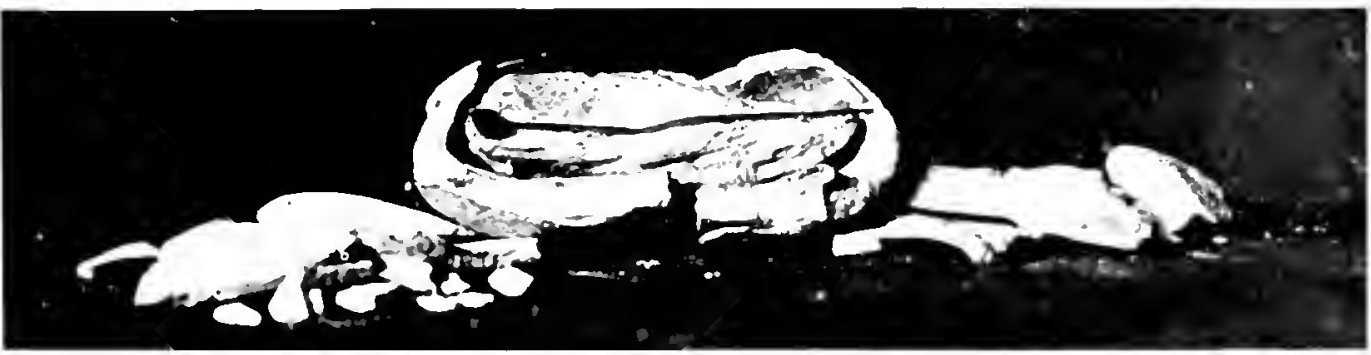


I'L.11\%: 111.

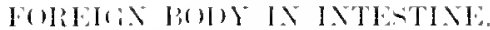

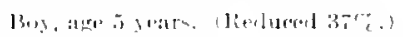

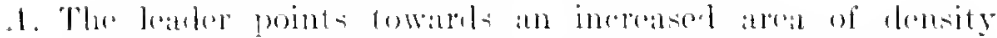

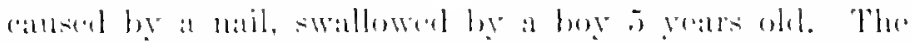

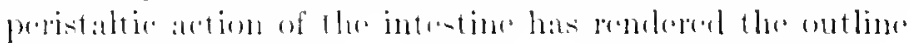
of the nail indrefinitr. 
PIATE: 114

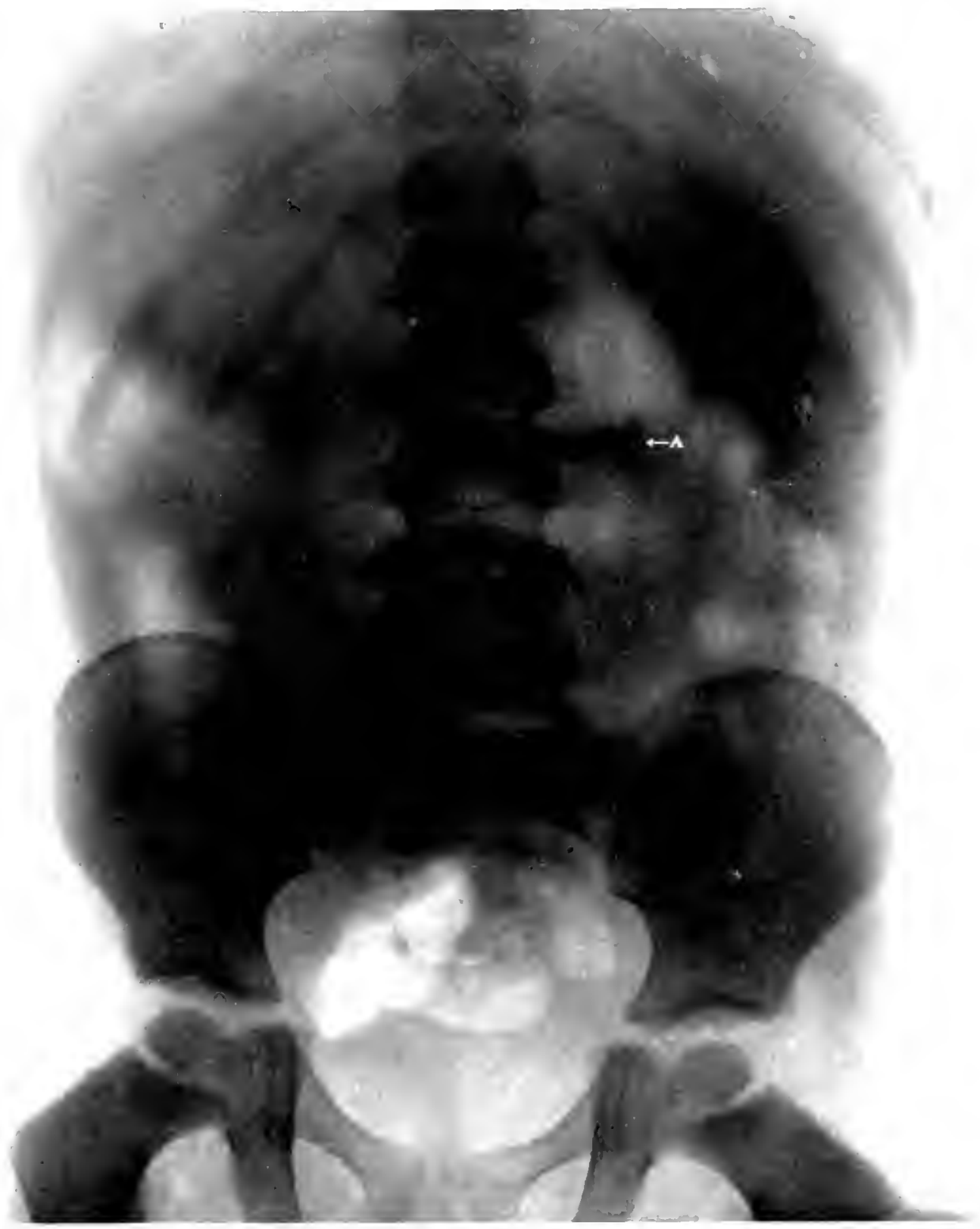


P!. V'l' 145.

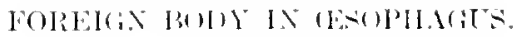

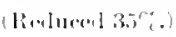

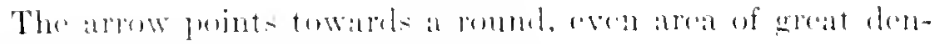

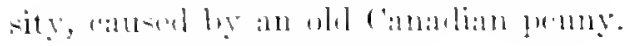


P'LATE 14.5

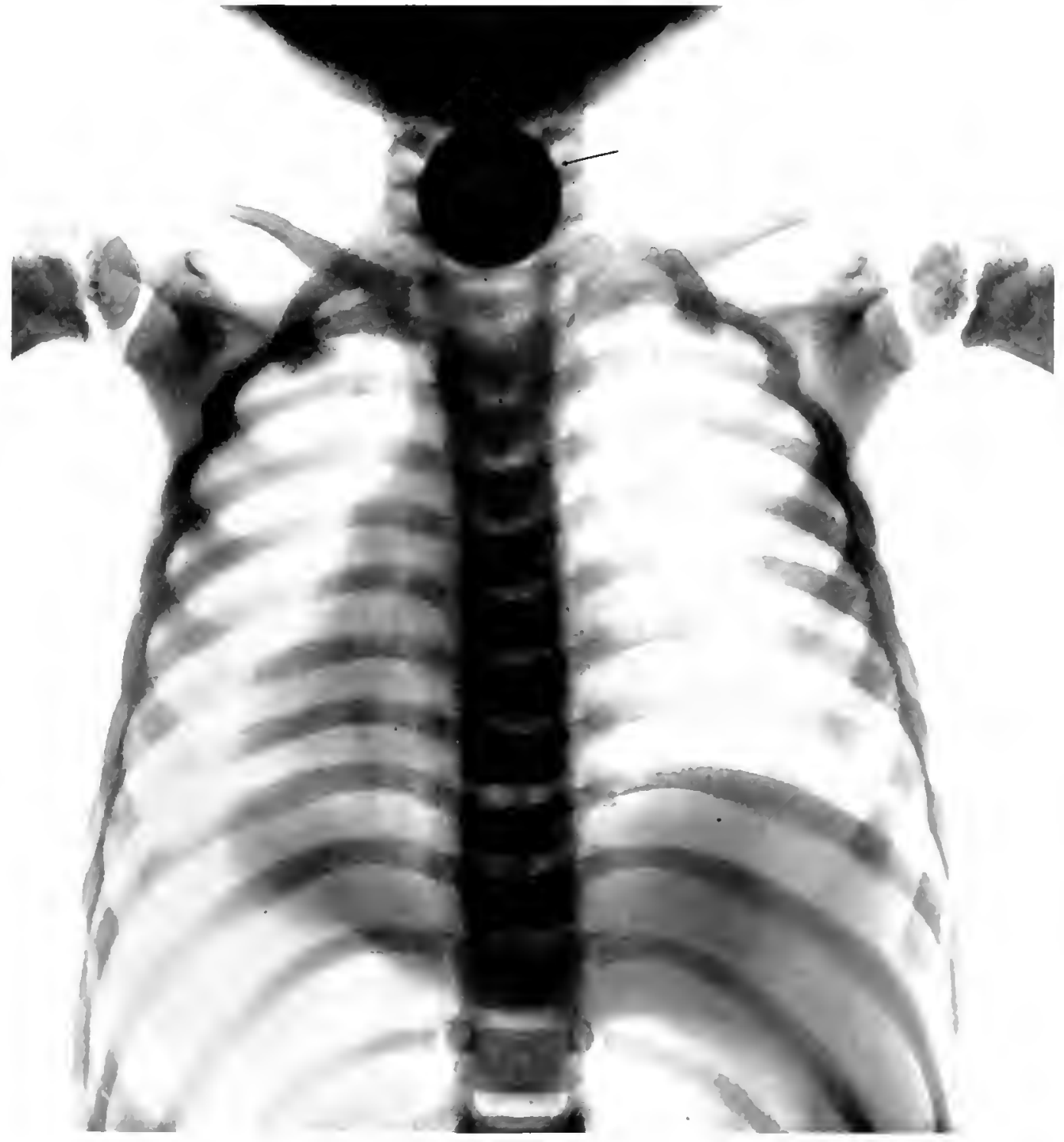


l'L.ITI: $111 \%$

HOMK IN THE LARYXX.

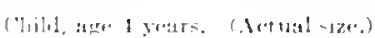


P'ITIS 1 |l

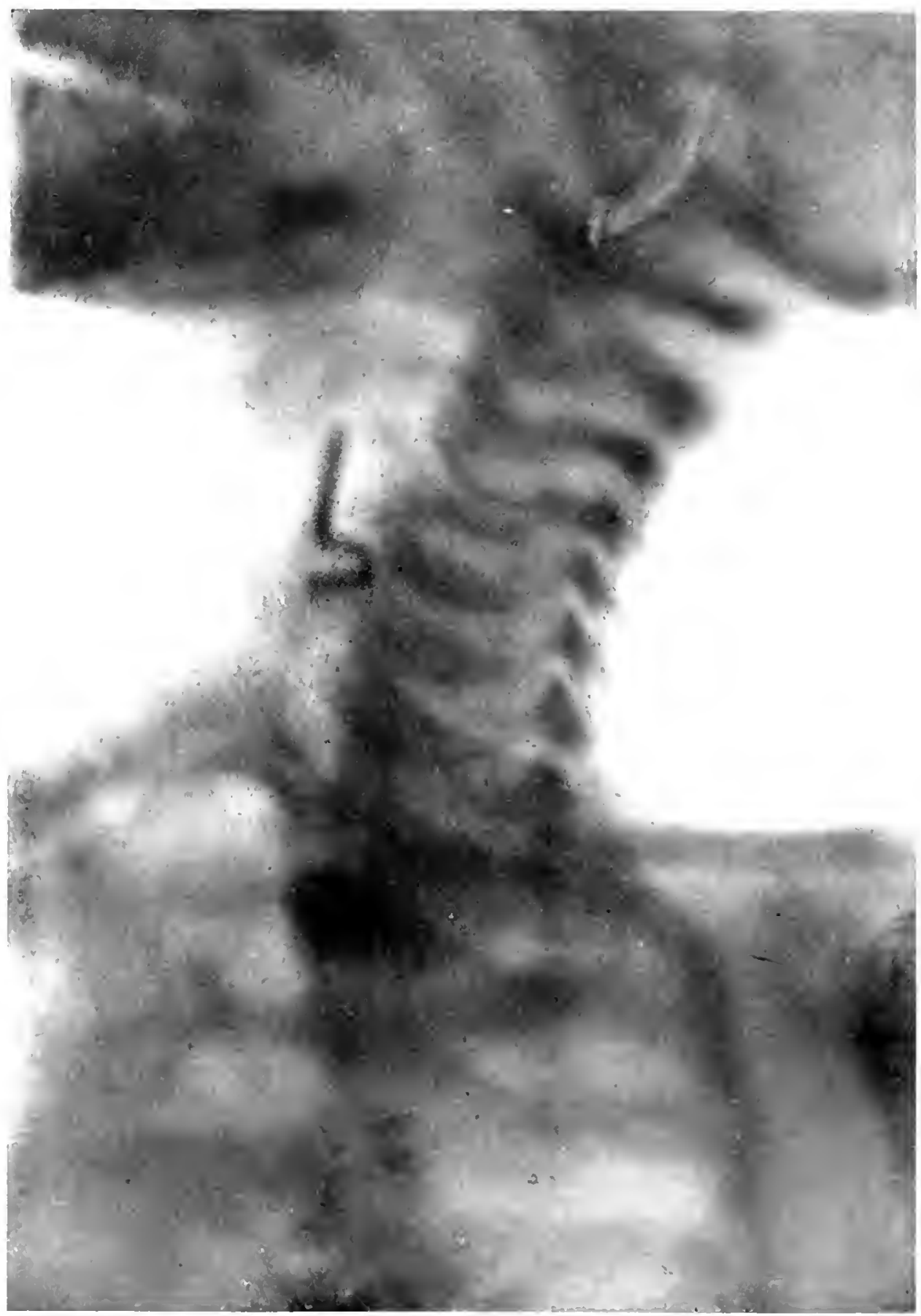


I'I.ATE: 117.

N.IL IN RIGHT L.TNG.

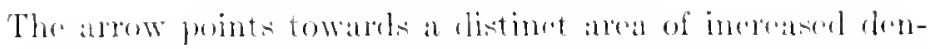
sity, hatring the shape of a ratil, and at about the junetion of the ninth ril, with the spine. Sust to the right, and al little above in the sixth interspater, is at sulall round area showing great density; this represents the shot used as a guide. 
Piate 147

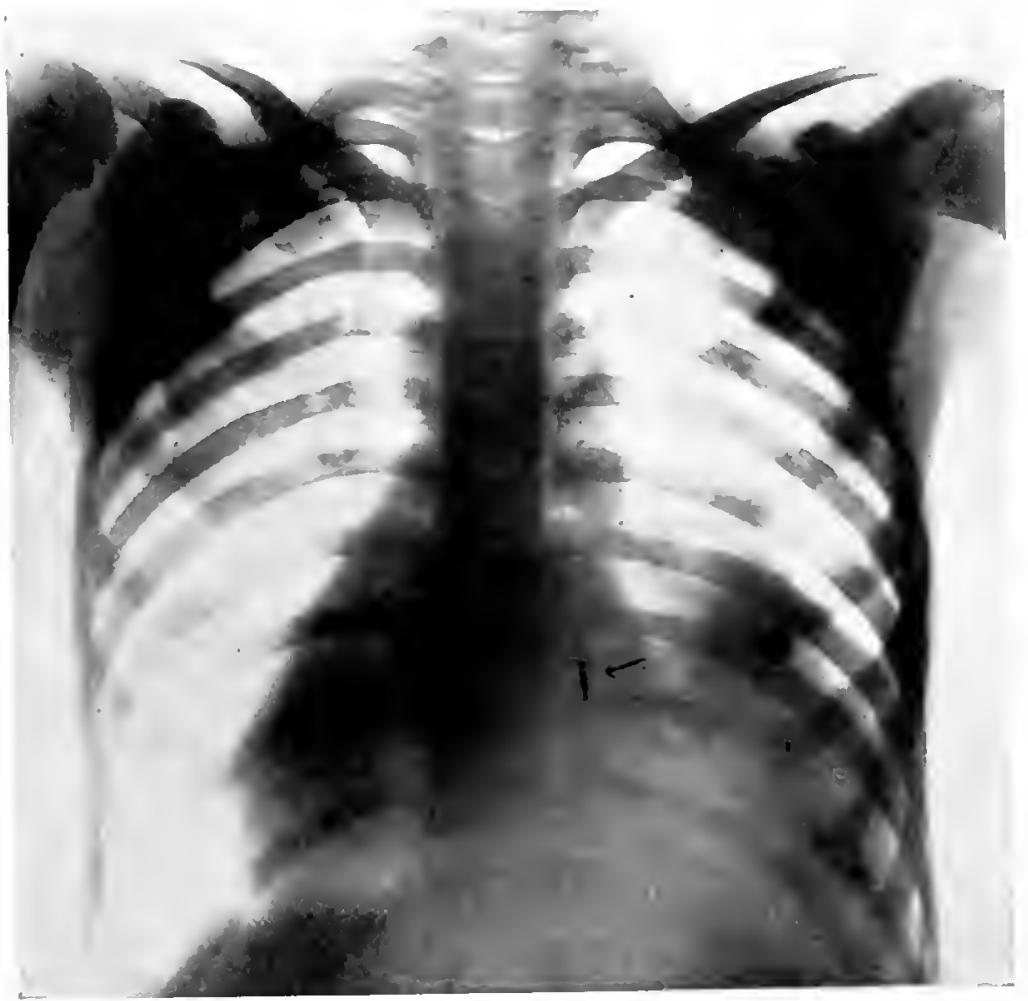


PLATT: 1K.

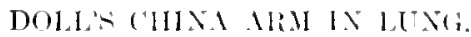

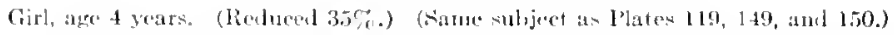

The arrow points to a small atera of increased dencity at alwout the junction of the seventh rib with the sternum, on the lift sille. 
I'I.1T: I IS

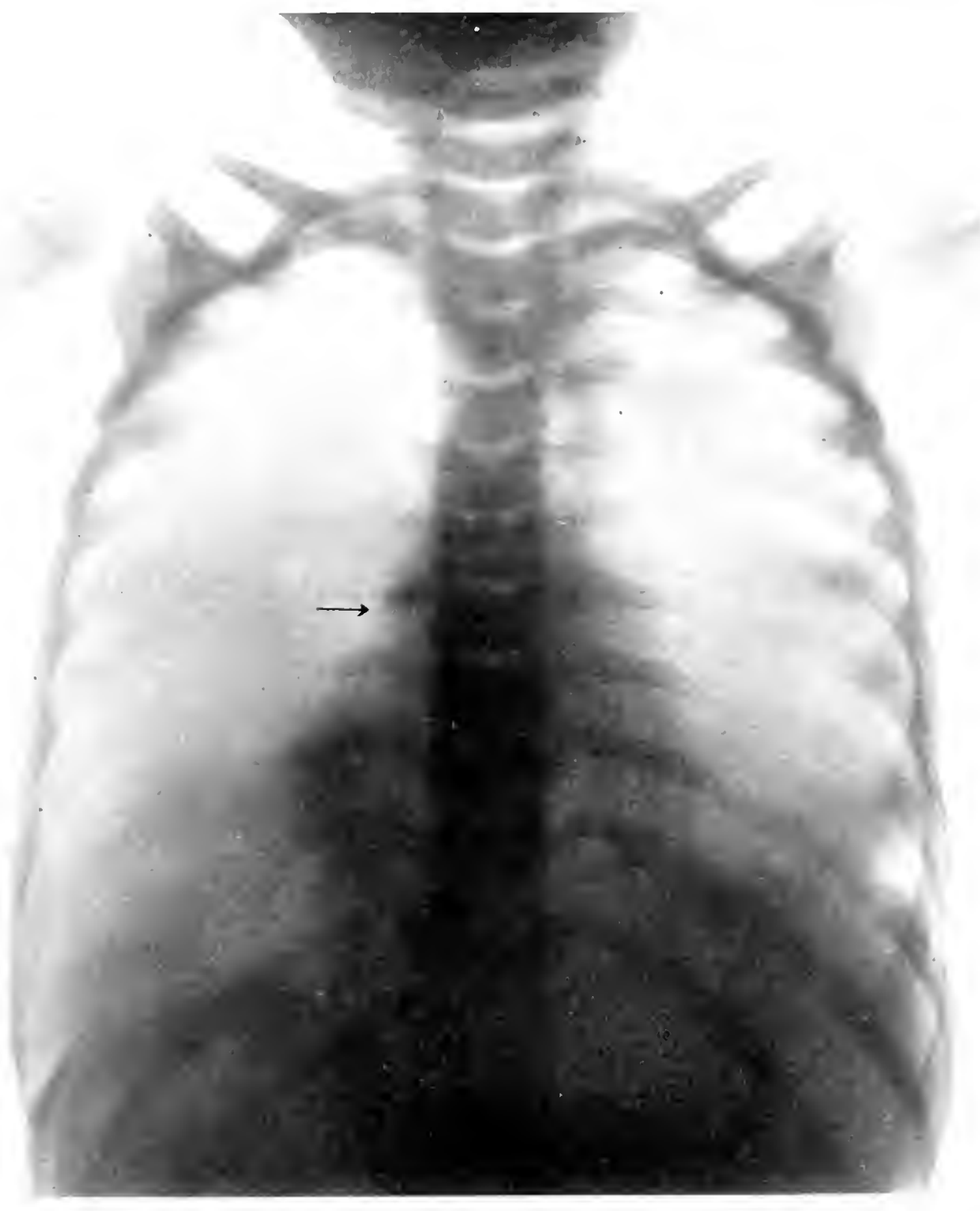




\section{PI..TTE 1.19.}

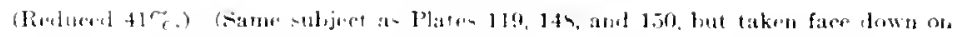
the plitte.

The area of chencity incliented by the arow is seen to be a litele hieher and about the junetion of the sixth rib with the loft redece of the sternum. 
I'I.ITE 1 I!

RIGHT

LEFT
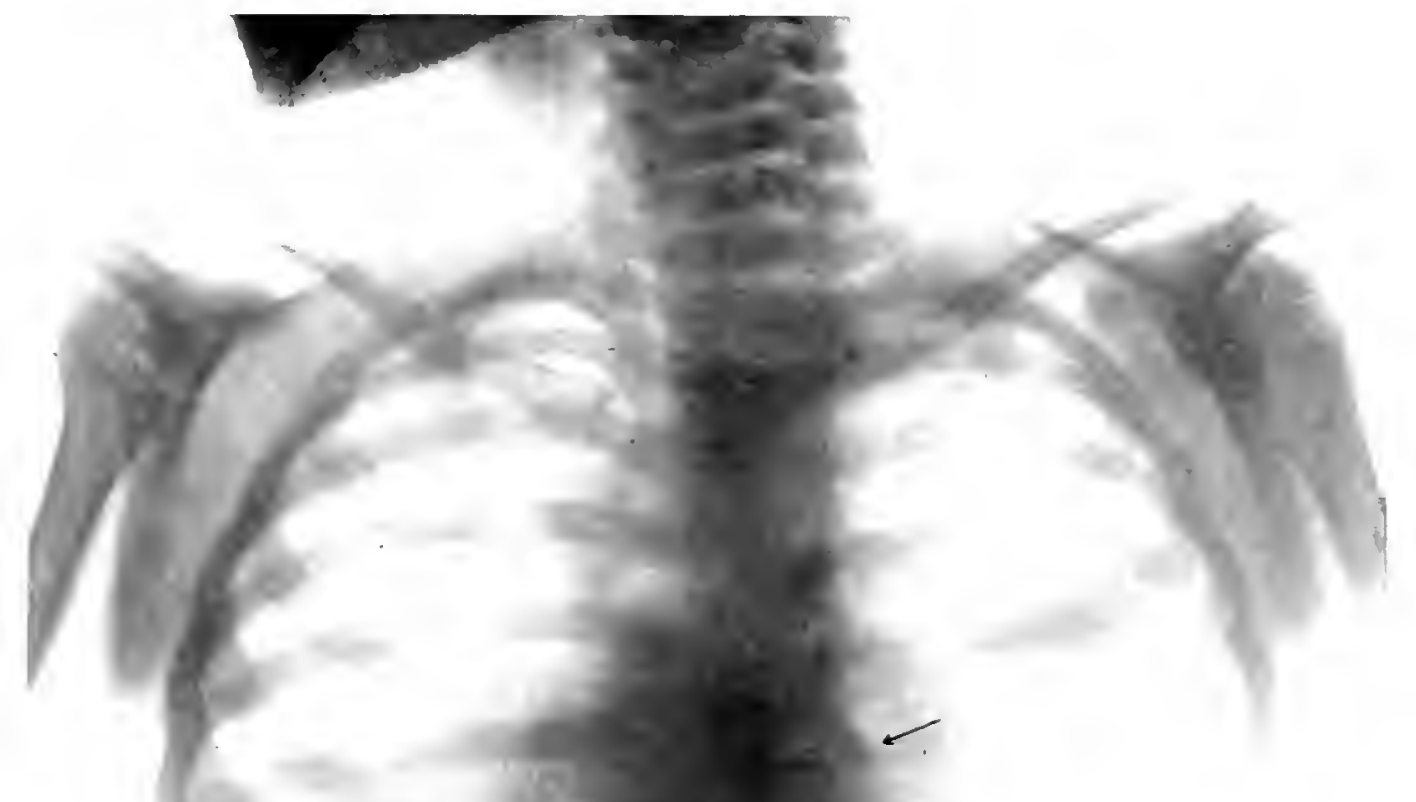
Pl.1PE: 1.00.

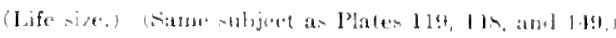

Showe the sande comblition a little mone alearly.

The arrow points to the china arus. 
PIATE 1.J0

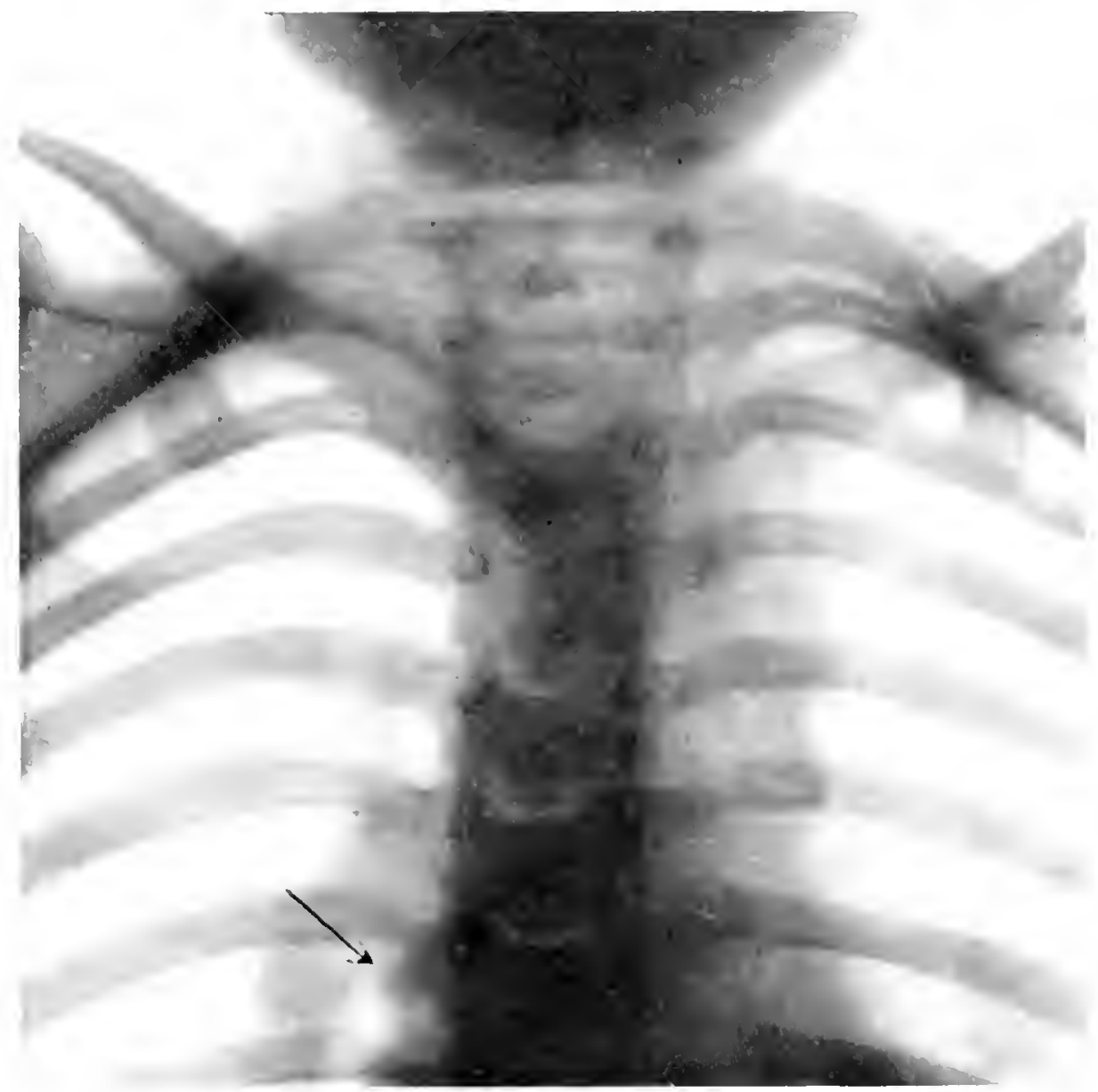


l'I.ITE 1.j1

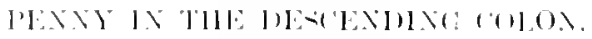

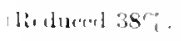

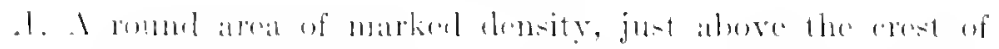
the ilimm (fement)

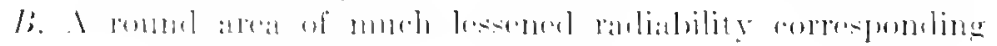
w the anils of onte of the intestines. 
PLATE 1.51

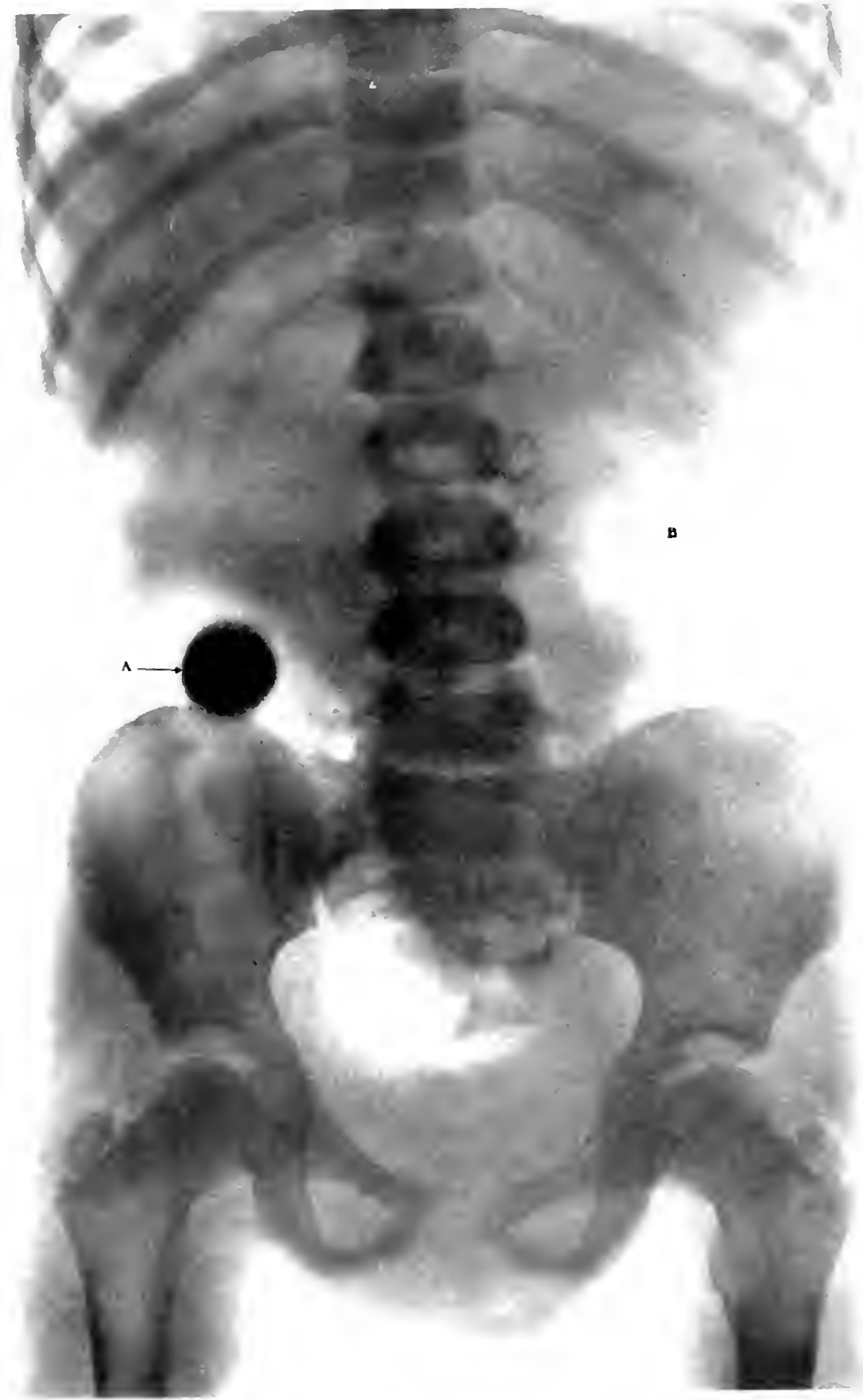


Pl.IPl: 1.'.

NGEDHE IN THE KNEL-JOAT.

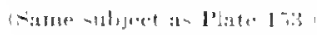

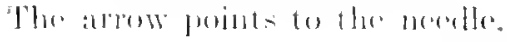


Piate 1.50

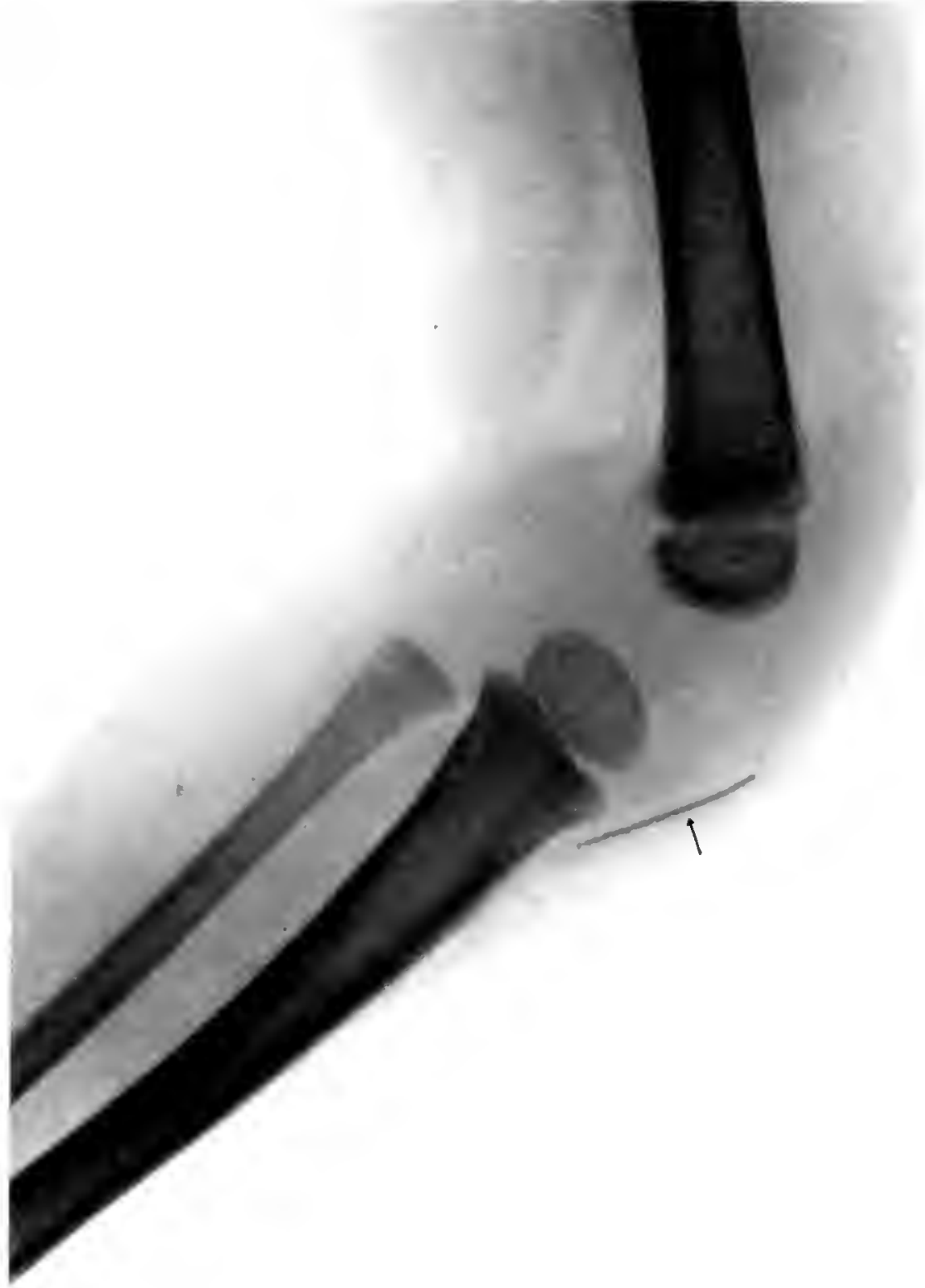


PI.ITI: 1.i)

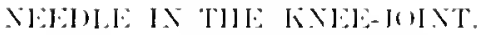

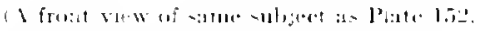

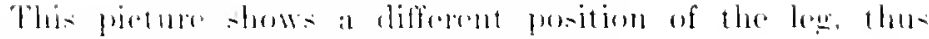
grivine the watet lowation of the nerelle. 
l'L.T' 1.3:3
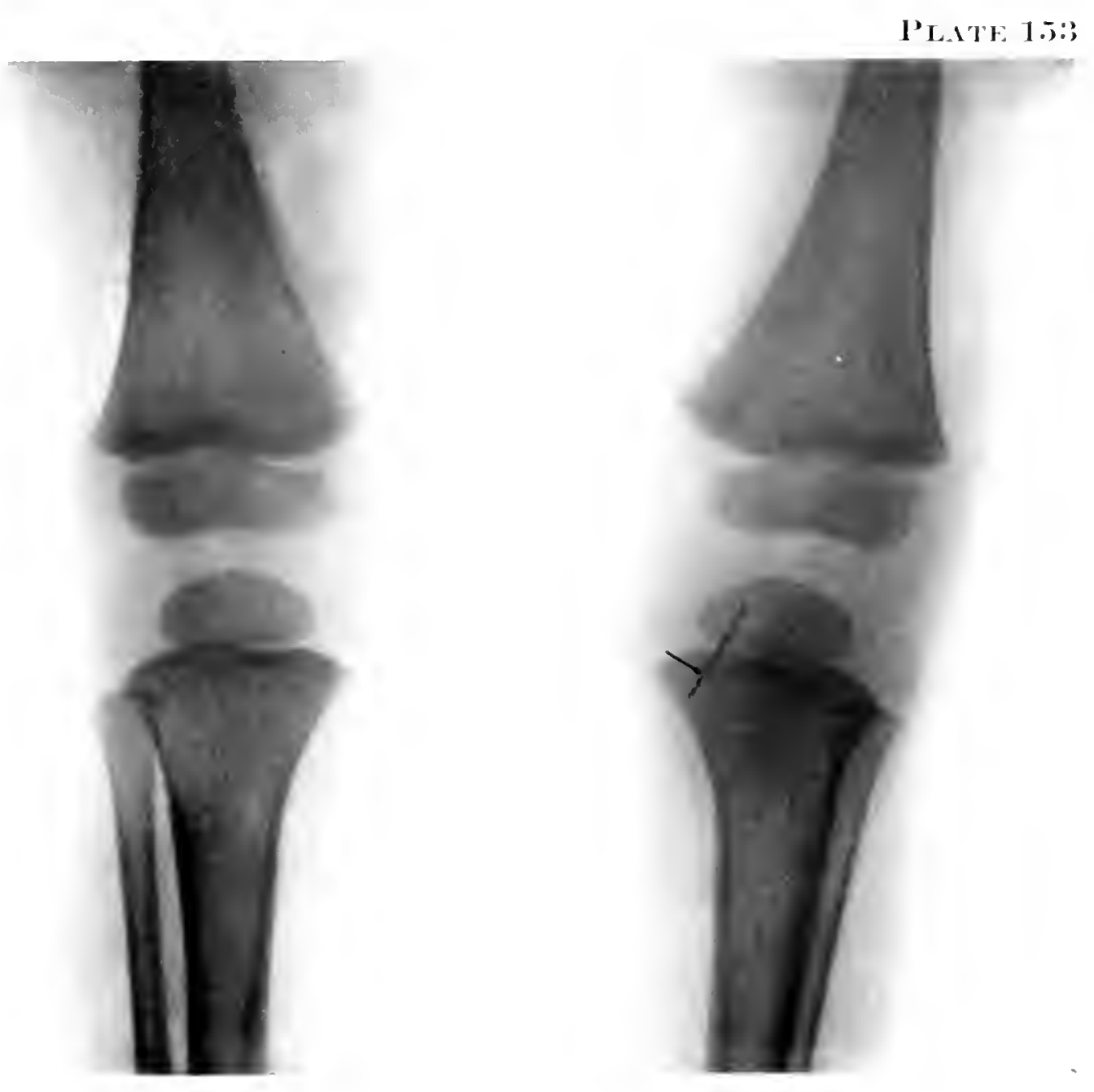
IPITTE IDI.

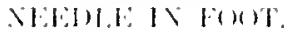

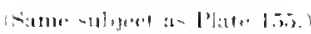

The : orow perinte to the heretle. 
Plate 1.jt

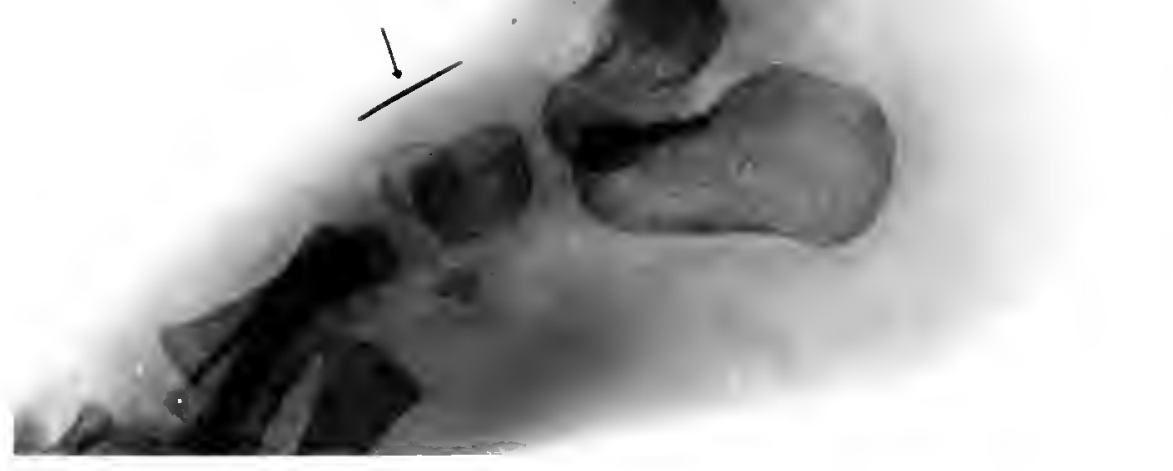


PLATE 1.T.

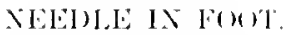

(Actual-ize.) (s:ame sntoject a- Platt list.)

This pieture shows a different viow of the foot, and then

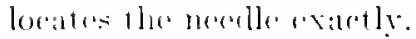

The atrow points to the neeslle. 


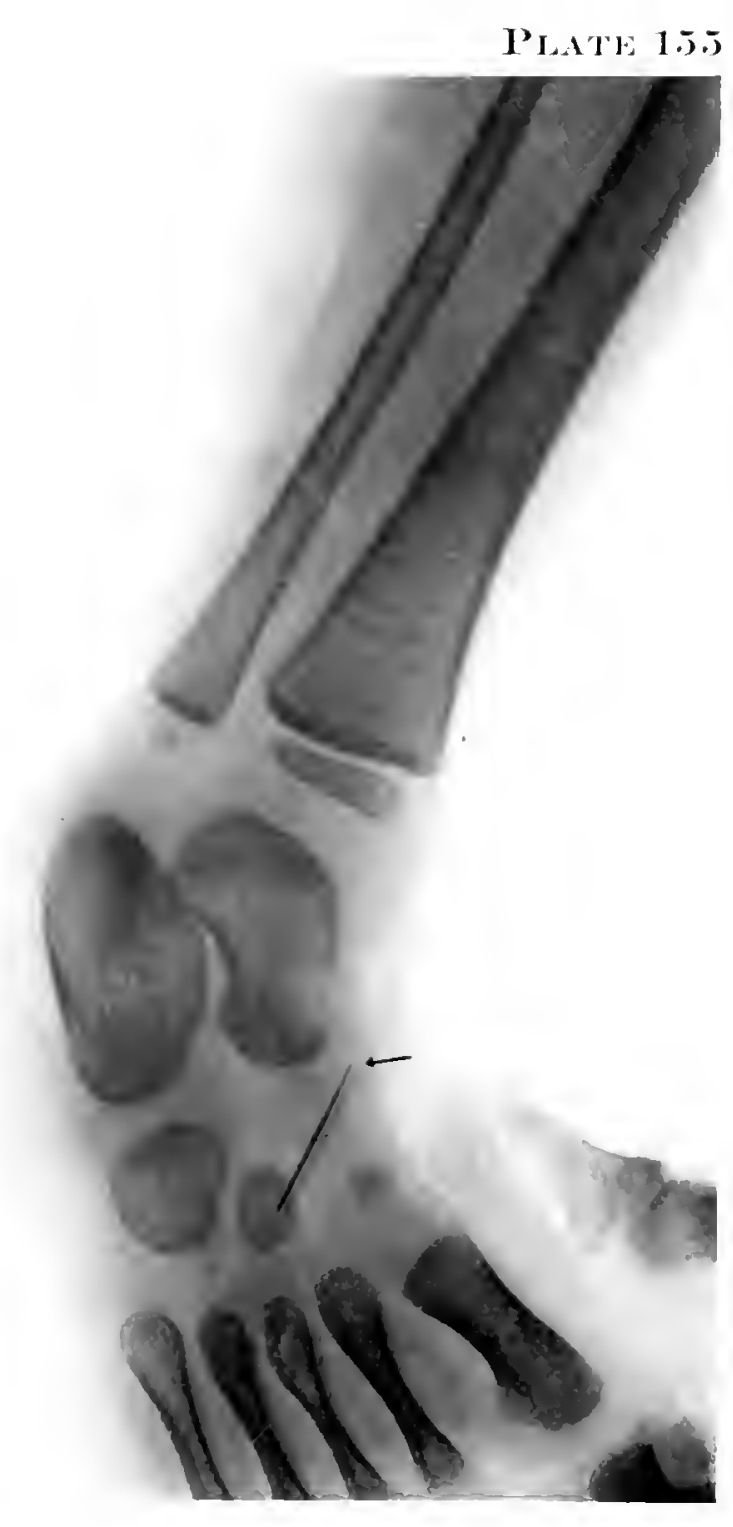


I'I.ITE 1.if.

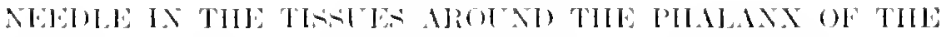
I.ITTILE TOE:

(16returiat 3.5\%

The arrow points to the nerolle. 
PLATE 156;

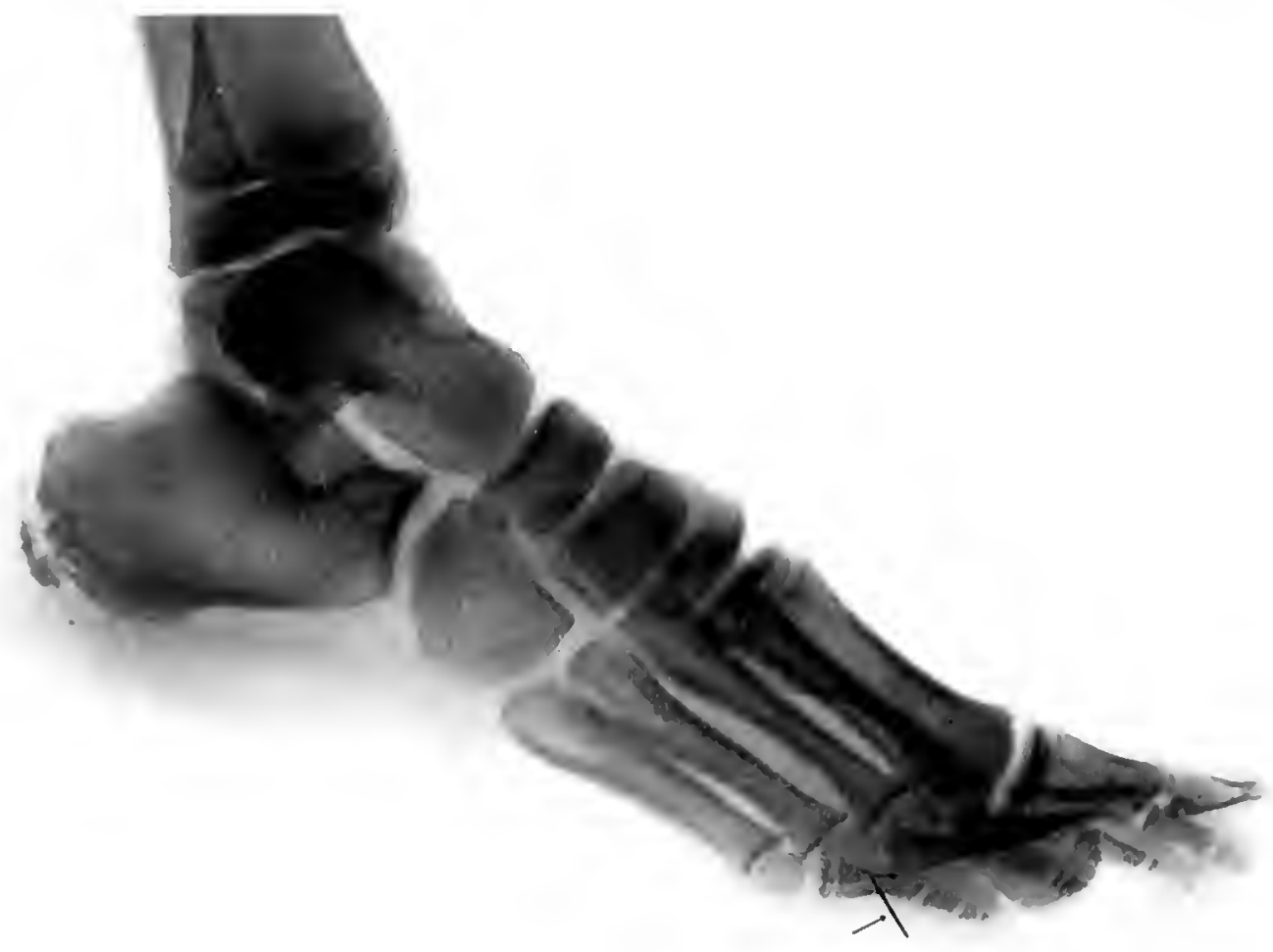





\section{Division IX}

\section{THE EXTREMITIES}

In describing the diseases of the extremities which can be detected by the Roentgen ray, we are dealing with at least one-half of the information about diseased conditions which can be obtained from Roentgenographs. In this varied class of cases it is especially necessary to use the utmost care in the differential analyses, the rules for which $I$ have already given in the Introduction. It is also apparent that this is the class of cases where it is absolutely necessary thoroughly to study and master at least the gross anatomy of the part investigated. This is all the more difficult and important, as the anatomic conditions change so rapidly in early life that it is not a question of the normal completed anatomy of the adult, represented by one single set of anatomic pictures, which we have to interpret. On the contrary, it is the numerous sets of frequently changing normal pictures which it is necessary for us to recognize as normal in order to determine what is abnormal. Of especial significance in this connection are the difficulties which arise in young subjects in distinguishing after an injury whether an epiphysis has been separated by trauma in a case where it should be united normally in accordance with the special stage of development of the individual. Often this can only be accomplished by the aid of the Roentgen ray. It is well in this class of cases to train the eye to recognize any differences from what we have learned to consider normal in our previous study of living anatomic conditions at different periods of development as described in Divisions I and II.

\section{IRREGULAR DEVELOPMENT}

Hand.-As an example of retarded development is the hand of a child eight years old shown in Plate 157. To determine whether 
or not this is a normal hand, we should first turn to the Roentgenographs of the normal hands, which have been described in Division II as indices of the rarious stages of development. From a comparison with these we should then select the group which corresponds to the hand of this child eight years old. On doing this we find that Group $\mathrm{C}$, which represents ehildren from two to three years old, approaches nearer to this picture than to any of the others; while the group which would correspond to the normal development of a child eight years old would be Group J. We see at once, however, that this hand showing delayed development is also anomalous in its type, even if the child were only two or three years old. An anomaly is especially noticeable in the very small epiphysis of the radius, which corresponds more in size to what would usually be found in Group $H$, that is, in children about six years old. The carpal bones, only three in number, as we have already stated, would place this hand naturally in Group C. On examining, however, Group C, which is marked by the os magnum, unciform and cuneiform bones, we see that the epiphyses of the metacarpal bones are present, and those of the phalanges also, while this hand shows a total absence of all the epiphyses of both metacarpal bones and phalanges. So far, however, as the bones themselves are concerned they simply show a slight deficiency in the lime salts.

Wrist.-In like manner if we compare, as is shown in Plate 158 , the anomalous radius of a child thirteen years old with that of the normal hand of thirteen years, as shown in Plate 40, Group M, some decided differences will be detected. This occurs in the carpal bones, which are quite as well developed or even more so than in Group M. They also show quite as great, if not greater development of the epiphyses of the metacarpal bones and of the proximal ends of the first phalanges. We also notice the early 
ossification of the epiphysis of the radius. On examining elosely we see that this epiphysis of the radius is already almost eompletely ossified. The epiphysis of the ulna, on the contrary, has not yet completed its growth and will be seen to show a decidedly delayed development in comparison with that of the normal ulna, Plate 40, Group II, where it has practically united with the epiphysis. On the other hand, this epiphysis of the ulna corresponds very much more to that seen in Group L, a child about twelve years old. It is very evident that the growth of this una is not yet completed, and its development will not be eompleted until its epiphysis has become completely ossified. The radius, on the other hand, having practically completed its growth as regards length, will always be shorter than normal in eomparison with the ulna. This early ossification of the radius probably depended upon an injury to the bone, which stopped the epiphyseal growth sooner than was normal, while the uninjured ulna continued to grow. It is interesting in this case to notice the sesamoid bone in the neighborhood of the metacarpal bone of the thumb and its counterpart in the normal hand, Plate 40, Group M.

Foot.-Plate 159 shows the seaphoid of the left foot of a boy six years old. For two or three weeks it had been noticed that this boy limped on walking and favored the left foot. The Roentgen examination showed a very mueh undeveloped seaphoid, which was not sufficiently supporting the areh of the foot, as the normal seaphoid of the same period of development should do. This boy had a twin brother in whom the Roentgen ray showed the same undeveloped condition of the scaphoid, but to whom this abnormality did not eause any trouble. After a properly adjusted shoe was applied to the foot, and exercises to strengthen the museles were carried out, the difficulty was obviated and the lameness passed away. 
Femur and Tibia.-Plate 160 shows the early ossification of the femur and tibia in a boy. Both these bones remained shorter than they should have been if their normal growth had continued. This is shown in the complete ossification of their epiphyses. On the other hand, the fibula is still growing, as its epiphysis is unossified. There is no involvement of the soft parts. The tendon of the quadriceps is distinetly shown. On the lower and outer surface of the femur there is seen to be a slight thickening of the periostenm.

Having learned to interpret these cases of delayed and carly ossification we can next study such conditions as are abnormal from various causes and which show a change of normal outline. This elass is represented by such conditions as swelling of the soft tissues, whether in the muscles or in the periosteum, and by exostoses of the bones. The recognition of these changes of normal outline and density is often of great value in that it clearly and surely shows whether the bones are affected, and indicates to the surgeon exactly what his operative procedure should be.

\section{ABSCESS}

Plate 161 shows the increased density and local increase of outline in the soft tissues around the left humerus in a boy nine years old. A subeutaneous injection had been given at this point, and there was so much tenderness, swelling, heat and redness that a Roentgenograph was taken to see if the bone was affected. As shown by the picture no disturbance of the bone was found, and the outline of the cortex was seen to be smooth, even, and normal. An operation disclosed an abscess of the soft tissues.

Plate 162 shows a swelling of the soft parts over the heel in a boy twelve years old. It will be seen that the density is inereased, the radiability being lessened on account of a hematoma just below the os caleis. The epiphysis of the os ealeis shows an increase in size beyond the normal epiphysis at this age, this being due to the 
increased amount of blood which the bone has received into its tissues. The other bones of the foot and ankle are normal.

\section{SARCOMA}

Myelogenous sarcomata are rare in children, but may occur in or about the larger of the long bones. They usually attack the shaft of the bone and produce changes similar to certain grades of osteomyelitis, differing only in that the latter presents an outline showing periosteal reaction. In medullary sarcomata certain areas of increased density appear which resemble spiculæ or islands of osseous material and show actual absorption of the bone, with none of the normal portions of the bone remaining about this point. Of the cases seen at the Children's Hospital, a marked increase of tissue has been apparent. In osteomyelitis we generally have a more definite proliferation of the periosteum, and a more definite formation of new bone about the necrosed area, or pieces of bone which have not been absorbed.

Thigh.-Plate 163 shows a boy four and a half years old with sarcoma of the left thigh.

Fig. 1 shows a Roentgenograph where a lateral view of the femur is seen with the tumor definitely encapsulated among the muscles of the thigh. The bone is shown to be perfectly normal and the joint is not involved in any way. The mass of the tumor can easily be seen through the surrounding muscles by the decreased radiability.

The photograph, Fig. 2, shows a swelling of the posterior region of the left thigh, with a bulging both on the inside and outside of the thigh extending down into the popliteal space.

The diagnosis of sarcoma by the ray was proved to be correct by operation.

Plate 164 shows a medullary sarcoma in the lower part of the femur of a child about twelve years old. 
Plate 165 shows a periosteal sarcoma in the lower part of the femur of a boy ten years old.

\section{ATROPHY}

Hand and Arm.-Plate 166 shows an outline of the bones of the hand, radius, and ulna, which is seen to be abnormal in comparison with the normal bones. This pieture was taken from a boy thirteen years old, and shows the outlines of extreme atrophy. Between the middle of the shafts of both bones there is a somewhat suspicious appearance suggesting the beginning of a periostitis. The cause of the condition in this child was unknown, although syphilis was suspected on account of the periosteal thickening of both radius and uha. There is an inereased radiability of both radius and ulna, aud also in parts of the bones of the hand. This hand and lower arm should be compared with the normal hand of about the same age shown in Plate 40, Group MI, where it will be noticed how much larger are the bones and how much greater is the density.

\section{POLIOMYELITIS}

Hand.-Plate 167 shows an atrophy in quality with its inereased radiability, and also an atrophy in size of the hand of an infant one and a half years old in its sixth week of an attack of poliomyelitis anterior. On comparing the bones of the right hand with those of the normal left hand the atrophy of quality is especially marked and is seen in all the bones, radius, ulna, epiphysis of the radius, os magnum, unciform, the metaearpal bones and the phalanges, with their respective epiphyses.

Shoulders.-Plate 168 represents the shoulders and upper arms of an infant twelve months old. The left shoulder and humerus show the results of an attack of acute poliomyelitis of six weeks' duration. The humerus, especially at its neek, is seen to show increased radiability as compared with the other side. This is also well shown in the epiphysis. The increase in the radiability extends 
the whole length of the bone. There is a slight evidence of decrease in the radiability of the scapula and of the acromial process. The thorax and chest are those of a perfectly normal child.

Elbow and Wrist.-Plate 169 shows the elbow, lower arm, and wrist of a child four years old, with marked atrophy of the muscles and bone following an attack of polionyelitis anterior. To be especially noted is the atrophy in size and also in quality of the carpal bones and of the radius and ulna. The atrophic condition of the muscle is not especially well shown in the reproduction, but in the original plate was well marked.

\section{SUBPERIOSTEAL HEMORRHAGE}

Thigh.-Plate 170 shows the change from the normal outline caused by an injury to the thigh followed by a subperiosteal hemorrhage. The periosteum is seen to be stripped from almost the entire length of the femur, and the resulting clot to have become organized. The plate represents a side view of the thigh.

\section{EXOSTOSES}

Exostoses of the bones and allied conditions are comparatively rare in children, though occasional cases are seen in which an examination by the Roentgen ray demonstrates that in all the epiphyses or near the epiphyses of the body rough irregularities or formations of new bone appear with distortion of the bone at these points. Occasionally only a single exostosis is seen. This condition usually occurs in children in or about the epiphyses. The exostoses show a definite bone structure generally, but they may take a more or less fantastic shape. The normal bone about the exostoses does not change in any way. The cause of these exostoses, whether the condition is multiple or single, is somewhat obscure. Infection and traumatism represent in part the cause of the single exostoses. They may arise from a number of infections, such as scarlet fever. 
Astragalus.-Plate 171 shows an exostosis of the astragalus in a child twelve years old. The outgrowth is shown on the anterior surface of the bone and appeared as a sequela of searlet fever.

Tibia and Fibula.-Plate 172 shows the lesions of a case of multiple exostoses in a child five years old. The change in the normal outline of the bones of the fibula and tibia will at once be recognized. Especially to be noted is the exostosis at the inner side of the upper diaphysis in comparison with the elear-eut line of the lower diaphysis of the right tibia. In this case practically all the bones of the body had similar exostoses.

Knee.-Plate 173 shows a well-marked case of multiple exostoses in the neighborhood of the knee-joint in a boy five years old. There is a very evident new formation of bone at the lower end of the femur. The upper end of the fibula shows an irregular and distorted appearance with a complete absence of its epiphysis. The imer and upper edge of the tibia also shows an exostosis. In this case there is seen to be a considerable ehange in the quality of the bones, as well as the change in shape.

Femur.-Plate 174 shows an exostosis of the lower end of the femur.

Plate 175 shows an exostosis of the upper part of the right tibia in a boy twelve years old. This pieture also shows a number of narrow transverse lines passing aeross the whole front of the diaphysis. These are supposed to be the remains of old ossified epiphyseal lines.

\section{CALLUS}

Plate 176 shows the feet of a girl ten years old. A year previous to being seen a set of steel plates had been prescribed for her by a physician, supposedly for the breaking down of the areh of her foot. She had worn the plates continuously and showed on the inner surface of each foot in the region of the scaphoids a hard thickened 
callus. The Roentgenograph was taken in order to discover whether there were any malformations or exostoses of the bones of the feet. The lesions evidently came from wearing badly fitting plates, and an examination with the Roentgen ray showed that the arch was not broken down, Plate 177, but on the contrary was higher than usual. It also showed the outer surface of the scaphoid on the left foot to be somewhat irregular, a little roughened and with lessened radiability. In comparison with the right foot, which is normal, it is seen how much smaller is the border line of the left tarsus, and the arch of the foot is rather higher than normal. The plates were removed and the thick callus formation gradually disappeared.

\section{FLAT-FOOT}

Plate 178 shows the condition of moderate flat-foot in comparison with the high instep just shown in Plate 177.

\section{TRAUMATISM}

Traumatism in children plays an important part, in that secondary changes due to injuries to the different tissues and bones of the skeleton may occur, which disturb, either by actual destruction of bone or by injury to the epiphyses, the growth and the future usefulness of a given bone or joint.

The lesions of the extremities which are most readily detected by the ray are those which are produced by traumatism. The most exact knowledge of the epiphyseal lines and of the epiphyses, as to their normal development at different periods of growth and when they become united to their diaphyses, is a prerequisite for the correct interpretation of the many disturbances which result from a traumatism of these parts in early life. In J. S. Stone's admirable monograph on acute epiphyseal and periosteal infections in infants and children, he states that among infants and children many lesions of the bones and joints are seen which differ materially from those which occur in adults. The character of all lesions inrolving 
the bones of infants and children depends largely on the anatomy of the epiphyses and to a less extent on that of the periosteum. This is true whether the cause is traumatic or infectious. The epiphyseal lines are a very frequent seat of infection, are a source of mechanical weakness, and are the seat of numerous lesions. The strong periosteum is a source of mechanical strength, but influences the spread of infectious processes. Epiphyseal separations are generally regarded as very similar to fractures. In infants and in young children there must be innumerable injuries to the epiphyses which pass unrecognized, since the lesions cannot be demonstrated clinically. In relatively severe injuries to the epiphyses localized tenderness, and perhaps some swelling with disturbance of function, are the only signs which can be detected. An intimate knowledge of the epiphyseal lines, therefore, and of the gradual appearance and ossification of the epiphyses is especially necessary. Of much interest also are the degrees of separation and the final permanent attachment of the condyles and of the tuberosities, abnormal conditions of which, such as separations and displacements, are of interest to both physician and surgeon. This is true not only from their relation to treatment, but from a medicolegal point of view, and from their liability to provide a nidus for the dissemination of infectious processes.

Since the Roentgen ray has come into use so extensively the study by it of injuries to the osseous system has been of great assistance and value to the surgeon. Fractures heretofore unrecognized or seldom recognized can now be ensily detected, indeed fractures of all kinds have been diagnosticated much more commonly since the Roentgen method laas been used systematically.

The technic in the Roentgen examination of cases of traumatism or suspected fractures of any of the bones is important in certain details. 
First, that the part to be examined, as for instance the wristjoint, should be carefully immobilized and the tube centred directly over the part suspected.

Second, two views, one at right angles to the other, should be taken, that is, an anteroposterior view and a lateral. In all cases of fractures of the bones in which these two views are accessible, such as the long bones, and with the exception of the shoulder and hip-joint, this examination should be made as a matter of routine.

Third, it is better to have the eases examined without dressings, such as splints or plaster. If this is contraindicated for any reason, it must be borne in mind that there will be an increase in the general size of the part under examination. Aside from this, however, there will be no actual distortion, so that the relations of the part will be the same one to another.

I shall present a few illustrations of the various forms of traumatism, such as separation of the epiphyses, dislocations, and fractures. These injuries are so eommon and so varied that I shall speak only of a few representative traumata, a thorough study of which will aid very much in the diagnosis of other injuries of the same parts.

Knee.-Plate 179 represents an injury to the knee-joint in a child twelve years old. The Roentgenograph shows that the inner condyle of the femur has been displaced backwards. The epiphyseal cartilage is rough and irregular along its posterior two-thirds. There is a slight tissue-reaction in the knee-joint as shown by haziness. The shafts of the femur, tibia, and fibula are perfectly normal. Epiphysis and Shaft of Humerus.-Plate 180 shows a complete dislocation of the epiphysis and a marked displacement of the shaft of the humerus. It also shows a complete fraeture of the head of the humerus.

Fracture of Humcrus.-Plate 181 shows a fracture of the shaft 
of the humerus in a boy cleven years old which gave the clinical picture of a dislocation rather than a fracture. There is, however, a complete transverse fracture at the anatomical neek of the humerus.

Fracture of Surgical Neck of II umerus.-Plate 182 shows a slight amount of displacement of the shaft of the humerus of a boy twelve years old. There is also a fracture of the surgieal neck of the humerus.

In regard to the loeality of various injuries it may be stated that fractures of the ribs in children are rather rare, as the ribs are very pliable and stand a comparatively greater strain than they do in adults.

Fractures of the bones that make up the shoulder in ehildren, with the exception of the clavicle, are comparatively rare, and here, again, the importanee of considering the normal anatomy of the shoulder-joint at different ages becomes importint.

Fracture of Humcrus.-Plate 183 shows a fracture of the humerus in a child four years old. This pieture was taken through the dressings and shows a break in the continuity of the outer border of the humerus in the region of the surgical neck with a slight impaction of the shaft. Otherwise the bones are normal.

Knee-joint: Dislocation and Fracturc.-Plate 184 represents a dislocation of the epiphysis of the femur and a fracture of the condyle in a boy five years old, and shows a lateral riew of the kneejoint. The picture shows a complete transverse fracture and backward dislocation of the condyle with new formation of bone at the lower end of the femur which unites with the dislocated epiphysis. No changes in the shafts of the bones are present.

Tibia: Creen-sticl Fracture.-Plate 185 shows the result of an untreated green-stick fracture at the lower third of the tibia. The tibia in this region seems to be irregular in outline, and there is an increase on its concive side. This consists of an increase of the 
periosteum and of the cortical substance of the bone. There is a slight increase in radiability at the lower end of the tibia with slightly decreased radiability in the same region of the fibula. The epiphyseal lines, however, are normal.

Femur: Intracapsular Fracture.-Plate 186 shows an intracapsular fracture of the femur in a boy ten years old. The whole joint is rather hazy, but along the acetabulum is seen the separated epiphysis of the femur. The femur itself shows some increased radiability.

Knee-joint; Femur: Dislocation of Epiphysis and Condyle.Plate 187 shows the knee-joint of a boy ten years old, with the epiphysis and the inner condyle dislocated from the lower end of the femur. Otherwise the structure of the bones is practically normal.

Humerus: Fracture.-Plate 188 represents a fracture of the lower end of the humerus in a child ten years old. The picture shows a transverse fracture of the lower end of the shaft of the humerus with displacement of the lower fragnents inwards. In this case the condition was considered clinically a perfect reduction.

Radius: Green-stick Fracture.-Plate 189 shows a green-stick fracture of the radius occurring in a child twelve years old. As shown by the grayish-white, faint transverse line in the lower third of the radius, there is a break, but the periosteum has not broken through, the break being in the cortical and medullary portion of the bone. The irregular areas in the plate show parts of the bandage, and we should remenber, as I have explained before, that although the details of the parts are not altered on this account the whole picture is somewhat larger than it otherwise would be.

Radius and Ulna: Impacted Fracture.-Plate 190 shows a complete transverse fracture with slight inpaction of both radius and ulna in a boy between three and four years old. At the top of the 
picture are to be noted the proximal ends of the first phalanges, and also the fingers of the hand of the assistant who wats holding the child's fingers down. Also to be noted is the development of the lower epiphysis of the radius, which corresponds to the anatomical Group D, from three to four years.

Radius and Ulna: Fracture-Plate 191 shows a complete fracture of the radius and ulna in a child three years old. The Roentgenograph is taken through the splint after the fracture had been reduced. The capitellum, as it appears in the elbow-joint close to the lower end of the humerus, is normal at this age.

Tibia: Fracture.-Plate 192 shows the otherwise normal foot of a child twelve months old, with a small incomplete fracture just above the lower epiphyseal line of the tibia on the anterior surface. The rupture of the tendo Achillis is well marked. The fracture occurred at the time the child was being treated for the correction of club-foot.

Astragalus: Fracture.-Plate 193 shows a fracture of the astragalus in a boy eleven years old. The bones of the foot are normal with the exception of the astragalus, which shows a complete fracture in its upper third. This fracture was due to eatching the foot in a revolving wheel.

Tibia and Fibula: Fracture and Abscess.-Plate 194 shows an abnormal condition at the lower end of the tibia and fibula with a bridge uniting the two, which seems to represent the osseous connection of an old fracture. The lower end of the tibia and the bones of the foot are seen to be very finely pencilled, indicating a marked lime absorption. Posterior to the epiphysis of the fibula there is seen to be a definite swelling of the soft parts due to the formation of an abscess. The whole foot illustrates a tubercular process with the formation of an abscess but without actual destruction of the bone. 
Os Calcis: Fracture of Epiphysis.-Plate 195 shows a fracture of the epiphysis of the os calcis in a child ten years old. Clinically this child had marked pain and tenderness over the region of the heel. In making a differential diagnosis of this lesion we must remember that the epiphysis of the os calcis sometimes unites by two centres and gives this same appearance at this age. Mobilization of the heel in this case gave relief, and the supposition therefore was that it was a fracture. Cases of this kind enunciate very strongly the importance of avoiding the overlooking of slight fractures by calling to our aid an examination by the Roentgen ray. Especially is this important in children, who often can be relieved of their pain and general discomfort at once as soon as the seat of the lesion has been located, while unless we do ascertain this by means of the ray the lesion not only might not recover rapidly, but might even be increased in its severity with a possible final result of impaired usefulness of the part.

Tibia: Fracture.-Plate 196 shows a condition arising from an ununited fracture of the tibia in the left leg of a girl twelve years old. The fracture is in the middle and upper third of the tibia and shows an attempt to unite. The whole tibia shows marked atrophy, not only in thickness but also in length and quality, as shown by the greatly increased radiability of the shaft. The fibula, however, shows a compensatory increase in size and decrease in radiability. It has practically taken up the function of the tibia. The right leg is normal in every respect for this age, and the injured leg should be carefully compared with it.

\section{JOINTS}

There has been a great deal of discussion concerning the proper classification of conditions in which the joints are affected. In our efforts to decide upon a rational and correct nomenclature, much opposition has arisen from the fact that physicians are loath to 
change the names by which they have been accustomed to recognize, or rather think that they recognize, certain groups of clinical symptoms. One of the reasons for this is that they do not appreciate that the same clinical symptons may be produced by a number of different etiologic factors, and that again these factors in their origin may simply arise from traumatism without specific infection, or again may arise from a large number of different specific infections. So long, therefore, as a classification on a clinical and symptomatic basis is retained, no advance ean be made in the proper and intelligent understanding of the different diseases. On the other hand, there are those who prefer a pathologic classification. Here, again, the mistake is made in thinking that preeise and exact pathologic conditions always correspond to a specific etiology. In quite a large number of pathologic conditions which are found at the autopsy there is no doubt that the lesions do not necessarily arise from one specific cause. On the contrary they are merely terminal lesions which maty represent a number of different primary conditions of a special disease. When we consider that in most eases we have had to wait until the patient has died in oreler to discover what the lesions were which corresponded to his special symptoms, it is no wonder that such confusion has arisen from our having to depend upon dead pathology. It is very evident also that now that we are enabled to study living pathology by means of the Roentgen method a great advance can be macle in elassification. We can of ten recognize the early lesions of a special disease, and taking advantage of this we can eventually sweep away the false position and resulting obscure ideas which we have arrived at from our study of the lesions represented by dead pathology. The truth of what I have just said is manifest when we come to consider disturbances of the joints, whether from simple congestion, trauma, without infection or followed by infection, or infection without trauma by direct 
infection of the part through the blood. In approaching this subjeet we would naturally begin with those affections of the joints which might be considered non-infectious, and to be the result of traumatism. In quite a number of eases where I have been unable to determine in a lesion of the joint that the resulting pathologic condition has arisen from an infection, and therefore is a so-called idiopathic disturbance of the joint, I have had to assume for the time being that I was dealing with an injury without infection. Certain acute conditions of a joint, especially of the knee, will, following an acute injury, present the symptoms of heat, swelling, tenderness, effusion into the joint, with absorption and complete recovery. This sequence of symptoms has so frequently been met with that it perhaps seems hard to believe that infection has taken place in any way etiologically. It is very probable, however, that in the future the number of eases which will be proved to be non-infectious will grow less and less as our knowledge of infectious processes increases, and when our power of detecting specific organisms is more perfected. A specific organism may have a brief elinical history. It may be so benign that its effect simply represents an acute disease rumning a very rapid course and tending to recover. We must for the present, therefore, allow that the whole subject is sub judice. I would, however, enunciate the general principle that the only safe course to take in any injury of the joint, whether from mild, or moderate, or severe trauma, is that it should be at once treated as though possibly an infection had taken place or might take place very shortly. Only in this way ean we aroid the mistakes which we see made over and over again, when a slight trauma is passed over without much notice.

I shall not attempt to make any elassification of this set of cases, as it is hardly within the seope of the work in which I am now engaged. I merely wish to show the living pathology of the 
joints with the same idea in view as when we study the dead pathology. For instance, I shall show what an inflamed congested joint looks like in a Roentgenograph: the appearances which are met with in the different disturbanees of the diaphysis: the epiphyseal line: the epiphyses: the capsular lining of the joint: the appearanee when an effusion is present; the appearance when resulting adhesions have taken place, or still further that villous formation which is the result of a number of pathologic conditions of the joints. IIaving learned to recognize the different lesions which may oceur in acute and chronic processes, we are in a position to discuss the lesions which result from various disturbances of the joints.

Following out this idea I shall now refer to some plates which represent a few of the lesions which are met early and late in disturbances of the knee-joint, whether traumatic or infeetious.

Knee: Congestion.-Plate 197 illustrates the results of a congestion of the knee of a boy six years old. There is seen to be an hypertrophy in the size of the epiphyses of both femur and tibia in the left knee in comparison with the right. There is also increased radiability in the epiphyses on the left. In addition to this there is some thickening of the soft parts and capsule around the kneejoint. The epiphyseal line of the femur and tibia is comparatively normal. The epiphysis of the fibula to the left has appeared, whereas it is absent on the right. This increase in size of the epiphyses of the left knee over that in the right, and the presence of the epiphysis of the fibula on the left, are due to the increased blood supply occurring in the process of a chronic infection. This picture also shows lines radiating transversely across the lower diaphysis of the femur and less marked in the upper diaphysis of the tibia. These narrow lines are supposed to represent the old lines of the epiphyses.

Knee; Epiphyseal Line: Epiphysitis and Osteochondritis.-Plate 195 illustrates an inflammation of the epiphyseal line in the knee 
of a child one year old. The soft parts in this case are normal, with the exception perhaps of a slight amount of thickening around the knee-joint. The structure of the femur is practically normal until the epiphyseal line is reached. It is to be noticed that the epiphyseal line is darker than normal, showing new formation of bone. The epiphyses of the tibia and fibula also show the same eharacteristic increased deposit of lime, which points strongly to inflammatory proliferation around the epiphyseal line. All the epiphyses in this case also show the same change in the epiphyseal lines. The condition is mostly one of epiphysitis, but there may also be a certain amount of osteochondritis.

Knee; Tibia: Old Trauma, Suppuration of Epiphysis.-Plate 199 illustrates the condition of a tibia due to an early trauma followed by suppuration of the epiphysis of the tibia. This occurred in a girl ten years old. The femur in this case is normal and its epiphyseal line is still easily seen and shows no evidence of ossification. Along the epiphyseal line of the tibia, however, there is complete ossification of its epiphysis. The fibula is seen to be longer than the tibia, which we would expeet, as its epiphysis is still unossified and it has continued to grow, while the tibia has ceased to grow.

Knee: Old Inflammation-Infectious Arthritis, Atrophic Variety.Plate 200 illustrates one of the results of inflammation of the kneejoint in a child three years old. There is marked thiekening of the soft parts around the knee-joint and a slight roughening of the femoral epiphysis. The shafts of the bones are normal, except that there is a rather fine pencilling at the lower end of the femur and at the upper end of the tibia. The plate illustrates the condition of infectious arthritis of the atrophie variety.

Knee: Inflammation-Villous Arthritis.-Plate 201 shows one of the end results following inflammatory disturbance of the kneejoint. This is the class of cases which is spoken of as villous arthritis, 
and may arise from a number of eauses, or rather follow a number of proeesses. Roentgenographs of cases of this kind have been rather confusing, owing to the attempt of certain writers to have this condition represent a class rather than the result of a number of inflammintory conditions. The tissues around the knee-joint are seen to be thickened, especially in the popliteal space. The capsule of the joint is greatly thickened and can be easily outlined. The joint itself is full of a roughened villous hypertrophic growth. This lesion was supposed to be the result of an acute septic infection of the knee, aceompanied by swelling, heat, and pain, but the symptoms did not subside under the usual treatment. Before operating the Roentgenograph showed a simple villous arthritis and at the operation no infection was discovered.

Knee: Inflammation, Ankylosis.-Plate 202 represents the end results of a severe case of inflammatory disturbance of the kneejoint not recognized in the early stages and resulting in ankylosis of the joint in a boy thirteen years old. The picture shovs complete ossification between the heads of the femur and tibia. To be noticed is the great change in the structure of the bone at the lower end of the femur and at the upper end of the tibia, and that a formation of new bone extends directly across the line of ossification. The patella is seen to be greatly atrophied in quality rather than in size, a natural result arising from disuse, as the joint was perfectly stiff and could not be bent.

Before speaking more in detail of the infections class of cases in connection with the joints, in other words, infections arthritis, a few explanatory suggestions may be of use to the student. Of especial significance are the results of Stone's investigations of these cases in early life, since he has had for many years unusual opportunities for examining and operating on infants and young children at the Infants' Hospital. 
He draws attention to the anatomical fact that there is a very free vaseular supply of the epiphyseal lines by terminal arteries and that this increases the liability of infections reaching these points through the blood. This, in addition to the supposition that infections entering through the ear, tonsil, and other sourees attack most readily those parts where there is an unusual blood supply, and where there are tissues especially susceptible to infection, makes it highly probable that most infections of the joints in early life begin in the epiphyseal lines. The underlying cause of these infections may often be traced directly to some preceding disease which not only impairs the general health but also aids to bring the infecting organisms into the circulation. Thus in young children it is common to see epiphyseal lesions after pneumonia, measles, or searlet fever. In very young infants the unhealed umbilieus seems to be a not infrequent source of infection. The opportunity for infection through the tonsils and lymphoid tissue of the nasopharynx is generally recognized. In this way a preceding disease which allows the entrance of the organism into the circulation leads to an infection of a vascular area, the resistance of which has been impaired by perhaps only a slight trauma. Since the pathologic process of this area may extend still further and may result in an exudate, it is well to study certain points in connection with the anatomy of the joints. In order to understand rightly the pieture of an exudate in the neighborhood of a joint, it is also important and even neessary to bear in mind the anatomic relation of the epiphysis to the capsule of the joint. When the epiphyseal line reaches within the joint-capsule an exudation of pus ean reach the joint whenerer the epiphysis is separated by suppuration. In the wrist the capsule is inserted into the epiphysis of the radius and una, and not into the sae, so that pus to reach the joint has to perforate the cartiliginous epiphysis. In the hip-joint and in the shoulder-joint the epiphyseal 
line reaches within the joint-capsule, and thus when the epiphysis is separated by suppuration the pus can enter the joint. The same reasoning is true of the upper epiphysis of the tibia, for the capsular ligament of the knee does not cross the epiphysis but is inserted into it, therefore when separation oecurs the pus burrows under the periosteum but does not break into the knee. Different parts around the knee-joint may be involved aceording to where the pus has the freest vent. Sometimes the extension may be into the extra-irticular tissues, or up into the shaft of the femur, or again down into the joint itself.

The point of especial interest in regard to this group of eases is the exact point of the original foeus of infection. At operation it is rarely possible to determine the location of the original focus aceurately. It seems probable that it is usually either the epiphyseal line close to the periosteum, or the periosteum near to the epiphyseal line. The reasons for this belief are:

This location seems usually to be the eentre of the abseess.

The condition is brought on by the same factors which bring on true epiphysitis, namely, slight trauma combined with the presence of an infecting organism.

The early symptoms are identieal with those of aeute epiphysitis. These abscesses oceur most frequently at the age at which epiphyseal injuries are most eommon.

The loeation of these abscesses, their eourse, their symptoms, and their whole clinical history differ absolutely from those of abscesses originating in lymph-nodes and also from those originating among the muscles and faseice.

On the other hand Stone has never found one of these extraarticular abseesses showing any erosion of the bones, though very frequently the periosteum is slightly frayed and ragged elose to the 
epiphyseal line, as though it might have been perforated at this point. It is, therefore, probable that in all these varicties of cases there is an infection beginning in or close to the epiphyseal line, and that when suppuration occurs the pus spreads along the anatomic lines of least resistance. These depend on:

The spot in the epiphyseal line at which infection occurs, which is determined presumably by the nature of the trauma.

The anatomy of the epiphysis.

If the original focus of infection is at the periphery of the epiphyseal line the pus is very likely to find a vent into the surrounding tissues before any extensive damage is done to the epiphyseal line itself. On the other hand, if the infection occurs at the centre of the epiphyseal line the chance of a complete separation and consequent necrosis of the whole epipliysis is much greater. Thus the entire question is one of anatomy.

The cases of dissecting periostitis usually occur in somewhat older children than do the cases with abscesses in or about the joints. While some apparently start at the epiphyseal line, in other cases the infection seems to be primary under the periostcum. Periosteal infections occur usually in the tibia, radius, or una. They are especially common about the ankle and wrist, and somewhat less common about the lower end of the femur. It scems reasonable to suppose under these circumstances that direct injury of exposed parts plays a most important rôle in the causation of these lesions.

In another class of cases connected with the periosteum and its separation from the bone we may have two results which should be carefully looked for in our Roentgen examination. For instance, the bone may become necrotic, not as a result of inflammation from infection of the marrow, but as a result of the interference with nutrition incident to the stripping off of the periosteum and 
the consequent destruction of the nutrient ressels. In this case there would be necrosis but not osteomyelitis.

Stone gives the following varieties of lesions originating in the epiphỵseal line:

Acute epriphysitis resulting in the sequestration of the epiphysis and incidental involvement of the joint.

Acute epiphysitis with perforation into the joint, resulting in an acute articular abscess.

Acute epiphysitis with perforation outside the joint into the extraartieular tissues (an extra-articular juxta-epiphyseal abscess).

Acute epiphysitis with extension beneath the periosteum (a dissceting periostitis).

In addition to this a dissecting periostitis is found occasionally away from the epiphyseal line and without evident lesion in the bone eren on careful search.

With this rather formidable array of the different etiological factors which may produce an arthritis, we should certainly appreriate how important it is that aid in our differential diagnosis by the Roentgen ray should be obtained early. During infancy the wide area of cartilage and the vulnerability of the epiphyseal line give ample opportunity for suppuration to spread outward rather than to enter the shaft. As development adrances, as the cartilage at the epiphyseal line becomes thinner, and as the epiphyseal line itself becomes less well defined, the likelihood of sepsis spreading into the medullary cavity increases. Thus the infections involving no other pirt of the bone than the epiphyseal line are particularly to be expected in the youngest patients and cannot possibly occur in adults. True osteomyelitis is more common in older children, and any infection beginning in the epiphyseal line in adults must of necessity be a true osteomyelitis. 
In older ehildren dissecting periostitis is relatively common. The pain, and especially the tenderness and swelling in such cases, is usually more widely diffused than in an acute osteomyelitis.

The value and the limitation of Roentgenographs in these cases should be clearly understood. In those taken early in the course of acute infections beginning in the epiphyseal line in infants and young ehildren there is no reason to expect any change from the normal. In such cases the disease involves the cartilage, which shows at best but vaguely even in the elearest Roentgenographs. The most that can be looked for is an irregularity of the end of the diaphysis, or a broadening or haziness between the epiphysis and diaphysis. Neither of these points can be determined accurately or even approximately without wide experience. Early in the course of infeetions under the periosteum the Roentgenograph will show no ehange in the bone, unless it is a slight degree of roughening of the outer part of the cortex. In due time the new forming periosteal bone may be seen separated very slightly from the underlying cortex. The negative value of a good Roentgenograph, however, as showing the absence of any advanced lesion within the diaphysis, is often of the utmost importance and should never be disregarded.

In connection with what will be said eoncerning congenital syphilis it is well to remember that this may often during infancy cause well-defined swelling and tenderness at the epiphyseal lines. Among older children, of course, periosteal syphilitic lesions are very common, and indeed it has long been noted that syphilitic periostitis is the marked lesion in older children, while syphilitic osteochondritis most commonly occurs in young infants. As a rule, however, especially if the Roentgen ray is used, there is not much probability of the syphilitic lesions being confused with the acute epiphyseal or periosteal infections.

In like manner we should bear in mind that the subperiosteal 
hemorrhages, so commonly oceurring in infantile scorbutus, at times with their extreme pain and tenderness simulate closely pus in the same locality, but in this ease the reneral clinical symptons would aid in the differential diagnosis. The symptoms of scorbutus show a slow onset and a relatively low temperature, in contrast with the acute attacks of subperiosteal effusion from other causes.

I believe that in all these eases Roentgenographs should be taken from time to time through the course of the disease, even when the symptoms are mild. The profession will gradually learn that the physical examination of these cases gives us infinitely less information than does the Roentgen. This method of examination is more valuable than any other, whether it be in cases of severe infantile scorbutus, or of osteomyelitis, infectious periostitis, infectious epiphysitis, or infeetious osteochondritis.

In regard to the special organisms which give rise to infectious arthritis and to osteomyelitis, we may say that in the chronic eases of low grade we find mostly the staphylococeus, while in rapidly septic cases the streptococeus is most common. The pneumococeus produces a comparatively chronic, while the bacillus of typhoid either an acute or chronic condition. We must remember also that the conditions in these infections, as seen in children up to the age of puberty, are not altogether like those of the adult, and that the characteristic pietures seen in adults of ten eannot be shown in children. It should be noted for the purpose of differential diagnosis that clinically the characteristic picture of an acute polyarthritis may be present, and yet it may be very difficult to determine the cause of the condition. The reason for this is that we at times see in the living individual terminal results of an acute, subacute, or chronic inflammatory process which may be slightly active or entirely quiescent.

It is also well to note that we can have a combination of both 
atrophy in quality and atrophy in size in certain cases which show simply a decrease in the size of the bone with an absorption of the lime salts. This is seen especially in the chronic forms of arthritis as well as in tubereulosis of the bone or in general tuberculosis. It is also seen in paralytic conditions, such as poliomyelitis anterior, as already shown in Plate 168; also in some of the malignant osteomata, where spontaneous fracture takes place, and very rapidly extends in both direetions, but usually towards the origin of the nutrient arteries. Here we find also a diminution in the size of the bone and an actual absorption of the substance of the bone without any tendency towards the formation of a callus.

In connection with those cases in which there is an infection of the periosteum, we should note that in acute infections by a pyogenic organism outside the joint-eapsule the process may start with a direct infection of the periosteum or through a roughened or ragged periosteal line. In the former case we may have the appearance in the Roentgenograph of a definite beginning of proliferation of the periosteum.

\section{EPIPHYSITIS}

Certain anatomic facts are worthy of note when we are considering inflammatory conditions of the epiphyses. There is a very marked difference between the epiphyses as they exist in the joint of a child and in an adult. It is very important that by means of the Roentgenograph we should not mistake a normal separation of the epiphysis in a young subject for a fracture, since the same picture in an adult would almost invariably represent the latter. It is also known that where a dislocation is produeed by trauma in an adult, it is much more likely in the ease of a child to cause separation of the epiphysis. It is all the more important to recognize these injuries of the epiphyses in young subjects since, unless properly treated, they are followed by suppuration and stiffness in a 
neighboring joint, resulting perhaps in a deformity or in an imperfect development of the limb. It is also to be noted that the epiphyses of the knee, wrist, and shoulder are of especial importance so far as the increase in length of the bones is concerned, because they are the last to join the shaft and the growth in them is consequently continued the longest. The striking growth of a small centre of ossification in the course of five or six years is quite remarkable. Hammond has done such excellent work regarding the epiphyses in this connection that I shall quote from his results quite freely.

Shoulder.-In comnection with the epiphyses of the shoulder the upper epiphysis of the humerus is found as a dome-shaped mass which appears to rest lightly on top of the shaft. It is composed of the centres for the head and for the greater and lesser trochanters, which unite to form this epiphysis presumably at about the fifth year, according to the old chronologic method of determining development. The epiphyseal line lies a little way above the surgieal neck, and is not horizontal, but is higher in the middle of the shaft than at the outer and inner sides. The inercase in length of the humerus takes place principally at this epiphysis, and hence its great importance.

Elbow.-The lower epiphysis of the humerus at the age of five or six years shows merely a small, round, bony mass, the centre for the capitellum. At twelve or thirteen years, however, the centres for the trochlea and the external epicondyle have appeared and have united with the centre for the eapitellum, forming the lower epiphysis. In an anteroposterior view of the elbow this is seen as a wedgeshaped mass, its lower surface being convex, and lying below the external condyle. The internal epicondyle is not a part of the lower epiphysis of the humerus, but is formed from a separate centre of ossification. In a Roentgenograph it appears as a small, oval mass higher up on the inner side of the humerus and intimately con- 
neeted with the internal condyle. The epiphysis of the head of the radius is seen as a small, flat disc lying just above that bone. In a lateral view of the elbow at about five years chronologically the lower epiphysis of the humerus appears to be semilunar in shape, fitting elosely the lower end of the shaft. The lower end of the humerus is bent forward so that at times the epiphysis appears to be slightly displaced. The epiphyseal line may appear as a cleft either at the front or at the back of the humerus, instead of a uniform line running all the way between the shaft and the epiphysis. This is quite confusing at times, as the epiphysis appears to be displaced either forward or backward. In these cases it is only by having a Roentgenograph of the normal joint that we can determine whether the epiphysis is separated. It is alway's well to have a Roentgenograph of the normal joint in all eases, but it is especially necessary in injuries to the elbow. The ray should strike the part at the same angle in each ease, so that we view both elbows as nearly as possible under exactly the same eonditions, otherwise an injured epiphysis may be overlooked, or a normal epiphysis may be mistaken for a separation. In the lateral view the picture of the internal epicondyle is usually merged with that of the internal condyle, or may be seen partly overlapping it. The epiphysis of the head of the radius is seen above that bone, and the epiphysis of the upper end of the ulna lies above the oleeranon. This latter epiphysis is an irregular, rounded or three-sided bony mass, and from its location and appearanee is of ten ealled the patella of the arm.

Wrist.-In conneetion with the wrist it is well to note that the epiphysis of the lower end of the radius is sometimes mistaken for a Colles's fracture. In an anteroposterior view it appears wedgeshaped and is thicker on the outer than on the inner side of the wrist. The epiphyseal line, though irregular and wavy, is nerer rough and jagged as in a fracture. Colles's fracture also is always found at a 
higher point on the shaft. This epiphysis has a great share in the inerease in length of the radius. The epiphysis of the lower end of the ulna is situated at a slightly higher level than that of the radius and shows the prominenee of the styloid process on its inner side. This epiphysis, though not commonly injured, is important because the increase in length of the ulna takes place almost entirely from it and any injury to this epiphysis may lead to serious deformity of the forearm, wrist, or hand. The epiphyses of the four inner metacarpal bones are situated at the distal end of the shafts, but in the phalanges and in the metacarpal bone of the thumb (which latter is to be regarded morphologically and developmentally as a phalanx) the epiphyses are found at the proximal ends of their respective shafts. In a lateral view of the wrist the epiphysis of the ulna is seen to be at a distinctly higher level than that of the radius.

Hip.-The epiphysis of the upper end of the femur includes merely the articular head of the bone and forms no part of the neck. In a Rocntgenograph it sometimes resembles in appearance the epiphysis of the upper end of the humerus. Both the greater and lesser trochanters arise from separate centres of ossification, but these are less frequently shown in the Roentgenograph than is the larger epiphysis.

Knee.-The epiphysis at the lower end of the femur is the largest epiphysis in the body, and is probably the one most frequently injured. It is the only epiphysis in which bone is formed before birth. In an anteroposterior Roentgenograph it is seen to be a large, irregular, bony mass, forming the entire lower end of the femur. The epiphyseal line is seen at the level of the abductor tuberele on the inner side. Its outline is wavy, rises rather sharply toward its centre, and has a slightly lower level at the outer side of the bone. In this view also are seen the upper epiphyses of the tibia and fibula. The epiphyseal line of the tibia somewhat resembles 
that of the lower end of the femur just above it. The upper epiphysis of the fibula is a small mass appearing to rest lightly on the top of the shaft. In the lateral view of the knee the epiphyseal lines of the femur and fibula are nearly horizontal. The epiphysis of the upper end of the tibia in this view is seen to have a tongue-like projection extending down the front of the bone to the tubercle of the tibia. Sometimes this tongue-like process does not reach so far as the tubercle, and the latter is seen developing from a separate centre of ossification. Its importance has increased since the Roentgen ray has shown it to be rather frequently the seat of injuries which present somewhat vague elinical signs and symptoms.

Ankle.-The lower epiphyses of the tibia and fibula are seen in an anteroposterior view of the ankle. The epiphyseal line is nearly horizontal in the ease of both bones, but that of the fibula is at a lower level and comes opposite the ankle-joint. The internal malleolus forms the imner portion of the lower tibial epiphysis, and the external malleolus is practically entirely composed of the lower epiphysis of the fibula. This latter epiphysis is greatly concerned in the increase in length of the fibula.

Os Calcis.-The os calcis has an epiphysis on its posterior surface, just below the attachment of the tendo Achillis, and is seen as a small, oval disc. This is to be remembered in studying Roentgenographs of the foot in childhood.

The epiphysis at the inner end of the clavicle, those of the acromion and coracoid processes of the scapula, and those of the ribs, vertebrce, and pelvic bones are of no practical importance and would rarely cause confusion in studying a Roentgenograph.

Hammond concludes his most valuable and interesting study of the normal cpiphyses by saying that in injuries of the joints in childhood the outline at the different stages of derelopment should always be remembered and the normal Roentgenographs should be familiar to every one. 
Young children, especially infants, are very susceptible to inflammations in the regrion of the joints whenever infection occurs, and the younger the individual the more apt is a joint to be involved. The small joints of the wrist, knee, and foot show the greatest susceptibility to infection. Pyogenic organisms may enter at the umbilicus or through the intestinal tract and give rise to arthritis of the jounts, as may also the infecting organisms of influenza, and the pneumococeus or the gonococcus. The infecting organism of rheumatic fever has a special predilection for the joints, and the bacillus of tubercle finds a favorable site for growth in the joints. Congenital syphilis very frequently attacks the joints. Age not only makes a difference as to the susceptibility of infection of the joints, but it is to be noted that the tissues around the joints are more liable to infeetion in infants than in older children. It has also been noted that children under five years of are are much less apt to have suppurative conditions in the region of the joints. When they occur in children over five years of age the infection is either tuberculous or rheumatic. Shortly after birth the infections most frequently met with are those due to sepsis, and somewhat later, though still during the early months, they are due to the gonococcus. These infections are apt to be multiple. To repeat somewhat, the infective agent may be the streptococeus, the staphylococcus, the pneumococcus, the bacillus of typhoid, the bacillus of influenza, and oceasionally the colon bacilhus. In regard to the infeetions which involve the joints themselves certain infections of the bone occur in infants and young children, and also in the cellukar tissues adjacent to the joints, which at times give rise to swelling and to those symptoms found in actual involvement of the joint. Osteomyelitis, periostitis, epiphysitis, and deep cellulitis may often occur near the joints and extend into the joint-capsule. On the other hand, infections beginning in the joint may quickly involve the 
tissues around the joint and these may beeome more inflamed than the joint itself. This follows the rule of probable infection in the epiphysis aecording as the are of the individual and the degree of breadth of the eartilage of the epiphyseal line is greater or less.

If the inflammation is acute it is somewhat difficult to make a diagnosis, as the loeal signs are very similar, whether the infeeting agent is the streptococeus or some other organism. If the inflammation is of the subaeute or ehronie type it is more likely to be due to the bacillus of tuberele or to the gonococeus. If the infeetion involves only one joint, or perhaps shows moderate reaction, the probability is that the organism is the baeillus of tuberele.

It may be said in regard to the infeetion of the hip-joint that König's collection of infections of this joint shows that there were 560 caused by the bacillus of tuberele and 110 by other organisms producing acute infeetion.

The infection of the epiphyses represents a characteristic picture. The knee is the most eommon seat of the infeetion, but the epiphyses of any of the bones may be attacked and the infection may be single or multiple. The eharacteristie appearance is shown in Plate 19S. There is swelling and thickening of the tissues around the joint, and the epiphyseal line is thickened and filled in. The density is inereased around the epiphysis and the diaphysis. The joint is not involved early unless in aceordance with the normal anatomic relation of the capsules of the joint as described on page 169.

\section{INFECTIOUS ARTHRITIS}

There are a number of inflammatory eonditions in the joints, in many of which the speeifie organism has been diseovered. There are, however, a large number in which the infeeting organism has not yet been determined, but the proeess is considered by analogy and eomparison to be caused by an organism. We know that the 
bacillus of tuberele is one of the organisms which primarily attacks the joints. We also know that the various organisms which may give rise to the general disease osteomyelitis, such as the streptococeus and staphylococeus, though they primarily attack first the shaft of the bone, yet often involve the joint. This depends on whether the capsule of the joint is inserted into the epiphysis itself or into the zone of proliferation. As instances of other causes of infectious arthritis are the pneumococeus, the organism of rheumatic fever, the gonococeus, and the etiologic factors of searlet fever and measles. These foci of infection may be in any part of the body, as in the tonsil or in the ear. The arthritis may be due to the presence of a specifie organism in the joint, or to the action of its toxins. In the former case the local process is more severe. It is thus seen that under the general term infectious arthritis should be included a very large number of diseases. Arthritis may be caused by a primary infection from an original focus, or through some portal of entrance, such as the tonsils, or it may be an accompanying condition of certain general diseases, such as scarlet fever, tỵphoid fever, syphilis, epidemic influenza, erysipelas, cerebrospinal meningitis, gonorrhoea, glanders, the infectious diarrhœas, and a number of others. In making a pathologic diagnosis by the Roentgen ray in these infeetions we should seek to determine the specifie lesions which characterize the special disease and the organism causing it. In the past it has been extremely difficult to accomplish this, as the post-mortem findings as I have said before almost invariably teach us only terminal results. It is well known, however. that the same terminal results may represent a number of primary conditions, the lesions of which differ very materially according to the specific infecting organism. It will in the future, therefore, by means of the Roentgen examination be possible to determine during life primary lesions and conditions and thus complete our knowledge 
of the various inflammations of the bones and of the joints. It is now pretty well accepted, from what we have learned by investigating diseases of the joints by means of the Roentgen method, that etiologically they are all infections, whether we have polyarticular disturbance or that of a single joint; also that these infeetions through some direct portal or from some definite focus are transmitted by the blood usually to the most highly organized anatomic areas, and that the infeetion takes place in an especial tissue, aceording to the predilection of the speeial organism for that tissue. Of course where the lesions are symmetrical, we should consider etiologically the possibility of trophic conditions. Infectious arthritis, therefore, may be the result of any of the infections or pusprodueing organisms. The severity of the attack depends not upon whether the joint is the primary seat of the lesion, but upon the special organism, and upon its rirulence, whether it is actually present in the joint, and also upon the idiosynerasy of the individual ehild. When we come to consider the more chronie processes in the joints, the determination of a satisfactory classification is difficult. From what we have learned, however, by studying these processes on the living subject we are led to believe that these chronic eonditions, which are when examined post mortem in most cases terminal conditions, are produced by the primary infection of specifie organisms. This in the future will probably prove to be true when it is possible to determine the especial infecting organism. As instances of the chronic form of arthritis, for we can eall these also arthritis, since the term infection implies inflammation and since by this term we understand reaction with cellular infiltration, are the chronic conditions represented by hypertrophy and atrophy of the tissues of the joints. The examination by the Roentgen method will in the future present to us the early lesions of these diseases in the living subject, but it at present merely shows that certain condi- 
tions exist at various stages of the tissue changes. Thus the Roentgenograph can show what in children is a secondary condition, namely, a villous arthritis. Again, the picture may show a swelling of the soft tissues about the joint, or again atrophy of the bony and cartilaginous parts. It may show hypertrophic changes in the bones and cartilages of the joints, Heberden's nodes, the urates of soda, and the deposits of gout. It is also to be noticed that atrophy of the surrounding soft tissues as an end result of ankylosis of the joint commonly oceurs.

Although details of the examination by the Roentgen method are not by any means completely developed, or in fact thoroughly understood, still it is well known by expert Roentgenologists that certain definite pathologic facts are being continually demonstrated in these cases. The change which oceurs in the joint is that of an increased blood supply. It is demonstrated in all of these cases, if seen early, that there is an increase, usually in the size of the bone, as is shown in the plate. If the knee-joint is infected the condyles will be increased in size in comparison with the opposite side. The epiphysis of the fibula if not already developed will appear earlier than on the unaffected side, and it is evident that this inflammation is due to a definite organism.

It is well to again enunciate the importance of bearing in mind that in children, especially in young children, the acute lesion of the bone is usually at the begimning an epiphysitis or a periostitis, and not an osteomyelitis, for the bone-marrow is not involved. The pus ceases to spread in bone or cartilage when once it has any other direction in which to spread, and it ceases to dissect up the periosteum when it can escape in any other direction. Hence in young subjects, in contradistinction from the later periods of development, the pus, having a freer vent into the line of the epiphyseal cartilage, does not so frequently attack the medulla, bone, and periosteum. 
Humerus: Infectious Arthritis, Atrophy of Shaft.-Plate 203 represents a case of infectious arthritis in a child and shows a marked atrophy of the shaft of the humerus, this atrophy being both of size and of quality. The outlines of the cortex and the medulla in the shaft are distinetly marked. The special infecting organism was not ascertained in this ease. The ehild aceording to the anatomic age should be placed in Group F or G.

Plate 204 represents the hands of the same case. These hands show the finely peneilled outlines of the bones. There is no absolute destruction of tissue, but simply absorption. The joint substances are not as yet disturbed. In the left hand there is a slight delay in the development of the carpal bones. There is also a slight amount of inflammation of the tissue in both joints.

Hand: Infectious Arthritis.-Plate 205 shows a case of infectious arthritis with the organism not determined, showing itself in the hand of a boy four and a half years old. The second and third metacarpal bones show some thickening of the periosteum along the shaft. The third metacarpal bone also shows two small areas of absorption of the lime salts. There is only a very slight inflammation of the tissues shown by decreased radiability in the palm of the hand. There is a thickening of the tissues about the wristjoint. The earpal bones are a little larger than normal and their structure is somewhat effaced.

The carpal bones are a little larger than normal, show less bony strueture.

Knee: Effusion.-Plate 206 shows a lateral view of an effusion in the knee-joint of a boy ten years old. There is no disturbance of the femur, tibia, or fibula, exeepting in the posterior aspect of the condyles of the femur, which are a little ragged. The patella has a few excrescences of bone on its superior border. This is probably due to irregular ossifieation. The tissues about the joint seen to be 
thickened as well as to contain fluid. The fluid is differentiated from the density of the knee-joint by the ballooning eharacter of the density as well as by the space oceupied by the subpatella fat pad, which is absent in this plate. It will be noticed that the ligamentum patella is pushed upwards and that it is convex on its anterior surface.

Femur: Infection - Non-tubercular. - Plate 207 shows the destruction of the head of the right femur from some septic infection, the specific organism of which was not determined, but in all probability was not tubercular.

Rheumatic Fever-Knee.-Plate 208 represents the left knee-joint of a case of rheumatic fever. In this case there was acute swelling, pain, and fever, and the Roentgen plate taken early in the attack showed slight thickening of the capsule. The reproduction, however, shows nothing abnormal and the illustration is merely given here to show how difficult it is to detect in some of the acute infections of the joints any pathologic process whatever.

Rheumatic Fever-Ankle.-Plate 209 shows the joint of the ankle of the same subject. Here also there was swelling, tenderness, and fever, and the case clinically scemed to be one of rheumatic fever. In this case the Roentgenographic plate also showed thickening of the capsule of the joint, but here also nothing special could be detected in the reproduction.

\section{INFECTIOUS PERIOSTITIS}

The diagnosis of an early infection of the periostcum by means of the Roentgen ray is attained principally by recognizing certain pathologic changes, such as thickening and bulging of the periosteum. Usually the line of the periosteum is less distinctly seen, particularly that of the normal bone. Again the periosteum may be seen to be broken and ragged, exposing the cortex. 


\section{OSTEOMYELITIS}

Although the disease osteomyelitis has been recognized for a number of years, yet we now have a much more definite knowledge of it than in the past. We know that it is not caused by any one special organism, but that it is a general term which covers a number of specific infections, and simply means that, instead of special organisms affecting the periosteum, the cortex of the bone and the medullary cavity are also affected. Now that the Roentgen ray has been brought to bear upon this general class of infections, and a special study has been made of these different organisms in connection with their infection of the bones, a great advance has been made in our knowledge of the living pathology of osteomyelitis. For this reason also the primary pathologic conditions in the bones in early life have become more prominent, as they show at that period the actual pathology, while at a later period the postmortem in many cases only shows terminal results. These terminal results may sometimes be characteristic of the special infection, but more often may represent conditions which are the result of a number of entirely different infectious organisms.

The type of infections which are grouped under the name osteomyelitis makes it in some respects the most important disease of the bones which occurs in early life. This is true on account of the tremendous destruction of bone which may occur, either terminating in death or in various degrees of deformity which may be permanent. The rapidity of the onset and the resulting rapid destruction of the bone give osteonyelitis a place in diseases of the bone which appendicitis holds in diseases of the abdomen. In the case of appendicitis delays in operation may mean death. In osteomyclitis, delay in diagnosis and in operative treatment may mean not only death, but resulting deformities which camnot be rectified, and in some cases may mean more than 
death. It is therefore important that an early diagnosis should be made of this class of cases, and that operative treatment if indicated should be decided upon at once. To accomplish this early diagnosis, the Roentgen ray is of inestimable value, for at times it tells us what the clinical examination fails to find.

Osteomyelitis is a general disease so far as its etiology is concerned, but in many cases it can only be diagnosticated surely by the Roentgen ray. Among the numerous organisms which may cause it, the most prominent is the staphylococeus in the chronie cases of low grade, while in rapidly septic cases the streptococcus is most commonly found. In certain cases the pneumococcus oceurs and produces a farly chronic condition, and finally the bacillus of typhoid may cause either an acute or chronic process.

I shall merely enunciate the fact that the Roentgen ray should be used at once during the earliest period of the symptoms, and that we should not be led astray in our diagnosis by thinking that the case may be one of rheumatic fever. There is more confusion in the mind of the greneral practitioner in regard to rheumatic fever in connection with this set of eases than arises when a differential diagnosis is to be made from any other disease. For this reason the organism which may be producing an osteomyelitis has an opportunity to thoroughly infect the bone, and of ten to such an extent that operative treatment becomes of little avail. This is especially the case in very young children, where the percentage of cases caused by infections of the epiphyses is very great.

As in other severe diseases of the bone, it is difficult to make a definite diagnosis of osteomyehitis unless the case is examined by the Roentgen method. When the Roentgenograph is used, however, the difficulty is rery greatly lessened.

Infectious ostcomyelitis may be single or multiple in its first appearance, and in its course may be acute, subacute, or chronie. 
An involvement of any of the bones may occur, but the knee is the most common seat of infection. The tissues usually show swelling and thickening, the epiphyseal line is thickened and filled in, and the radiability surrounding the epiphysis and diaphysis is increased. The infection commonly attacks the long bones, and it is usually the extremities of the bones which are involved. Aceording to the site of the infection and of the tissues involved, the primary infection may be of the periosteum or of the marrow. When the infection is seen early by the Roentgen ray the principal pathologie change is found in the periosteum. The change consists of thickening and hypertrophy of the periosteum with its line less distinctly seen. Again the periosteum may be thickened and ragged, exposing the cortex. When the infection is of the marrow the Roentgenograph shows very early in the process the infected area to be represented by one or more definite degrees of density, varying in size from a pinhead to several times that size. There is also found, at times, an increased area of radiability in which the strueture of the bone is being destroyed and absorption of the lime salts is probably taking place.

Osteomyelitis, as distinctive from tuberculosis or syphilis of the bone, is shown by a more definite proliferation of the periosteum, a more definite formation of new bone in the area of necrosis, or this area may show large pieces of bone which have not been absorbed.

In the subacute and chronic cases of osteomyelitis the structure of the bone shows less distinctly and is accompanied by atrophy below the point of infection. This atrophy is not particularly in size, but rather in quality, and is aceompanied by an excessive amount of proliferation of the periosteum. At times the process goes so far, as is shown in Plate 210, Fig. 2, that there is a definite area of exposed bone surrounded by an involucrum.

Tibia: Early Stage.-Plate 210, Fig. 1, represents the knee of a 
child nine years old, showing one of the earliest manifestations of osteomyelitis. The child was kicked on the tibia, the injury having taken place two days before being scen. The Roentgenograph was taken on the third day and showed an increased radiability of bone about one inch below the epiphyseal line of the tibia. Below this there was a slightly boggy periosteum running down almost the whole length of the tibia, especially in front, and showing evidently an exudation of fluid under the periosteum, proved later by operation not to be blood. The elinical symptoms were extrene pain, swelling, no fluctuation or redness, tenderness, and a varying temperature.

Tibia: Same Case, Later Stage.-Plate 210, Fig. 2, shows a later stage of the same case. Owing to a delay in making a correct diagnosis, an operation was postponed until infection had taken place, and the process went on to such an extent that the whole bone became involved. The plate shows proliferation of the periosteum, with formation of sequestra.

This case was evidently one of simple trauma in the beginning, and if it had been recognized that an early infection had taken place operative treatment would have been very simple and would have preserved the leg from the extensive lesions shown in the Roentgenograph.

Femur.--Plate 211 represents the leg of a child ten years old who entered the hospital for rheumatic fever. The clinical symptoms were referred to the knee, where there was swelling and tendemess, but nothing localized was detected in the lower part of the femur. The Roentgenograph showed increased radiability of the diaphysis of the femur with proliferation of the periosteum. The operation was delayed too long and the infection went so far that the disease lasted for over a year. Several operations had to be performed for the removal of the sequestra, and although the child finally recovered it was left with irreparable deformity. 
Femur.-Plate 212, Fig. 1, is the pieture of a child twelve years old. The Roentgenograph of this ease shows the permanent results of acute osteomyelitis of the left femur. It was treated for tuberculosis of the hip. All of the destruction took place within two or three months. An carly operation would have obviated this result. In the course of the differential diagnosis the process was aspirated and the infection was proved to be from the staphylococcus.

Hip.-Fig. 2 is the pieture of a ehild five years old. There was a history of swelling in the region of the right hip with slight limitation of motion and pain about the hip. It was sent to the hospital with a diagnosis of tubereulosis of the hip. The Roentgenograph showed an infiltration, with the formation of an abseess resulting from infection of the neck of the femur. There was proliferation of the periosteum about midway between the greater and lesser trochanter and epiphyseal line. There was here also an area of increased radiability. The infection was so eompletely outside of the capsule of the joint that the case was evidently not one of tuberculosis but of osteomyelitis.

Tibia.-Plate 213, Fig. 1, represents a ease of osteomyelitis of the tibia in a boy eight years old. In this picture it will be seen that the capsule and soft parts about the right knee are thickened. The femur is eomparatively normal, as is also the fibula, but at the upper end of the tibia there is seen to be an area of destruction of the bone with a formation of sequestrum. The epiphyseal line, as well as the epiphysis, is involved in the destructive process, which starts in the epiphyseal line of the tibia. Operation was delayed and the process extended into the epiphysis. This ease shows how important it is to recognize the very early lesions of an osteomyelitis. This could have been done if the Roentgen method of examination had been employed at once.

Tibia: Bone Plug.-Fig. 2 shows the same case. An operation 
was performed later and a bone plug inserted in the carity. The Roentgenograph illustrates that the operation must necessarily be a failure for there is a sequestrum still remaining in the eavity. The tibia shows below the point sinply absorption of the lime salts, which is also seen in the fibula, in both cases there being a lessened radiability. This Roentgenograph is a striking illustration of the great use of the ray, not only in detecting the nidus of the disease, and its early portrayal of destruction of the tissues, but also how a series of pictures will aid the surgeon by elucidating why his operation is a failure.

Femur.-Plate 214 represents a case of acute infectious osteomyelitis in a girl two and a half years old, twelve hours after the onset of the first symptoms. There is a haziness of the outline of the right femur with marked inflammatory reaction of the soft parts about the thigh. The process went on, as is shown in Plate 215, to a general destruction of the entire shaft of the femur. This plate illustrates how early changes in the tissues and bones can be demonstrated by the Roentgen ray.

Femur: Plate 215 shows the same case as Plate 214, but five weeks later. In the middle of the shaft is an area of inflammation; somewhat lower, and on the outer edge of the bone, there is newly formed periosteal tissue. Further down in the upper part of the diaphysis is the point of attachment of the eapsule and periosteum to the shaft of the femur. It is to be noticed that the joints have not yet become infected, but that the infection of the bone is confined within the limits of the periosteum. There was a considerable reaction of the soft parts not shown in this plate but clearly seen in the original plate. All the other bones shown in the pieture are normal.

Elbow.-Plate 216 is the picture of a boy nine years old, with osteonyelitis of the elbow. There is seen to be marked atrophy 
in the quality of the bones, but not in the size. The epiphyses of all the bones in the elbow, but especially of the radius and of the ulna, are almost completely eroded and the joint is filled with necrotic material.

Humeri-Plate 217 shows marked destruction of both humeri in a boy eight years old. The whole structure of the bone and periosteum of the upper half of both bones is seen to be markedly involved. There is active destruction with formation of sequestra at both upper ends. The joints of both shoulders are affected. The head of the bone on the right is more involved than on the left. The lower ends of the humeri on both sides show a marked increase in radiability. The thorax is normal. The infecting organism was found to be the bacillus of typhoid.

Radius.-Plate 218 represents an osteomyelitis of the lower third of the radius in a boy six years old. The soft tissues are greatly thickened. There is marked thickening of the periosteum on the lower half of the radius, with a general necrosis of the bone in the lower third, and with the formation of a sequestrum at both points. There is an almost complete separation at the lower end of the radius. The epiphyseal line is involved and the proximal surface of the epiphysis is roughened.

Radius.-Plate 219, Fig. 1, represents the pnotograph of a child with acute osteomyelitis of the radius. There is seen to be a considerable swelling of the soft parts of the wrist and of the whole lower arm, starting at the bend of the elbow.

The Roentgenographs, Figs. 2 and 3, do not show periosteal reaction on account of the destruction of the bone. The lower twothirds of the radius seems to be almost completely a mass of necrotic material. The upper part of the radius seems to be out of place. The surface of the joint of the elbow is apparently not infected. The two carpal bones that are present are not involved. 
Hip.-Plate 220 shows an acute infectious arthritis in a child two and a half years old. The soft tissues around the left hip are seen to have a greater density than on the right. The shaft of the femur shows slightly increased radiability, and the radiability of the epiphysis is also marked. The primary focus of the disease in this case is in the acetabulum, where at the junction of the ischium and ilium there is a marked destruction. There is a small formation of sequestrum at this point.

Hip.-Plate 221 shows a marked increase in the radiability of the soft tissues around the hip-joint in a boy seven years old. The epiphysis of the greater trochanter is irregular and partly absorbed. The medullary eanal at the upper end of the femur shows marked absorption of lime salts; periosteal reaction and proliferation are strongly marked. The epiphyseal line is seen to be rery ragged, although there is no aetual destruetion at this point. The lower portion of the acetabulum is also roughened.

Femur.-Plate 222 represents the results of an infectious osteomyelitis of the upper part of the femur, occurring in a girl three and a half years old and produced by the pneumococeus. The soft tissues appear to be rather hazy, but of inereased radiability, and indicate an inflammatory action. Just outside the eapsule of the joint and in the region of the surgical neek of the femur there is seen to be an area of destruction of bone with the formation of a sequestrum. The line of demarcation at the lower end of the involved area is elearly outlined. There is a slight increase of periosteum along the upper line of the femur. The head of the femur and the acetabulum are perfectly normal. No apparent decrease in the lime salts is shown.

Femur.-Plate 223 shows a marked disturbance in the neck of the femur in a girl four and a half years old. There is an almost complete absorption of lime in this area and marked periosteal 
thickening at the upper end of the femur on both sides. There is apparently no involvement of the epiphyseal line, but there is a marked inflammatory reaction of the soft parts around the greater trochanter. This infection was due to the staphylococcus.

Hip-joint.-Plate 224 shows a mixed infection of the hip-joint in a girl six years old. The soft parts around the right hip-joint are seen to be greatly thickened and the density is much increased. The shaft of the femur shows no atrophy of size, but a slight one of quality. The neck and epiphysis of the femur, however, are almost completely destroyed or absorbed. The acetabulum also is markedly affected. There is thickening of the periosteum along the iliopectineal line. The rami of the os pubis and ischium show the same process. There is a partial dislocation.

Tibia.-Plate 225 represents osteomyelitis of the lower end of the tibia in a boy twelve years old. It is to be noted that there is an area of necrotic bone which is surrounded by a dense zone of new bone formation which walls the process completely off. This Roentgenograph illustrates the condition which occurs in a low grade of staphylococcus infection.

Femur.-Plate 226 is the picture of a boy seven years old with osteomyelitis of the right femur. To be noted is the slight amount of swelling in the soft parts around the right knee-joint. The lower end of the femur is larger in size than the left femur, and shows along its inner edge an absorption of lime salts. There is hypertrophy in addition to absorption. The epiphysis of the right femur shows an irregular deposit characterized by its ragged appearance. The primary focus of the infection is seemingly in this epiphysis.

Tibia.-Plate 227 represents osteomyelitis of the left tibia at its upper end in a boy twelve years old. The picture shows a marked proliferation of the periosteum of the upper third of the tibia, with numerous sequestra. An operation showed this to be a staphylococeus infection. 
Tibia: Early Staye of Infection.-Plate $22 S$ shows at practically normal tibia and foot exeept at one point in a boy twelve years old. At the lower end of the tibia close to its epiphyseal line there is a small area with inereased radiability. This condition shows an early stage of osteomyelitis.

Fibula.-Plate 229 is the pieture of a girl eleven years old with osteomyelitis of the fibula. It will be seen that the periosteum of the fibula is greatly thickened and that in the lower third there is also quite an area of increased radiability, which is the focus of the infection. It will also be seen that there is a begimning rim of new formation of bone around this area, nature's process of walling off. The other bones in this pieture are normal.

Tibia.-Plate 230 shows an undetermined infection of the lower epiphysis of the tibia in a boy ten years old. The strueture of the bone is perfectly normal exeept at the point of infection. At this point in the epiphysis of the tibia there is a small area of increased radiability. As yet there is no atrophy of any of the bones of the foot.

Tibia.--Plate 231 shows a case of chronic infectious osteomyelitis in a boy six years old. The soft tissues around the kneejoint are shown to be greatly thickened. The epiphyseal line of the tibia is ragged and irregular with some increase in radiability. Along the inner margin of the upper end of the tibia there is a beginning destruction of the bone. The femur and fibula simply show slight increased radiability.

HYPERTROPHIC AND ATROPHIC PROCESSES OF THE JOINTS

The chronic hypertrophic conditions of the joints which occur in later life are very rare in early life. The pathologic condition which is most common in this chronic class of cases in early life is represented by an atrophic condition of the joints. It is a slow, progressive disease, and most commonly manifests itself in some of 
the smaller joints, usually in the proximal row of the phalangeal articulations. At first one or more joints are affected, but gradually other joints become involved until in many instances every joint of the body is included in the destructive process. The progress of the disease lasts for a period of years. A great deal of work has been done by different investigators on this subject, but I shall not attempt to discuss the subject of classification here, as we are simply interested in what we may expect to see in the Roentgenographs of these cases.

The chronic atrophic cases show a swelling of the soft tissues about the joints due to symovial fluid, and subsequent atrophy of the cartilaginous parts of the bones. The Roentgen ray is especially valuable in these cases for the purpose of differentiating them from certain forms of infectious arthritis, where there are spindle-cell swellings but where the ray shows there is not the characteristic atrophy.

Chronic Atrophic Wrists, Hands, Arms, Knees, Legs, and Ankles. -Plate 232 represents the hands of a boy, showing a chronic atrophic condition of the joints of the wrists and hands. There is marked atrophy of the forearm, with considerable fusiform swelling in the carpal region as well as in the third and fourth fingers of the right hand and the first, second, third, and probably the fourth of the left hand. Clinically this was a case of infectious arthritis followed by atrophy.

Plate 233 shows the knces of a boy eight years old. There is marked thickening about the knee-joint, contrasting with the extreme atrophy of the leg. The same condition of the knce-joint will be noticed in the Roentgenograph; all the bones of the body were affected in a similar way.

Knee: Chronic Atrophy.-Plate 234 shows a Roentgenograph of the same boy. It is to be noted that the bone substance is more 
compact and smaller than normal, and that there is an atrophy of the substance of the museles. The joint itself is partly involved and the surfaces of the joint are seen to be greatly roughened, especially the epiphysis of the femur. The cortex is very sharply defined. The patella shows an atrophie condition with increased radiability. 'The outline of the articular surface of the condyles is irregular, and is due in part to the irregular ossification of the substance of the bone, as well as to some disease, as shown by erosion. There is also thickening about the joint but no fluid. There is marked atrophy of the femur, tibia, and fibula, not only of size but also of quality, and characterized by finely pencilled cortical bone seen in all the bones. There is a sharp outline of the condytes and of the epiphysis of the tibia. There is fine trabeculation of the bone, and the patella shows fine pencilling.

\section{SYPHILIS}

The lesions of syphilis which ean be shown in Roentgenographs are mostly of the osseous tissues. Although all the organs of the body may be affected by the Spirocheta pallida, yet the special lesions by which the diagnosis of the disease can be made when the Roentgen method is employed are essentially those of the bones. The living pathology of the lesions of the bones differs aecording to whether we are dealing with a ease of congenital syphilis, which corresponds to the secondary symptoms of acquired syphilis, or with the retarded and later form of syphilis in childhood, which corresponds to the tertiary lesions of acquired syphilis. In the former case, the congenital, we find that the Roentgenograph shows the picture of an acute epiphysitis and its accompanying periostitis, leading later to separation of the epiphyses and to pseudoparalysis, simulating also in many cases fracture. To those who are not especially aequainted with the many ways in which syphilis simulates other diseases, these lesions of the bones are apt to be misleading. I have 
seen cases where a separation of the epiphysis of the wrist in young infants has been mistaken and treated for a fracture, the thickened periosteum being mistaken for a callus. In such cases as these the Roentgen method aids us to make a differential diagnosis. The lesions which are mostly to be confounded with syphilis of the bones are those of tuberculosis. It is in these cases which are often obscure that special means for a differential diagnosis should be employed. By means of the Roentgenograph we can distinguish the destruction of the cortex of the bone and the primary lesions occurring in the joint in tuberculosis from the marked thickening of the periosteum, layer on layer, significant of syphilis.

In syphilitic lesions of the osseous system which occur in children there are at first disturbinces of the periosteum with marked periosteal overgrowth and much laying down of new bone. Usually changes in shape occur more along the middle of the shaft of the bone than near the epiphyses. The overgrowth of periosteum is out of all proportion to what would be expected from simple periostitis, or without any change in the bone excepting an increase of the cortex.

The close simulation of syphilitic dactylitis and tubercular dactylitis makes the Roentgen method of especial value in the differential diagnosis of the two diseases. Plate 237 shows a syphilitic dactylitis with its periosteal lesion, and Plate 242 represents a tubercular dactylitis with its special destruction of bone.

Syphilitic Dactylitis. - The differential diagnosis by the Roentgen method shows that in syphilis there is a definite change in the exterior of the bone without any change in the interior unless suppuration has taken place. The periosteum is thickened and increased in size, so that we may have in a phalanx the characteristic changes that take place in the long bones.

Cases have been seen where the condition of one hand was that 
of a syphilitic dactylitis and of the other hand that of a tubercular dactylitis.

The point of diagnosis generally in favor of syphilitic dactylitis and against tubereular dactylitis is that the long bones in other parts of the body do not show in tubereular dactylitis disturbances such as are expected to be found in retarded or congenital syphilis.

Elbow.--Plate 235 represents syphilis in the elbow of a child eleven years old. The lower end of the humerus along its inner border shows great thickening of the periosteum due to syphilis (overgrowth), and it is to be noted that there is no change in the shaft of the bone with this exception. There is no change in the soft tissues.

Elbow.-Plate 236, Fig. 1, represents a syphilitic elbow in a girl two and a half years old. There is increased thickening of the periosteum along the inner border of the humerus. There is some decrease in the lime salts at the lower end of the bone. The greatest area of involvement, however, is seen in the ulna, where the periosteum is markedly thickened, being almost as thick as the structure of the bone itself. On the outer edge of the olecranon there is seen to be a beginning destruction of the periosteum. The whole olecranon at its upper third is completely changed from its normal structure. The radius, however, shows little disturbance beyond the periosteal reaction to be expected in this condition. There is a marked inerease in the radiability of all the bones in this plate.

Ulna.-Fig. 2 represents a syphilitic lower arm in a child four and a half years old. There is a very thick layer of periosteum on either side of the lower part of the shaft of the ulna. On the outer side of the ulna the tissues are thickened and there is a formation of abseess in the soft parts. Such a bone as this is absolutely characteristic of retarded syphilis. 
Dactylitis.-Plate 237 shows a syphilitic dactylitis in a ehild two years old. There is infection of the first and third metacarpal bones and of the first phalanx of the fourth finger. It is to be noted that there is very little ehange in the strueture of the bone, but a great change is seen in the thickened periosteum. There is considerable tissue reaction and much fine pencilling of the bone tissue.

Tibia: Periostitis.-Plate 238 represents a syphilitic periostitis of the tibia in a ehild five years old. There is bulging of the soft parts on either side of the tibia and fibula. The periosteum along the whole of the tibia, and also of the fibula, is thickened in a characteristic manner. There is also apparently some thickening of the eortical substance.

Tibia: Periostitis.-Plate 239 shows a syphilitic periostitis of the tibia in a ehild eight years old. This is a lateral view. The outline of the bone along the anterior border of the tibia is slightly more convex than normal, with a slight prominenee in the upper third of the bone posteriorly. On the anterior surface the thickening of the periosteum extends along the shaft and down towards the lower third.

Ostcoperiostitis and Osteochondritis. - Plate 240 shows a syphilitie osteoperiostitis and osteochondritis in a case of congenital syphilis in a colored ehild six weeks old. The infunt was breast-fed. When four weeks old it developed a pseudoparalysis of the left arm and leg, with marked tender swellings at the elbows and knees. There was an enlargement of the spleen and lirer, and a squamous desquamation of the soles of the feet and palms of the hands. There were fissures at the anus. The enlargement of the upper ends of the tibia and fibula, and of the lower ends of the femur, was very much marked. The Roentgenograph shows an osteochondritis with involvement of the diaphyses of the femora and of the tibia and fibula, and great thickening of the periosteum. 
Retarded Syphilis, Tibia and Fibula.-Plate 241 was a case of retarded syphilis in a child twelve years old. This child had the lesions of retarded syphilis in other bones of the body. This plate shows the left side of the tibia and fibula. The lower third of the tibia shows an area of active suppuration with beginning formation of sequestrum. The whole lower part of the tibia is seen to have areas of increased radiability. The periosteum is seen to be thickened along the whole length of the tibia, and of the fibula also. The process in the tibia extends apparently down to the epiphyseal line but does not involve the ankle-joint. There is also increased radiability of the shaft of the fibula. The bones of the foot are seemingly normal.

\section{TUBERCULOSIS}

Much has been studied in connection with and much progress made in tuberculosis of the lungs, but we should understand that of equal, if not greater, importance is a consideration of tubereulosis of the bones.

There are a number of facts which make a study and consequently a knowledge of tuberculosis of the bones of great importance.

In order to diagnosticate tubercular conditions and to enable us to differentiate tubercular from non-tubercular lesions it is of the utmost moment that we should recognize the normal living anatomy of the bones at different periods of their development, as portrayed by the Roentgen ray. This is especially necessary in the early stages of tubercular disease, where the pathologic changes are often slight and obscure, and yet where an early diagnosis is extremely valuable. This of ten enables us to arrest the disease by treatment, and to determine whether the condition is merely a slight anomaly of healthy bone or some non-tubereular affection. In both these latter conditions we can often decide that the case is not tubercular, and therefore not one to be sent to tubereular 
hospitals or homes. It is also important that the treatment in tuberculosis should be begun very early, so as to protect the ear and other organs from secondary infeetion.

There is no diagnostic means so valuable for the detection of tubereulosis of the bones as the Roentgen ray in the hands of experts in its technic. Over and over again it becomes a most important factor in the chain of evidence which may lead, unless refuted by the ray, to stamping an individual as syphilitie, and thus perhaps ruining his social life.

Witness the possible differentiation by the ray of the tubereular lesions of the bones from the periosteal lesions of syphilis and the especial lesions represented by dactylitis. Again, the importance of differentiating by the Roentgen method a non-tubercular from a tubercular arthritis, and a tubereular hip from an osteomyelitis or an acute trauma, is very great.

In the past, and very often in the present, many cases have been considered and treated as tubercular which have really been non-tubercular. The Roentgen method often corrects us when we have been mistaken in making a diagnosis of tuberculosis, and shows us that if the true diagnosis had been made, an entirely different treatment would have been indieated.

We may tabulate our knowledge of the pathologie findings, and understand the terminal lesions at the autopsy; we may make advances in the treatment of tuberculosis; but how significant is the announcement in the sanatoria that early eases of tuberculosis are preferably taken, or of ten only taken! The diagnosis of these early cases, especially where the bones are affected, is exceedingly difficult without the aid of the Roentgen ray, even when a thorough elinical examination has been made, and especially in those cases where the tuberculin test has failed. Most important of all, next to the actual operative indications which the Roentgen ray gives 
us, is its differentiation of tubereulosis of the bones in doubtful and obseure cases from conditions caused by osteomyelitis, syphilis, and trauma. Probably thousands of lives could be saved if eases of tubereulosis could be recognized before the later and more advanced lesions have appeared, with their almost hopeless prognosis.

There are certain early lesions in tubereulosis of the bones which ean be deteeted by means of the Roentgen ray. One of the first manifestations of a tuberculous lesion of the bones is atrophy of the shaft of the bone, especially of the femur or of the humerus. This may oceur long before a definite focus of disease is discermible, and this atrophy of size as well as of quality of the bone is noticed to increase progressively.

The infection commonly attacks the epiplyses of the long bones, and is first detected by the Roentgen method as a small area of increased radiability. This area slowly increases in size and shows an atrophy of the bone about the point of infeetion disproportionate to the amount of diseased bone apparent. This atrophy may be due partly to the infeetion, but is also due to the slow progress of the disease and to disuse of the part.

The value of the Roentgen ray in detecting diseases of the bones is very great, because it indicates the exact location of the infection, and the details of the living gross pathology. Primary infeetion of a bone in children by the bacillus of tubercle always oceurs in the epiphyses exeept where the ehild is of an age at which the given bone has no ossific centre. The early lesions of tubereulosis of the bones which ean be detected by means of the Roentgen ray are:

Atrophy in size of the bone at the upper part of the femur as a rule is present. In the joints we find frequently hypertrophy in the early stages followed later by atrophy. Atrophy in quality of the substance of the bone.

A definite area of necrosis of the bone. 
Examination by the Roentgen method shows just at what portion of the bone the disease begins in a given case, whether in the epiphysis or in the diaphysis. It will show also the relative size of the discased areas. It will show the lack of definiteness of the structure of the bone which accompanies atrophy, change in quality due to an absorption of lime salts, or a disintegration of the bone into caseous material. Where a joint is infected by the bacillus of tubercle, erosion of the surface of the joint usually begins, by either a definite focus at one point or a definite erosion at some portion.

In cases of tuberculosis of the linec-joint one of the earliest manifestations is usually atrophy of the soft parts. In the epiphyses, however, there is at first apparent increase in size but atrophy in the texture of the bones. There is an increased density in the capsule, due in all probability to thinning or possibly fluid within the joint; there is also squaring and atrophy of the epiphyses. A focus may be defined, although in the majority of cases it is not found early. We also notice an early stimulation of epiphyseal growth and an appearance of hypertrophy of the shaft and of the epiphyses. This is usually accounted for by the marked increase of the blood supply, which would naturally produce an hypertrophy. This accounts also for the increased length of a given part.

As the process becomes more cxtensive the characteristic features are generally atrophy of the soft parts, atrophy of the cortex, increased density of the cortex, increase of the medullary canal at the expense of the cortex, and a lack of definition of the structure of the bone. Within the joint affected we find more or less actual destruction of bone with detritus about the focus of infection, erosion of the surfaces of the joints, increase in the density of the capsule, and lack of definition of the structure of the bone generally.

One of the first manifestations of an infection by the bacillus of tubercle is usually atrophy of the size of the bone. This occurs 
long before a definite focus of disease is discernible either by the eye or in the Roentgenographs. The epiphyses of the long bones, or the acetabulum, are first infected, and this is detected by means of the Rocntgen ray as a small area or areas of increased radiability. This area slowly increases in size and shows an atrophy of the bone about the infected point, so that the dense qualities of the bone are reduced to that of its surrounding soft parts.

At times the only noticeable change from the normal is in the marked atrophy of size without evidence of destruction of the bone, and at times an infiltration of the tissues and capsule with the formation of an abscess. The bacilli of tubercle do not cause periosteal reaction, and when this reaction is seen in the Roentgenograph it shows that there has been a mixed infection.

A point of interest in the examination of a large number of cases of tuberculosis in children by the Roentgen method is that it demonstrates repeatedly the clinical manifestations of tuberculosis of the joint without definite foci being detected in the bone, though a great deal of capsular thickening may be evident. It is unusual to find a distinct early necrosis of the bone.

In the very chronic cases, when examined by the Roentgen method, we find the characteristic reconstruction going on to ankylosis and to new formation of bone.

In connection with the differential diagnosis between osteomyelitis and tuberculosis, it is well known that various pathogenic infections may result in acute and chronic osteomyelitis. If the specific cause of these infections is recognized early, the treatment often differs decidedly from that which should be cmployed where the infection is tubercular. By means of the Roentgen method we can of ten localize the pathologic process before the infected area has increased very much. We can also almost at once determine whether the infection has attacked the bone to any great extent. 
Atrophy.-Atrophy of bone as demonstrated by the Roentgen method has become important enough to be spoken of in detail. It is evident that three kinds of atrophy are seen,-(1) atrophy of size, (2) atrophy of the quality of the substance of the bone, or (3) a combination of both.

It is characteristie that certain organisms produce eharacteristic changes in a bone which are more or less constant. In infection of a bone by the bacillus of tuberele, particularly about a large joint, such as that of the hip, one of the early manifestations of the infection will be a marked atrophy in the size of the femur. This is demonstrated long before any actual disease is apparent, unless it is a mixed infection.

Certain authorities have demonstrated by Roentgenographs that an atrophy of bones in juxtaposition, such as the os pubis and ischium, takes place with an apparent hypertrophy of the ilium. In a great majority of these cases misinterpretation may arise resulting from a deformity of the pelvis brought on by postural or struetural change which the femur undergoes, due to traction applied in carrying out the treatment. Under these cireumstances the pelvis may be distorted in its relation to the healthy side, so that a twist of the innominate bone on the side of the infection, whereby the greatest width is brought into riew, will give an apparent atrophy of the os pubis and of the ischium.

Atrophy of the size of the bone will be erident in the bones adjacent to the larger joints. The question has come up repeatedly whether atrophy of size is due to a speeifie disease or to disuse. Experimentally, Dr. A. T. Legg elaims that atrophy of size is not due to disease but to disuse. However, the fact is recognized that the osseous and muscular systems of individuals suffering from diseases such as general tuberculosis are not able to withstand immobilization as well as healthy individuals. It is reasonable therefore to sup- 
pose that a person suffering from tubereulosis, and for some reason treated by immobilization of a given part, would have more atrophy of the size of the bone than would be evident in an individual who was perfectly well having the same amount of immobilization.

The first change that takes place with immobilization either for a fracture or experimentally is the marked re-arrangement of the structure of the bone, so that the bone becomes apparently porous, the interspaces between the trabecule being demonstrated more elearly. Under these conditions the trabeeula stand out a little more definitely without any apparent ehange in size. This appearance is typical of the atrophy seen in a foot where there is a Pott's fracture, or in a fracture where immobilization has been used. In a healthy individual this appearance will progress more or less indefinitely. In a person suffering from some constitutional disease, or infection, where the nutrition of the given part is disturbed or has not the equivalent of a healthy individual's blood supply, there is on the contrary a change in the natural size of the bone with a marked absorption of the strueture of the bone.

Where, for any reason, there is an increased supply of blood to a given part, and the case becomes more or less ehronic, it is demonstrated that the part enlarges or hypertrophies in comparison with the healthy and unaffeeted side. This is seen in chronic osteomyelitis and in chronic synovitis.

We ean have also a combination of both atrophy of quality and atrophy of size in which there is a decrease of the size of the bone with an absorption of the lime salts. This is seen in the chronic forms of arthritis, as well as in tuberculosis of the bone or in general tuberculosis. It is also seen in paralytic conditions, such as poliomyelitis anterior, and in some of the malignant osteomata, where spontaneous fracture takes place usually near the origin of the nutrient artery. This fracture usually extends in both directions, 
causing diminution in the size of the bone, and actual absorption of the bone substance without any tendency towards the formation of a callus.

Tubercular Dactylitis.- This is a condition in which there is a fusiform dactylitis of the fingers or toes, which may attack one or more bones in one or both hands. It is characterized in the early stage by a beginning destruction about a focus in the interior of the bone and sometimes has the appearance of a cystic formation of the bone.

In a later stage of the disease, destruction, or more definitely, absorption of the whole interior of the medullary canal of the bone takes place with a re-arrangement of the structure of the bone. This at times looks as if it were one big cyst or multiple cysts, and the bone is larger, and usually has a very thin cortex if any.

The adjacent joints are not usually affected. This condition goes on to thickening, enlarging of the phalanx, and sometimes sequestrum in its interior. Suppuration may occur with discharge of one or more sequestra, leaving a permanent deformity, usually a shortening of the affected finger. Commonly, however, healing takes place without much change in the growth.

Metatarsal Bone.-Plate 242, Fig. 1, represents a destruction of eortical bone and a slight eavity in the fourth metatarsal bone in a girl twelve years old. The lesions suggest a tubereular process, or possibly a benign eyst of the bone.

Tubercular Dactylitis.-Fig. 2 represents tubercular dactylitis in a boy; age, three years; parents healthy. There was no tuberculosis in the family history. The nurse was probably tubereular. The child was well until five months old, when a swelling appeared in the upper part of the tibia, just below the patella; at this time also a swelling of the first phalanx of the middle finger of the right hand and the second phalanx of the middle finger of the left hand appeared. 
Through a misapprehension of the serious nature of the case by the physician in charge (who was inclined to make the diagnosis of syphilis on account of the dactylitis) nothing especial was done, except to live at a sanatorium until January, 1907, when a Roentgenograph was taken. The true lesions were, therefore, not recognized for some time, and in March, 1907, a sceondary infection of the dorsal vertebrie took place.

The plate shows a distinct area of destruction of the bone in the middle phalanx of the middle finger of the right hand, without any evidence of proliferation of the periosteum. The first phalanx of the middle finger in the left hand shows the result of a previous destructive process, but at the time when the Roentgenograph was taken there was new formation of the bone.

Atrophy from Disuse. - Plate 243 represents the knces of a child twelve years old. A clinical diagnosis of tuberculosis of the knec was made in this case, and it was treated as such for two years. A series of Roentgenographs of this case were then taken at intervals, and at no time was any change noticed in the bones which would suggest tuberculosis. On the contrary, the marked atrophy of the bone and its lessened radiability pointed more to disuse than to disease. That is, it was an atrophy of the quality of the bone substance rather than the atrophy of size which is characteristic of tubcrculosis. It was the treatment with a stiff plaster bandage which had produced this condition.

Tubercular Dactylitis.-Plate 244 represents a tubercular dactylitis occurring in a child two and a half years old. It shows the enlargement of the first phalanx of the second and fourth fingers. It will be scen that there is a marked change in the structure of the bone, also giving it the appearance of a cystic formation, there being considerable absorption. It is to be noted that there is very little involvement of the periosteum, apparently none. The other boncs of the hand show in certain areas an osteoporosis. 
Tubercular Dactylitis.-Plate 245 represents a case of tubercular dactylitis The soft parts around the infected areas are seen to be markedly thickened, the fingers having the spindle-shaped appearancc. The first and third metacarpal bones are affected, and there is marked disturbance of the first phalanx of the fourth finger, with apparently an almost cystic formation. It is to be noted that there is very little periosteal reaction. In this way it differs markedly from syphilitic dactylitis.

Ulna: Necrosis.-Plate 246 shows an area of necrosis of the bone in the lower end of the ulna with a small formation of sequestrum. There is also some atrophy of quality.

Carpal Bones. - Plate 247 represents a tubercular condition of the carpal bones of the hands of a child five years old. In the left hand (1) it is to be noted that the soft parts are greatly thickened and that there is destruction in some of the carpal bones. This process was undoubtedly due to a tubercular infection. The right hand (2) is normal.

Elbou-joint.-Plate 248 represents a tubercular process in the elbow-joint of a child eight years old. The soft parts show a thickening and haziness. The lower end of the humerus shows some periosteal reaction, although the contour is regular. The ulna on the outer edge of the olecranon shows an area of increased radiability, due to absorption of the lime salts and to the gradual extension of the tubercular process. The periosteum of the ulna is somewhat thickened and the radius shows some decrease in its lime salts.

Hip-joint: Mixed Infection.-Plate 249 represents a tubercular hip-joint caused by a mixed infection in a child five years old. The density of the soft parts around the left hip is markedly increased. It will be seen that the shaft of the femur shows a decreased radiability, due to absorption of the substance of the bone. 
The periosteum along the iliopectineal line is greatly thickened. This periosteal thickening is probably not due to the bacilus of tuberele, but to marked reaction, indicating probably a mixed infection.

Acetabulum.-Plate 250 represents a boy twelve years old showing marked disease of the whole acetabulum and a mottled appearance of a decreased radiability. The head of the femur has practically disappeared and there is a general involvement of the whole neek and greater trochanter, as is also shown by the mottled appearance. There is practically no tissue reaction to be scen in this plate. The shaft of the femur is negative.

Hip: Mixed Infection.--Plate 251 shows a mixed infection of the hip with great involvement of the whole head of the bone and destruction of the upper epiphysis of the femur.

Hip. - Plate 252 represents the result of an old tubereular process in the hip of a child fourteen years old. The dotted line shows where the head of the femur would naturally be. The acetabulum is practically destroyed by the disease. There is no regular outline, but the process can be seen to be inactive. The femur is seen to be ossified to the ilium just above the acetabulum and there is atrophy in the region of the greater trochanter. The greater trochanter, however, is not to be seen.

Acetabulum and Fcmur: Necrosis. - Plate 253 represents a tubercular condition of the diaphysis of the femur in a boy eight years old. The picture shows a focus of the diaphysis of the right femur marked as an area of increased radiability, clue to absorption of the lime salts and to necrosis of the bone. This area is surrounded by a finely peneilled line denoting the formation of new bone, corresponding to nature's effort to wall off the discased area. The epiphysis also shows the presence of disease by its irregular articulating surface and the destruction of bone that has taken place. 
The outline of the acetabulum is also irregular, due to the same process.

Hip.-Plate 254 represents an infectious hip in a child five years old. In examining the two hip-joints in this case it will be seen in the first place that the soft parts of the left hip-joint have a markedly increased radiability. The outline of this increased area of density is clearly marked. There is also an area of infection in the neck of the right femur. It will be noted in comparing this femur with the femur on the other side that there is no atrophy of the shaft of the femur nor of the bones of the pelvis. It will be seen that the epiphyseal line of the left femur shows lessened radiability. The epiphysis on the left will be seen to have an increased radiability as compared with the right side. The dotted lines on the left show diagrammatically the arrangement of the joint-capsules made by the anterior and the posterior insertions of the capsule. The dotted lines on the right represent the capsule distended with pus. On an earlier examination the disease was noted outside of the eapsule, but when this picture was taken it had extended into the capsule.

Hip-joint.-Plate 255 represents the pieture of tuberculosis of the hip-joint in a girl eight years old. There is a marked density of the soft parts around the left hip-joint. There is atrophy of both size and quality of the shaft of the left femur. There is partial absorption of the neck and head of the femur and marked involvement is shown by the increased radiability and destruetion of outline of the acetabulum. There is also atrophy of all the bones of the pelvis on this side as compared with the other.

Knee-joint.-Plate 256 shows an early tuberculosis of the knee-joint of a child five years old. There is increased density about the knee-joint, which shows thickening of the soft parts and capsule. There is a roughening of the epiphysis of the femur with 
as yet no destruction of bone. There is, however, a slight amount of atrophy in the shafts.

Knee-joint. - Plate 257 represents a tuberculous knee-joint in a child eight years old. There is a marked thickening of the soft parts and of the capsule around the knee-joint. There is atrophy of quality as well as of size of the bones. The condyles are irregular in outline and there is an irregular development of the patella. The epiphysis of the femur is irregular in outline.

Thigh: Abscess.-Plate 258 shows an encapsulated abseess of the thigh in a boy six years old. The lateral view of the thigh shows a definitely encapsulated abscess in the anterior lower aspect. The tissues around the knee-joint are markedly thickened. The condyles and epiphysis of the femur are very irregular in outline, as is also the upper end of the tibia. There is atrophy of quality as well as of size of the femur and also of the tibia and fibula. There is no absolute destruction of bone.

Tibia, Epiphysis. - Plate 259 represents tubereulosis of the epiphysis of the tibia. The shaft of the tibia and fibula and the epiphyseal line of the tibia are perfectly normal. There will be seen a small focus on the posterior aspect of the epiphysis of the tibia. There is almost complete absorption of bone in this area. There is no formation of sequestrum. The bones of the foot are normal.

Os Calcis: Abscess.-Plate 260 represents tuberculosis of the os calcis in the foot of a child two and a half years old. On the dorsum of the foot is a well-defined abscess. The primary foeus is on the anterior aspect of the os calcis, where there is scen to be actual destruction of about one-half of the bone. The other bones of the foot show an osteoporosis.

Ankle and Foot: Abscess.-Plate 261 shows in the ankle-joint of a girl four years old a definitely walled abscess consisting either of fluid or thickened tissue. The lower end of the tibia shows marked 
absorption of lime salts and increased radiability. The epiphysis of the tibia and the astragalus show marked destruction of the structure of the bone. They are apparently within the cavity of the abscess. The metatarsal bones show the same absorption of lime salts as does the lower end of the tibia. There is an atrophy of size as well as of quality. The change in quality is shown in the os calcis by its finely pencilled outline. The case was probably tubercular.

Ankle: Tuberculosis.-Plate 262 shows a tubercular ankle in a child five years old. The soft tissues surrounding the ankle are greatly thickened and infiltrated. There is a slight amount of increased radiability of the tibia as well as of the os calcis. The epiphysis of the tibia shows an actual destruction in its lower end and an involvement of the astragalus on its articulating surface.

Astragalus and Os Calcis: Tuberculosis.-Plate 263 represents a tuberculosis of the astragalus and of the os calcis in a boy twelve years old. There is a thickening of the soft parts around the ankle and an area of actual destruction of the posterior-inferior portion of the astragalus and of the superior border of the os calcis. There has been both destruction and absorption. The bones of the foot show a finely pencilled outline, as does the lower end of the tibia, representing atrophy in quality in these parts, although there is no actual involvement.

\section{NON-TUBERCULAR INFECTIONS}

In the acute pyogenic infections within the joint we have classical symptoms of acute infection of the joint, acute onset, rapid distention of the joint, much pain, and constitutional disturbance.

The subacute or chronic cases of intra-articular pyogenic infection may differ in no respect clinically from tuberculosis in its early stages, but will clear up much earlier than tuberculosis, with possibly 
a perfect recovery of function. This type, however, may show an ultimate permanent lengthening from the irritation of the epiphyseal cartilage.

Little need be said of the early diagnosis of the acute pyogenic infections. exeept of its importanee in demanding operative interference.

In the chronic cases of pyogenic infection with symptoms identical with those of tuberenlosis of the hip, we should hesitate to make a differential cliagnosis until, after careful watching and repeated Roentgenographs, we could detect the beginning of a destruetive process demanding operative interference.

These particular cases of pyogenic infection resulting in acute and chronic osteomyelitis vary in no detail as to our conception of osteomyelitis in its pathologic proeess. Aceording to Legg and Gorge it is apparent that these cases are very commonly confused with tubereulosis, but if recognized the subsequent early treatment will differ radically from that of tuberculosis. The ultimate result as to early recovery and as to the function of the part will be, however, rastly better.

In order to recognize this class of eases early we must use all the means at our disposal and make a most careful clinical examination; the Roentgen ray, however, plays the most important part ii the diagnosis:

1. In the location of the pathologic process.

2. In the early recognition of the extent of the pathologic process.

1. Location. - An infection by a pyogenic organism, though it may occur in the epiphysis, is most commonly found in the diaphysis, or shaft of the bone, and it will usually be outside of the insertion of the joint-capsule and within the boundaries of the periosteum. Statisties, however, do not altogether agree with this, 
as Becker states that he has found the ratio of infection in the epiphyseal line to be $1: 4$ of that of infection along the shaft. Here, again, we have an illustration of how a similar location of the focus of a non-tubercular disease may give almost identical clinical signs with tuberculosis. But a pyogenic infection may occur along the diaphyseal side of the epiphyseal line, in which case, depending on the virulence of the organism, the cartilage acts more or less successfully as a barrier against the complete involvement of the epiphysis or joint.

It is generally accepted that infection of bone by the bacillus of tubercle occurs in the epiphysis in children witl rare exceptions, and that these are either when the child is under an age in which a given bone has no ossific centre, or by direct infection from a focus in the epiphysis.

2. The Roentgen ray shows:

(1) Apparent infection of the periosteum.

(2) Apparent infection of the marrow.

Pcriosteum.-In acute infections by pyogenic organisms outside of the joint-capsule the infection may start either with direct infection of the periosteum, so that we may have the appearance of a definite thickening of the periosteum, or with proliferation, or as a broken or ragged periosteal outline.

Marrow.--In infections of the marrow we see in the Roentgenographs a disturbance of the normal densities of the bone, and an area, or areas, varying in size from the smallest possible point to half an inch or an inch in diameter. We find that the light easily penetrates these points, and we have then the so-called "punchedout" appearance, or area of increased radiability.

In the chronic conditions produced by these pyogenic infections we see definite areas of actual destruction of the bone with or without periosteal reaction, and usually with proliferation of the perios- 
teum. This depends to a certain extent on the proximity to the joint-capsule. A formation of sequestrum may or may not take place. Commonly the bone is thickened and the periosteum is proliferated to a great extent, forming an involucrum about the shaft with an area of rarefaction and with sequestrated pieces of bone in the centre of these areas. Sometimes the bone becomes very dense and only here and there by careful examination can areas of rarefaction be detected. Areas of suppuration surround the sequestrum.

Plate 264 is an example of non-tubercular infection manifested in a child five years old. This case represented a mistake in diagnosis. There were classical signs of tuberculosis of the hip, and the diagnosis was tuberculosis of the hip. The Roentgenograph showed a focus of disease in the neck of the femur, extending to the epiphyseal line, but not into the epiphysis. The lesion represented a low grade of infection, probably non-tubercular. Tuberculosis very seldom comes primarily in the shaft of a bone. 



\section{PLATE 157. \\ RETARDED DEVELOPMENT OF HAND. \\ Boy, age $S$ years. (Life size.)}

The only carpal bones present are the os magnum, unciform, and semilunar.

A. Points towards the very slightly developed lower epiphysis of the radius.

The epiphyses of the metacarpal bones and of the phalanges are absent. 
Pente

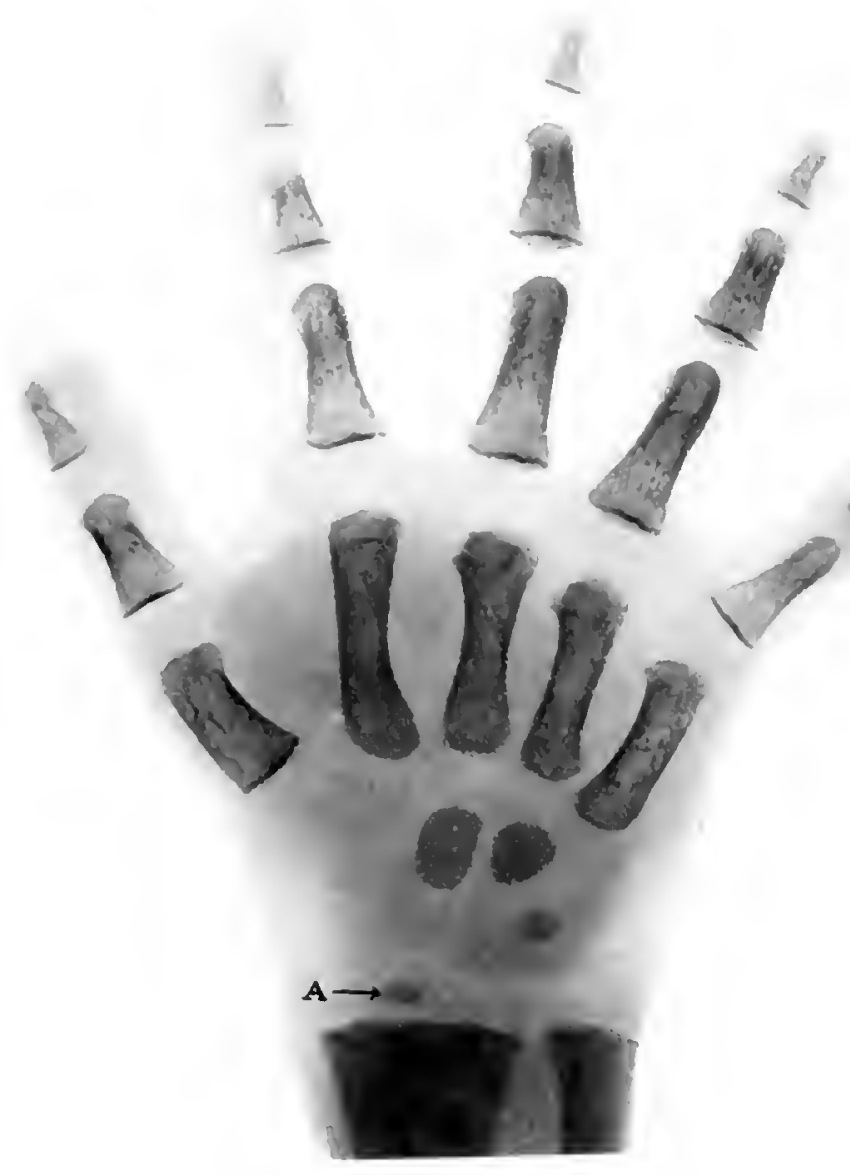


PHATE 1.S.

flee la year. (Lifes size.)

1. Premature osification of the lewer epholyeis of the radius.

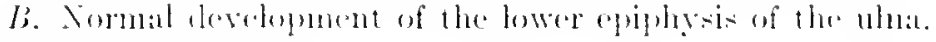

r. Serammind bone.

The other bons atre normal. 


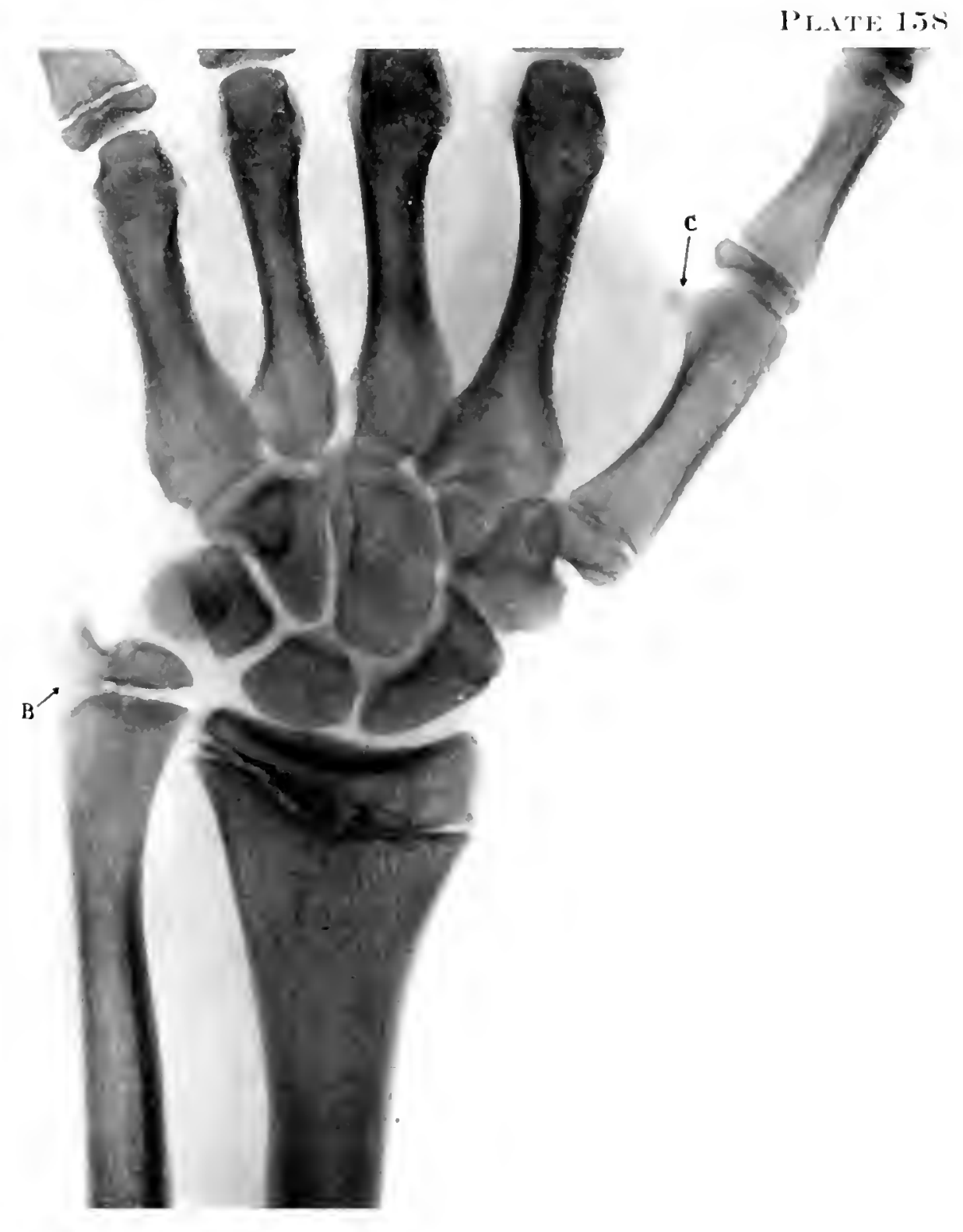


PI.ITE 1.,

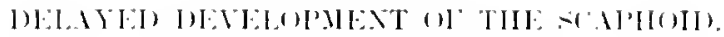

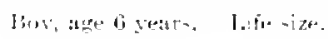

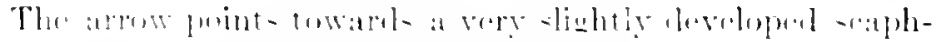

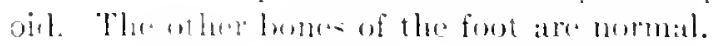


Pilte: 1.j9

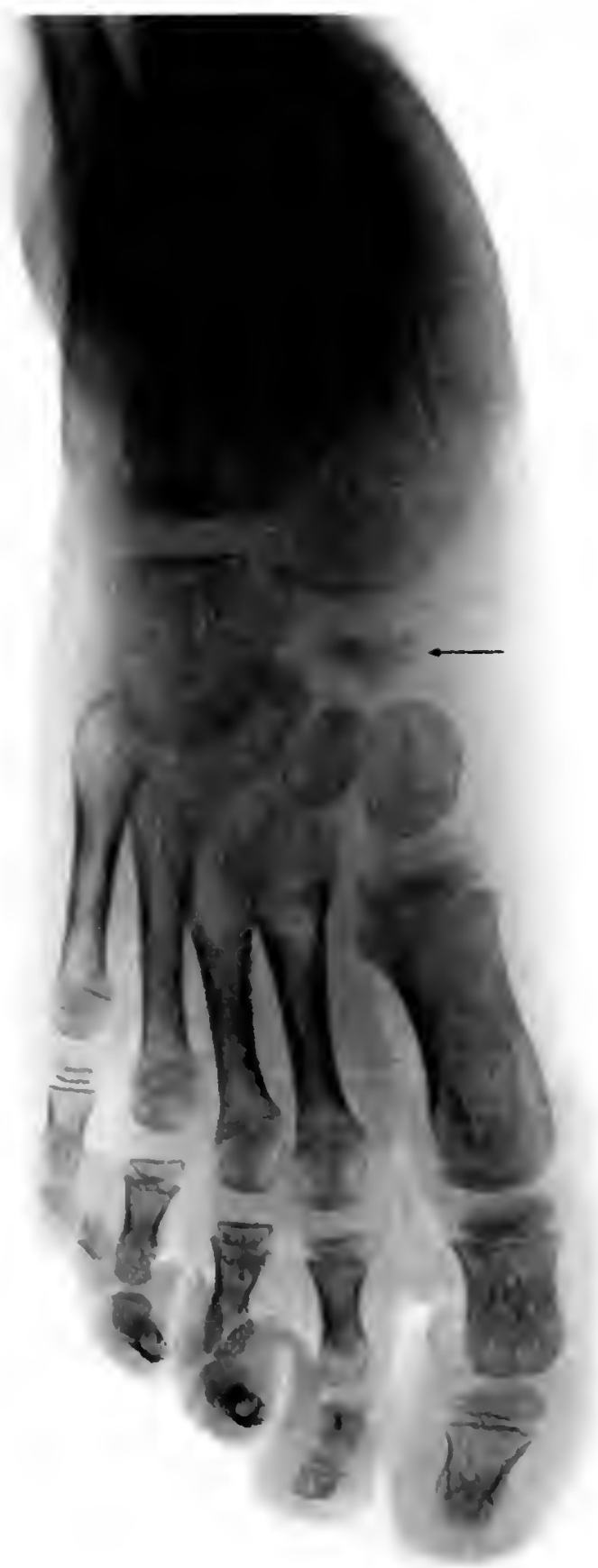




\section{V'I. IT': 160.}

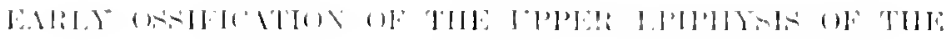
TIIST.

life size.)

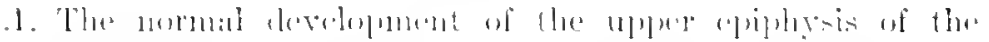
[i])ilat.

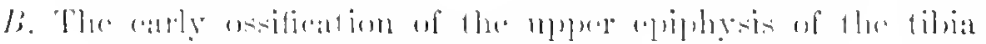

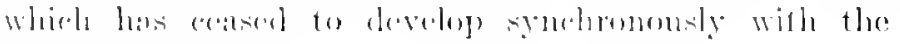
filıulal.

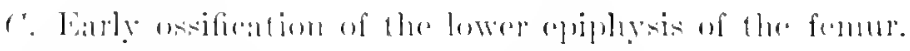

1). Musiale.

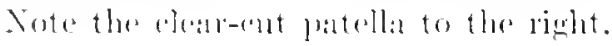


PLATE I60)

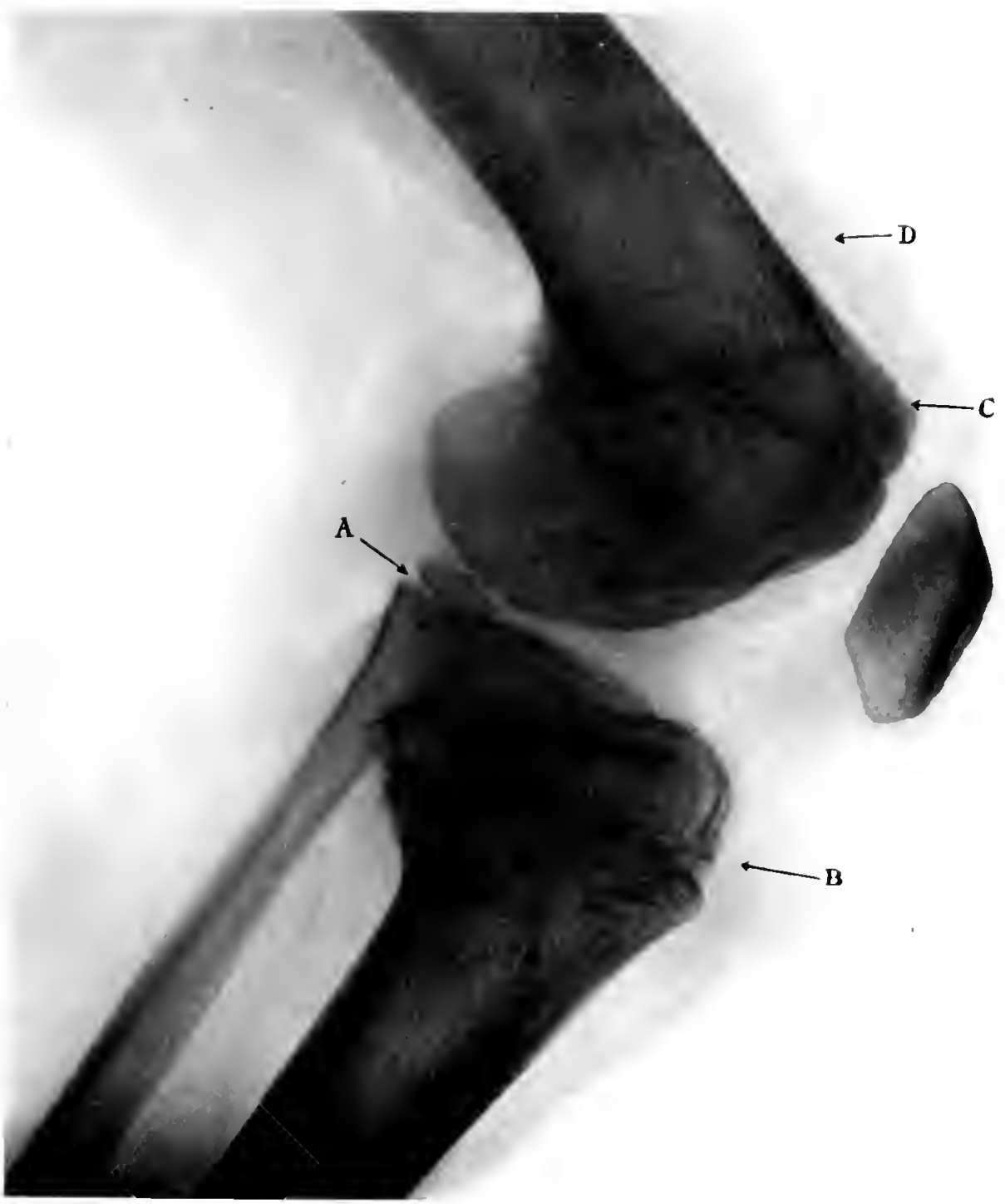


PLATE: 161.

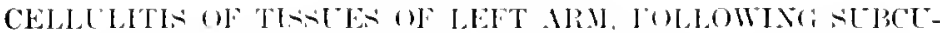
TANEOE IXIETTON.

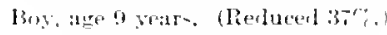

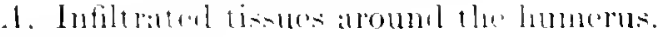

I). ('i),it.llum. 
Pente 16i

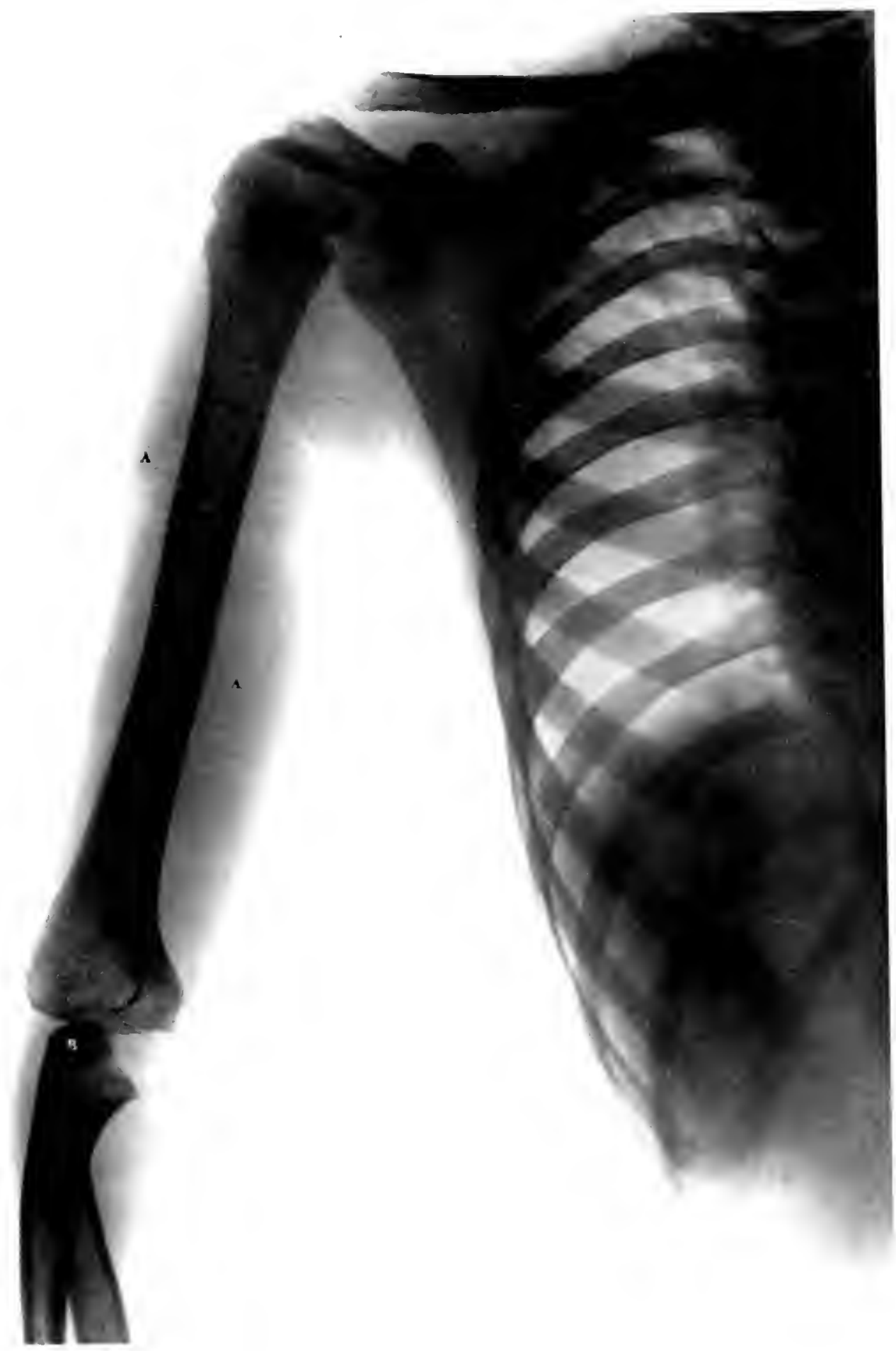


PI.XTE 11i:.

II.EYTT(M) , IIE IIEL.

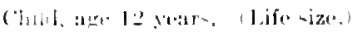

1. Infiltrated tis-mene of the laned.

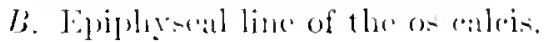


I'I ITE $16 \%$

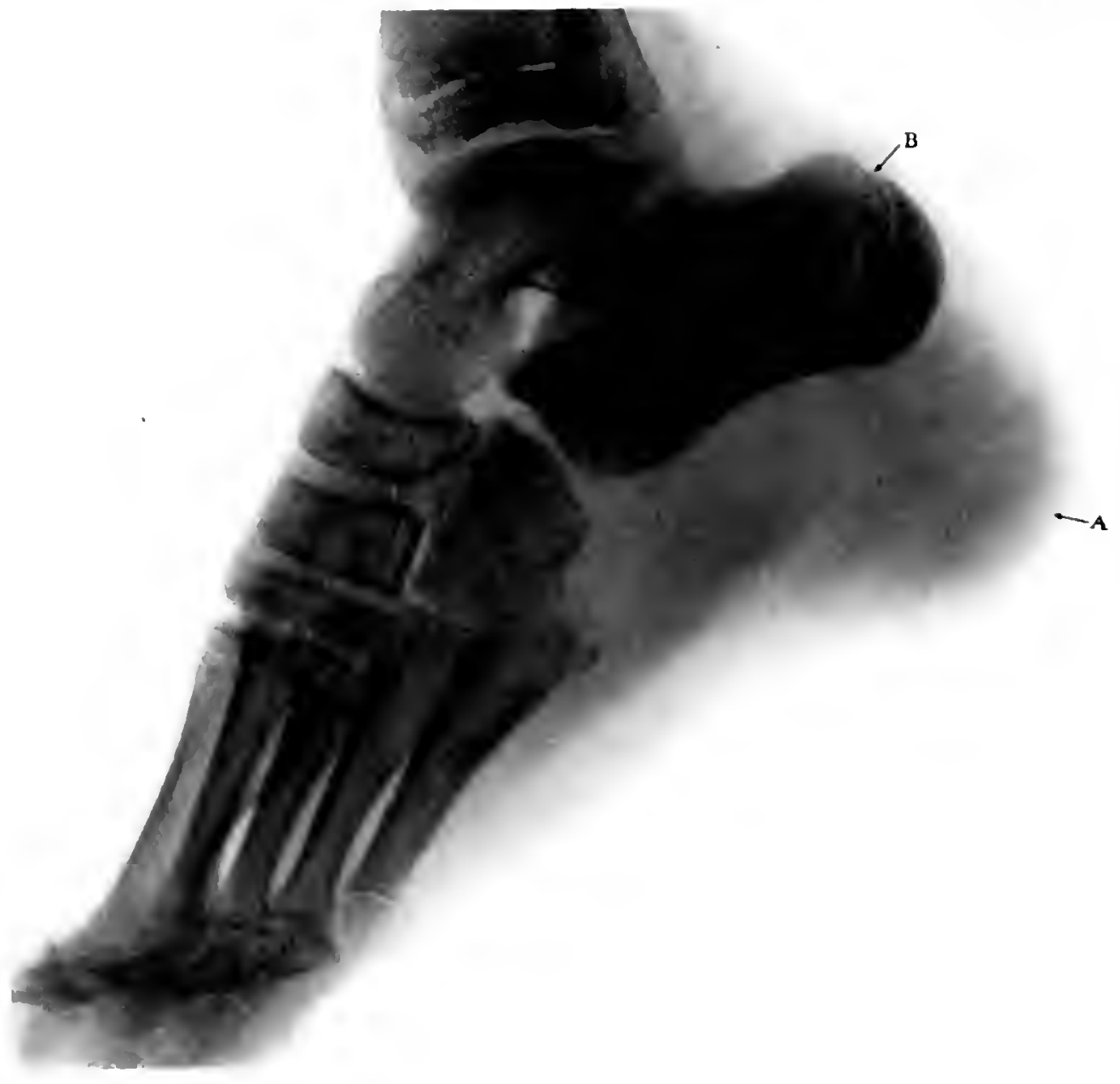


PJATE Li:?.

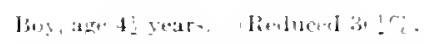

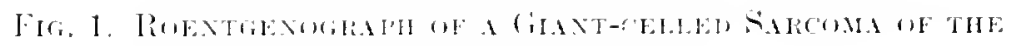

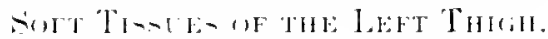

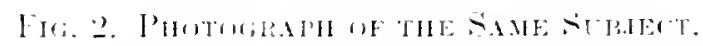

The alrow foint- towatris the sareomatous growth in the lisitu- of the thigh. 
FIG. 1.

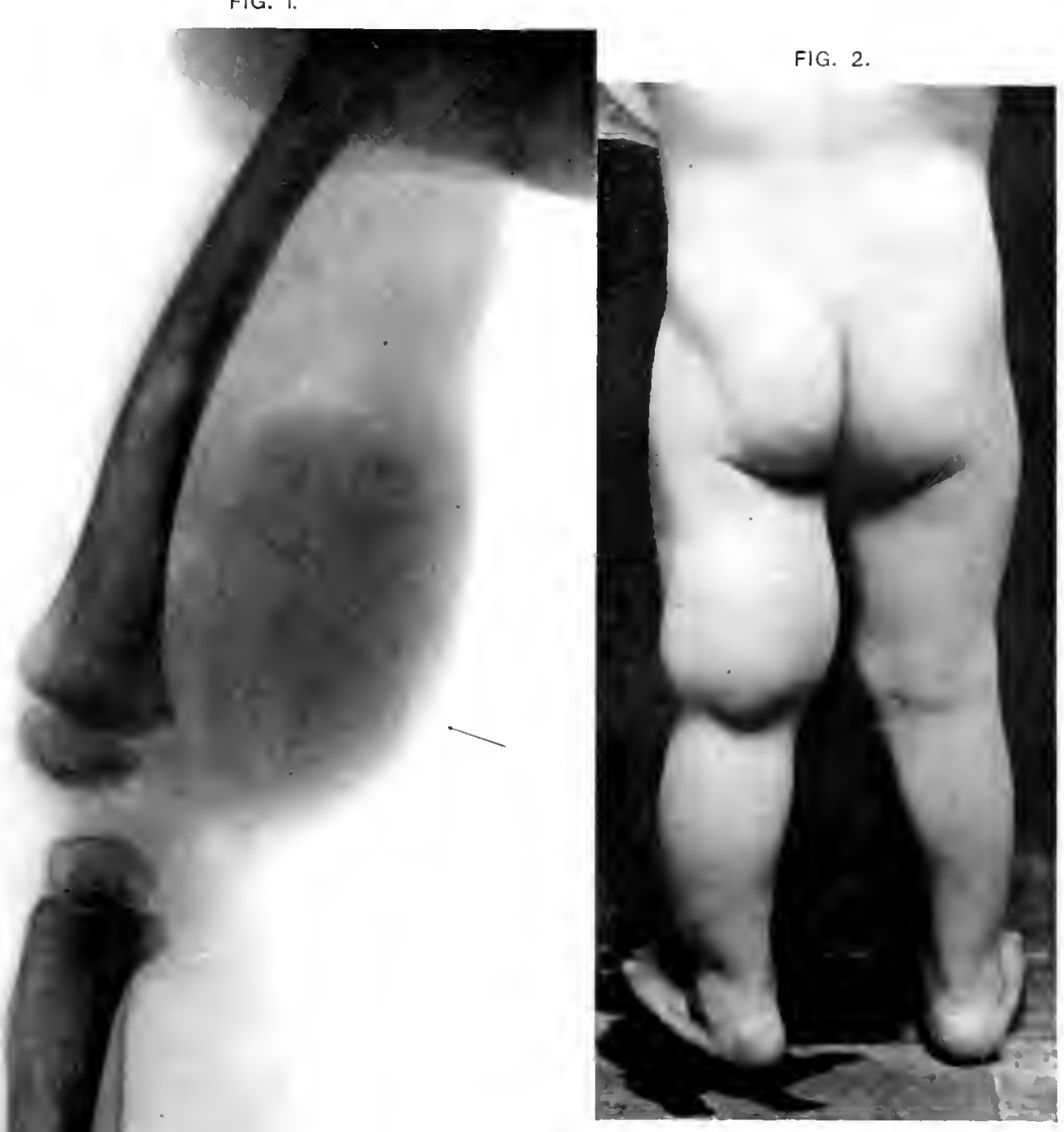


I'I.TTE 1tit.

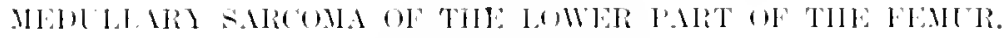

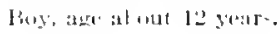


PLATE 16;

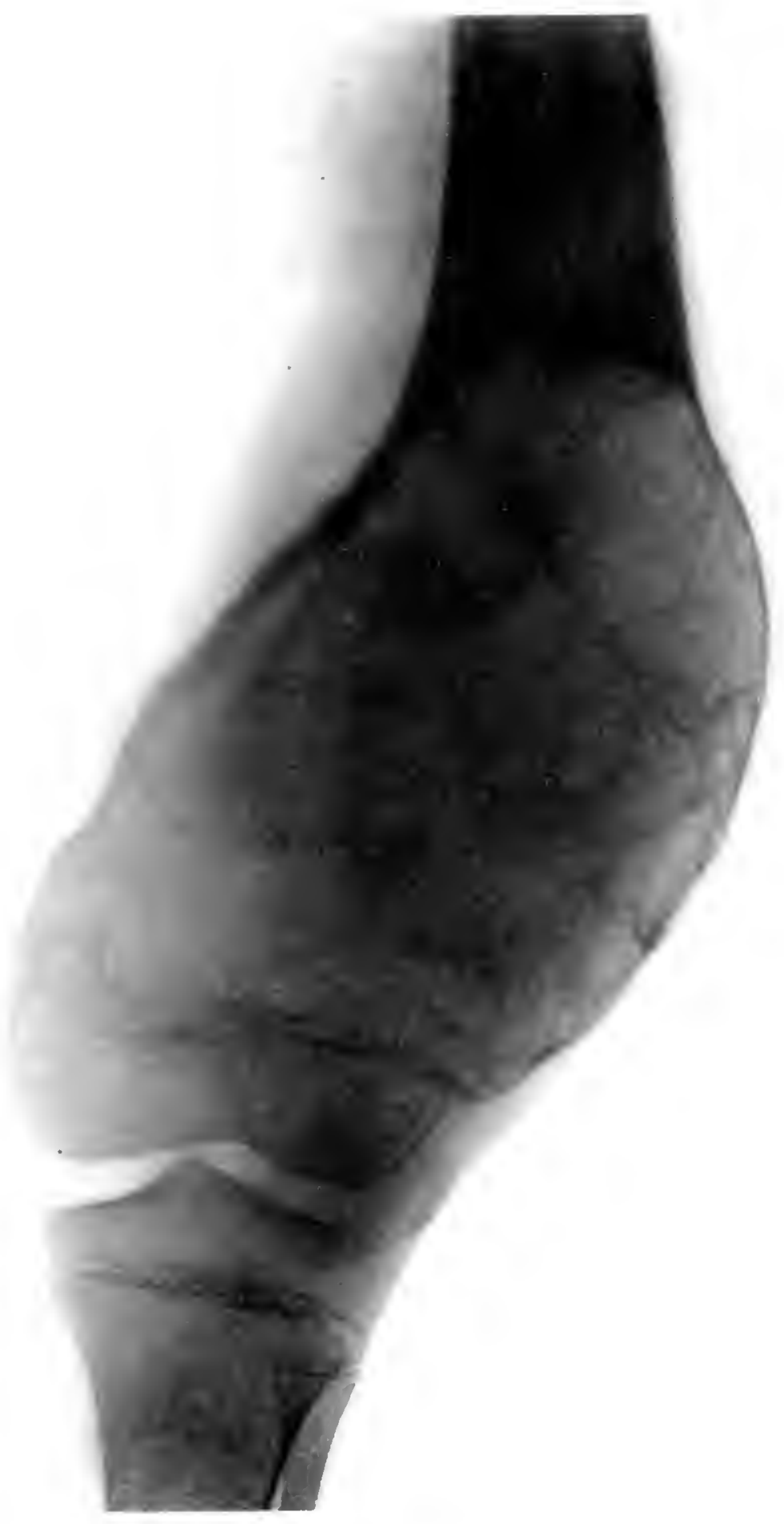


P'l. ITl: 16.5.

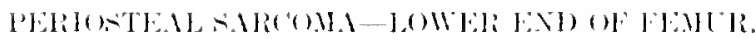

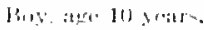

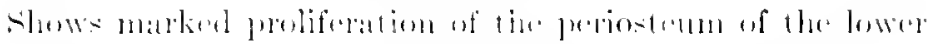

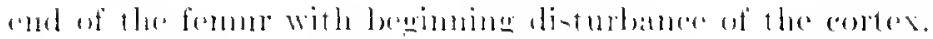


PI. ITE 1:5 
P'. ITE: 166.

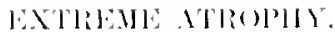

13,

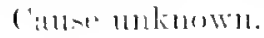


Puate 16G

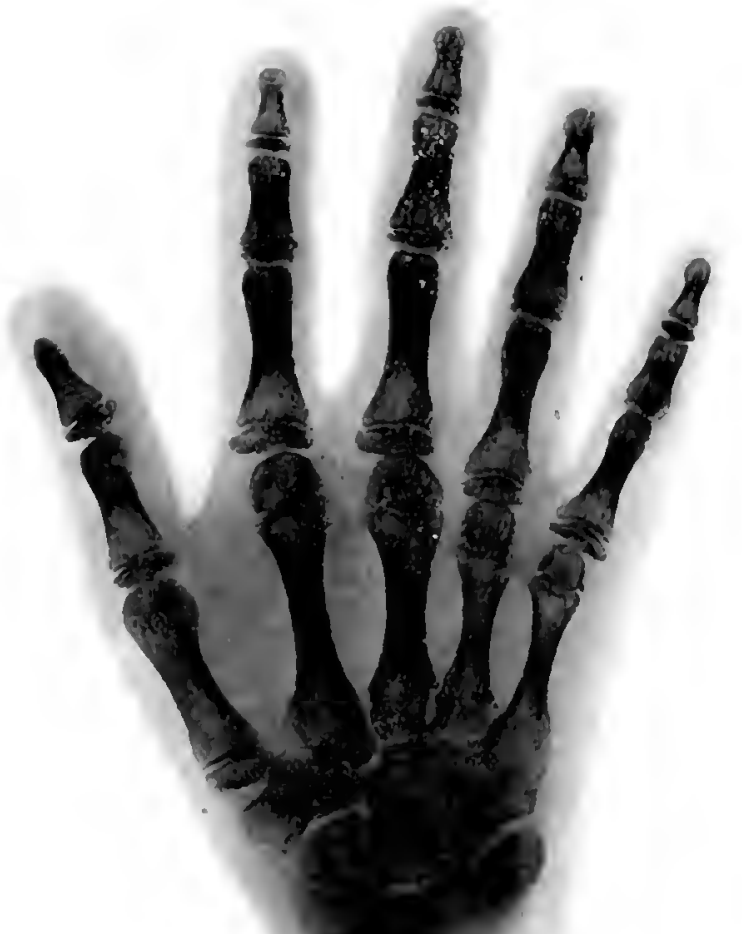


Pl.STE, 16is.

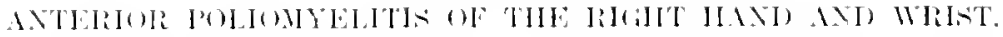

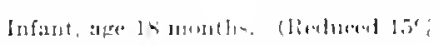

1. The left normal hatud and wrist.

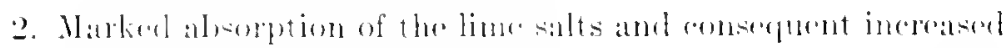
radialoility in the right hamt. 
PIATE 167
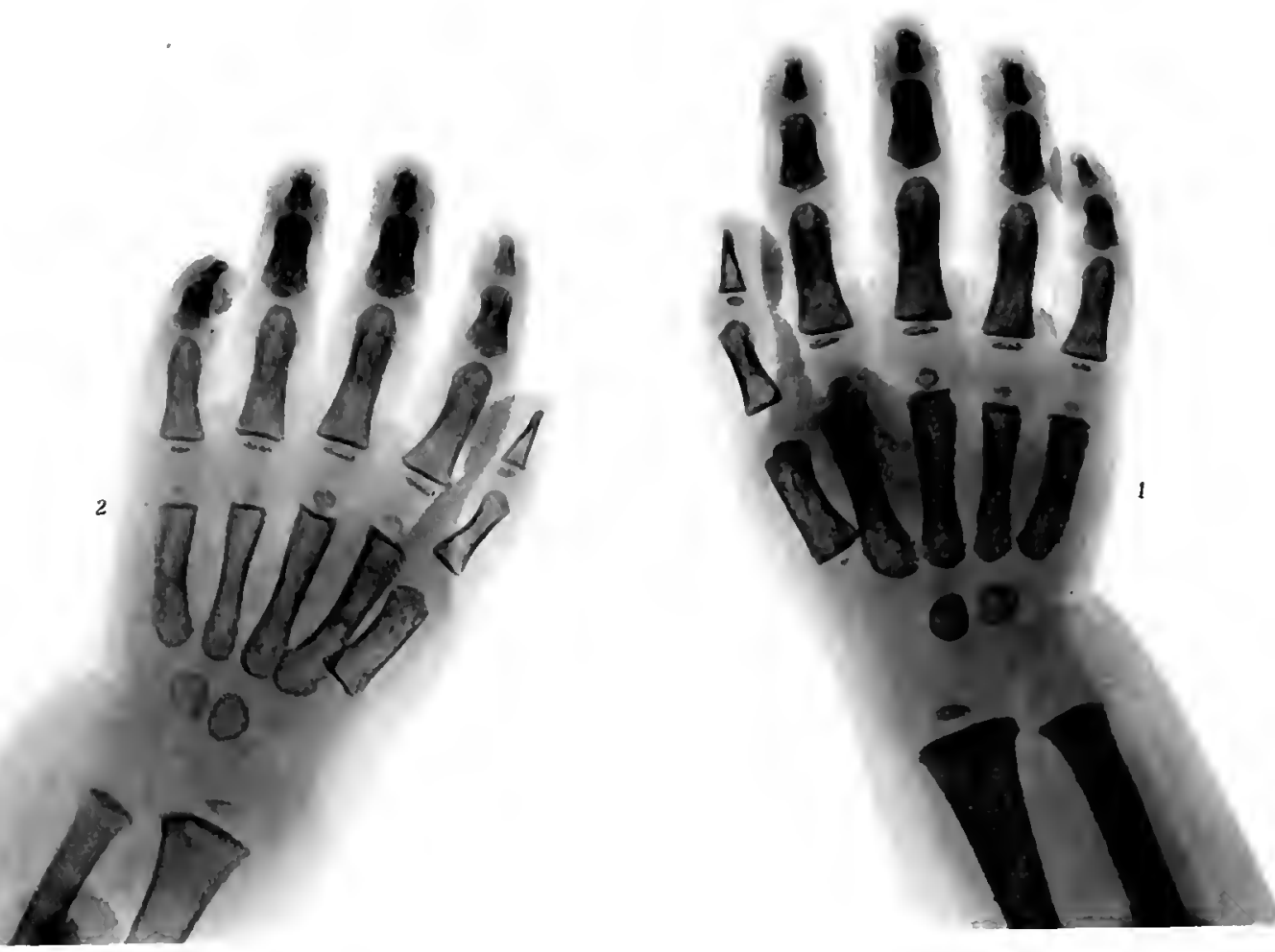
PLATT 16S.

ANTEIIOR POLOMYLLTIS OF TIE LFFT SHOTLDER.

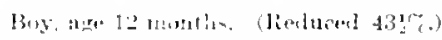

The arrow points towate the ill-dereloped opiphysis of the l.ft humerue, and hows the atrophy in size amel quality in comparison with the right arm. 
Phate $16 \mathrm{~S}$

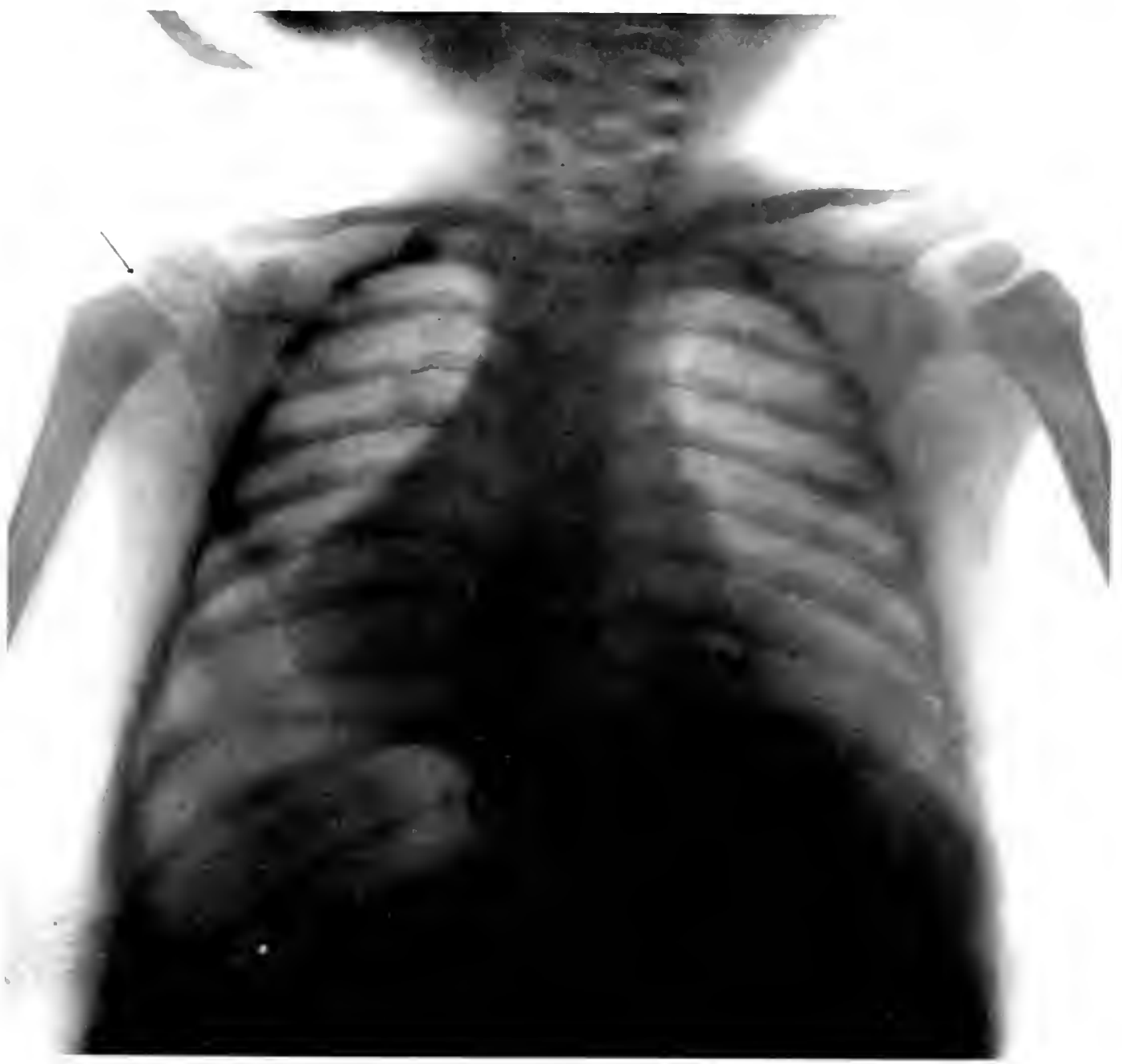


PLATE: 16!.

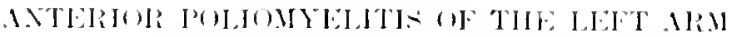

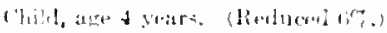

I)ecreaterel elemaity of all the homes.

The arrow peint to the atrophicerl musele. 
Plate 169

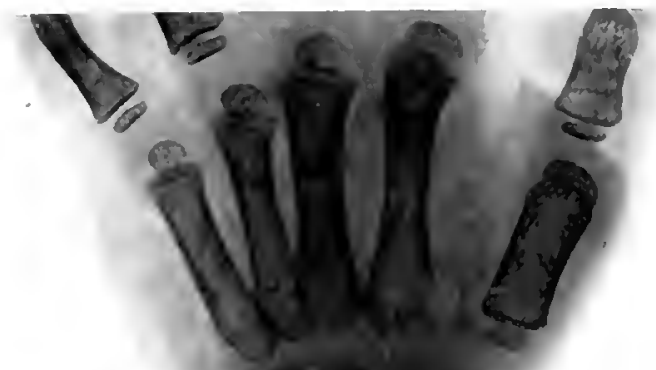


11.191: 170.

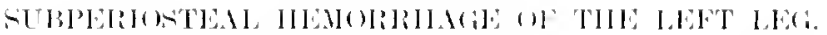

(lialuend s s l'; )

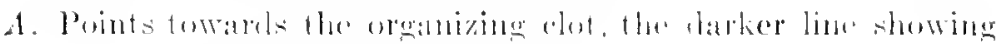

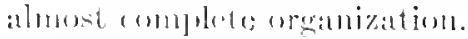


PuATe 170

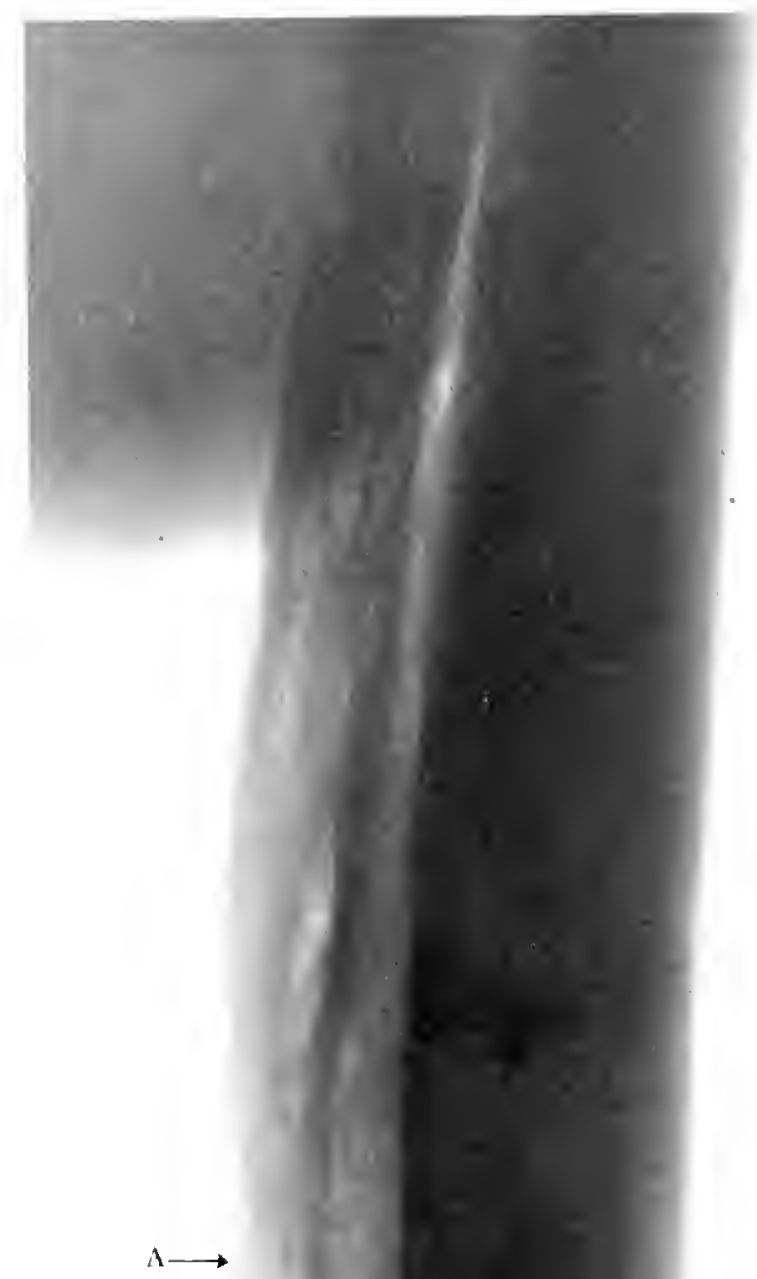


PI.TTL 171.

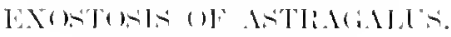

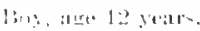

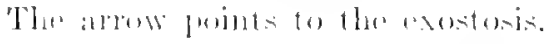


PIATE 171

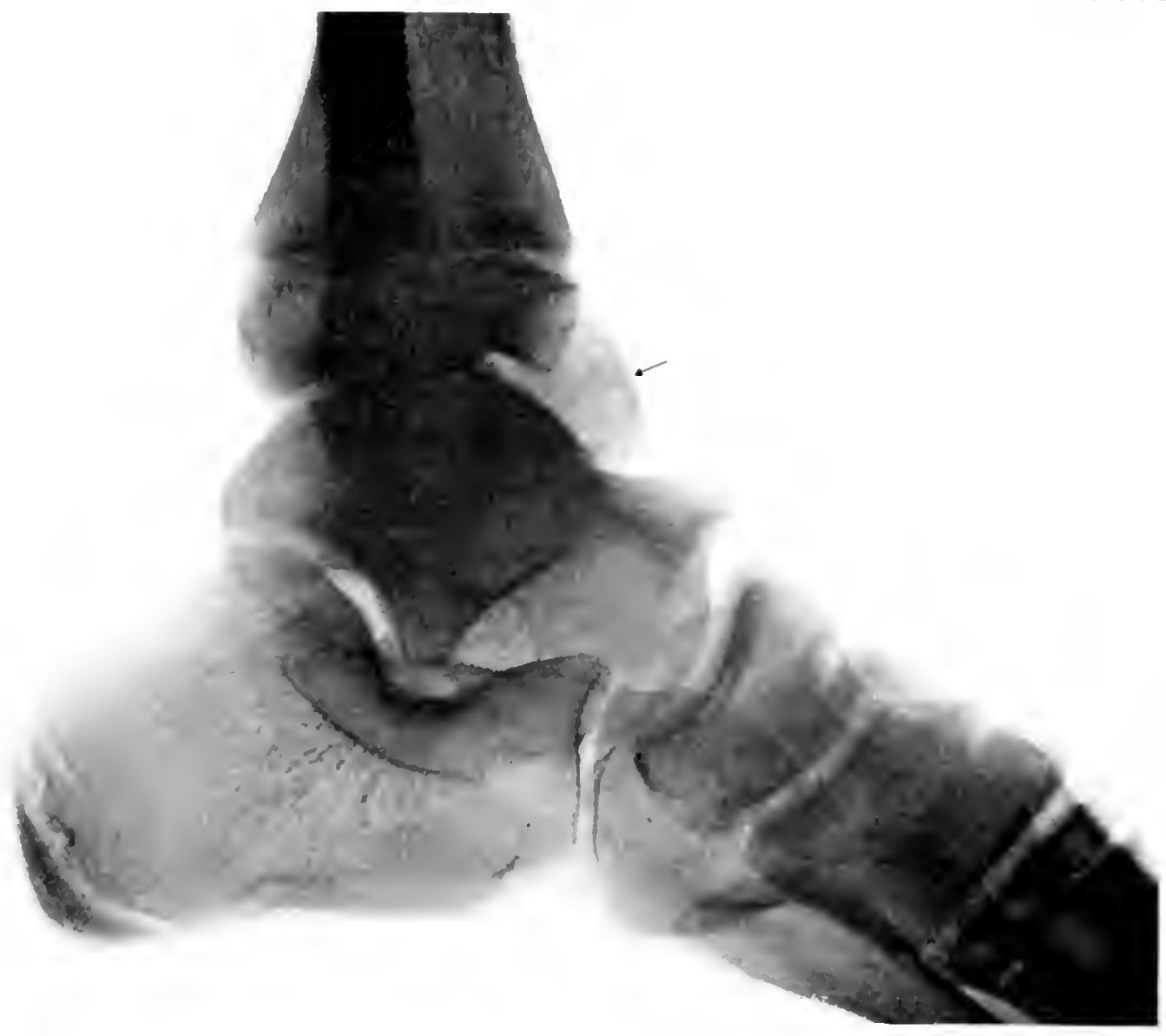


PLATE: 172.

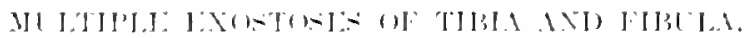

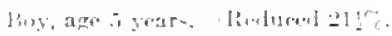

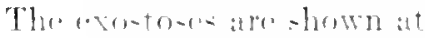

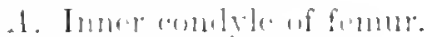

L). Onter liart of fitmlit.

c. Inwer patt of filmlat. 
PLATE 170
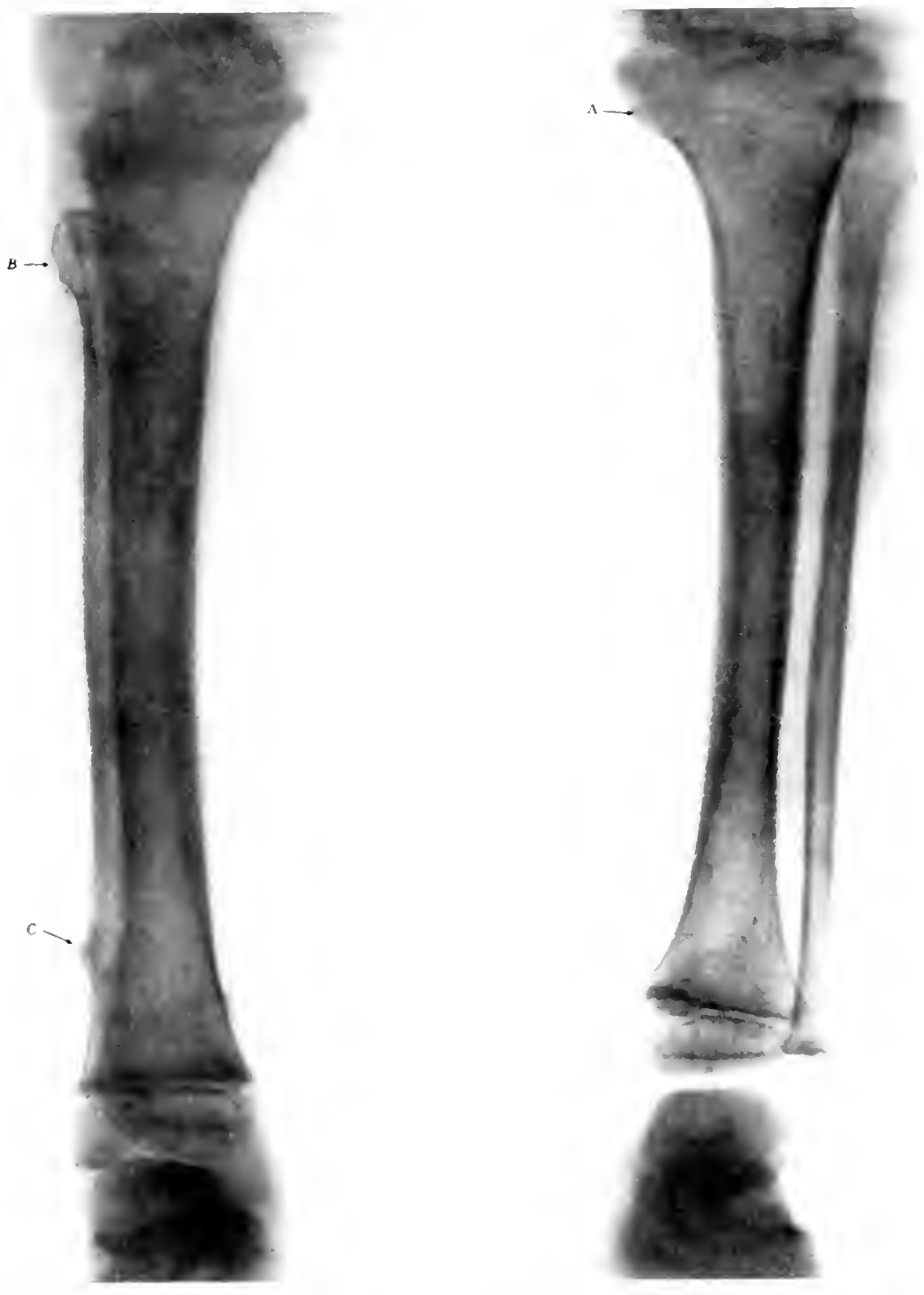
PISTI: 17:3.

\section{MULTIPLE EXONTOSL}

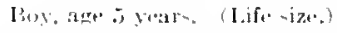

1. Point 40 exostose of the loweg part of the fomur.

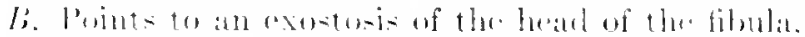

('. I'oints to an exo-tonis of the ulper patt of the tilia. 
PLATE $17: ;$

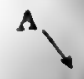

A

$\leftarrow A$

$$
\text { B }
$$


PIATE 17.

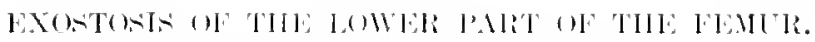

The arow jerints to the exostosis. 
PLATE 174

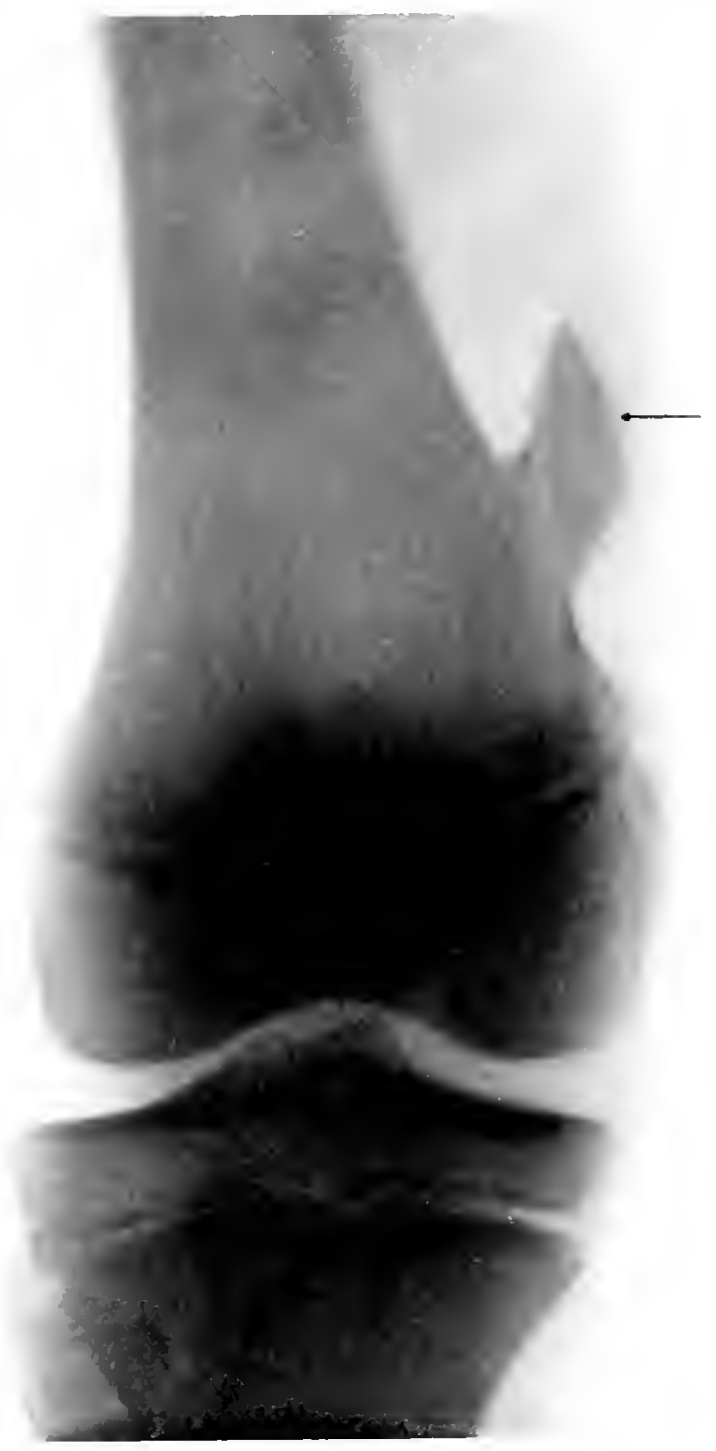


PI. IT1: 17.

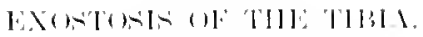

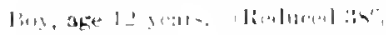

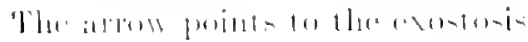


PJATE 17.5
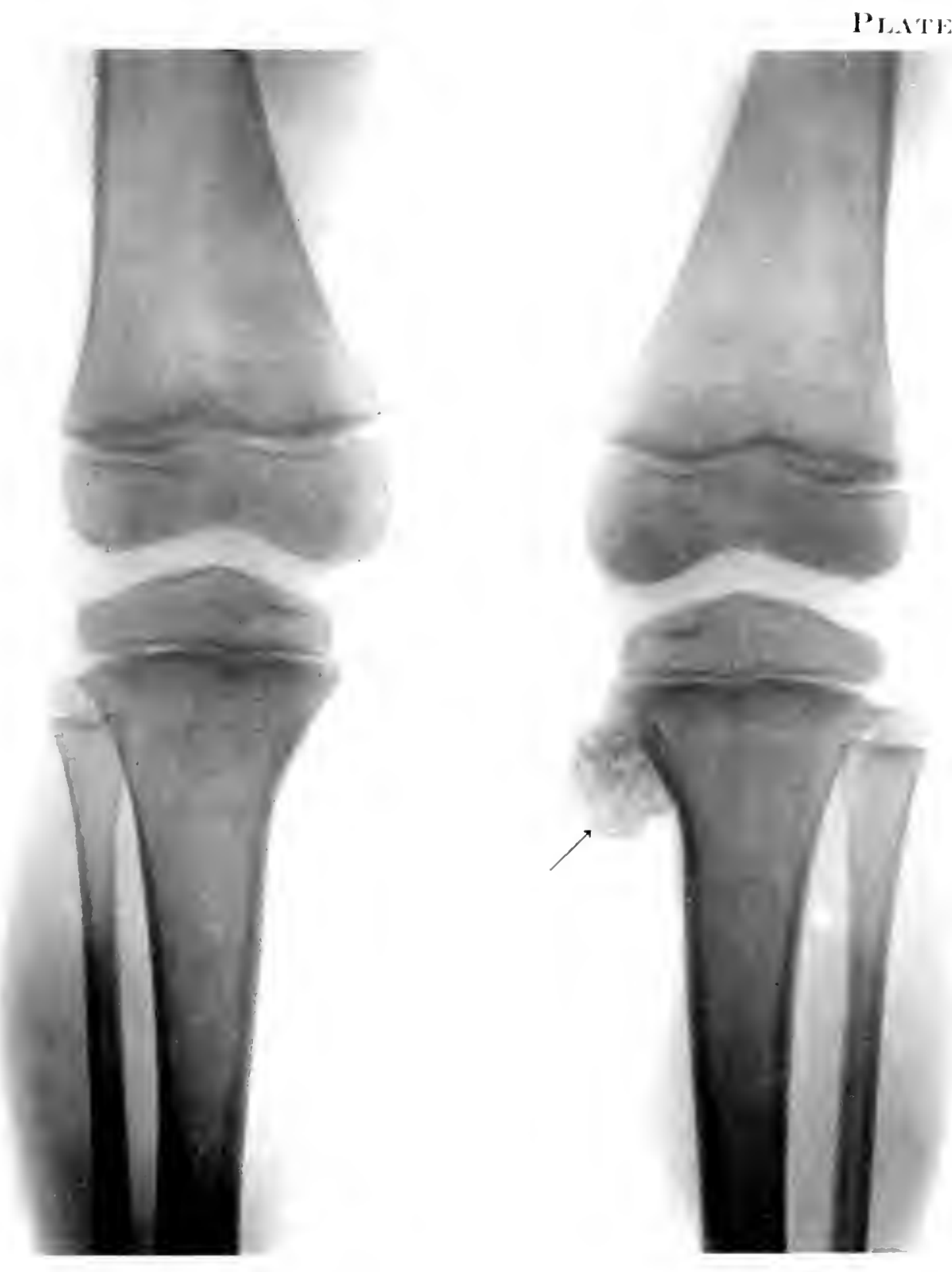
PI. TTI I76.

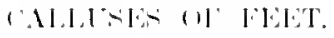

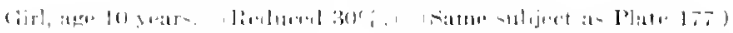

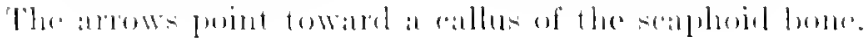


PIAI $17 \%$
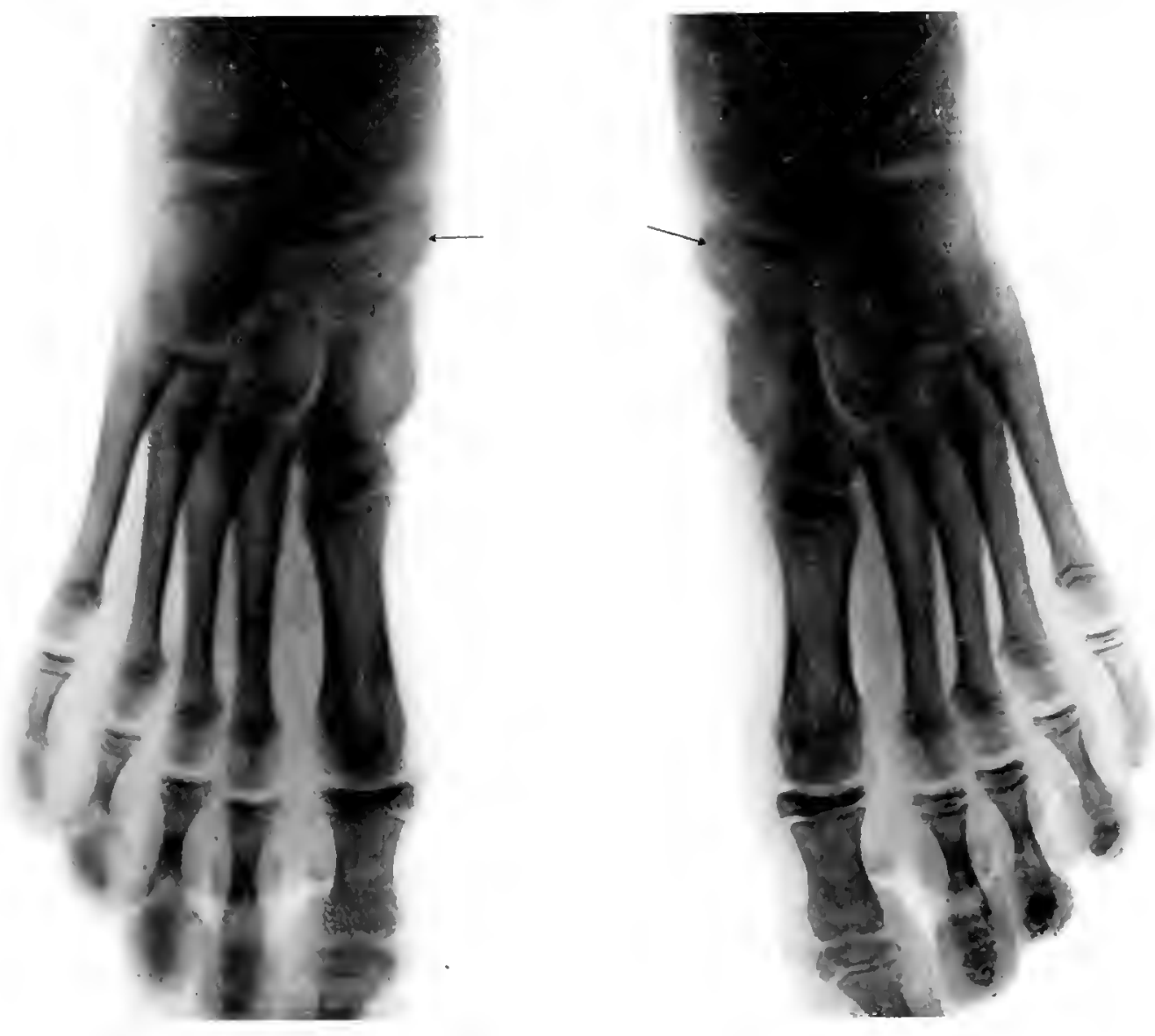
PI.ITE 17.

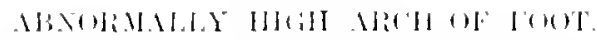

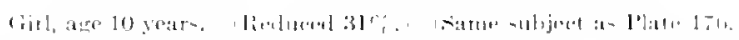

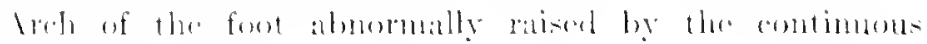
wearine of at bat plitte. 
PLATE 17\%

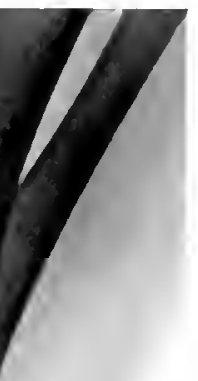




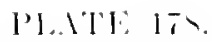

MOHER.ATE FHAT-FOMT.

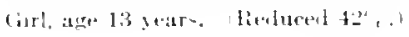


PUATE 1TS

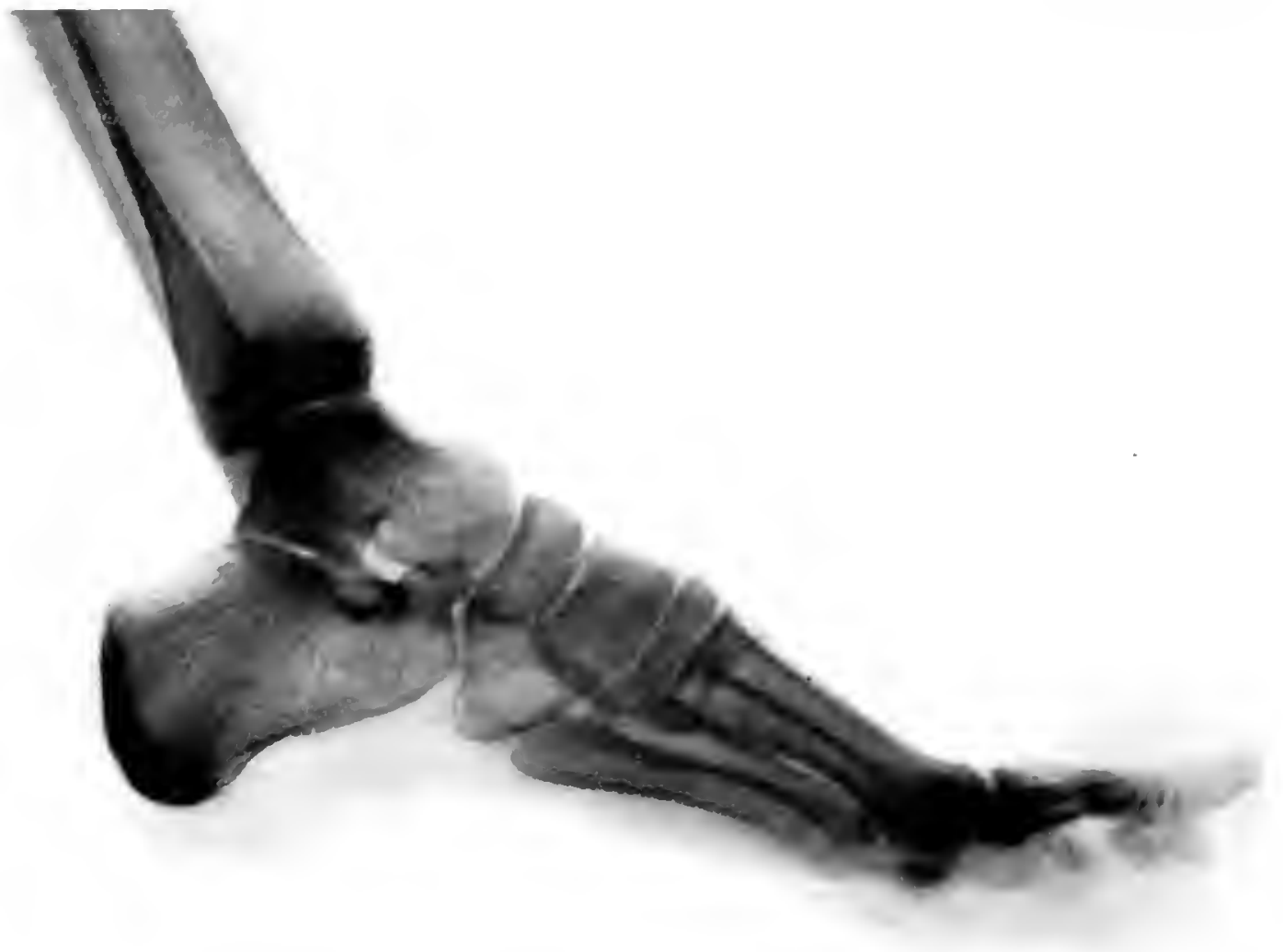


PI.STE $17 !$.

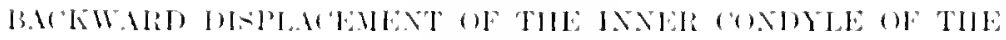
I'lillels.

How, aw" 12 yrar- (Lile siza.)

l. P'atrilla.

li. Di-platerel condylu.

('. binpleyeal line. 
PIATI: 17!

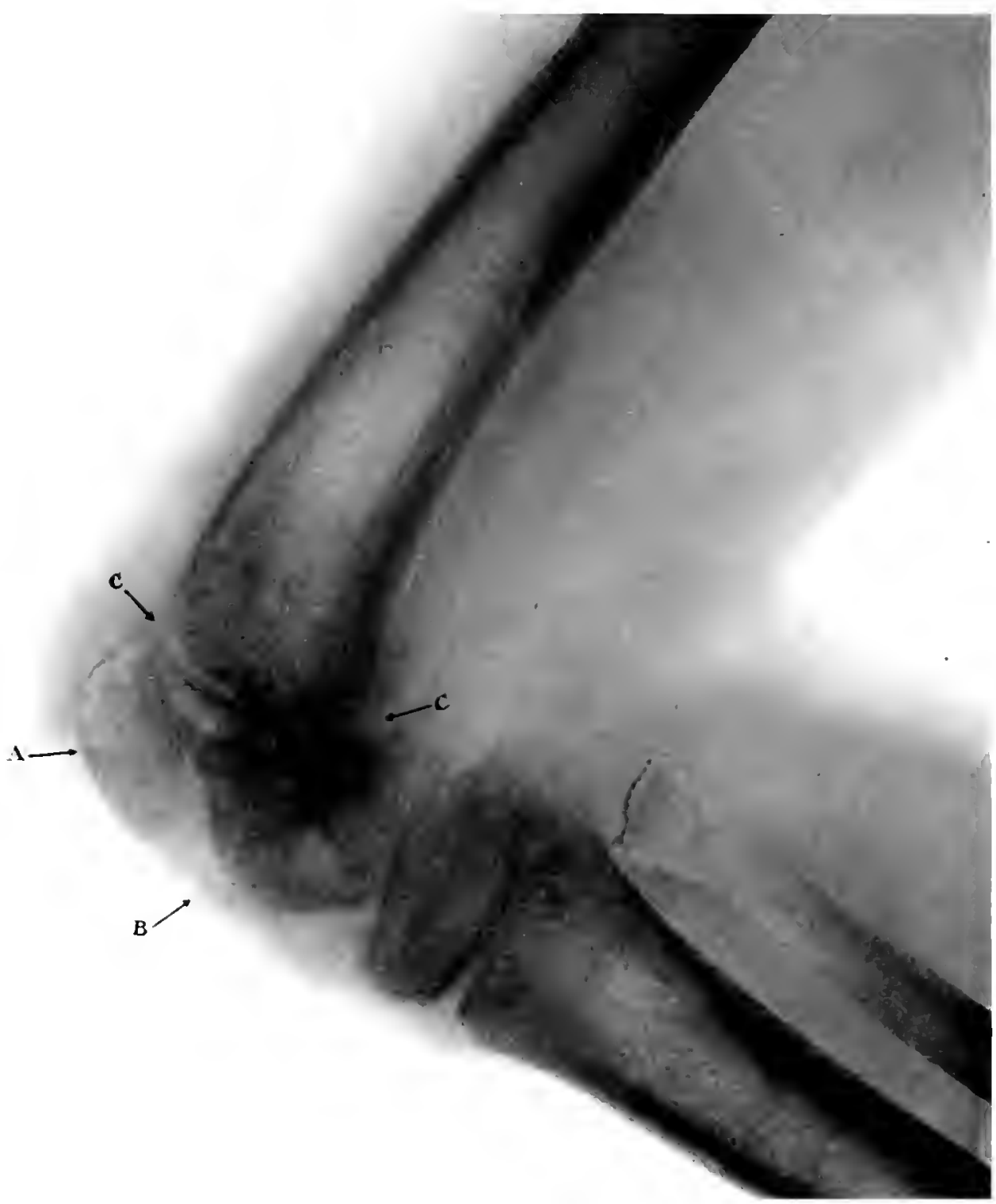




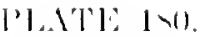

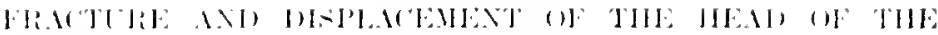
Jll MIRIS.

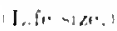

1. Hated of the humarats.

li. Shaft of the loumerus. 
PIATE 1 SO

A 
PI.ITI: 1 S1.

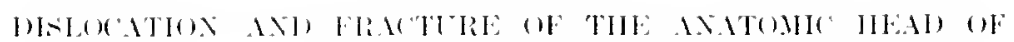
TIIE IIIMHEIS.

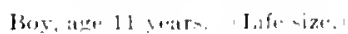

A. Eniphyeal line. 
P'LATE INI

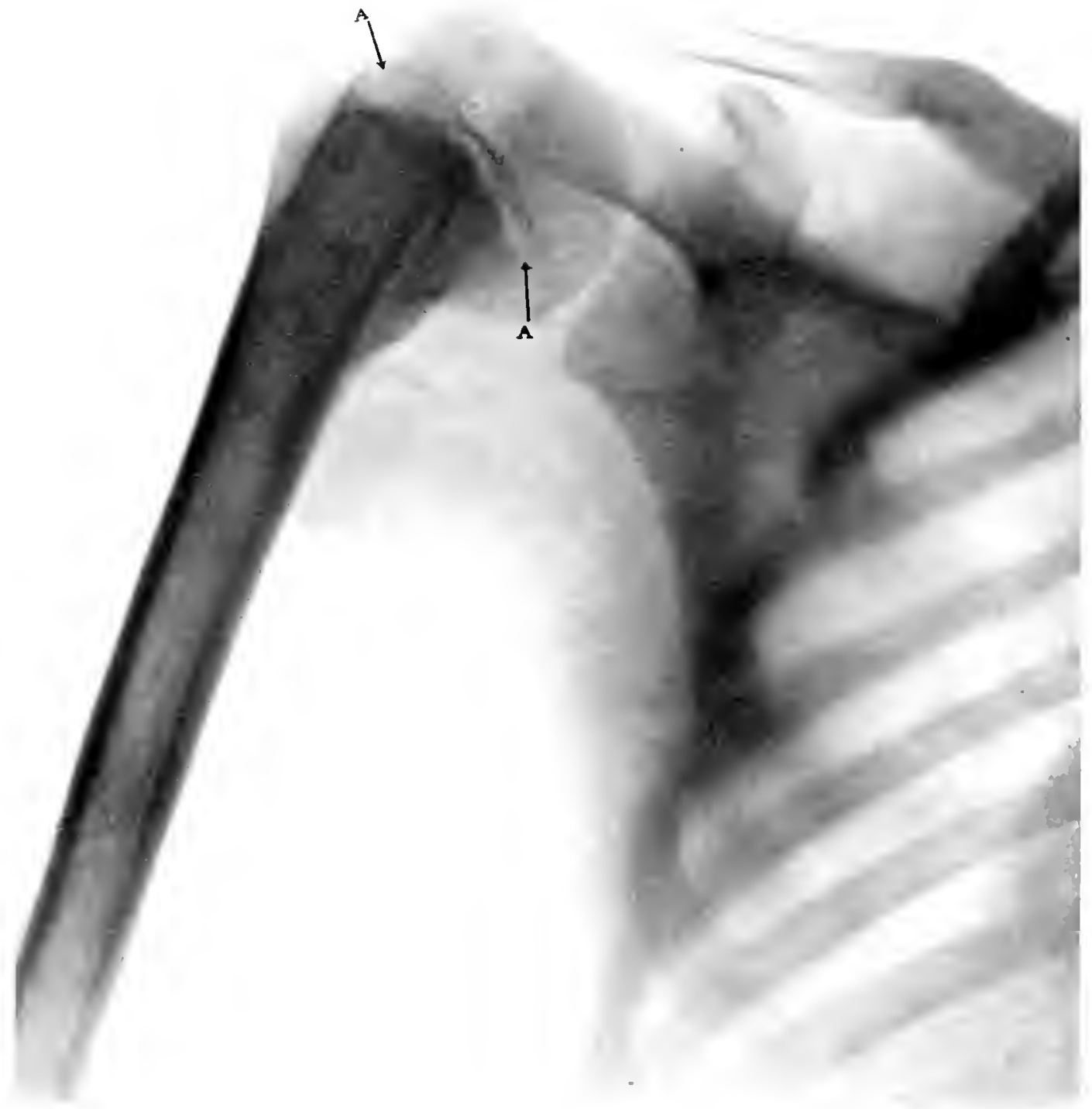


II.TTE: 142.

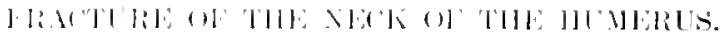

Ism,

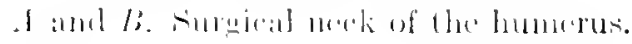


PISTE ISE

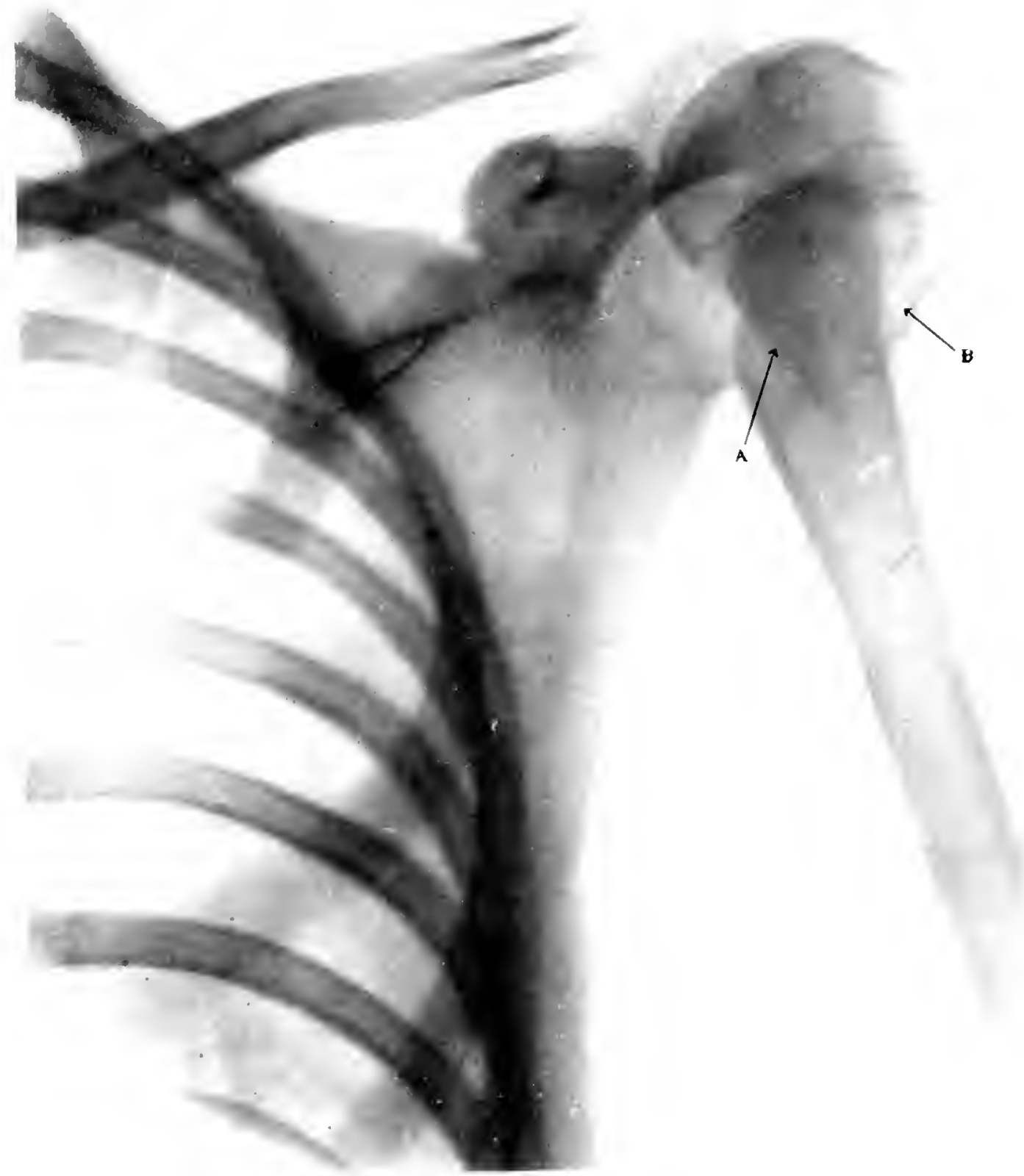




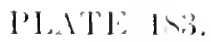

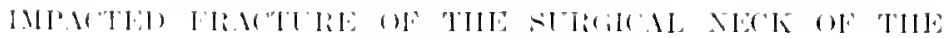
III:IIEI:IS.

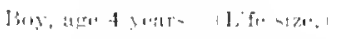

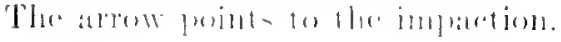


PIATE 1S:3

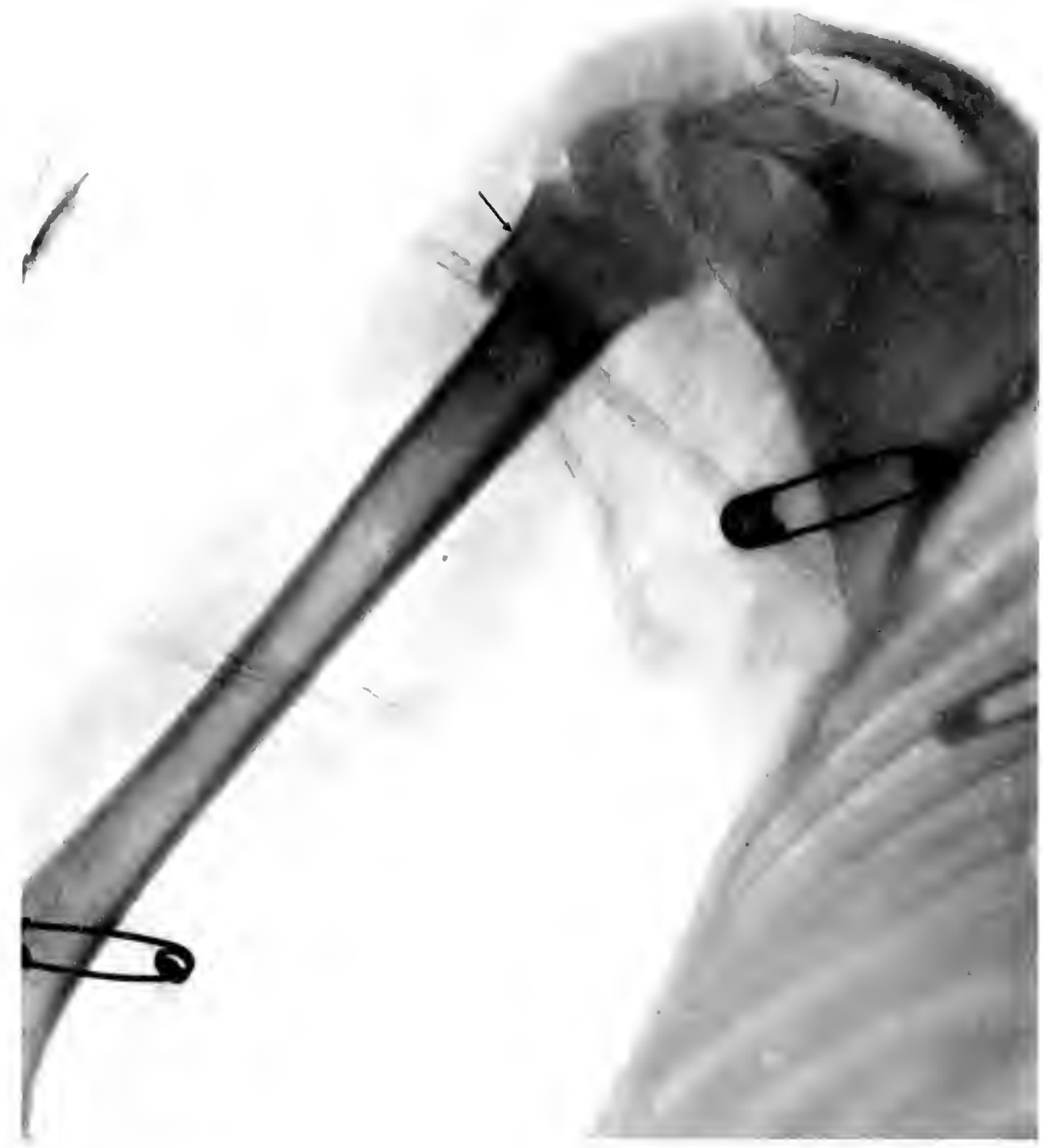


PI.ITF $1 \mathrm{St}$

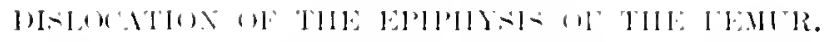

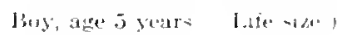

1. Vipilisis.

li. Irrenular diaphysis.

('. 1:illus.

O. I'atrillit. 


$$
\text { PLATE } 1 \text { St }
$$

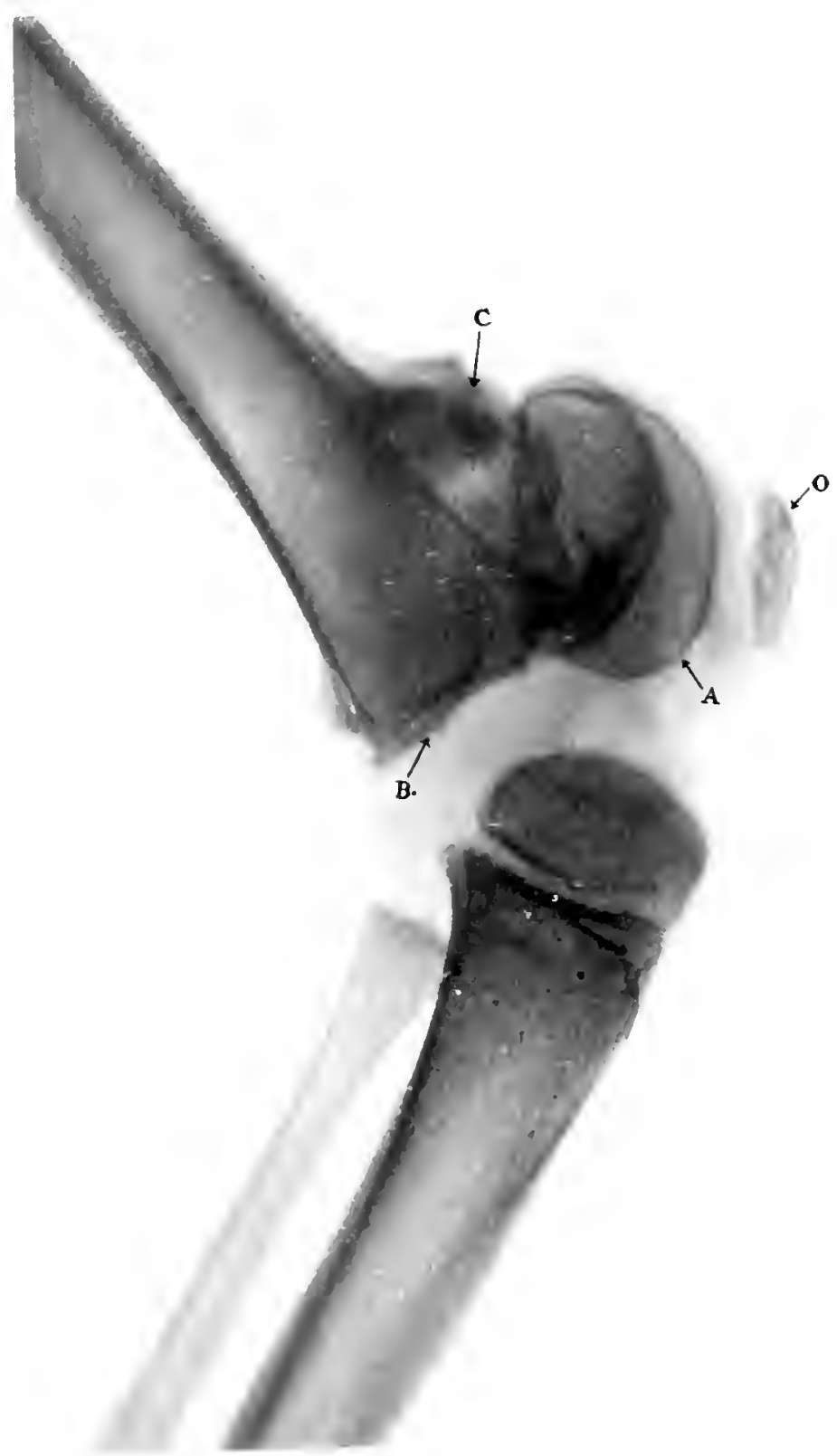




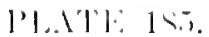

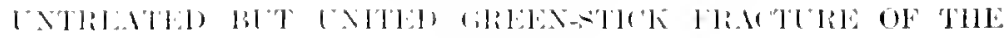
T'11:1.1.

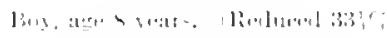

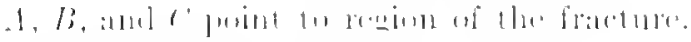

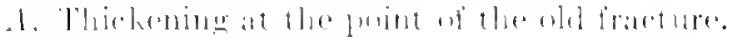

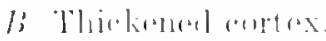

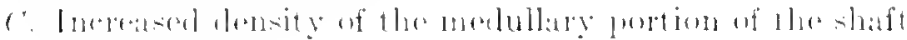


I'I.I'J: 15.5
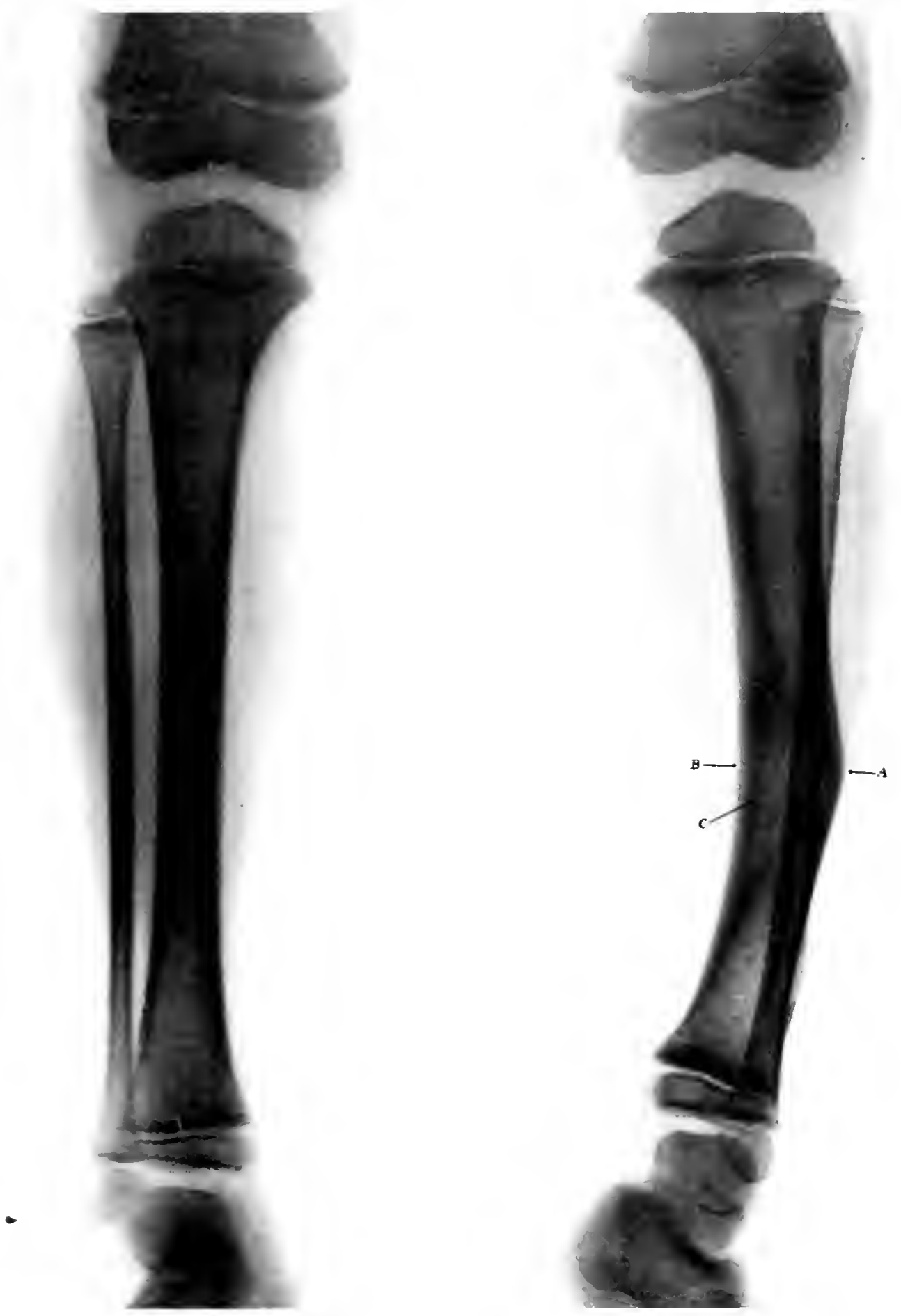
PI..T'l: JVi.

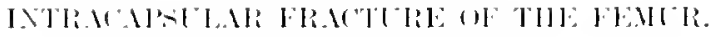

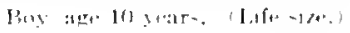


I'IATE 186

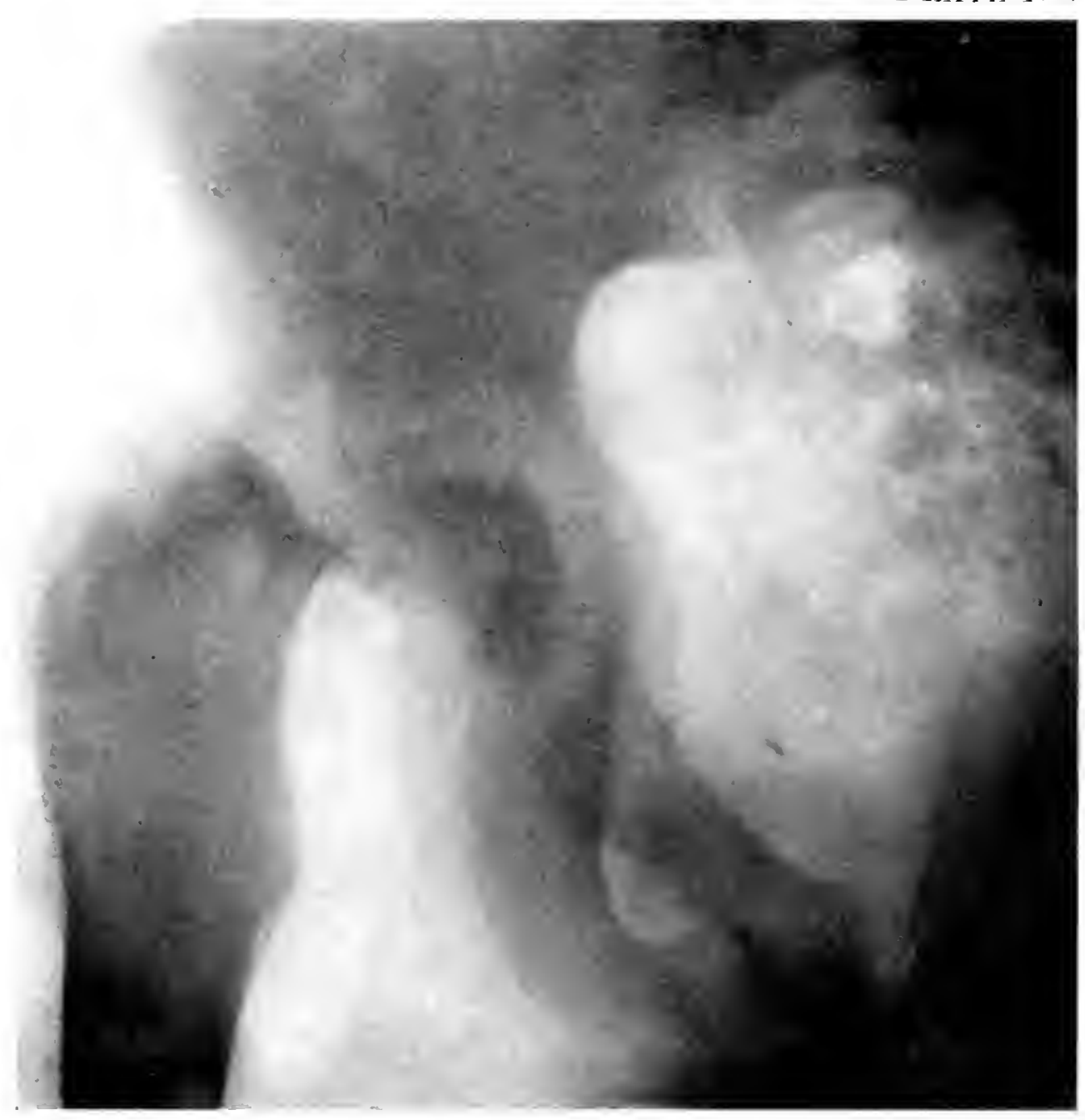


I'ITE 1N.

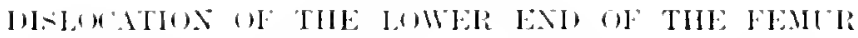

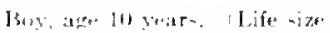

A. The comlyle disloeated barkwarl. 
T'ATE

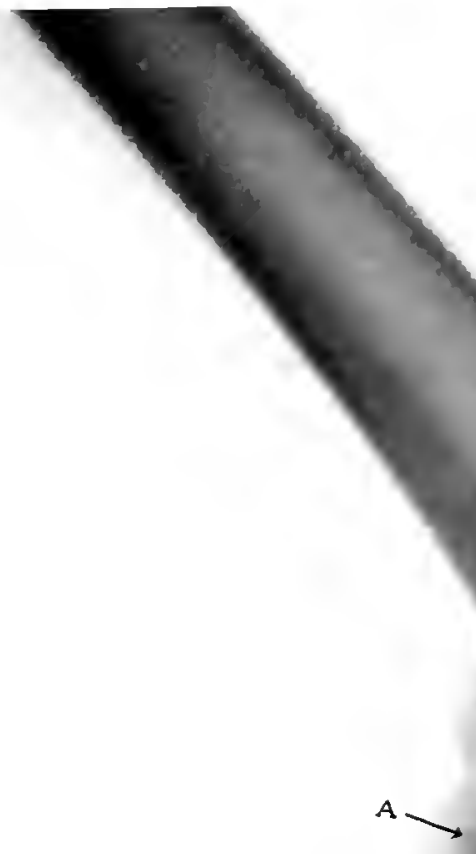


PhATE ISS.

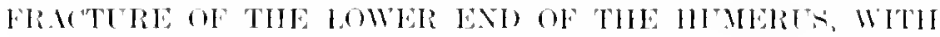

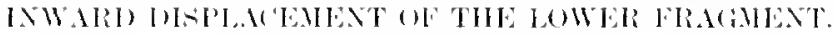

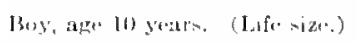

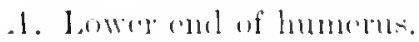

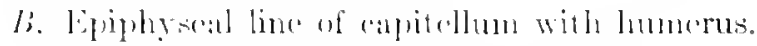

('. Epiphysis of the alecramon process.

I). Epiphysic of malius.

b. cispitillum.

F. Tiu splint. 
PLATE INS

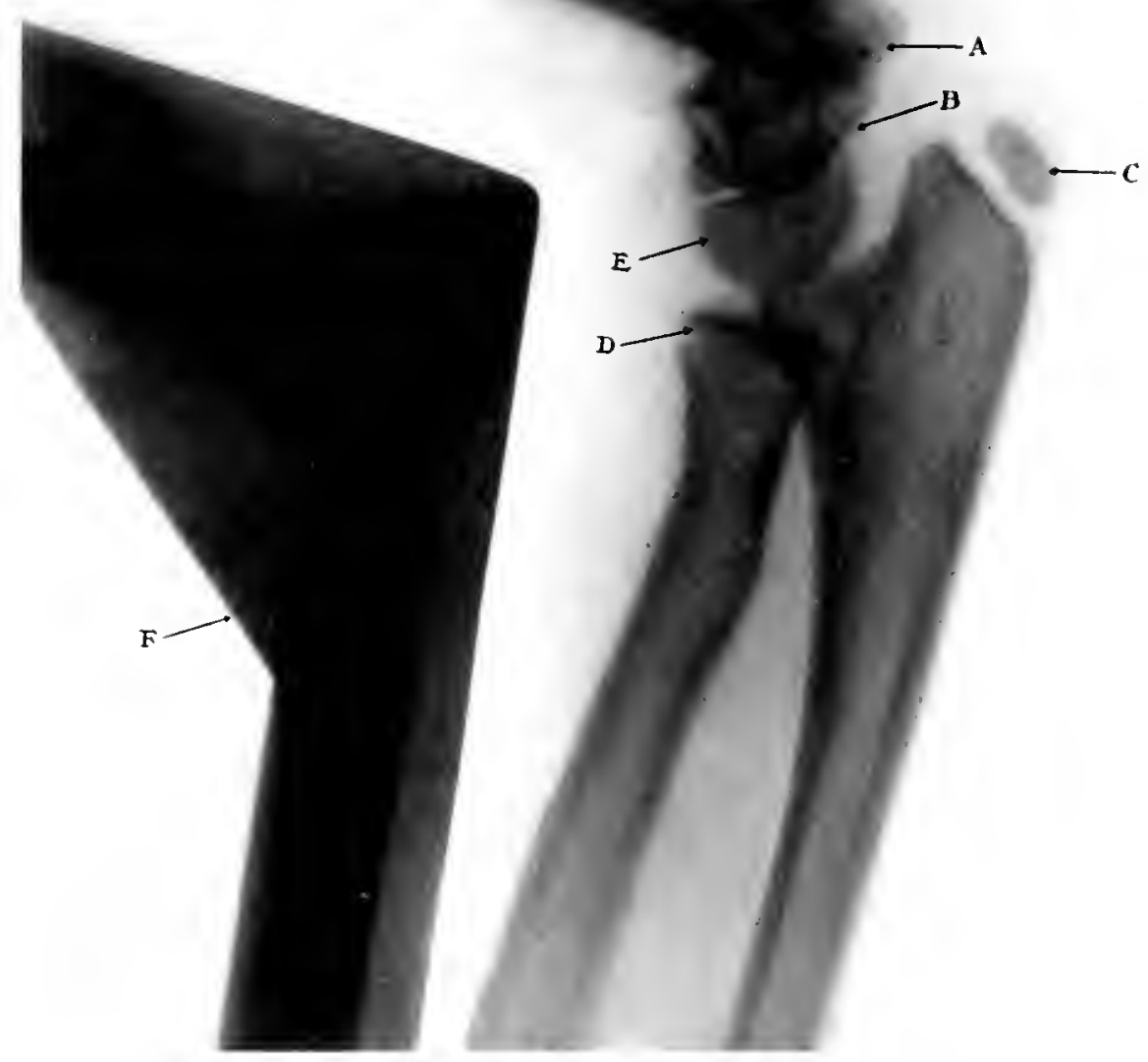


I'ATE: 1S!.

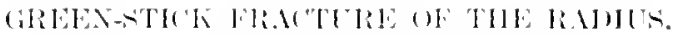

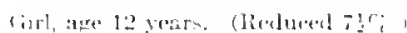

The arrew proints to the fracture.

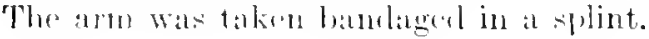


PIATH: 1S!

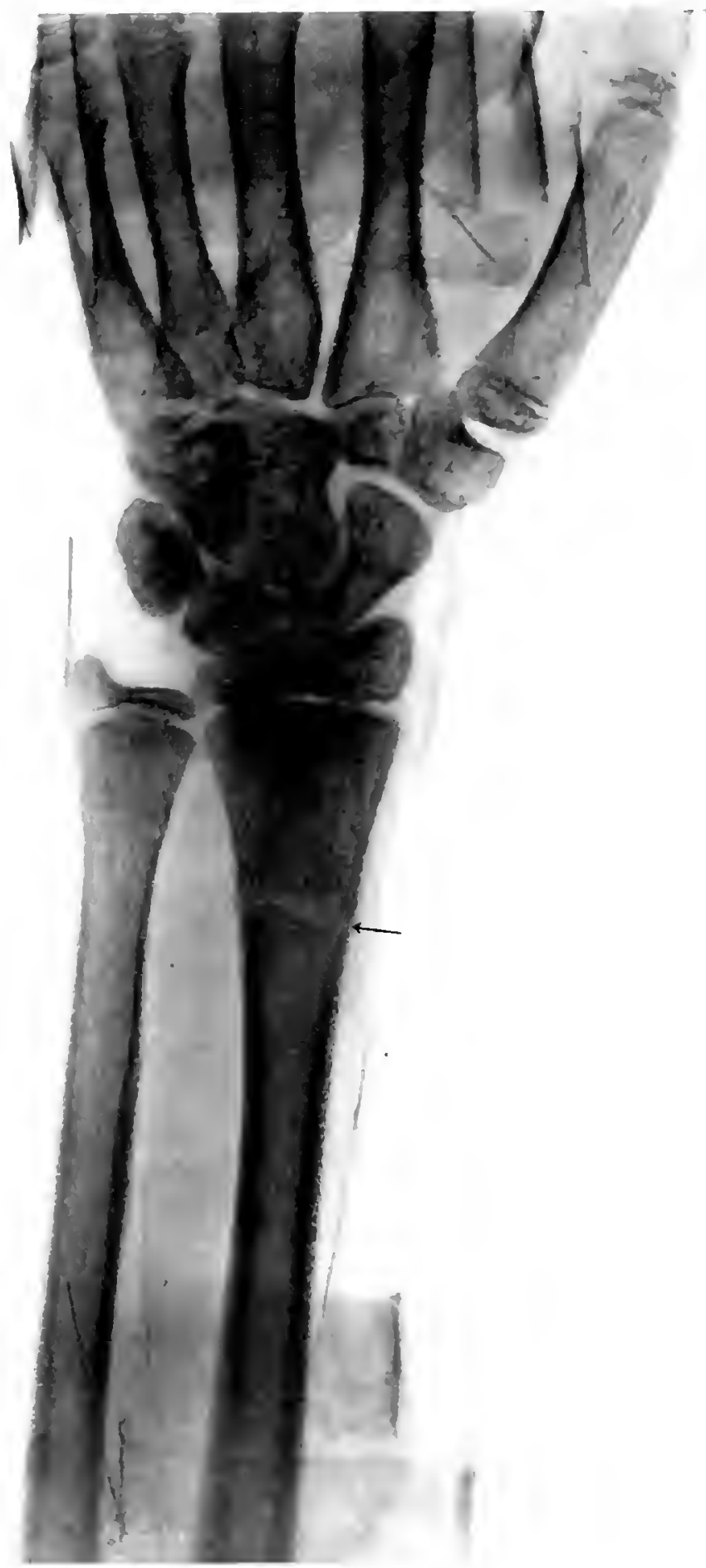


PATI: 190.

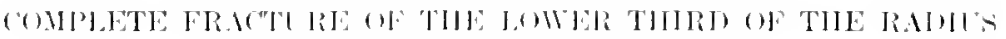

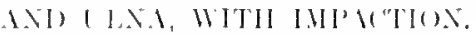

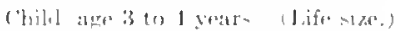

1. Impartion of the ralius.

I. Impration of the nitua. 


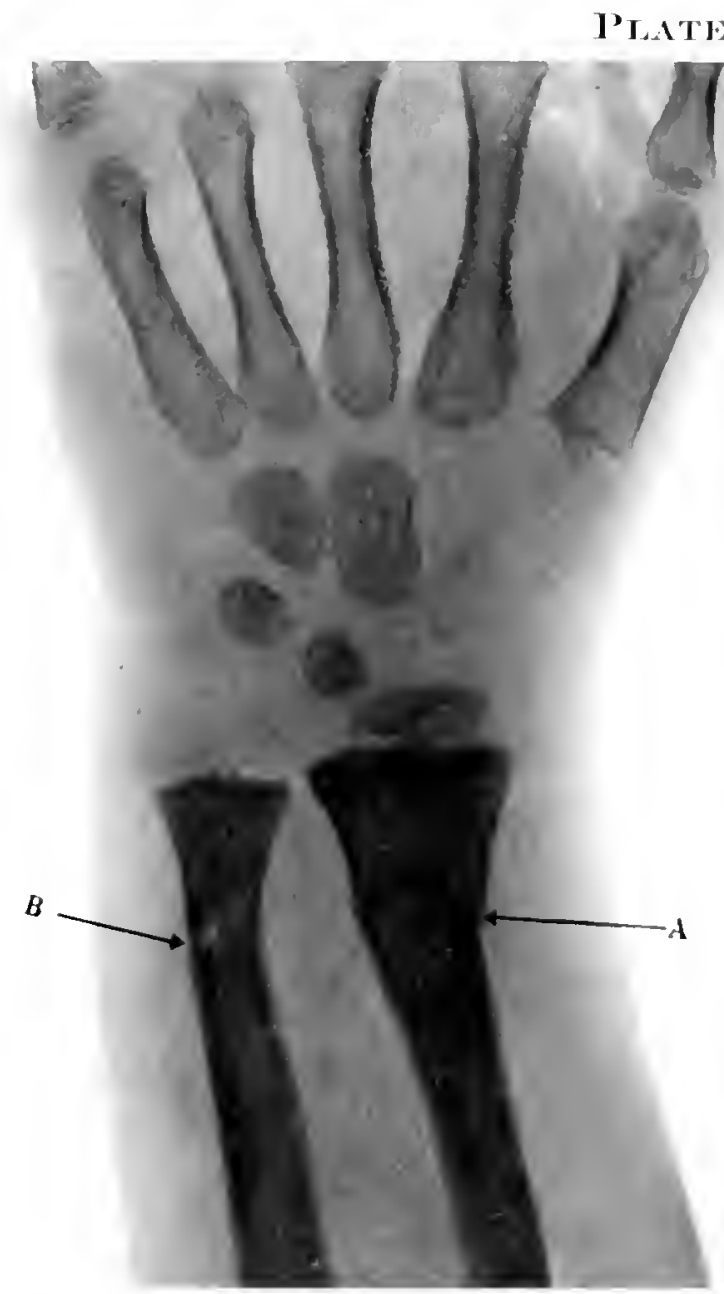


PLATT: 191.

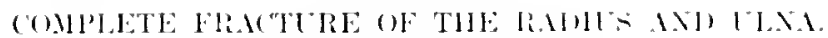

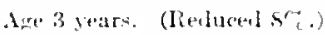

1. Frateture of the ratlins.

B. Frateture of the whit.

C. Calpitutlum, 


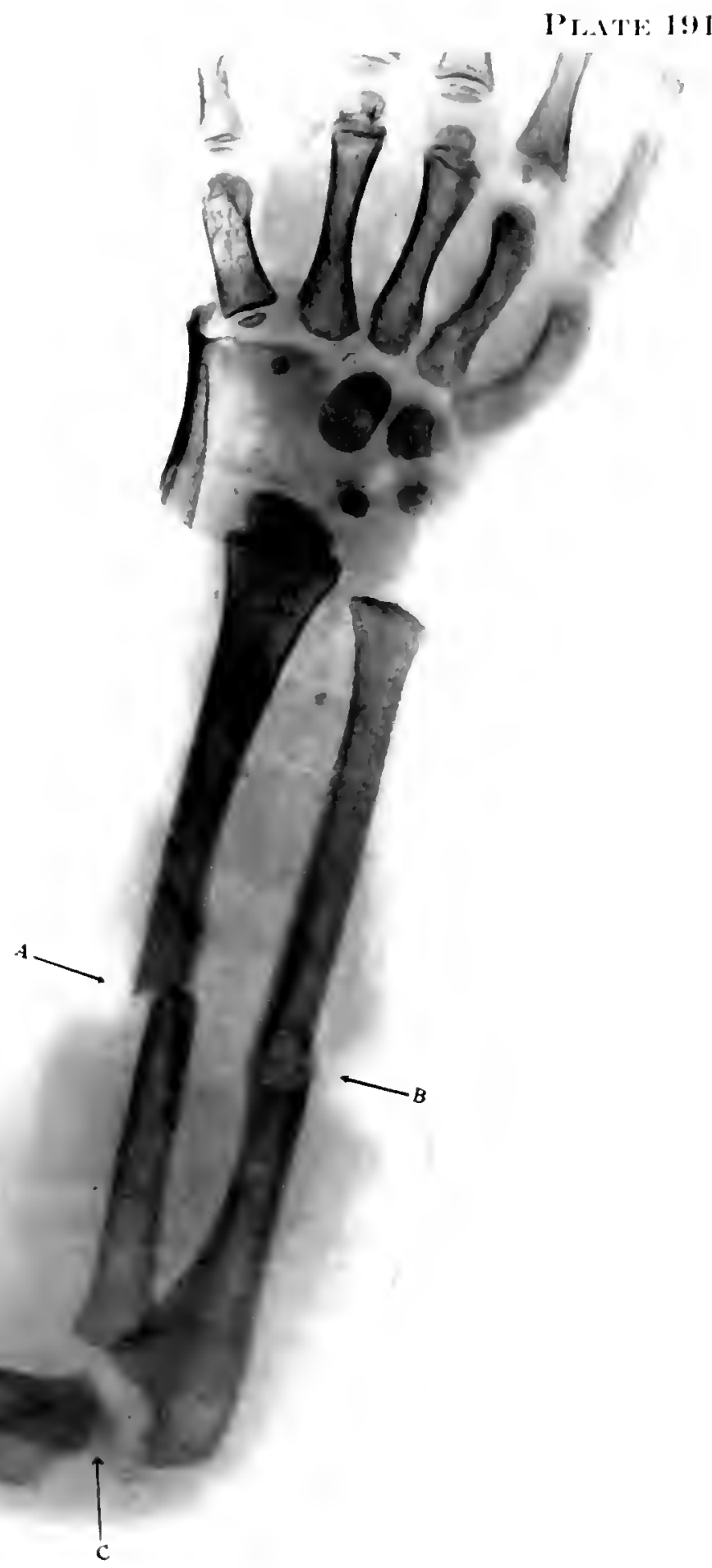


PIATE 1!2.

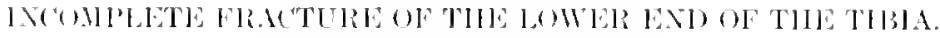

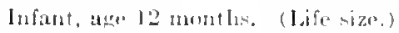

1. Friature.

b. Showing ruphume of the temen Anhillis. 
PI.TE 19:

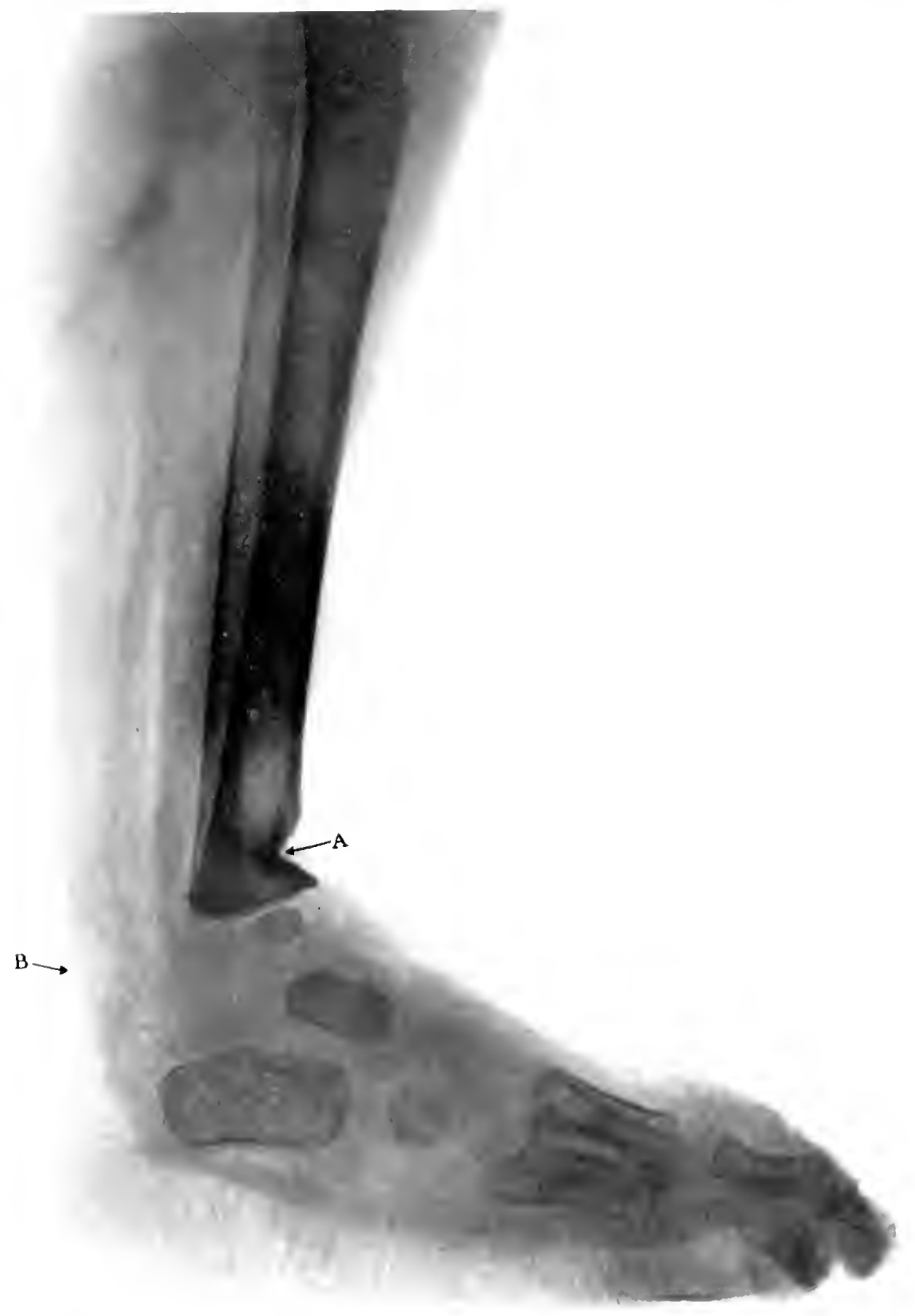


Pl.TPl: 1:6.

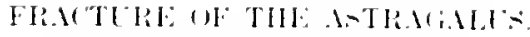

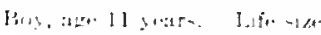

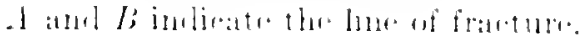


PIATE 19:;

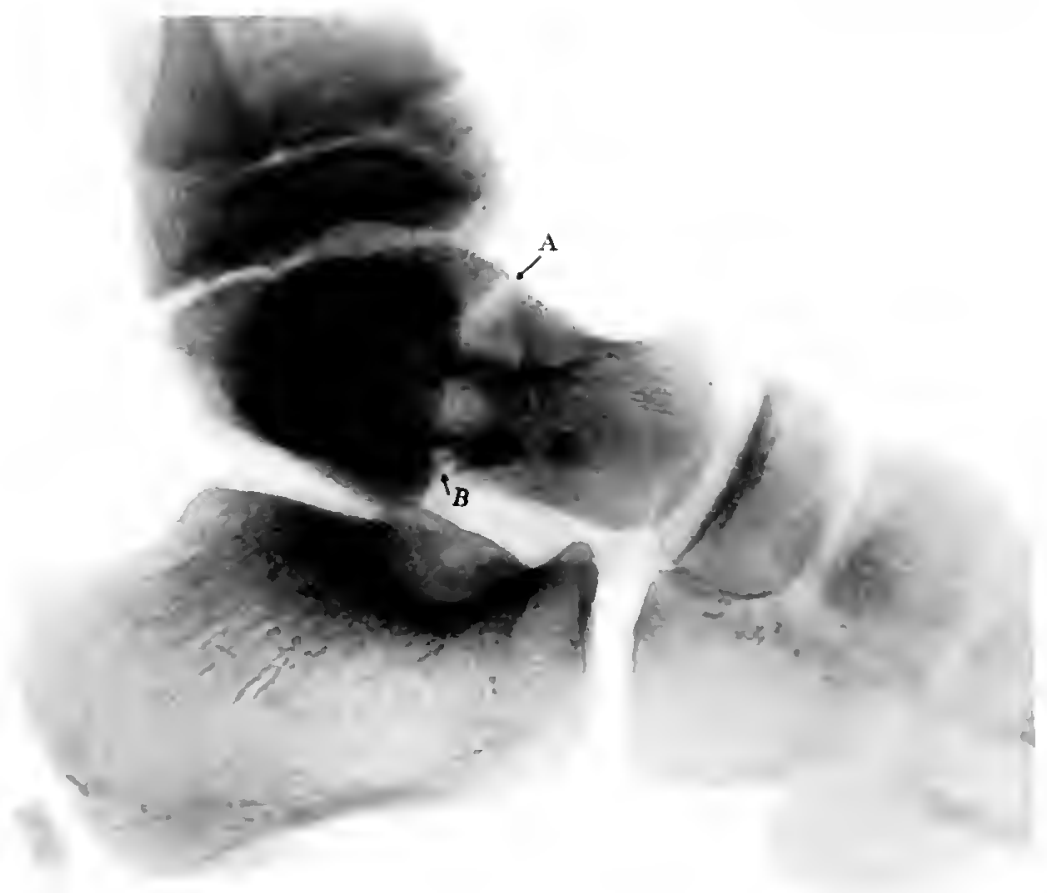


PI.ITY 1!1.

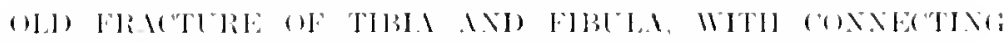

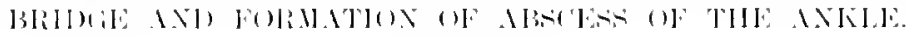

I. $f_{1} \cdot$ size.

1. Brisler.

di. Fombition of the abserse of the tistues of the ankle. 
PLATE 194

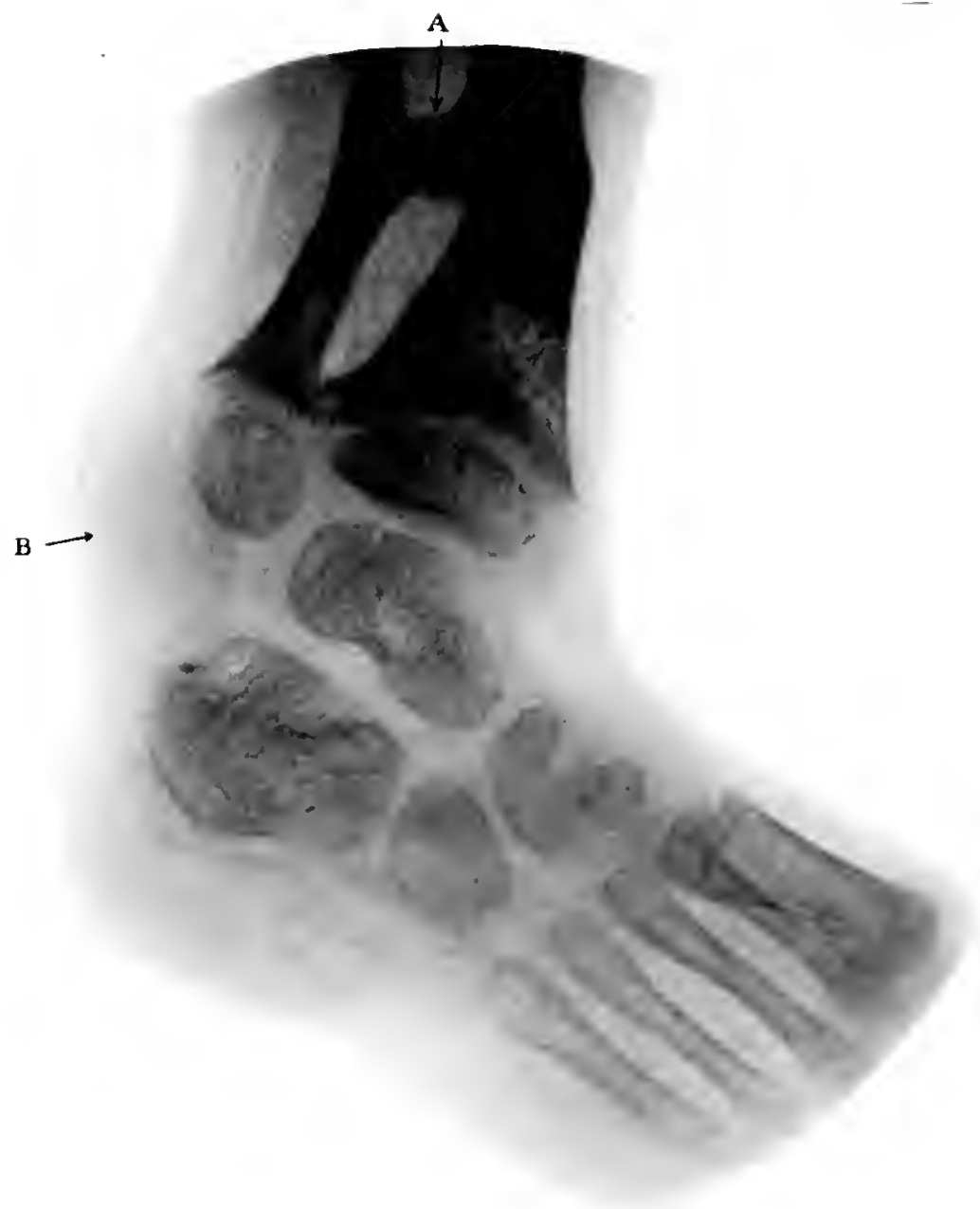




\section{PIAT'E 19. \\ Solithe form.}

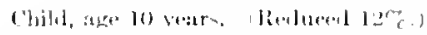

Shows the epiphysis of the os calcis desceloping from two centres, which clinirally aty) eatrell 10 bo a flatedure.

The arrow pointe to the two csifie centres of the as caleis. 


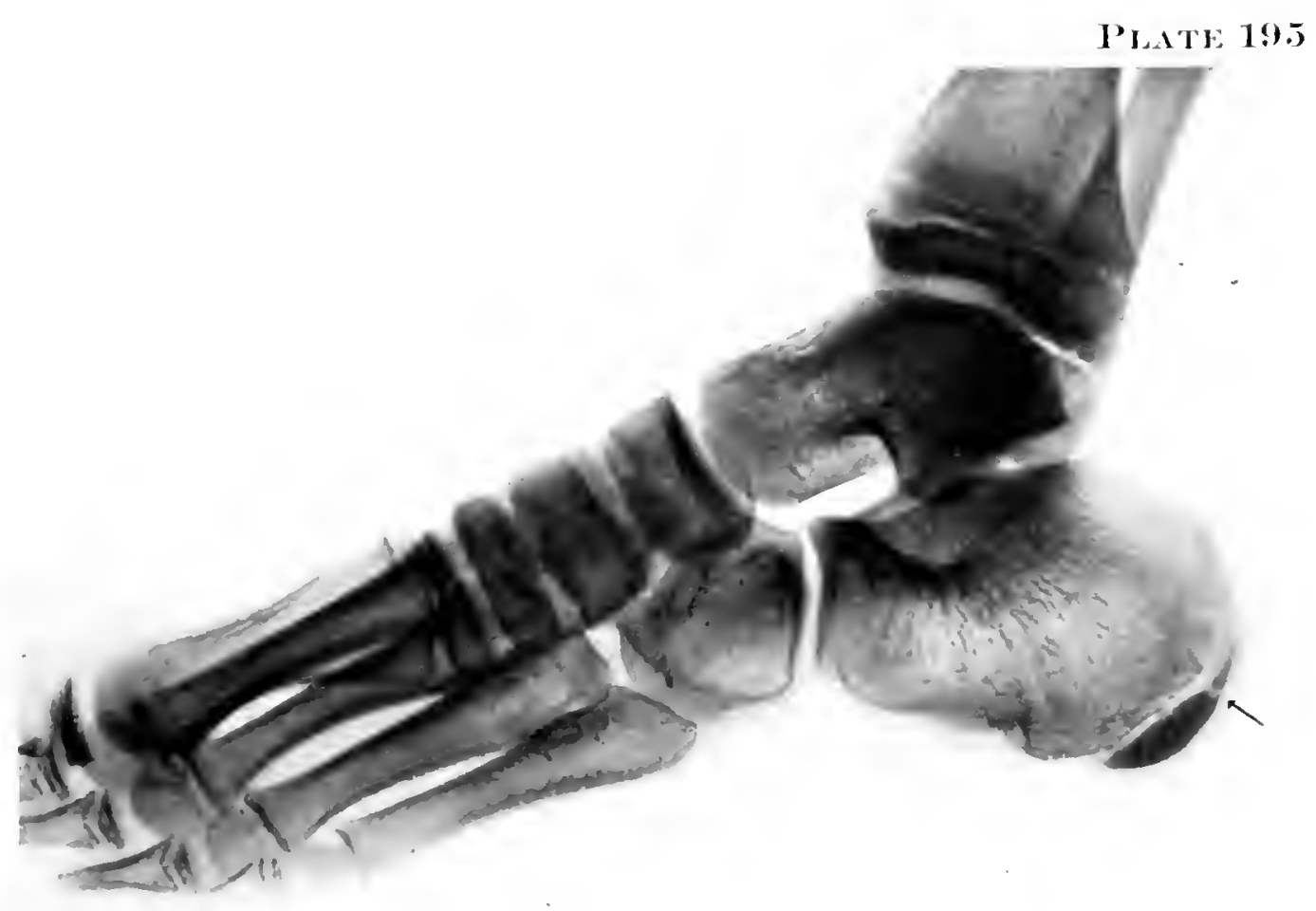


PI. ITI $1 ! K$.

FRACTERE OF THBIA WTH COMPENATOHY GOUTII OF IIBLIAL.

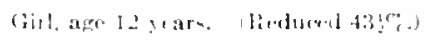

1. Points towards ununitod fratedure of the tibiat.

B. "The very mueh increased cortionl bont of the fibmlat. Note the marked increase in elensity of the whole filmal on the left side, and the matred deregentse of radtialidity of the bowere ond of the fomme amel of the tibiat of the loft lear. 
PIATE I!)
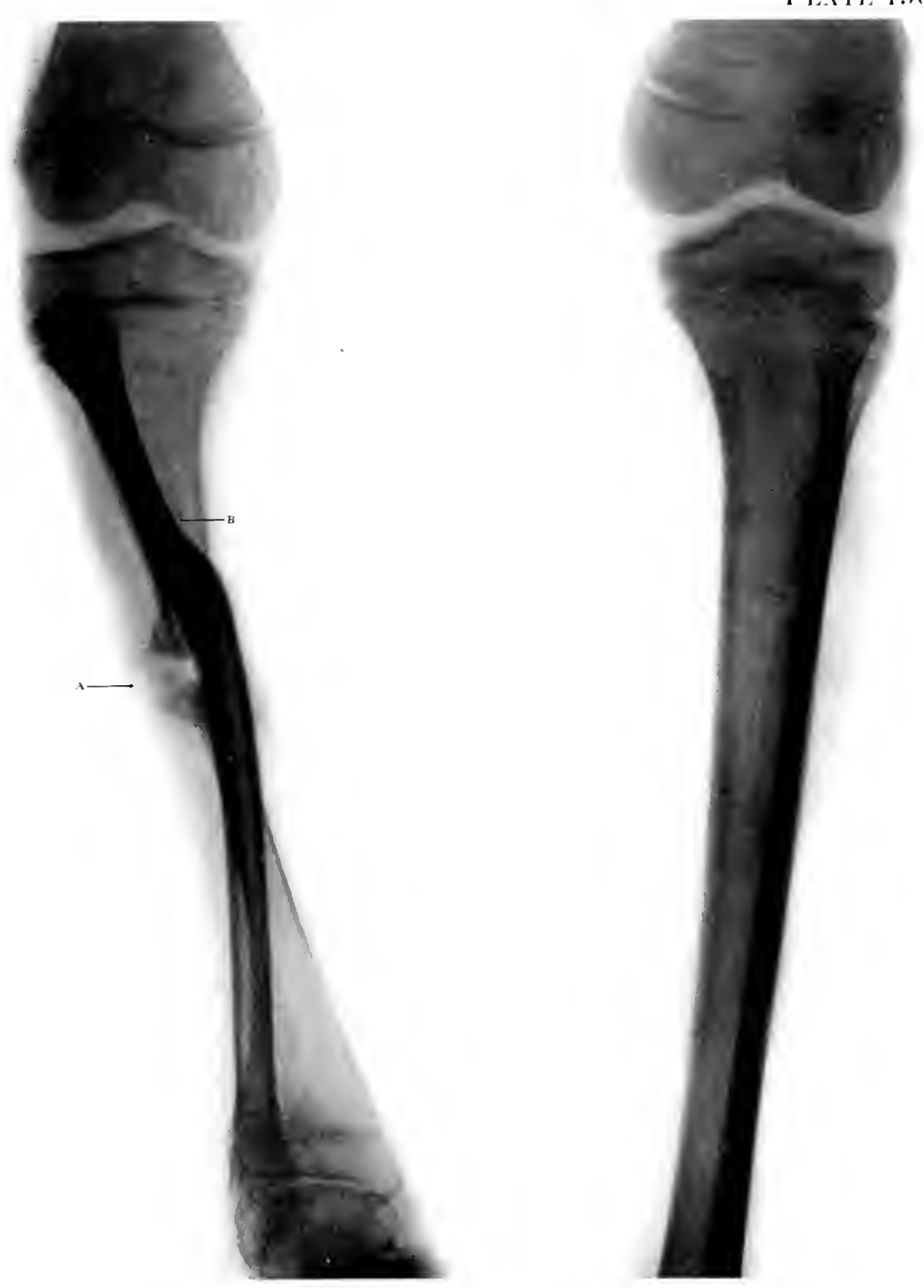
PI.NTE 19T.

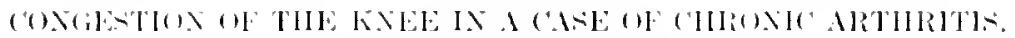

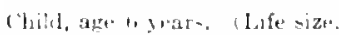

1. Points to the slight eitpulat thickening and the colarged ppiphyses of the fomure and tiliat.

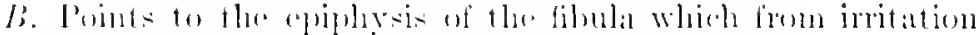
has dereloped sormere than on the riglit side.

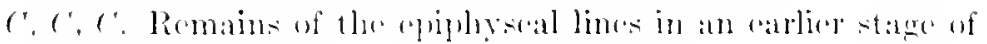
development. 

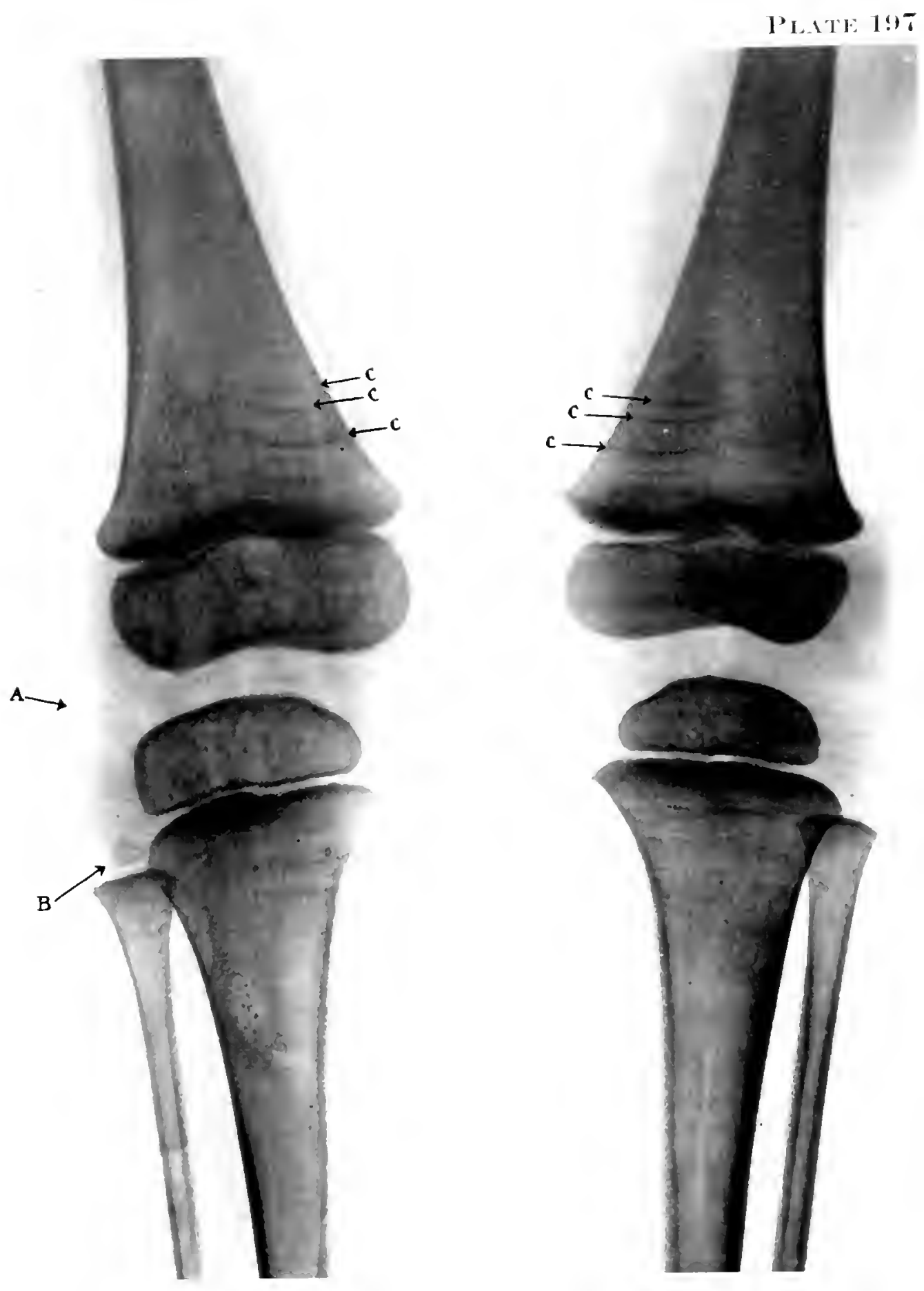
I'I.X'1: 1!)

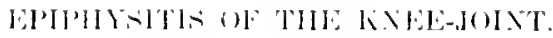

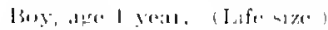

The arrow points lowatels the diaphysis of the fomul which is matkrelly irregular. 


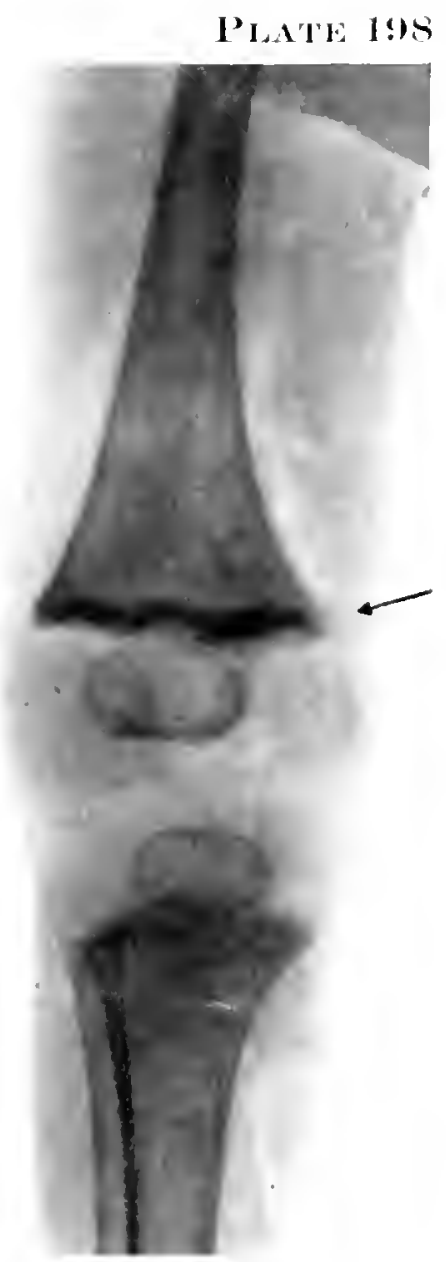


PI.ATI: 1!n,

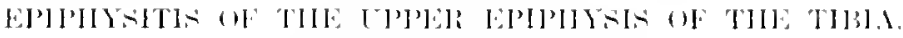

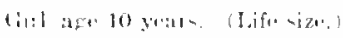

1. Points to the early ascifieation of the epriphysis of the tikilit.

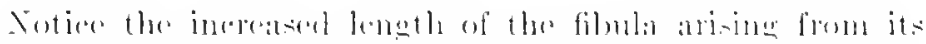

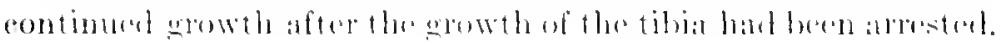


I'I.ATH 1!)

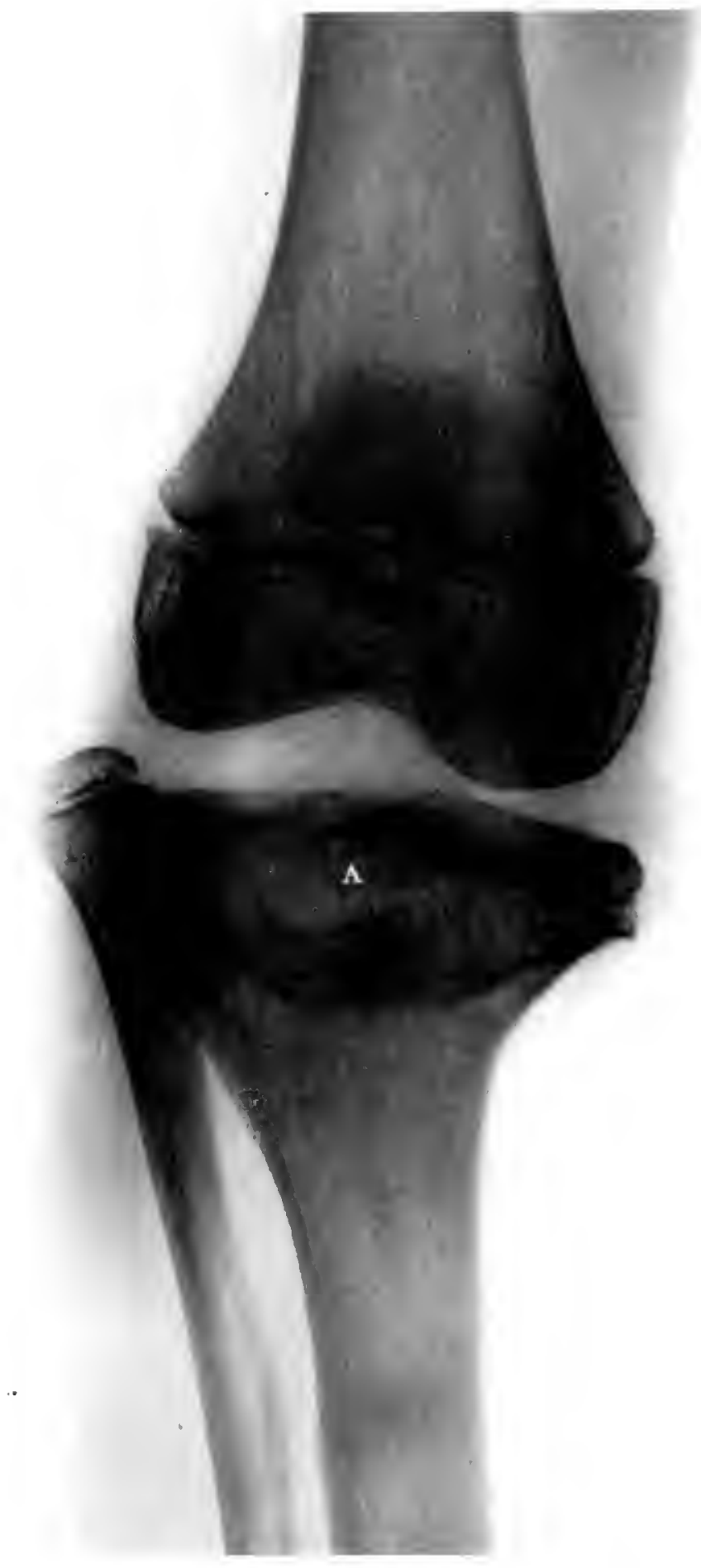




\section{I'1.191: 200.

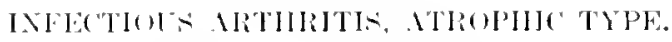

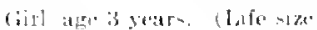

Notre the erencral ostenporosis.

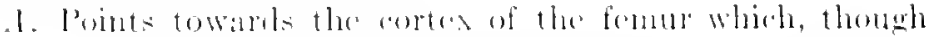

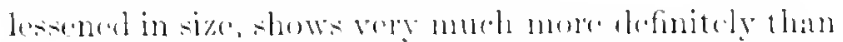
a nomat routex at this ane

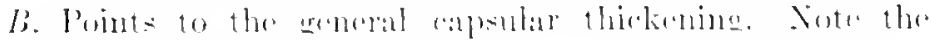

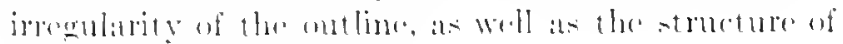

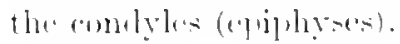




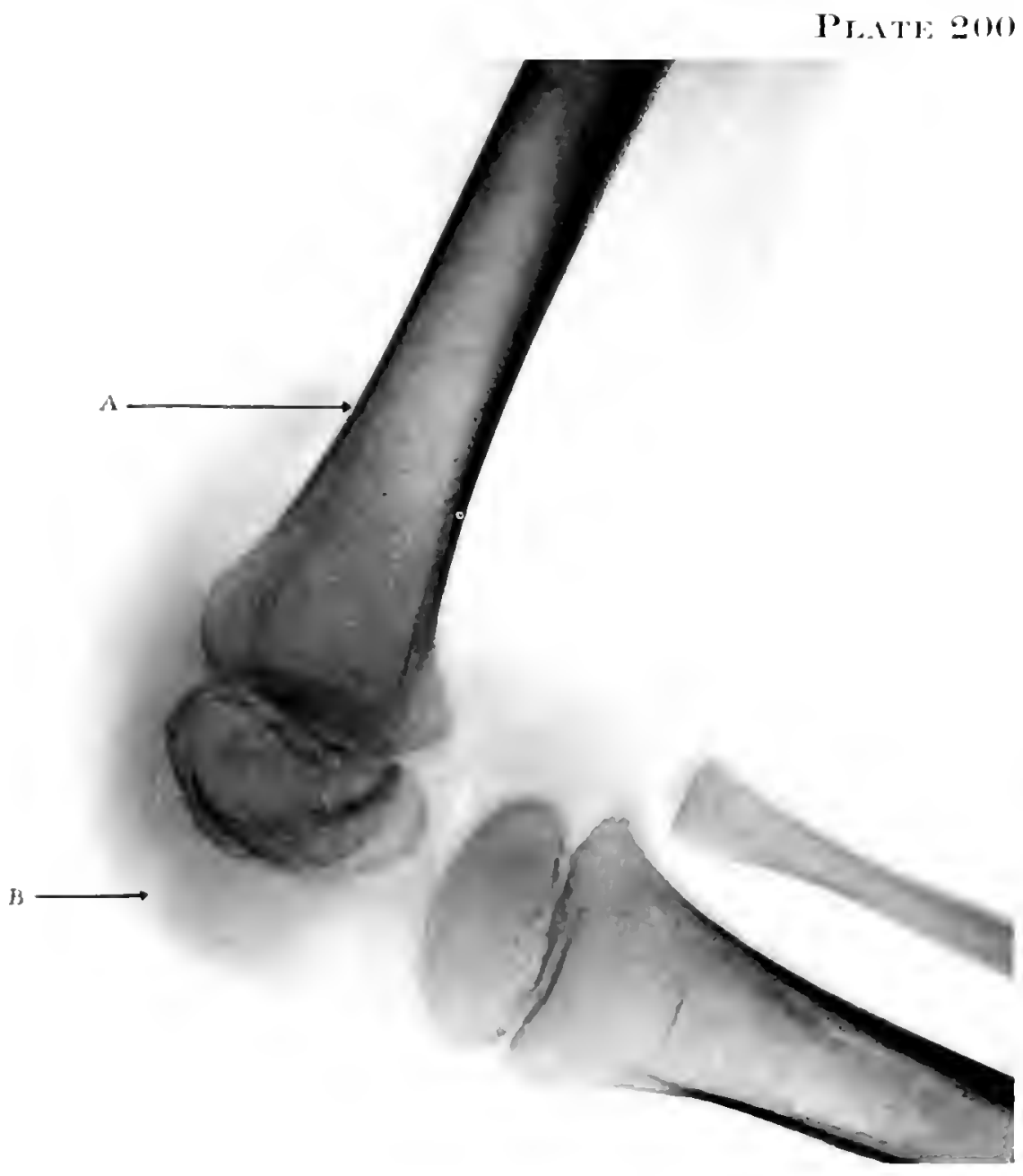


PI.ITI: 211.

MILUL' MRTHITL

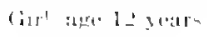

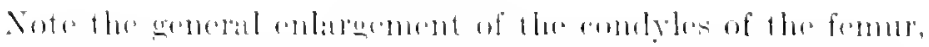

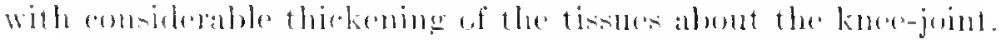


PIATI: 201

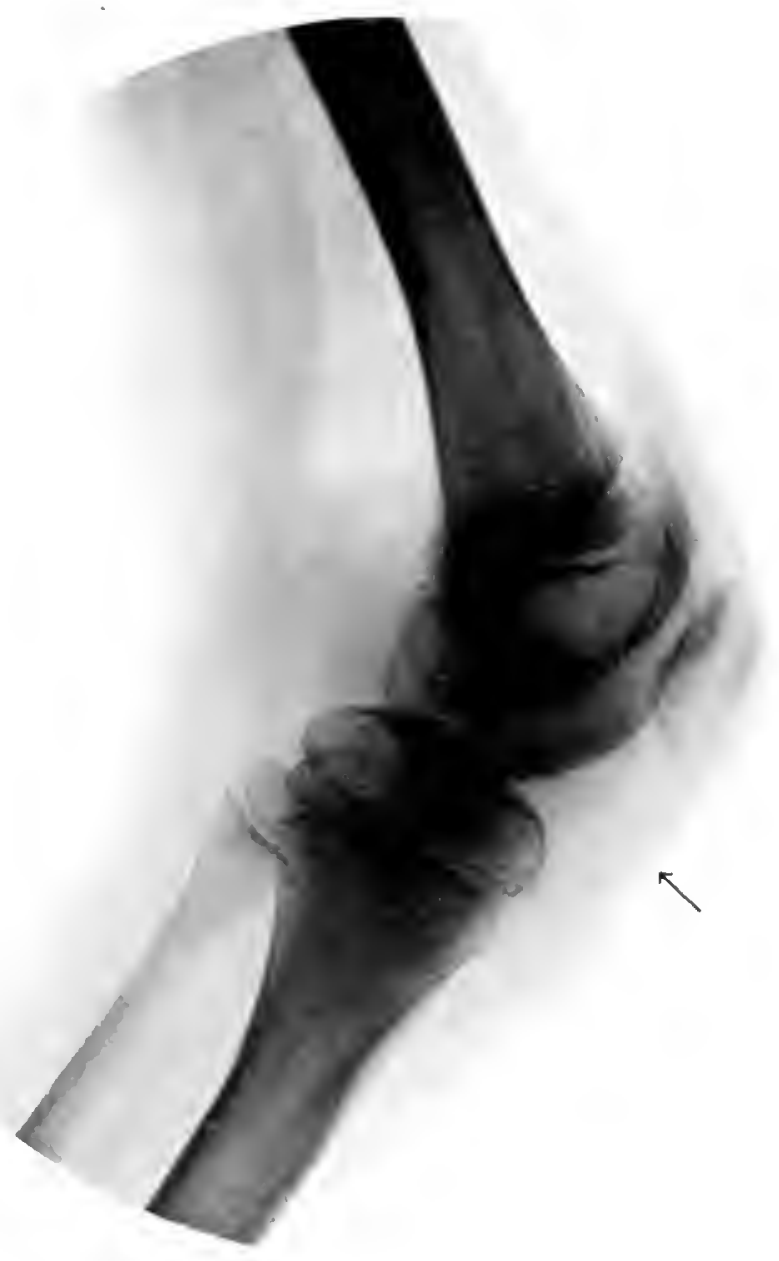


PLATE: 20

AXIIUAS OF KNEE-JUAT.

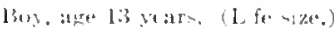

1. Patcllia.

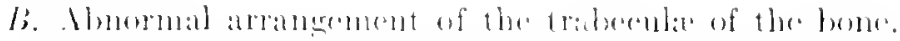

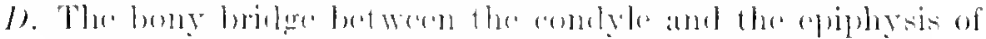
the tilitis.

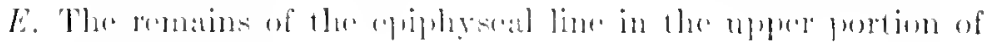
the tillial.

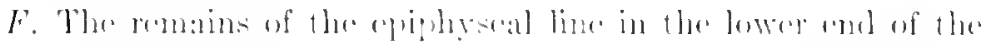
fromml.

Note in this instane how the structure of the bone is and

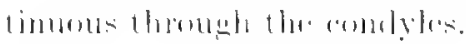


PLATF $\because(1)$

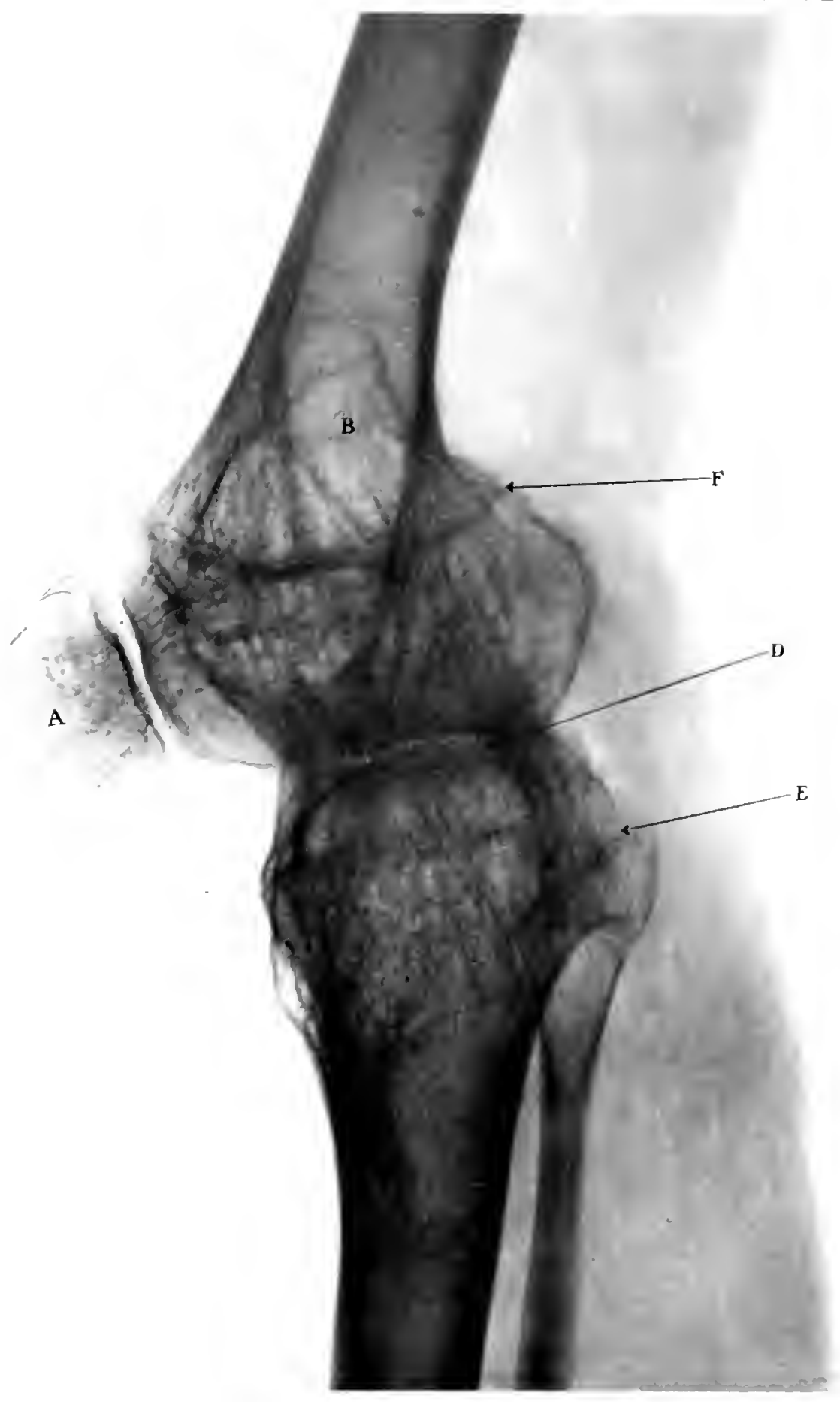


PJ.ITT: 2013.

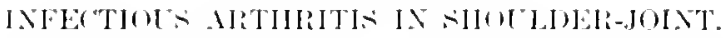

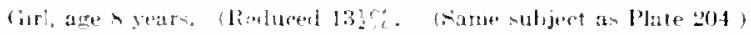

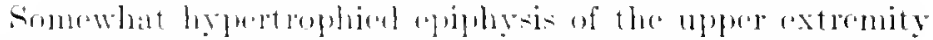
of the homeme, with atrophy of the shaft of the bone, both in sizu aml quality. 
PI.ATE: $20: 3$

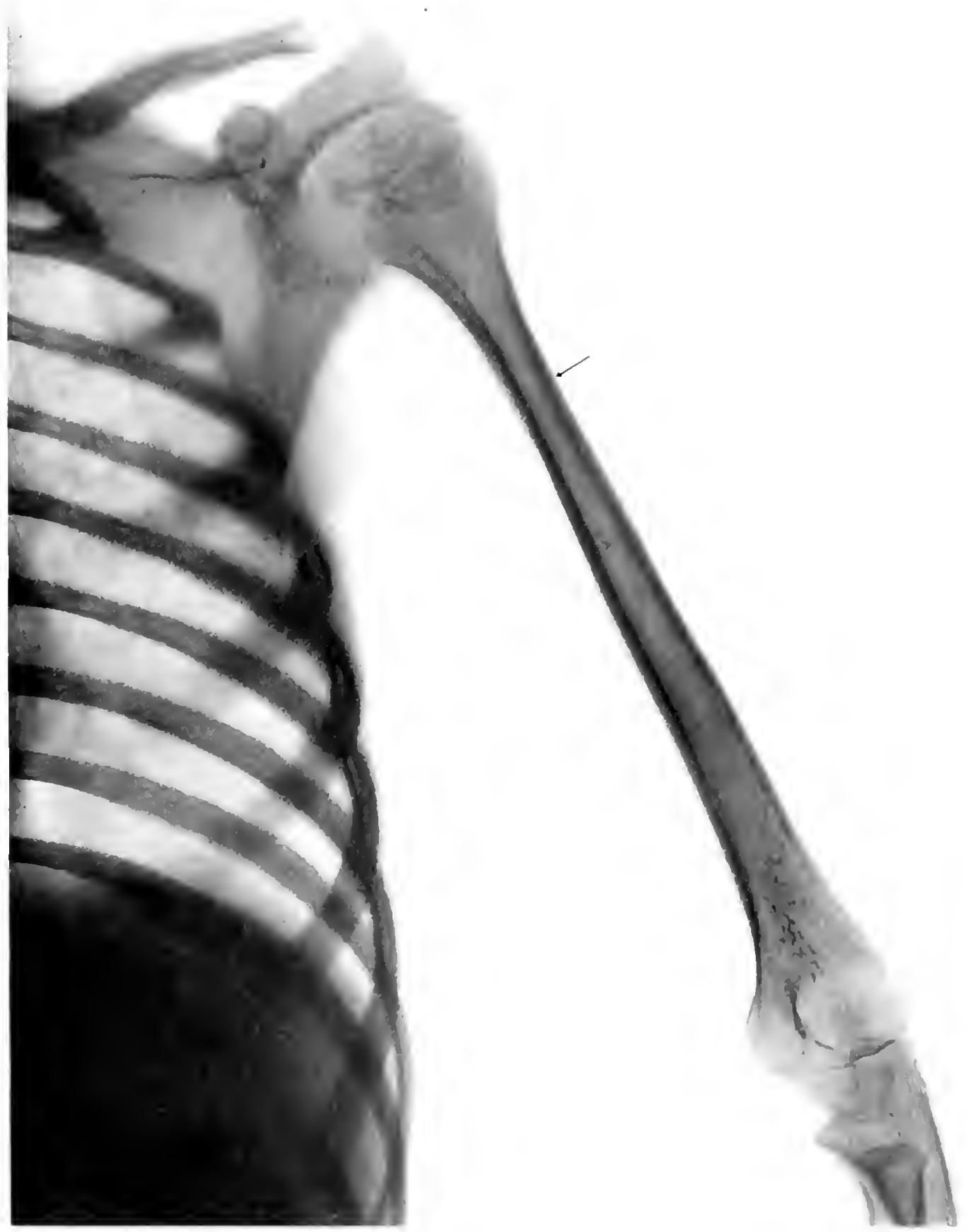


PIATE: 201 .

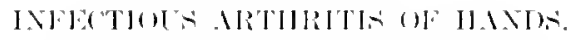

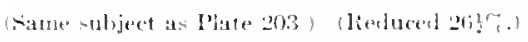

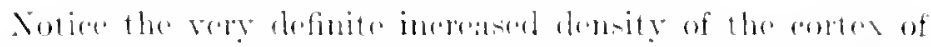

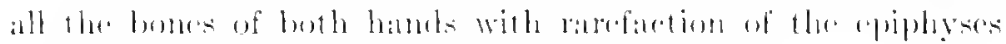

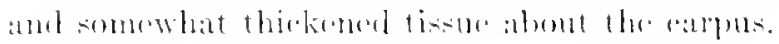


Plate 204
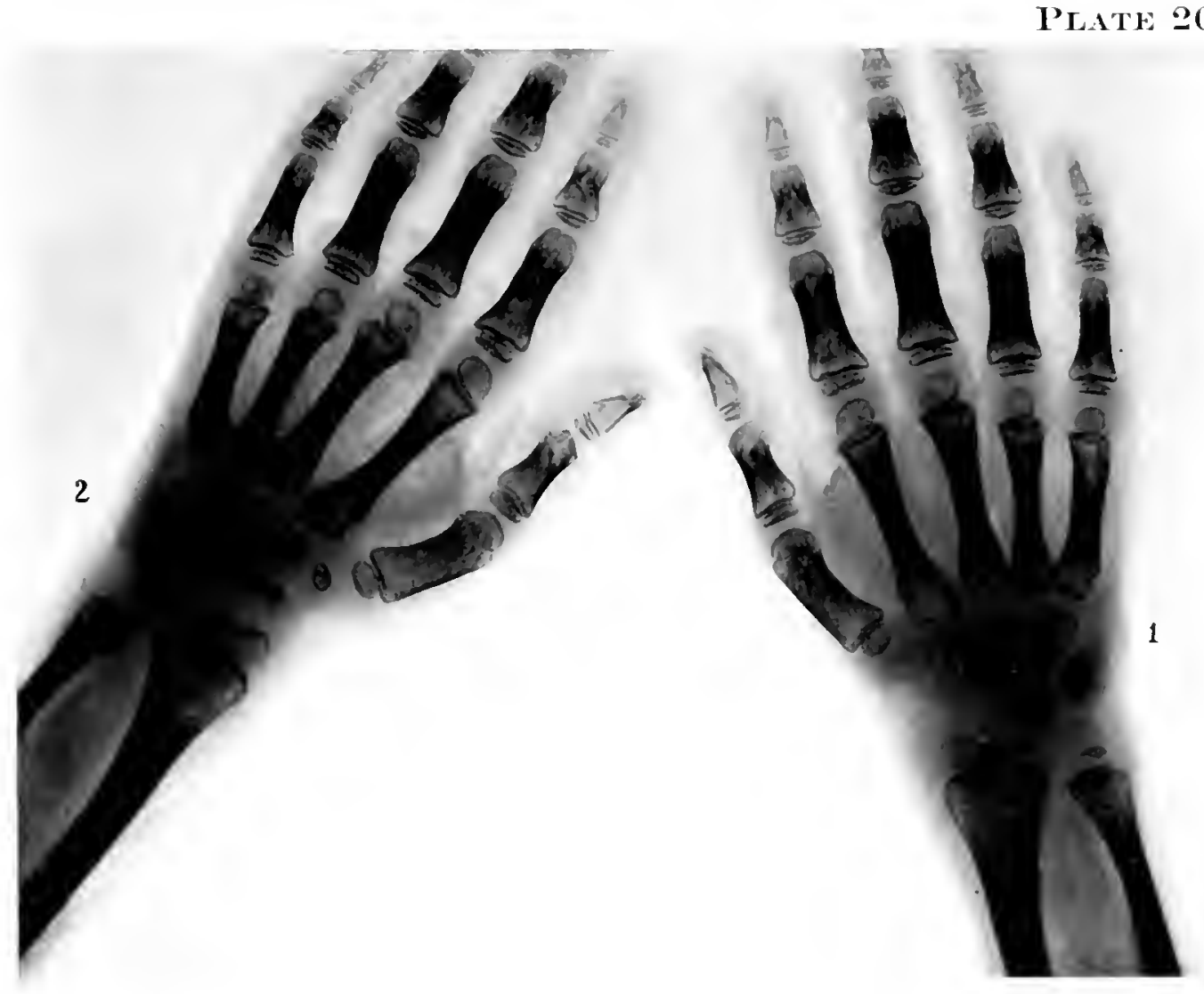
PI.ATE 20.5.

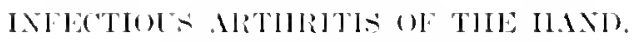

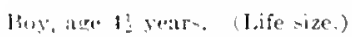

1. Points to a periostand reartion along the shat of the thim metalcarpall bome.

D. Points to the sinne (ondition in a less dregere in the second Inctalrarpal bome.

The proximal purtions of these two bomes show slight noedo-

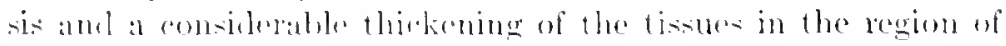
the carpal bones. 
PIATE 20.5

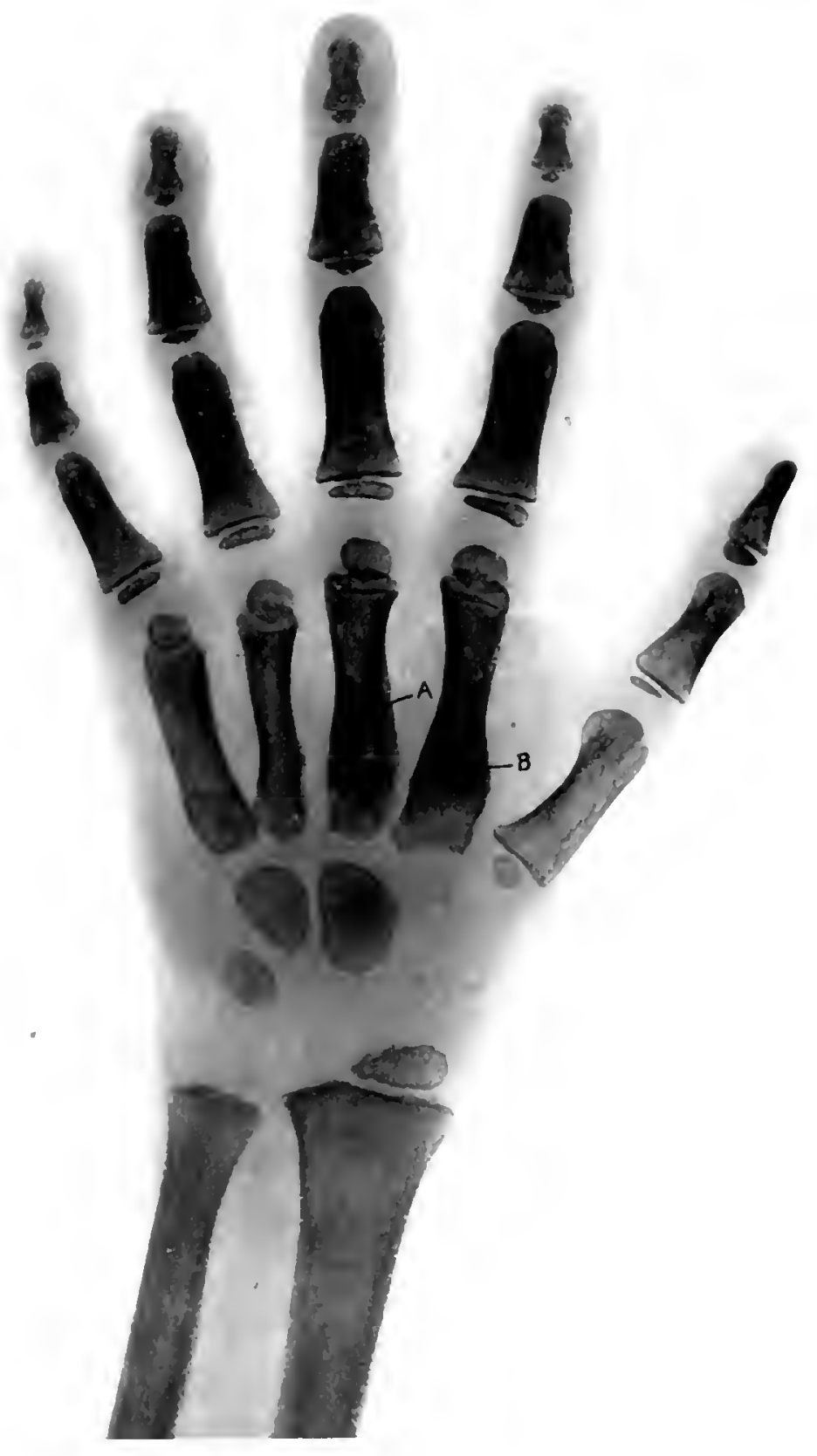


PJ.\TE: 206.

EFHTSON IN THE RNEL-JOAT.

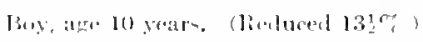

Note the definite outline of the density of the catpsule. 
PI, TRE 206 ; 
PLATE $21 \%$.

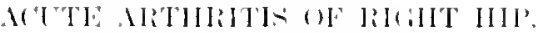

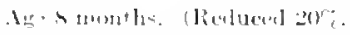

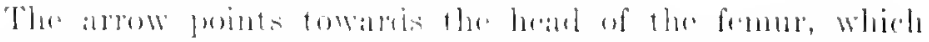

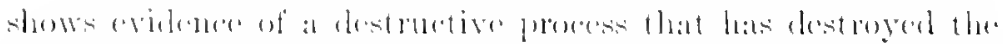
epriphys and part of the nerk, amsing a distoration of the firmtit:

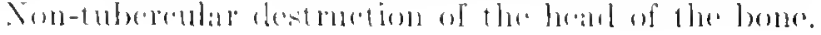


P'LATE 207

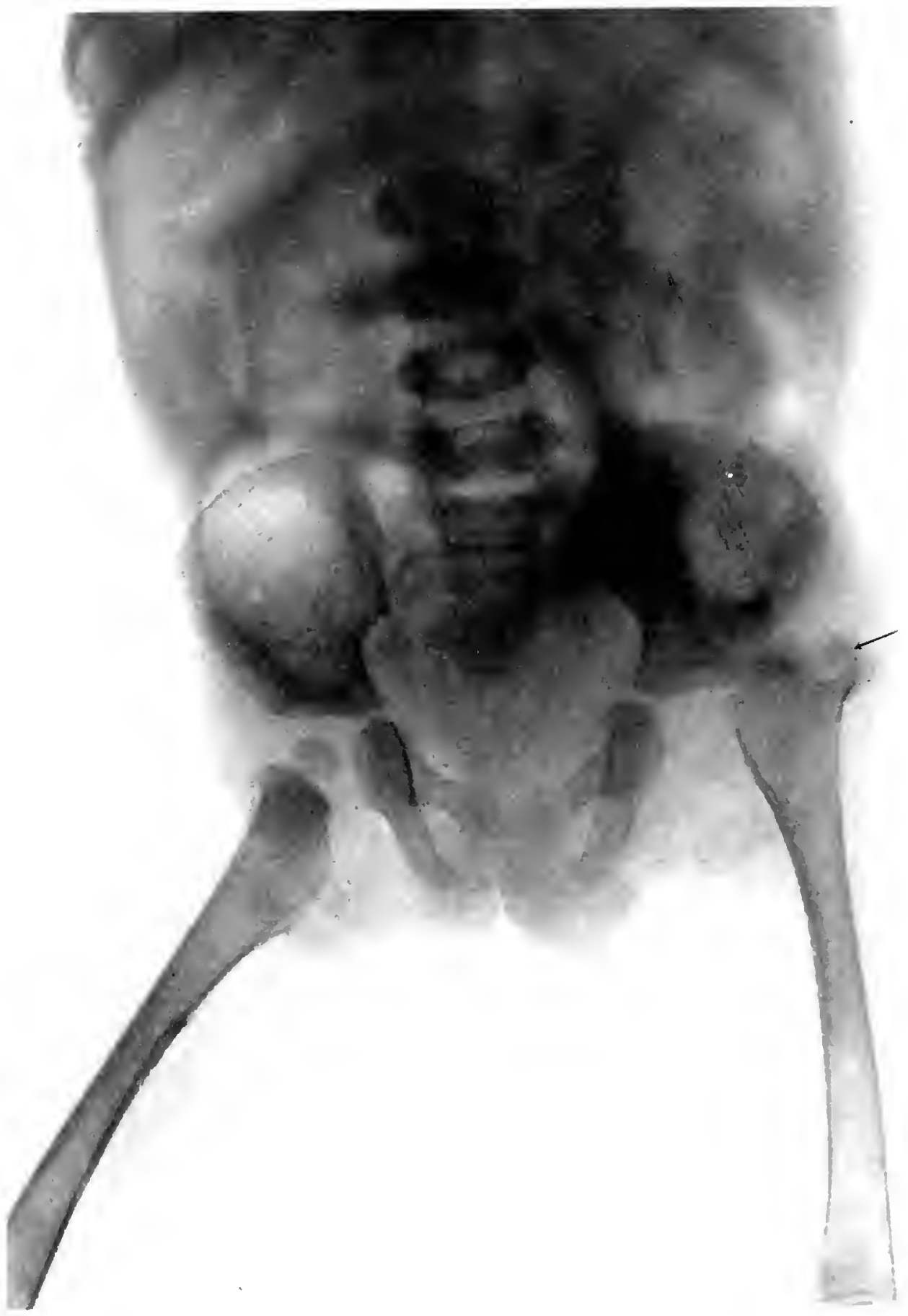


PI.XTE 2018.

RHETILTH TLVER-KNEL-JOLT.

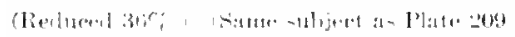

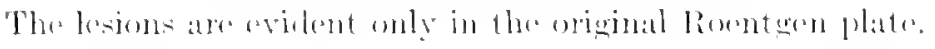


I'L.TE ״US
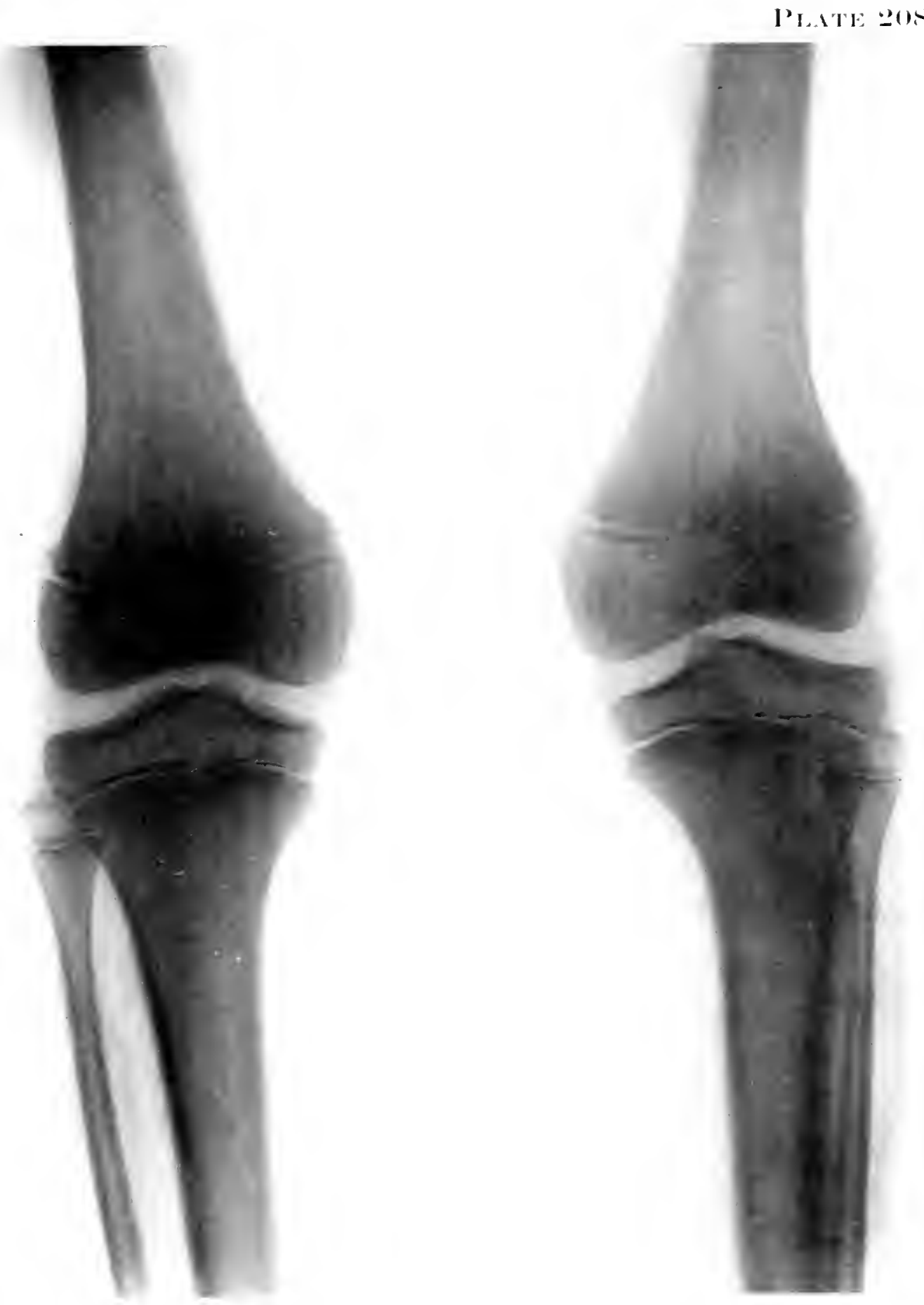
PLITIE: 200.

RIEUMATH FEVER-ANRLE-JOINT.

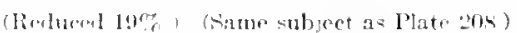

The lesions are evident only in the original looenteren plate. 
P'LATE: 209

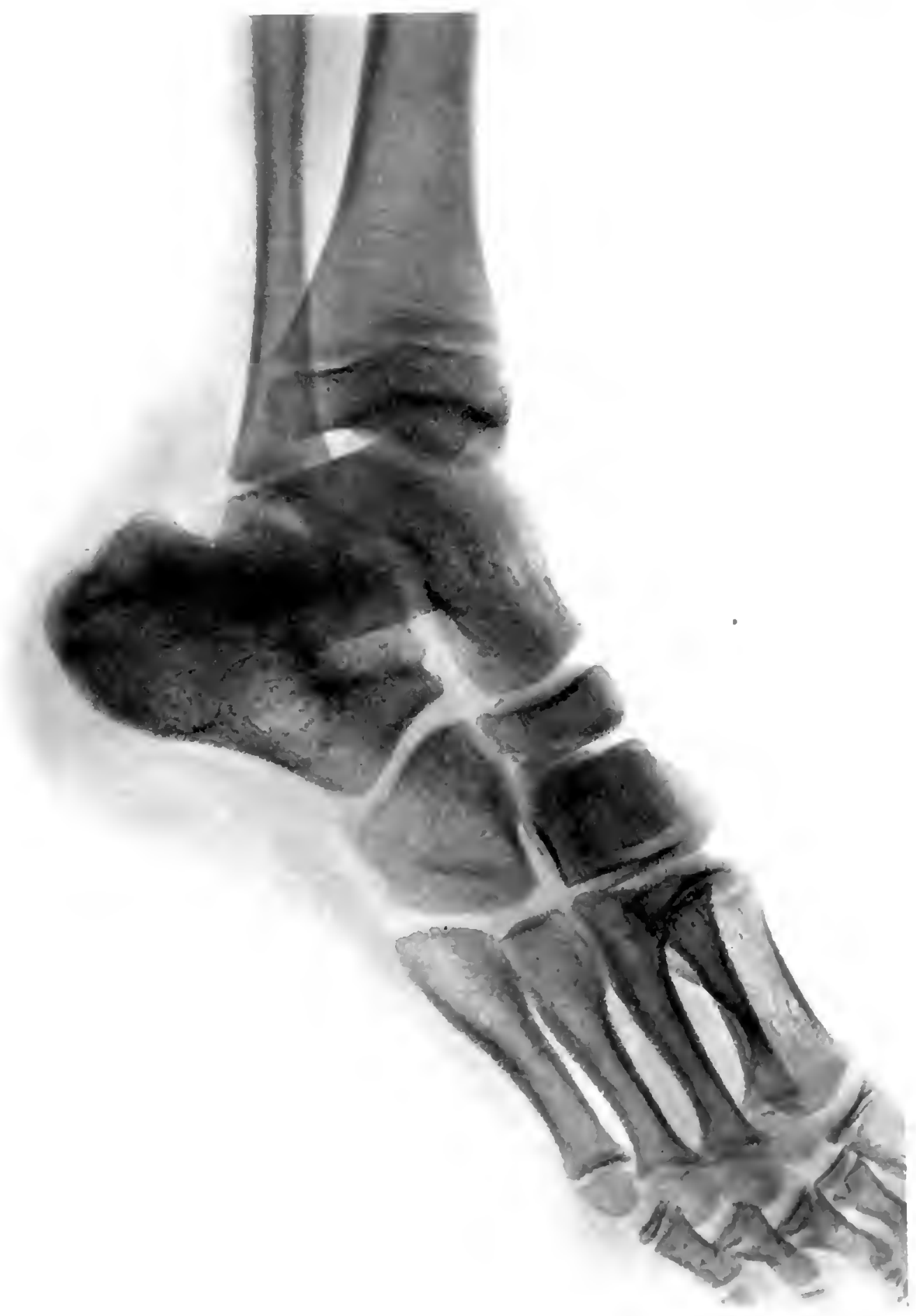




\section{PLATE 210.}

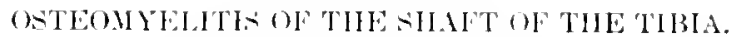

(Arehiv. I'ndiatrics. July, 1900).)

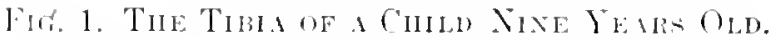

The arrow points towams a fow small arras of increasul ralialility.

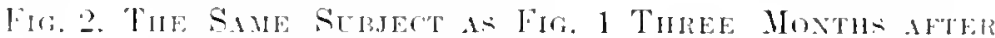
FIMT Plate WA- T.LKL.

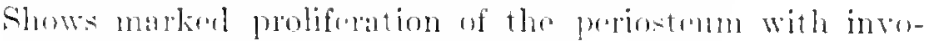
homen formation aluent the whole shaft of the this. 
PLATE :10

FIG. 1 .

FIG. 2 .

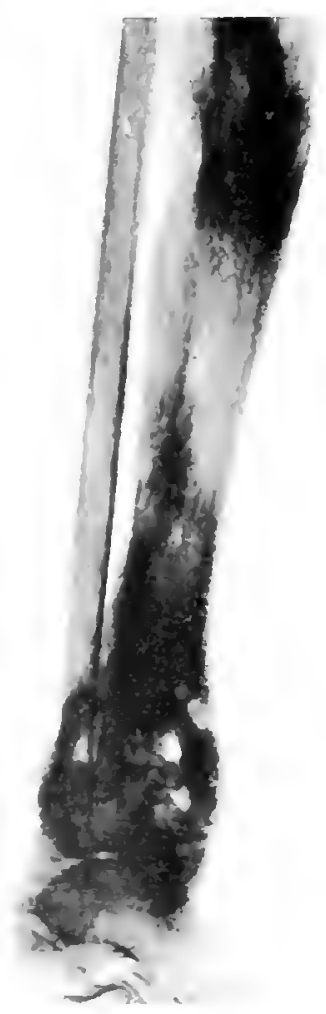


Pl. ITl: 211.

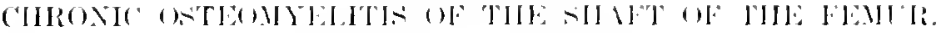

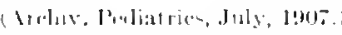

The arrom points tomatris the formation of an involuedum of the whole shaft of the fomme with the fommtion of at secpuretrmm.

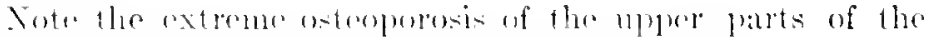
shaft and of the epophyses of the tibia and fibulat. 
PI.ITE 211

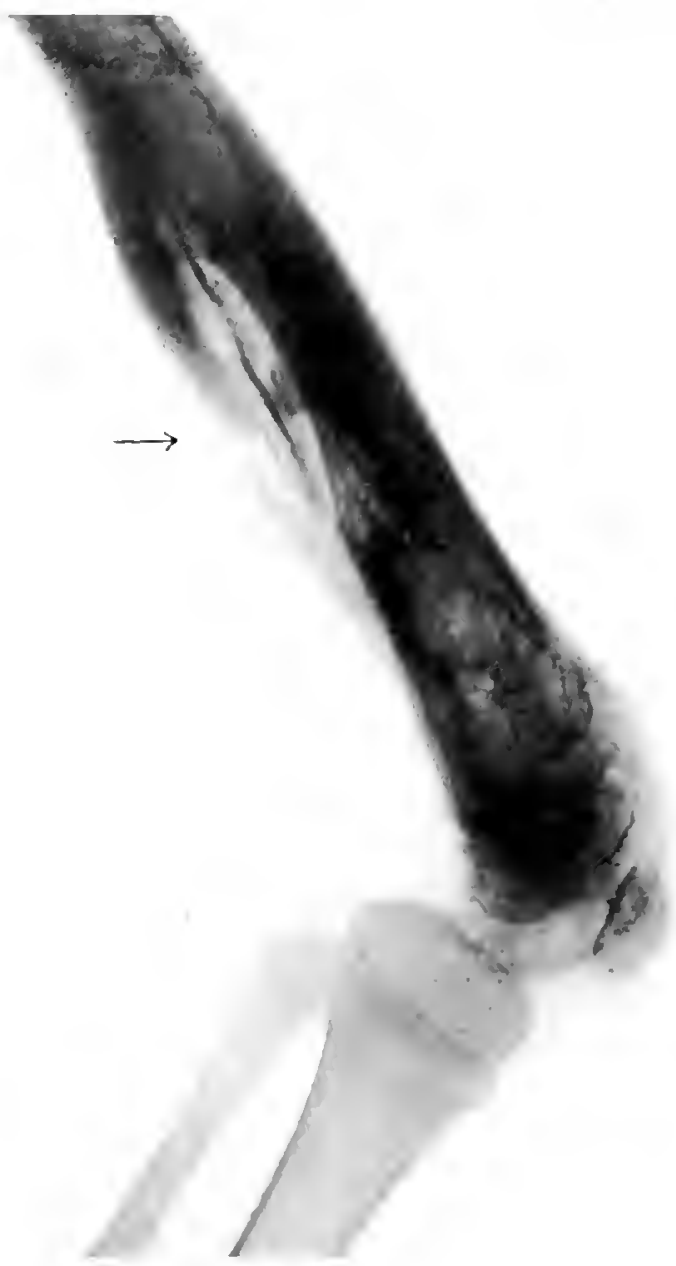


PLITE: 212.

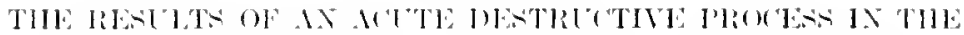
IIIIPS.

(Arohiv. Iיwlattice, July, 1907.)

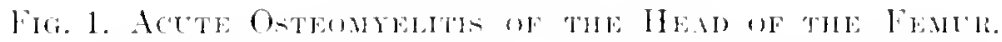

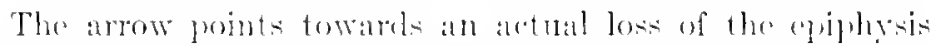
and part of the neck of the fromen, with imenularity of the acetabulum due to an early arute non-tulererular infection.

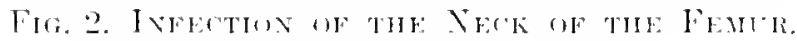

The arrow prints towatsds an abress. 
FIG. 1.

PL.TE: 212

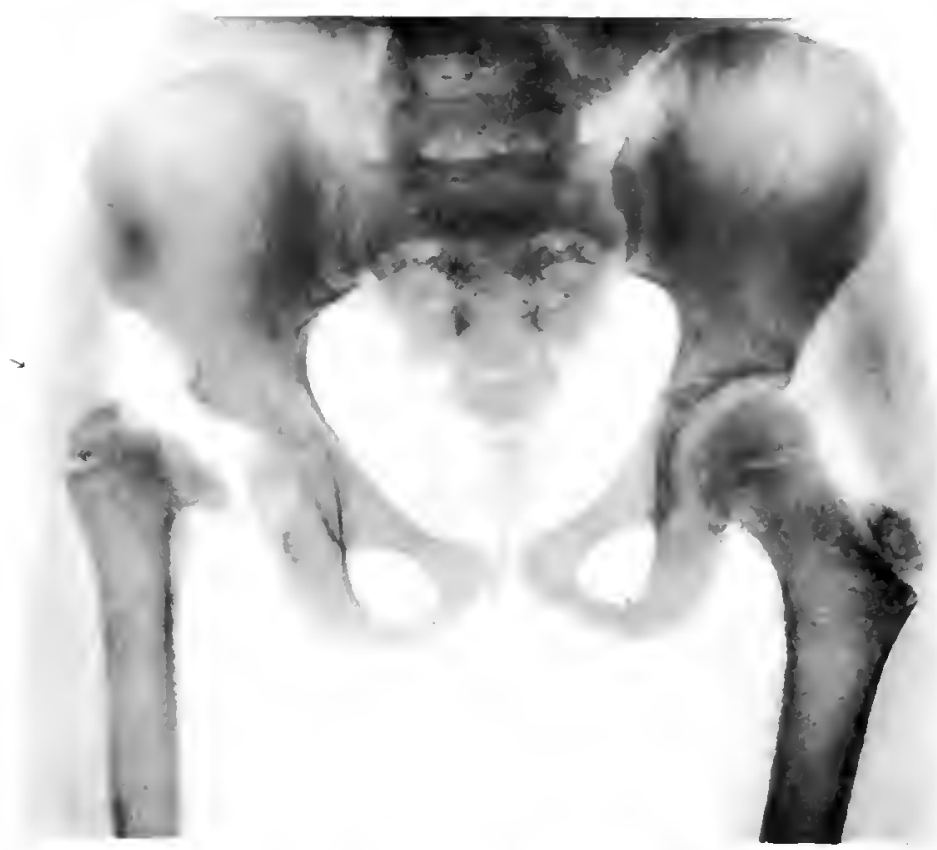

FIG. 2 .

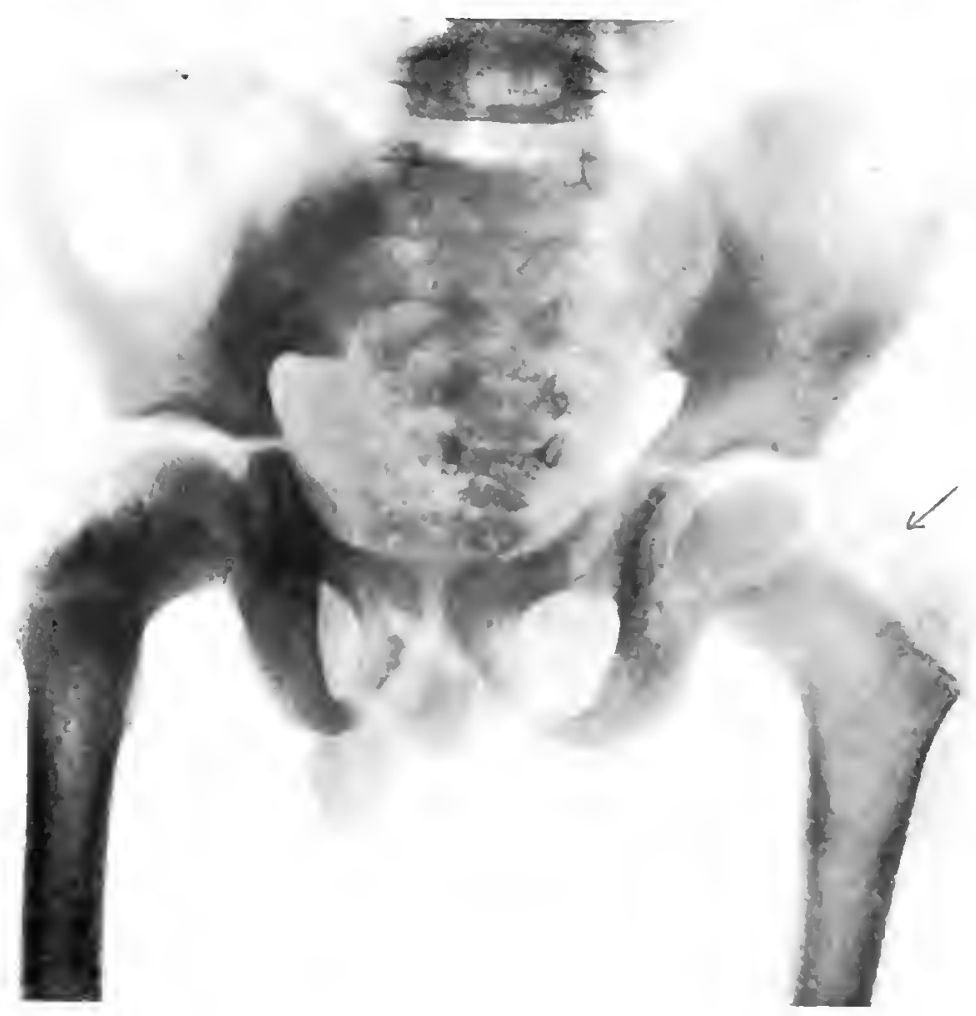


PI.ATF: 221:3.

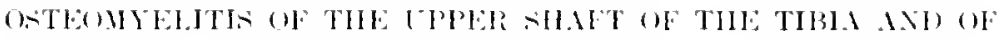
THL: LPPIHSE:

(Arehiv. Pewiatries, duly, 190-.)

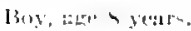

Fus. 1. Neerosis with formation of serpuestmin in the shaft and somerwat in the epiphyses.

The arrow points towards the necrotic area.

Fra. 2. The arrow minte to an area of aletnal neerosis within the niper shatt of the this as woll as in the opiphysis.

The very dene area partly whin the diaphysis and party. wathin the epiphysis is a plug. 
I'I ITH: :21:'

FIG. 2.

FIG. i.
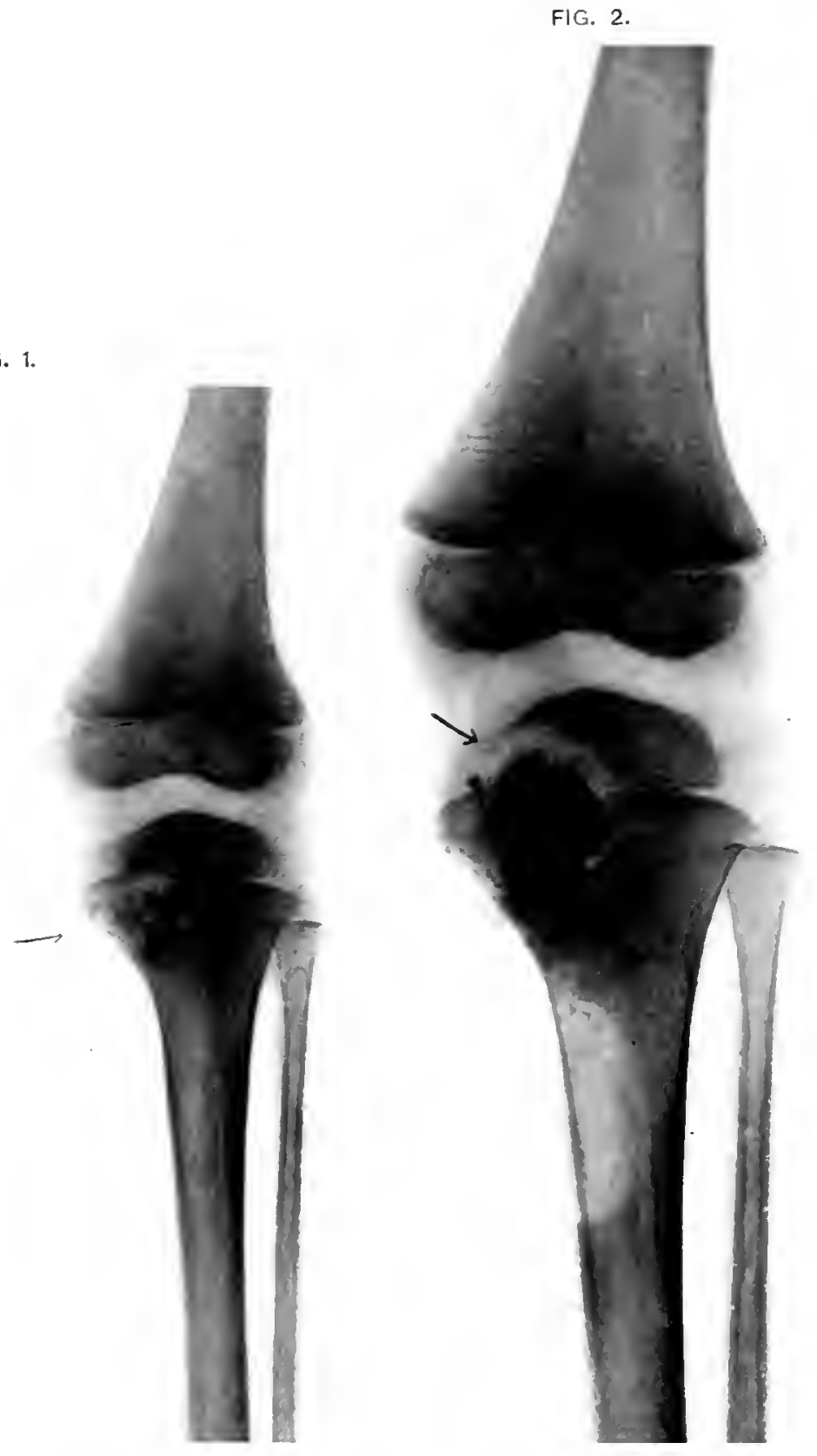
I'L. ITE: 211.

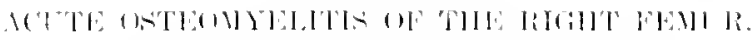

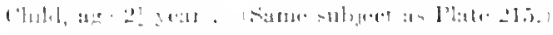

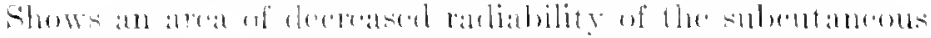

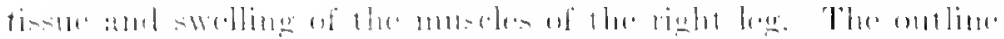

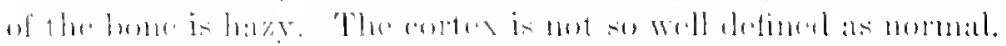

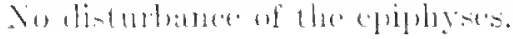


PLATE 214

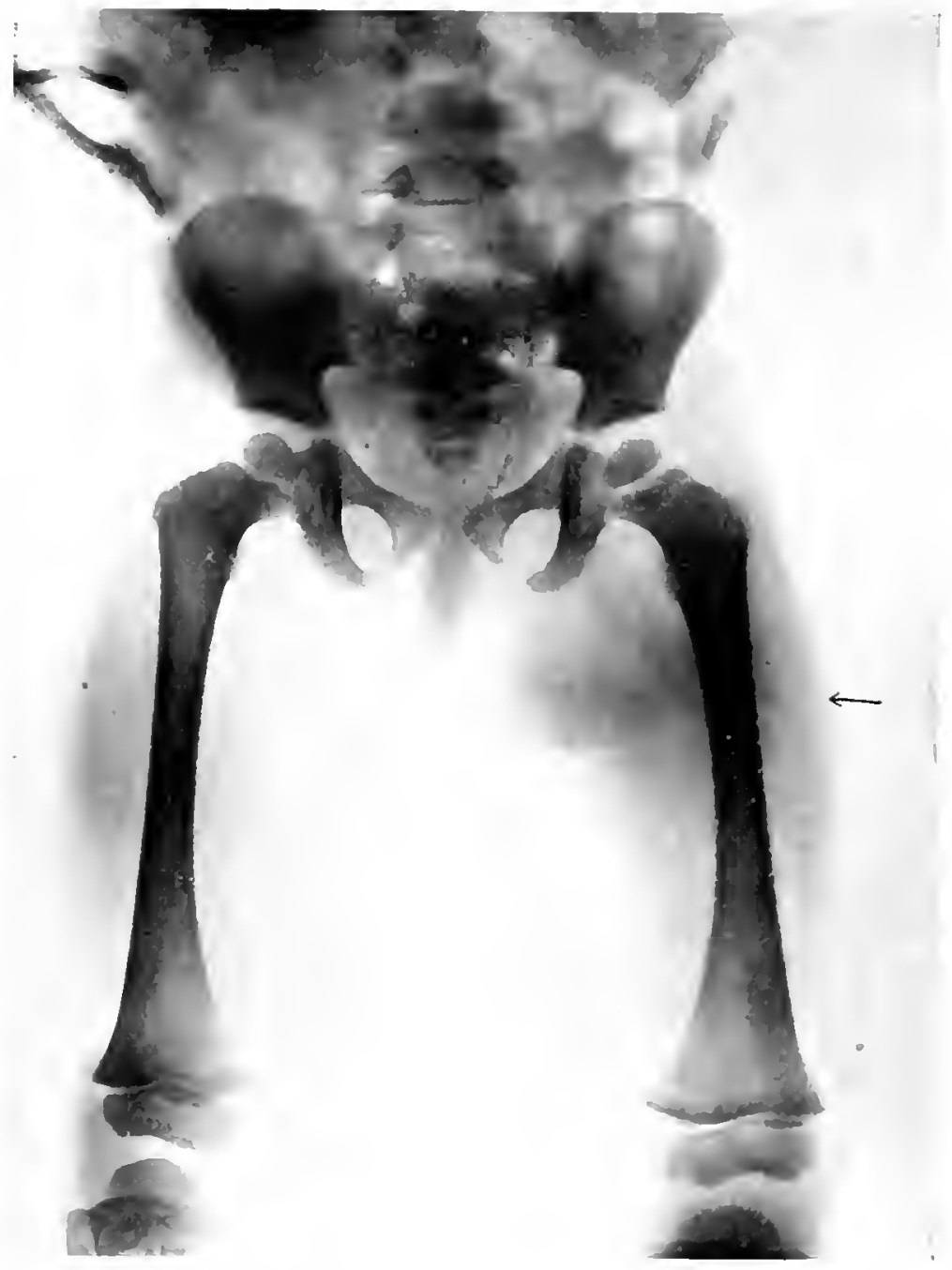


I'LATE: 2-1.

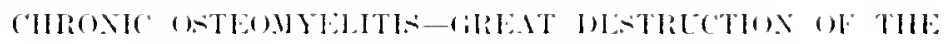
IEXI ?.

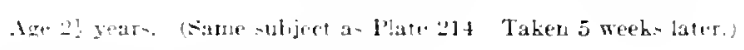

1. In arrat of -uppuration.

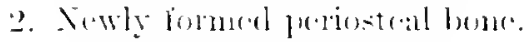

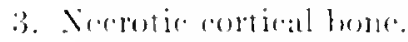

The arrows show points of attachmont of the catpende and periesteum. The shaft of the fomme, the hip- anel the knere joints are not inbolved: the infertion is confined within the limit- of the periostemm. 
Plate 215

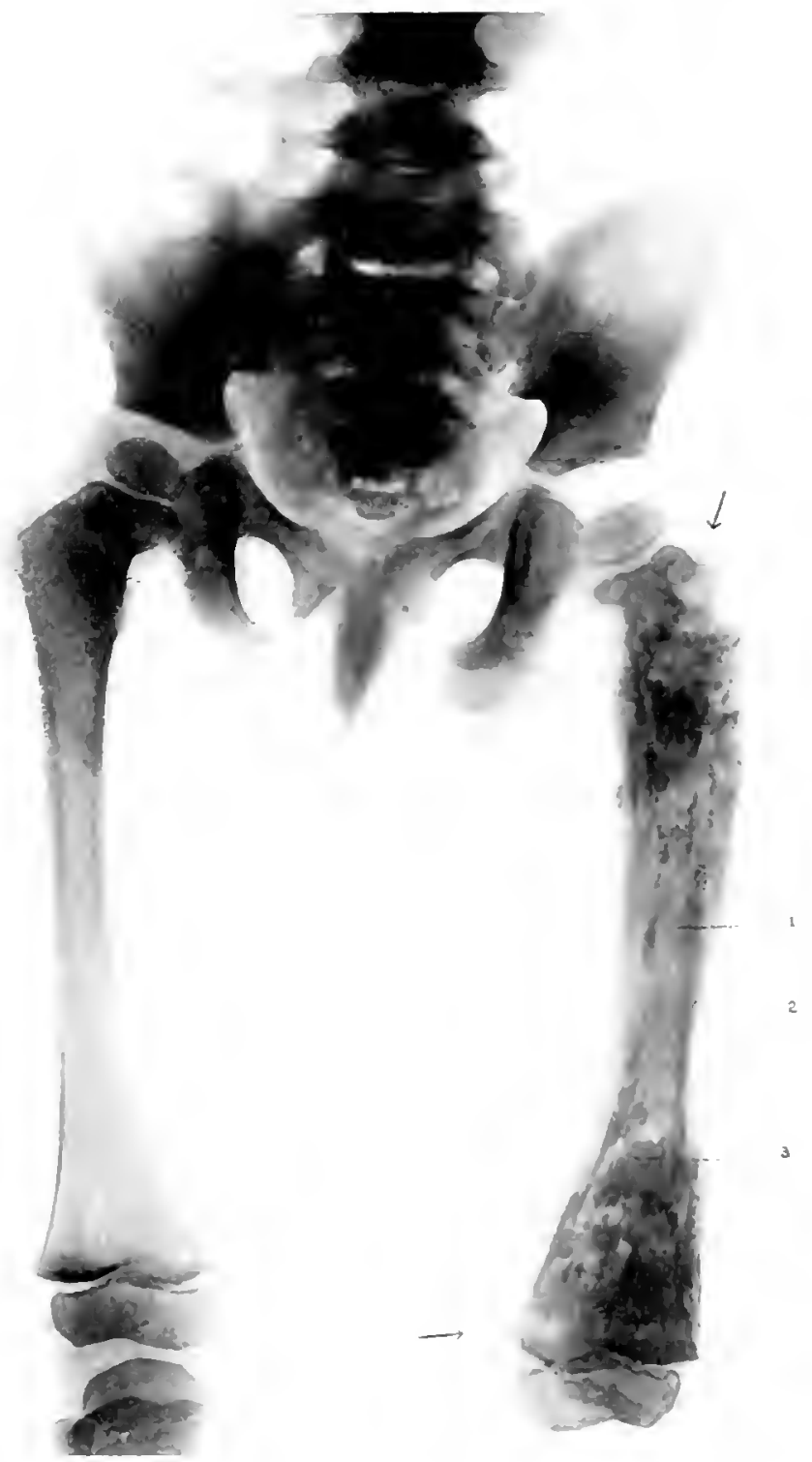


1'l. ITE: 211.

(NEEMVELTTS QF ELBOW.

bur. an a year. (tife -ize

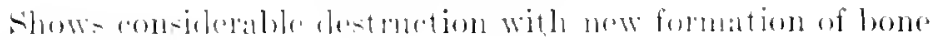
within the joint.

1. Ratertaction of the thaft of the humelus. 


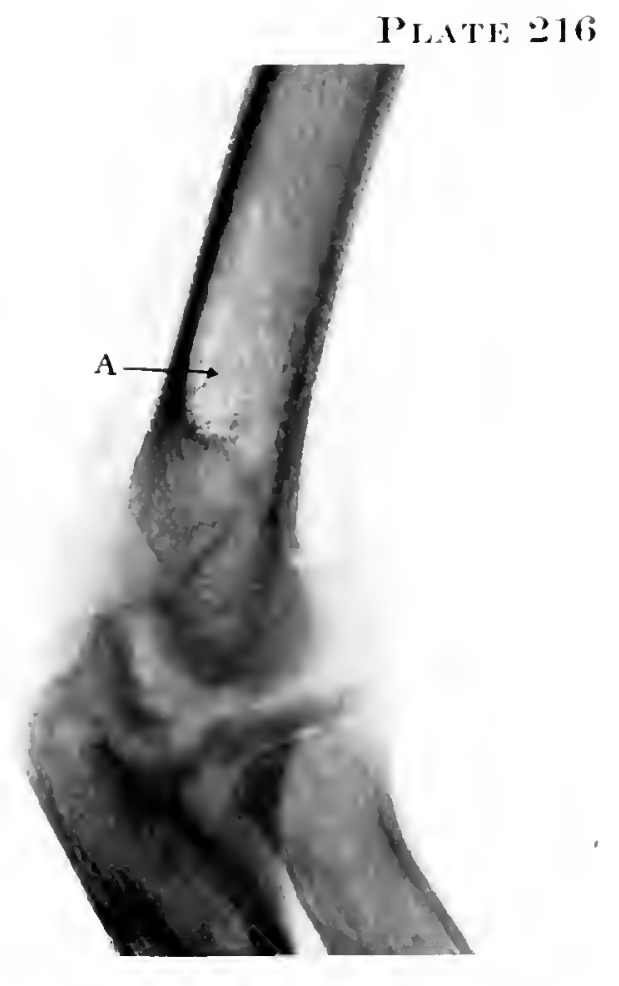


Pl.1Tl: 217.

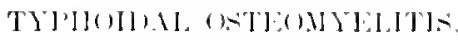

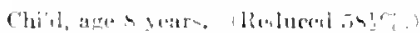

The arrow point towark the humeri, wheh show matked disturbane of the shafts here to nererosis and new formation of bone. 


$$
\text { PLATE } 217
$$

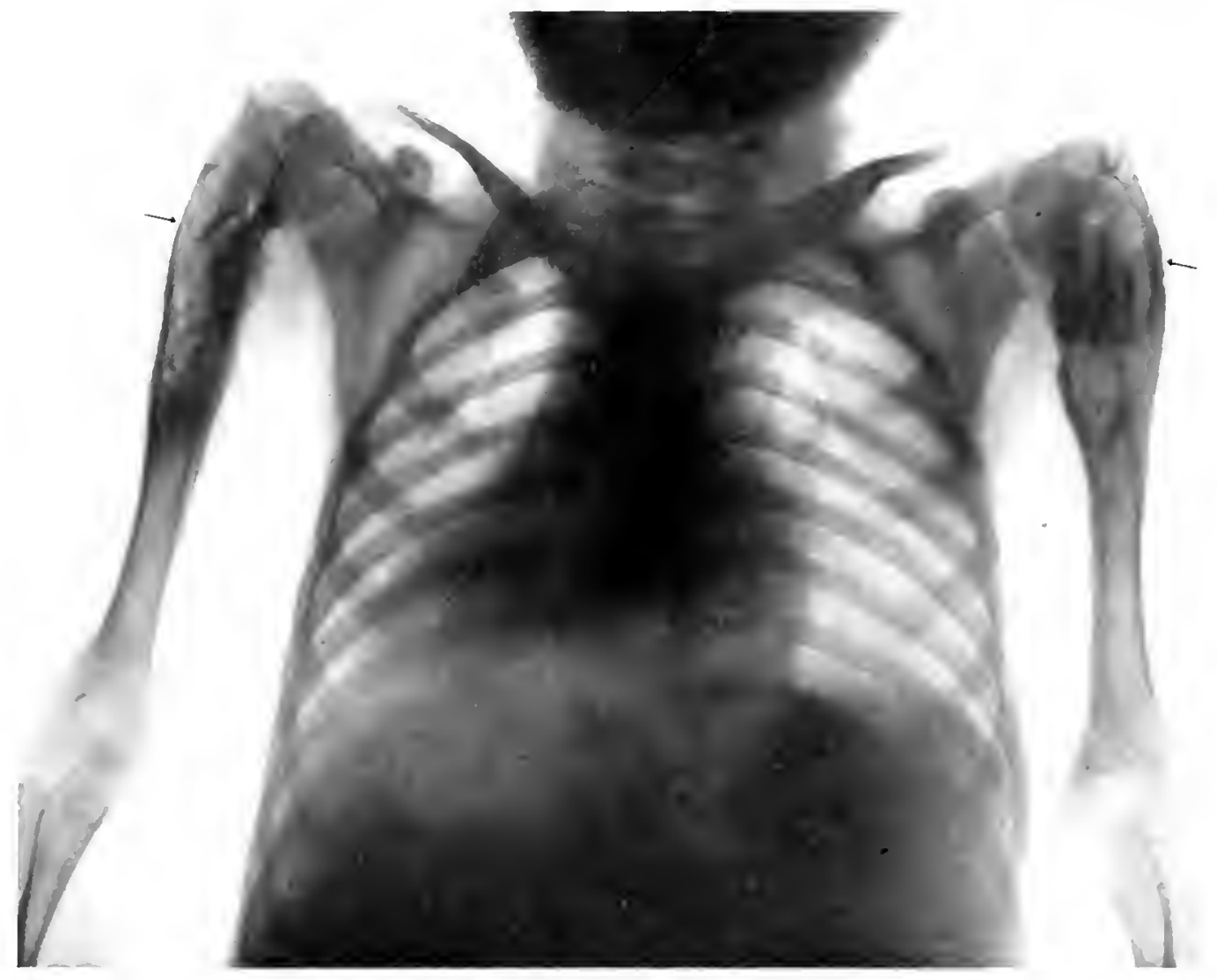


II.XT: 21 S.

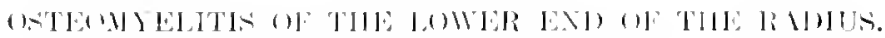
ing a a

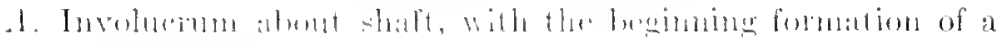
-rymut rum. 
Pinte 218

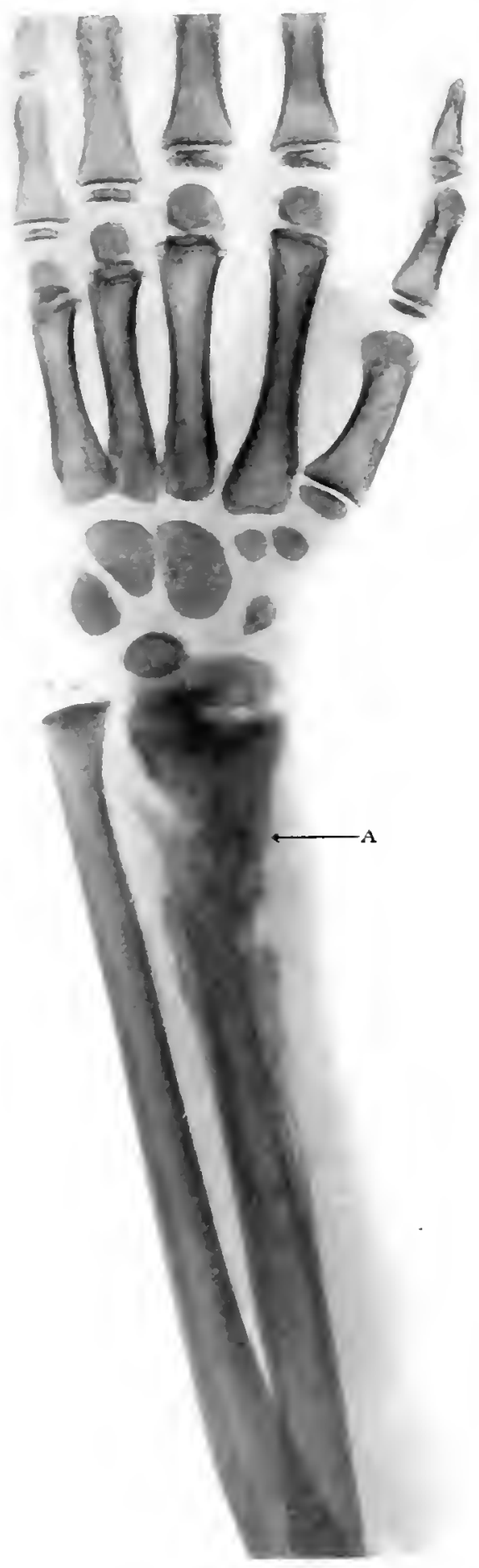


IHATE: 219.

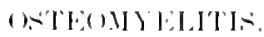

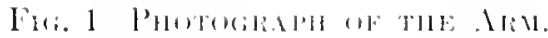

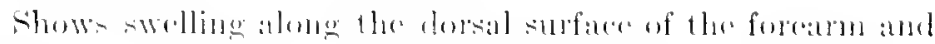
l::1!1.

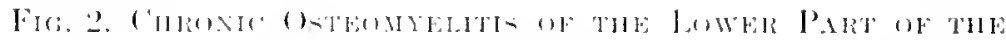

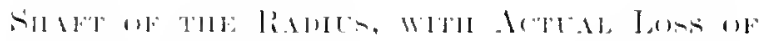
MOST OH THE SHILT.

1. Points to : an ontline of thiekenest tistur.

li. Xew formation of lmme.

e. The ent of the shaft.

1). The (alrpus.

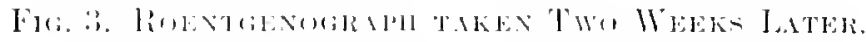

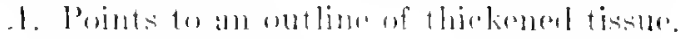

1). The (")

E. Vex formation of lom, 
FIG. 1.

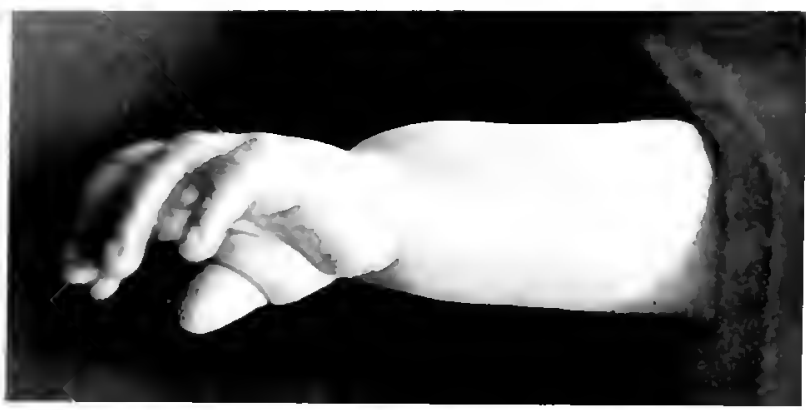

FIG. 2 .

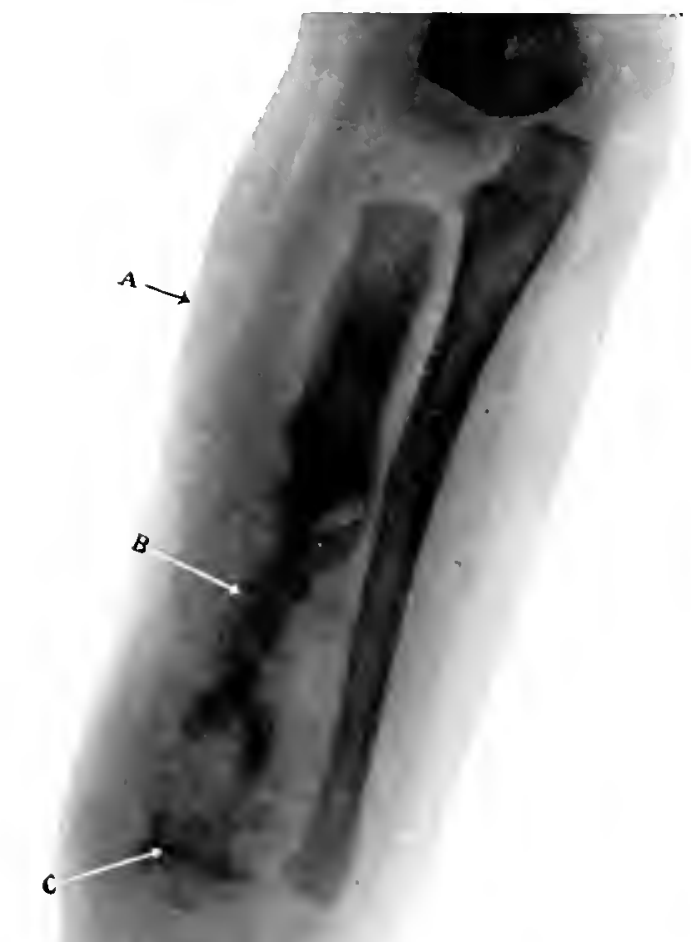

$$
x-D
$$

a)
PIATE: II

FIG. 3.
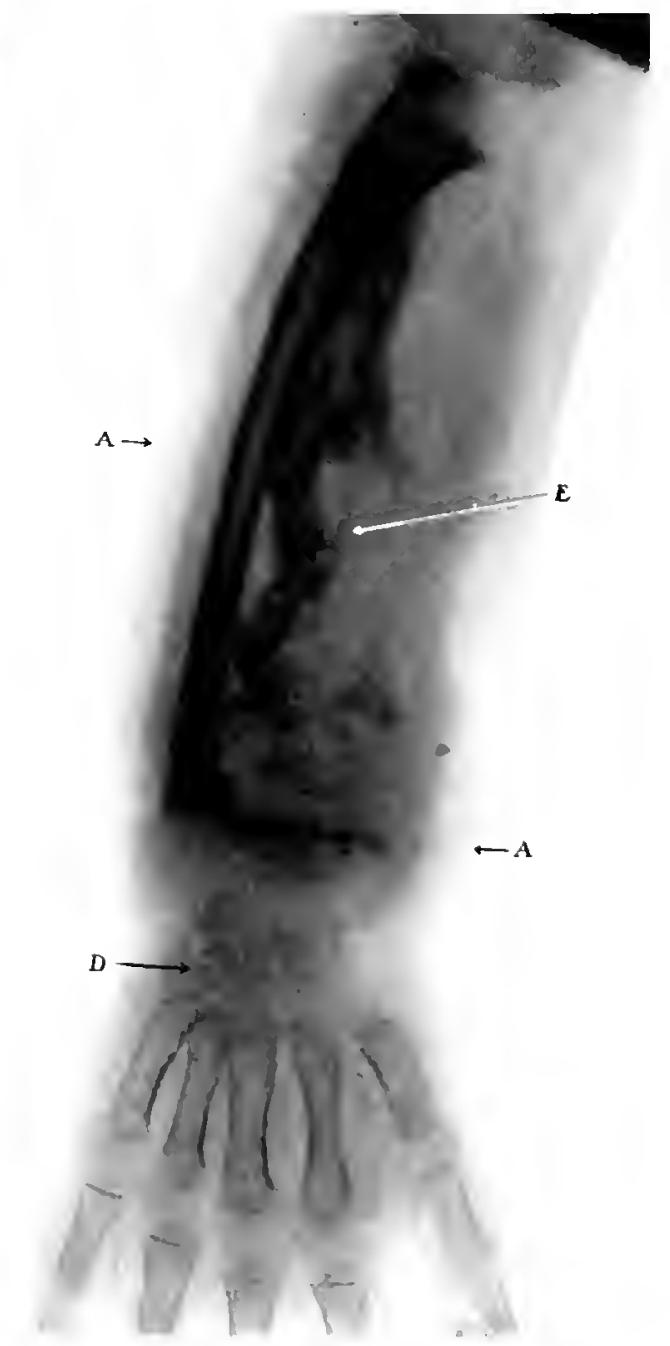
PI.TTY: 2:20.

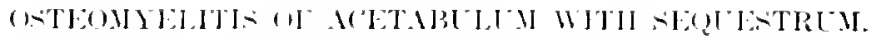

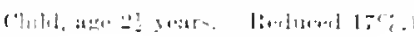

The armo points to a repuretmus in the region of the acrotulum of the left hip.

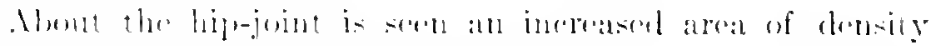

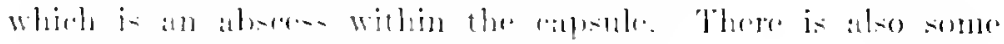
atrophy of the quality of the egiphy-ix. 
PI.TTE 200 
PI.IT': 21.

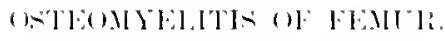

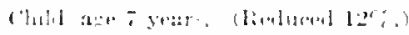

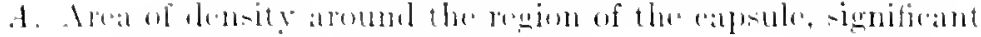
of abserse within the joint.

B. Alrorptim of the diaphysis.

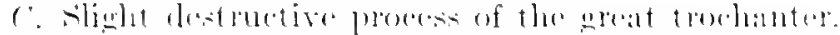

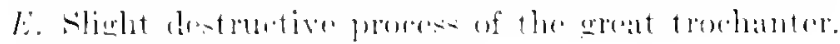

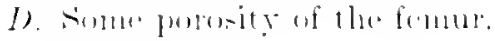




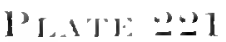

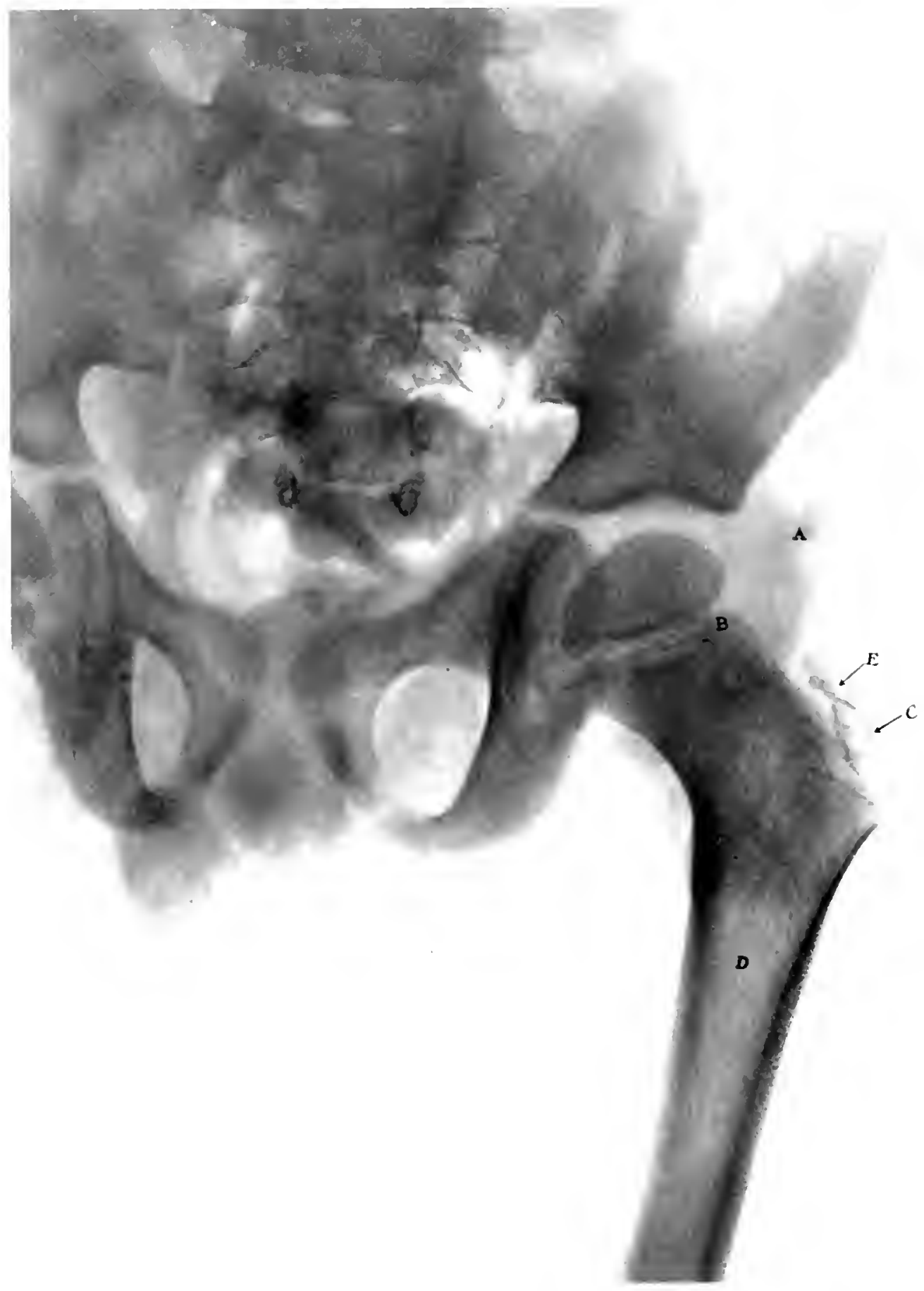




\section{P'I.1'1: 2.:.'.}

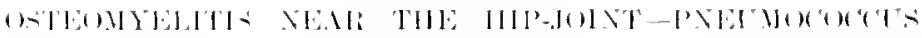
INTETHE.

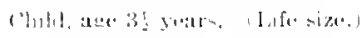

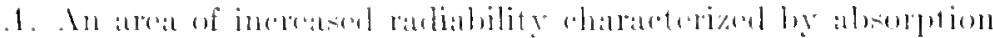
anel formation of a sefurstrum within the shaft of the lonte. 
PIATE 202

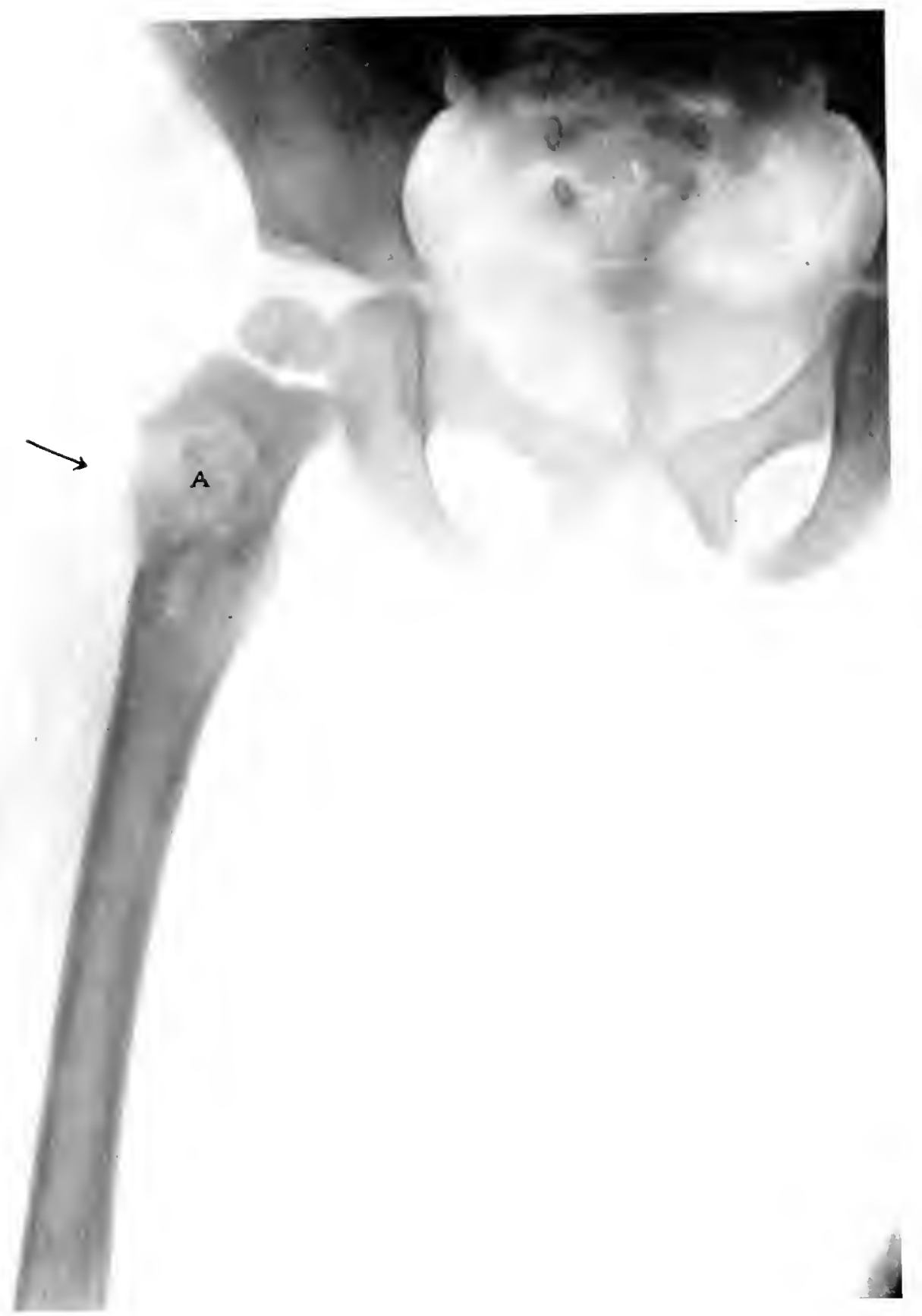


PLATl: 2.:3.

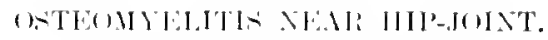

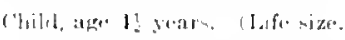

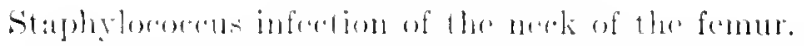

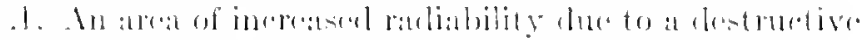
proceses within the bome. Cimatly thickened

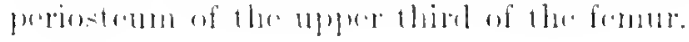




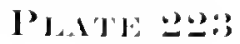

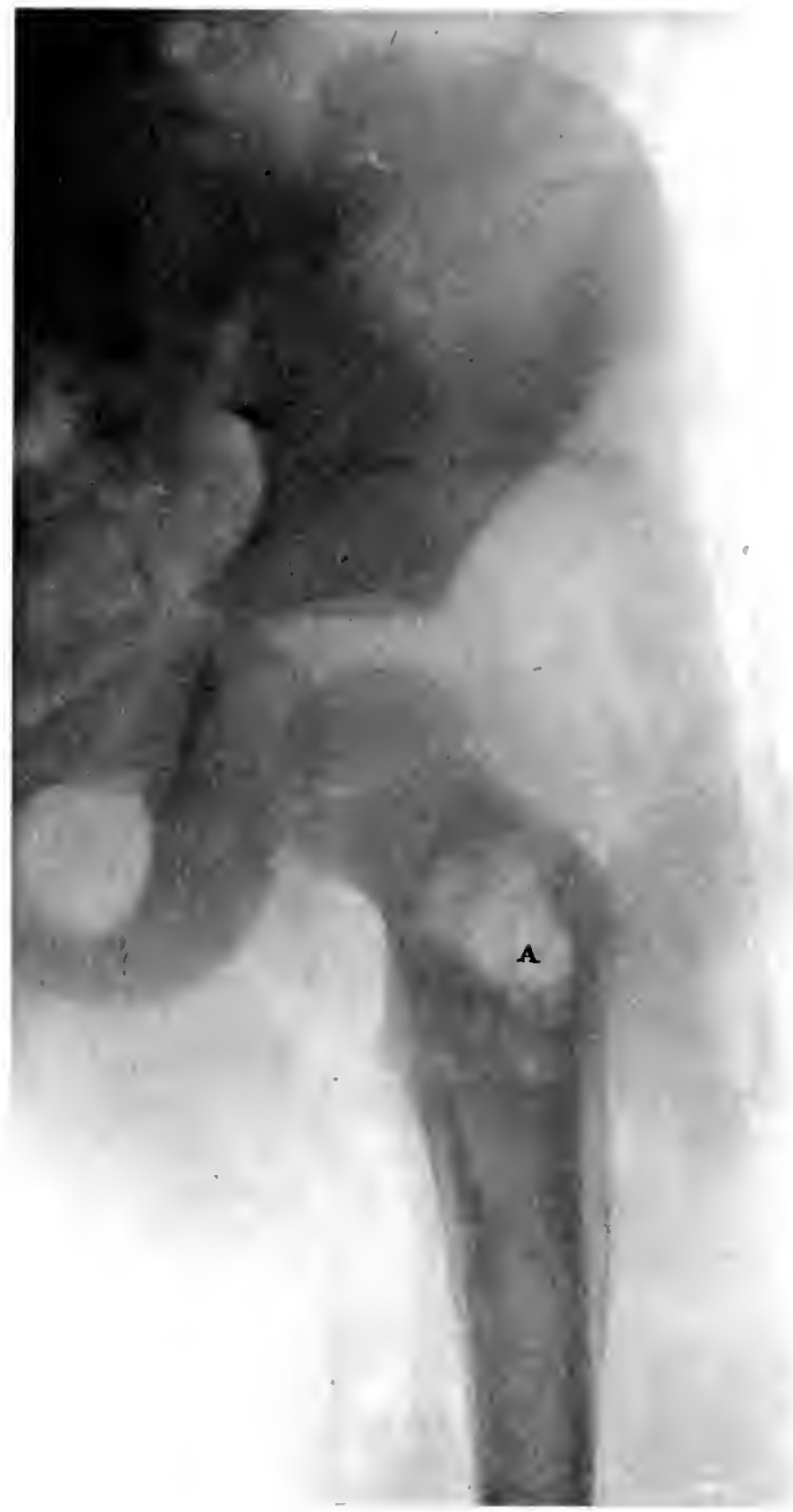


I'I.ITI: 2.2!

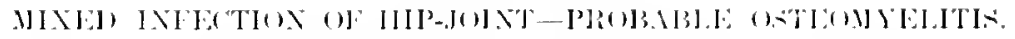

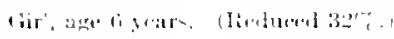

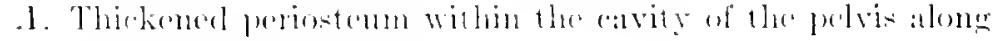
the iliopertimeal lin'.

li. Marked alsorption of the norek and heat of the fommr;

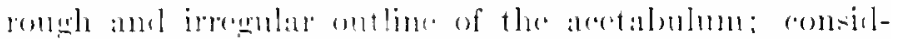

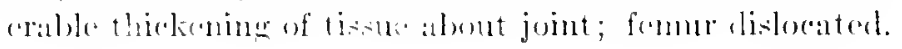


PIATE 204

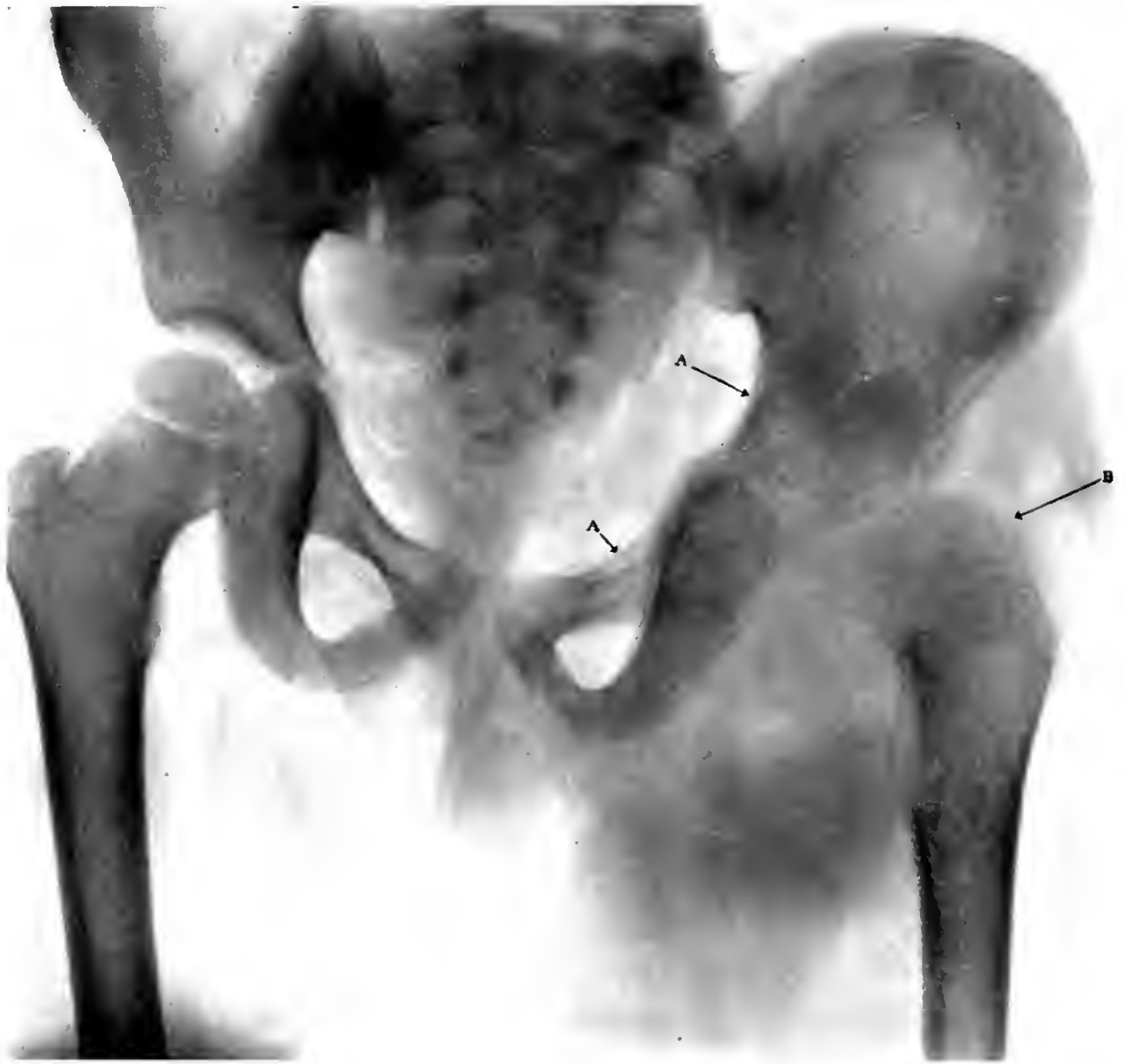


II.1TE 2.25.

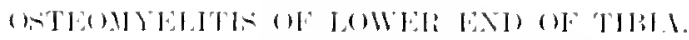

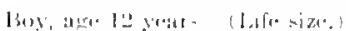

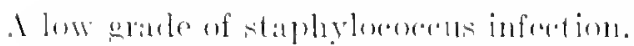

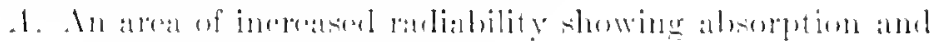
destruetion of the tibiat. 
PIATL: 20.5

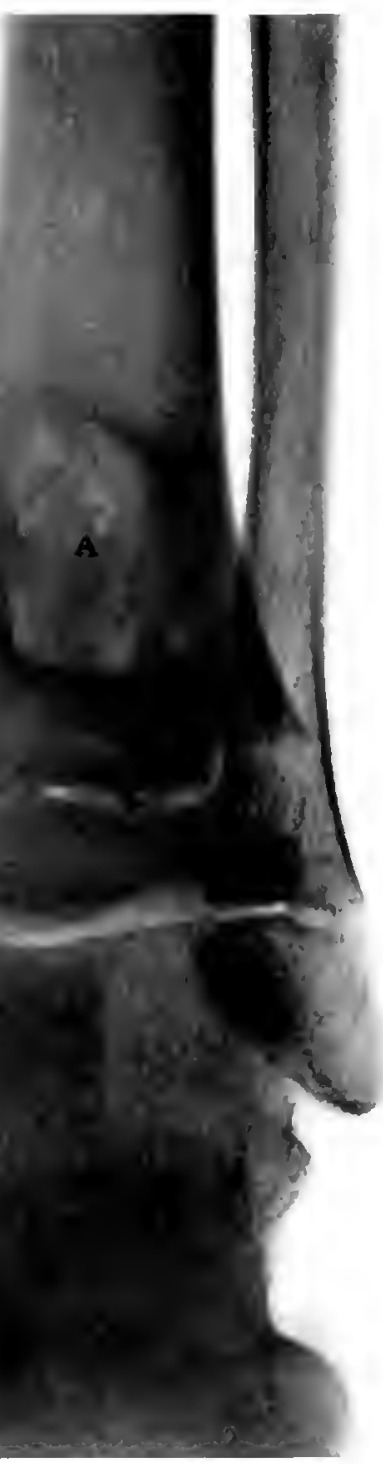




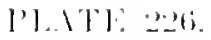

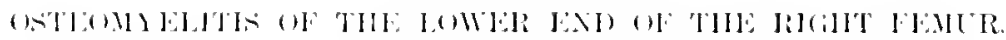

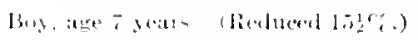

The arowe groint to a proess in the lower end of the fommer

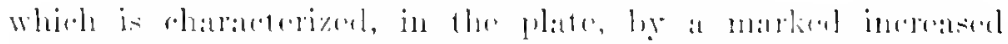

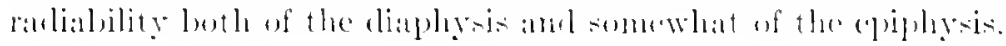




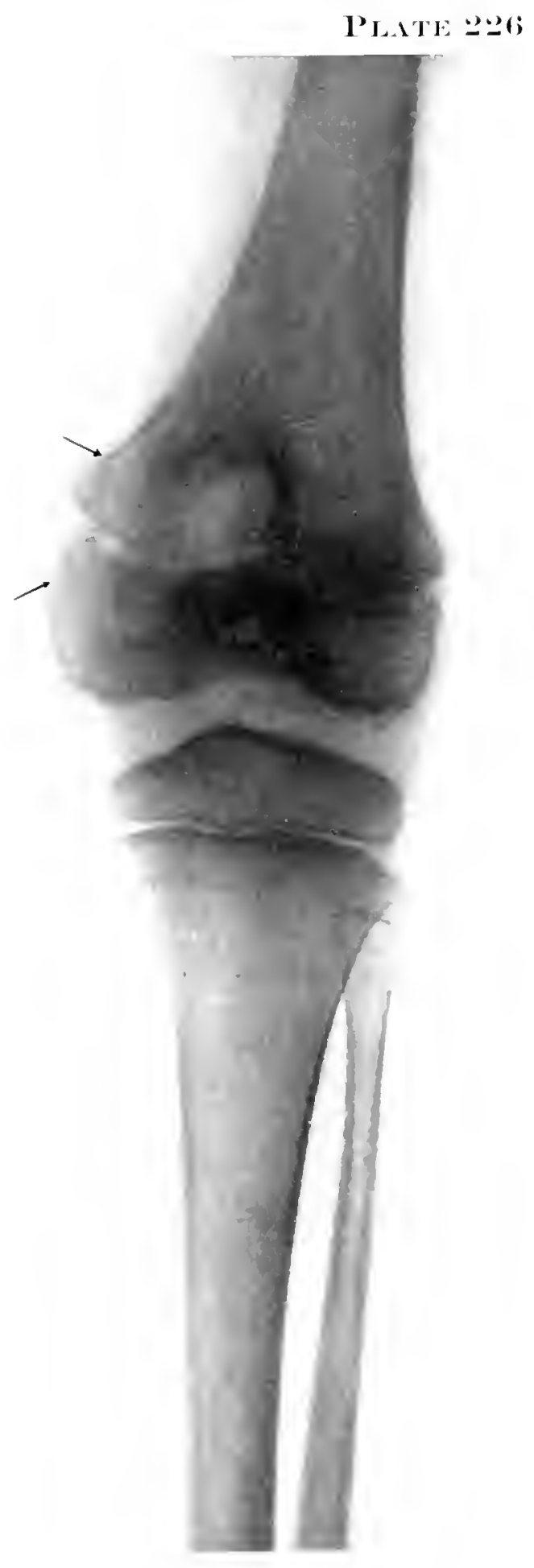




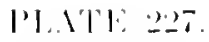

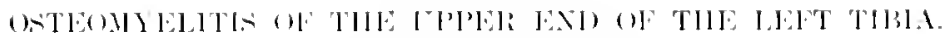

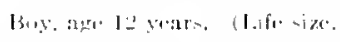

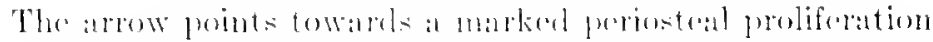

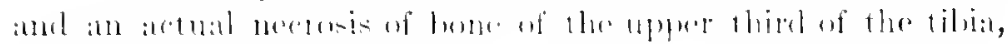

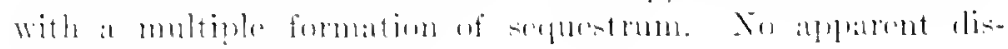

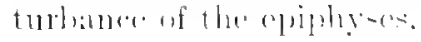


PLATE
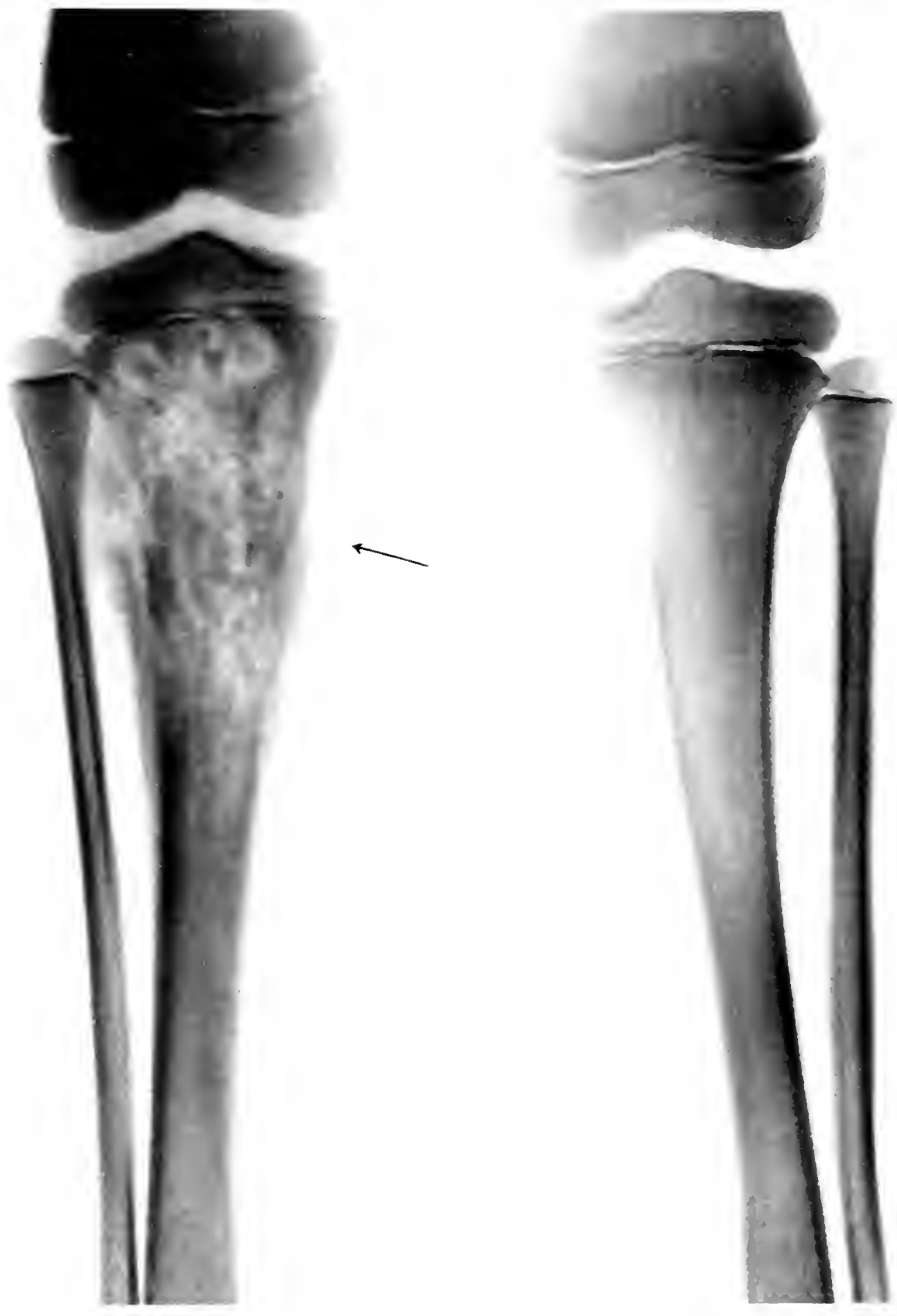
I'ATt: 2.2.

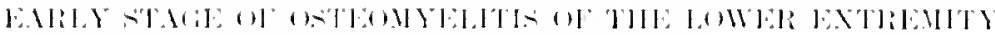
(1) TIIL: TIBA.

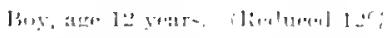

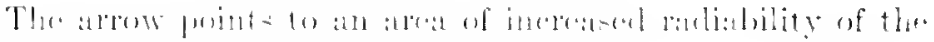

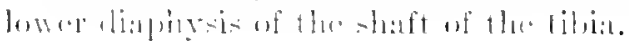




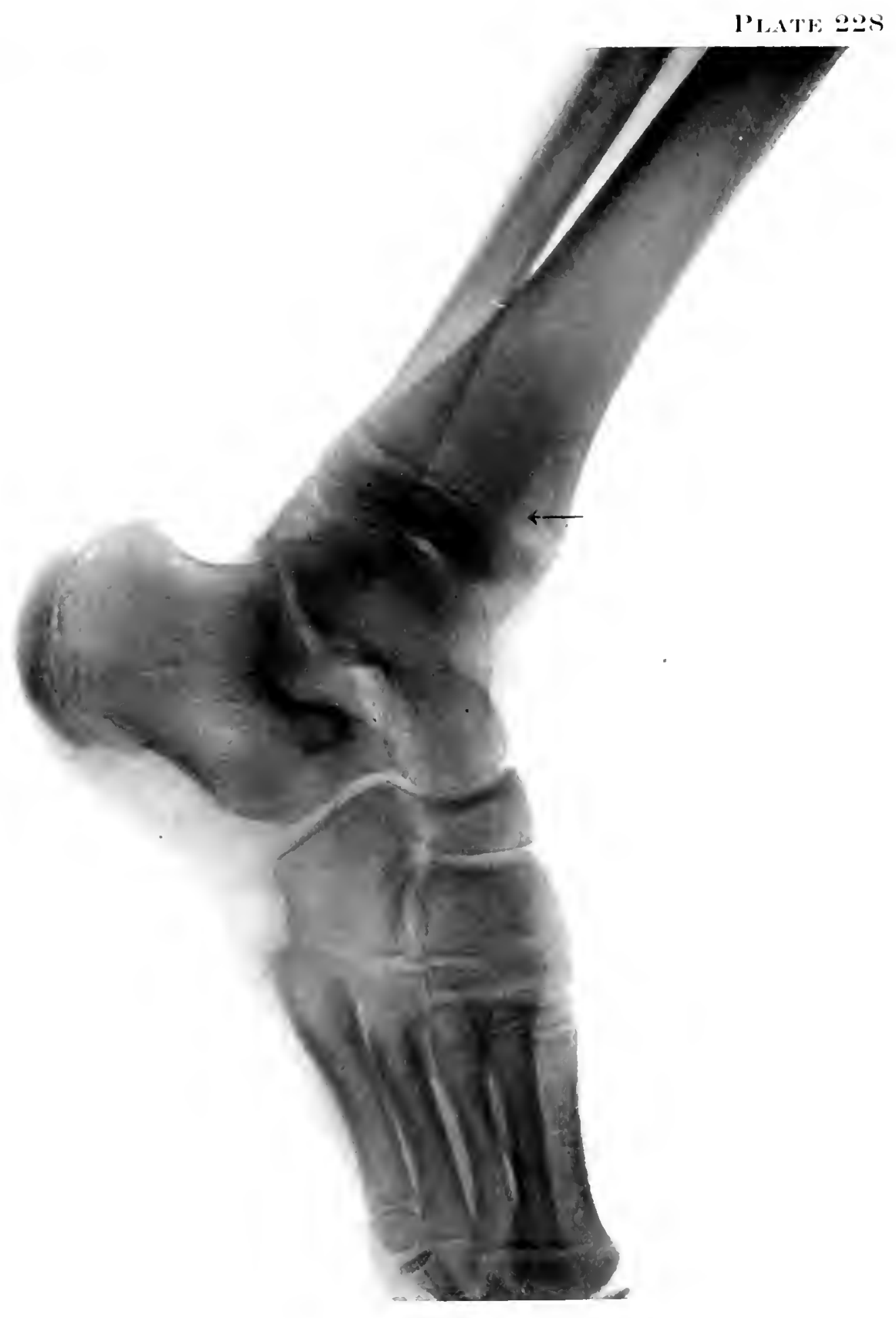


Pl.AT'T: 2.2!!

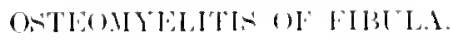

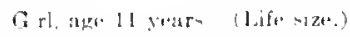

The arrew peints towank the lower thied of the fibula in

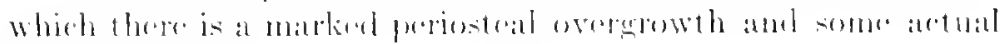
boute nerosis. 
PISTE 20!

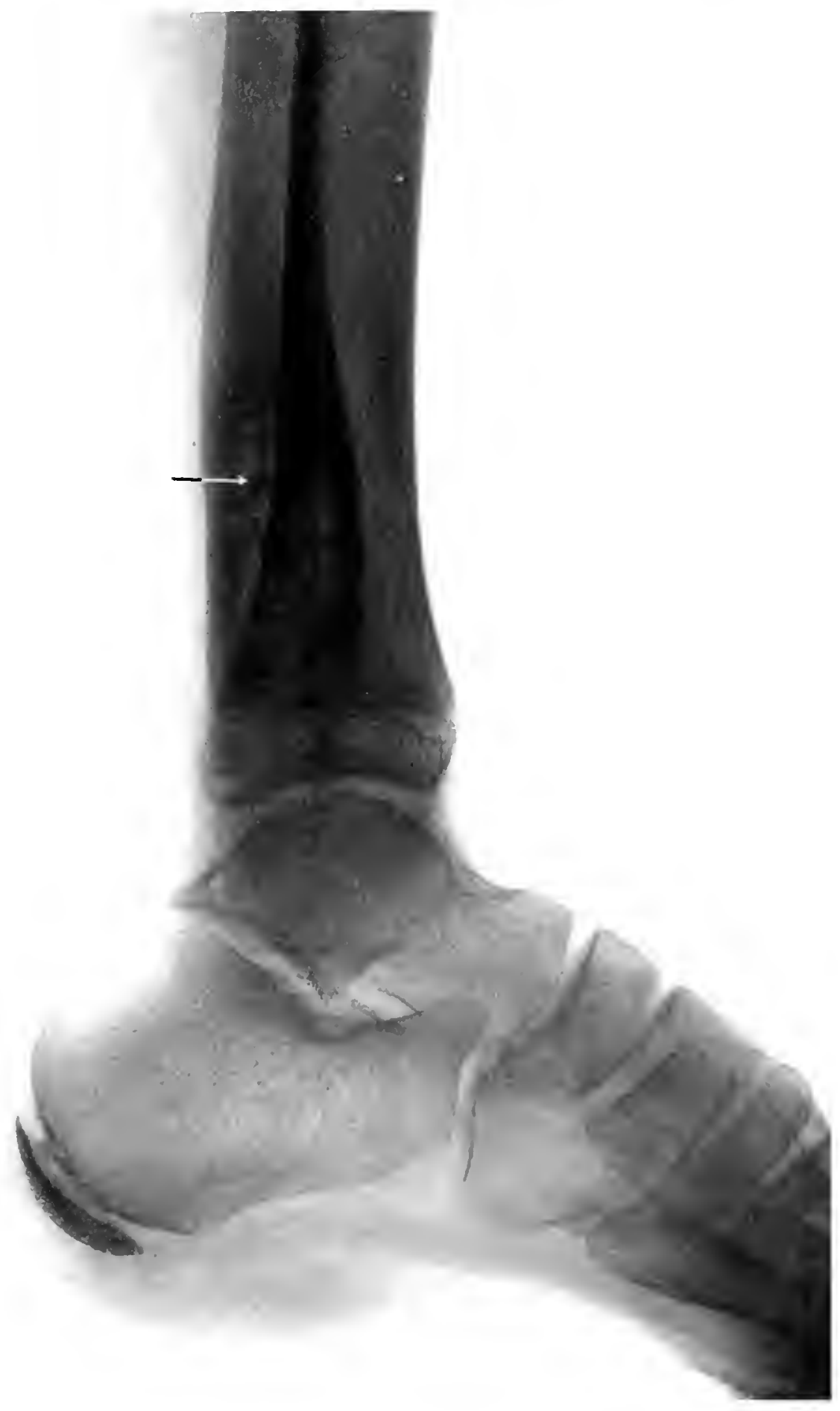


PLATY: 2:30.

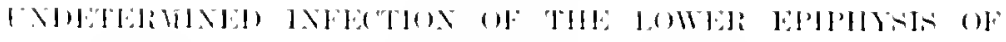
THES JIBI.L.

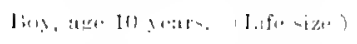

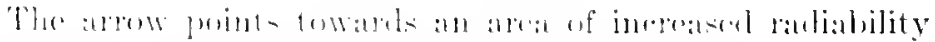

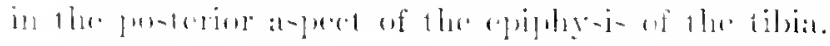


P'LATE 2830

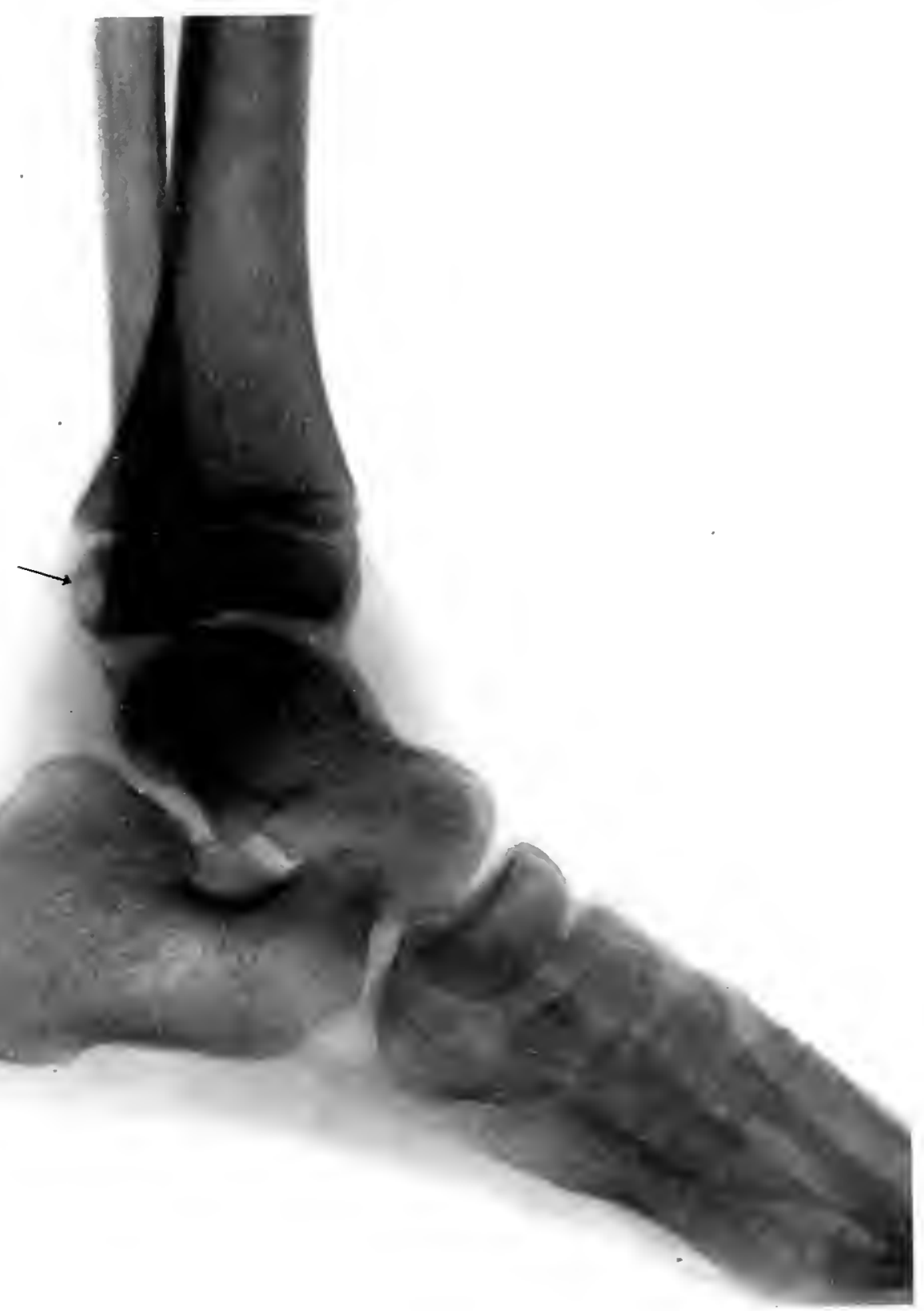




\section{I1.1T1:2:31.

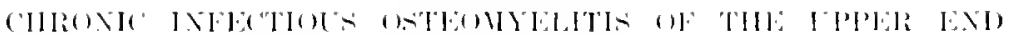 (1) THL: TIIII.

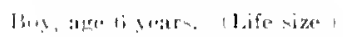

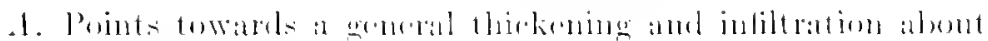
the knce-joint.

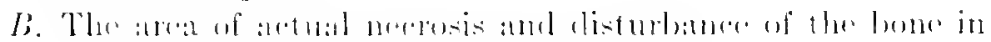

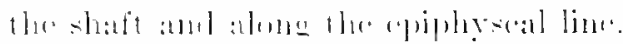

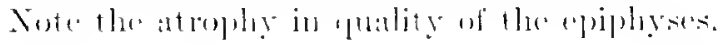


PLATE Z:31

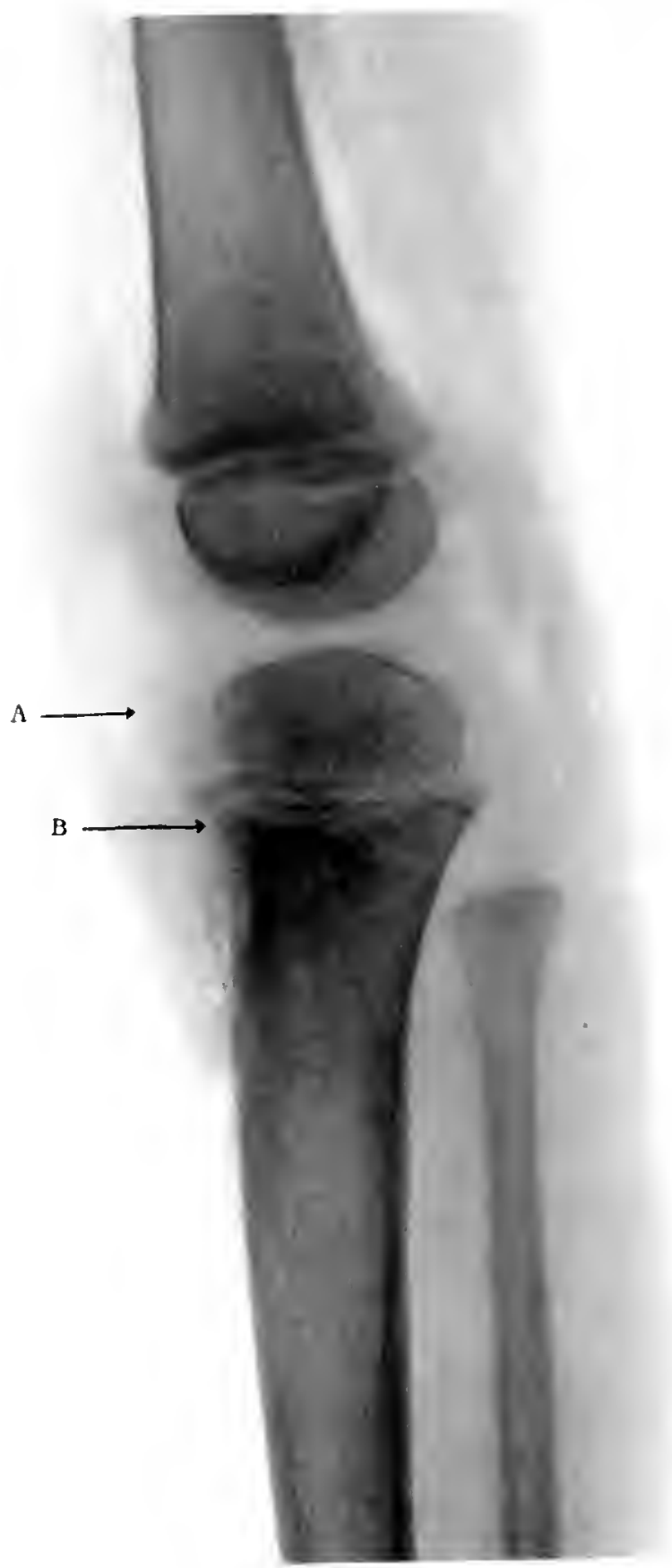


I'L.1T1: 23:3.

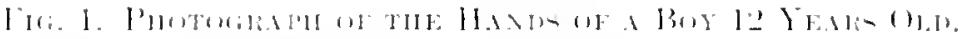

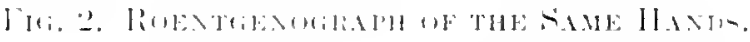

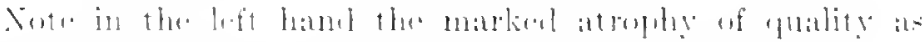

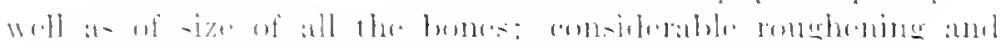

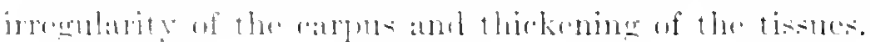


FIG. 1.

PJATE: $2: 3 \%$

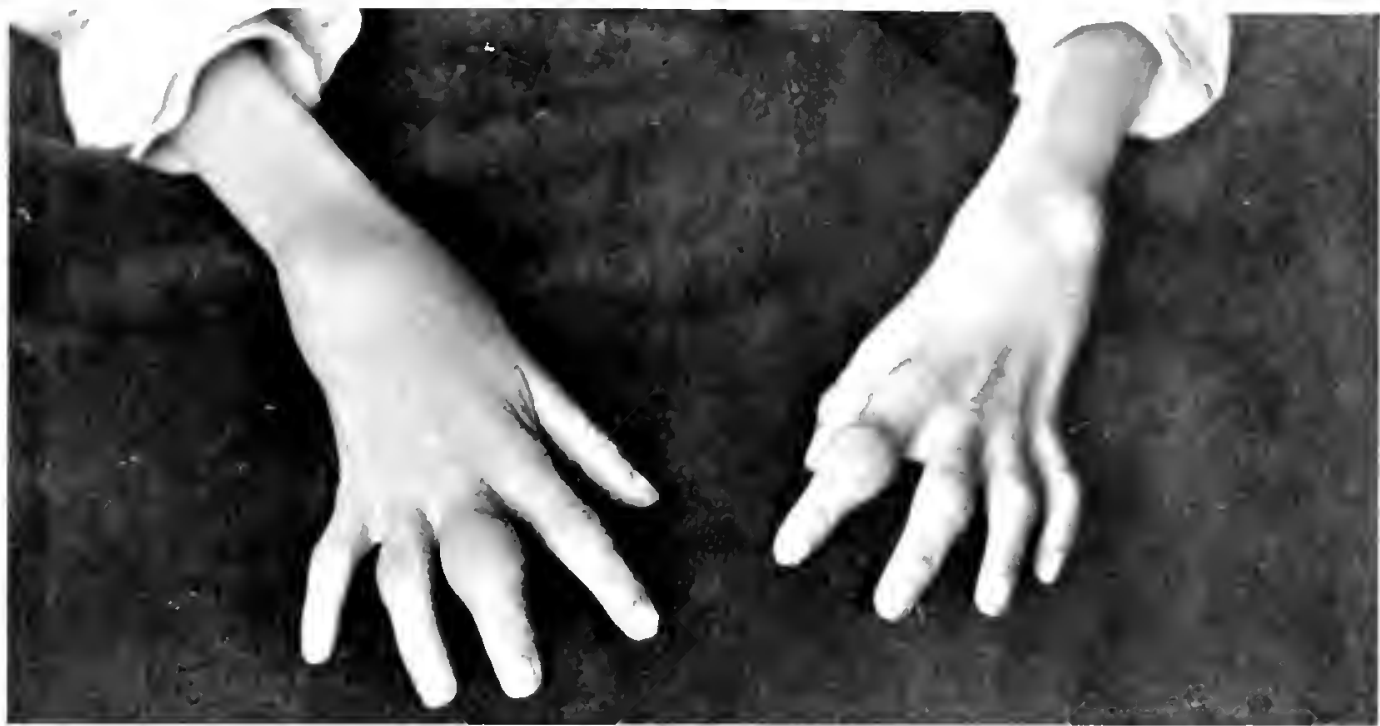

FIG. 2.
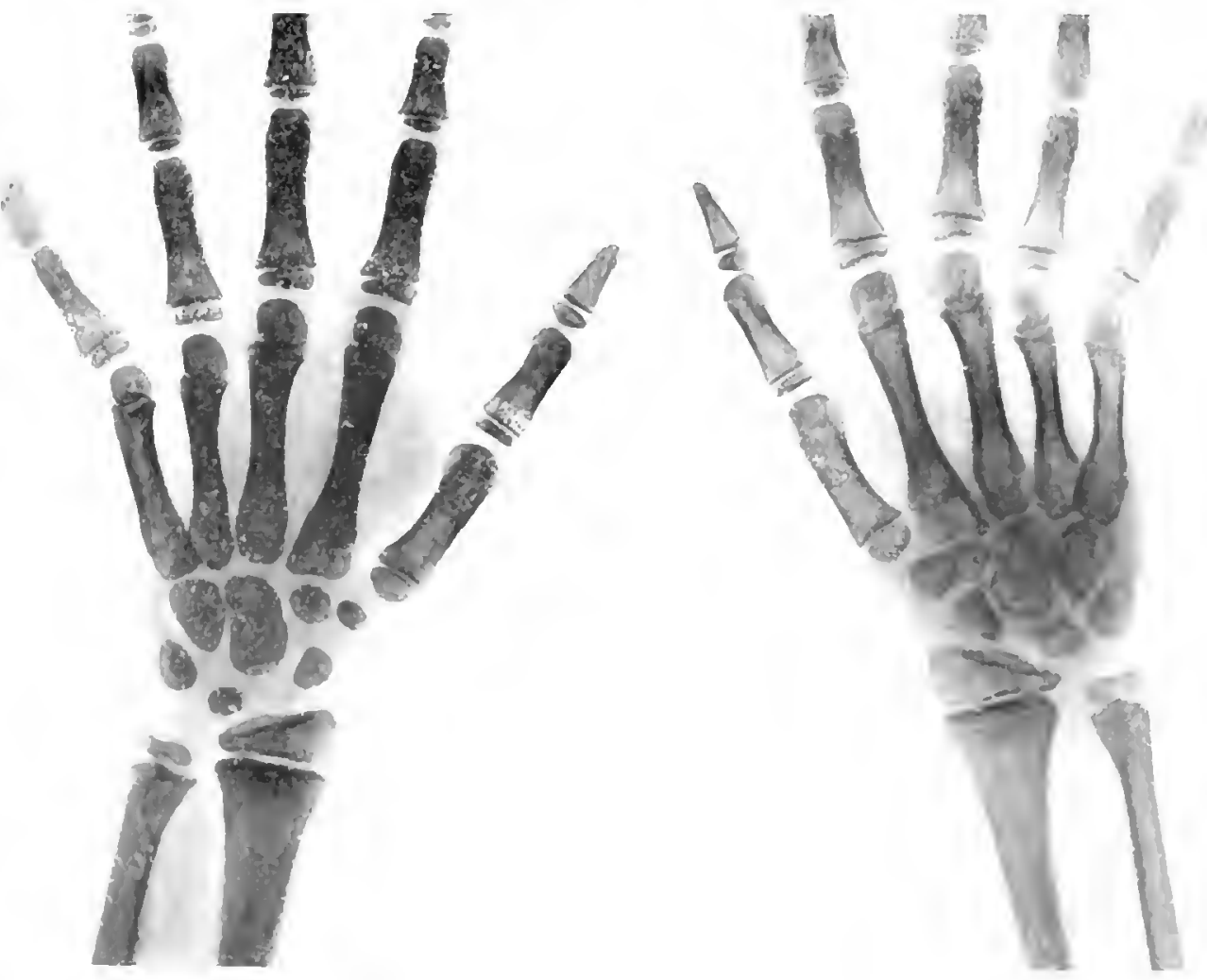
PLATH

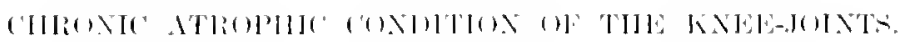

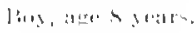

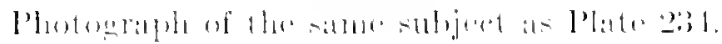


PJATE $2: 3: 3$

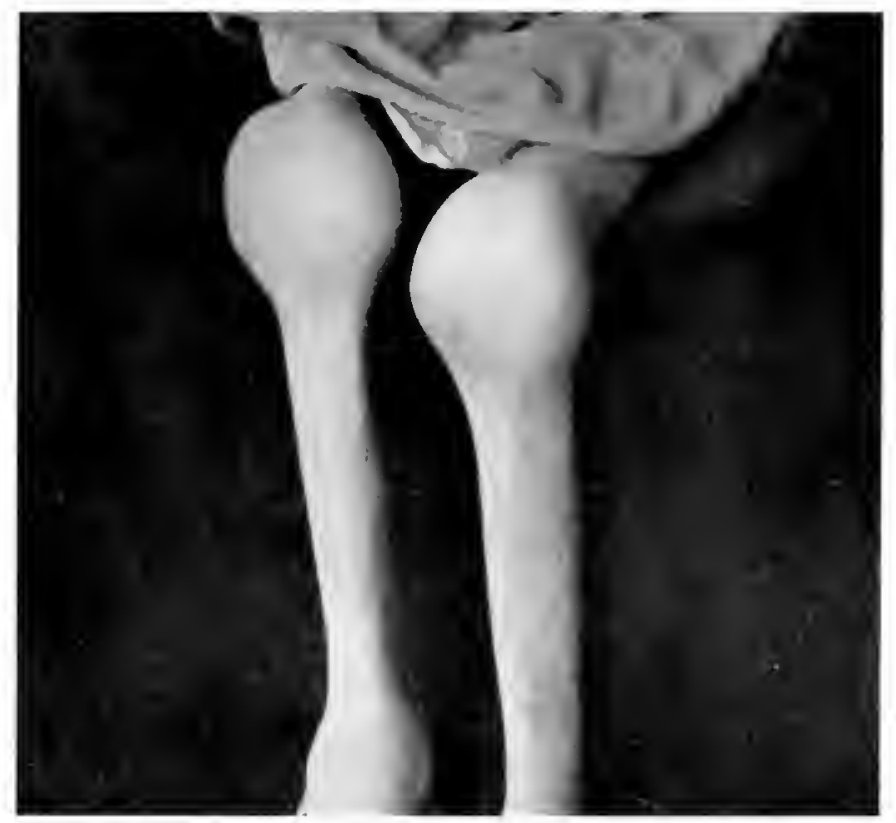


PI. ITI:-31.

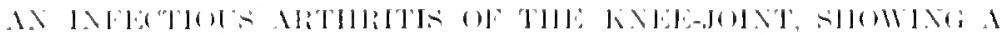

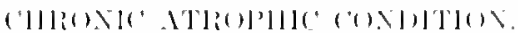

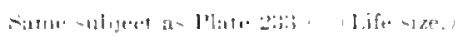

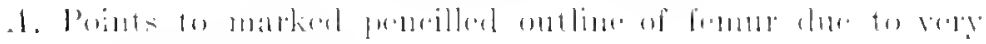

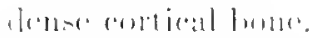

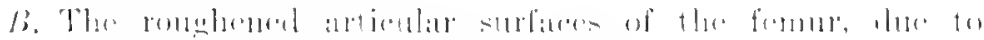

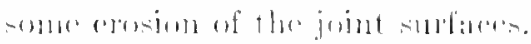

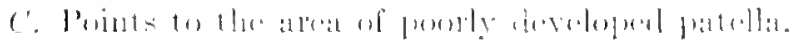


I'LATE: :3:4

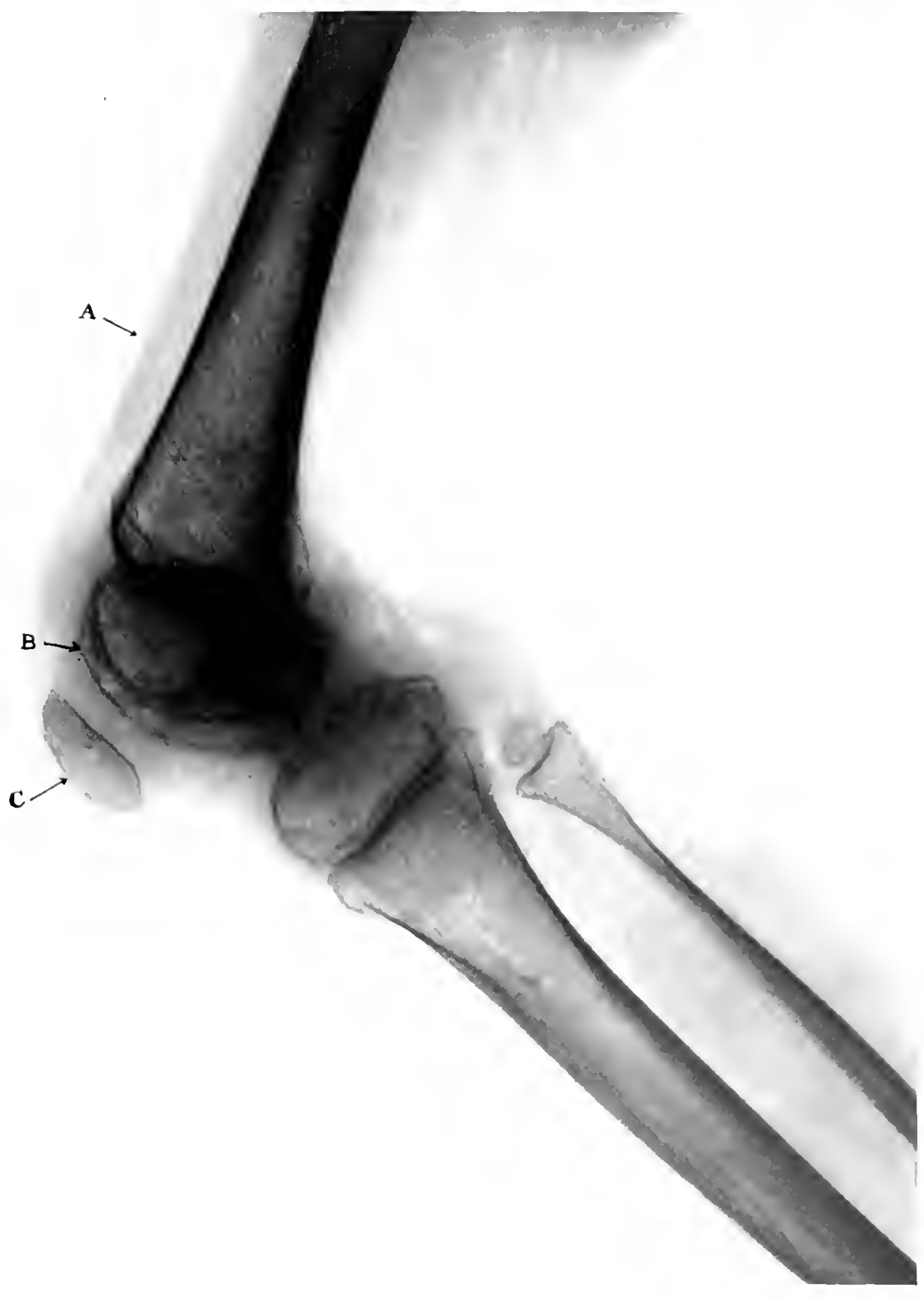


PLATE: 23.5.

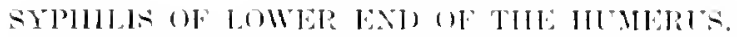

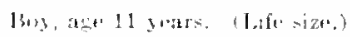

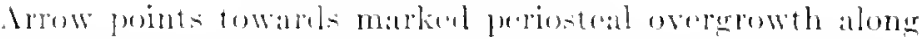
the inner borker of the humerux. "There is no destruction of the bone. 
I'I.ATH: :Z:5

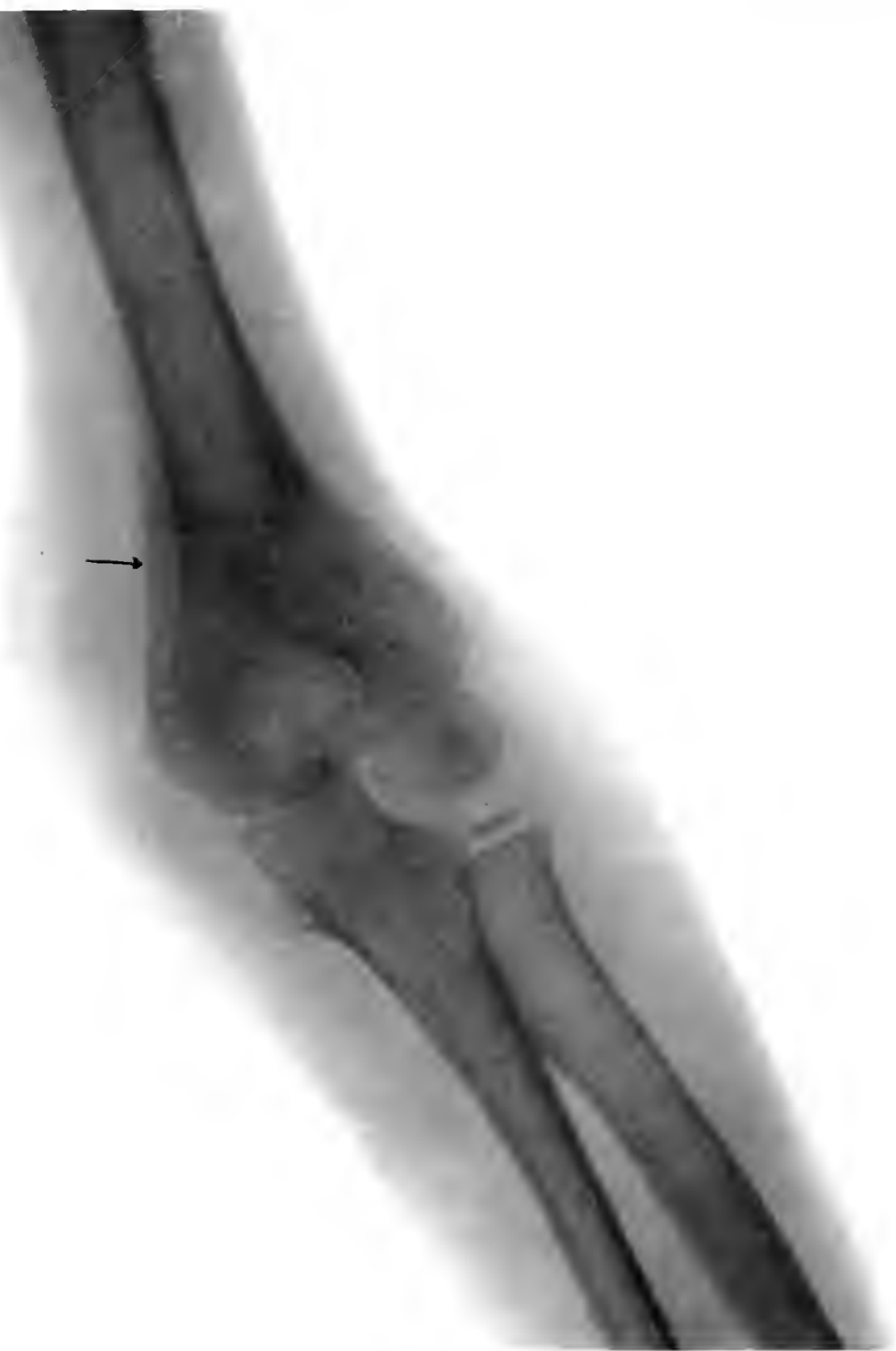


PI.ITE: 236.

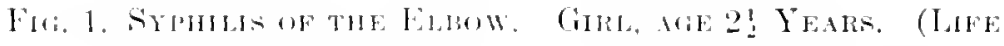
- -I $Z$ E.)

1. Points to marker preriostral overerowth, with an area of destructive promen.

b. Very deno new hase-forming periostemom.

'. Ouline of the arierinal bome.

Siste that there is no acelual destruetion of the ulna.

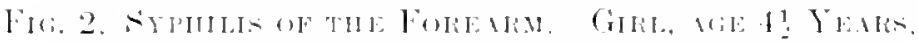

1. Points to markod juriostral rearetion.

13. Shaft of uluit.

(". Thinkming of tisturs probably due to abserses. 
PILATE : 2:36

FIG. 1.

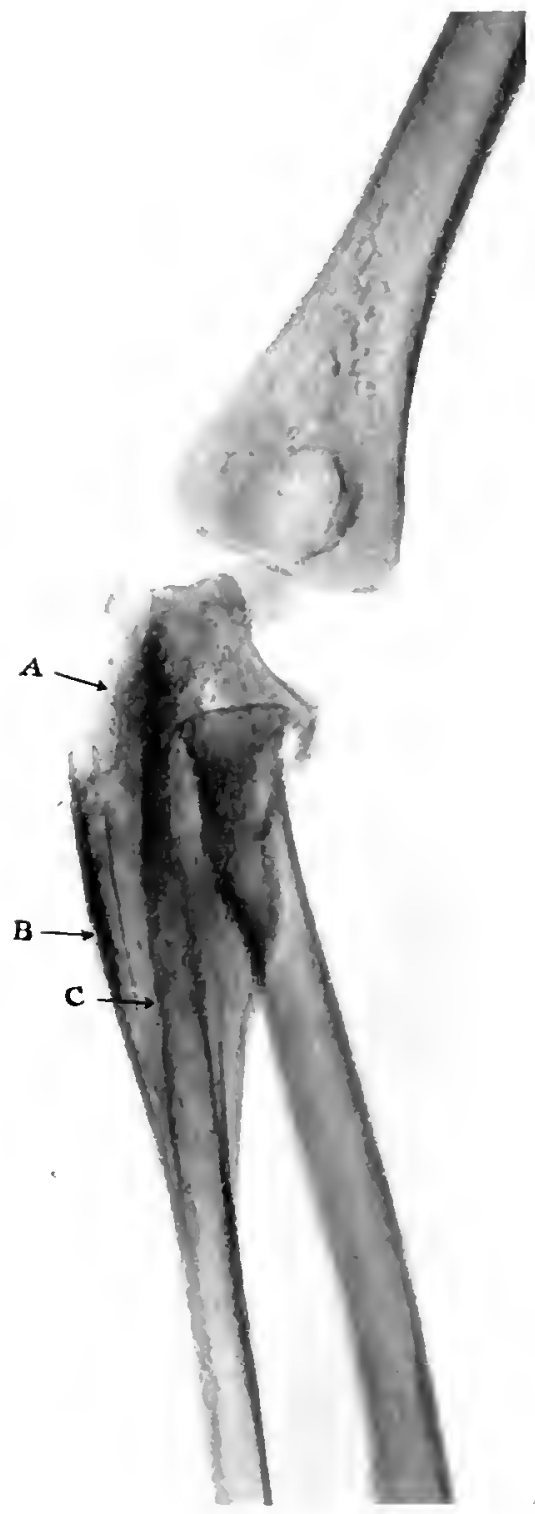

FIG. 2.

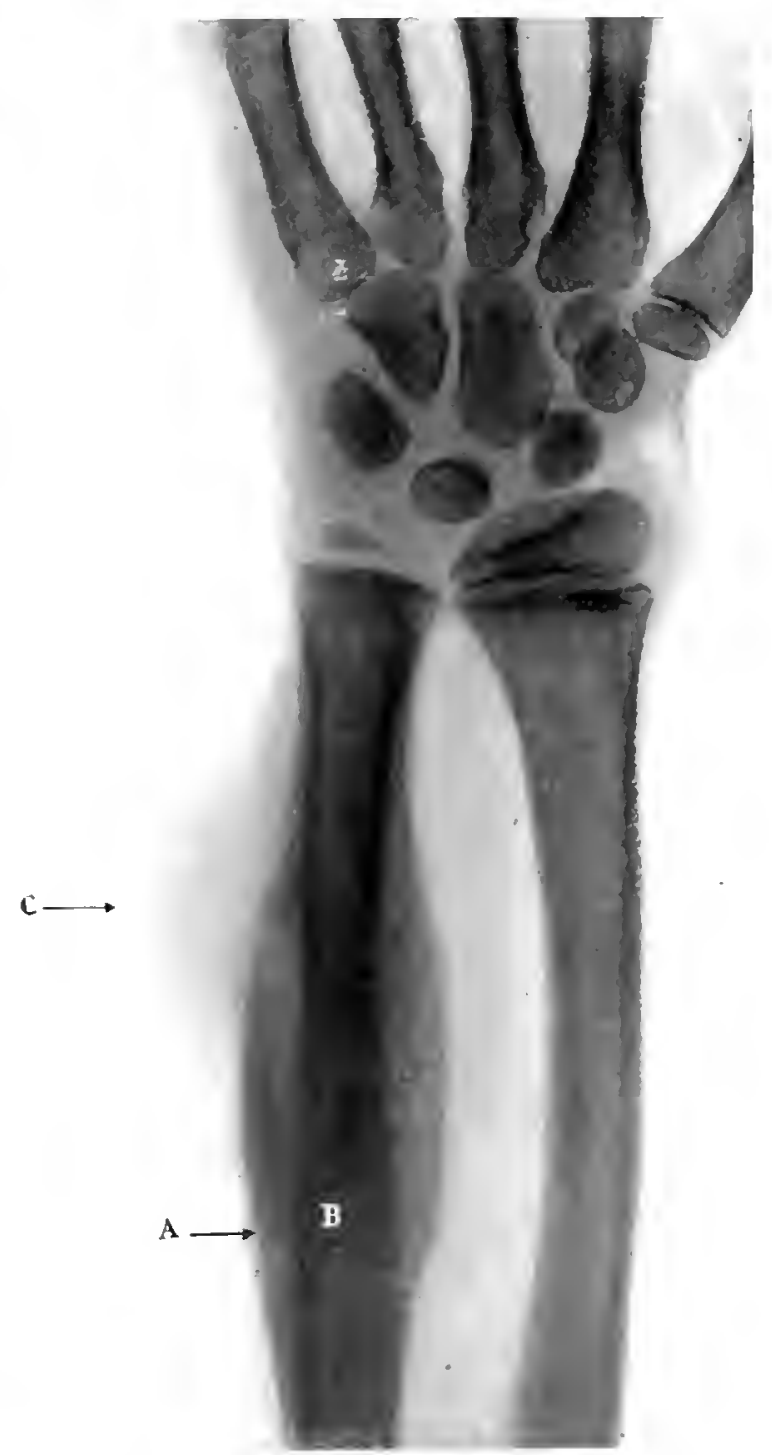


J'AT'P:BT.

\section{SYPHH.ITIC DACTYLTIS.}

Chilh, are 2 yesrs. (Life sizc.)

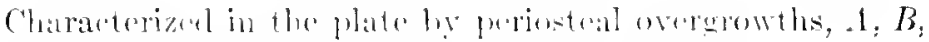
C. D, whth no apparent disturbance of the bone itself. 
PLATE:

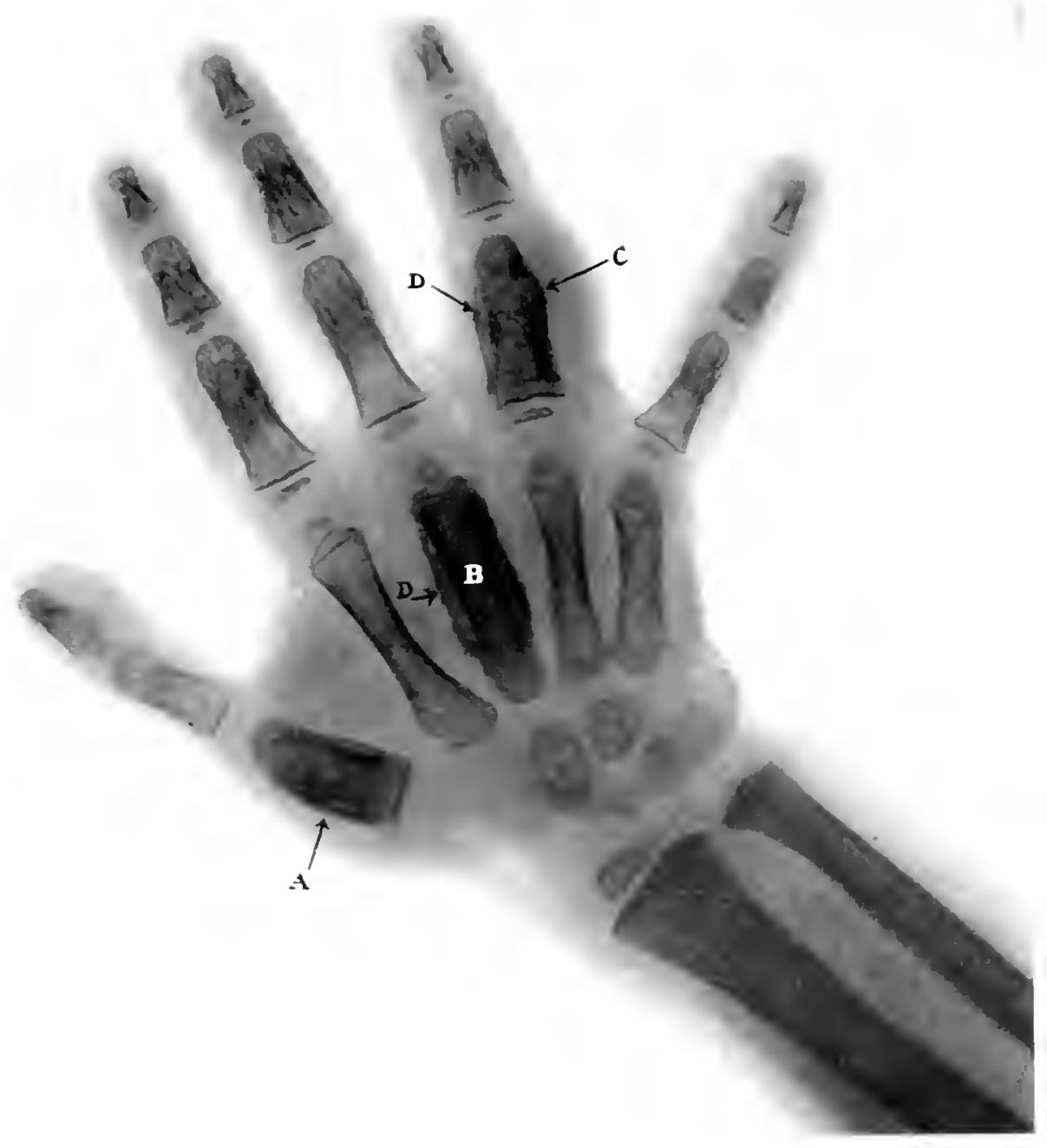


I'LATL: 22:3.

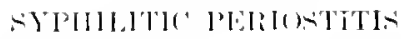

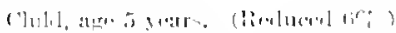

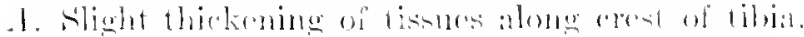

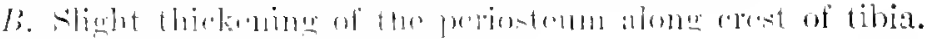

( . Slingt thickeninge alume fibulit. 
Plate: 2:38

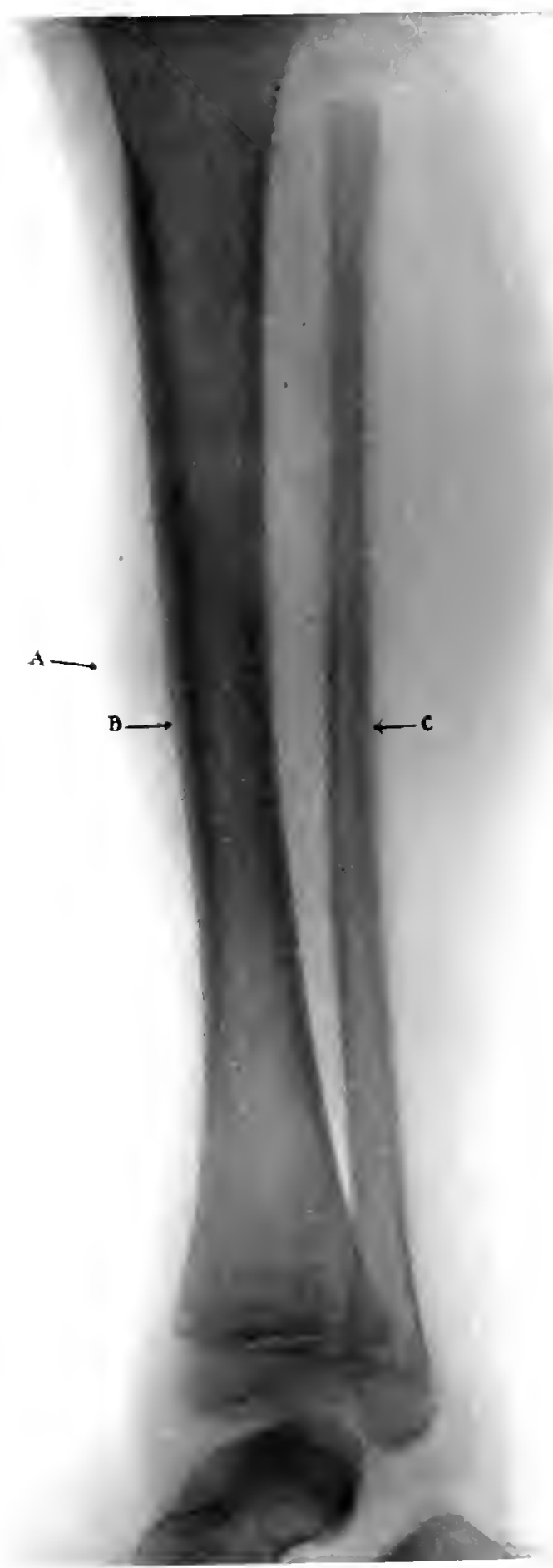


PLATI: 293.

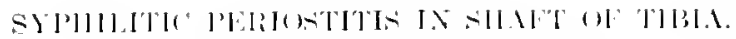

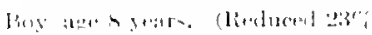

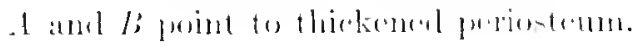


P'LATE $2: 391$

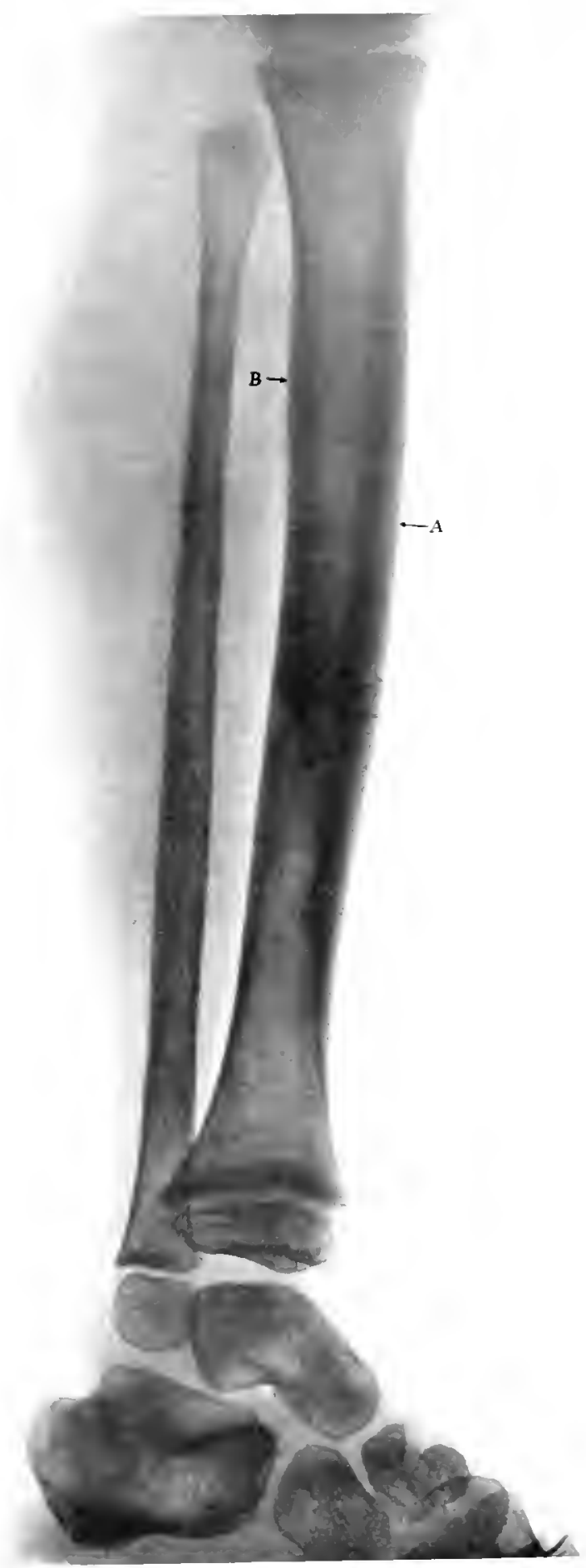


PI.191: 210.

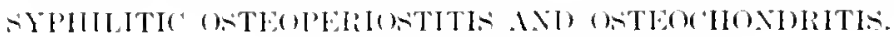

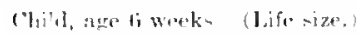

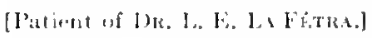

Plate shows extreme preliforative proens along the shafts

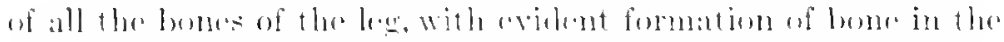

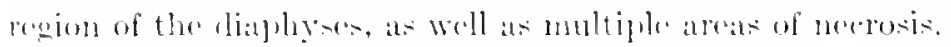


PIATE 240

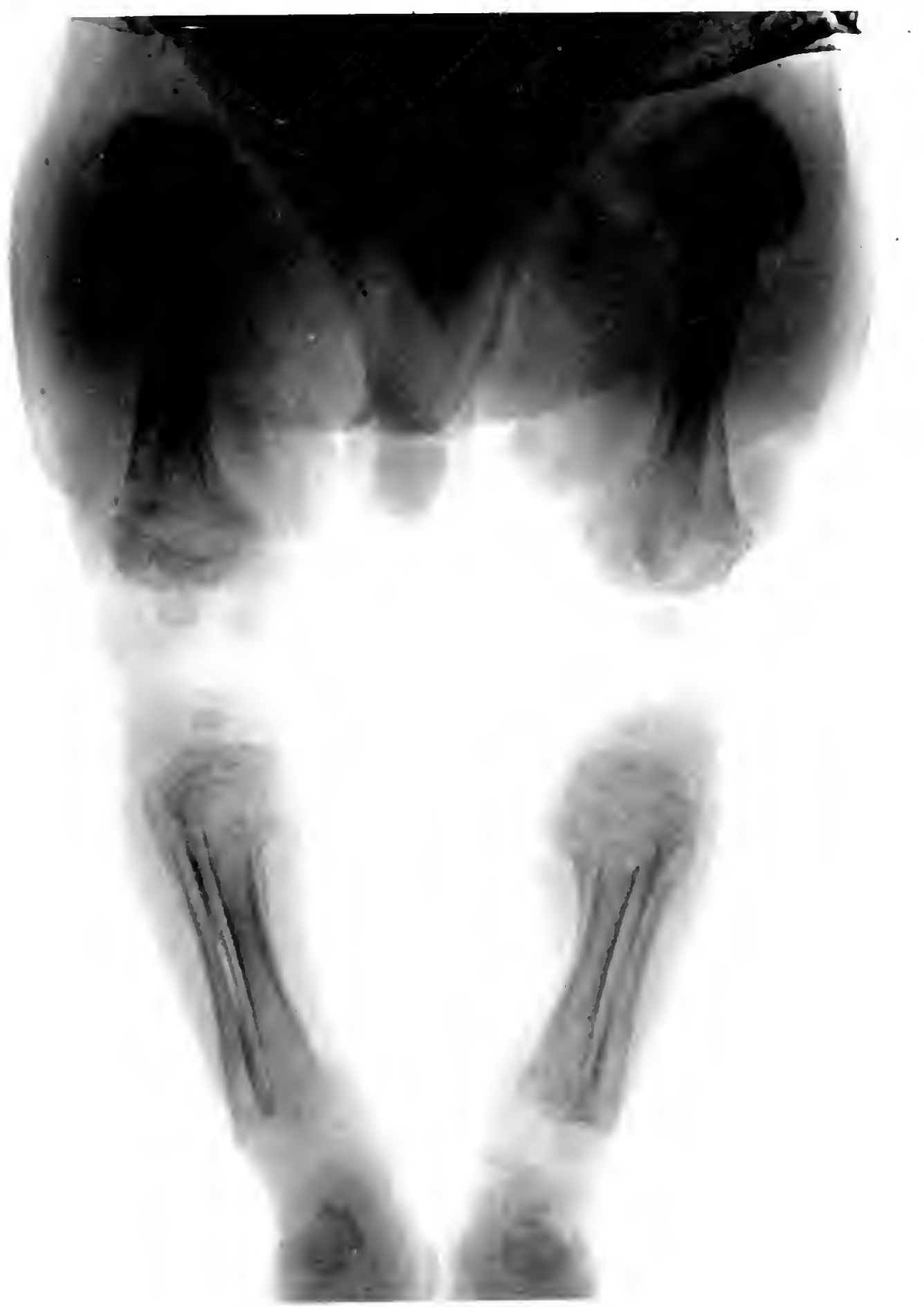


PI.ITL: 211.

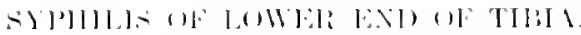

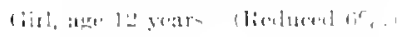

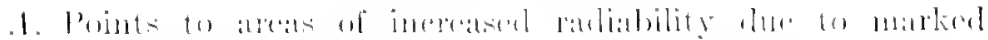

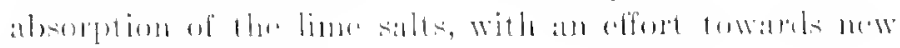
formatilion of losme.

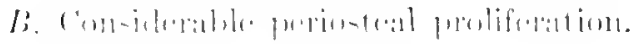

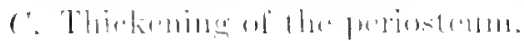


PH. TT: $\because 41$

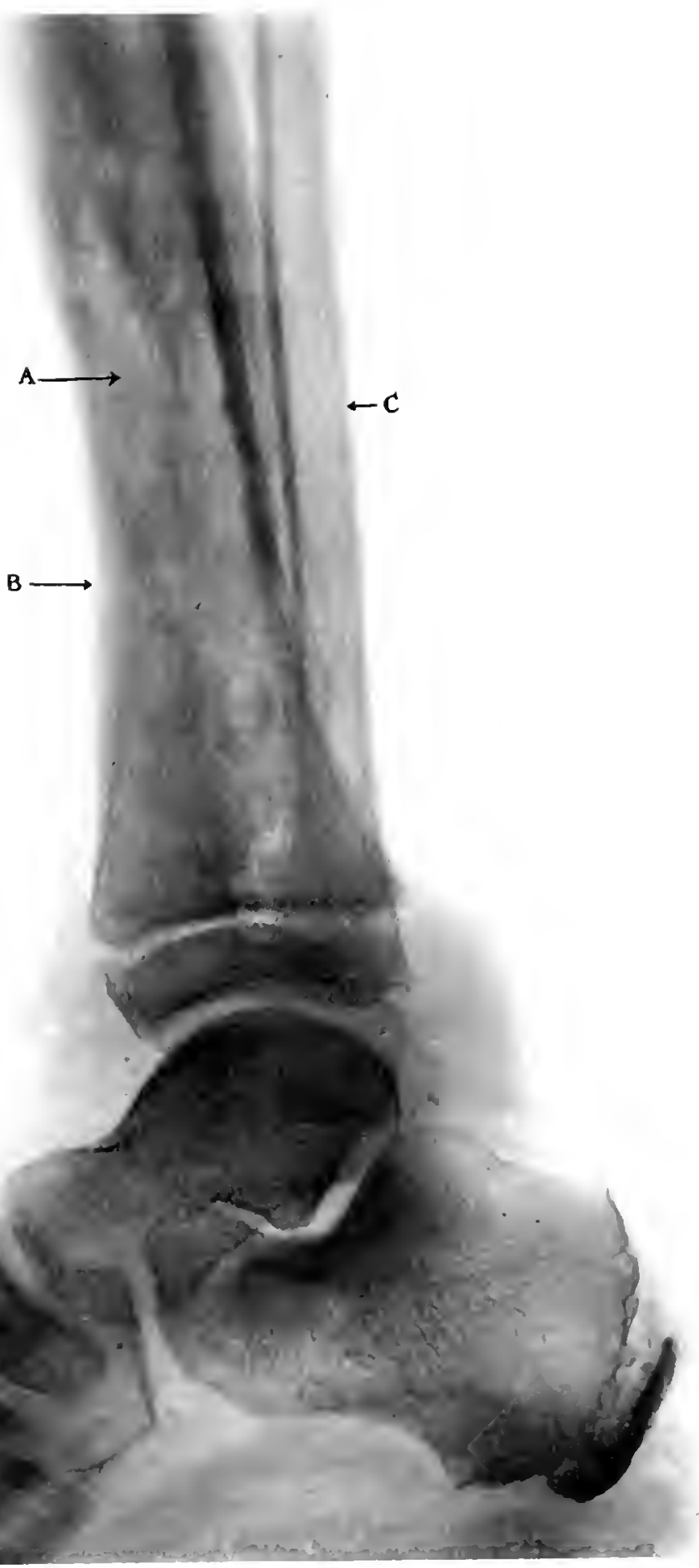


PL..TT: 212.

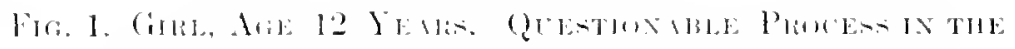

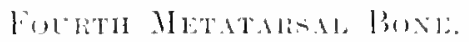

(lhataleterizenl be matkel new bone formation arouml the shaft of the metatiasus.

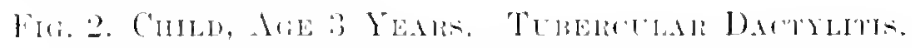

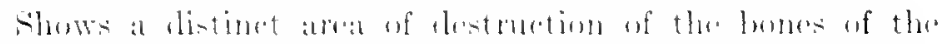

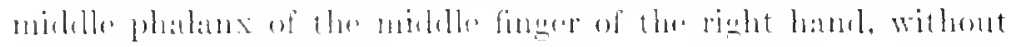
any leriostanl matetion.

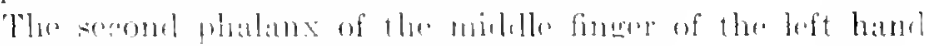

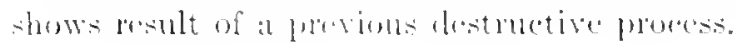


FIG. 1.

PIATE 242

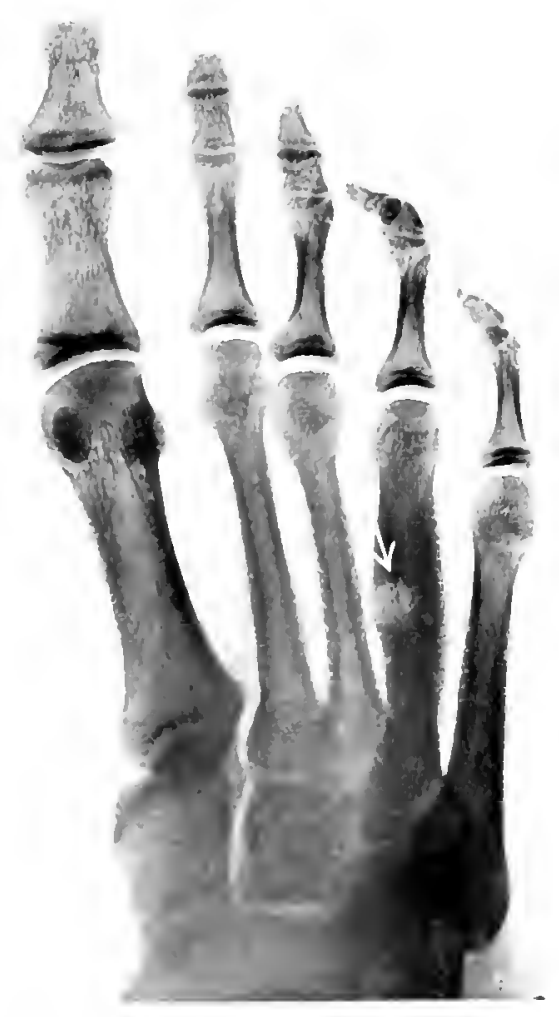

FIG. 2.
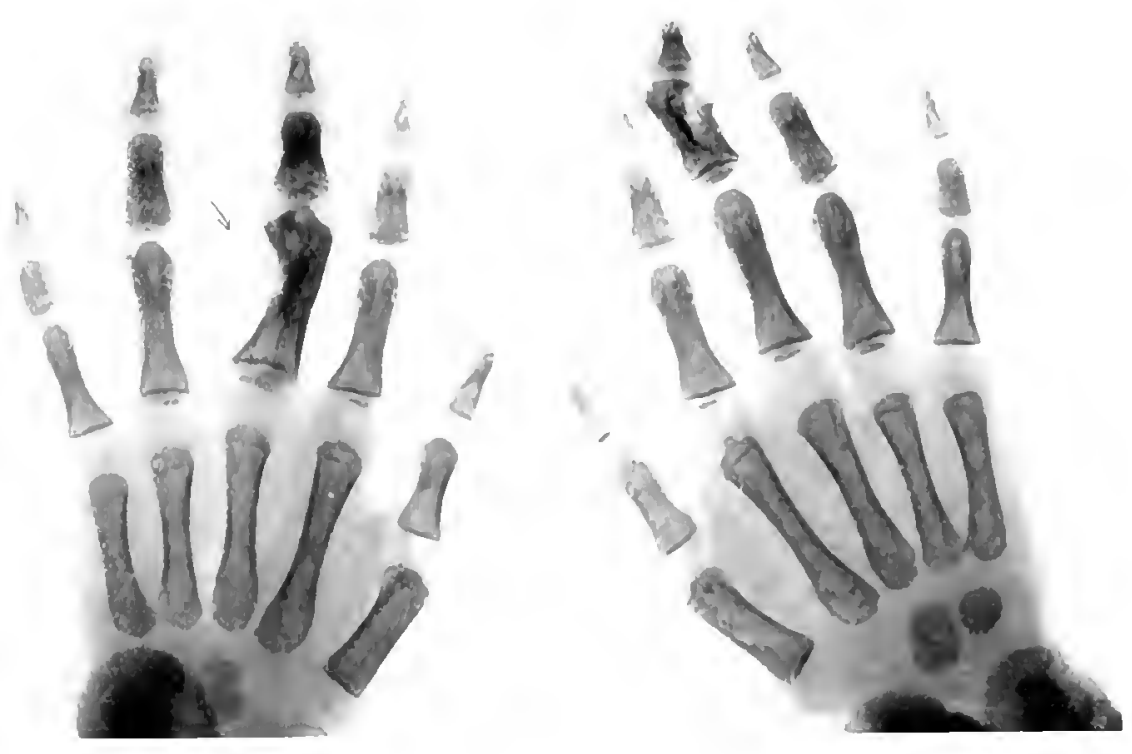
IPLATI: 213.

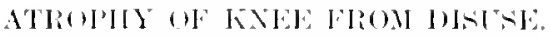

Girl, aler 12 yourm.

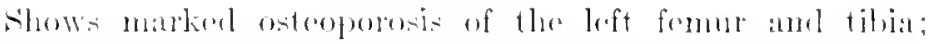

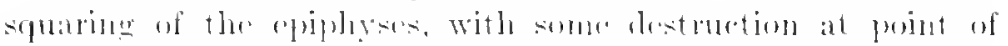
armow. Atrophy of chatity of the bomer. 
PLATE $\because 4: 3$
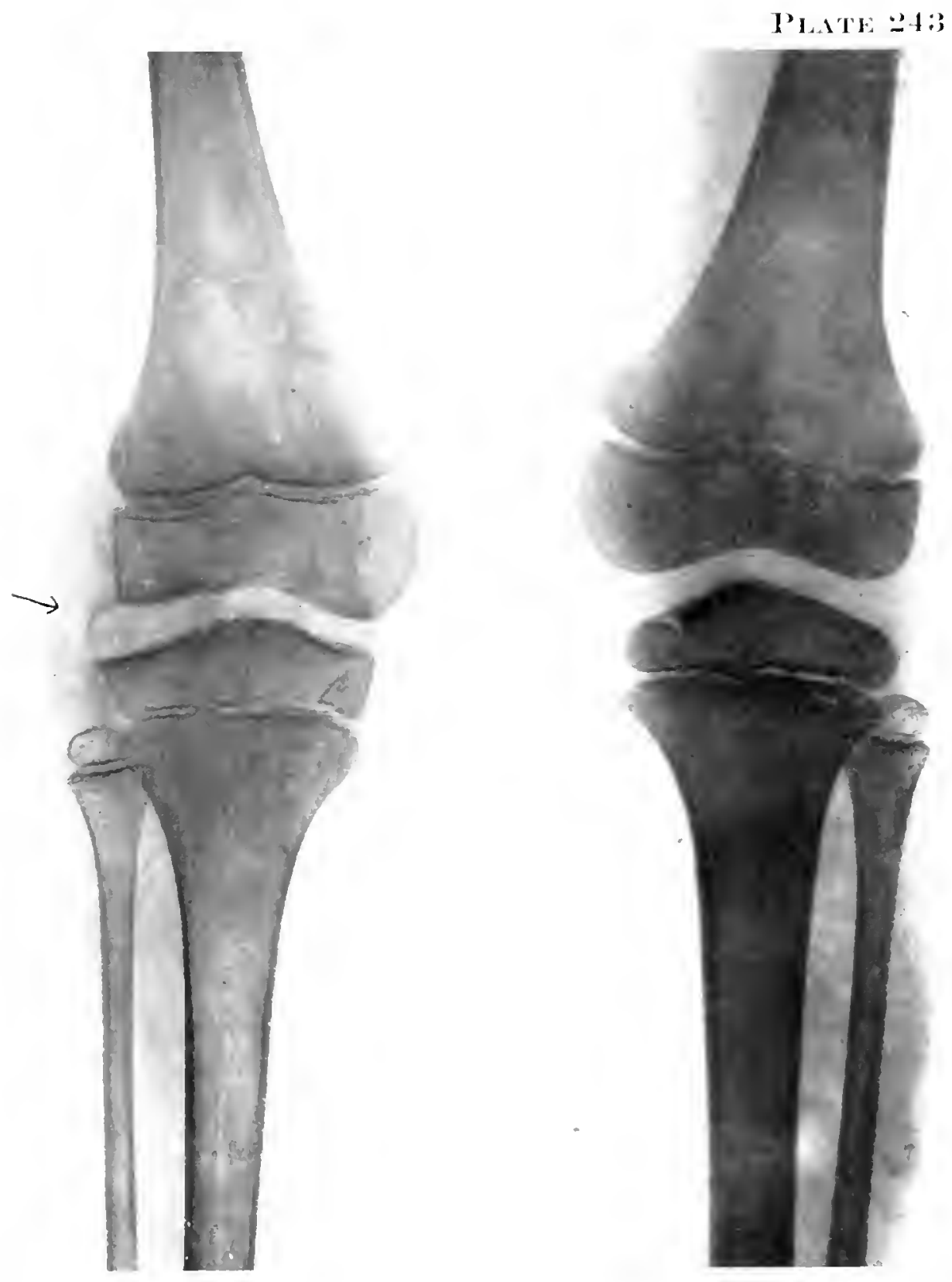
PI.TY:"2t1.

TIBERETI.AL H.A TYLITIS.

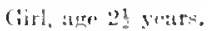

The first phalanx of the soromel and thind fineress show markind onlatrement of the shaft, with re-arrangement of the strueture of that bome resembling "ystomation. 
PLate 244
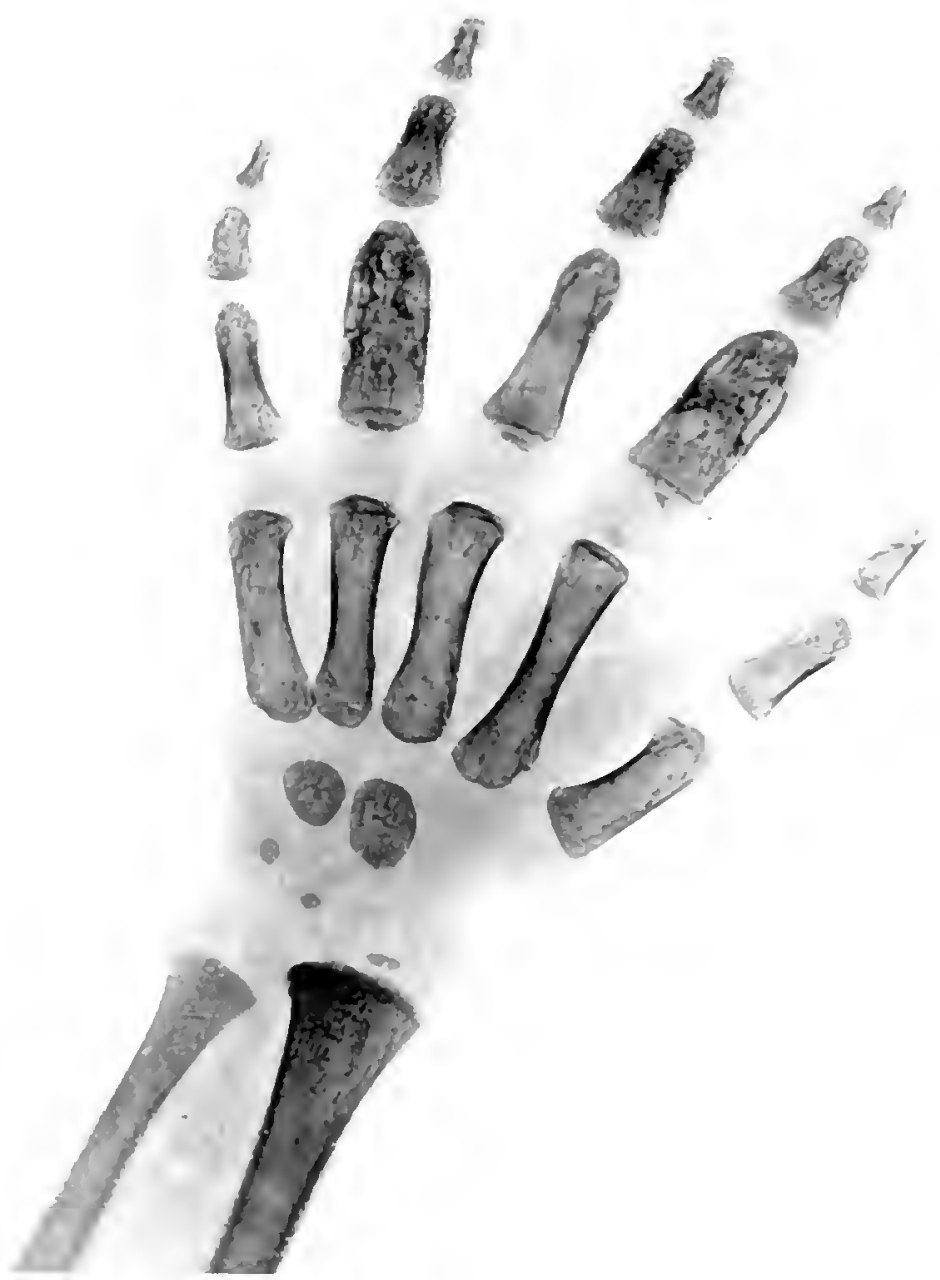
PI.ATE: 215.

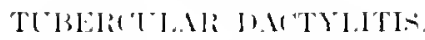

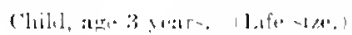

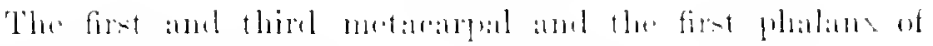

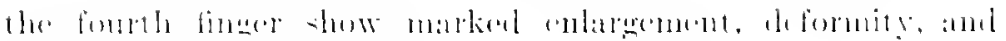
der-trution of bune li-cule. 


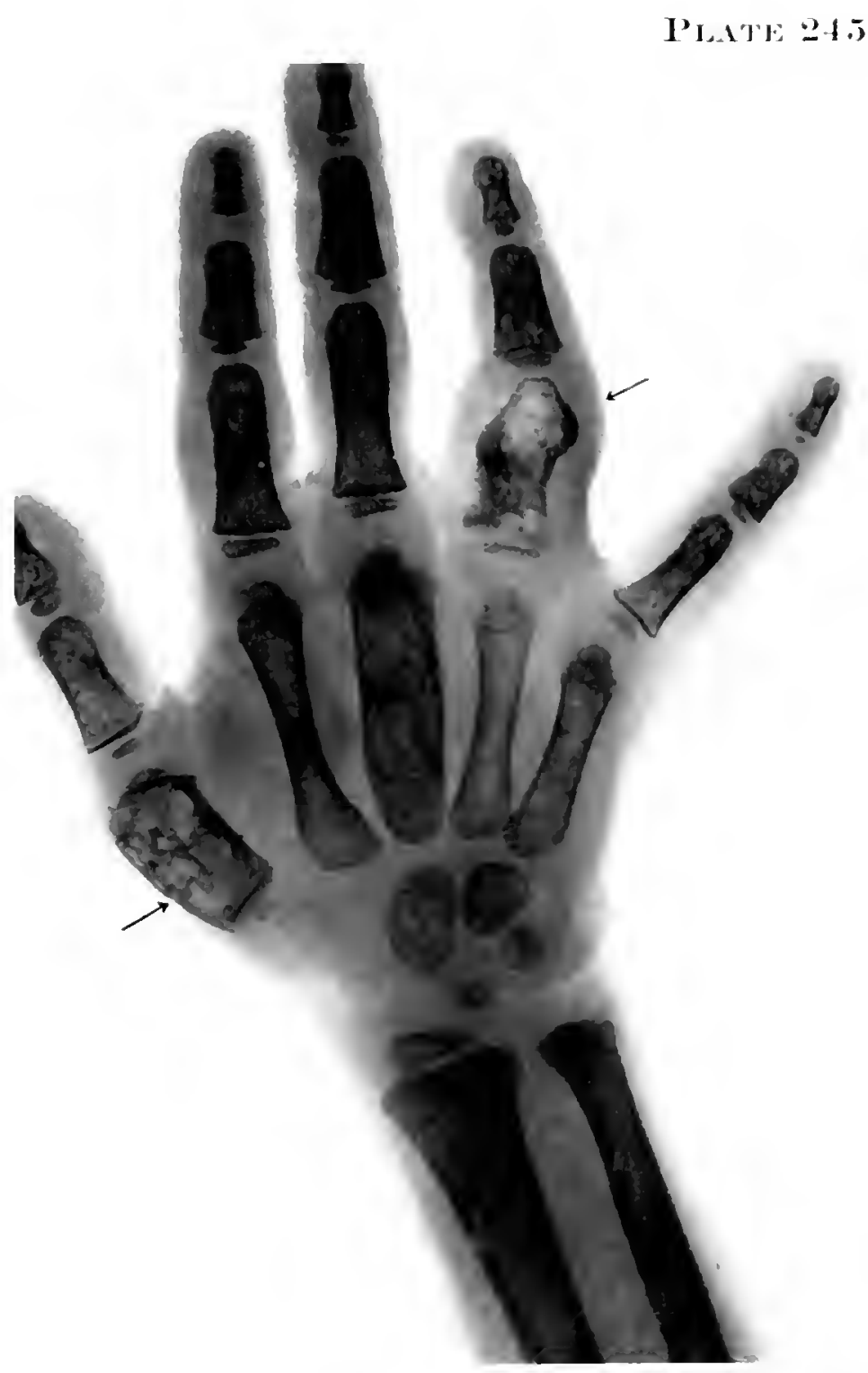


P1.191: 216.

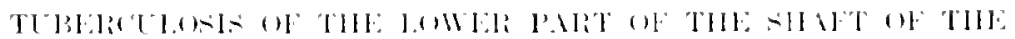
I.I.I.

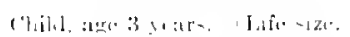

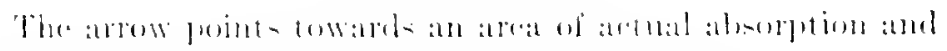

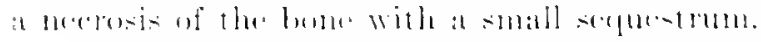

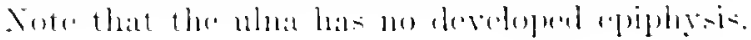



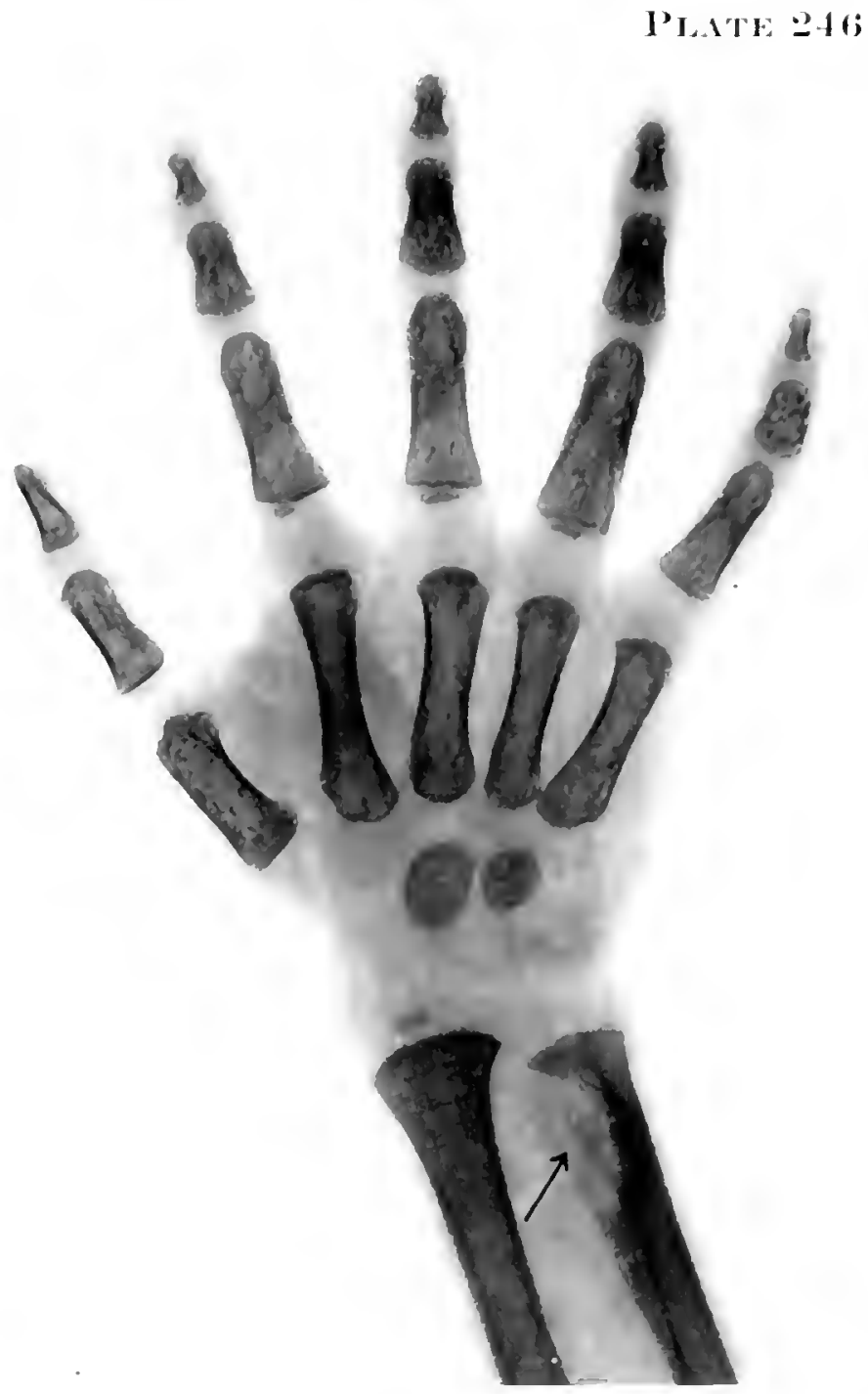
J'I.1T2: 217.

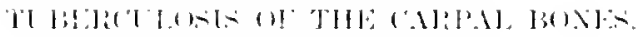

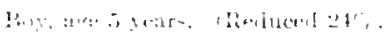

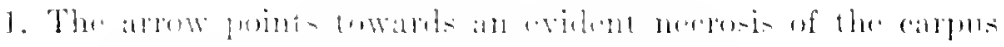

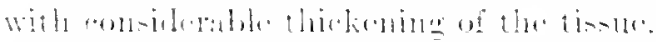

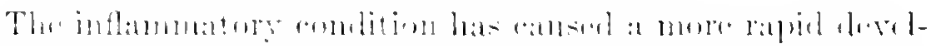

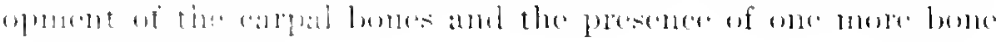

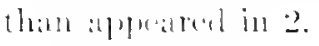

2. Sintutal hand. 

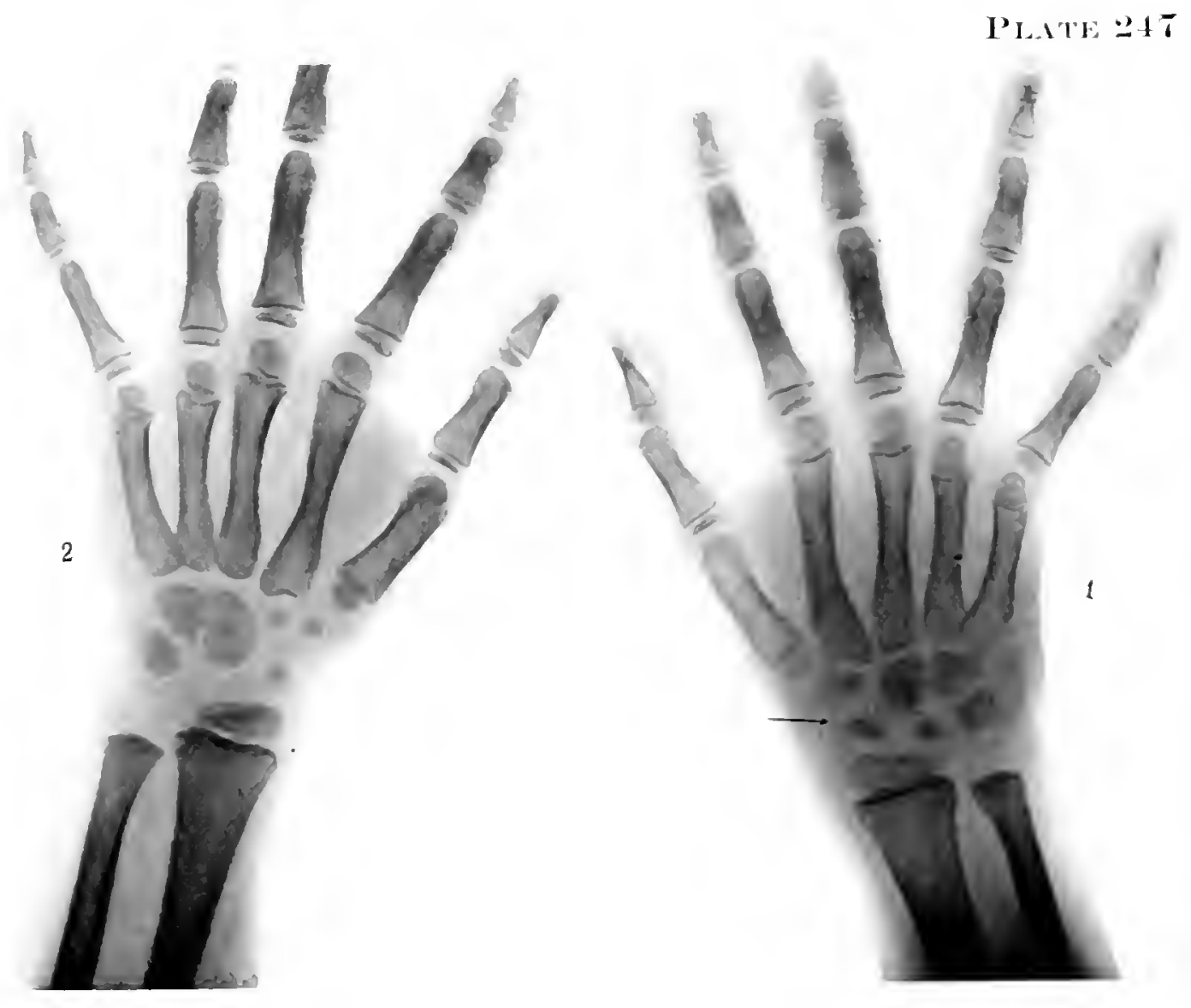
PIATL: 2 .

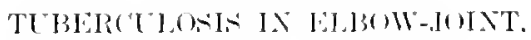

Girl, and byars. (life ily

A. Hlumerus.

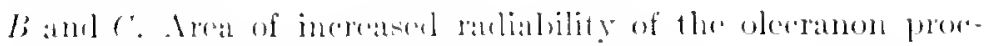
ass of the ulna within the joint.

I). Radius. 


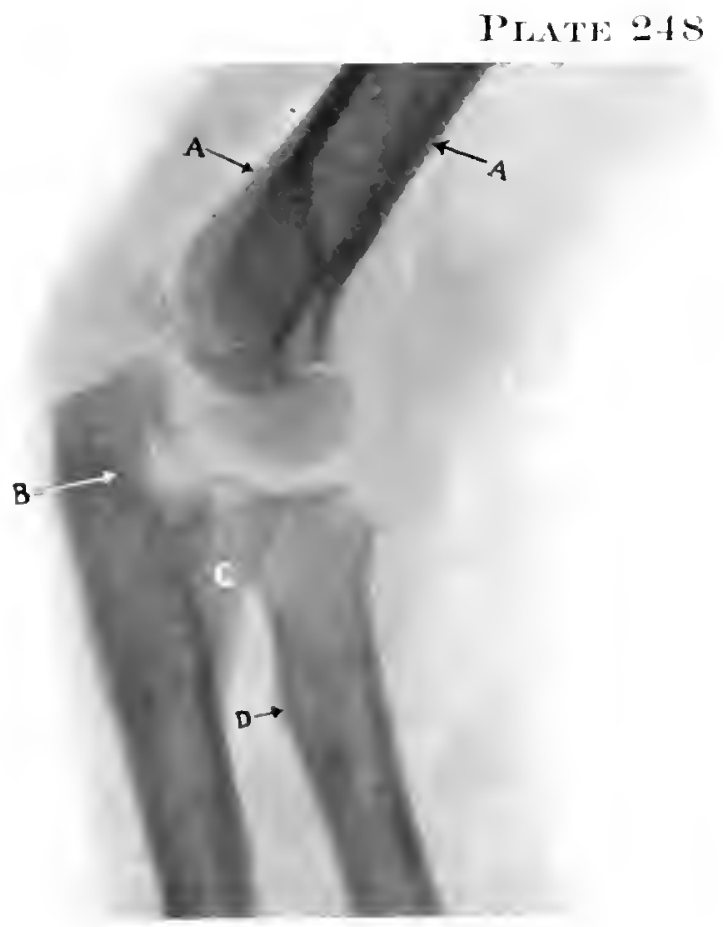


l'L.1T1: 21!.

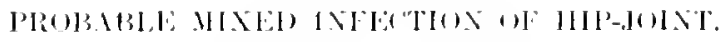

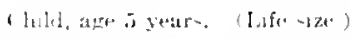

A. Periosteal proliforation within the pelvis.

b. Marked infiltration and thirkeninge of tistur. The hearl

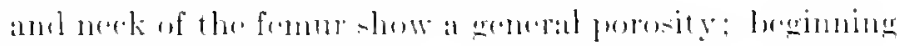
dislocation of the firmur. 
P'LATE 249

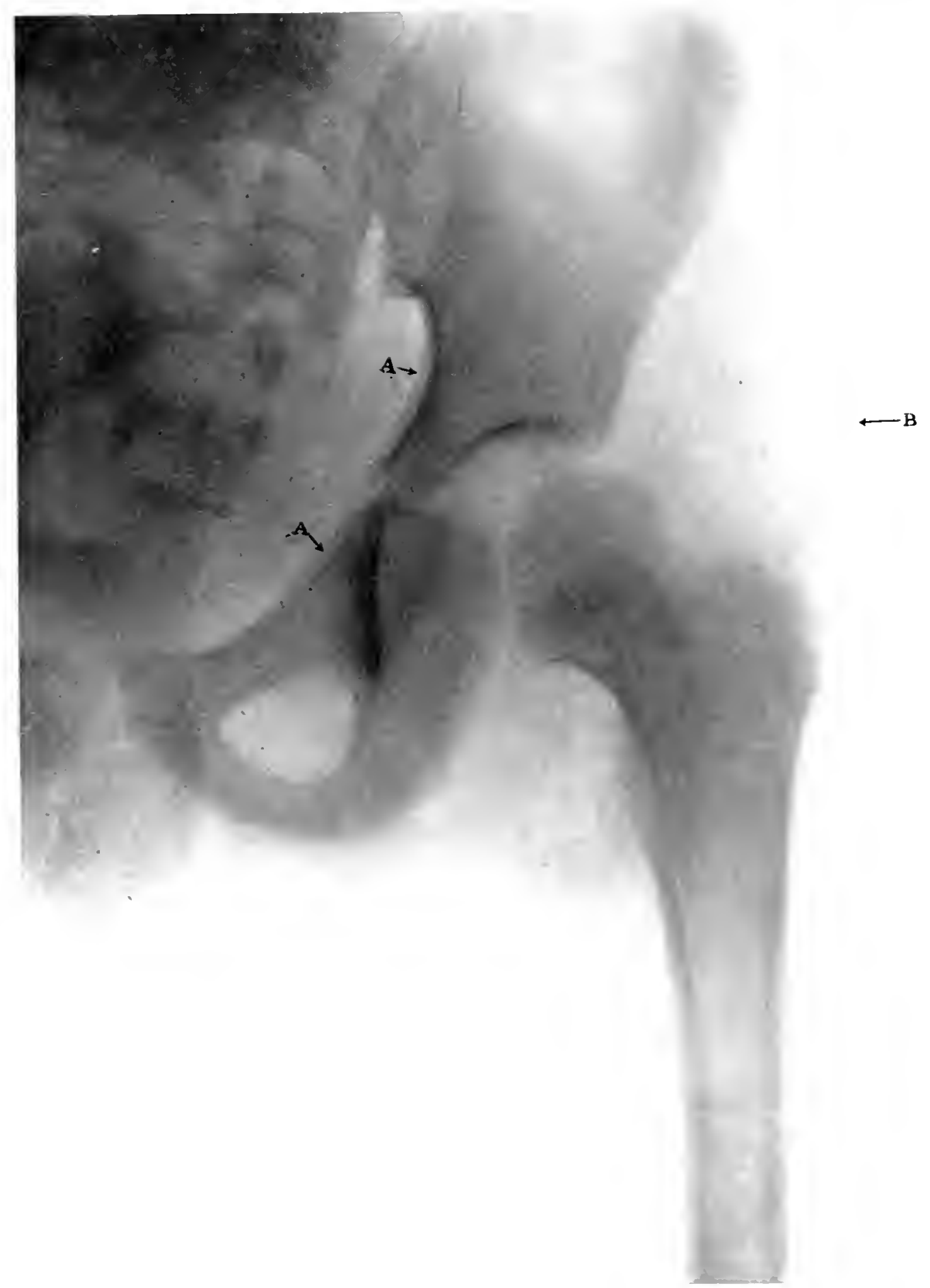


PLATl: 20.

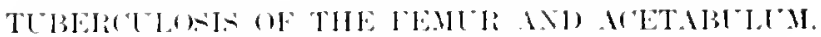

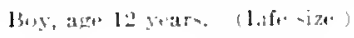

This plate -how: the end result of at fong-iandine tuber-

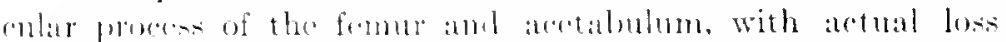

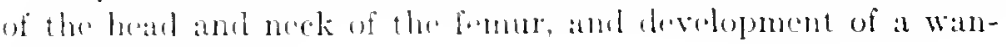
derine alcotabulum.

1. Antabulum.

b. Rematins of the neck.

$\therefore$ cireat trochanter. 
PIATE :-50

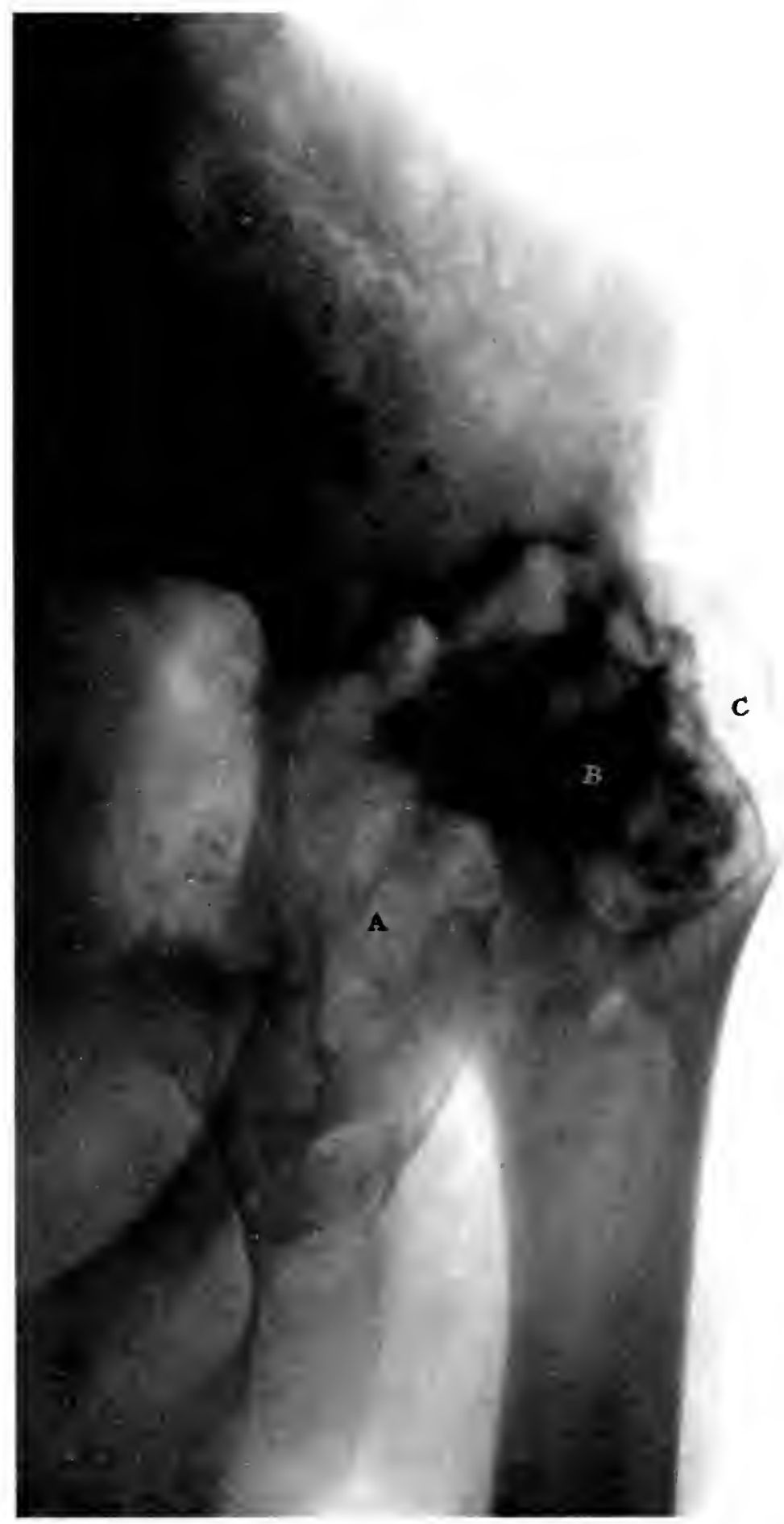


Pl.ATE 2.i.

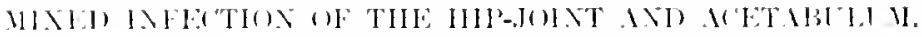

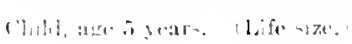

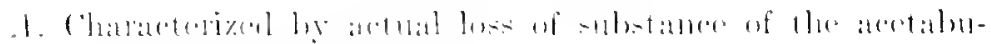

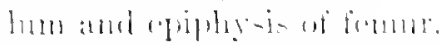

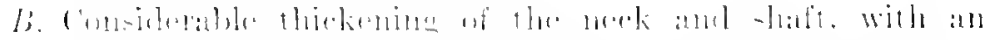

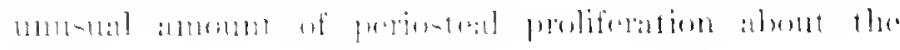
mek :and - butf of the bume. 
PHATE 2.51

A.

$\longleftarrow$ B 


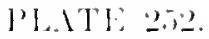

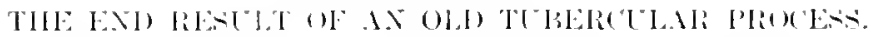

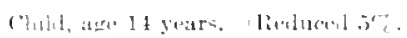

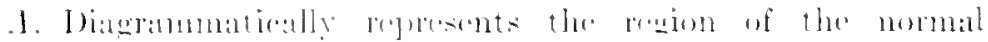

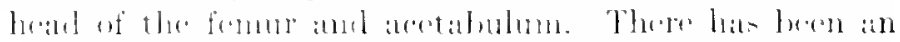

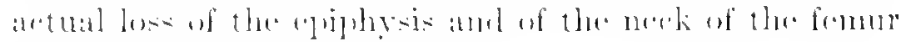
int this ("itine.

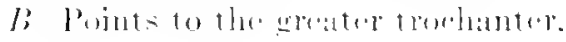


P小TE

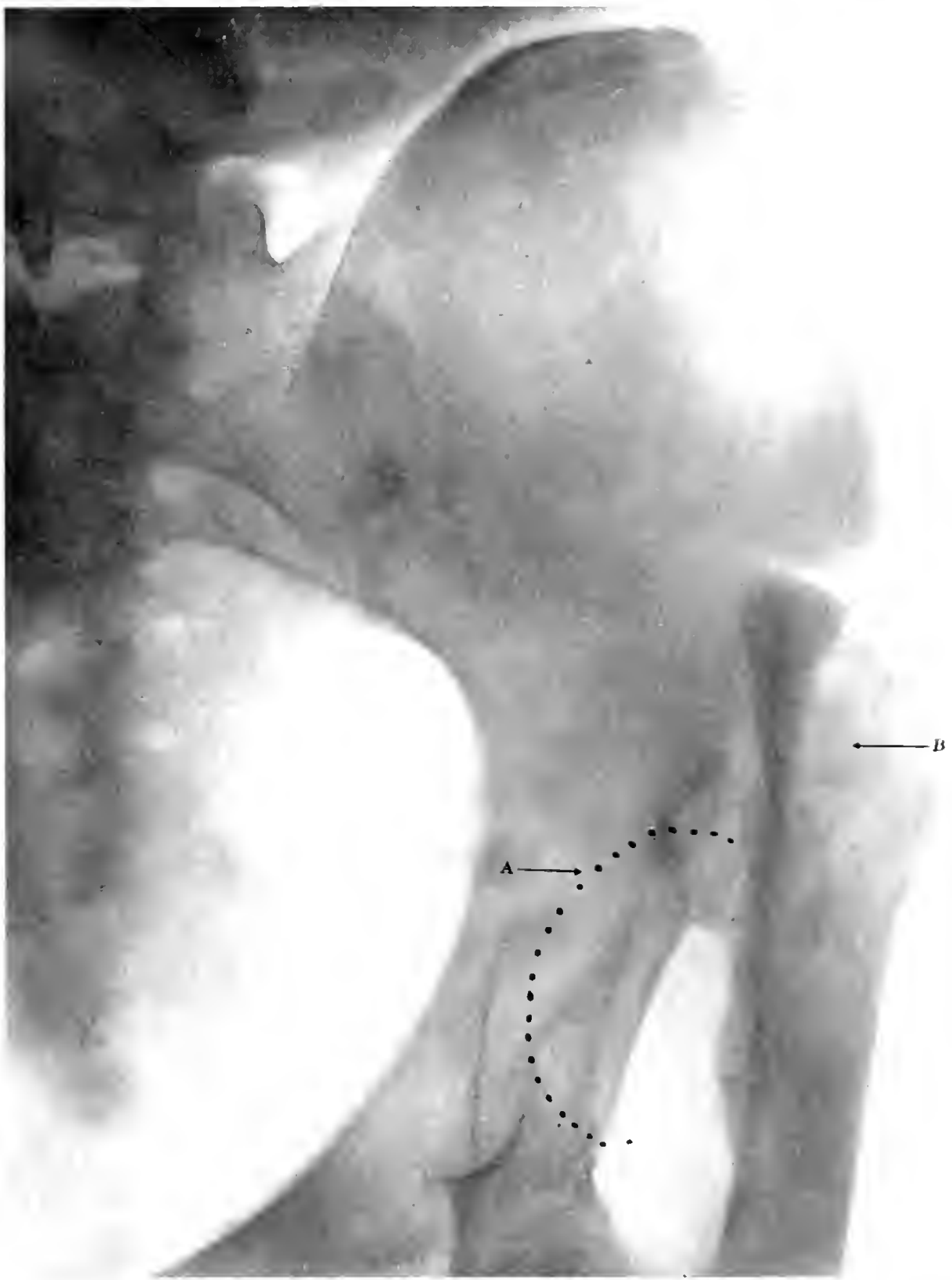


P.IT'T: 2:R.

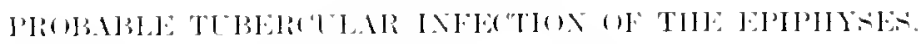

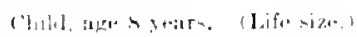

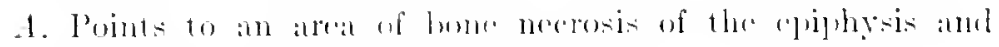
necle of the formut.

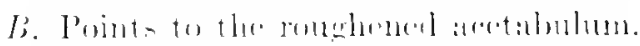

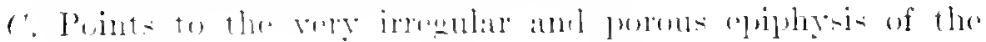
fermul: 
PI,ITE:3:3

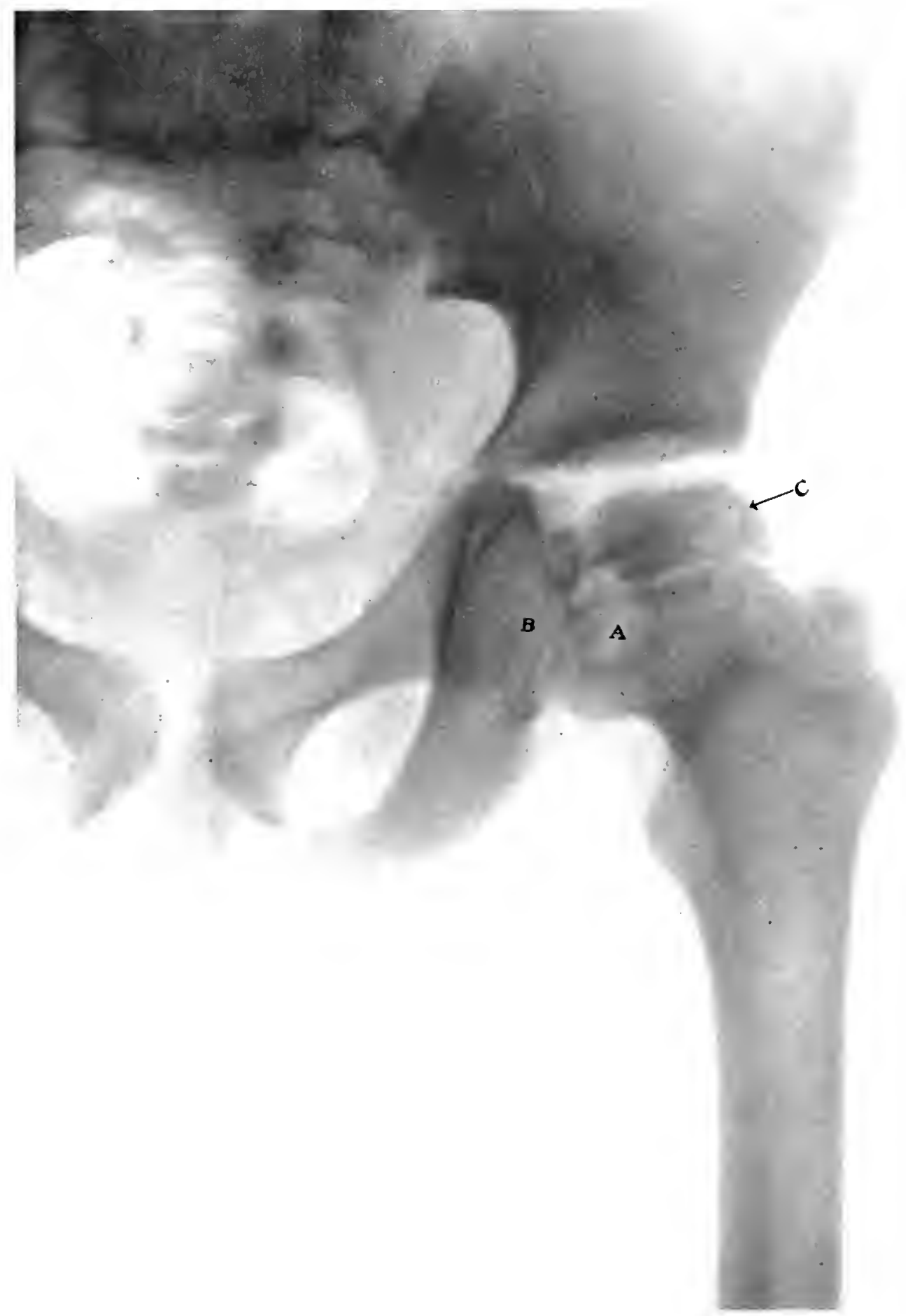




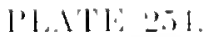

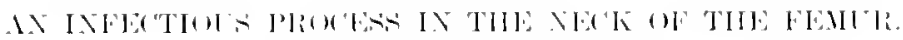

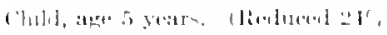

The alrow prointe to an alseress within the loft hip-joint,

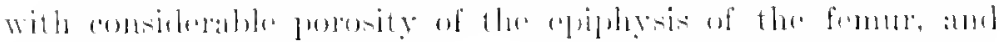

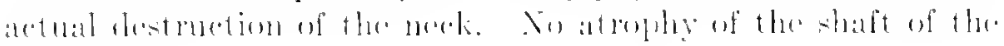
limul'.

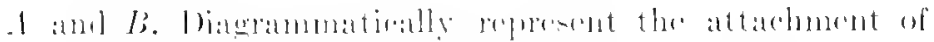
the atpilu of the rieht hip-joint. 
PLATE "ZJ!

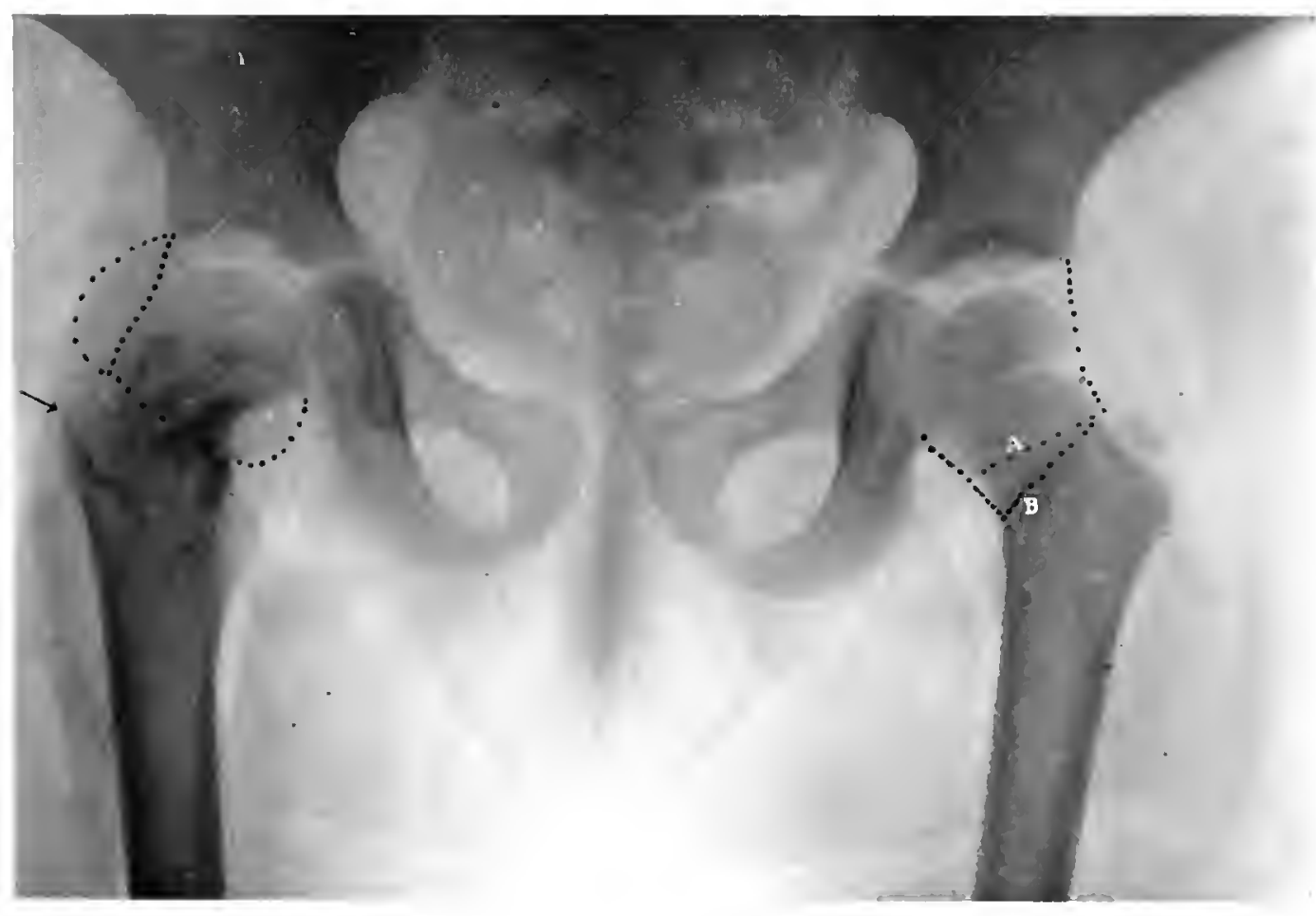




\section{Pl.1T2 2.in.}

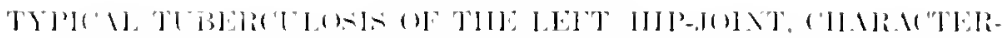

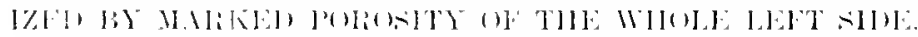

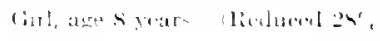

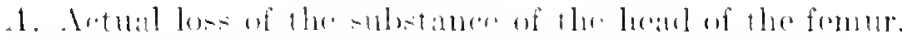

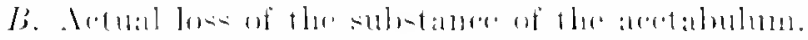

C. Bxtreme atropley of the shaft of the bome. in both puality and size. 
Plate 255

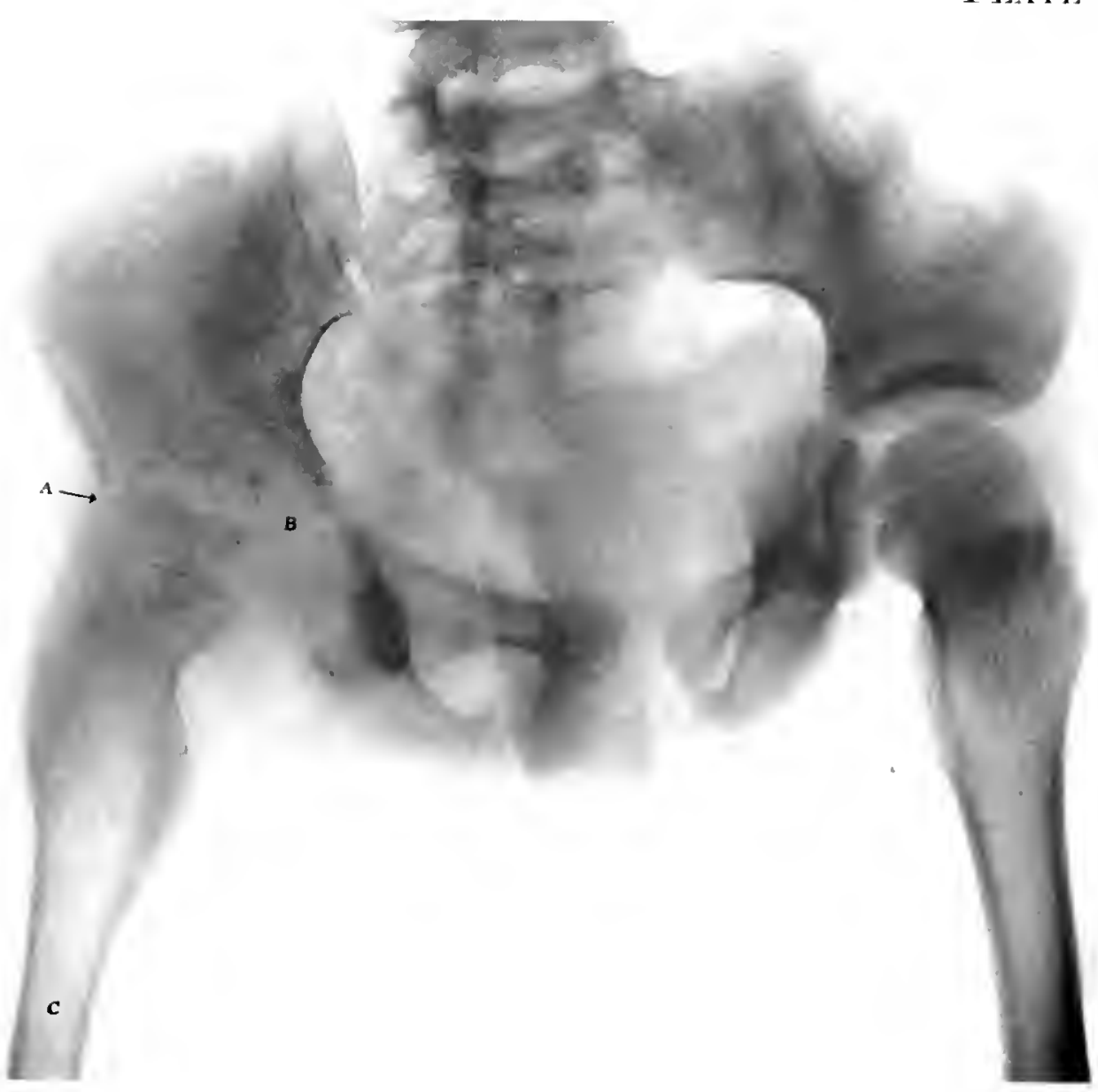


PI..TP: 2.iti.

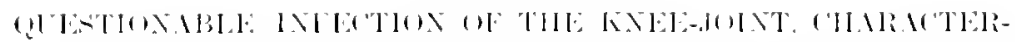

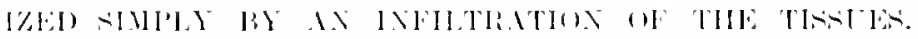

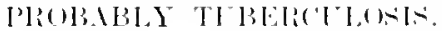

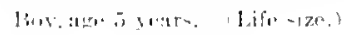

1. Pruints to the intiltrated tin-lues 


$$
\text { Plate } 20 \text { (5) }
$$

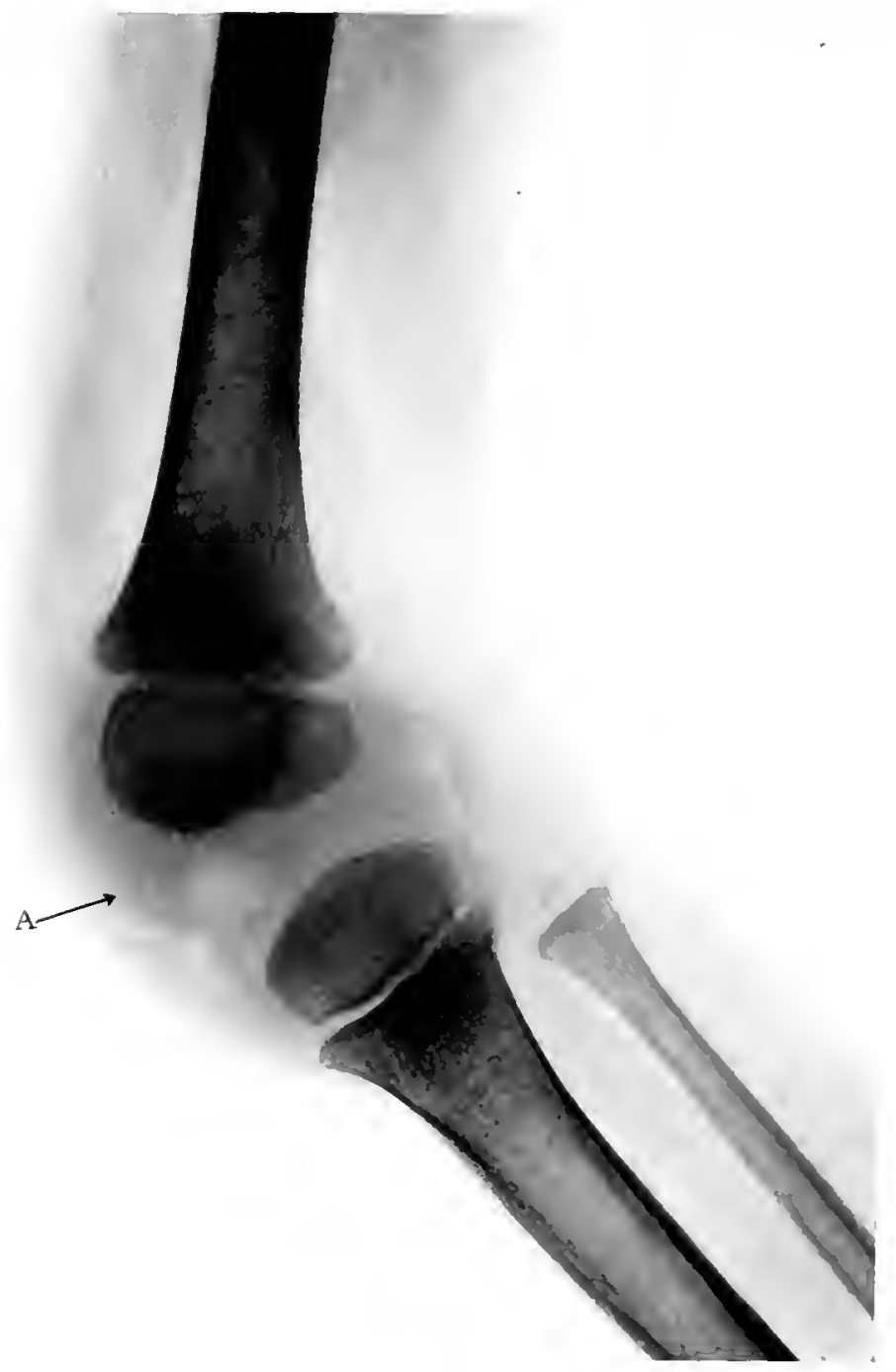




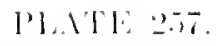

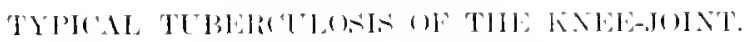

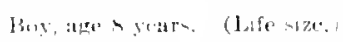

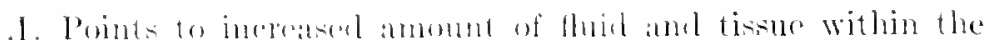
ralumise.

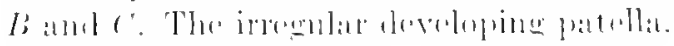

1). The andyles, whinh show very irmentar formation. 
PLATE 2.57

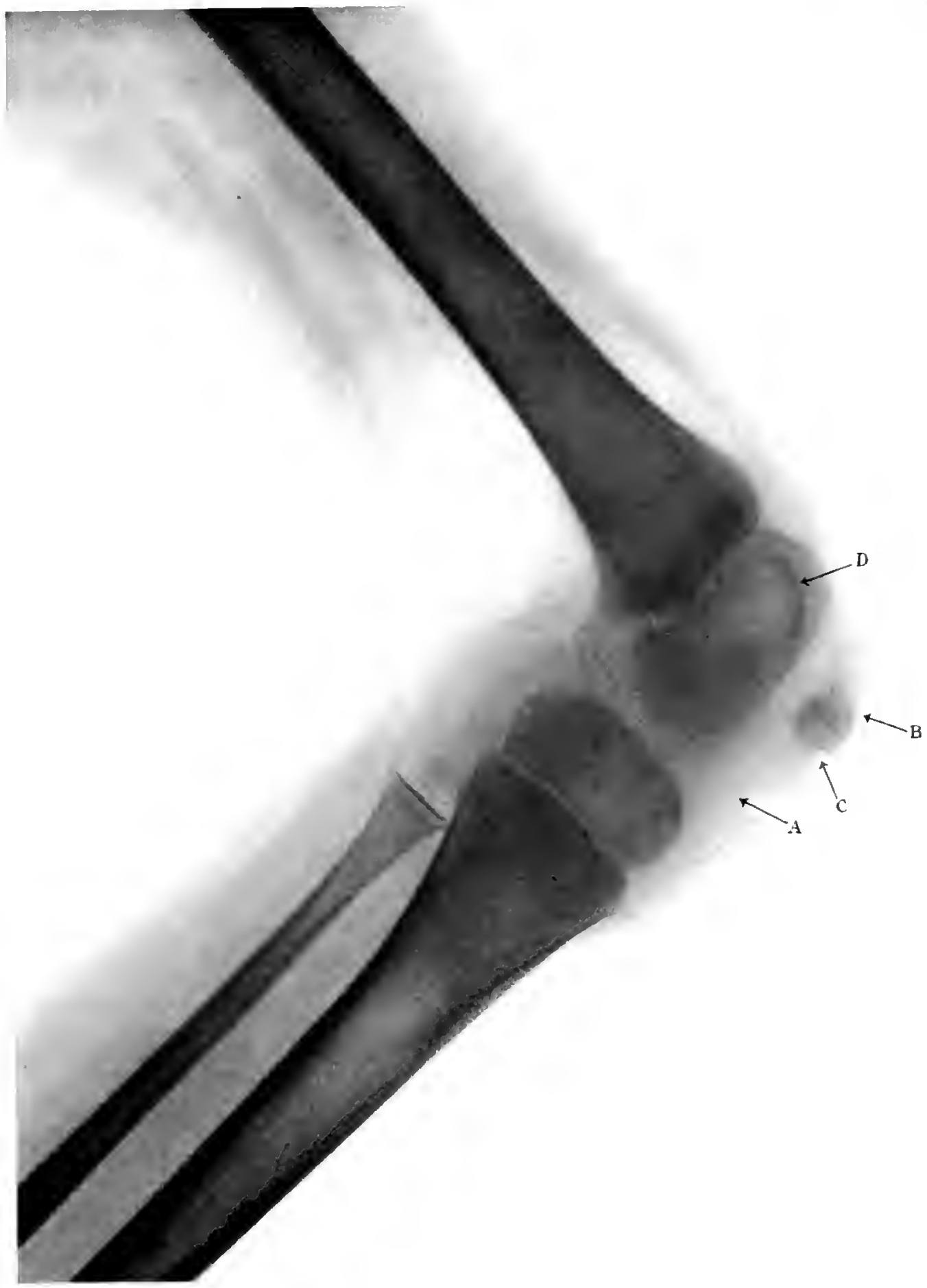


PHAT: 2.S.

ABSTES IF THE THMH.

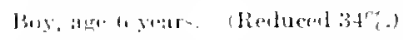

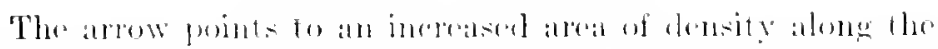
anterise anpert of the thigh probally hatmatoma from tratemati-nl, 
PLATL:

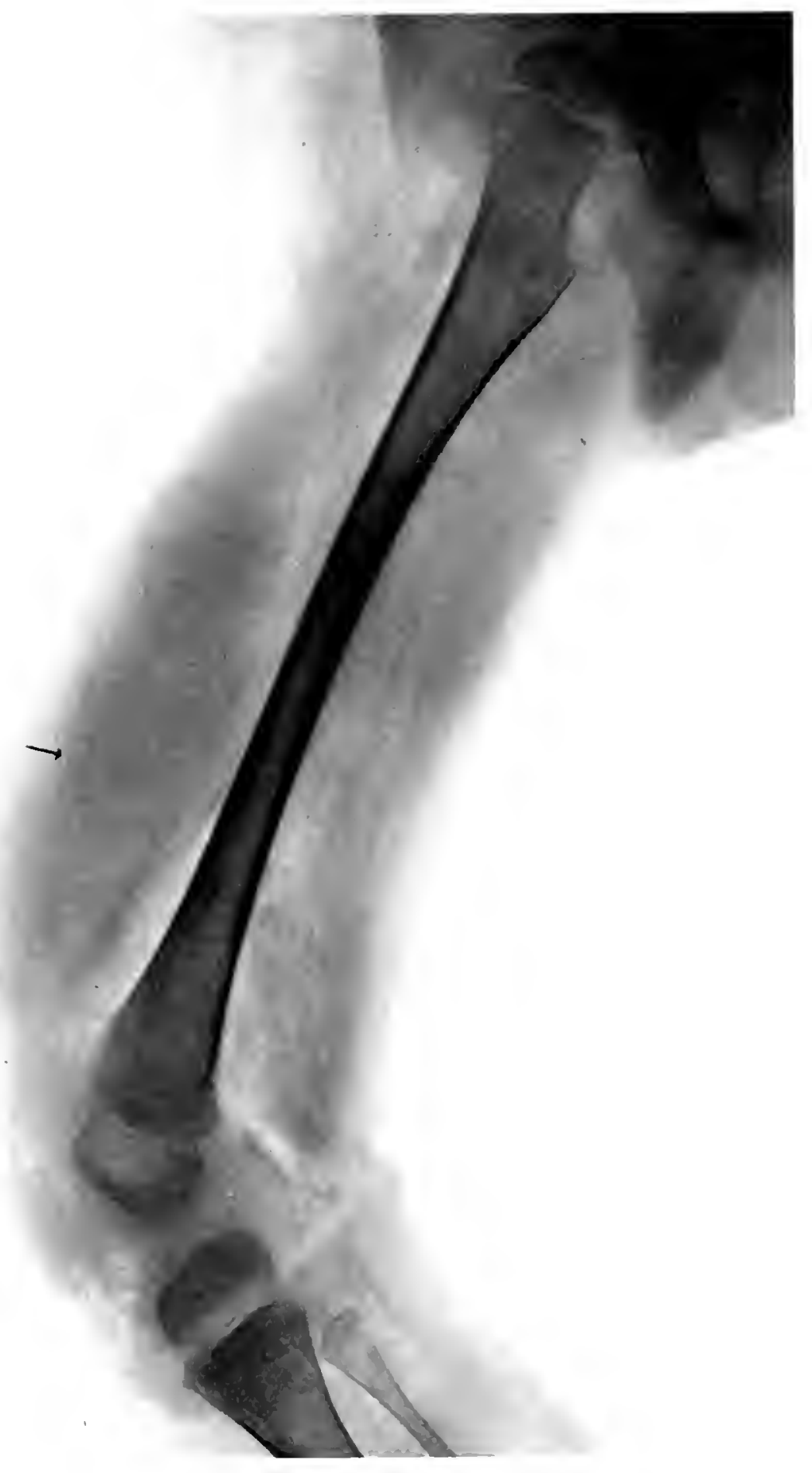


PLATE: 2.99.

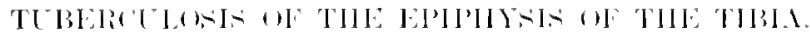

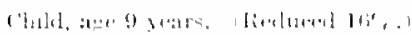

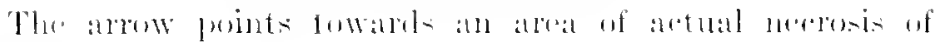
the butso. 
PI, ITH: 2.59

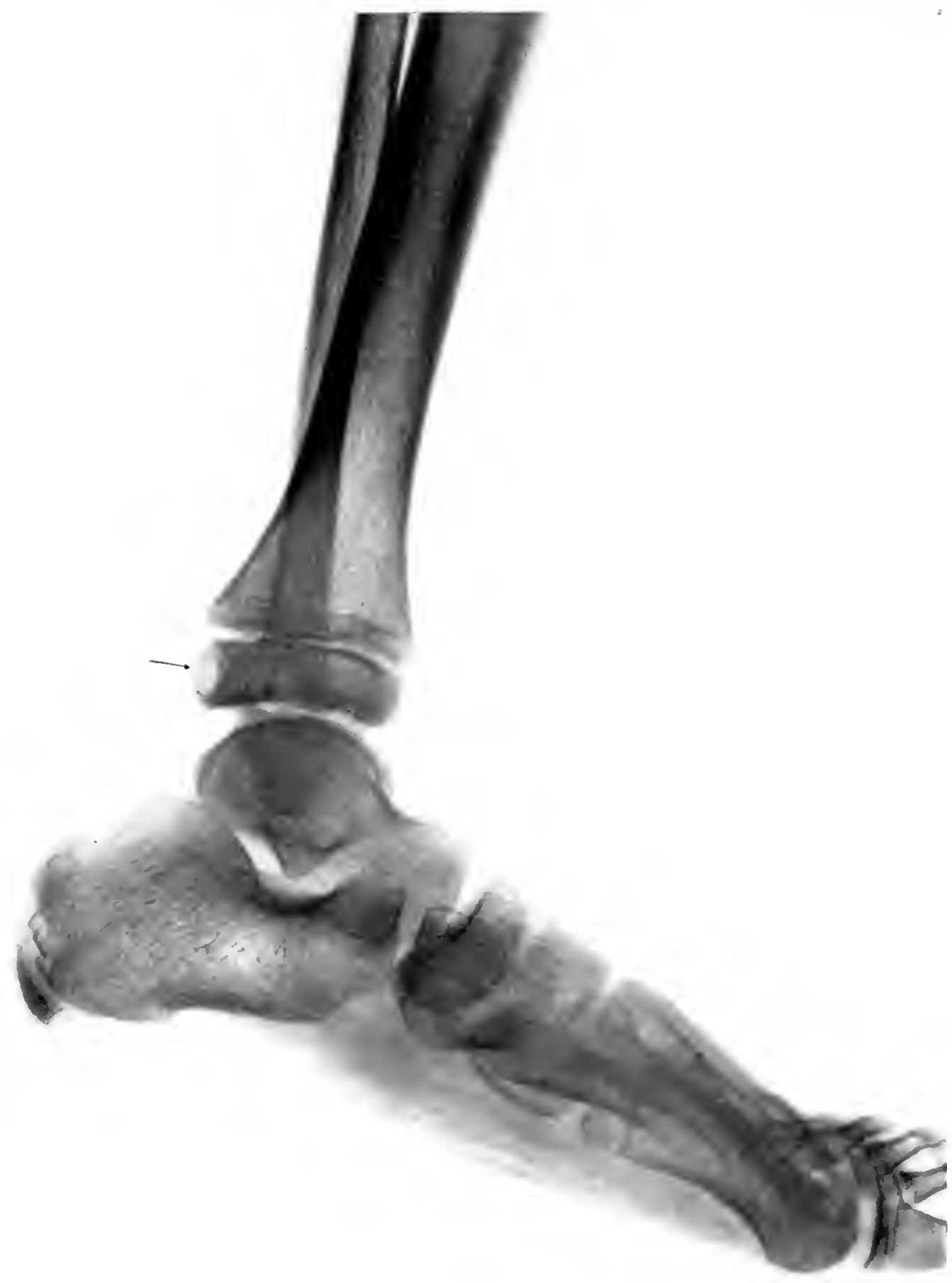


PI.ITE: 2(io.

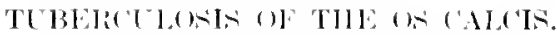

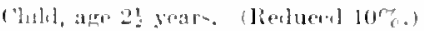

The bower arrow printe to a markerl nereresis of the bone of the burly of the as atcis.

The upper arrow points to a thickening of the tissur. 
PIAte 260

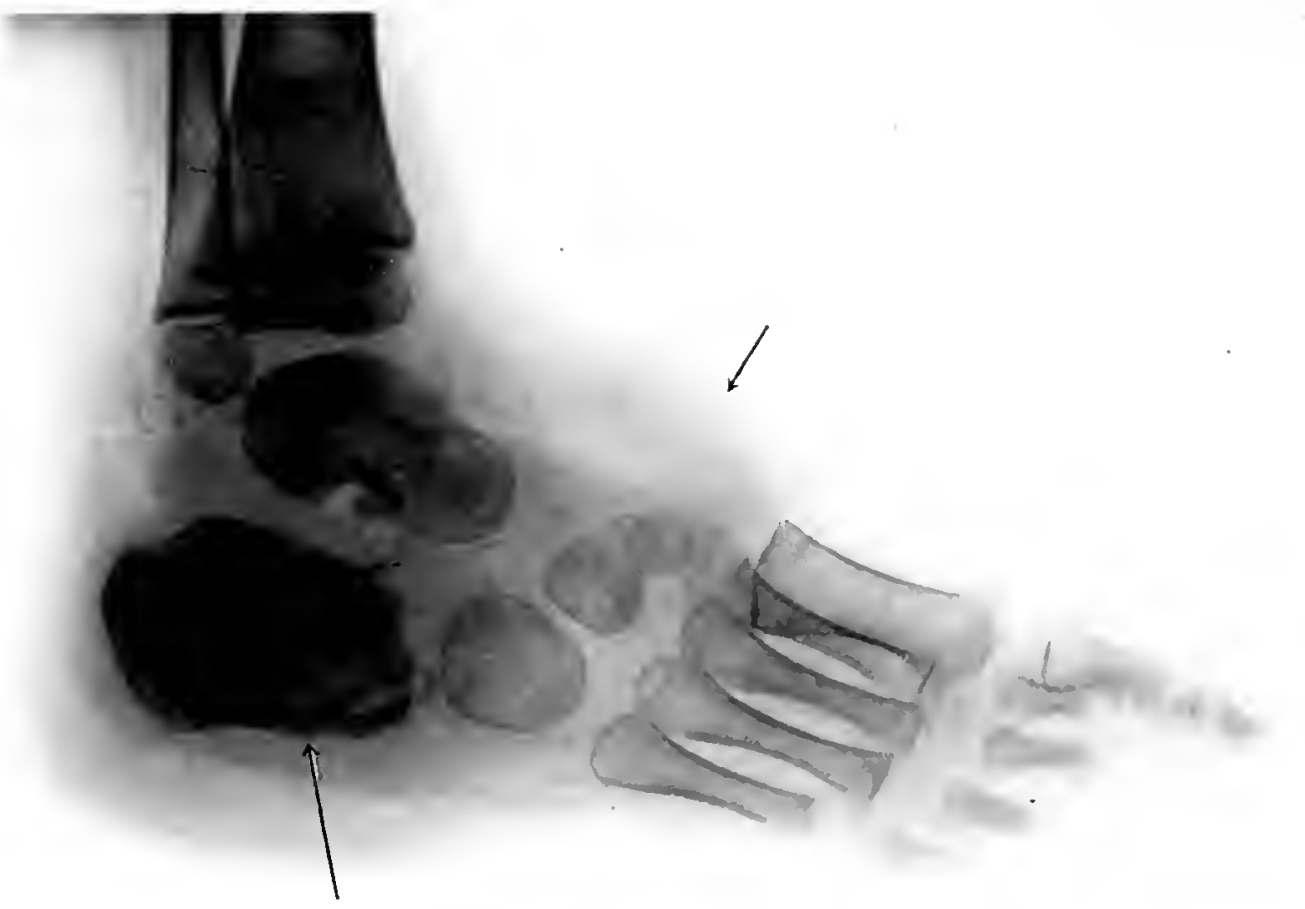


1'1.17\%:2061.

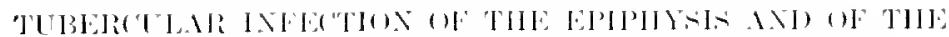

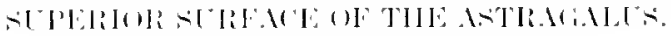

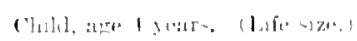

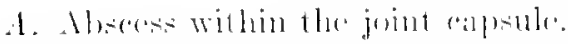

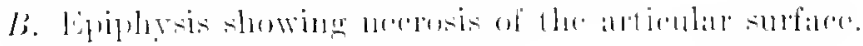

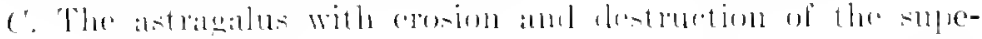
rior stafila.

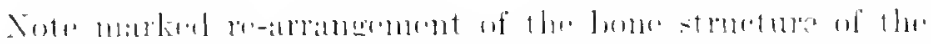

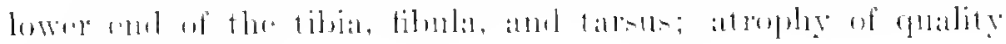
as well al - ol $-i z c$. 
PIATE Q

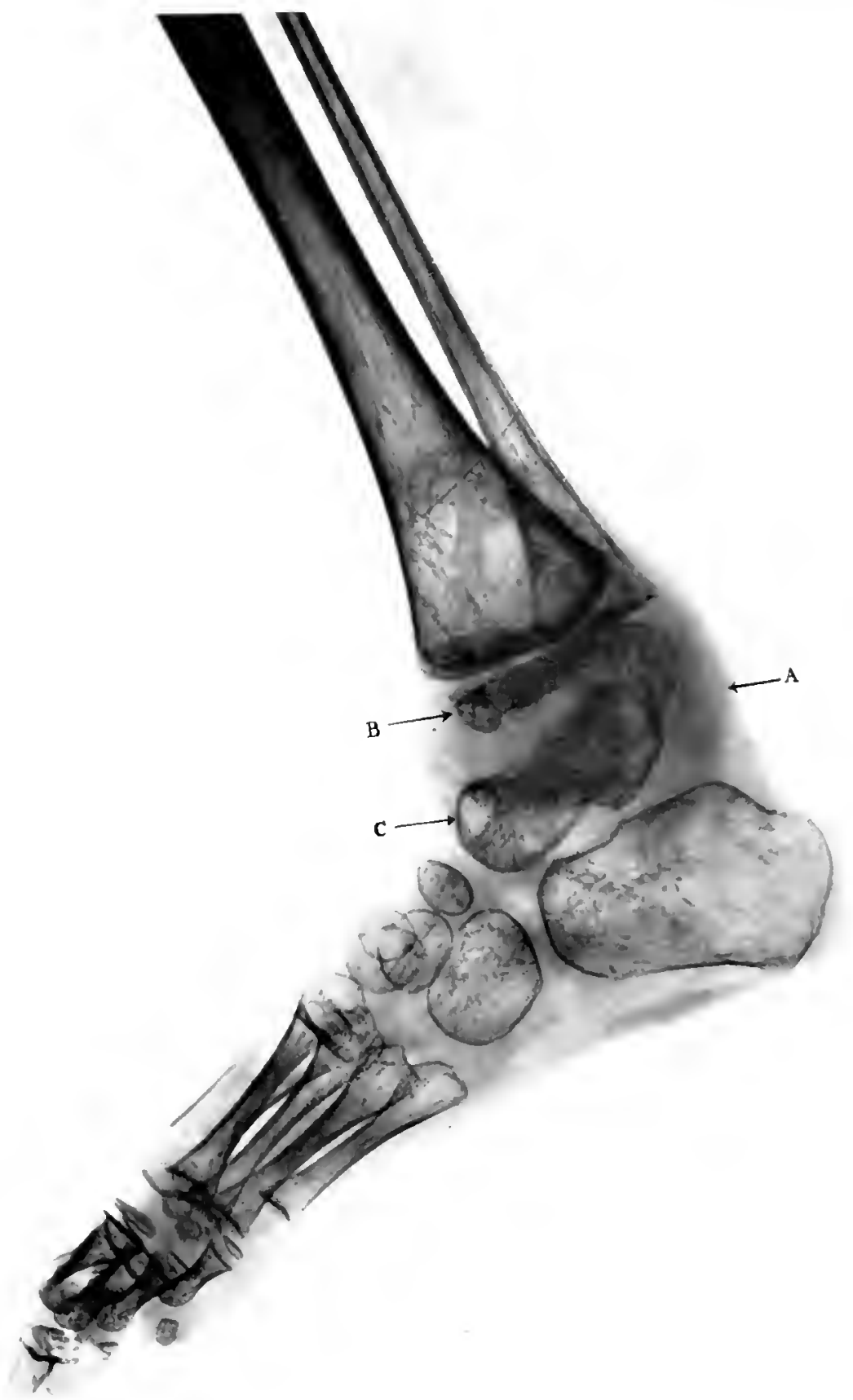




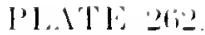

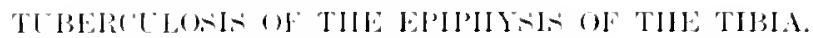

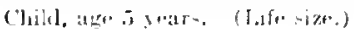

1. Merestis.

li. Lirosion al tha epiphy-io.

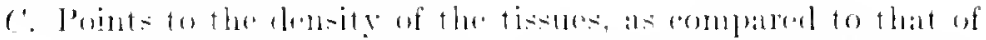

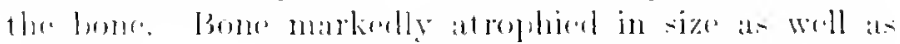
in quality.

1). (1): calleis. 


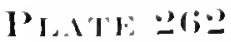

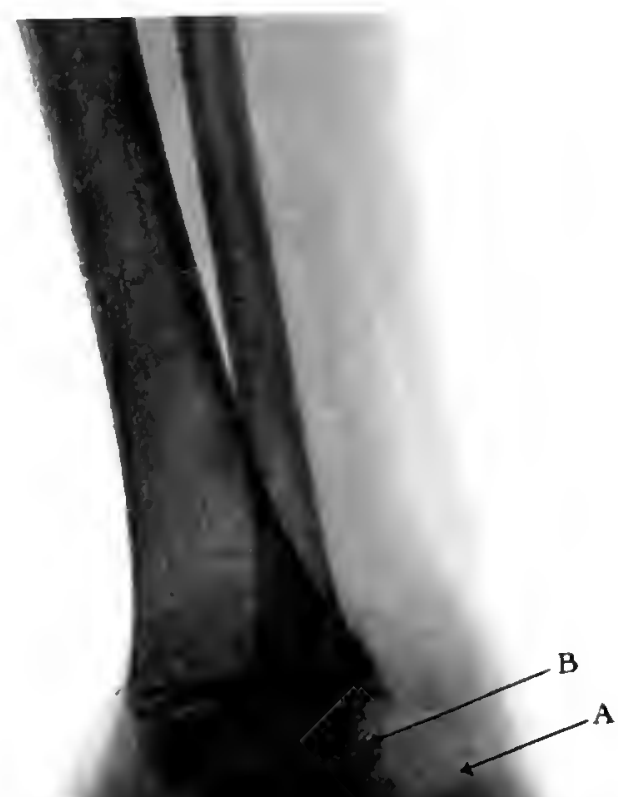

$\uparrow_{C}$

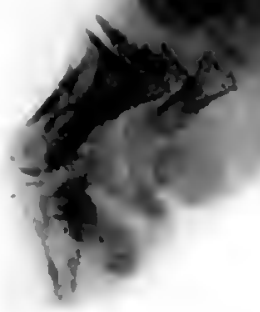


PI.ITE: 263.

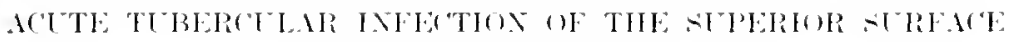
WF THF US COLOT:

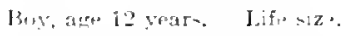

The arrow buints to the area of infortion. 


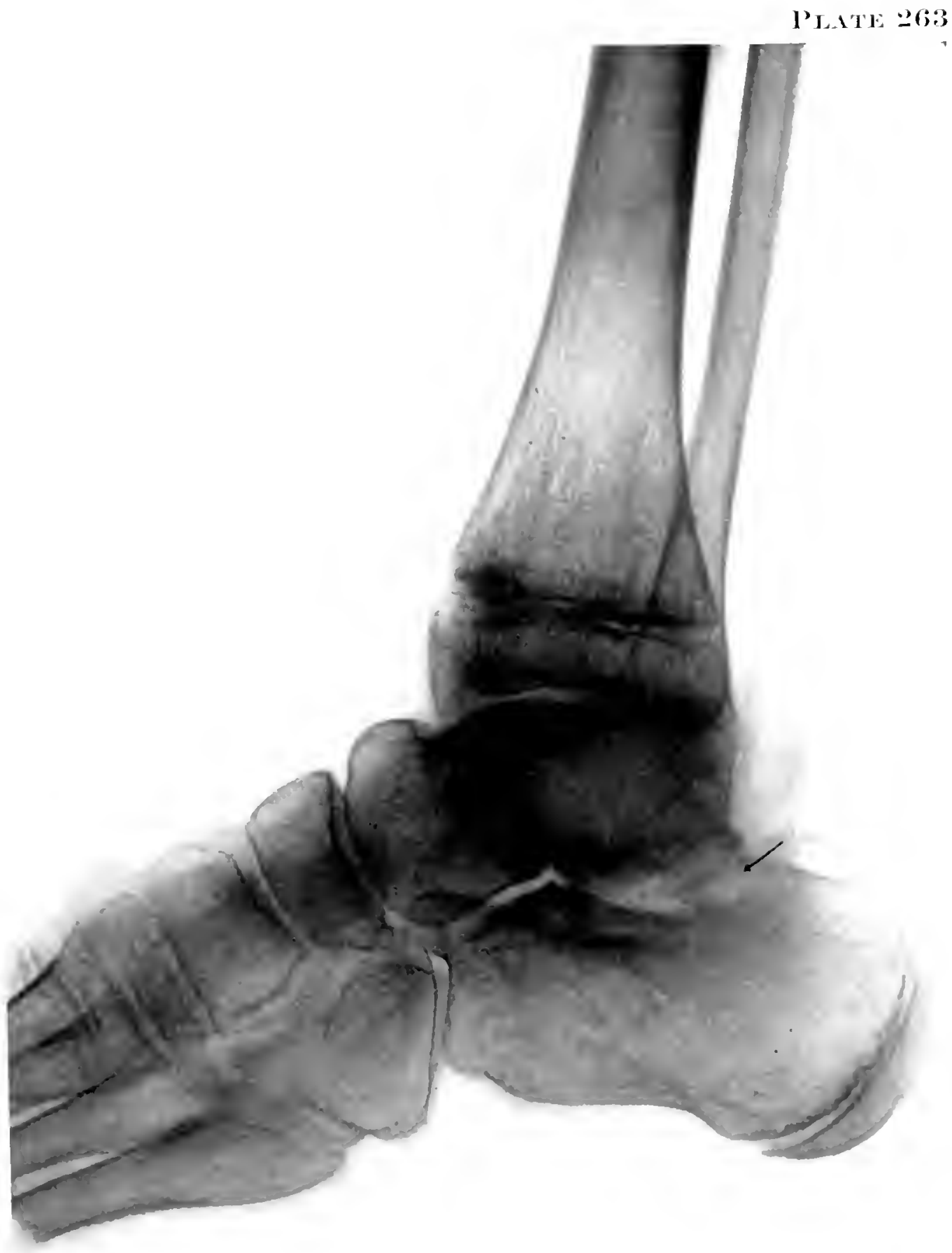


I'I.ATL:2lit.

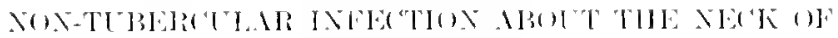
THL Fitho

Ihilit, ang is searn.

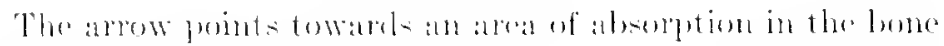

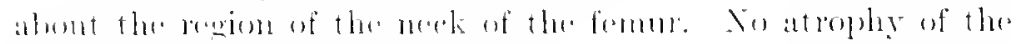
shaft or uf the apiphysis is mored. 
l'J.1T1:

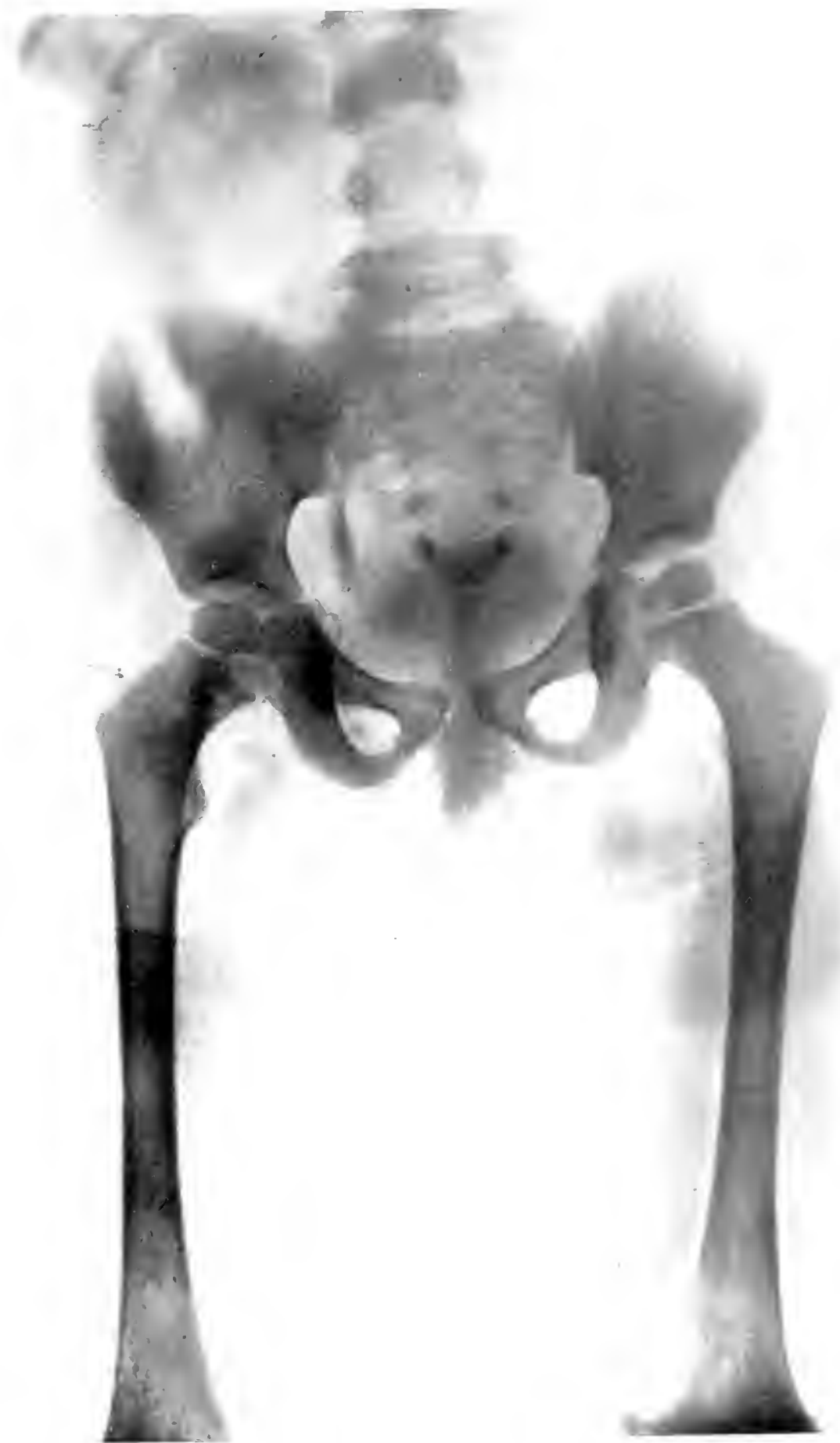





\section{INDEX}

Abdomen, 141, 142

Abscess of fibula, 162

of humerus, 152

mediastinal, 134

of os calcis, 214

of spine, 128

of thigh, 214

of tibia, 162

Abt, 62

Acetabulum, necrosis, 212

tubercular, 212

tuberculous, 212

Achondroplasia, S1

Acromion process, epiphysis of, 179

Adolescence, rhachitis of, 112

Anatomy, living normal, illustrative use of, 49

Aneurism, 131, 140

Ankle, chronic atrophic, 197

epiphysis of, 179

rheumatic fever, 186

tuberculosis of, 215

Ankylosis of knce, 165

Anomalies of the extremities, 76,77

of the head, 71

of the pelvis, 80

of the ribs, 75

of the spinc, 71

intra-abdominal, 76

intra-thoracic, 76

Arm, atrophy of, 154

china doll's, 136

chronic atrophic, 197

normal child 10 years, 45

Arthritis, infectious, $1 \mathrm{~S} 1$

atrophic variety, 167

villous, 167

Ascites, 142

Astragalns, devclopment of, 31

exostoses of, 156

fracture of, 162

tuberculosis of, 215
Atelectasis of lung, 133

Athleties, 64

Atrophy of arm, 154

fron disuse, 210

of elbow, 155

of hand, 154

infantile, 99

of quality in tuberculosis, 208

of shoulder, 154

of size in tubcrculosis, 208

of wrist, 155

Bismuth stomach, 141

Bone, constituents of, 24

description of, 24

developinent of, 26

examination, 13

structure of, 14

Boston Medical and Surgical Journal, obstetrical paralysis, 91

Boy, 9 years, normal hands of, 45

Bronchi, 131

description of, 133

Bronchial nodes, 131, 132

description of, 132

tuberculosis of, 132

Bronchoscope, 147

Brown, Percy, 131, 141

Buck and Bryant, osteomalacia, 97

Roentgen pictures of osteomalacia, $9 \mathrm{~S}$

Bullard, obstetrical paralysis, 91

Callus of feet, 156

Cardiohepatic angle, deseription of, 23 significance of, 2:2

Carpal bones, tubercular, 211

Carpus, development of, $2 \mathrm{~s}$

Child, five years, normal foot of, 4.4

labor, 64

laws, 65

State lan's, 66-68 
Child, six years, knce of, 45

normal thorax of, 45

slioulder of, 45

skeleton of 44

ten years, normal arm of, 45

normal hand of, 45

pelvis of, 45

thorax of, 45

upper legs of, 45

three years, knees of, $\mathbf{4 4}$

lower limbs of, 44

Chondrodystrophia, description of the Roentgen pictures, $\mathrm{S6}$

examination by Roentgen method, 86

fotalis, 81

cases of, 83

types, $S 2$

hyperplastica, $\$ 2$

hypoplastica, 82

malacica, $\$ 2$

résumé of, 85

Clavicle, epiphysis of, 179

Club-hand, 78

Coracoid process, epiphysis of, 179

Cortex, examination, 14

Crampton, correlation of weight, height, and strength with scholarship, 52

cruption of teeth in 1000 elementary scliool-boys, 53

growth of pubic hair, 51

height, weight, appearance of teeth, and strength, 51

neenstrual function, 51

physiologic development correlates closely

with anatomie normal development, 55

Cretinism, 80

recognition of, 83

Cuboid, development of, 31

Cuneiform, development of, 29

external, development of, 31

internal, development of, 31

middle, development of, 31

(yst, tubercular, 209

Dactylitis, 199

syphilitic, 199, 201

tubercular, 199, 209

Deformities of jaws, 36

of lower extremities, is
Deformities of upper extremities, 77

Dentition, first, temporary teeth, 35 second, permanent tecth, 35

Development, backward mental, 80

Diaphyses, description of, 25

Digit, separate, 77

Digits, webbed, 77

Diserepancies of development in anatomies, 59

Diseases of the head, 115 of the spine, 123 of nutrition, 95

Durante, 84

Early years, mental care of, 61 physical care of, 61

Educators, 62

Effusion of knee, 185 pericardial, 139, 140

Elbow, at rophy of, 155 epiphysis of, 176 infant 2-3 months, 41 normal ehild 12 years, 46 osteomyelitis of, 192 poliomyelitis of, 155 syphilis of, 200

Emphysema, 137 compensatory, $13 \mathrm{~s}$

Empyema, encapsulated, 138

Enamel, formation of, 33

Epiphyscal cartilage, description of, 27 line or zone of proliferation, examination, 15

Epiphysis or epiphyses:-

of acromion process, 179

of ankle, 179

of cluvicle, 179

of coracoid process, 179

description of, 25

dislocation of, 159

of elbow, 176

examination, 15

of hip, 17s

of knee, $17 \mathrm{~S}$

of os calcis, 179

of pelvic bones, 179

of ribs, 179

of scapula, 179

of shoulder, 176

time of appearance, 26

of vertebræ, 179 
Epiphysis of wrist, 177

Epiphysitis, 175

Ethmoiditis of skull, 116

Exostoses, 155

of astragalus, 156

of femur, 156

of fibula, 156

of knee, 156

of tibia, 156

Exposure, great advantage of short over long, 131,141

Extremities, the, 119 anomalies of, 76,77

Factories, 65

Femur, congenital dislocation of, 80

development of, 29

dislocation of, 80

early ossification of, 152

exost oses of, 156

intercapsular fracture, 161

necrosis, 212

non-tubercular infection, 186

osteomyelitis of, 190, 192

tubercular, 212

Fetal rhachitis, 90

intra-uterine, 113

recognition of, 83

Fibula, abscess of, 162

development of, 30

exostoses of, 156

fracture of, 162

asteomyelitis of, 196

First permanent nolar, calcification of, 37

Flat foot, $15 \%$

Foot, backward development of, 151

normal child 5 years, 44

one toe, 79

valqus of, 89

Foreign bodies, 143

in intestine, 143-147

in larynx, 144

in lung, 144,147

in asophagus, 143

Fragilitas ossium, 87

\section{Gangrene, 137}

George, Dr., study of 1000 cases of healthy children, 55
Girl, six months, skeleton of, 41

twelve months, trunk of, 42

about twenty-four months, skeleton of, 42

Gymnastics, 64

IIancl or hands:-

atroplay of, 154

chronic atrophic, 197

infant three months old, 41

infectious arthritis of, 185

irregular development of, 149

normal, child ten years, 45

normal, boy nine years, 45

poliomyelitis of, 154

Hearl, anomalies of, 71

cliseases of, 115

infant ten weeks old, 41

of normal infant, 40

Healthy children, study of, 55

Ileart, 131

enlarged, 139

enlargement of, 139

transposition of, 132

Hemorrhage, subperiosteal, 155

IIip, epiphysis of, $17 \mathrm{~s}$

mixed infection, 212

osteomyelitis of, 191, 194

tubercular, 212, 213

Ifip-joint, infection of, 181

mixed infection, 211

Hour-glass contraction of stomach, 141

Howship, 97

IIumerus, abscess of, 152

development of, 27

displacement of, 1.59

fracture of, 159, 160, 161 of surgical neck of, 160

infant two to three months, 41

infectious arthritis of, 185

osteomyelitis of, 193

1 y ydropneumothorax, 137

Idiot, Mongolian, 135

lium, development of, 32

Incisors, central, calcification of, 37

lateral, calcification of, 37

permanent, calcification of, 37

Index development a key to, 57

Intant, head of normal, 40 


\section{INDEX.}

Infant, head of ten weeks old, 41 nremature, deseription of, 39 ten days, upper legs of, 40 ten weeks olul, head of, 41 lower extremity of, 41 three and a half years, skeleton of, 43 three months old, hand of, 41 two to three months, elbows of, 41 humeri of, 41 thorax of, 41

Infantile at rophy, 95, 99 differential hagnosis [rom general tubereulosis, 100

Infection of hip-joint, $\mathbf{1 S 1}$

non-tubercular, of marrow, 217 of periosteum, 217

Infectious arthritis, 181 atrophie variety, 167 of hand, 185 of humerus, 185 periostitis, 186

Intestine, enngenital malformations of, 141 fibrous bands of, 141 nail in, 143 penny in, 147

Intra-abdominal anomalies, 76

Intra-thoracie anomalies, 76

Intra-uterine rhachitis, 113

Ischium, development of, 32

Jaws, deformities of, 36 osteomyelitis of, 116

Joints, 163

atrophie, 196

classification, 165

lypertrophie, 196

original focus, 170

Kassowitz, rhachitis, 106

Kaufm:an, 81

Kerley, 62

Knee, ankylosis of, 168 ehild! six years, 45

three years, 44

chronic atrophie, 197

at rophy of, 197

congestion of, 166

effusion of, 185

epiphyseal line, 166
Knee, epiphysis of, $17 \mathrm{~S}$ suppuration of, 167

epiphysitis, 166

exostoses of, 156

injury to, 159

osteochondritis, 166

rheumatic fever, 186

villous arthritis, 167

lince-joint, dislocation of epiphysis and condyle, 161

disloeation and fraeture of, 160

early tubereulosis, 213

tuberculous, 214

liönig's collection of infeetions of the hipjoint, 181

Kyphosis, 126

La Fetra, plate presented by, plate 240

Larynx, hook in, 144

Legg, Dr. A. T., at rophy from disuse, 207

Legs, congenital paralysis of, 80

chronic at rophic, 197

upper, of infant ten days, 40

of a child ten years old, 45

Liver, transposition of, 132

Lordosis, 126

Lovett, Dr. R. W., \$S

Lower extremities, deformities of, 78

extremity, delayed development of, Morse's case, 79

inlant ten weeks old, 41

limbs, child three years, 44

Lungs, 131

atelectasis of, 133

collapse of, 134

compression of, 139

doll's china arm in, 145

general description, 133

nail in, 144

Malformations of the feet, 77

of the hands, 77

of the cesophagus, 141

of the seapula, 75

of the stomaeh, 141

congenital, of the intestines, 141

Marrow, non-tubercular infeetions, 217

Mediastinal abscess, 134

Medullary canal, examination, 14 
Mental care of early years, 61

devclopment, backward, 80

Metacarpal bones, development of, 29

Iletatarsal boncs, absent, 79

tuberculosis in, 209

Metatarsus, development of, 31

Molar, first permanent, development of, $3 \pi$ calcification of, 37

Wongolian idiot, 135

Morse, patient of, plate 57

MIullen's case of osteogenesis imperfecta, 85

Muller, rhachitis, 106

Muscle, examination, 13

Myxœdema, so

New-born, diseases of, 69

Nichols, ss

Nodes, bronchial, 132

mesenteric, 142

Non-tubercular infections, 215

of femur, 186

of marrow, 217

of spine, 126

location of, 216

Roentgen ray in, 217

Normal living anatomy, chronologic examples of, 38

Nutrition, diseases of, 95

Obstet rical paraly'sis, 90

Esophagus, malformations of, 141 penny in, 143

Os calcis, abscess of, 214

developinent of, 31

cpiphysis of, 179

fracture of epiphysis, 163

tubercular, 214

innominatum, development of, 32

magnum, development of, 28

pubis, development of, 32

Osgood, $10 \mathrm{~S}$

Osteitis of spine, 126

tubercular, 127

Osteochondritis, syphilis in, 201

Osteogenesis imperfecta, British Medical Journal, $\$ 9$

comparison with osteomalacia, 98

Lovett's case of, 58

Mlullen's case of, 85
Osteogenesis imperfecta, Nichols' case of, 88 recognition of, 83 synonyms of, $8 T$

Osteomalacia, 95,96 comparison with osteogenesis imperfecta, 98

Ostcomyelitis, 187

of elbow, 192

of femur, 190, 191, 192

of fibula, 196

of hip, 191

of humerus, 193

of jaw, 116

of radius, 193

of spine, 126,127

of tibia, 159-191

Osteoperiostitis, syphilis in, 201

Osteoporosis, \$2

Ostcopsathyrosis, idiopathic, 87

Osteosclerosis, $\$ 2$

Paralysis, obstetrical, 90

Parrot, s1

Patella, development of, 30

Pelvic bones, epiphyses of, 179

Pelvis, anomalies of, 80 normal child ten years, 45

Pericardium, 131, 139

Periosteum, acute infections by pyogenic organisms, 217

description of, 24

examination, 15

Periostitis of spine, 126 syphilitic, 201

Pcrmanent incisors, calcification of, 37 teeth, 35

Phalanges, development of, 29, 31

Physical care of early years, 61

Pirnmes, rhachitis, 106

Pisiform, development of, 29

Pleura, 131, 137

Pneumonia, broncho-, 136

lobar, $13 j$

unresolved, 135

Pneumothorax, 137

Polionyelitis, 154

of elbow, 155

of hancl, 154

of shoullers, 154

of wrist, 155 
Porier, 60

Postpubeseents, height of, 52 seholarship of, 52 strength of, 52 weight of, 52

Pott's disease, 125

Preeocious and bright ehildren, 63

Premature infant, deseription of, 39

Prepubescents, seholarship of, 52

Primary areolic, 27

Pryor, 5s

Radius, deformity of, is development of, 28

fracture of, 162 green-stick fraeture, 161

impaeted fraeture, 161 osteonyelitis of, 193

Rhachitis, 95

of adoleseence, 112

eompared with chondrodystrophia foctalis, 107

deseription of, 104

epiphysis and zone of proliferation, $10 \mathrm{~s}$

fetal, 90

fraeture, 109

general structure of the bone, $10 \mathrm{~s}$

Kascowitz, 100

marked deformities of, 109

medullary eanal, $10 \mathrm{~S}$

Muller, 106

osseous system, 108

with osteomalacia, 107

out line of the cortex and periosteum, 108

Pimmes, 106

types of, 110

Vierordt, 106

Virchow, 106

Zicgler, 106

zone of proliferation, 107

Rheumatie fever of ankle, 186 of knee, 186

Rilss, anomalies of, 75

eollapsed, 135

epiphyses of, 179

Roentgen method, determination of teeth by: 35

Roteh method of study of development by, 56
Roentgen plate, $s$

ray in dentistry, 33

Sacro-iliae joint, tubereular proeess of, 125

Sareoma, medullary, 153

myelogenous, 153

periosteal, 154

Seaphoid, delayed development, 151 development of, 29, 31

Seapula, elevation of, 76

epiphysis of, 179

malformations of, 75

Seoliosis, 126

Seorbutus, 95

hemorrhage in, 100

Roentgenograph compared with sareoma, 103

Semilunar, development of, 29

Shoulder, atrophy of, $15 \mathrm{k}$

elilk six years, 45

epiphyses of, 176

poliomyelitis of, 15-I

Skeleton, child about six years, 44

girl six months, 41

about $t$ wenty-four months, 42

infant about three and a hall years, 43

normal ehild twelve years, 40

Skull, ethmoiditis of, 116

fracture of, 115

Spina bifida, 73 oceulta, 74

Spine, abseess of, 128

anomalies of, 71

diseases of, 123

non-tubereular infections of, 126

normal ehild ten years, 46

osteitis of, 126

osteomyelitis of, 126, 127

periostitis of, 126

tuberculosis of, 127

Stomach, bismuth, 141

hour-glass eontraetion, $1+1$

malformations of, 141

Stone, 157

Stransky, obstetrieal paralysis, 91

Strueture of the bone, examination, 1 .

Stupid children, 64

Subcutaneous tissue, examination, I3

Subperiosteal hemorrhage, 155 
Supernumerary teeth, 119

Syphilis, 198

of elbow, 200

retarded, 202

of tibia, 201

of ulna, 200

Syphilitic osteochondritis, 201

ostcoperiostitis, 201

periostitis, 201

Tarsus, development of, 31

Teeth, 32

normal permanent, 47

permanent, 35

normal development of, 38

supernumerary, 119

temporary, 35

separation of, 36

Thigh, abscess of, 214 subperiosteal hemorrhage of, 155

Thomas, 91

Thorax, infant, ten days, 40

two to three months, 41

normal boy twelve years, 46

child, six years, 45

ten years, 45

Tibia, abscess of, 162

development of, 30

early ossification of, 152

epiphysis, 214

suppuration of, 167

exostoses of, 156

fracture of, 162, 163

green-stick fracture, 160

osteomyelitis of, 1S9, 191

early stages of infection, 196

syphilis of, 201

tubercular, 214

Tissues, at omic weight of, 8

elements of, $\mathrm{S}$

radiability of, $\mathrm{S}$

subcutaneous, examination of, 13

Torticollis, 75

Trapezium, development of, 29

Trapezoid, development of, 29

Traumatism, 157

technic of, 158, 159
Trunk, girl $t$ welve months, 42

Tubercular dactylitis, $199,209,210$

Tuberculosis, 137, 202

of acetabulum, 212

acute miliary, 136, 137

of ankle, 215

of astragalus, 215

atrophy in, 204, 20.5, 206, 207

of quality in, $20 \mathrm{~S}$

of bronchial nodes, 132

of carpus, 211

cyst, 209

of elbow, 211

of hip-joint, 211, 212

of knee, 213

of metatarsus, 209

of os calcis, 214,215

of spine, 127

Ulna, deformity of, 78

development of, 28

fracture of, 162

impacted fracture of, 161

necrosis of, 211

syphilis of, 200

Unciform, development of, $2 s$

Upper extremities, deformities of, 77

legs, infant ten days, 40 normal child ten years, 45

Urethra, stone in, 143

Valgus of foot, 89

Vertebra, epiphyses of, $\mathbf{1 7 9}$

Vierordt, rhachitis, 106

Villous arthritis, 167

Virchow, rhachitis, 106

Wolff's law, 110, 111

Wrist, anomaly of, 150

atrophy of, 155

chronic atrophic, 197

epiphysis of, 177

poliomyelitis of, 155

Ziegler, osteomalacia, 96

rhachitis, 106

Zonc of proliferation, description of, 27 


LVIVERSITI OF C.ILIFORNIA LIBRARY

I, os Angeles

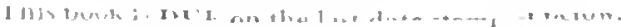

\section{BIOMED JUH 06 '87}


